\title{
CENTER FOR DRUG EVALUATION AND RESEARCH
}

\author{
APPLICATION NUMBER: \\ 2022930rig1s000
}

STATISTICAL REVIEW(S) 
U.S. Department of Health and Human Services

Food and Drug Administration

Center for Drug Evaluation and Research

Office of Translational Sciences

Office of Biostatistics

\section{STATISTICAL REVIEW AND EVALUATION \\ Clinical STUdies}

NDA:

Drug Name:

Indication(s):

Applicant:

Date(s):

Review Priority:

Biometrics Division:

Statistical Reviewer:

Concurring Reviewers:

Medical Division:

Clinical Team:

Project Manager:
202293

Dapagliflozin

Treatment of type 2 diabetes mellitus

Bristol-Myers Squibb and Astra Zeneca

Receipt date: July 11, 2013

Goal Date: January 11, 2014

Priority

Division of Biometrics VII

Eugenio Andraca-Carrera, Ph.D.

Mat Soukup, Ph.D.

Aloka Chakravarty, Ph.D.

Division of Metabolism and Endocrinology Products (DMEP)

Medical Officer: Frank Pucino, MD

Medical Team Leader: Karen Mahoney, MD

Bola Adeolu (DMEP)

Keywords: type 2 diabetes mellitus, cardiovascular safety, meta-analysis 


\section{Executive Summary}

The proposed indication of dapagliflozin is as an adjunct to diet and exercise to improve glycemic control in adult patients with type 2 diabetes mellitus (T2DM). The proposed therapeutic dosage is $10 \mathrm{mg}$ for most patients and a $5 \mathrm{mg}$ starting dose for patients at risk for volume depletion due to coexisting conditions or concomitant medications such as loop diuretics. Per the request of the Division of Metabolism and Endocrinology Products, this statistical review evaluates the cardiovascular (CV) safety of dapagliflozin in 21 Phase 2 and Phase 3 randomized clinical trials. The cardiovascular safety of this product has been previously discussed at an Advisory Committee Meeting held on July 19, 2011, and was re-evaluated with the additional data submitted as part of a Major Amendment in October 2011. This review focuses on the pre-marketing evaluation of cardiovascular safety of dapagliflozin based on an updated submission dated July 11 , 2013. A separate statistical review addressing the efficacy and glycemic control of dapagliflozin is being conducted by Dr. Wei Liu.

\subsection{Conclusions and Recommendations}

Bristol-Myers Squibb and Astra Zeneca evaluated the cardiovascular safety of dapagliflozin through a meta-analysis of 21 randomized clinical trials. These trials had different inclusion and exclusion criteria as described in Section 3.1 of this review. The Agency considered two trials to be of special interest for the evaluation of cardiovascular safety: trials 18 and 19. These two trials enrolled subjects with prior cardiovascular disease and were analyzed separately upon request from the Agency.

The agreed upon primary meta-analysis population consisted of the randomized, controlled, short term plus long term periods of the 21 trials. The analyses included data following initiation of any rescue therapy. The dapagliflozin treatment group $(n=5936)$ was comprised of subjects randomized to any dose of dapagliflozin, excluding dapagliflozin $1 \mathrm{mg}$ daily. The comparator group $(n=3403)$ in the meta-analysis was comprised of all non-dapagliflozin randomized groups and included subjects randomized to placebo, metformin, glipizide and HCTZ. The primary agreed upon cardiovascular endpoint of interest was the composite of the following 4 adjudicated outcomes: cardiovascular death, myocardial infarction, stroke, and hospitalization for unstable angina. All potential events were adjudicated by the Clinical Event Committee

There were 97 primary events observed among 5936 subjects randomized to dapagliflozin and 81 primary events observed among 3403 subjects randomized to comparators in the 21 trials used in the meta-analysis. The pre-specified Cox proportional hazards model stratified by each of the 21 trials yielded an estimated hazard ratio of dapagliflozin vs. all comparators of $\mathbf{0 . 8 1}$ with a $95 \%$ confidence interval of $(\mathbf{0 . 5 9}, \mathbf{1 . 0 9})$. The upper bound of this $95 \%$ confidence interval meets the risk margin of 1.8 necessary to show adequate pre-marketing cardiovascular safety of new antidiabetic products in accordance to the FDA Diabetes Guidance of 2008. 
The Agency requested that secondary analyses of MACE be conducted on all analyses populations. MACE is a composite of CV Death, myocardial infarction and stroke. There were 73 MACE observed 5936 among subjects randomized to dapagliflozin and 62 MACE among 3403 subjects randomized to comparators in the primary analysis population of all 21 trials. The corresponding estimated hazard ratio of MACE associated with dapagliflozin relative to all comparators in these trials was $\mathbf{0 . 7 8}$ with a 95\% confidence interval of $(\mathbf{0 . 5 5}, \mathbf{1 . 1 1})$. The results of analyses for the primary endpoint and MACE are summarized in Table 1.

Table 1. Primary Composite Endpoint and MACE in the Primary Meta-Analysis Population

\begin{tabular}{|c|c|c|c|}
\hline & $\begin{array}{c}\text { Comparators } \\
\mathrm{N}=3403 \\
\mathrm{PY}=3831\end{array}$ & $\begin{array}{c}\text { Dapagliflozin } \\
\mathrm{N}=5936 \\
\mathrm{PY}=6594\end{array}$ & $\begin{array}{c}\text { Hazard Ratio } \\
(95 \% \mathrm{Cl})\end{array}$ \\
\hline Primary events $^{1}{\text { (rate per } 1000 \mathrm{PY})^{2}}^{2}$ & $81(21.1)$ & $97(14.7)$ & $0.81(0.59,1.09)$ \\
\hline MACE $^{3}$ & $62(16.2)$ & $73(11.1)$ & $0.78(0.55,1.11)$ \\
\hline
\end{tabular}

${ }^{1} \mathrm{CV}$ death, MI, stroke, hospitalization for unstable angina

${ }^{2}$ Unadjusted rate of events pooled across all trials

${ }^{3} \mathrm{CV}$ death, MI, stroke

Source: Created by reviewer. Dataset: adcv5.xpt

Subgroup analyses were consistent with the results shown in Table 1. There was no evidence of an interaction between the use of dapagliflozin and any of the following variables in terms of risk of the primary composite cardiovascular endpoint: gender, race, age, country of randomization, BMI, baseline eGFR or duration of diabetes at baseline.

In the two trials with high baseline cardiovascular risk, trials 18 and 19, there were 43 primary events observed among 942 subjects randomized to dapagliflozin and 44 primary events among 945 subjects randomized to placebo. The corresponding estimated hazard ratio of the primary endpoint associated with dapagliflozin relative to all comparators in trials 18 and 19 was 0.98 with a 95\% confidence interval of $(\mathbf{0 . 6 4}, \mathbf{1 . 4 9 )}$. Similarly there were 32 MACE observed among subjects randomized to dapagliflozin and 29 among subjects randomized to placebo. The estimated hazard ratio of MACE associated with dapagliflozin in these two trials was 1.11 with $95 \%$ confidence interval $(\mathbf{0 . 6 7}, \mathbf{1 . 8 3})$. These results are summarized in Table 2 .

Table 2. Primary Composite Endpoint and MACE in Trials 18 and 19

\begin{tabular}{|c|c|c|c|}
\hline & $\begin{array}{c}\text { Placebo } \\
\mathrm{N}=945 \\
\mathrm{PY}=1119\end{array}$ & $\begin{array}{c}\text { Dapagliflozin } \\
\mathrm{N}=942 \\
\mathrm{PY}=1118\end{array}$ & $\begin{array}{c}\text { Hazard Ratio } \\
(95 \% \mathrm{CI})\end{array}$ \\
\hline Primary events $^{1}$ (rate per 1000 PY) $^{2}$ & $44(39.3)$ & $43(38.5)$ & $0.98(0.64,1.49)$ \\
\hline MACE $^{3}$ & $29(25.9)$ & $32(28.6)$ & $1.11(0.67,1.83)$ \\
\hline
\end{tabular}

${ }^{1} \mathrm{CV}$ death, MI, stroke, hospitalization for unstable angina

'Unadjusted rate of events pooled across all trials

${ }^{3} \mathrm{CV}$ death, MI, stroke

Source: Created by reviewer. Dataset: adcv5.xpt 
A post-hoc analysis was conducted to evaluate the cardiovascular risk of dapagliflozin within the first 30 days after randomization in the 21 trials in the meta-analysis. This analysis was motivated by an imbalance of cardiovascular events observed within the first 30 days of randomization in CANVAS, a dedicated cardiovascular outcomes trial for canagliflozin. Canagliflozin is the only SGLT2 inhibitor currently approved in the US market. In the dapagliflozin program, a small imbalance of primary events (CV death, MI, stroke, and hospitalization for unstable angina) was observed within the first 30 days after randomization in the 21 trials: 8 primary events were observed among 5936 subjects randomized to dapagliflozin $(0.13 \%)$ compared to 2 primary events among 3403 subjects randomized to comparators $(0.06 \%)$. Due to the small number of events, it is not possible to determine whether this imbalance is attributable to dapagliflozin or whether it might have been caused by chance.

\subsection{Brief Overview of Clinical Studies}

The Sponsors submitted data from 7 Phase 2 trials (MB102008, MB102009, MB102035, $\mathrm{MB} 102045$, D1692C00005, MB102013 and MB102032) and 14 Phase 3 trials (MB102014, D1690C00012, D1690C00006, MB102030, D1690C00005, D1690C00010, D1690C00004, MB102034, MB102021, MB102029, D1690C00018, D1690C00019, D1692C00006 and MB102054). The trials differed in their primary objectives, design, choice of comparators, populations of interest and inclusion criteria. Of particular interest for the evaluation of cardiovascular safety; trials D1690C00018, D1690C00019 enrolled subjects with a history of cardiovascular disease. A detailed discussion of these trials is provided in Section 3.1.1.

\subsection{Statistical Issues and Findings}

The Statistical Analysis Plan (SAP) to evaluate the cardiovascular safety of dapagliflozin was dated March $3^{\text {rd }}, 2010$. In the agreed upon SAP, it was determined that the cardiovascular safety of dapagliflozin would be evaluated through a meta-analysis of Phase 2 and Phase 3 randomized clinical trials. A meta-analysis of cardiovascular safety was submitted in 2011 as part of the original NDA submission for this product. Based on this submission, the Agency denied the approval of dapagliflozin at that time. The regulatory history of this product is summarized in Section 2. The current submission, dated 11 July 2013, includes an updated meta-analysis of cardiovascular outcomes based on data available from 21 randomized clinical trials for dapagliflozin with a data cut-off date of 15 November 2012.

The meta-analysis was designed to demonstrate that the hazard ratio of the primary composite endpoint of CV death, MI, stroke, and hospitalization for unstable angina associated with dapagliflozin relative to all comparators is smaller than the risk margin of 1.8 set forth in the FDA Diabetes Guidance for assessing cardiovascular safety. The prespecified primary model was a Cox proportional hazards model stratified by trial. The 
estimated hazard ratio was $\mathbf{0 . 8 1}$ with $95 \%$ confidence interval $(\mathbf{0 . 6 8}, \mathbf{1 . 2 1})$ as discussed in Section 1.1.

The Sponsors propose to evaluate the cardiovascular safety of dapagliflozin after approval through a dedicated cardiovascular outcomes trial, "D1693C00001". This trial was started in April 2013 with the goal of showing that the upper bound of the 95\% confidence interval for the hazard ratio of MACE associated with dapagliflozin is smaller than 1.3 relative to placebo. This trial plans to randomize up to 17000 subjects to dapagliflozin $10 \mathrm{mg}$ or placebo and has a median expected follow-up time of 4.5 years. Data from Trial D1693C00001 is not available and as such is not part of the updated meta-analysis discussed in this document.

Secondary analyses assessed the hazard ratio of MACE and individual components of the primary endpoint associated with dapagliflozin in the primary population of all 21 trials in the meta-analysis and the secondary population of trials 18 and 19 alone. A summary of these findings is shown in Table 3 and Table 4. The estimated hazard ratio and 95\% confidence interval for MACE, cardiovascular death, myocardial infarction, stroke and hospitalized unstable angina show no statistical evidence of increased risk associated with dapagliflozin. A detailed discussion of these results is provided in Section 3.1.6.

Table 3. Analysis of MACE and the Individual Components of the Primary Composite Outcome in the Population of All 21 Trials

\begin{tabular}{|c|c|c|c|}
\hline \multirow[b]{2}{*}{ Endpoint } & \multicolumn{2}{|c|}{ Events } & \multirow[b]{2}{*}{$\begin{array}{c}\text { Estimated HR } \\
(95 \% \mathrm{Cl}) \\
\end{array}$} \\
\hline & $\begin{array}{c}\text { Comparators } \\
\mathrm{N}=3403\end{array}$ & $\begin{array}{c}\text { Dapagliflozin } \\
\mathrm{N}=5936\end{array}$ & \\
\hline MACE & 62 & 73 & $0.78(0.55,1.11)$ \\
\hline CV Death & 18 & 20 & $0.71(0.37,1.37)$ \\
\hline Ml & 33 & 31 & $0.59(0.35,0.97)$ \\
\hline Stroke & 18 & 25 & $1.00(0.54,1.86)$ \\
\hline Hospitalization for unstable angina & 20 & 27 & $0.91(0.50,1.66)$ \\
\hline
\end{tabular}

Source: Created by reviewer. Dataset: adcv5.xpt

Table 4. Analysis of MACE and the Individual Components of the Primary Composite Outcome in Trials 18 and 19

\begin{tabular}{|lccc|}
\hline & \multicolumn{3}{c|}{ Events } \\
Endpoint & $\begin{array}{c}\text { Comparators } \\
\mathbf{N}=\mathbf{9 4 5}\end{array}$ & $\begin{array}{c}\text { Dapagliflozin } \\
\mathbf{N}=\mathbf{9 4 2}\end{array}$ & $\begin{array}{c}\text { Estimated HR } \\
\mathbf{( 9 5 \%} \mathbf{~ C l )}\end{array}$ \\
\hline MACE & 29 & 32 & $1.11(0.67,1.83)$ \\
CV Death & 9 & 8 & $0.89(0.34,2.30)$ \\
Ml & 12 & 12 & $1.00(0.45,2.23)$ \\
Stroke & 10 & 12 & $1.21(0.52,2.80)$ \\
Hospitalization for unstable angina & 15 & 12 & $0.80(0.37,1.70)$ \\
\hline
\end{tabular}

Source: Created by reviewer. Dataset: adcv5.xpt 


\section{Introduction}

\subsection{Product Description and Regulatory Background}

Dapagliflozin is a subtype 2 sodium-glucose transport protein (SGLT2) inhibitor. The proposed indication of dapagliflozin is as an adjunct to diet and exercise to improve glycemic control in adult patients with type 2 diabetes mellitus (T2DM). The proposed therapeutic dosage is $10 \mathrm{mg}$ for most patients and a $5 \mathrm{mg}$ starting dose for patients at risk for volume depletion due to coexisting conditions or concomitant medications such as loop diuretics.

The Endocrinologic and Metabolic Drugs Advisory Committee met on July 19, 2011 to discuss the efficacy and safety of dapagliflozin. At the meeting, the cardiovascular safety of dapagliflozin was evaluated through a meta-analysis of 14 randomized clinical trials. The primary endpoint of this meta-analysis was a composite of CV death, myocardial infarction (MI), stroke and hospitalization for unstable angina. The meta-analysis included 78 subjects with an event (48 of 4287 randomized to dapagliflozin and 30 of 1941 randomized to comparators). The estimated hazard ratio and $98 \%$ confidence interval for this endpoint associated with dapagliflozin was $0.67(0.38,1.18)$. The 1.18 upper bound of this confidence interval was smaller than the margin of 1.8 set forth in the FDA Guidance for evaluating cardiovascular risk in new antidiabetic therapies to treat type 2 diabetes $(2008)^{1}$. This $98 \%$ confidence interval was pre-specified (instead of a $95 \%$ interval) as part of a two part sequential testing strategy. A statistical review of this meta-analysis was completed by Dr. Anita Abraham on 07 September, 2011.

In October 2011, Bristol-Myers Squibb and Astra Zeneca submitted an updated metaanalysis of 19 trials to support the cardiovascular safety of dapagliflozin. A statistical review of this meta-analysis was completed by Dr. Anita Abraham on November 22, 2011. The updated 2011 meta-analysis produced an estimated hazard ratio and $95 \%$ confidence interval of $0.82(0.59,1.38)$ associated with dapagliflozin relative to all comparators on the risk of the primary pre-specified composite endpoint of: CV death, MI, stroke and hospitalization for unstable angina. In her review, Dr. Abraham concluded that the upper bound of the $95 \%$ confidence interval for the risk ratio of CV events "meets the stated 1.8 non-inferiority margin" in accordance with the FDA Guidance for cardiovascular safety of products intended to treat type 2 diabetes.

The updated 2011 meta-analysis included data up to the first 52 weeks from the then ongoing trials D1690C00018 and D1690C00019. These two trials were conducted in subjects with a history of cardiovascular disease and hypertension. Per request from the FDA, a secondary analysis of cardiovascular safety was conducted in these two trials alone. The results of this analysis are summarized in Table 5. In this review, we will refer to trials D1690C00018 and D1690C00019 as trial '18' and '19' respectively. 
Table 5. Analysis of Primary Composite Endpoint and MACE in Studies D1690C00018 and D1690C00019 Based on Data Submitted for the 2011 Meta-Analysis

\begin{tabular}{|ccc|}
\hline Endpoint & Total Events & $\begin{array}{c}\text { Estimated HR } \\
\mathbf{( 9 5 \% ~ C l )}\end{array}$ \\
\hline Primary composite endpoint ${ }^{1}$ & 60 & $1.07(0.64,1.77)$ \\
MACE $^{2}$ & 43 & $1.27(0.69,2.31)$ \\
\hline${ }^{1}$ Composite of CV death, MI, stroke and hospitalization for unstable angina. \\
${ }^{2}$ Composite endpoint of CV death, Ml and stroke.
\end{tabular}

Based on the totality of the data submitted in support of the 2011 application, the FDA denied the approval of dapagliflozin in the United States at that time. In the complete response letter (CRL) dated 17 January 2011, the FDA expressed concern that:

"While we cannot conclude that dapagliflozin is associated with an excess $C V$ risk based on an analysis of only these two trials [Studies D1690C00018 and D1690C00019], the findings from these two large, adequate and well-designed trials in a relevant patient population cannot be ignored. More importantly, we cannot include any suggested $C V$ benefit observed in the original meta-analysis in a risk-benefit consideration in regard to cancer and liver safety signals."

In the same CRL, the FDA provided the following path forward for this application:

"To address the above deficiencies, you will need to submit additional clinical trial data to increase the patient-years of exposure to dapagliflozin and comparators. At a minimum, the resubmission must include data from patients in Studies D1690C00018 and D1690C00019 who have completed at least 52 weeks in these studies."

In response to this CRL, the sponsors submitted a request for formal dispute resolution to the Office of New Drugs on 17 July 2012. The first item of the request addressed cardiovascular safety; in it the sponsors proposed: "that the $C V$ data from studies D1690C00018 and D1690C00019 be viewed in the context of the overall CV risk assessment of dapagliflozin and not as stand-alone studies."

The dispute appeal was denied by the Agency. The response letter stated that:

1. The path forward as written in the CR letter is reasonable under the circumstances posed by the data in the NDA and stands as written. This includes the request for updated safety analyses of the NDA database, including at least 52 weeks of data from Studies 18 and 19, but given information that you shared with the review team about event rates at 52 weeks, the full study data, which are expected to be completed with two years of data are imminent and would be far preferable.

And 
2. A resubmitted NDA should be brought before an Advisory Committee. A second AC meeting on this drug is important because data and the overall spectrum of risk have shifted since their first meeting... More importantly, the CV data and how it evolved to shift expectations for dapagliflozin, from having hope for $C V$ benefit to concern about a signal of $C V$ risk, warrants discussion. It raises questions about how to factor in new data to a meta-analysis that has already been used to make a decision, that the FDA Guidance on CV risk for diabetes drugs does not fully address. Also, how should clinical trials that are highly enriched for $C V$ risk be considered in the overall premarketing risk assessment for a diabetes treatment? If they are to play a major role, should such enriched trials be required earlier in development than was planned for dapagliflozin? Should they carry more weight than studies in patients with fewer CV risk factors? These questions, being raised in the context of this NDA, should not be answered without public discussion.

In July 11, 2013, Bristol-Myers Squibb and Astra Zeneca submitted an updated metaanalysis of cardiovascular adverse events conducted in 21 randomized clinical trials for dapagliflozin as part of their application package for NDA 202293. This document presents a statistical review of this meta-analysis.

\subsection{Clinical Trial Overview}

The 2013 updated meta-analysis consisted of 21 trials: 7 Phase 2 trials and 14 Phase 3 trials. It included the complete data from trials 18 and 19, including their full 28 and 52 week extension periods. The full duration of these two trials, including their extensions, was 104 weeks. This submission fulfills the condition established in the path forward to include at least 52 weeks of data from trials 18 and 19. The 21 trials in this meta-analysis of cardiovascular safety are summarized in Table 6 . The table highlights the differences between the current submission and the October 2011 meta-analysis:

1. Two new trials were included in the current meta-analysis: D1692C00006 and MB102054. These were regional trials conducted in Japan and China respectively.

2. The current meta-analysis includes additional patient-years from the extension periods of trials D1690C00012, D1690C00010, D1690C00004, D1690C00018 and D1690C00019.

The data cutoff date for this meta-analysis was 15 November 2012. At that time, all trials had been completed except for trial D1690C00004, which had completed its short term period, but was undergoing its long term extension period. This trial was completed in January 2013. According to the sponsors, two ongoing Phase 3 trials (MB102055 and D1692C00005) were not included in the meta-analysis of cardiovascular events because they were ongoing and blinded at the data cutoff date. 
Table 6. List of Trials Included in the 2013 Updated Meta-Analysis

\begin{tabular}{|c|c|c|c|c|c|c|}
\hline Trial ID & $\begin{array}{c}\text { Duration in Weeks } \\
\text { Short Terms + } \\
\text { (Long Terms) }\end{array}$ & $\begin{array}{l}\text { Total } \\
\text { Sample } \\
\text { Size }\end{array}$ & $\begin{array}{l}\text { Dapagliflozin groups by daily } \\
\text { dosage }(\mathrm{mg})\end{array}$ & $\begin{array}{l}\text { Dapagliflozin } \\
\text { (N) }\end{array}$ & Placebo $^{1}$ (N) & $\begin{array}{l}\text { Active } \\
\text { Control } \\
\text { (N) }\end{array}$ \\
\hline \multicolumn{7}{|l|}{ Core Phase 2b Studies } \\
\hline MB102008 & 12 & 389 & $2.5,5,10,20,50$ & 279 & 54 & 56 \\
\hline MB102009 & 12 & 71 & 10,20 & 48 & 23 & - \\
\hline MB102035 & 12 & 75 & 10 & 24 & 25 & 26 \\
\hline MB102045 & 12 & 44 & 5 & 23 & 21 & - \\
\hline D1692C00005² & 12 & 220 & $2.5,5,10$ & 166 & 54 & - \\
\hline \multicolumn{7}{|l|}{ Core Phase $2 \mathrm{~b}$ Studies } \\
\hline MB102013 & $24+(78)$ & 485 & $2.5,5,10$ & 410 & 75 & - \\
\hline MB102032² & 24 & 210 & $2.5,5$ & 142 & 68 & - \\
\hline \multicolumn{7}{|c|}{ Add-on Studies (Placebo-controlled) } \\
\hline MB102014 & $24+(78)$ & 546 & $2.5,5,10$ & 409 & 137 & - \\
\hline D1690C00012 & $24+(78)$ & 182 & 10 & 91 & 91 & - \\
\hline D1690C00006 & $24+(24)+(56)$ & 807 & $2.5,5,10$ & 610 & 197 & - \\
\hline MB102030 & $24+(24)$ & 420 & 5,10 & 281 & 139 & - \\
\hline D1690C00005 & $24+(24)$ & 596 & $2.5,5,10$ & 450 & 146 & - \\
\hline $\mathrm{D} 1690 \mathrm{C00010}$ & $24+(24)$ & 451 & 10 & 225 & 226 & - \\
\hline \multicolumn{7}{|c|}{ Active Comparator Study vs. Sulfonylurea } \\
\hline D1690C00004 & $52+(52)+(104)$ & 814 & Titrated dose of $2.5-5-10 \mathrm{mg}$ & 406 & - & 408 \\
\hline \multicolumn{7}{|c|}{ Initial Combination Study with Metformin (Active Comparator vs. Metformin) } \\
\hline MB102034 & 24 & 638 & 10 & 430 & - & 208 \\
\hline \multicolumn{7}{|c|}{ Initial Combination Study with Metformin } \\
\hline MB102021 & 24 & 598 & 5 & 397 & - & 201 \\
\hline \multicolumn{7}{|l|}{ Special Populations } \\
\hline MB102029 & $24+(28)+(52)$ & 252 & 5,10 & 168 & 84 & - \\
\hline D1690C00018 & $24+(28)+(52)$ & 922 & 10 & 460 & 462 & - \\
\hline D1690C00019 & $24+(28)+(52)$ & 965 & 10 & 482 & 483 & - \\
\hline \multicolumn{7}{|c|}{ Regional Phase 3 Studies } \\
\hline D1692C00006 & 24 & 261 & 5,10 & 174 & 87 & - \\
\hline MB102054 & 24 & 393 & 5,10 & 261 & 132 & - \\
\hline
\end{tabular}

Source: Created by reviewer. Dataset: adcv5.xpt

*Bold type denotes information added or updated since the latest 2011 meta-analysis.

1 Placebo arm with or without background medication.

${ }^{2}$ Trial randomized subjects to Dapagliflozin $1 \mathrm{mg}$. These subjects are excluded from this table and from all analyses in this review.

${ }^{3}$ Trial included an uncontrolled group randomized to dapagliflozin only. These subjects are excluded from this table and from all analyses in this review. 
Table 7 shows the total number of subjects randomized to each dose of dapagliflozin in the 21 trials. The most common dose was $10 \mathrm{mg}$ daily.

Table 7. Pooled sample size by daily dosage of dapagliflozin

\begin{tabular}{|c|c|c|c|c|c|c|}
\hline & $2.5 \mathrm{mg}$ & $5 \mathrm{mg}$ & $10 \mathrm{mg}$ & $20 \mathrm{mg}$ & $50 \mathrm{mg}$ & $\begin{array}{c}\text { Titrated } \\
2.5 \mathrm{mg} \\
\text { to } 10 \mathrm{mg}\end{array}$ \\
\hline Total & 814 & 1668 & 2909 & 83 & 56 & 406 \\
\hline
\end{tabular}

\subsection{Data Sources}

The applicants compiled the data necessary to conduct time to event analyses of cardiovascular endpoints across these 21 trials into one dataset: adcv5.xpt. This dataset contained information on baseline characteristics, dosage, reason for discontinuation, and time and type of cardiovascular event.

The following file folder available within the CDER Electronic Document Room (EDR) was used in this review:

\section{$\underline{\text { Ilcdsesub1 } 1 \text { evsprod } \backslash N D A 202293 \backslash 0000 \backslash m 5}$}

The format, content and documentation of the data submitted in support of this application was adequate to conduct a statistical review of the cardiovascular risk associated with dapagliflozin.

\section{Statistical Evaluation}

This review focuses on the meta-analysis of 21 trials to assess the cardiovascular risk of dapagliflozin. For an updated statistical evaluation of the efficacy of dapagliflozin, please refer to the review authored by Dr. Wei Liu.

\subsection{Evaluation of Safety}

\subsubsection{Trial Designs}

Nineteen of the 21 trials included in the current meta-analysis were included in the October 2011 meta-analysis submission. Please refer to Dr. Abraham's statistical review dated 22 November 2011 for a thorough description of these trials. The description of trials 18 and 19 below is based on Dr. Abraham's review and is provided here because these two trials are analyzed and discussed separately in the present review: 
Trial D1690C00018 was a Phase 3, multi-center randomized, double-blind, placebo controlled superiority trial with a 24 -week short-term treatment period followed by a 28- week long-term extension period and a second 52-week long-term extension period to evaluate the efficacy and safety of dapagliflozin $10 \mathrm{mg}$ once daily in adults with T2DM, cardiovascular disease and hypertension, who exhibit inadequate glycemic control on standard of care. Trial D1690C00018 was conducted in 171 centers in Europe, North America, Latin America and Asia, in male subjects $>45$ years of age and female subjects $>50$ years of age diagnosed with T2DM, CVD and hypertension with inadequate glycemic control. A total of 922 subjects were randomized in a 1:1 fashion using 8 strata based on a combination of age, insulin use and time to most recent qualifying $\mathrm{CV}$ event to the placebo group and $10 \mathrm{mg}$ dosages of dapagliflozin. Trial D1690C00018 was powered to provide $90 \%$ or greater power to demonstrate the superiority of dapagliflozin compared to placebo to (sic) with a difference of $15 \%$ between dapagliflozin and placebo for changes in clinical benefit using a 3-item endpoint (absolute drop of $0.5 \%$ or more from baseline $\mathrm{HbAlc}$, relative drop of $3 \%$ or more from baseline for total body weight, and an absolute drop of $3 \mathrm{mmHg}$ or more from baseline in seated systolic blood pressure); and to provide $>99 \%$ power to detect a difference of $0.5 \%$ between dapagliflozin and placebo for mean change in HbA1c from baseline to week 24 at a significance level of 0.0125 for both primary objectives. Subjects had to be scheduled for a follow-up visit 3 weeks after discontinuation of investigational product. Trial D1690C00018 was completed on December 2012.

Trial D1690C00019 was a Phase 3, multi-center randomized, double-blind, placebo controlled superiority trial with a 24 -week short-term treatment period followed by a 28- week long-term extension period and a second 52-week long-term extension period to evaluate the efficacy and safety of dapagliflozin $10 \mathrm{mg}$ once daily in adults with T2DM and cardiovascular disease who exhibit inadequate glycemic control on standard of care. Trial D1690C00019 was conducted in 173 centers in Europe, North America, South America and Australia, in male subjects $>45$ years of age and female subjects $>50$ years of age diagnosed with T2DM, and CVD with inadequate glycemic control. A total of 965 subjects were randomized in a 1:1 fashion using 8 strata based on a combination of age, insulin use and time to most recent qualifying $\mathrm{CV}$ event to the placebo group and $10 \mathrm{mg}$ dosages of dapagliflozin. Trial D1690C00019 was powered to provide $90 \%$ or greater power to demonstrate the superiority of dapagliflozin compared to placebo to (sic) with a difference of $15 \%$ between dapagliflozin and placebo for changes in clinical benefit using a 3 -item endpoint(absolute drop of $0.5 \%$ or more from baseline $\mathrm{HbA} 1 \mathrm{c}$, relative drop of $3 \%$ or more from baseline for total body weight, and an absolute drop of $3 \mathrm{mmHg}$ or more from baseline in seated systolic blood pressure); and provided $>99 \%$ power to detect a difference of $0.5 \%$ between dapagliflozin and placebo for mean change in HbA1c from baseline to week 24 at a significance level of 0.0125 for both primary objectives. Subjects had to be scheduled for a follow-up visit 3 weeks after discontinuation of investigational product. Trial D1690C00019 was completed on December 2012. 
The current submission includes the complete short term and long term periods of trials 18 and 19, which were ongoing at the time of the 2011 meta-analysis. Additionally, two new trials have been included in the current submission:

D1692C00006 was a Phase 3 randomized, double-blind, clinical trial conducted in 30 centers in Japan. The objective of the trial was to show superiority of dapagliflozin $5 \mathrm{mg}$ or $10 \mathrm{mg}$ relative to placebo on the change of $\mathrm{HbAlc}$ after 24 weeks of treatment. The trial randomized 174 subjects to dapagliflozin and 87 subjects to placebo. The inclusion criteria was drug naïve subjects with $\mathrm{HbAlc}$ between $6.5 \%$ and $10 \%$ or subjects ongoing anti-diabetic treatment with a baseline $\mathrm{HbA} 1 \mathrm{c} \leq 8 \%$. The trial was conducted between February 2011 and March 2012.

MB102054 was a Phase 3 randomized, double-blind, clinical trials conducted in patients in China $(\mathrm{N}=326)$ with a few additional patients randomized in India $(\mathrm{N}=25)$, South Korea $(\mathrm{N}=17)$ and Taiwan $(\mathrm{N}=25)$. The objective of the trial was to show superiority of dapagliflozin $5 \mathrm{mg}$ or $10 \mathrm{mg}$ relative to placebo on the change of HbAlc after 24 weeks of treatment. The trial randomized 261 subjects to dapagliflozin and 132 to placebo. The inclusion criteria was Asian drug naïve subjects with $\mathrm{HbAlc}$ between $7.5 \%$ and $10.5 \%$ at the time of enrollment. The trial was conducted between June 2010 and March 2012.

\subsubsection{Populations}

The primary meta-analysis population consisted of the randomized, controlled, short term plus long term periods of the 21 trials in Table 6 . The analyses included data following initiation of any rescue therapy.

Per request from the FDA, a secondary analysis was conducted that incorporated only data from the two trials conducted in a population of subjects with cardiovascular disease and hypertension (trials 18 and 19). This analysis was intended to assess the cardiovascular risk of dapagliflozin in two trials with a large sample size, long follow-up, and increased baseline cardiovascular risk.

The sponsors conducted a secondary analysis in the population of subjects with prior CV disease in the 21 trials, including trials 18 and 19. Prior CV disease was defined as history of any of the following at baseline: MI, congestive heart failure (CHF), hospitalization for unstable angina, stable angina, percutaneous coronary intervention, coronary artery bypass graft, coronary artery disease, cerebrovascular accident, carotid artery. Table 8 shows that trials 18 and 19 had a different distribution of cardiovascular risk factors than the subjects with a history of $\mathrm{CV}$ disease in the other trials in the program. Consequently, the secondary analyses in this review were conducted in these two populations separately:

- Trials 18 and 19 alone, and

- Subjects with a history of CV disease in other trials. 
Table 8. Baseline Cardiovascular Risk Factors in Subjects with Prior CV Disease

\begin{tabular}{|ccc|}
\hline & $\begin{array}{c}\text { Subjects with history } \\
\text { of CV disease in other } \\
\text { trials } \\
(N=1336)\end{array}$ & Trials 18 and 19 \\
\hline History of Hypertension (\%) & $83.8 \%$ & $96.2 \%$ \\
History of Dyslipidemia (\%) & $66.8 \%$ & $84.4 \%$ \\
History of Congestive HF (\%) & $9.8 \%$ & $14.4 \%$ \\
Smokers (current or former) (\%) & $47.5 \%$ & $59.7 \%$ \\
Statin Use & $60.7 \%$ & $81.3 \%$ \\
SBP $\geq 140$ mmHg $^{1}$ & $34.7 \%$ & $37.7 \%$ \\
Diabetes Duration $\geq 10$ years & $36.7 \%$ & $58.2 \%$ \\
\hline Baseline eGFR (ml/min) & & \\
\hline$<60$ & $21.6 \%$ & $17.4 \%$ \\
$61-90$ & $54.6 \%$ & $59.4 \%$ \\
\hline$\geq 90$ & $23.8 \%$ & $23.3 \%$ \\
\hline
\end{tabular}

Source: Created by reviewer. Dataset: adcv5.xpt

'Statin Use was defined as use at any time during the trial. Therefore statin use may

be associated with randomized treatment and may not be representative of baseline use.

\subsubsection{Endpoints and Adjudication Methods}

\subsubsection{Primary Composite Endpoint}

The agreed upon primary composite endpoint to assess CV safety was defined as the time until the first of the following adjudicated events:

a CV death

- Myocardial infarction (MI)

- Stroke

- Hospitalization for unstable angina.

The time to event analyses for all endpoints used the following censoring rules. Subjects without an event in AstraZeneca studies (studies with a ' $D$ ' in the beginning of the study code) were censored at the earliest of the date of last contact or date of death. Subjects without an event in BMS studies (studies with a 'MB' in the beginning of the study code) were censored at the earliest of the following: end of treatment +30 days, date of death, date of discontinuation due to loss of follow-up.

\subsubsection{Secondary Composite Endpoint}

A secondary composite endpoint was defined as the time until the first of the following adjudicated events:

a CV death

๑ MI 
a Stroke

- Hospitalization for unstable angina

- Unplanned coronary revascularization

a Hospitalization for heart failure

Separate analyses were also conducted on Major Adverse Cardiovascular Endpoints (MACE), defined as the composite of CV death, MI and Stroke.

\subsubsection{Adjudication Methods}

Potential cardiovascular events were identified through a combination of Standardized MedDRA queries (SMQs) and custom SMQs. For each potential event, the principal investigators of each trial prepared a case submission report and sent it to the Clinical Event Committee (CEC) (b) (4) for adjudication. The CEC consists of two cardiologists and two neurologists. All events were adjudicated by the $\mathrm{CEC}$ in a blinded fashion. The adjudication was done prospectively in all trials, except for MB102008 and MB102009 which were completed before the adjudication process was started. The adjudication of these two trials was conducted retrospectively.

\subsubsection{Statistical Methodologies}

The 2011 meta-analysis pre-specified a two part sequential testing strategy to show that the hazard ratio of the primary outcome associated with dapagliflozin was no larger than 1.8 with an overall $\alpha=0.05$. The first pre-specified test of the 2011 meta-analysis produced an estimated hazard ratio of 0.67 and a $98 \%$ confidence interval of $(0.38,1.18)$. The upper bound of this interval successfully ruled out a risk margin greater than 1.8 associated with dapagliflozin.

The current 2013 meta-analysis updates the 2011 meta-analysis by adding two new trials and additional patient-years from the extension phase of trials that were ongoing in 2011. Because the 2011 meta-analysis was successful in ruling out a hazard ratio margin of 1.8 with an overall $\alpha=0.05$, the current 2013 meta-analysis does not include any type-I error adjustments for multiple looks. The current meta-analysis will compute a single nominal $95 \%$ confidence interval for the hazard ratio of the primary endpoint and the hazard ratio of secondary endpoints in each analysis.

\subsubsection{Primary Analysis}

The agreed upon primary meta-analysis compares the hazard ratio of the primary cardiovascular composite endpoint (CV death, MI, stroke, and hospitalization for unstable angina) in subjects randomized to all doses of dapagliflozin, excluding $1 \mathrm{mg}$ daily, to subjects randomized to all comparators using a Cox proportional hazards model stratified by trial. The model has a single covariate for treatment: dapagliflozin vs. comparators. The proportional hazards assumption of the primary Cox model will be 
evaluated graphically by plotting the scaled-Schoenfeld residuals of the model against time.

This Cox proportional hazards model will be fit in the primary population of all 21 trials in the meta-analysis and in the secondary populations of trials 18 and 19 alone, and in subjects with baseline history of CV disease excluding trials 18 and 19.

\subsubsection{Secondary Analyses}

A Cox proportional hazards model similar to the primary model described in Section 3.1.3.2 will be fit to the secondary cardiovascular endpoint and to the MACE composite. Similarly, the individual components of the primary endpoint: CV death, MI, stroke, and hospitalization due to unstable angina will be analyzed separately through the same model.

These analyses will be repeated in the population of all 21 trials, the population of trials 18 and 19 alone, and the population of subjects with a baseline history of CV disease excluding trials 18 and 19 .

\subsubsection{Sensitivity Analyses}

A sensitivity analysis pre-specified by the sponsors will estimate the Mantel-Haenszel incidence rate ratio of events associated with dapagliflozin. The results of this analysis are discussed in Section 3.1.6.6.

A post-hoc sensitivity analysis will be conducted to examine the risk of cardiovascular events comparing dapagliflozin $10 \mathrm{mg}$ to all comparators through a stratified Cox proportional hazards model. The comparator group for this analysis will include only subjects randomized to comparators in trials that included a dapagliflozin $10 \mathrm{mg}$ treatment arm.

\subsubsection{Post-hoc Analyses}

A post-hoc analysis will be conducted to examine the risk of cardiovascular events associated with dapagliflozin within the first 30 days after randomization in the 21 trials in the meta-analysis. This analysis was motivated by an imbalance of early cardiovascular events observed in the development program for canagliflozin, the only SGLT2 inhibitor currently approved in the United States. Canagliflozin was approved in March 2013 in the United States under NDA 204042, as an adjunct to diet and exercise to improve glycemic control in adult patients with type 2 diabetes mellitus. The early imbalance of cardiovascular events observed in the canagliflozin program is discussed in the Appendix. 


\subsubsection{Evaluation of Heterogeneity between Trials}

The stratified Cox model allows for different baseline hazards across strata (trials), but assumes that the effect of treatment, the hazard ratio, is constant across strata. Formal statistical testing for a difference in hazard ratios is equivalent to testing for an interaction of treatment by trial in the Cox model. Given that none of the trials in the meta-analysis were powered to evaluate cardiovascular safety by themselves, few events are expected to be observed in each individual trial in the meta-analysis. Therefore a test for interaction of treatment by trial in the primary Cox model would have limited power to detect differences in the hazard ratios between trials. Consequently, we do not test for the interaction of treatment and strata in this review.

The trials' populations were heterogeneous by design:

- The inclusion criteria for trial MB102035 was based on baseline blood pressure, not $\mathrm{HbA1c}$, like the other 20 trials in the meta-analysis.

- Trials 18 and 19 enrolled subjects with higher cardiovascular baseline risk.

- Trials D1692C00006 and MB102054 were regional trials only.

A random effects model for the rate ratio of the primary composite event will be fit. Its results will be compared with the results of the primary Cox proportional hazards model to assess the impact of trial heterogeneity on estimates of cardiovascular risk. No formal statistical test will be conducted to determine which model fits the data better.

\subsubsection{Evaluation of Cardiovascular Safety to Meet the 1.3 Margin after Approval}

The Sponsors propose to evaluate the cardiovascular safety of dapagliflozin after approval through a dedicated cardiovascular outcomes trial, "D1693C00001". This trial was started in April 2013 with the goal of showing that the upper bound of the $95 \%$ confidence interval for the hazard ratio of MACE associated with dapagliflozin is smaller than 1.3 relative to placebo. The trial plans to randomize up to 17000 subjects to dapagliflozin $10 \mathrm{mg}$ or placebo and has a median expected follow-up time of 4.5 years.

Trial D1693C00001 is not part of the updated meta-analysis discussed in this document. Therefore it will not be discussed any further. For a detailed discussion of the protocol and study design of trial D1693C00001, please refer to the review authored by Dr. Janelle Charles dated 01 June, 2012. 


\subsubsection{Subject Disposition, Demographics and Baseline Characteristics}

\subsubsection{Characteristics of All Trials in the Meta-Analysis}

Table 9 shows baseline demographic characteristics by treatment arm pooled across the 21 trials in the meta-analysis. Small pooled differences in these characteristics were caused by different inclusion criteria and randomization ratios between trials. Overall, baseline characteristics appeared balanced between treatment arms within each individual trial in the meta-analysis (not shown here).

Table 10 shows baseline cardiovascular risk factors by treatment arm pooled across trials. Again, the differences between treatment arms are caused by heterogeneous baseline characteristics and unequal randomization ratios across trials.

Table 9. Baseline Characteristics Pooled across All Trials in the Meta-Analysis

\begin{tabular}{|c|c|c|}
\hline & $\begin{array}{l}\text { All Comparators } \\
(N=3403)\end{array}$ & $\begin{array}{c}\text { Dapagliflozin } \\
(N=5936)\end{array}$ \\
\hline Percent Female & $42.3 \%$ & $45.4 \%$ \\
\hline Age \pm SD (years) & $58.1 \pm 10.3$ & $56.9 \pm 10.4$ \\
\hline$\leq 50$ years & $22.0 \%$ & $25.6 \%$ \\
\hline $51-65$ years & $52.6 \%$ & $53.8 \%$ \\
\hline 66 - 75 years & $22.9 \%$ & $17.7 \%$ \\
\hline$>75$ years & $2.5 \%$ & $2.9 \%$ \\
\hline $\mathrm{BMI} \pm \mathrm{SD}\left(\mathrm{kg} / \mathrm{m}^{2}\right)$ & $31.6 \pm 5.8$ & $31.3 \pm 5.7$ \\
\hline$\leq 25$ & $12.3 \%$ & $12.6 \%$ \\
\hline $26-30$ & $31.2 \%$ & $31.3 \%$ \\
\hline$>30$ & $56.5 \%$ & $56.2 \%$ \\
\hline \multicolumn{3}{|l|}{ Race $^{1}$} \\
\hline White & $77.7 \%$ & $75.9 \%$ \\
\hline Black / African American & $3.7 \%$ & $3.5 \%$ \\
\hline Asian & $15.1 \%$ & $17.7 \%$ \\
\hline Other / Multiracial & $3.6 \%$ & $2.9 \%$ \\
\hline $\begin{array}{l}\text { Percent randomized in } \\
\text { the USA }\end{array}$ & $21.4 \%$ & $21.6 \%$ \\
\hline \multicolumn{3}{|l|}{ Region } \\
\hline North America & $28.0 \%$ & $30.1 \%$ \\
\hline Europe & $40.2 \%$ & $34.5 \%$ \\
\hline Latin America & $17.9 \%$ & $18.6 \%$ \\
\hline Asia/Pacific & $13.9 \%$ & $16.8 \%$ \\
\hline
\end{tabular}

Source: Created by reviewer. Dataset: adcv5.xpt

'Race categories were defined by the sponsor. We grouped "Asian" (689), "Asian Indian" (25), "Asian Other" (2), "Chinese" (348), "Japanese" (483) and "Korean" (16) into the category "Asian" (1563). The categories "White" and "Black / African American" were defined by the sponsor. All other race categories were grouped into "Other / Multiracial". 
Table 10. Baseline Cardiovascular Risk Factors Pooled across All Trials in the MetaAnalysis

\begin{tabular}{|ccc|}
\hline & $\begin{array}{c}\text { All Comparators } \\
(N=3403)\end{array}$ & $\begin{array}{c}\text { Dapagliflozin } \\
(N=5936)\end{array}$ \\
\hline History of CVD (\%) & $39.9 \%$ & $31.3 \%$ \\
History of Hypertension (\%) & $71.9 \%$ & $65.7 \%$ \\
History of Dyslipidemia (\%) & $59.6 \%$ & $55.2 \%$ \\
History of Congestive HF (\%) & $4.8 \%$ & $4.0 \%$ \\
Smokers (current or former) (\%) & $46.3 \%$ & $43.3 \%$ \\
Statin Use ${ }^{1}$ & $50.5 \%$ & $44.2 \%$ \\
SBP $\geq 140$ mmHg & $28.5 \%$ & $26.5 \%$ \\
Diabetes Duration $\geq 10$ years & $29.9 \%$ & $26.9 \%$ \\
\hline Baseline eGFR (ml/min) & & \\
\hline$<60$ & $11.8 \%$ & $11.7 \%$ \\
$61-90$ & $52.5 \%$ & $52.1 \%$ \\
$\geq 90$ & $35.7 \%$ & $36.2 \%$ \\
\hline
\end{tabular}

Source: Created by reviewer. Dataset: adcv5.xpt

1Statin Use was defined as use at any time during the trial. Therefore statin use may be associated with randomized treatment and may not be representative of baseline use.

\subsubsection{Characteristics of Trials 18 and 19}

The baseline demographic and cardiovascular characteristics of subjects in trials 18 and 19 are summarized in Table 11 and Table 12. These characteristics appear balanced between the two treatment arms: dapagliflozin and placebo. Table 12 shows that the population of these two trials had high baseline cardiovascular risk, as intended by design.

Table 11. Baseline Characteristics in Trials 18 and 19

\begin{tabular}{|c|c|c|}
\hline & $\begin{array}{c}\text { Placebo } \\
(N=945)\end{array}$ & $\begin{array}{l}\text { Dapagliflozin } \\
(N=942)\end{array}$ \\
\hline Percent Female & $32.4 \%$ & $32.7 \%$ \\
\hline \multicolumn{3}{|l|}{ Age \pm SD (years) } \\
\hline$\leq 50$ years & $3.1 \%$ & $3.5 \%$ \\
\hline $51-65$ years & $57.4 \%$ & $56.2 \%$ \\
\hline $66-75$ years & $34.6 \%$ & $33.6 \%$ \\
\hline$>75$ years & $4.9 \%$ & $6.7 \%$ \\
\hline \multicolumn{3}{|l|}{$B M I \pm S D\left(k g / m^{2}\right)$} \\
\hline$\leq 25$ & $6.9 \%$ & $4.9 \%$ \\
\hline $26-30$ & $26.7 \%$ & $28.1 \%$ \\
\hline$>30$ & $66.4 \%$ & $67.0 \%$ \\
\hline \multicolumn{3}{|l|}{ Race $^{1}$} \\
\hline White & $89.3 \%$ & $88.9 \%$ \\
\hline Black / African American & $3.9 \%$ & $3.7 \%$ \\
\hline Asian & $4.8 \%$ & $5.8 \%$ \\
\hline Other / Multiracial & $2.0 \%$ & $1.6 \%$ \\
\hline
\end{tabular}




\begin{tabular}{|ccc|}
\hline $\begin{array}{l}\text { Percent randomized in } \\
\text { the USA }\end{array}$ & $23.9 \%$ & $25.0 \%$ \\
\hline Region & & \\
\hline North America & $31.4 \%$ & $34.5 \%$ \\
Europe & $50.9 \%$ & $47.4 \%$ \\
Latin America & $10.8 \%$ & $9.7 \%$ \\
Asia/Pacific & $6.9 \%$ & $8.4 \%$ \\
\hline
\end{tabular}

Source: Created by reviewer. Dataset: adcv5.xpt

Table 12. Baseline Cardiovascular Risk Factors in Trials 18 and 19

\begin{tabular}{|c|c|c|}
\hline & $\begin{array}{l}\text { Placebo } \\
(N=945)\end{array}$ & $\begin{array}{l}\text { Dapagliflozin } \\
(N=942)\end{array}$ \\
\hline History of CVD (\%) & $99.6 \%$ & $99.5 \%$ \\
\hline History of Hypertension (\%) & $96.2 \%$ & $96.3 \%$ \\
\hline History of Dyslipidemia (\%) & $84.0 \%$ & $84.4 \%$ \\
\hline History of Congestive HF (\%) & $13.5 \%$ & $15.1 \%$ \\
\hline Smokers (current or former) (\%) & $59.2 \%$ & $60.1 \%$ \\
\hline Statin Use ${ }^{1}$ & $82.0 \%$ & $80.4 \%$ \\
\hline $\mathrm{SBP} \geq 140 \mathrm{mmHg}$ & $36.9 \%$ & $38.3 \%$ \\
\hline Diabetes Duration $\geq 10$ years & $56.5 \%$ & $59.6 \%$ \\
\hline \multicolumn{3}{|l|}{ Baseline eGFR (ml/min) } \\
\hline$<60$ & $16.9 \%$ & $17.8 \%$ \\
\hline $61-90$ & $59.8 \%$ & $58.9 \%$ \\
\hline$\geq 90$ & $23.3 \%$ & $23.3 \%$ \\
\hline
\end{tabular}

Source: Created by reviewer. Dataset: adcv5.xpt

'Statin Use was defined as use at any time during the trial. Therefore statin use may

be associated with randomized treatment and may not be representative of baseline use.

\subsubsection{Follow-up Time by Treatment Arm}

Table 13 shows the mean days of follow-up by treatment arm including both short term and long term extension periods within each trial in the meta-analysis. The mean followup time in these trials ranged from just over 12 weeks (MB102009) to almost 2.4 years (D1690C00004).

Table 14 shows the number of patient-years contributed to the meta-analysis by each trial. The four largest trials contributed more than 1000 patient years of exposure each to the meta-analysis: D1690C00004 (1938 PY), D1690C00006 (1275 PY), trial 19 (1177 PY) and trial 18 (1060 PY). 
Figure 1 shows a plot of the time until the primary composite event, trial discontinuation, or death, by treatment arm in the pooled trials 18 and 19. The figure shows that subjects dropped out of these two trials at a similar rate in both treatment arms. No evidence of differential discontinuation by treatment was observed.

Table 15 shows the distribution of reasons for trial discontinuation in the 21 trials in the meta-analysis. Some imbalances were observed within trials, for example, the table shows that $9.5 \%$ of subjects in the comparator arm of trial MB102045 were lost to follow-up compared to $0 \%$ in the dapagliflozin arm. Some of these differences are explained by the small sample size of some trials: the $9.5 \%$ of subjects lost to follow-up among subjects randomized to comparators in trial MB102045 represents only 2 subjects. No consistent pattern of discontinuation associated with dapagliflozin was observed across the 21 trials.

Table 13. Mean (SD) Days of Follow-up by Trial

\begin{tabular}{|c|c|c|}
\hline Trial ID & All Comparators & Dapagliflozin \\
\hline D1690C00004 & $844(523)$ & $895(526)$ \\
D1690C00005 & $329(84)$ & $331(79)$ \\
D1690C00006 & $531(265)$ & $591(239)$ \\
D1690C00010 & $329(76)$ & $335(75)$ \\
D1690C00012 & $665(173)$ & $635(207)$ \\
D1690C00018 & $421(208)$ & $419(208)$ \\
D1690C00019 & $444(211)$ & $447(214)$ \\
D1692C00005 & $111(8)$ & $112(9)$ \\
D1692C00006 & $181(32)$ & $182(32)$ \\
MB102008 & $108(22)$ & $111(19)$ \\
MB102009 & $101(28)$ & $111(17)$ \\
MB102013 & $543(265)$ & $584(250)$ \\
MB102014 & $550(250)$ & $608(222)$ \\
MB102021 & $183(42)$ & $185(41)$ \\
MB102029 & $493(292)$ & $558(257)$ \\
MB102030 & $320(93)$ & $334(81)$ \\
MB102032 & $199(7)$ & $190(33)$ \\
MB102034 & $182(38)$ & $180(41)$ \\
MB102035 & $116(8)$ & $115(5)$ \\
MB102045 & $112(17)$ & $118(4)$ \\
MB102054 & $186(38)$ & $186(41)$ \\
\hline Pooled: & $411(320)$ & $406(301)$ \\
\hline
\end{tabular}

Source: Created by reviewer. Dataset: adcv5.xpt 
Table 14. Patient-Years Used in the Meta-Analysis by Trial

\begin{tabular}{|c|c|c|}
\hline Trial ID & All Comparators & Dapagliflozin \\
\hline D1690C00004 & 943 & 995 \\
D1690C00005 & 131 & 408 \\
D1690C00006 & 287 & 988 \\
D1690C00010 & 204 & 206 \\
D1690C00012 & 166 & 158 \\
D1690C00018 & 532 & 528 \\
D1690C00019 & 587 & 590 \\
D1692C00005 & 16 & 51 \\
D1692C00006 & 43 & 87 \\
MB102008 & 32 & 85 \\
MB102009 & 6 & 15 \\
MB102013 & 111 & 655 \\
MB102014 & 206 & 681 \\
MB102021 & 101 & 201 \\
MB102029 & 113 & 257 \\
MB102030 & 122 & 257 \\
MB102032 & 37 & 74 \\
MB102034 & 103 & 212 \\
MB102035 & 16 & 8 \\
MB102045 & 6 & 7 \\
MB102054 & 67 & 133 \\
\hline Pooled: & $\mathbf{3 8 3 1}$ & $\mathbf{6 5 9 4}$ \\
\hline
\end{tabular}

Figure 1. Follow-up Time in Trials 18 and 19, Censored at First Primary Event, Death or Study Discontinuation

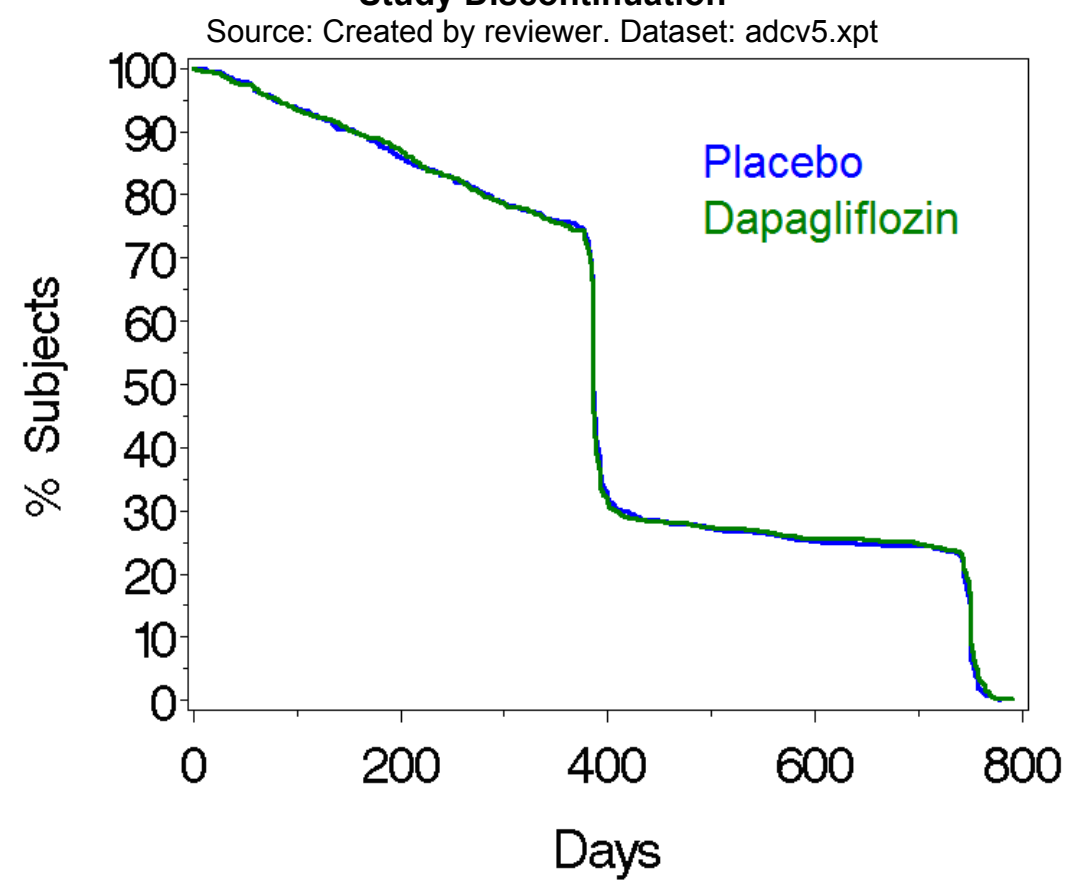


Table 15. Reason for Discontinuation in All Trials in the Meta-Analysis

\begin{tabular}{|c|c|c|c|c|c|c|c|c|}
\hline \multirow[t]{2}{*}{ Trial } & \multicolumn{2}{|c|}{ Adverse Event } & \multicolumn{2}{|c|}{ Lost to Follow-Up } & \multicolumn{2}{|c|}{$\begin{array}{c}\text { No Longer Meets Trial } \\
\text { Criteria }\end{array}$} & \multicolumn{2}{|c|}{ Other } \\
\hline & Comparators & Dapagliflozin & Comparators & Dapagliflozin & Comparators & Dapagliflozin & Comparators & Dapagliflozin \\
\hline D1690C00004 & $10.5 \%$ & $13.1 \%$ & $1.2 \%$ & $1.2 \%$ & $29.2 \%$ & $18.2 \%$ & $24.5 \%$ & $27.8 \%$ \\
\hline D1690C00005 & $3.4 \%$ & $2.7 \%$ & $1.4 \%$ & $1.6 \%$ & $2.1 \%$ & $1.1 \%$ & $6.9 \%$ & $9.3 \%$ \\
\hline D1690C00006 & $6.6 \%$ & $6.4 \%$ & $1.0 \%$ & $0.2 \%$ & $0.5 \%$ & $0.3 \%$ & $37.1 \%$ & $26.9 \%$ \\
\hline D1690C00010 & $1.8 \%$ & $3.1 \%$ & $1.3 \%$ & $3.1 \%$ & $4.0 \%$ & $0.4 \%$ & $12.0 \%$ & $3.6 \%$ \\
\hline D1690C00012 & 0 & $4.4 \%$ & 0 & 0 & $14.3 \%$ & $12.1 \%$ & $7.7 \%$ & $7.7 \%$ \\
\hline D1690C00018 & $3.0 \%$ & $5.4 \%$ & $2.8 \%$ & $0.9 \%$ & $8.4 \%$ & $10.2 \%$ & $19.9 \%$ & $17.6 \%$ \\
\hline D1690C00019 & $5.0 \%$ & $2.9 \%$ & $1.2 \%$ & $0.2 \%$ & $5.2 \%$ & $9.1 \%$ & $19.7 \%$ & $20.1 \%$ \\
\hline D1692C00005 & 0 & $1.2 \%$ & 0 & 0 & $9.3 \%$ & $1.2 \%$ & $7.4 \%$ & $1.8 \%$ \\
\hline D1692C00006 & $3.5 \%$ & $1.7 \%$ & 0 & 0 & $2.3 \%$ & $4.0 \%$ & $3.5 \%$ & $2.3 \%$ \\
\hline MB102008 & $1.8 \%$ & $2.5 \%$ & $3.6 \%$ & $4.3 \%$ & $1.8 \%$ & $0.4 \%$ & $7.3 \%$ & $3.9 \%$ \\
\hline MB102009 & $4.4 \%$ & $4.2 \%$ & $4.4 \%$ & 0 & 0 & 0 & $21.7 \%$ & $4.2 \%$ \\
\hline MB102013 & $5.3 \%$ & $6.6 \%$ & $10.7 \%$ & $5.6 \%$ & 0 & $0.7 \%$ & $26.7 \%$ & $24.9 \%$ \\
\hline MB102014 & $6.6 \%$ & $4.2 \%$ & $6.6 \%$ & $6.9 \%$ & $0.7 \%$ & $1.7 \%$ & $29.9 \%$ & $21.3 \%$ \\
\hline MB102021 & $3.0 \%$ & $2.0 \%$ & $4.5 \%$ & $2.5 \%$ & $1.0 \%$ & 0 & $6.5 \%$ & $8.1 \%$ \\
\hline MB102029 & $25.0 \%$ & $15.5 \%$ & $1.2 \%$ & $2.4 \%$ & $3.6 \%$ & $0.6 \%$ & $17.9 \%$ & $18.5 \%$ \\
\hline MB102030 & $3.6 \%$ & $2.9 \%$ & $4.3 \%$ & $3.2 \%$ & 0 & $0.7 \%$ & $15.8 \%$ & $8.5 \%$ \\
\hline MB102032 & 0 & $0.7 \%$ & 0 & $3.5 \%$ & 0 & 0 & $4.4 \%$ & $5.6 \%$ \\
\hline MB102034 & $3.9 \%$ & $3.0 \%$ & $2.4 \%$ & $4.9 \%$ & 0 & $0.2 \%$ & $6.7 \%$ & $5.6 \%$ \\
\hline MB102035 & 0 & 0 & 0 & 0 & 0 & 0 & 0 & $4.2 \%$ \\
\hline MB102045 & 0 & 0 & $9.5 \%$ & 0 & 0 & $4.4 \%$ & 0 & 0 \\
\hline MB102054 & $0.8 \%$ & $3.1 \%$ & $0.8 \%$ & $1.5 \%$ & 0 & $1.5 \%$ & $14.4 \%$ & $6.9 \%$ \\
\hline
\end{tabular}

Source: Created by reviewer. Dataset: adcv5.xpt 


\subsubsection{Analysis Results}

\subsubsection{Descriptive Statistics of the Composite Primary, Secondary and MACE Endpoints}

A total of 178 primary composite endpoints were observed in the 21 trials in the current meta-analysis. This represents 33 additional primary events than the October 2011 metaanalysis (15 on dapagliflozin, 18 on comparators). A total of 237 secondary events and 135 MACE were observed in the current meta-analysis. Table 16 shows the number of events observed by trial and treatment arm.

Table 16. Number of Subjects with a Composite Primary, Secondary or MACE Endpoint

\begin{tabular}{|c|c|c|c|c|c|c|}
\hline \multirow[b]{2}{*}{ Trial } & \multicolumn{2}{|c|}{ Primary Composite $^{1}$} & \multicolumn{2}{|c|}{ Secondary Composite ${ }^{2}$} & \multicolumn{2}{|c|}{ MACE $^{3}$} \\
\hline & $\begin{array}{c}\text { Comparators } \\
\qquad \begin{array}{c}\mathrm{N}=3403 \\
\mathrm{PY}=3831\end{array}\end{array}$ & $\begin{array}{c}\text { Dapagliflozin } \\
\mathrm{N}=5936 \\
\mathrm{PY}=6594\end{array}$ & $\begin{array}{c}\text { Comparators } \\
N=3403 \\
P Y=3820\end{array}$ & $\begin{array}{c}\text { Dapagliflozin } \\
\quad N=5936 \\
P Y=6584\end{array}$ & $\begin{array}{c}\text { Comparators } \\
\begin{array}{c}N=3403 \\
\text { PY }=3843\end{array}\end{array}$ & $\begin{array}{c}\text { Dapagliflozin } \\
\text { N }=5936 \\
\text { PY }=6606\end{array}$ \\
\hline D1690C00004 & 10 & 8 & 14 & 12 & 10 & 6 \\
\hline D1690C00005 & 1 & 6 & 4 & 7 & 1 & 3 \\
\hline D1690C00006 & 6 & 11 & 8 & 18 & 5 & 8 \\
\hline D1690C00010 & 1 & 1 & 1 & 1 & 1 & 0 \\
\hline D1690C00012 & 1 & 1 & 1 & 1 & 1 & 1 \\
\hline D1690C00018 & 18 & 21 & 27 & 27 & 12 & 16 \\
\hline D1690C00019 & 26 & 22 & 36 & 29 & 17 & 16 \\
\hline D1692C00005 & 0 & 1 & 0 & 1 & 0 & 1 \\
\hline D1692C00006 & 1 & 0 & 1 & 0 & 1 & 0 \\
\hline MB102008 & 0 & 1 & 0 & 1 & 0 & 1 \\
\hline MB102009 & 0 & 0 & 0 & 0 & 0 & 0 \\
\hline MB102013 & 0 & 6 & 0 & 6 & 0 & 5 \\
\hline MB102014 & 6 & 5 & 7 & 6 & 4 & 4 \\
\hline MB102021 & 1 & 2 & 1 & 2 & 1 & 2 \\
\hline MB102029 & 8 & 7 & 9 & 10 & 7 & 7 \\
\hline MB102030 & 0 & 1 & 0 & 1 & 0 & 0 \\
\hline MB102032 & 0 & 0 & 0 & 0 & 0 & 0 \\
\hline MB102034 & 1 & 2 & 1 & 2 & 1 & 1 \\
\hline MB102035 & 0 & 0 & 0 & 0 & 0 & 0 \\
\hline MB102045 & 0 & 0 & 0 & 0 & 0 & 0 \\
\hline MB102054 & 1 & 2 & 1 & 2 & 1 & 2 \\
\hline Total & 81 & 97 & 111 & 126 & 62 & 73 \\
\hline
\end{tabular}

Source: Created by reviewer. Dataset: adcv5.xpt

${ }^{1}$ Composite endpoint of CV death, MI, stroke and hospitalization for unstable angina.

${ }^{2}$ Composite endpoint of CV death, MI, stroke, hospitalization for unstable angina, unplanned coronary revascularization and hospitalization for heart failure.

${ }^{3}$ Composite endpoint of CV death, MI and stroke. 
Figure 2 shows the pooled cumulative probability plots (Kaplan-Meier) for the primary composite event across all 21 trials in the meta-analysis on the left panel and trials 18 and 19 alone on the right panel. Note that the left panel pools trials with unequal randomization ratio and therefore should be interpreted with caution. This plot may provide an inaccurate representation of the true risk associated with each treatment arm because trials with a population with higher baseline risk may contribute a different number of subjects to each pooled treatment group. The right panel shows little overall difference, graphically, in the risk of the primary composite cardiovascular endpoint between both treatment arms in trials 18 and 19 through the full duration of the trials. A formal statistical comparison of dapagliflozin versus comparators is presented in the following sections.

Figure 2. Observed Cumulative Probability of Primary CV Composite Endpoint

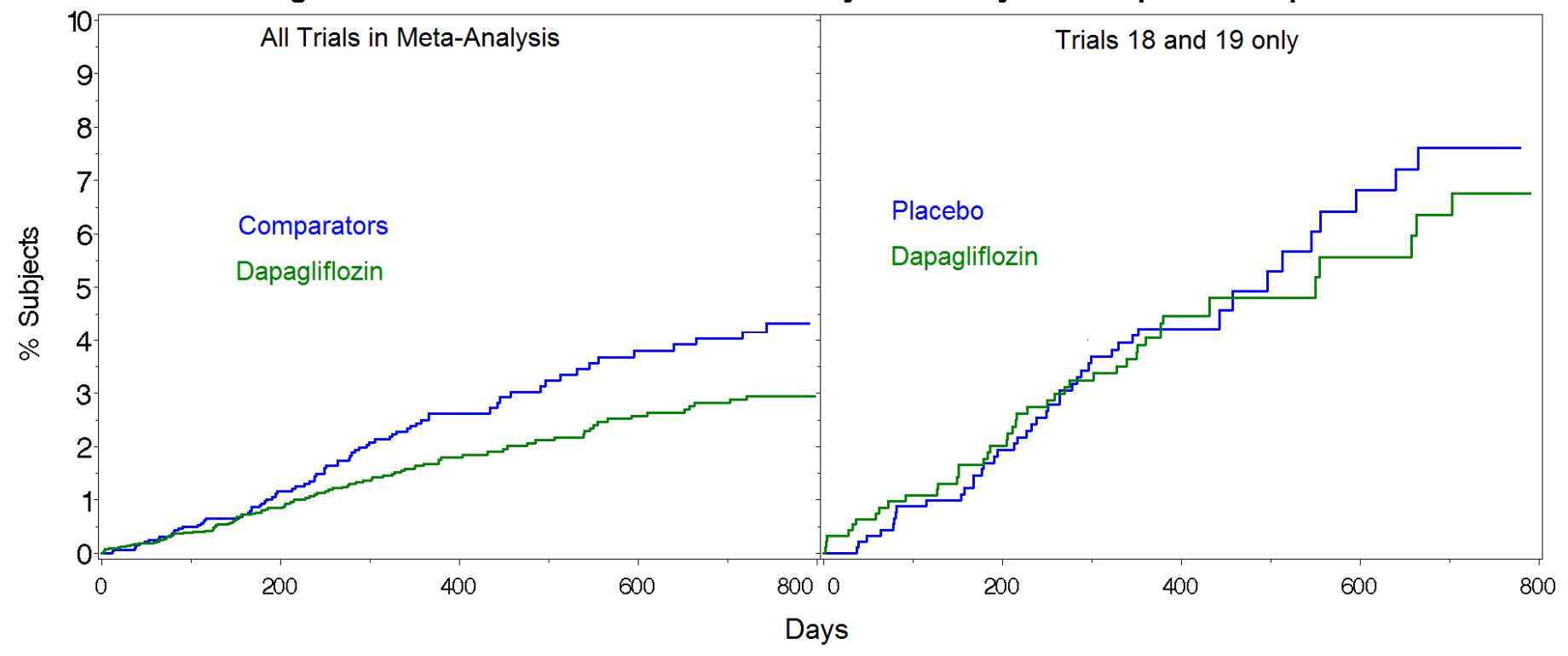

\subsubsection{Analysis of Primary Cardiovascular Composite Endpoint}

Results of the pre-specified meta-analysis using a stratified Cox proportional hazards model are shown in Table 17. Eighty one (81) events were observed among 3403 subjects in the comparator arm and 97 events were observed among 5936 subjects in the dapagliflozin arm. The estimated hazard ratio of the primary composite endpoint associated with dapagliflozin based on this model was $\mathbf{0 . 8 1}$ with $95 \%$ confidence interval (0.59, 1.09). Based on this result alone, the upper bound of the $95 \%$ confidence for the hazard ratio successfully ruled out a hazard ratio of the primary composite endpoint larger than 1.8 associated with dapagliflozin. No formal test to rule out a hazard ratio of 1.3 was pre-specified or conducted. 
Table 17. Analysis of the Primary Composite Endpoint in

All Trials in the Meta-Analysis

\begin{tabular}{|c|c|c|c|}
\hline & $\begin{array}{c}\text { Comparators } \\
\mathrm{N}=3403 \\
\mathrm{PY}=3831\end{array}$ & $\begin{array}{c}\text { Dapagliflozin } \\
\mathrm{N}=5936 \\
\mathrm{PY}=6594\end{array}$ & $\begin{array}{c}\text { Hazard Ratio } \\
(95 \% \mathrm{Cl})\end{array}$ \\
\hline Events (rate per 1000 PY) & $\mathbf{8 1 ( 2 1 . 1 )}$ & $\mathbf{9 7}(\mathbf{1 4 . 7})$ & $\mathbf{0 . 8 1}(\mathbf{0 . 5 9} \mathbf{1 . 0 9})$ \\
\hline
\end{tabular}

${ }^{1}$ Unadjusted rate of events pooled across all trials

Source: Created by reviewer. Dataset: adcv5.xpt

Figure 3 is a forest plot of trial specific hazard ratios for the primary composite endpoint in the meta-analysis. The $95 \%$ confidence intervals for each individual trial included 1.0, except for trial MB102014, which showed potential evidence of cardiovascular protection associated with dapagliflozin (HR 0.25 with 95\% CI 0.08-0.82). The two trials with the largest number of events were trials 19 (48 events) and 18 (39 events). The forest plot shows no evidence of heterogeneity of hazard ratios across trials.

Figure 3. Forest Plot of Estimated HR and $95 \% \mathrm{Cl}$ for the Primary Composite Endpoint

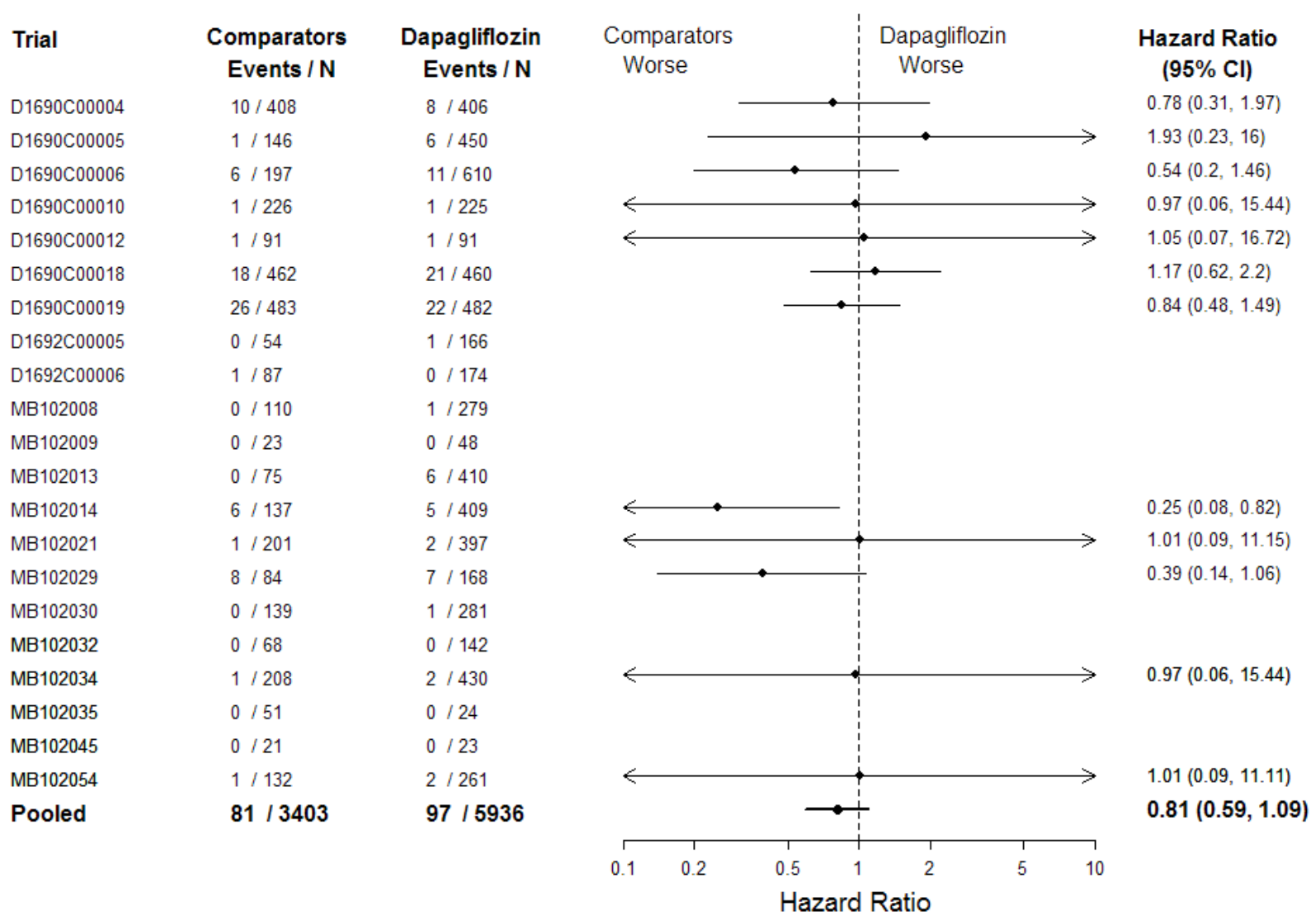

Figure 6 in the Appendix shows the plot of the scaled Schoenfeld residuals corresponding to the primary Cox model discussed above including all 21 trials in the meta-analysis. A plot of the scaled Schoenfeld residuals as a function of time was created to evaluate the 
assumption of proportional hazards. In this type of plot, a non-zero slope indicates a potential violation of the proportionality assumption. The slope of the loess curve in Figure 6 appears approximately flat through time and shows no evidence of nonproportional hazards.

\subsubsection{Analysis of the Primary Cardiovascular Composite Endpoint in Secondary Populations}

Table 18 shows results of the Cox proportional hazards model for the primary composite cardiovascular event in trials 18 and 19 alone. There were 44 events observed among 945 subjects randomized to placebo and 43 events among 942 subjects randomized to dapagliflozin in these two trials. The estimated hazard ratio and 95\% confidence interval for the primary composite endpoint associated with dapagliflozin was $\mathbf{0 . 9 8}$ with 95\% confidence interval $(\mathbf{0 . 6 4}, \mathbf{1 . 4 9})$. Figure 7 in the Appendix shows the plot of the scaled Schoenfeld residuals for the Cox proportional hazards model in trials 18 and 19. The plot shows no evidence of non-proportional hazards.

Table 19 shows results of a similar analysis conducted in the population of subjects with a history of CV disease, excluding those in trials 18 and 19. The estimated hazard ratio and $95 \%$ confidence interval in this population was $\mathbf{0 . 5 3}(\mathbf{0 . 2 8}, \mathbf{1 . 0 2})$.

Both sets of analyses show no evidence of increased cardiovascular risk associated with dapagliflozin.

Table 18. Analysis of the Primary Composite Endpoint in Trials 18 and 19 (Stratified)

\begin{tabular}{|c|c|c|c|}
\hline & $\begin{array}{c}\text { Placebo } \\
\mathrm{N}=945 \\
\mathrm{PY}=1119\end{array}$ & $\begin{array}{c}\text { Dapagliflozin } \\
\mathrm{N}=942 \\
\mathrm{PY}=1118\end{array}$ & $\begin{array}{c}\text { Hazard Ratio } \\
(95 \% \mathrm{Cl})\end{array}$ \\
\hline Events (rate per 1000 PY) & $\mathbf{4 4}(\mathbf{3 9 . 3})$ & $\mathbf{4 3 \mathbf { 3 8 . 5 }}$ & $\mathbf{0 . 9 8} \mathbf{( 0 . 6 4 , \mathbf { 1 . 4 9 } )}$ \\
\hline
\end{tabular}

1 Unadjusted rate of events across both trials

Source: Created by reviewer. Dataset: adcv5.xpt

Table 19. Analysis of the Primary Composite Endpoint in Subjects with a History of CV Disease, Excluding Trials 18 and 19

\begin{tabular}{|c|c|c|c|}
\hline & $\begin{array}{c}\text { Comparators } \\
\qquad \begin{array}{l}N=417 \\
P Y=510\end{array}\end{array}$ & $\begin{array}{c}\text { Dapagliflozin } \\
N=919 \\
P Y=1166\end{array}$ & $\begin{array}{l}\text { Hazard Ratio } \\
(95 \% \mathrm{Cl})\end{array}$ \\
\hline Events (rate per 1000 PY) ${ }^{1}$ & $17(33.3)$ & $25(21.4)$ & $0.53(0.28,1.02)$ \\
\hline
\end{tabular}

${ }^{1}$ Unadjusted rate of events pooled across all trials

Source: Created by reviewer. Dataset: adcv5.xpt 


\subsubsection{Analysis of the Secondary Cardiovascular Composite Endpoints}

Table 20 summarizes the analyses conducted on the secondary cardiovascular composite and MACE in the three populations of interest: all trials in the meta-analysis, trials 18 and 19 alone, and subjects with a history of CV disease excluding trials 18 and 19.

Five of the 6 estimated hazard ratios shown on Table 20 were smaller than 1.0. The only estimated hazard ratio greater than 1.0 was that of MACE in trials 18 and 19 (HR 1.11, $95 \%$ CI 0.67-1.83). Overall, the analyses summarized in Table 20 are consistent with the primary analysis and show no evidence of increased cardiovascular risk associated with dapagliflozin.

Table 20. Analysis of the Secondary Composite Endpoint and MACE in All Populations

\begin{tabular}{|c|c|c|c|}
\hline \multirow{2}{*}{$\begin{array}{l}\text { Population } \\
\text { Endpoint }\end{array}$} & \multicolumn{2}{|c|}{ Events } & \multirow{2}{*}{$\begin{array}{c}\text { Estimated HR } \\
(95 \% \mathrm{CI})\end{array}$} \\
\hline & Comparators & Dapagliflozin & \\
\hline All Trials in the Meta-Analysis & $N=3403$ & $N=5936$ & \\
\hline Secondary composite endpoint & 111 & 126 & $0.76(0.59,1.00)$ \\
\hline MACE & 62 & 73 & $0.78(0.55,1.11)$ \\
\hline Trials 18 and 19 (stratified) & $N=945$ & $N=942$ & \\
\hline Secondary composite endpoint & 63 & 56 & $0.89(0.62,1.27)$ \\
\hline MACE & 29 & 32 & $1.11(0.67,1.83)$ \\
\hline $\begin{array}{l}\text { Subjects with a history of CV disease, excluding } \\
\text { Trials } 18 \text { and } 19\end{array}$ & $N=417$ & $\mathbf{N}=919$ & \\
\hline Secondary composite endpoint & 24 & 38 & $0.57(0.33,0.97)$ \\
\hline MACE & 16 & 18 & $0.41(0.20,0.84)$ \\
\hline
\end{tabular}

*Secondary composite event: CV death, MI, stroke, hospitalization for unstable angina, unplanned coronary revascularization and hospitalization for heart failure

*MACE composite endpoint: CV death, MI, stroke

Source: Created by reviewer. Dataset: adcv5.xpt

\subsubsection{Analysis of Individual Components of the Primary Endpoint}

The primary cardiovascular endpoint is a composite of $\mathrm{CV}$ death, MI, stroke, and hospitalization for unstable angina. Table 21 shows hazard ratios and corresponding 95\% confidence intervals for time to event analyses for each of these individual components in the three populations of interest.

In the primary population of all 21 trials in the meta-analysis, the estimated hazard ratio was smaller than 1.0 for each of the four components of the primary composite. In the population of trials 18 and 19, there were 2 more strokes observed in the dapagliflozin arm than the placebo arm (HR 1.21, 95\% CI 0.52-2.80), but there were 3 more hospitalizations for unstable angina observed in the placebo arm (HR 0.80, 95\% CI 0.371.70). The population of subjects with a history of CV disease excluding trials 18 and 19 
experienced a small number of total events that produced wide confidence intervals for the hazard ratios of $\mathrm{CV}$ death, stroke and hospitalization for unstable angina.

The upper bound of the nominal $95 \%$ confidence interval for the hazards ratio of MI was smaller than 1.0 in the full population and the population of subjects with prior CV disease excluding trials 18 and 19, and suggested a potential decrease in the risk of MI associated with dapagliflozin. However, the estimated hazard ratio for MI in the population of trials 18 and 19 was 1.0 and showed no evidence of benefit.

Overall, the analyses of the individual components of the primary composite outcome are consistent with the primary analysis and show no evidence of increased cardiovascular risk for dapagliflozin.

Table 21. Analysis of the Individual Components of the Primary Composite Outcome

\begin{tabular}{|c|c|c|c|}
\hline \multirow{2}{*}{$\begin{array}{l}\text { Population } \\
\text { Endpoint } \\
\end{array}$} & \multicolumn{2}{|c|}{ Events } & \multirow{2}{*}{$\begin{array}{l}\text { Estimated HR } \\
\quad(95 \% \mathrm{Cl})\end{array}$} \\
\hline & Comparators & Dapagliflozin & \\
\hline All Trials in the Meta-Analysis & $N=3403$ & $N=5936$ & \\
\hline CV Death & 18 & 20 & $0.71(0.37,1.37)$ \\
\hline $\mathrm{Ml}$ & 33 & 31 & $0.59(0.35,0.97)$ \\
\hline Stroke & 18 & 25 & $1.00(0.54,1.86)$ \\
\hline Hospitalization for unstable angina & 20 & 27 & $0.91(0.50,1.66)$ \\
\hline Trials 18 and 19 (stratified) & $N=945$ & $N=942$ & \\
\hline CV Death & 9 & 8 & $0.89(0.34,2.30)$ \\
\hline $\mathrm{Ml}$ & 12 & 12 & $1.00(0.45,2.23)$ \\
\hline Stroke & 10 & 12 & $1.21(0.52,2.80)$ \\
\hline Hospitalization for unstable angina & 15 & 12 & $0.80(0.37,1.70)$ \\
\hline $\begin{array}{l}\text { Subjects with a history of CV disease, excluding } \\
\text { Trials } 18 \text { and } 19\end{array}$ & $N=417$ & $\mathbf{N}=919$ & \\
\hline CV Death & 4 & 8 & $0.65(0.19,2.22)$ \\
\hline $\mathrm{Ml}$ & 10 & 6 & $0.24(0.08,0.68)$ \\
\hline Stroke & 4 & 6 & $0.62(0.16,2.37)$ \\
\hline Hospitalization for unstable angina & 1 & 7 & $2.52(0.30,21.33)$ \\
\hline
\end{tabular}

Source: Created by reviewer. Dataset: adcv5.xpt

\subsubsection{Sensitivity Analyses using Mantel-Haenszel Incidence Rate Ratio and Random Effects Incidence Rate Ratio}

A pre-specified sensitivity analysis estimated the Mantel-Haenszel incidence rate ratio for the primary composite, the secondary composite and MACE associated with dapagliflozin. Table 22 shows the estimated Mantel-Haenszel incidence rate ratios and corresponding $95 \%$ confidence intervals. In every case, the estimated incidence rate ratios in Table 22 were within $2 \%$ of the estimated hazard ratios shown in Tables 13-16. 
Table 22. Mantel-Haenszel Incidence Rate Ratio

\begin{tabular}{|c|c|c|c|}
\hline \multirow{2}{*}{$\begin{array}{r}\text { Population } \\
\text { Endpoint } \\
\end{array}$} & \multicolumn{2}{|c|}{ Events } & \multirow{2}{*}{$\begin{array}{c}\text { MH Incidence } \\
\text { Rate Ratio } \\
(95 \% \mathrm{Cl}) \\
\end{array}$} \\
\hline & Comparators & Dapagliflozin & \\
\hline All Trials in the Meta-Analysis & $P Y^{*}=3831$ & $P Y^{*}=6594$ & \\
\hline Primary composite endpoint & 81 & 97 & $0.80(0.59,1.09)$ \\
\hline Secondary composite endpoint & 111 & 126 & $0.76(0.58,0.99)$ \\
\hline MACE & 62 & 73 & $0.78(0.55,1.10)$ \\
\hline Trials 18 and 19 (stratified) & $P Y^{*}=1119$ & $P Y^{*}=1118$ & \\
\hline Primary composite endpoint & 44 & 43 & $0.98(0.64,1.50)$ \\
\hline Secondary composite endpoint & 63 & 56 & $0.88(0.61,1.28)$ \\
\hline MACE & 29 & 32 & $1.11(0.67,1.85)$ \\
\hline $\begin{array}{l}\text { Subjects with a history of CV disease, excluding } \\
\text { Trials } 18 \text { and } 19\end{array}$ & $P Y^{*}=510$ & $P Y^{*}=1166$ & \\
\hline Primary composite endpoint & 17 & 25 & $0.52(0.27,1.00)$ \\
\hline Secondary composite endpoint & 24 & 38 & $0.57(0.33,0.98)$ \\
\hline MACE & 16 & 18 & $0.40(0.19,0.82)$ \\
\hline
\end{tabular}

${ }^{*}$ Patient Years for primary composite endpoint. The number of patient years for the secondary endpoints are slightly different.

Source: Created by reviewer. Dataset: adcv5.xpt

Table 23 shows the estimated random effects incidence rate ratios and corresponding $95 \%$ confidence intervals for the primary composite, the secondary composite and MACE. These rate ratios were calculated to assess the impact of potential trial heterogeneity on estimates of cardiovascular risk. Random effects models tend to produce wider confidence intervals in the presence of heterogeneity. The estimated random effects rate ratios and corresponding $95 \%$ confidence intervals were very similar to the hazard ratios in Tables 13-16, particularly in the primary population and the population of trials 18 and 19.

The $\tau^{2}$ statistic was calculated for the random effects models of the primary composite endpoint. This statistic is a measure of the variability between trials. The method of moments estimator of $\tau^{2}$ for the primary endpoint in the population of all trials and the population of trials 18 and 19 alone was zero and indicates no evidence of heterogeneity. The estimator of $\tau^{2}$ in the population of subjects with a history of CV disease excluding trials 18 and 19 was 0.41 and indicates moderate heterogeneity in this population.

The estimates of both the Mantel-Haenszel incidence rate ratio and the random effects incidence rate ratio were consistent with the primary pre-specified analyses. These analyses show no evidence of increased cardiovascular risk associated with dapagliflozin. 
Table 23. Random Effects Incidence Rate Ratio

\begin{tabular}{|lccc|}
\hline Population & \multicolumn{2}{c}{ Events } & $\begin{array}{c}\text { Random Effects } \\
\text { Rate Ratio } \\
\text { Endpoint }\end{array}$ \\
\hline All Trials in the Meta-Analysis
\end{tabular}

${ }^{*}$ Patient Years for primary composite endpoint. The number of patient years for the secondary endpoints are slightly different.

Source: Created by reviewer. Dataset: adcv5.xpt

\subsubsection{Sensitivity Analysis of Cardiovascular Risk Comparing Dapagliflozin $10 \mathrm{mg}$ to all Comparators}

There were 2909 subjects randomized to dapagliflozin $10 \mathrm{mg}$ in 17 trials in the metaanalysis. Three trials did not randomize any subjects to dapagliflozin $10 \mathrm{mg}$ : MB102021, MB102032 and MB102045. Trial D1690C00004 titrated subjects to dapagliflozin doses between $2.5 \mathrm{mg}$ and $10 \mathrm{mg}$, and is not included in this sensitivity analysis. Table 24 shows the estimated hazard ratios for the primary and secondary endpoints and MACE comparing subjects randomized to dapagliflozin $10 \mathrm{mg}$ to all comparators in the remaining 17 trials in the meta-analysis. The results of this analysis are consistent with the primary analysis.

Table 24. Cardiovascular Risk Comparing Dapagliflozin $10 \mathrm{mg}$ to All Comparators

\begin{tabular}{|lccc|}
\hline & \multicolumn{2}{c}{ Events } & Estimated HR \\
Endpoint & Comparators & $\begin{array}{c}\text { Dapagliflozin } \\
\mathbf{1 0} \mathbf{~ m g}\end{array}$ & \begin{tabular}{c}
$\mathbf{( 9 5 \%} \mathbf{~ C l})$ \\
\hline
\end{tabular} \\
Primary composite endpoint & $\mathbf{N}=\mathbf{2 7 0 5}$ & $\mathbf{N}=\mathbf{2 9 0 9}$ & \\
Secondary composite endpoint & 70 & 61 & $0.84(0.60,1.19)$ \\
MACE & 96 & 76 & $0.76(0.56,1.03)$ \\
\hline
\end{tabular}

Source: Created by reviewer. Dataset: adcv5.xpt 


\subsubsection{Cardiovascular Endpoints Observed within the First $\mathbf{3 0}$ Days after Randomization in the Dapagliflozin Program}

As mentioned in Section 3.1.3.4, a post-hoc analysis was conducted to examine the risk of cardiovascular events associated with dapagliflozin within the first 30 days after randomization in the 21 trials in the meta-analysis. This analysis was motivated by an imbalance of early cardiovascular events observed in the development program for canagliflozin, a recently approved SGLT2 inhibitor for the treatment of Type 2 diabetes mellitus.

An imbalance of cardiovascular events was observed within the first 30 days of treatment with dapagliflozin in the meta-analysis as shown in Table 25. There were 8 primary events observed among 5936 subjects randomized to dapagliflozin $(0.13 \%)$ and 2 primary events (plus 2 secondary events) among 3403 subjects randomized to comparators $(0.06 \%)$. Four of the 8 events in the dapagliflozin arm occurred within the first week after randomization. The corresponding estimated hazard ratio and $95 \%$ confidence interval for primary events associated with dapagliflozin within the first 30 days in the 21 trials was $2.77(0.57,13.33)$.

Table 25. List of Primary and Secondary Composite Cardiovascular Events within the First 30 Days after Randomization in the Dapagliflozin Program

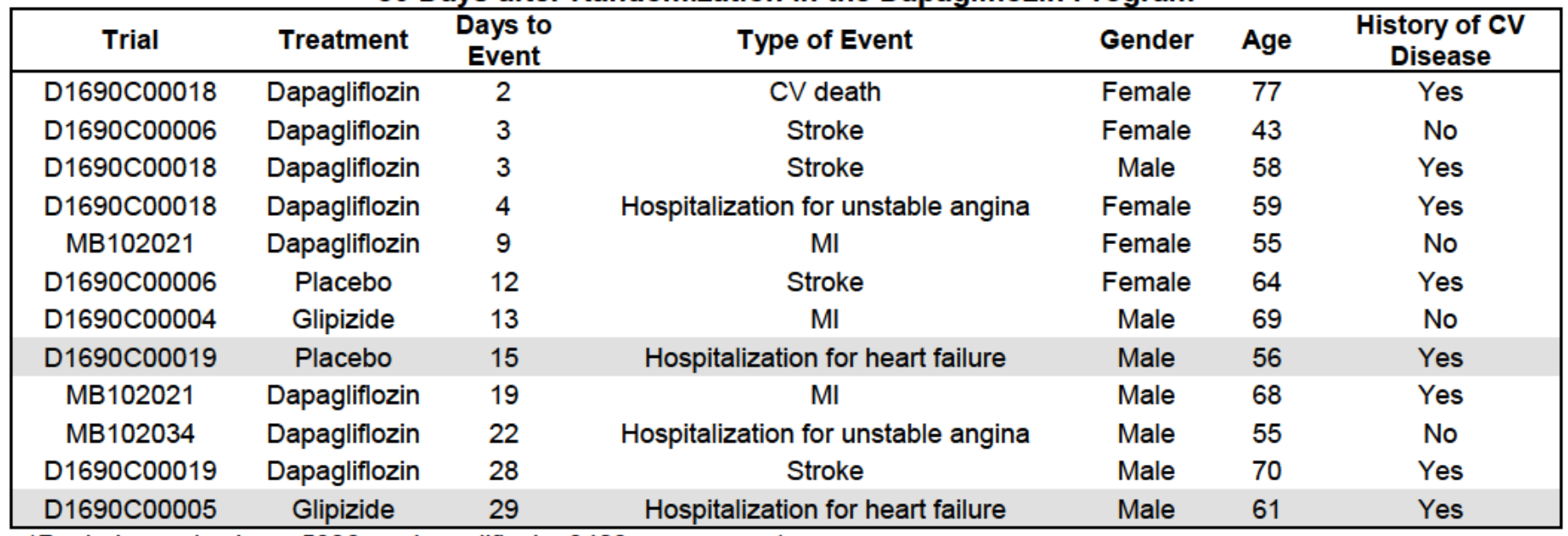

${ }^{\text {*}}$ Pooled sample size $=5936$ on dapagliflozin, 3403 on comparators

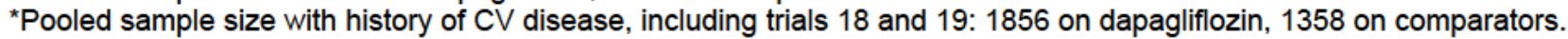

The two subjects highlighted in gray experienced the secondary composite outcome but no the primary composite outcome.

In trials 18 and 19 alone, there were 4 primary events observed among 942 subjects randomized to dapagliflozin and 0 primary events (plus 1 secondary) among 945 subjects randomized to placebo within the first 30 days after randomization. Figure 4 shows the Kaplan-Meier plots of the observed cumulative probability of primary composite events in trials 18 and 19 alone. The plot shows the imbalance of early events in favor of the placebo arm. However, throughout the full duration of these two trials, both treatment arms showed a comparable risk profile: HR $0.98(0.64,1.49)$, logrank test p-value 0.92 . 
Figure 4. Observed Cumulative Probability of Primary Composite Endpoint in Trials 18 and 19

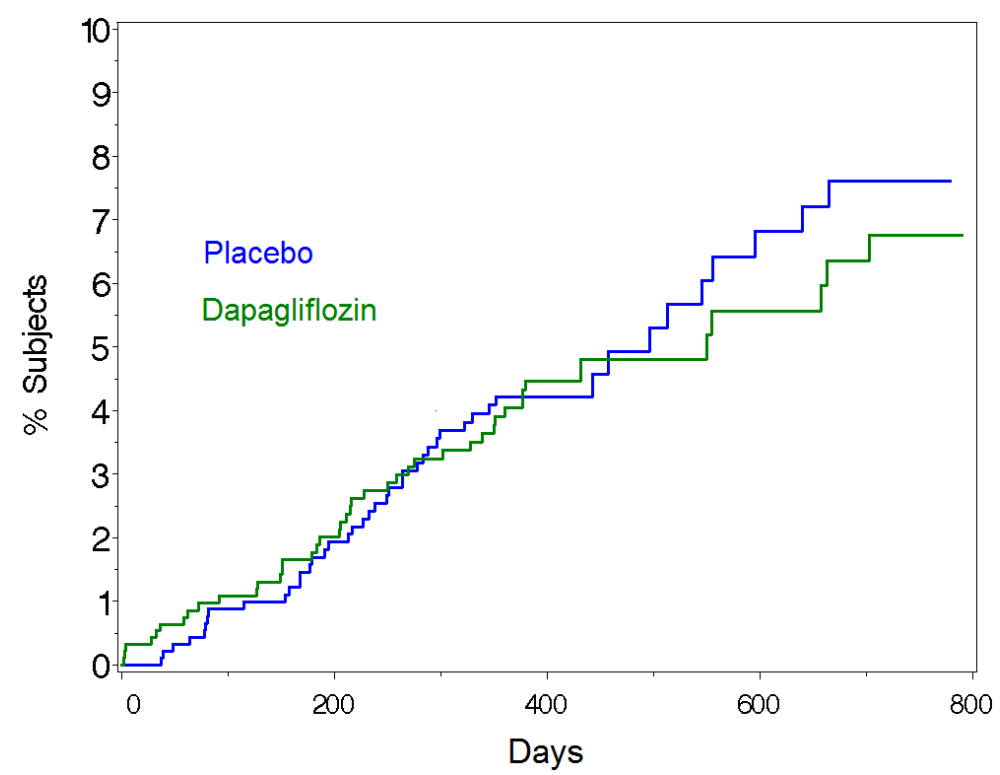

It is not clear whether the early imbalance of cardiovascular events observed in the canagliflozin and dapagliflozin programs may be associated with the use of these products or may be attributable to chance.

\section{Findings in Special/Subgroup Populations}

In this Section, the risk of the primary composite endpoint associated with dapagliflozin is evaluated within subgroups defined by gender, race, age and country of randomization. Results are presented for the population of all 21 trials in the meta-analysis and the population of trials 18 and 19 alone. Note that these subgroup analyses were not prespecified in the Statistical Analysis Plan. Unadjusted 95\% confidence intervals are presented for each estimated hazard ratio. Therefore, the results of these subgroup analyses should be used for hypothesis generation only.

\subsection{Gender, Race, Age and Country of Randomization}

\section{Gender}

Among the 9339 subjects in the meta-analysis (5936 on dapagliflozin and 3403 on comparators), 56\% were male and 34\% were female. The estimated HR of the primary endpoint associated with dapagliflozin among males in the population of all trials was 0.81 with $95 \%$ CI $(0.56,1.17)$, and $0.87(0.52,1.44)$ in trials 18 and 19 alone. Among females, the estimated HR of the primary endpoint was $0.81(0.47,1.38)$ in the 
population of all trials and $1.31(0.60,2.85)$ in trials 18 and 19 alone. These data showed no statistically significant evidence of differential cardiovascular risk associated with dapagliflozin by gender.

Table 26. Analysis of the Primary Endpoint, by Gender

\begin{tabular}{|c|c|c|c|}
\hline \multicolumn{4}{|c|}{ Gender $=$ Male $(\mathrm{N}=5207)$} \\
\hline & All Comparators & Dapagliflozin & Hazard Ratio \\
\hline All Trials in the Meta-Analysis & $55 / 1964 \quad(2.8 \%)$ & $65 / 3243 \quad(2.0 \%)$ & $0.81(0.56,1.17)$ \\
\hline Trials 18 and 19 & $33 / 639 \quad(5.2 \%)$ & $28 / 634 \quad(4.4 \%)$ & $0.87(0.52,1.44)$ \\
\hline \multicolumn{4}{|c|}{ Gender $=$ Female $(\mathrm{N}=4132)$} \\
\hline & All Comparators & Dapagliflozin & Hazard Ratio \\
\hline All Trials in the Meta-Analysis & $26 / 1439(1.8 \%)$ & $32 / 2693 \quad(1.2 \%)$ & $0.81(0.47,1.38)$ \\
\hline Trials 18 and 19 & $11 / 306 \quad(3.6 \%)$ & $15 / 308 \quad(4.9 \%)$ & $1.31(0.60,2.85)$ \\
\hline
\end{tabular}

Source: Created by reviewer. Dataset: adcv5.xpt

\section{Race}

$76.5 \%$ of the subjects in the meta-analysis were White, $16.7 \%$ were Asian, $3.6 \%$ were Black and the remaining $3.2 \%$ were identified as other races. The estimated HR of the primary endpoint associated with dapagliflozin among Whites in the population of all trials was $0.77(0.55,1.07)$ and $1.00(0.64,1.57)$ in trials 18 and 19 alone. Table 27 shows that the confidence intervals for the hazard ratio of the primary endpoint were very wide among subjects whose race was not White, due to their relatively small sample size. The data showed no evidence of differential risk of the primary composite endpoint associated with dapagliflozin between subgroups defined by race.

Table 27. Analysis of the Primary Endpoint, by Race

\begin{tabular}{|c|c|c|c|}
\hline \multicolumn{4}{|c|}{ Race = Asian ( $\mathrm{N}=1563)$} \\
\hline & All Comparators & Dapagliflozin & Hazard Ratio \\
\hline All Trials in the Meta-Analysis & $3 / 513 \quad(0.6 \%)$ & $12 / 1050(1.1 \%)$ & $2.43(0.67,8.85)$ \\
\hline Trials 18 and 19 & $1 / 45 \quad(2.2 \%)$ & $4 / 55 \quad(7.3 \%)$ & $3.38(0.38,30.39)$ \\
\hline \multicolumn{4}{|c|}{ Race $=$ Black $(\mathrm{N}=333)$} \\
\hline & All Comparators & Dapagliflozin & Hazard Ratio \\
\hline All Trials in the Meta-Analysis & $4 / 125 \quad(0.03 \%)$ & $2 / 208 \quad(1.0 \%)$ & $0.29(0.04,1.91)$ \\
\hline Trials 18 and 19 & $3 / 37 \quad(8.1 \%)$ & $1 / 35 \quad(2.9 \%)$ & $0.36(0.04,3.55)$ \\
\hline \multicolumn{4}{|c|}{ Race $=$ White $(\mathrm{N}=7149)$} \\
\hline All Trials in the Meta-Analysis & $71 / 2644 \quad(2.7 \%)$ & $81 / 4505(1.8 \%)$ & $0.77(0.55,1.07)$ \\
\hline Trials 18 and 19 & $38 / 844 \quad(4.5 \%)$ & $38 / 837 \quad(4.5 \%)$ & $1.00(0.64,1.57)$ \\
\hline \multicolumn{4}{|c|}{ Race $=$ Other $(\mathrm{N}=294)$} \\
\hline All Trials in the Meta-Analysis & $3 / 121 \quad(2.5 \%)$ & $2 / 173 \quad(1.2 \%)$ & $0.74(0.12,4.58)$ \\
\hline Trials 18 and 19 & $2 / 19 \quad(10.5 \%)$ & $0 / 15 \quad(0 \%)$ & - \\
\hline
\end{tabular}

Source: Created by reviewer. Dataset: adcv5.xpt 


\section{Age at Baseline}

$59.4 \%$ of the subjects in the meta-analysis were 60 years old or younger at the time of randomization, and $40.6 \%$ were older than 60 years. The estimated hazard ratio of the primary endpoint in the population of all trials in the meta-analysis was 0.82 in both age groups. The estimated hazard ratio was numerically higher in the younger group (HR $1.37,95 \%$ CI $0.71-2.66$ ) than in the older group (HR $0.77,95 \%$ CI $0.44-1.35$ ) in trials 18 and 19 , but the difference was not statistically significant.

Table 28. Analysis of the Primary Endpoint, by Age at Baseline

\begin{tabular}{|lccc|}
\hline \multicolumn{5}{|c|}{ Age $\leq 60$ (N=5552) } \\
\hline & All Comparators & Dapagliflozin & Hazard Ratio \\
\hline All Trials in the Meta-Analysis & $30 / 1896(1.6 \%)$ & $36 / 3656(1.0 \%)$ & $0.82(0.50,1.35)$ \\
Trials 18 and 19 & $15 / 334(4.5 \%)$ & $21 / 342(6.1 \%)$ & $1.37(0.71,2.66)$ \\
\hline \multicolumn{5}{c}{ Age $>60(\mathrm{~N}=3787)$} \\
\hline
\end{tabular}

Source: Created by reviewer. Dataset: adcv5.xpt

\section{Country of Randomization}

$21.5 \%$ of the subjects in the meta-analysis were randomized in the United States. In the population of trials 18 and 19 , this proportion was $24.4 \%$. The estimated hazard ratio and $95 \%$ confidence interval for the primary endpoint associated with dapagliflozin among subjects randomized in the United States was $0.53(0.26,1.09)$ in the population of all trials in the meta-analysis, and $0.36(0.13,1.05)$ in trials 18 and 19. Among subjects randomized outside of the United States, the estimated hazard ratios were $0.88(0.62$, $1.23)$ and $1.19(0.75,1.90)$ respectively.

Table 29. Analysis of the Primary Endpoint, by Country of Randomization

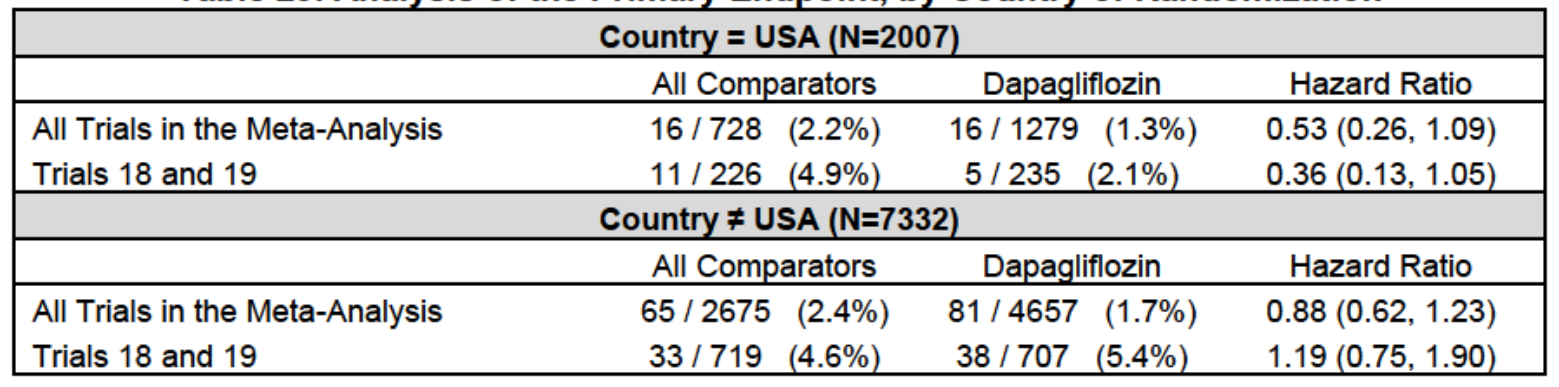

Source: Created by reviewer. Dataset: adcv5.xpt 
Overall, no statistically significant differences in the risk of the primary cardiovascular composite endpoint associated with dapagliflozin were observed among subgroups defined by gender, race, age or country of randomization. These subgroup analyses are summarized in Figure 5 for the population of all 21 trials in the meta-analysis.

Figure 5. Hazard Ratio of the Primary Composite Endpoint by Subgroups in All Trials

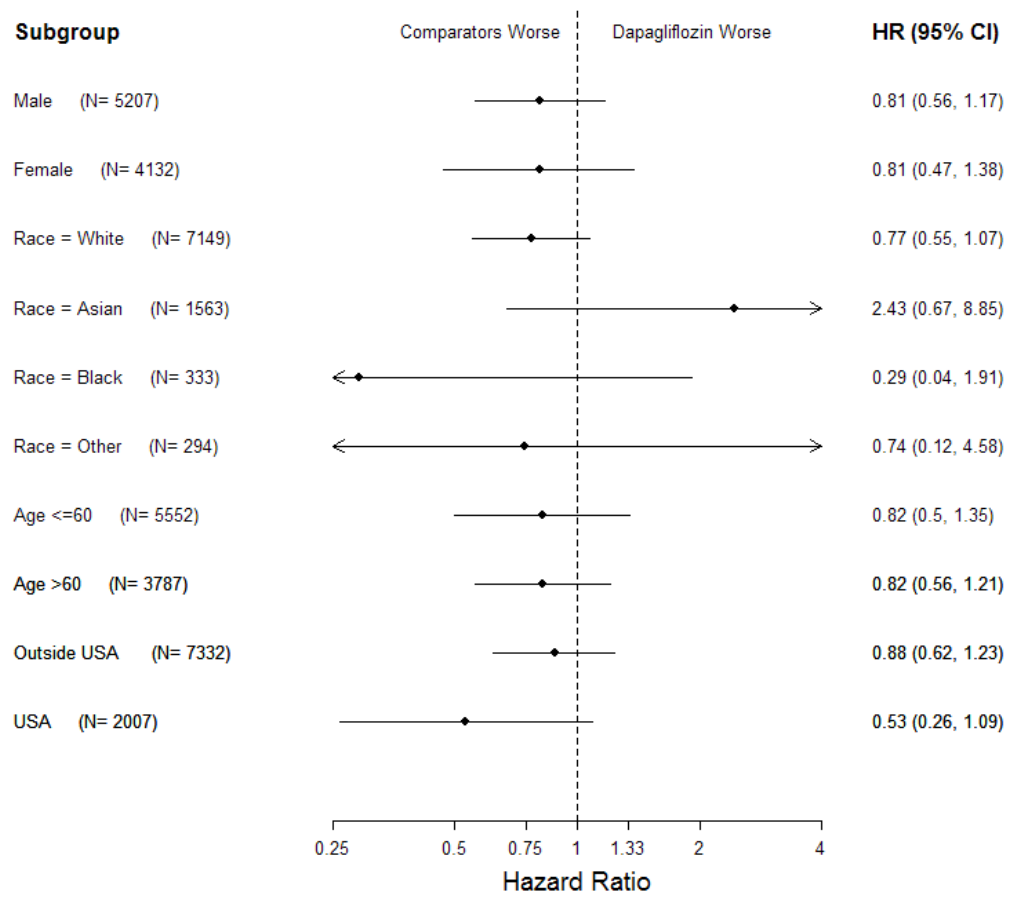

\subsection{Other Special/Subgroup Populations}

This Section discusses analyses of the risk of the primary composite endpoint associated with dapagliflozin in subgroups defined by baseline categories of: BMI, eGFR, and duration of diabetes. These subgroup analyses are presented for the population of all trials in the meta-analysis and trials 18 and 19 alone. These subgroup analyses were not prespecified in the Statistical Analysis Plan and should be used for hypothesis generation only. Unadjusted $95 \%$ confidence intervals are presented for each estimated hazard ratio.

\section{BMI at Baseline}

Approximately $43.7 \%$ of all subjects in the meta-analysis had a baseline BMI less than or equal to $30 \mathrm{~kg} / \mathrm{m}^{2}$. The remaining $56.3 \%$ of subjects had a baseline BMI larger than 30 $\mathrm{kg} / \mathrm{m}^{2}$. The estimated hazard ratio of the primary composite endpoint associated with dapagliflozin was not statistically different between subgroups defined by baseline BMI 
in both the population of all trials in the meta-analysis and in trials 18 and 19 alone. The estimated hazard ratios and $95 \%$ confidence intervals are shown in Table 30 .

Table 30. Analysis of the Primary Endpoint, by Baseline BMI

\begin{tabular}{|c|c|c|c|}
\hline \multicolumn{4}{|c|}{$\mathrm{BMI} \leq 30 \mathrm{~kg} / \mathrm{m}^{2}(\mathrm{~N}=4082)$} \\
\hline & All Comparators & Dapagliflozin & Hazard Ratio \\
\hline All Trials in the Meta-Analysis & $31 / 1479 \quad(2.1 \%)$ & $45 / 2603 \quad(1.7 \%)$ & $0.99(0.62,1.59)$ \\
\hline Trials 18 and 19 & $14 / 317 \quad(4.4 \%)$ & $16 / 311(5.1 \%)$ & $1.25(0.61,2.56)$ \\
\hline \multicolumn{4}{|c|}{$\mathrm{BMI}>30 \mathrm{~kg} / \mathrm{m}^{2}(\mathrm{~N}=5257)$} \\
\hline All Trials in the Meta-Analysis & $50 / 1924 \quad(2.6 \%)$ & $52 / 3333(1.6 \%)$ & $0.70(0.47,1.04)$ \\
\hline Trials 18 and 19 & $30 / 628 \quad(4.8 \%)$ & $27 / 631 \quad(4.3 \%)$ & $0.87(0.52,1.46)$ \\
\hline
\end{tabular}

Source: Created by reviewer. Dataset: adcv5.xpt

\section{Baseline eGFR}

9077 out of 9339 subjects in the meta-analysis had a recorded eGFR measurement at baseline. Approximately $11.7 \%$ of these subjects had a baseline eGFR measurement of less than $60 \mathrm{ml} / \mathrm{min}$. Subjects with a lower baseline eGFR had numerically lower hazard ratios for the primary composite endpoint associated with dapagliflozin in both the population of all trials and in trials 18 and 19 alone as shown in Table 31. However, the difference was not statistically significant.

Table 31. Analysis of the Primary Endpoint, by Baseline eGFR

\begin{tabular}{|c|c|c|c|}
\hline \multicolumn{4}{|c|}{ Baseline eGFR $<60 \mathrm{ml} / \mathrm{min}(\mathrm{N}=1064)$} \\
\hline & All Comparators & Dapagliflozin & Hazard Ratio \\
\hline All Trials in the Meta-Analysis & $24 / 391 \quad(6.1 \%)$ & $26 / 673 \quad(3.9 \%)$ & $0.58(0.32,1.05)$ \\
\hline Trials 18 and 19 & $11 / 160 \quad(6.9 \%)$ & $8 / 168 \quad(4.8 \%)$ & $0.74(0.30,1.85)$ \\
\hline \multicolumn{4}{|c|}{ Baseline eGFR $\geq 60 \mathrm{ml} / \mathrm{min}(\mathrm{N}=8013)$} \\
\hline All Trials in the Meta-Analysis & $56 / 2924 \quad(1.9 \%)$ & $71 / 5089 \quad(1.4 \%)$ & $0.92(0.64,1.32)$ \\
\hline Trials 18 and 19 & $33 / 784 \quad(4.2 \%)$ & $35 / 774 \quad(4.5 \%)$ & $1.05(0.66,1.70)$ \\
\hline
\end{tabular}

Source: Created by reviewer. Dataset: adcv5.xpt

\section{Diabetes Duration}

Approximately $72.5 \%$ of the subjects in the meta-analysis and $43.1 \%$ of subjects in trials 18 and 19 had diabetes for fewer than 10 years at the time of randomization. Among these subjects, the estimated hazard ratio of the primary composite endpoint associated with dapagliflozin was $0.99(0.63,1.54)$ in the population of all trials and $1.32(0.70$, $2.48)$ in trials 18 and 19 alone. 
Among the $27.5 \%$ of subjects in the meta-analysis and $56.9 \%$ in trials 18 and 19 with diabetes durations longer than 10 years at baseline, the estimated hazard ratios and $95 \%$ confidence intervals were $0.67(0.43,1.03)$ and $0.77(0.44,1.37)$ respectively.

Table 32. Analysis of the Primary Endpoint, by Diabetes Duration at Baseline

\begin{tabular}{|c|c|c|c|}
\hline \multicolumn{4}{|c|}{ Diabetes duration $\leq 10$ years $(\mathrm{N}=6772)$} \\
\hline & All Comparators & Dapagliflozin & Hazard Ratio \\
\hline All Trials in the Meta-Analysis & $36 / 2414 \quad(1.5 \%)$ & $52 / 4358$ (1.2\%) & $0.99(0.63,1.54)$ \\
\hline Trials 18 and 19 & $18 / 426 \quad(4.2 \%)$ & $21 / 387 \quad(5.4 \%)$ & $1.32(0.70,2.48)$ \\
\hline \multicolumn{4}{|c|}{ Diabetes duration $>10$ years $(\mathrm{N}=2567)$} \\
\hline & All Comparators & Dapagliflozin & Hazard Ratio \\
\hline All Trials in the Meta-Analysis & $45 / 989 \quad(4.6 \%)$ & $45 / 1578 \quad(2.9 \%)$ & $0.67(0.43,1.03)$ \\
\hline Trials 18 and 19 & $26 / 519(5.0 \%)$ & $22 / 555(4.0 \%)$ & $0.77(0.44,1.37)$ \\
\hline
\end{tabular}

Source: Created by reviewer. Dataset: adcv5.xpt

The subgroup analyses defined by baseline categories of BMI, eGFR, and duration of diabetes showed no evidence of differential cardiovascular risk associated with dapagliflozin in these subgroups.

\section{$5 \quad$ Summary and Conclusions}

\subsection{Statistical Issues and Collective Evidence}

Based on the Statistical Analysis Plan (SAP) to assess Cardiovascular Safety dated March $3^{\text {rd }}, 2010$ and agreed upon by the FDA, the cardiovascular safety of dapagliflozin was evaluated through a meta-analysis of 21 Phase 2 and Phase 3 randomized clinical trials. The agreed upon primary population in the meta-analysis consisted of the randomized, controlled, short term plus long term periods of the 21 trials. A secondary analysis population consisted of two trials, trials ' 18 ' and '19', that enrolled subjects with a prior history of cardiovascular disease. The primary agreed upon safety endpoint of interest was the composite of CV death, MI, stroke and hospitalization for unstable angina. There were two secondary endpoints:

1. the composite of $\mathrm{CV}$ death, MI, stroke, hospitalization for unstable angina, unplanned coronary revascularization, and hospitalization for heart failure; and

2. MACE, a composite of CV death, $\mathrm{MI}$ and stroke.

The meta-analysis was designed to demonstrate that the hazard ratio of the primary composite endpoint of $\mathrm{CV}$ death, MI, stroke, and hospitalization for unstable angina associated with dapagliflozin relative to all comparators is smaller than the risk margin of 1.8 set forth in the FDA Diabetes Guidance for assessing cardiovascular safety. The prespecified primary model was a Cox proportional hazards model stratified by trial. 
There were 97 primary events observed among 5936 subjects randomized to dapagliflozin and 81 primary events observed among 3403 subjects randomized to comparators in the 21 trials used in the meta-analysis. The pre-specified Cox proportional hazards model stratified by each of the 21 trials yielded an estimated hazard ratio of dapagliflozin relative to all comparators of $\mathbf{0 . 8 1}$ with a $95 \%$ confidence interval of $\mathbf{( 0 . 5 9}$, 1.09). The upper bound of this $95 \%$ confidence interval meets the risk margin of 1.8 necessary to show adequate pre-marketing cardiovascular safety of new antidiabetic products in accordance to the FDA Diabetes Guidance of 2008.

The Agency requested that secondary analyses of MACE be conducted on all analyses populations. MACE is a composite of CV Death, myocardial infarction and stroke. There were 73 MACE observed 5936 among subjects randomized to dapagliflozin and 62 MACE among 3403 subjects randomized to comparators in the primary analysis population of all 21 trials. The corresponding estimated hazard ratio of MACE associated with dapagliflozin relative to all comparators in these 21 trials was $\mathbf{0 . 7 8}$ with a 95\% confidence interval of $(\mathbf{0 . 5 5}, \mathbf{1 . 1 1})$.

In the two trials with high baseline cardiovascular risk, trials 18 and 19, there were 43 primary events observed among 942 subjects randomized to dapagliflozin and 44 primary events among 945 subjects randomized to placebo. The corresponding estimated hazard ratio of the primary endpoint associated with dapagliflozin relative to all comparators in trials 18 and 19 was $\mathbf{0 . 9 8}$ with a 95\% confidence interval of $(\mathbf{0 . 6 4}, \mathbf{1 . 4 9})$. Similarly there were 32 MACE observed among subjects randomized to dapagliflozin and 29 among subjects randomized to placebo in these two trials. The estimated hazard ratio of MACE associated with dapagliflozin in trials 18 and 19 was $\mathbf{1 . 1 1}$ with $95 \%$ confidence interval $(0.67,1.83)$.

A summary of these analyses is presented in Table 33. A detailed discussion of these results is provided in Section 3.1.6 of this review.

Table 33. Analyses of the Primary Composite Endpoint and MACE

\begin{tabular}{|c|c|c|c|}
\hline & $\begin{array}{c}\text { Comparators } \\
N=3403 \\
P Y=3831\end{array}$ & $\begin{array}{c}\text { Dapagliflozin } \\
N=5936 \\
P Y=6594\end{array}$ & $\begin{array}{l}\text { Hazard Ratio } \\
(95 \% \mathrm{Cl})\end{array}$ \\
\hline \multicolumn{4}{|l|}{ All Trials in the Meta-Analysis } \\
\hline Primary events $^{1}{\text { (rate per } 1000 \mathrm{PY})^{2}}^{2}$ & $81(21.1)$ & $97(14.7)$ & $0.81(0.59,1.09)$ \\
\hline $\mathrm{MACE}^{3}$ & $62(16.2)$ & $73(11.1)$ & $0.78(0.55,1.11)$ \\
\hline \multicolumn{4}{|l|}{ Trials 18 and 19} \\
\hline Primary events $^{1}{\text { (rate per } 1000 \mathrm{PY})^{2}}^{2}$ & $44(39.3)$ & $43(38.5)$ & $0.98(0.64,1.49)$ \\
\hline $\mathrm{MACE}^{3}$ & $29(25.9)$ & $32(28.6)$ & $1.11(0.67,1.83)$ \\
\hline
\end{tabular}

${ }^{1} \mathrm{CV}$ death, MI, stroke, hospitalization for unstable angina

${ }^{2}$ Unadjusted rate of events pooled across all trials

${ }^{3} \mathrm{CV}$ death, MI, stroke

Source: Created by reviewer. Dataset: adcv5.xpt 


\subsection{Conclusions and Recommendations}

Bristol-Myers Squibb and Astra Zeneca evaluated the cardiovascular safety of dapagliflozin through a meta-analysis of 21 randomized clinical trials. The pre-specified primary Cox proportional hazards model for this meta-analysis obtained an estimated hazard ratio for the primary cardiovascular endpoint (composite of CV death, MI, stroke, and hospitalization for unstable angina) of $\mathbf{0 . 8 1}$ with corresponding 95\% confidence interval $(\mathbf{0 . 5 9}, \mathbf{1 . 0 9})$. The upper bound of this $95 \%$ confidence interval was smaller than 1.8 and therefore met the hazard ratio risk margin set forth in the FDA Guidance to establish cardiovascular safety of new antidiabetic products pre-marketing.

A secondary analysis of the primary endpoint was conducted in the two trials that enrolled subjects with a history of cardiovascular disease, trials 18 and 19. The results of this analysis were consistent with the results of the primary analysis. The estimated hazard ratio for the primary event in trials 18 and 19 was $\mathbf{0 . 9 8}$ with corresponding 95\% confidence interval $(\mathbf{0 . 6 4}, \mathbf{1 . 4 9})$.

Analyses of MACE and of the secondary composite endpoint (which included unplanned coronary revascularization and hospitalization for heart failure) in the population of all trials in the meta-analysis and in the population of trials 18 and 19 were consistent with the primary analysis. Overall, the data submitted in support of this application show no evidence of increased cardiovascular risk associated with dapagliflozin.

A small imbalance of primary events was observed within the first 30 days after randomization in the 21 trials: 8 primary events were observed among 5936 subjects randomized to dapagliflozin $(0.13 \%)$ compared to 2 primary events among 3403 subjects randomized to comparators $(0.06 \%)$. This post-hoc analysis was motivated by a similar imbalance observed during the first 30 days of the dedicated outcomes trial "CANVAS" for canagliflozin. Due to the small number of events, it is not possible to determine whether this imbalance is attributable to dapagliflozin or whether it might have been caused by chance.

The Sponsors propose to evaluate the cardiovascular safety of dapagliflozin after approval through a dedicated cardiovascular outcomes trial, "D1693C00001". This trial was started in April 2013 with the goal of showing that the upper bound of the 95\% confidence interval for the hazard ratio of MACE associated with dapagliflozin is smaller than 1.3 relative to placebo. This trial is not part of the updated meta-analysis discussed in this document.

\section{$6 \quad$ References}

1. Food and Drug Administration. Guidance for industry: Diabetes mellitus - evaluating cardiovascular risk in new antidiabetic therapies to treat type 2 diabetes. December 2008. (www.fda.gov/downloads/Drugs/GuidanceComplianceRegulatorylnformation/Guidances/ucm 071627.pdf) 


\section{$7 \quad$ Appendix}

\section{A.1 Evaluation of Proportional Hazards Assumption}

Figure 6. Evaluation of Proportional Hazards: Schoenfeld Residuals Plots in All Trials

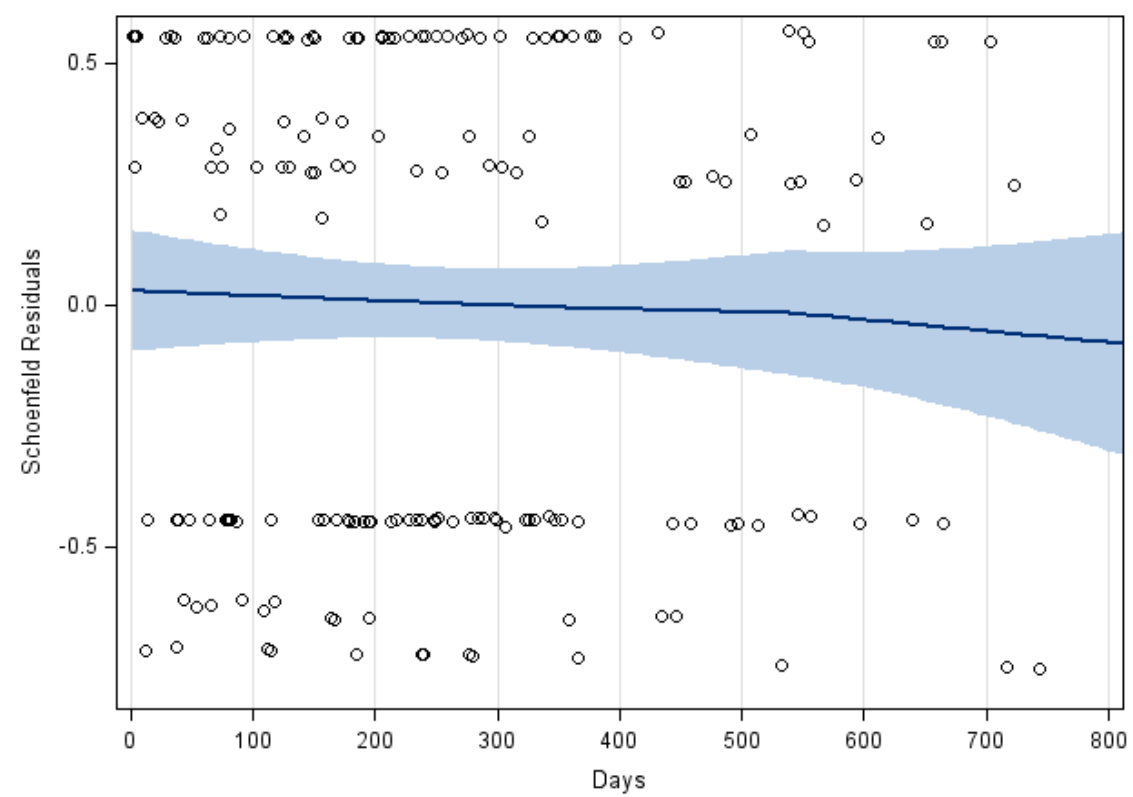

Figure 7. Evaluation of Proportional Hazards: Schoenfeld Residual Plots in Trials 18 and 19

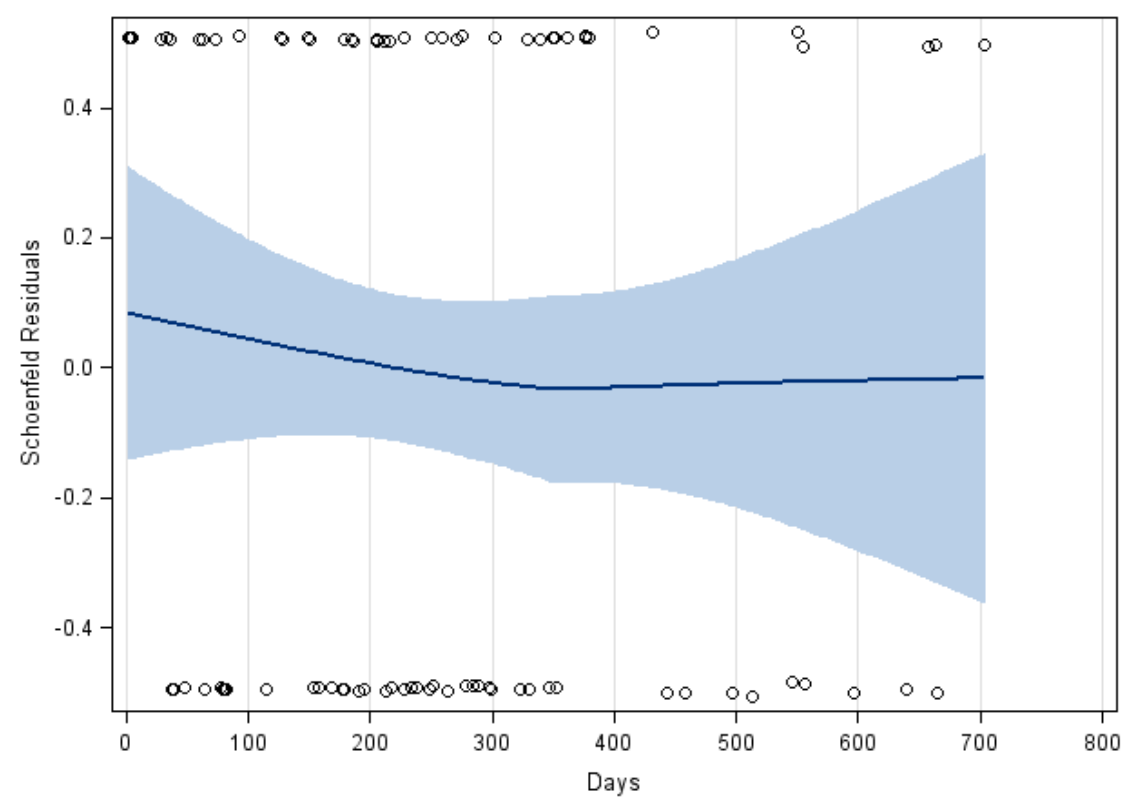




\section{A.2 Early Cardiovascular Events in the Canagliflozin Program}

On 31 May 2012, Janssen submitted a meta-analysis of cardiovascular events conducted in nine randomized clinical trials for canagliflozin as part of their application package for NDA 204042. The meta-analysis included one Phase 2 trial, seven Phase 3 trials and one ongoing Phase 3 dedicated cardiovascular safety trial, 'CANVAS'. CANVAS enrolled subjects with history or high risk of CV disease defined as either (1) age $\geq 30$ with documented symptomatic atherosclerotic $\mathrm{CV}$ disease or (2) age $\geq 50$ with 2 or more risk factors for $\mathrm{CV}$ disease at the time of screening.

The pre-specified primary Cox proportional hazards model in the meta-analysis obtained an estimated hazard ratio and $95 \%$ confidence interval of $0.91(0.68,1.22)$ for the risk of MACE-plus comparing canagliflozin to all comparators. MACE-plus was defined as the composite of cardiovascular death, MI, stroke and hospitalized unstable angina. The results of this meta-analysis were discussed at an Advisory Committee Meeting held on January 10, 2013.

An imbalance of MACE-plus was observed within the first 30 days after randomization in the dedicated cardiovascular safety trial CANVAS: 13 MACE-plus were observed among 2886 subjects randomized to canagliflozin $(0.45 \%)$ and 1 MACE-plus was observed among 1441 subjects randomized to placebo $(0.07 \%)$. Seven of the events in the canagliflozin arm were observed within the first week after randomization. A summary of these events is shown in Table 34.

The early imbalance of MACE-plus was not observed in the non-CANVAS trials in the meta-analysis. During the first 30 days after randomization, 2 events were observed in 3510 subjects randomized to canagliflozin $(0.057 \%)$ and 4 events were observed in 1886 subjects randomized to comparators $(0.21 \%)$. Therefore, although there were only 6 MACE-plus observed within the first 30 days in the non-CANVAS trials, a higher proportion of events was observed in the comparator arm than in the canagliflozin arm $(0.21 \%$ vs. $0.057 \%)$.

The imbalance of early MACE-plus observed in CANVAS may have been caused by an early increased cardiovascular risk associated with canagliflozin among subjects with high background risk or it may have been attributable to chance. 
Table 34. List of MACE-plus in CANVAS during the First 30 Days After Randomization

\begin{tabular}{|ccc|}
\hline Treatment & Day of Event & Type of Event \\
\hline Cana $300 \mathrm{mg}$ & 2 & Nonfatal Stroke \\
Cana $100 \mathrm{mg}$ & 2 & Hospitalized Unstable Angina \\
Cana $100 \mathrm{mg}$ & 2 & Nonfatal Stroke \\
Cana $300 \mathrm{mg}$ & 6 & Nonfatal Myocardial Infarction \\
Cana $300 \mathrm{mg}$ & 6 & Nonfatal Myocardial Infarction \\
Cana $300 \mathrm{mg}$ & 7 & Cardiovascular Death \\
Cana $100 \mathrm{mg}$ & 7 & Nonfatal Stroke \\
Cana $300 \mathrm{mg}$ & 12 & Nonfatal Myocardial Infarction \\
Cana $100 \mathrm{mg}$ & 14 & Nonfatal Myocardial Infarction \\
Cana $100 \mathrm{mg}$ & 21 & Nonfatal Myocardial Infarction \\
Placebo & 23 & Nonfatal Myocardial Infarction \\
Cana $100 \mathrm{mg}$ & 24 & Nonfatal Stroke \\
Cana $100 \mathrm{mg}$ & 26 & Nonfatal Stroke \\
Cana $300 \mathrm{mg}$ & 29 &
\end{tabular}

\section{SIGNATURES/DISTRIBUTION LIST}

Primary Statistical Reviewer: Eugenio Andraca-Carrera

Date: $12 / 16 / 2013$

Concurring Reviewers: Mat Soukup, Aloka Chakravarty

Statistical Team Leader: Mat Soukup

Biometrics VII Division Director: Aloka Chakravarty

cc:

HFD-750

Aloka Chakravarty (Director, Division of Biometrics VII)

Mat Soukup (Division of Biometrics VII)

Eugenio Andraca-Carrera (Division of Biometrics VII)

Lillian Patrician (Office of Biostatistics) 
Appears this way on original

Reference ID: 3422426 
This is a representation of an electronic record that was signed electronically and this page is the manifestation of the electronic signature.

/s/

EUGENIO ANDRACA-CARRERA

$12 / 16 / 2013$

MATTHEW J SOUKUP

$12 / 16 / 2013$

Concur with review

ALOKA G CHAKRAVARTY

$12 / 16 / 2013$ 
U.S. Department of Health and Human Services

Food and Drug Administration

Center for Drug Evaluation and Research

Office of Translational Sciences

Office of Biostatistics

\title{
Statistical Review and Evaluation
}

\author{
Clinical Studies
}

NDA/BLA \#:

Supplement \#:

Drug Name:

Indication(s):

Applicant:

Date(s):

Review Priority:

Biometrics Division:

Statistical Reviewer:

Concurring Reviewers:

Medical Division:

Clinical Team:

Project Manager:
NDA 202293/0095

NA

Dapagliflozin propanediol tablets

Improve glycemic control in adults with type 2 diabetes mellitus

Bristol-Myers Squibb

Received on $7 / 11 / 2013$

Standard (10-month)

Division of Biometrics II

Wei Liu, Ph.D.

Mark D. Rothmann, Ph.D. (Team Leader)

Metabolism and Endocrinological Products (HFD-510, DMEP)

Frank Pucino, M.D.

Karen Mahoney, M.D. (Team Leader)

Adeolu, Abolade

Keywords: NDA review, clinical studies, add-on design, analysis of covariance, double-blind, labeling, longitudinal data analysis, missing data, sensitivity analyses, stratification, 


\section{Table of Contents}

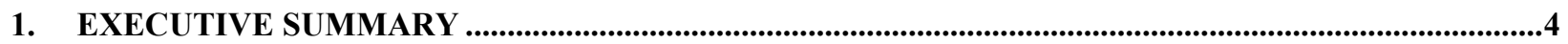

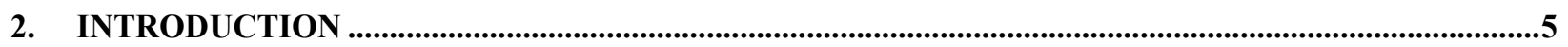

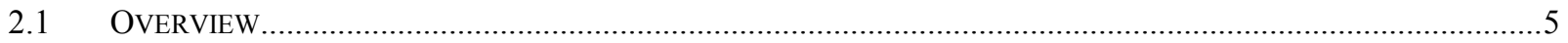

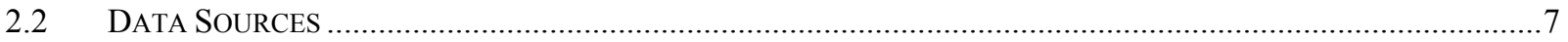

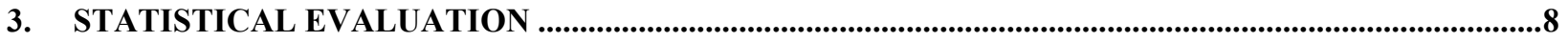

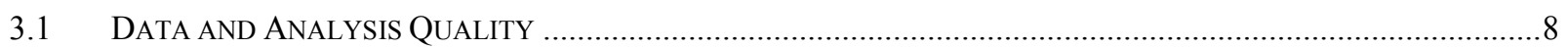

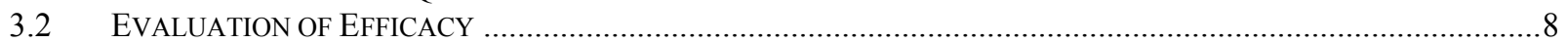

3.2.1 Study D1690C00010 - In combination with Sitagliptin or Sitagliptin plus Metformin ..........................9

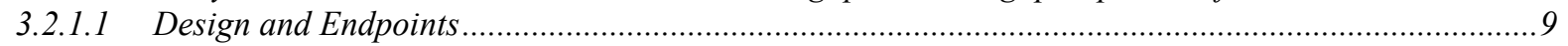

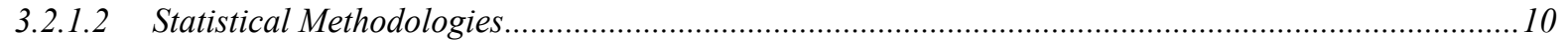

3.2.1.3 Patient Disposition, Demographic and Baseline Characteristics................................................11

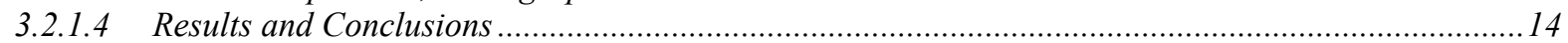

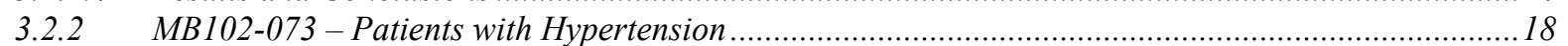

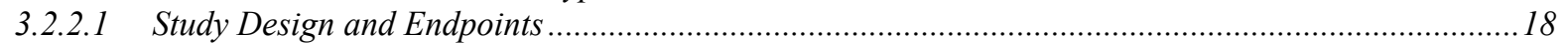

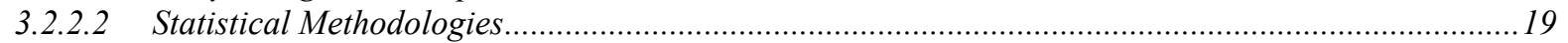

3.2.2.3 Patient Disposition, Demographic and Baseline Characteristics.................................................20

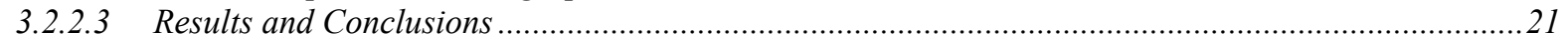

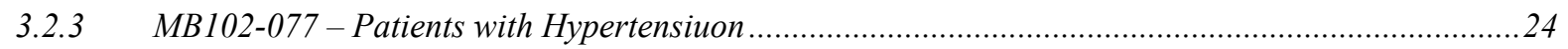

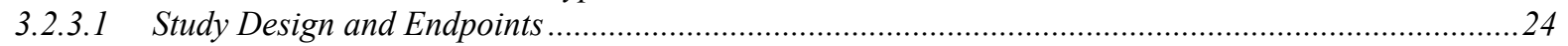

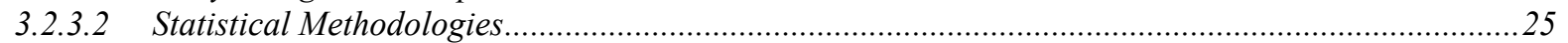

3.2.3.3 Patient Disposition, Demographic and Baseline Characteristics..................................................26

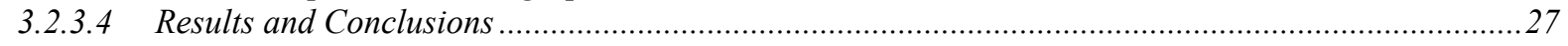

3.3 INTEGRATED ANALYSIS OF EFFICACY IN PLACEBO-CONTROLLED STUDIES ..............................................30

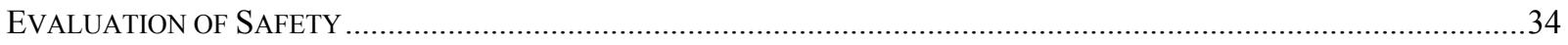

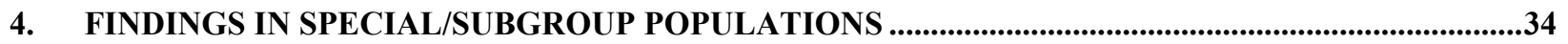

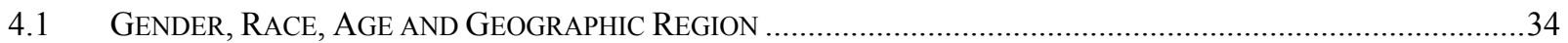

4.2 Other SPECIAL/SUBgroup POPULATIONS - INTEGRATED ANALYSIS OF HBA1C BY SUBGROUPS IN

PATIENTS BASED ON THEIR BASELINE EGFR LEVELS IN PlACEBO-CONTROLLED STUDIES ...................................34

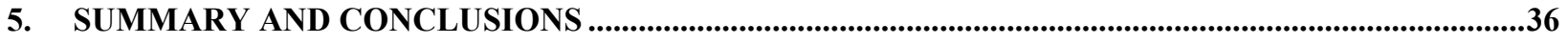

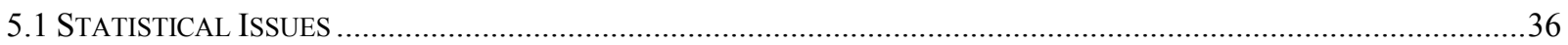

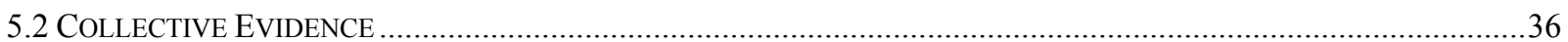

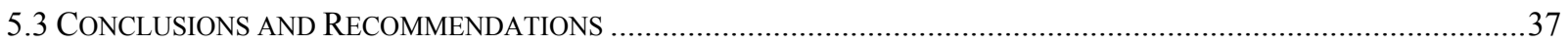

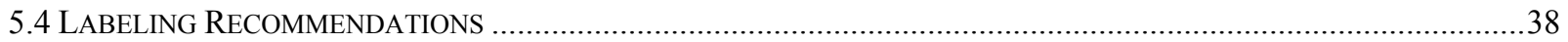




\section{LIST OF TABLES}

Table 2.1 Trial Overview

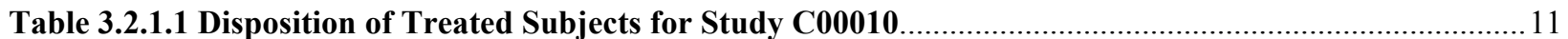

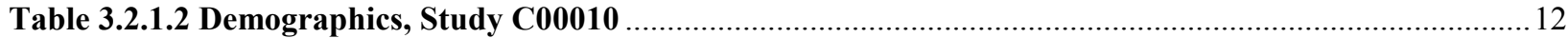

Table 3.2.1.3 Summary of Primary and Key Secondary Efficacy Endpoints, 24-Week Short-term, Double-blind Treatment Period Excluding Data After Rescue (Including Data After Rescue for SBP), Study C00010 (full analysis set, LOCF) (Source: Clinical Study Report24-week, Table 21)

Table 3.2.1.4 Placebo-corrected mean changes from baseline in HbA1c at week 24 by subgroups (Excluding Data After Rescue, full analysis set) (Source: Clinical Study Report-24-week, Table 11.2.2.3.2)

Table 3.2.1.5 Placebo-corrected mean changes from baseline in HbA1c at week 24 and at week 48 by subgroups (full analysis set) (Source: Clinical Study Report-48-week, Table 21).....

Table 3.2.2.1 Subject Disposition for 12-Week Double-blind Treatment Period of Study MB102073

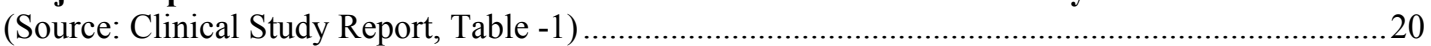

Table 3.2.2.2 Demographic Characteristics, Randomized Subjects - MB102073 ........................................20

Table 3.2.2.3 Adjusted mean change from baseline in Primary and Secondary Efficacy Endpoints at Week 12, Randomized Subjects (MB102073)

.

Table 3.2.3.1 Subject Disposition for 12-Week Double-blind Treatment Period- MB102077 .....................26

Table 3.2.3.2 Demographic Characteristics, Randomized Subjects - Study MB102077 ...............................26

Table 3.2.3.3 Seated Systolic Blood Pressure (mmHg) and HbA1c (Percent) Longitudinal Repeated Measures Analysis: 12-Week Double-blind Treatment Period, Excluding Data After Rescue, Randomized Subjects (Study MB102077) 28

Table 3.3.1 Efficacy Parameters After 24 Weeks of Treatment in Pooled Placebo-Controlled Studies for DAPA $5 \mathrm{mg}$ and $10 \mathrm{mg}$ in Patients with Type 2 Diabetes (FAS/LOCF) ............................. 31

Table 3.3.2 Information of Data Missing in Resubmitted Phase 3 Trials .................................................. 33 Table 4.1 Subgroup Analaysis in HbA1c for Dapagliflozin (5 mg and $10 \mathrm{mg}$ ) in Patients with Type 2 Diabetes by eGFR Levels (FAS/LOCF)

\section{LIST OF FIGURES}

Figure 3.2.1.1 Flow chart of study design ....

Figure 3.2.1.2 Time to discontinuation for lack of glycemic control or rescue for failing to achieve pre-

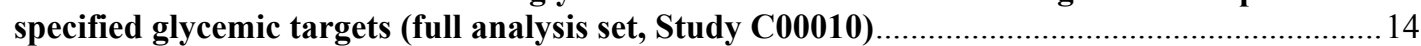

Figure 3.2.2.1 Overview of the study design, MB102073 (Source: Clinical Study Report, Figure 3.1-1)............ 18

Figure 3.2.2.2 Seated Systolic Blood Pressure (mmHg) Adjusted Mean Change for Baseline at Week 12 Evaluation in Subgroups Excluding Data After Rescue, Randomized Subjects (MB102073) ...23

Figure 3.2.2.3 HbA1c (Percent) Adjusted Mean Change from Baseline at Week 12 Evaluation in Subgroups Including Data After Rescue, Randomized Subjects (MB102073)........................24

Figure 3.2.3.1 Overview of the study design, 2077 (Source: Clinical Study Report, Figure 3.1-1) ....................25 Figure 3.2.3.2 Seated Systolic Blood Pressure (mmHg) Adjusted Mean Change for Baseline at Week 12 Evaluation in Subgroups Excluding Data After Rescue, Randomized Subjects (MB102077)...29

Figure 3.2.3.3 HbA1c (Percent) Adjusted Mean Change from Baseline at Week 12 Evaluation in Subgroups, Including Data After Rescue, Randomized Subjects (MB102077). 30 


\section{EXECUTIVE SUMMARY}

The Applicant has resubmitted a new drug application for dapagliflozin propanediol (DAPA) as a treatment for type 2 diabetes mellitus. The previous submission included data from eleven Phase 3 studies evaluating the efficacy of DAPA and two additional Phase 3 studies in patients with cardiovascular disease in an amendment. See the statistical reviews of Dr. Jonathan Norton dated September 8, 2011 and November 21, 2011 for the details and efficacy summary and conclusions based on the previous submission. Dr. Norton concluded that "The submitted clinical studies provide strong evidence that dapagliflozin is effective in subjects with normal renal function or mild impairment."

The resubmission includes data from three additional phase 3 studies. This review primarily focused on the efficacy data from these three studies. Integrated analyses based on studies from both submissions are also performed.

Confirmation of efficacy:

The resubmitted data of phase 3 studies provide convincing evidence that dapagliflozin is efficacious for both of the proposed once daily doses, $5 \mathrm{mg}$ and $10 \mathrm{mg}$. For majority of the phase 3 studies, the efficacy analyses were based on LOCF as the primary method for accounting for missing data, disregarding observations recorded after any rescue treatment. Results from analyses using MMRM performed by this reviewer to selected studies were consistent with the primary analysis results with LOCF. However, the efficacy of dapagliflozin based on HbA1c reduction is modest.

In all the placebo-controlled studies but study MB102029, both the $5 \mathrm{mg}$ and $10 \mathrm{mg}$ doses of dapagliflozin were shown to be superior to placebo on the primary endpoint, using the planned primary analysis. The renal impairment study MB102029 failed to show superiority of dapagliflozin, at doses of both the $5 \mathrm{mg}$ and $10 \mathrm{mg}$, to placebo. As seen in Dr. Norton's review dated September 8, 2011, the active-controlled study showed that titrated doses of dapagliflozin and glipizide yielded quite similar results at Week 52. Although dapagliflozin was statistically non-inferior at Week 52, it should be noted that glipizide was clearly superior at some earlier time points.

The efficacy of DAPA ( $5 \mathrm{mg}$ and $10 \mathrm{mg}$ ) versus placebo is observed in the meta-analysis of the integrated placebo-controlled data for the primary efficacy endpoint $\mathrm{HbA} 1 \mathrm{c}$ and interested secondary efficacy endpoints (seated systolic blood pressure, body weight, and FPG) without family-wise type 1 error control. However, the results in the LDL change after 24 weeks treatment suggested elevated LDL levels in patients treated with DAPA (5 mg and $10 \mathrm{mg}$ ) while the LDL level in placebo arm remained close to the baseline level. This may raise a safety concern.

The results from meta-analyses on pooled phase 3 placebo-controlled studies in this resubmission are supportive to the efficacy of dapagliflozin based on changes from baseline to Week 24 in blood pressure, weight, and glycemic control. However, the efficacy of dapagliflozin based on HbA1c reduction is modest. Moreover, the results in the LDL change after 24 weeks treatment suggested elevated LDL levels in patients treated with DAPA (5 mg and $10 \mathrm{mg}$ ) while 
the LDL level in placebo arm remained close to the baseline level. This may raise a safety concern.

Subgroups analyses of $\mathrm{HbA1c}$ based on a pooled patient population from the 13 Phase 3 placebo-controlled studies with the primary endpoint at Week 24 suggests that dapagliflozin ( 5 $\mathrm{mg}$ and $10 \mathrm{mg}$ ) is effective only in subjects with normal renal function or mild impairment (eGFR $\geq 45 \mathrm{~mL} / \mathrm{min} / 1.73 \mathrm{~m}^{2}$ ). The size of the treatment effect should be considered when considering whether treatment with dapagliflozin is appropriate. There is no clear dose response of dapagliflozin (DAPA $5 \mathrm{mg}$ and $10 \mathrm{mg}$ ) versus placebo in HbA1c reduction from baseline to Week 24.

Limitations:

There are issues involving the bias and reliability of an estimate of a treatment difference when carrying forward an intermediate measurement and treating it as an actual measurement at the end point.

The data-missing rates for the three studies are:

$\begin{array}{ll}\text { D1690C00010 (24 weeks) } & \text { DAPA } 10 \mathrm{mg} 23 \% \text {, placebo } 47 \% \\ \text { MB102073 (12 weeks) } & \text { DAPA } 10 \mathrm{mg} 8 \% \text {, placebo } 11 \% \\ \text { MB102077 (12 weeks) } & \text { DAPA } 10 \mathrm{mg} 9 \% \text {, placebo } 13 \%\end{array}$

The average percent missing 24 -week data in all placebo-controlled phase 3 studies is $21 \%$ with a range from $9 \%$ to $47 \%$.

The meta-analysis of the integrated placebo-controlled data for the primary efficacy endpoint $\mathrm{HbA1c}$ and interested secondary efficacy endpoints (seated systolic blood pressure, body weight, and FPG) are performed without family-wise type 1 error control. The overall efficacy of DAPA will be evaluated in the adversary committee meeting on December 12, 2013 based on the statistical reviews by Dr. Norton and this reviewer.

\section{INTRODUCTION}

\subsection{Overview}

The Applicant, Bristol-Myers Squibb, has resubmitted the new drug application NDA 202-293 on 7/11/2013 in response to the Complete Response Letter (CRL) dated 1/17/2012 for the use of dapagliflozin (BMS-512148, DAPA) in adult patients with type 2 diabetes mellitus and inadequate glycemic control. There are original/updated and new data from 16 phase 3 efficacy and safety studies in this resubmission as shown in Table 2.1. There were eleven Phase 3 studies evaluating the efficacy of DAPA in the original submission of NDA 202-293 (submitted on $12 / 28 / 2010$ ). The efficacy results from these studies were discussed in the advisory committee meeting held 7/19/2011. The data of two additional Phase 3 studies, D1690C00018 (patients with cardiovascular disease and hypertension) and D1690C00019 (patients with cardiovascular disease), were submitted after the AC meeting (10/20/2011, 11/2/2011, and 11/9/2011). The efficacy of DAPA from these studies had been reviewed by Dr. Jonathan Norton who summarized that "I find these studies to provide convincing evidence that dapagliflozin is efficacious for both of the proposed daily doses, $5 \mathrm{mg}$ and $10 \mathrm{mg}$. In the four placebo-controlled studies (out of the six that I focused on), both the $5 \mathrm{mg}$ and $10 \mathrm{mg}$ doses of dapagliflozin were shown to be superior to the comparator on the primary endpoint, using the planned primary analysis. The active- 
controlled study showed that titrated doses of dapagliflozin and glipizide yielded quite similar results at Week 52. Although dapagliflozin was statistically non-inferior at Week 52, it should be noted that glipizide was clearly superior at some earlier time points. (See Figure 3 at the end of this report.) Findings for fasting plasma glucose (FPG) also support a finding of efficacy for both doses." Dr. Norton concluded that "The submitted clinical studies provide strong evidence that dapagliflozin is effective in subjects with normal renal function or mild impairment." For the details, please see his statistical reviews dated September 8, 2011 and November 21, 2011 and efficacy summary and conclusions based on the previous submission.

This submission includes efficacy data from three additional studies, D1690C00010 (add-on to DPP4 inhibitor, sitagliptin), MB102-073 and MB102-077 (in patients with type 2 diabetes and hypertension). The efficacy data of the three studies are reviewed by this reviewer. The design features of the three studies are also shown in Table 2.1.

Table 2.1 Trial Overview

\begin{tabular}{|c|c|c|c|c|c|}
\hline $\begin{array}{l}\text { Study ID (Brief } \\
\text { Name) }\end{array}$ & Population & Dose (mg) & $\begin{array}{l}\text { Background Tx } \\
\text { (Rescue) }\end{array}$ & Comparator & Weeks \\
\hline \multicolumn{6}{|l|}{ Monotherapy } \\
\hline $\begin{array}{l}\text { MB102013 } \\
(2013)\end{array}$ & Drug-naïve & $\begin{array}{l}\text { DAPA } 2.5,5, \\
10\end{array}$ & $\begin{array}{l}\text { None } \\
(\mathrm{MET})\end{array}$ & Placebo & $24+$ ext. \\
\hline $\begin{array}{l}\text { MB102032 } \\
(2032)\end{array}$ & Drug-naïve & $\begin{array}{l}\text { DAPA } 1,2.5, \\
5\end{array}$ & $\begin{array}{l}\text { None } \\
\text { (MET) }\end{array}$ & Placebo & 24 \\
\hline \multicolumn{6}{|c|}{ Combination Therapy } \\
\hline $\begin{array}{l}\text { MB102014 } \\
(2014)\end{array}$ & $\begin{array}{l}\text { Inadequate control } \\
\text { on background }\end{array}$ & $\begin{array}{l}\text { DAPA } 2.5,5, \\
10\end{array}$ & $\begin{array}{l}\text { MET (Pioglitazone or } \\
\text { acarbose) }\end{array}$ & Placebo & $24+$ ext. \\
\hline $\begin{array}{l}\text { D1690C00005 } \\
\text { (C00005) }\end{array}$ & $\begin{array}{l}\text { Inadequate control } \\
\text { on background }\end{array}$ & $\begin{array}{l}\text { DAPA } 2.5,5, \\
10\end{array}$ & $\begin{array}{l}\text { Glimepiride } \\
\text { (MET or TZD) }\end{array}$ & Placebo & $24+$ ext. \\
\hline $\begin{array}{l}\text { MB102030 } \\
(2030)\end{array}$ & $\begin{array}{l}\text { Inadequate control } \\
\text { on background }\end{array}$ & DAPA 5,10 & $\begin{array}{l}\text { Pioglitazone (MET or } \\
\text { SU) }\end{array}$ & Placebo & $24+$ ext. \\
\hline $\begin{array}{l}\text { D1690C00006 } \\
(\mathrm{C} 00006)\end{array}$ & $\begin{array}{l}\text { Inadequate control } \\
\text { on background }\end{array}$ & $\begin{array}{l}\text { DAPA } 2.5,5, \\
10\end{array}$ & $\begin{array}{l}\text { Insulin and up to two } \\
\text { OAD (Insulin up- } \\
\text { titration) }\end{array}$ & Placebo & $24+$ ext. \\
\hline $\begin{array}{l}\text { D1690C00004 } \\
(\mathrm{C} 00004)\end{array}$ & $\begin{array}{l}\text { Inadequate control } \\
\text { on background }\end{array}$ & $\begin{array}{l}\text { DAPA } 2.5,5, \\
10 \text { (titrated) }\end{array}$ & MET (None) & Glipizide* & $52+$ ext. \\
\hline $\begin{array}{l}\mathrm{D} 1690 \mathrm{C} 00012 \\
(\mathrm{C} 00012)\end{array}$ & $\begin{array}{l}\text { Inadequate control } \\
\text { on background }\end{array}$ & DAPA 10 & MET (Sitagliptin) & Placebo** & $24+$ ext. \\
\hline $\begin{array}{l}\text { D1690C00010 } \\
(\mathrm{C} 00010)^{\wedge}\end{array}$ & $\begin{array}{l}\text { Drug-naïve or } \\
\text { inadequate control } \\
\text { on background }\end{array}$ & $\begin{array}{l}\text { DAPA 10+ } \\
\text { sitagliptin }\end{array}$ & $\begin{array}{l}\text { Sitagliptin and/or MET } \\
\text { (glimepiride) }\end{array}$ & Placebo & $24+24$ \\
\hline $\begin{array}{l}\text { MB102021 } \\
(2021)\end{array}$ & $\begin{array}{l}\text { Drug-naïve with } \\
\text { higher } \mathrm{HbAlc}\end{array}$ & $\begin{array}{l}\text { DAPA 5+ } \\
\text { MET }\end{array}$ & $\begin{array}{l}\text { None (Pioglitazone, } \\
\text { acarbose, or sitagliptin) }\end{array}$ & $\begin{array}{l}\text { DAPA } 5 \mathrm{mg} \text {, } \\
\text { MET }\end{array}$ & 24 \\
\hline $\begin{array}{l}\text { MB102034 } \\
(2034)\end{array}$ & $\begin{array}{l}\text { Drug-naïve } \\
\text { with higher } \mathrm{HbA} 1 \mathrm{c}\end{array}$ & $\begin{array}{l}\text { DAPA } 10+ \\
\text { MET }\end{array}$ & $\begin{array}{l}\text { None (Pioglitazone, } \\
\text { acarbose, or sitagliptin) }\end{array}$ & $\begin{array}{l}\text { DAPA } 10 \\
\text { mg, MET }\end{array}$ & 24 \\
\hline \multicolumn{6}{|c|}{ Special Populations } \\
\hline MB102029 & Moderate renal & DAPA 5,10 & Any except MET (Any & Placebo & $24+$ ext. \\
\hline
\end{tabular}




\begin{tabular}{|c|c|c|c|c|c|}
\hline (2029) & impairment & & except MET) & & \\
\hline $\begin{array}{l}\text { MB102-073 } \\
(2073)^{\wedge}\end{array}$ & Hypertension & DAPA 10 & $\begin{array}{l}\text { OAD and/or insulin } \\
\text { (anti-hypertension) }\end{array}$ & Placebo & 12 \\
\hline $\begin{array}{l}\text { MB102-077 } \\
(2077)^{\wedge}\end{array}$ & Hypertension & DAPA 10 & $\begin{array}{l}\text { OAD and/or insulin } \\
\text { (anti-hypertension) }\end{array}$ & Placebo & 12 \\
\hline $\begin{array}{l}\text { D1690C00018 } \\
(\mathrm{C} 00018)\end{array}$ & $\begin{array}{l}\text { Cardiovascular } \\
\text { disease and } \\
\text { hypertension }\end{array}$ & DAPA 10 & $\begin{array}{l}\text { OAD and/or insulin } \\
\text { (Various) }\end{array}$ & Placebo & $24+$ ext. \\
\hline $\begin{array}{l}\text { D1690C00019 } \\
\text { (C00019) }\end{array}$ & $\begin{array}{l}\text { Cardiovascular } \\
\text { disease }\end{array}$ & DAPA 10 & $\begin{array}{l}\text { OAD and/or insulin } \\
\text { (Various) }\end{array}$ & Placebo & $24+$ ext. \\
\hline
\end{tabular}

Notes: DAPA $=$ dapagliflozin. Proposed once daily doses are 5, $10 \mathrm{mg} . \mathrm{OAD}=$ oral anti-diabetic drug. $\mathrm{SU}=$ sulfonylurea, MET=metformin, TZD = thiazolidinedione.

$\wedge$ New data

*Noninferiority comparison; other studies used superiority comparison.

**Primary endpoint is change in body weight; other studies used change in HbAlc.

To evaluate the overall efficacy of DAPA in a larger group of T2DM subjects, this reviewer performed meta-analyses based on pooled phase 3 placebo-controlled studies from 16 clinical trials in this resubmission on changes from baseline to Week 24 in blood pressure, weight, glycemic control, and lipid parameters. This reviewer also performed subgroup analysis by baseline eGFR on the pooled phase 3 placebo-controlled data at Week 24.

\subsection{Data Sources}

The sponsor submitted this NDA including the study data to the FDA CDER Electronic Document Room (EDR). The submission is recorded in the EDR with the link shown below. The data were submitted in SAS Xport transport format.

\begin{tabular}{|l|l|}
\hline Application: & NDA 202293/0095 \\
\hline Company & Bristol-Myers Squibb \\
\hline Drug & Dapagliflozin propanediol tablets \\
\hline CDER EDR link & $\backslash$ CCDSESUB1 \EVSPROD $\backslash$ NDA202293 $\backslash 0095$ \\
\hline Letter date & $7 / 11 / 2013$ \\
\hline
\end{tabular}

The Applicant submitted both analysis and tabulation data sets.

The applicant's electronic submission was well-organized. Parallel structure in the presentation of the results across all studies was well-done and appreciated by the reviewer.

All graphs and tables in the review were created by this reviewer unless otherwise noted. 


\section{STATISTICAL EVALUATION}

\subsection{Data and Analysis Quality}

Review the quality and integrity of the submitted data. Relevant issues include:

- It is possible to reproduce the primary analysis dataset from tabulation or "raw" datasets.

- It is possible to trace how the primary endpoint was derived from the original data source (e.g., case report form).

- It is possible to verify the randomized treatment assignments.

I did not encounter any major data quality issues in the course of this review. Due to time constraints, however, I did not attempt to reproduce all of the Applicant's key results from the submitted data.

\subsection{Evaluation of Efficacy}

Although the efficacy analyses of the three studies were produced by the Applicant, I verified the values in the tables that I include in this review from sponsor's Clinical Study Reports.

The primary endpoint for all the phase 3 studies was the change in HbAlc from baseline to the end of the study (Week 24 in C00010 and Week 12 in both studies 2073 and 2077). An additional co-primary endpoint was pre-specified in the following studies:

- 2073 and 2077: change from baseline at Week 12 in seated systolic blood pressure (SBP)

- C18 and C19: proportion of responders for a 3-item endpoint of clinical benefit, defined as: (1) an absolute drop of $0.5 \%$ or more from baseline in HbAlc, and (2) a relative drop of $3 \%$ or more from baseline in total body weight, and (3) an absolute drop of $3 \mathrm{mmHg}$ or more from baseline in seated SBP after 24 weeks of oral administration of double-blind treatment.

The key secondary endpoints of study C00010 were:

- Change in total body weight from baseline to week 24 (LOCF)

- Change in HbA1c in subjects with baseline HbA1c $\geq 8 \%$ from baseline to week 24 (LOCF)

- Change in FPG from baseline to week 24 (LOCF)

- Change in seated systolic blood pressure (SBP) in subjects with baseline seated SBP $\geq 130$ $\mathrm{mmHg}$ from baseline to week 8 (LOCF)

- Change in 2-hour post liquid meal glucose achieved with dapagliflozin versus placebo from baseline to week 24 (LOCF)

The key secondary endpoints of studies 2073 and 2077 were: the change from baseline at Week 12 in 24-hour mean ambulatory SBP, seated DBP, 24-hour mean ambulatory DBP, and serum uric acid.

The sponsor defined the following analysis sets for the evaluation of efficacy: 
- The full analysis set includes all randomized subjects (as randomized) who received at least one dose of study medication during the double-blind short-term treatment period who had a non-missing baseline value and at least one post-baseline value. (primary analysis set)

- The per-protocol analysis set is a subset of the full analysis set consisting of subjects who did not violate the terms of the protocol, which could affect the primary efficacy endpoints significantly.

The primary analyses disregarded observations recorded after receiving rescue treatment.

This reviewer performed integrated analyses on pooled phase 3 Placebo-controlled studies (PC) with endpoints of 24 weeks. This assessment of efficacy includes the Phase 3 placebo-controlled studies (except the renal impairment study MB102029, cohort 1 of Study MB102009 and group 2 of Study MB102013, both of which were uncontrolled, and the two 12-week studies MB102073 and MB102077 for hypertension patients) plus selected data from the initial combination studies (the dapagliflozin plus metformin and placebo plus metformin arms of Studies MB102021 and MB102034). Two doses, DAPA $5 \mathrm{mg}$ and $10 \mathrm{mg}$, are selected for the meta-analyses of the pooled 12 phase 3 data on changes from baseline to Week 24 in blood pressure, weight, glycemic control, and lipid parameters. Analyses were stratified by study.

This reviewer also performed subgroup analysis by baseline eGFR on a pooled dataset PC + data from the renal impairment study MB102029.

\subsubsection{Study D1690C00010 - In combination with Sitagliptin or Sitagliptin plus Metformin}

\subsubsection{Design and Endpoints}

This study was an international, multicenter, randomized, double-blind, placebo-controlled, parallel-group, Phase III study with a 24-week short-term (ST) treatment period followed by a 24-week long-term (LT) extension period, to evaluate safety and efficacy of dapagliflozin $10 \mathrm{mg}$ once daily (qd) in combination with sitagliptin or sitagliptin plus metformin on glycosylated hemoglobin A1c ( $\mathrm{HbAlc})$ in adult subjects with type 2 diabetes mellitus (T2DM) who have inadequate glycemic control.

The primary objective of this study was to compare the change from baseline in $\mathrm{HbAlc}$ at 24 weeks between dapagliflozin and placebo in subjects with T2DM who were inadequately controlled on sitagliptin alone or on sitagliptin plus metformin. Figure 3.2.1.1 shows the design for Study C00010.

Figure 3.2.1.1 Flow chart of study design (Source: Clinical Study Report-48-week, Figure 1) 


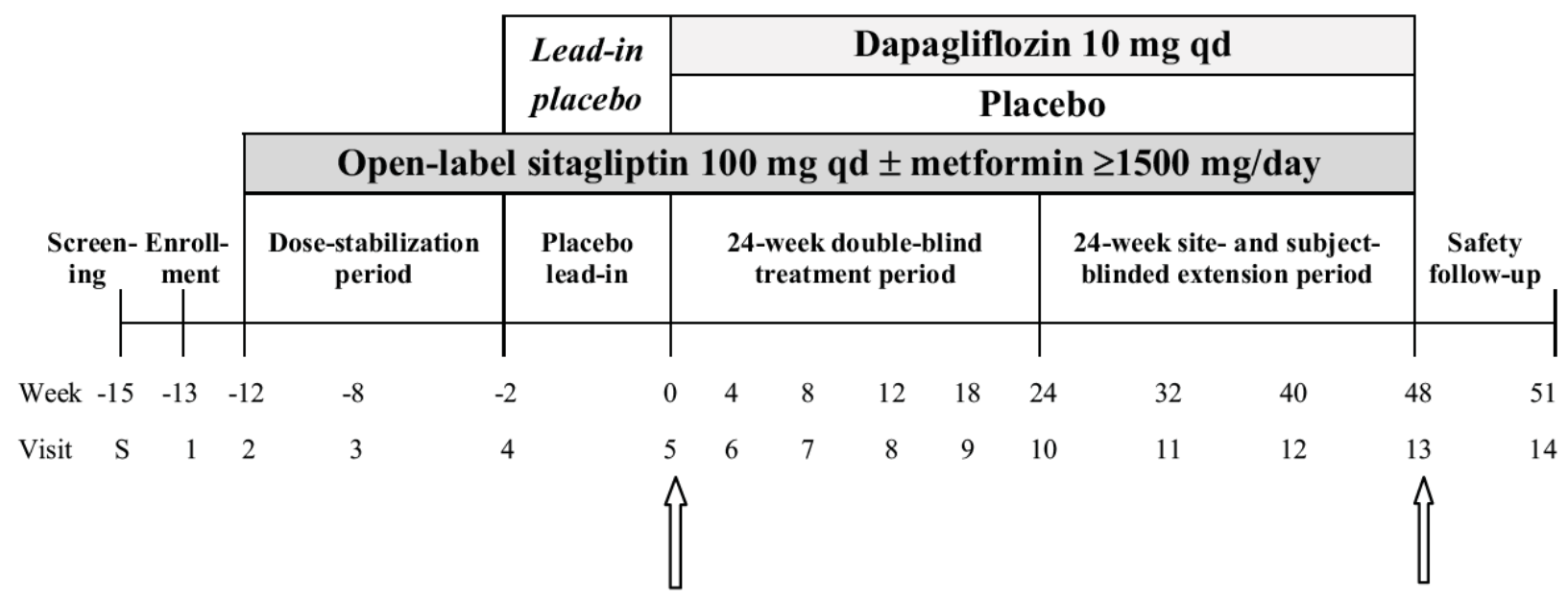

Randomization 1:1

End of randomized treatment

The primary efficacy endpoint was the change in hemoglobin A1c (HbA1c) from baseline to Week 24. The key secondary efficacy variables are the following:

- Change in total body weight from baseline to week 24 (LOCF)

- Change in HbA1c in subjects with baseline HbA1c $\geq 8 \%$ from baseline to week 24 (LOCF)

- Change in FPG from baseline to week 24 (LOCF)

- Change in seated systolic blood pressure (SBP) in subjects with baseline seated SBP $\geq 130$ $\mathrm{mmHg}$ from baseline to week 8 (LOCF)

- Change in 2-hour post liquid meal glucose achieved with dapagliflozin versus placebo from baseline to week 24 (LOCF)

- Proportion of subjects with a therapeutic glycemic response, defined as a reduction in HbAlc of $\geq 0.7 \%$ compared to baseline, at week 24 (LOCF)

\subsubsection{Statistical Methodologies}

In Study C00010, subjects eligible for the study were stratified according to their use of metformin, and within each stratum they were randomized to dapagliflozin or placebo treatment.

Sponsor's primary efficacy analysis was an ANCOVA of the primary efficacy variable, the change in hemoglobin A1c (HbA1c) from baseline to week 24, in the short-term double-blind period using last observation carried forward method (LOCF), with treatment group and stratum (sitagliptin monotherapy vs. sitagliptin plus metformin) as fixed effects and baseline HbA1c as a covariate in the model. For subjects with rescue medication, the last post-baseline measurement prior to the first dose of rescue medication was used in this analysis.

This reviewer performed a second analysis using Mixed Model Repeated Measure (MMRM) method (using the same factors as the ANOVA model along with factors for visit and treatmentby-visit interaction) to verify the primary analysis result.

The sponsor pre-specified a hierarchical closed testing procedure in order to control the type I error rate across the primary and key secondary objectives, based on data from the two strata 
combined. Analyses were also conducted within each stratum to separately evaluate the effect in the two back-ground treatment regimens. For variables found to be significant with the combined strata analysis, corresponding within-stratum treatment comparisons were individually tested at a two-sided significance level of 0.050 . For all other variables, nominal p-values were reported for both overall and within-strata comparisons without significance testing.

The flow of testing the secondary endpoints was based on the list order shown in "Study Design and Endpoints" from one objective to the next depending upon the overall results. Results within each stratum were also examined.

\subsubsection{Patient Disposition, Demographic and Baseline Characteristics}

Table 3.2.1.1 shows the disposition of the double-blind treated subjects, in particular whether they completed the short-term period, whether they continued into the long-term extension, and the reasons for discontinuation at each stage. (From this point on, the phrase treated subjects refers to subjects who were randomized and received double-blind treatment.)

Table 3.2.1.1 Disposition of Treated Subjects for Study C00010 (Source: Clinical Study Report-48-week, Table 10).

\begin{tabular}{|c|c|c|c|}
\hline & $P L A+S I T$ & DAPA $10 \mathrm{MG}+\mathrm{SIT}$ & Total \\
\hline SUBJECTS & 226 & 225 & 451 \\
\hline SUBJECTS COMPLETING THE ST PERIOD (\%) & $203(89.8)$ & $208 \quad(92.4)$ & 411 (91.1) \\
\hline SUBJECTS NOT COMPLETING THE ST PERIOD (음) & $23(10.2)$ & $17(7.6)$ & $40(8.9)$ \\
\hline \multicolumn{4}{|l|}{ REASON FOR NOT COMPLETING THE ST PERIOD (\%) } \\
\hline ADVERSE EVENT & $3(1.3)$ & $7(3.1)$ & $10(2.2)$ \\
\hline SUBJECT NO LONGER MEETS STUDY CRITERIA & $1(0.4)$ & $1(0.4)$ & $2(0.4)$ \\
\hline SUBJECT WITHDREW CONSENT & $13(5.8)$ & $4(1.8)$ & $17(3.8)$ \\
\hline LOST TO FOLLOW-UP & $3(1.3)$ & $4(1.8)$ & $7(1.6)$ \\
\hline POOR/NON-COMPLIANCE & 0 & $1(0.4)$ & $1(0.2)$ \\
\hline DEATH & $1(0.4)$ & 0 & $1(0.2)$ \\
\hline OTHER & $2(0.9)$ & 0 & $2(0.4)$ \\
\hline SUBJECTS CONTINUING IN THE STUDY (\%) & $202(89.4)$ & $208(92.4)$ & $410 \quad(90.9)$ \\
\hline SUBJECTS NOT CONTINUING IN THE STUDY (\%) & $24(10.6)$ & $17(7.6)$ & $41(9.1)$ \\
\hline \multicolumn{4}{|l|}{ REASON FOR NOT CONTINUING IN THE STUDY (\%) } \\
\hline ADVERSE EVENT & $4(1.8)$ & $7(3.1)$ & $11(2.4)$ \\
\hline SUBJECT NO LONGER MEETS STUDY CRITERIA & $1(0.4)$ & $1(0.4)$ & $2(0.4)$ \\
\hline SUBJECT WITHDREW CONSENT & $13(5.8)$ & $4(1.8)$ & $17(3.8)$ \\
\hline LOST TO FOLLOW-UP & $3(1.3)$ & $4(1.8)$ & $7(1.6)$ \\
\hline POOR/NON-COMPLIANCE & 0 & $1(0.4)$ & $1(0.2)$ \\
\hline DEATH & $1(0.4)$ & 0 & $1(0.2)$ \\
\hline OTHER & $2(0.9)$ & 0 & $2(0.4)$ \\
\hline
\end{tabular}




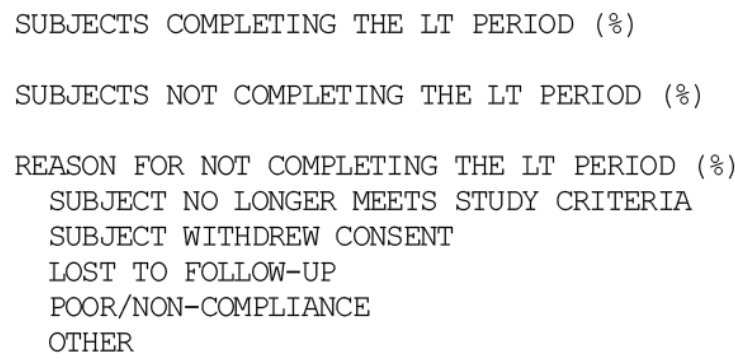

OTHER

The Applicant reported fairly high completion rates for the 24-week period in the two treatment arms: $92 \%$ (208/225) for DAPA $10 \mathrm{mg}$ and 90\% (203/226) for placebo. The denominators for these results are from the safety analysis set. Considering the small number of dropouts, there was not a substantial imbalance across arms in the reasons for discontinuation.

Table 3.2.1.2 shows selected demographic characteristics for the Full Analysis Set. The table was provided by the Applicant. They also reported that $26 \%$ of subjects were in the United States.

Table 3.2.1.2 Demographics, Study C00010 (Source: Clinical Study Report-48-week, Tables 14 and 15) 


\begin{tabular}{|c|c|c|c|c|c|c|}
\hline \multirow[b]{2}{*}{ AGE } & \multicolumn{2}{|c|}{$\begin{array}{l}\mathrm{PLA}+\mathrm{SIT} \\
\mathrm{N}=224\end{array}$} & \multicolumn{2}{|c|}{$\begin{array}{c}\text { DAPA 10MG + SIT } \\
N=223\end{array}$} & \multicolumn{2}{|c|}{$\begin{array}{c}\text { Total } \\
\mathrm{N}=447\end{array}$} \\
\hline & & & & & & \\
\hline $\mathrm{N}$ & & 224 & & 223 & & 447 \\
\hline MEAN & & 55.0 & & 54.8 & & 54.9 \\
\hline MEDIAN & & 55.0 & & 55.0 & & 55.0 \\
\hline MIN , MAX & $22 ，$ & 75 & 28, & 78 & $22 ，$ & 78 \\
\hline $\mathrm{Q} 1, \mathrm{Q3}$ & 48.5, & 62.0 & $47.0 ，$ & 63.0 & 48.0, & 62.0 \\
\hline STANDARD DEVIATION & & 10.20 & & 10.42 & & 10.30 \\
\hline \multicolumn{7}{|l|}{ AGE CATEGORIZATION (\%) } \\
\hline$<65$ & 182 & $(81.3)$ & 185 & $(83.0)$ & 367 & $(82.1)$ \\
\hline$>=65$ AND $<75$ & 41 & $(18.3)$ & 32 & $(14.4)$ & 73 & ( 16.3$)$ \\
\hline$>=75$ & 1 & $(0.4)$ & 6 & $(2.7)$ & 7 & $(1.6)$ \\
\hline NOT REPORTED & 0 & & 0 & & 0 & \\
\hline \multicolumn{7}{|l|}{ GENDER (\%) } \\
\hline MALE & 118 & $(52.7)$ & 127 & $(57.0)$ & 245 & $(54.8)$ \\
\hline FEMALE & 106 & $(47.3)$ & 96 & $(43.0)$ & 202 & $(45.2)$ \\
\hline NOT REPORTED & 0 & & 0 & & 0 & \\
\hline \multicolumn{7}{|l|}{$\operatorname{RACE}(\%)$} \\
\hline WHITE & 171 & $(76.3)$ & 161 & $(72.2)$ & 332 & $(74.3)$ \\
\hline BLACK/AFRICAN AMERICAN & 6 & $(2.7)$ & 11 & $(4.9)$ & 17 & $(3.8)$ \\
\hline ASIAN & 2 & $(0.9)$ & 2 & $(0.9)$ & 4 & $(0.9)$ \\
\hline OTHER & 45 & (20.1) & 49 & $(22.0)$ & 94 & $(21.0)$ \\
\hline NOT REPORTED & 0 & & 0 & & 0 & \\
\hline \multicolumn{7}{|l|}{ ETHNICITY (\%) } \\
\hline HISPANIC/LATINO & 124 & $(55.4)$ & 121 & $(54.3)$ & 245 & $(54.8)$ \\
\hline NOT HISPANIC/LATINO & 100 & $(44.6)$ & 102 & $(45.7)$ & 202 & $(45.2)$ \\
\hline NOT REPORTED & 0 & & 0 & & 0 & \\
\hline
\end{tabular}

The time to dropout by the Applicant is shown in Figure 3.2.1.2. Dropout rate in the placebo arm was obviously higher than the DAPA arm over time up to 52 weeks. 
Figure 3.2.1.2 Time to discontinuation for lack of glycemic control or rescue for failing to achieve pre-specified glycemic targets (full analysis set, Study C00010)

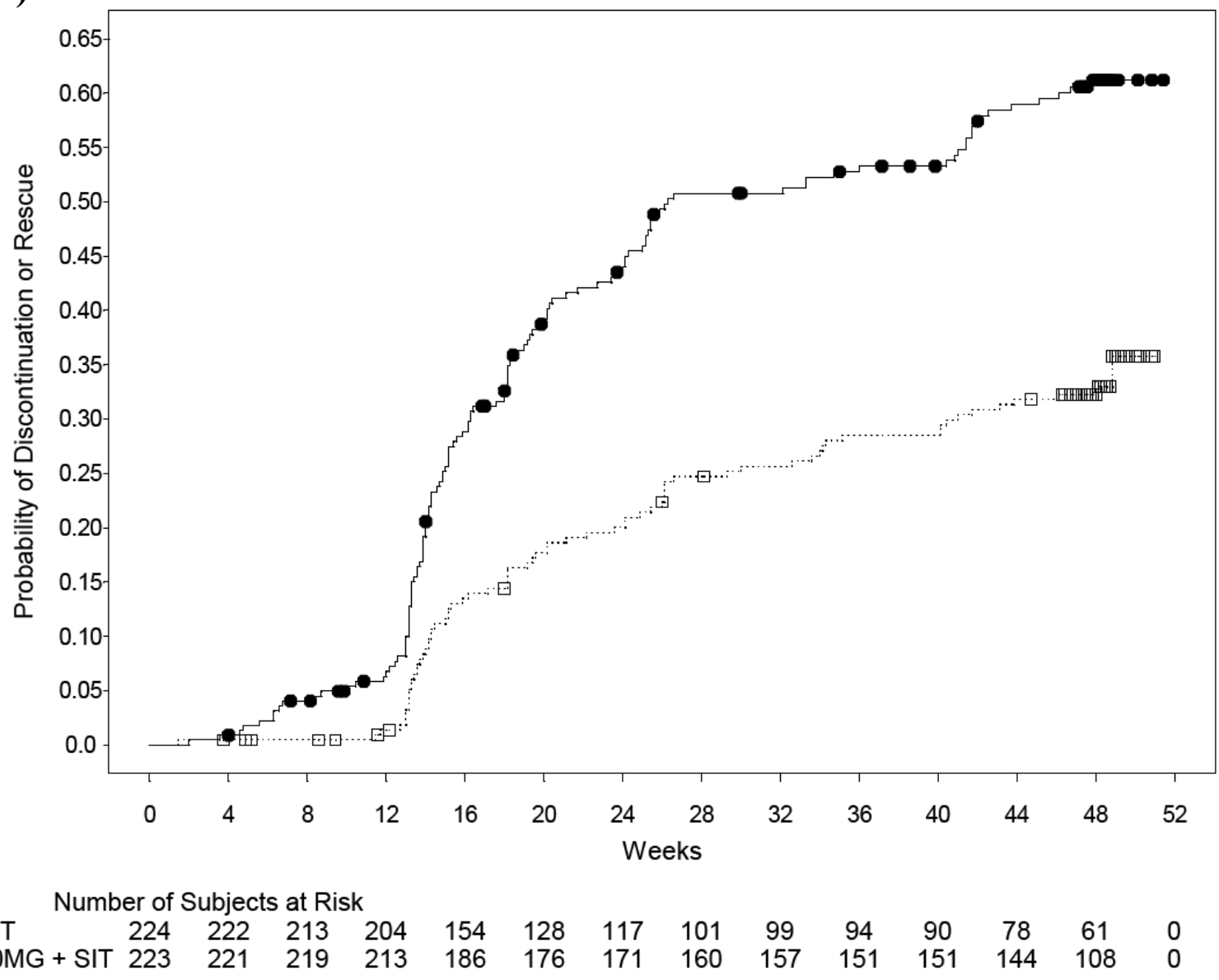

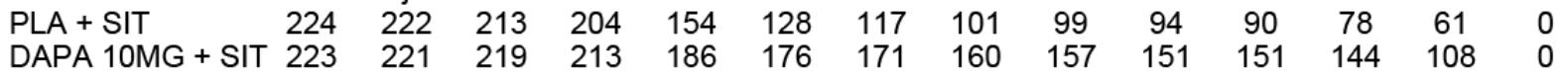

\subsubsection{Results and Conclusions}

The sponsor's results of primary and secondary analyses were shown in Table 3.2.1.3. The sponsor also showed that the efficacy of dapagliflozin remained at Week 48. This reviewer verified the primary analysis and performed the MMRM analysis which supports the results by the ANCOVA method using LOCF for missing observations.

Table 3.2.1.3 Summary of Primary and Key Secondary Efficacy Endpoints, 24-Week Short-term, Double-blind Treatment Period Excluding Data After 


\section{Rescue (Including Data After Rescue for SBP), Study C00010 (full analysis set, LOCF) (Source: Clinical Study Report-24-week, Table 21)}

\begin{tabular}{|c|c|c|}
\hline $\begin{array}{l}\text { EFFICACY ENDPOINT } \\
\text { STATISTICS }\end{array}$ & $\begin{array}{l}\text { PLA + SIT } \\
(\mathrm{N}=224)\end{array}$ & $\begin{array}{c}\text { DAPA } 10 \mathrm{MG}+\mathrm{SIT} \\
(\mathrm{N}=223)\end{array}$ \\
\hline PRIMARY EFFICACY ENDPOINT & & \\
\hline $\begin{array}{l}\text { HBA1C (\%) AT WEEK } 24 \text { (LOCF) } \\
\text { N\# } \\
\text { BASELINE MEAN (SD) } \\
\text { WEEK } 24 \text { LOCF MEAN (SD) } \\
\text { MEAN CHANGE FROM BASELINE (SD) } \\
\text { ADJ. MEAN CHANGE FROM BSL. (SE) (a) } \\
\text { 95\% CI FOR ADJ. MEAN CHANGE FROM BSL. } \\
\text { DIFFERENCE FROM PLA + SIT (SE) (b) } \\
\text { 95\% CI FOR DIFFERENCE FROM PLA + SIT } \\
\text { P-VALUE VS. PLA + SIT (*) }\end{array}$ & 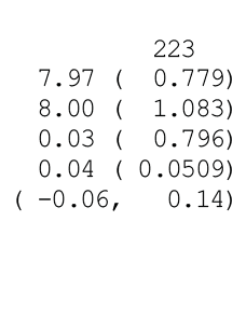 & $\begin{array}{ll}7.90 & (0.806) \\
7.46( & (0.833) \\
-0.44( & (0.770) \\
-0.45(0.0509) \\
(-0.55, \quad-0.35) \\
-0.48(0.0720) \\
(-0.62, \quad-0.34) \\
<.0001 *\end{array}$ \\
\hline KEY SECONDARY EFFICACY ENDPOINTS & & \\
\hline $\begin{array}{l}\text { TOTAL BODY WEIGHT (KG) AT WEEK } 24 \text { (LOCE) } \\
\text { N\# } \\
\text { BASELINE MEAN (SD) } \\
\text { WEEK } 24 \text { LOCF MEAN (SD) } \\
\text { MEAN CHANGE FROM BASELINE (SD) } \\
\text { ADJ. MEAN CHANGE FROM BSL. (SE) (a) } \\
\text { 95\% CI FOR ADJ. MEAN CHANGE FROM BSL. } \\
\text { DIFFERENCE FROM PLA + SIT (SE) (b) } \\
\text { 95\% CI FOR DIFFERENCE FROM PLA + SIT } \\
\text { P-VALUE VS. PLA + SIT (*) }\end{array}$ & $\begin{array}{rr} & 224 \\
89.23 & (20.887) \\
88.99 & (20.719) \\
-0.24 & (2.435) \\
-0.26 & (0.1741) \\
(-0.60, & 0.09)\end{array}$ & $\begin{array}{l}91.02(21.637) \\
88.86(21.294) \\
-2.16(2.822) \\
-2.14(0.1745) \\
(-2.48,-1.80) \\
-1.89(0.2466) \\
(-2.37,-1.40) \\
<.0001 *\end{array}$ \\
\hline $\begin{array}{l}\text { HBA1C (\%) AT WEEK } 24 \text { (LOCE) IN SUBJECTS WITH BASELINE HBA1C >=8\% } \\
\text { N\# } \\
\text { BASELINE MEAN (SD) } \\
\text { WEEK } 24 \text { LOCF MEAN (SD) } \\
\text { MEAN CHANGE FROM BASELINE (SD) } \\
\text { ADJ. MEAN CHANGE FROM BSL. (SE) (a) } \\
\text { 95\% CI FOR ADJ. MEAN CHANGE FROM BSL. } \\
\text { DIFFERENCE FROM PLA + SIT (SE) (b) } \\
\text { 95\% CI FOR DIFFERENCE FROM PLA + SIT } \\
\text { P-VALUE VS. PLA + SIT (*) }\end{array}$ & 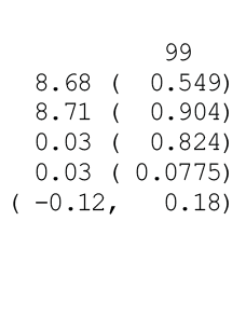 & 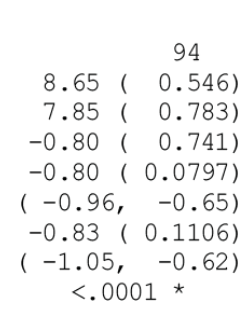 \\
\hline $\begin{array}{l}\text { FPG (MG/DL) AT WEEK } 24 \text { (LOCF) } \\
\text { N\# } \\
\text { BASELINE MEAN (SD) } \\
\text { WEEK } 24 \text { LOCE MEAN (SD) } \\
\text { MEAN CHANGE FROM BASELINE (SD) } \\
\text { ADJ. MEAN CHANGE FROM BSL. (SE) (a) } \\
\text { 95\% CI FOR ADJ. MEAN CHANGE FROM BSL. } \\
\text { DIFFERENCE FROM PLA + SIT (SE) (b) } \\
\text { 95\% CI FOR DIFFERENCE FROM PLA + SIT } \\
\text { P-VALUE VS. PLA + SIT (*) }\end{array}$ & $\begin{array}{rc} & 222 \\
163.08 & (34.587) \\
166.55 & (45.826) \\
3.47 & (38.806) \\
3.81 & (2.3474) \\
(-0.80, & 8.42)\end{array}$ & 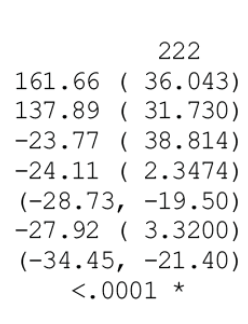 \\
\hline
\end{tabular}

For the analysis on the secondary efficacy endpoints, the efficacy of DAPA was supported by the change from baseline to week 24 (LOCF) in total body weight, HbAlc in subjects with baseline $\mathrm{HbA} 1 \mathrm{c} \geq 8 \%$, and FPG according to the sponsor's pre-specified multiplicity adjustment procedure at level of 0.05 . Testing of all other secondary endpoints failed to show a difference and claims on these endpoints should not be included in the product label.

Sponsor's results at week 48 by MMRM were supportive to the week-24 LOCF results on HbA1c and secondary endpoints. 
The Applicant reported the results of three sensitivity analyses for the primary endpoint: 1) ANCOVA of completers, excluding observations after rescue, 2) Mixed-effects model for repeated measures (MMRM), excluding observations after rescue, and 3) MMRM, including observations after rescue, with a rescue term in the model. These sensitivity analyses yielded similar estimates of the treatment effect to that from the LOCF analysis.

The sponsor did subgroup analyses for change in HbAlc based on baseline HbA1c, BMI, and eGRF levels, and also on ethnicity and age, as shown in Tables 3.2.1.4 and 3.2.1.5. These subgroup analyses showed a consistency in effect across the subgroups, supportive to the efficacy of DAPA across subgroups. Subjects with a higher baseline HbAlc showed a larger effect of dapagliflozin treatment than subjects with a lower baseline HbA1c. There was a tendency for a larger effect of dapagliflozin treatment in hispanics/latinos than in non hispanics/latinos and in subjects $<65$ years than in subjects $\geq 65$ years.

Table 3.2.1.4 Placebo-corrected mean changes from baseline in HbA1c at week 24 by subgroups (Excluding Data After Rescue, full analysis set) (Source: Clinical Study Report-24-week, Table 11.2.2.3.2)

\begin{tabular}{|c|c|c|}
\hline $\begin{array}{l}\text { SUBGROUP } \\
\text { STATISTICS }\end{array}$ & $\begin{array}{c}\mathrm{PLA}+\mathrm{SIT} \\
\mathrm{N}=224\end{array}$ & $\begin{array}{ll}\text { DAPA } & 10 \mathrm{MG}+\mathrm{SIT} \\
\mathrm{N}=223\end{array}$ \\
\hline \multicolumn{3}{|l|}{ P-VALUE $(*): 0.0895$} \\
\hline \multicolumn{3}{|l|}{ AGE $($ YEARS) $:<65$} \\
\hline & 181 & 185 \\
\hline BASELINE MEAN (SD) & $7.99(0.774)$ & $7.93(0.841)$ \\
\hline WEEK 24 LOCE MEAN (SD) & $8.03(1.105)$ & $7.44(0.878)$ \\
\hline MEAN CHANGE FROM BASELINE (SD) & $0.04(0.819)$ & $-0.48(0.799)$ \\
\hline ADJUSTED MEAN CHANGE FROM BASELINE (SE) & $0.05(0.0565)$ & $-0.48(0.0560)$ \\
\hline $\begin{array}{l}\text { 95\% CI OF ADJUSTED MEAN CHANGE FROM BASELINE } \\
\text { DIFFERENCE VS PLA + SIT (SE) }\end{array}$ & {$\left[\begin{array}{ll}-0.06, & 0.17\end{array}\right]$} & $\begin{array}{l}{\left[\begin{array}{ll}-0.59, & -0.37\end{array}\right]} \\
-0.53(0.0794)\end{array}$ \\
\hline 95\% CI OF DIFFERENCE VS PLA + SIT & & {$\left[\begin{array}{ll}-0.69, & -0.38\end{array}\right]$} \\
\hline \multicolumn{3}{|l|}{ AGE (YEARS) : > $=65$} \\
\hline & 42 & 38 \\
\hline BASELINE MEAN (SD) & $7.88(0.804)$ & $7.74(0.589)$ \\
\hline WEEK 24 LOCF MEAN (SD) & $7.86(0.984)$ & $7.53(0.572)$ \\
\hline MEAN CHANGE FROM BASELINE (SD) & $-0.02(0.697)$ & $-0.21(0.563)$ \\
\hline ADJUSTED MEAN CHANGE FROM BASELINE (SE) & $-0.04(0.1171)$ & $-0.25(0.1236)$ \\
\hline 95\% CI OF ADJUSTED MEAN CHANGE FROM BASELINE & {$\left[\begin{array}{ll}-0.27, & 0.19\end{array}\right]$} & {$\left[\begin{array}{ll}-0.50, & -0.01]\end{array}\right]$} \\
\hline DIFFERENCE VS PLA + SIT (SE) & & $-0.21(0.1702)$ \\
\hline 95\% CI OF DIFFERENCE VS PLA + SIT & & {$\left[\begin{array}{ll}-0.55, & 0.12\end{array}\right]$} \\
\hline
\end{tabular}


ETHNICITY: HISPANIC/LATINO

BASELINE MEAN (SD)

WEEK 24 LOCF MEAN (SD)

MEAN CHANGE FROM BASELINE (SD)

ADJUSTED MEAN CHANGE FROM BASELINE (SE)

95\% CI OF ADJUSTED MEAN CHANGE FROM BASELINE

DIFFERENCE VS PLA + SIT (SE)

95\% CI OF DIFFERENCE VS PLA + SIT

ETHNICITY: NOT HISPANIC/LATINO

N\#

BASELINE MEAN (SD)

WEEK 24 LOCF MEAN (SD)

MEAN CHANGE FROM BASELINE (SD)

ADJUSTED MEAN CHANGE FROM BASELTNE (SE)

95\% CI OF ADJUSTED MEAN CHANGE FROM BASELINE

DIFFERENCE VS PLA + SIT (SE)

95\% CI OF DIFFERENCE VS PLA + SIT

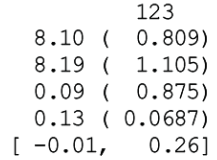

100

$7.81(0.714)$

$7.76(1.013)$

$-0.05(0.686)$

$-0.07(0.0762)$

$\left[\begin{array}{ll}-0.22,0.08 & 0.07\end{array}\right.$
121

$7.96(0.871)$

$7.46(0.903)$

$-0.50(0.827)$

$-0.49(0.0690)$

$[-0.63,-0.35]$

$-0.62(0.0974)$

$[-0.81,-0.43]$

102

$7.82(0.719)$

$7.46(0.746)$

$-0.36(0.692)$

$-0.39(0.0753)$

$[-0.53,-0.24]$

$-0.31(0.1066)$

$[-0.52,-0.10]$

The sponsor's longitudinal repeated measures analyses of change in HbAlc from baseline over time during the ST + LT period until week 48 are presented for all subjects excluding data after rescue by subgroups of $\mathrm{HbA1c}, \mathrm{BMI}$, and eGFR at baseline. An overview of placebo-corrected mean changes from baseline in HbAlc at week 24 and at week 48 for all subjects excluding data after rescue by subgroups of HbA1c, BMI, and eGFR at baseline is presented in Table 3.2.1.5.

Table 3.2.1.5 Placebo-corrected mean changes from baseline in HbA1c at week 24 and at week 48 by subgroups (full analysis set) (Source: Clinical Study

Report-48-week, Table 21)

\begin{tabular}{|c|c|c|}
\hline Subgroups & Week 24 & Week 48 \\
\hline Baseline $\mathrm{HbA} 1 \mathrm{c} \geq 9 \%$ & N.C. & N.C. \\
\hline Baseline $\mathrm{HbAlc} \geq 8 \%$ and $<9 \%$ & $\begin{array}{c}-0.98 \% \\
(-1.32 \%,-0.64 \%)\end{array}$ & $\begin{array}{c}-0.94 \% \\
(-1.45 \%,-0.42 \%)\end{array}$ \\
\hline Baseline HbAlc <8\% & $\begin{array}{c}-0.19 \% \\
(-0.38 \%, 0.01 \%)\end{array}$ & $\begin{array}{c}-0.41 \% \\
(-0.63 \%,-0.18 \%)\end{array}$ \\
\hline Baseline $\mathrm{HbA} 1 \mathrm{c} \geq 8 \%$ & $\begin{array}{c}-0.93 \% \\
(-1.26 \%,-0.60 \%)\end{array}$ & $\begin{array}{c}-0.98 \% \\
(-1.50 \%,-0.46 \%)\end{array}$ \\
\hline Baseline $\mathrm{BMI} \geq 27 \mathrm{~kg} / \mathrm{m}^{2}$ & $\begin{array}{c}-0.44 \% \\
(-0.62 \%,-0.26 \%)\end{array}$ & $\begin{array}{c}-0.62 \% \\
(-0.85 \%,-0.39 \%)\end{array}$ \\
\hline Baseline $\mathrm{BMI} \geq 30 \mathrm{~kg} / \mathrm{m}^{2}$ & $\begin{array}{c}-0.49 \% \\
(-0.71 \%,-0.27 \%)\end{array}$ & $\begin{array}{c}-0.69 \% \\
(-0.98 \%,-0.41 \%)\end{array}$ \\
\hline Baseline eGFR $<30 \mathrm{~mL} / \mathrm{min} / 1.73 \mathrm{~m}^{2}$ & N.A. & N.A. \\
\hline Baseline eGFR $\geq 30$ and $<60 \mathrm{~mL} / \mathrm{min} / 1.73 \mathrm{~m}^{2}$ & N.C. & N.C. \\
\hline Baseline eGFR $\geq 60$ and $<90 \mathrm{~mL} / \mathrm{min} / 1.73 \mathrm{~m}^{2}$ & $\begin{array}{c}-0.34 \% \\
(-0.54 \%,-0.13 \%)\end{array}$ & $\begin{array}{c}-0.64 \% \\
(-0.90 \%,-0.38 \%)\end{array}$ \\
\hline Baseline eGFR $\geq 90 \mathrm{~mL} / \mathrm{min} / 1.73 \mathrm{~m}^{2}$ & $\begin{array}{c}-0.61 \% \\
(-0.89 \%,-0.33 \%)\end{array}$ & $\begin{array}{c}-0.64 \% \\
(-0.98 \%,-0.31 \%)\end{array}$ \\
\hline
\end{tabular}


N.A., not applicable because no subject showed an eGFR $<30 \mathrm{~mL} / \mathrm{min} / 1.73 \mathrm{~m}^{2}$ at baseline

N.C., not calculated because less than 10 subjects had measurements of $\mathrm{HbA1c}$ both at baseline and at week $\mathrm{t}$ in both treatment groups. Numbers in brackets are $95 \%$ CIs of placebo-corrected mean changes from baseline in

$\mathrm{HbA1c}$.

This reviewer performed an additional subgroup analysis on patients with baseline eGFR $<90$ $\mathrm{mL} / \mathrm{min} / 1.73 \mathrm{~m}^{2}$ as shown below:

\begin{tabular}{|l|c|c|}
\hline Subgroup & Week 24 & Week 48 \\
\hline Baseline eGFR $<90 \mathrm{~mL} / \mathrm{min} / 1.73 \mathrm{~m}^{2}$ & $-0.34 \%$ & $-0.60 \%$ \\
& $(-0.55 \%,-0.13 \%)$ & $(-0.85 \%,-0.34 \%)$ \\
\hline
\end{tabular}

Note. Placebo: $\mathrm{n}=119$; DAPA $10 \mathrm{mg}$ : $\mathrm{n}=127$

In summary, the data of this study support the efficacy of dapagliflozin $10 \mathrm{mg}$ in reducing HbA1c, FPG, and body weight at Week 24. In addition, dapagliflozin is efficacious in T2DM patients with $\mathrm{eGFR} \geq 60 \mathrm{~mL} / \mathrm{min} / 1.73 \mathrm{~m}^{2}$.

\subsubsection{MB102-073 - Patients with Hypertension}

\subsubsection{Study Design and Endpoints}

This was a randomized, double-blind, 2-arm, parallel-group, placebo-controlled Phase 3 study to evaluate the effect of dapagliflozin $10 \mathrm{mg}$, given orally, to subjects with T2DM who have inadequate glycemic control and inadequately-controlled BP, while on a stable dose of an oral antidiabetic drug(s) (OAD) and/or insulin and a stable therapeutic dose of an ACEI or ARB.

The primary study objectives were:

- To compare the change from baseline in seated SBP after 12 weeks of double-blind treatment between the dapagliflozin $10 \mathrm{mg}$ treatment group and the placebo treatment group.

- To compare the change from baseline in HbAlc after 12 weeks of double-blind treatment between the dapagliflozin $10 \mathrm{mg}$ treatment group and the placebo treatment group.

Figure 3.2.2.1 shows the design for study MB102073.

Figure 3.2.2.1 Overview of the study design, MB102073 (Source: Clinical Study Report, Figure 3.1-1) 


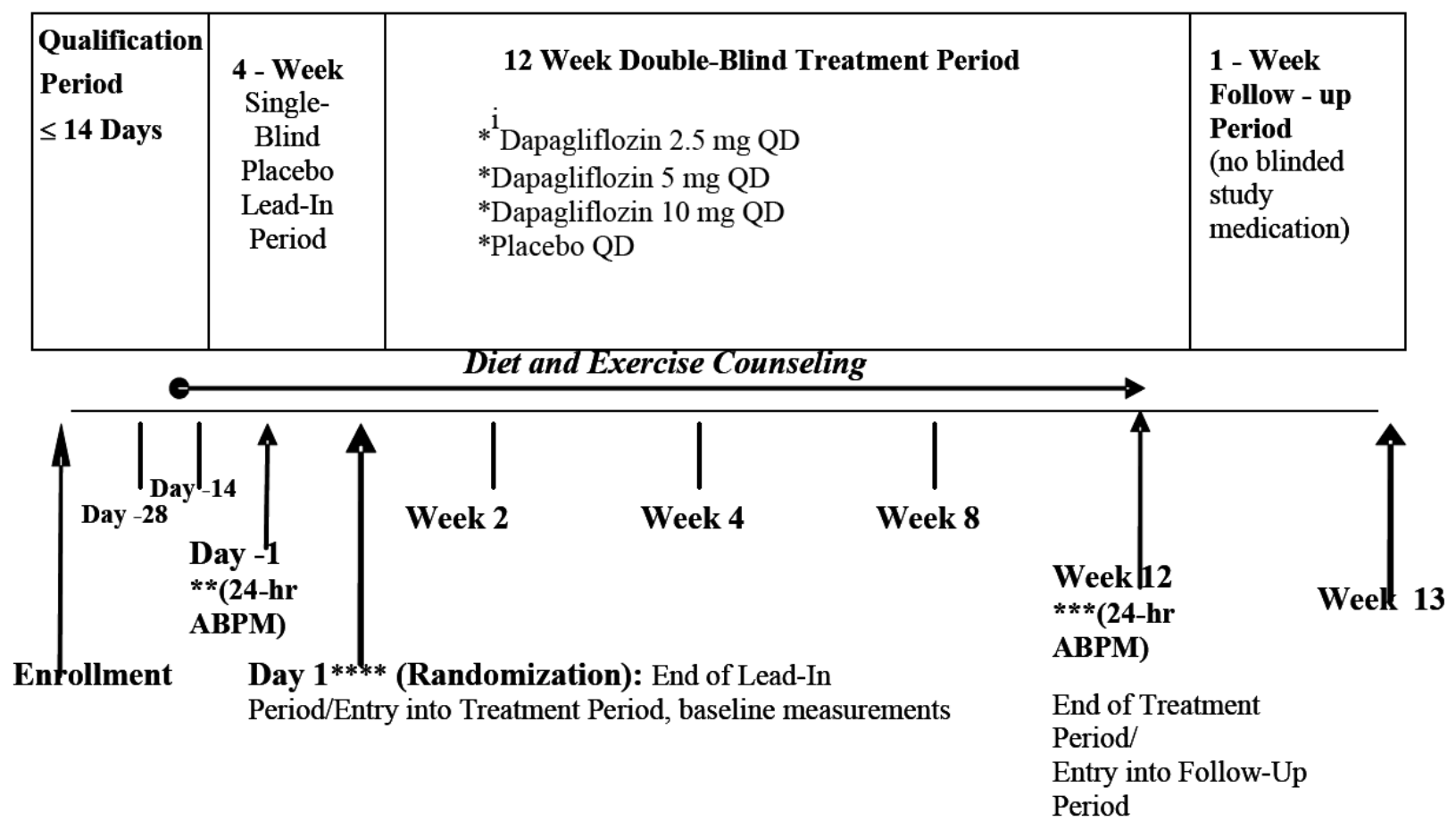

The co-primary endpoints were the change from baseline at Week 12 in seated systolic blood pressure (SBP) and HbAlc.

The secondary endpoints were the change from baseline at Week 12 in 24-hour mean ambulatory SBP, seated DBP, 24-hour mean ambulatory DBP, and serum uric acid.

\subsubsection{Statistical Methodologies}

Randomization was stratified according to insulin use at baseline. Based on the sponsor's study report, the primary efficacy analysis evaluated the change from baseline in seated SBP at Week 12 for the dapagliflozin $10 \mathrm{mg}$ treatment group compared to placebo based on a longitudinal repeated measures analysis using direct likelihood with fixed categorical effects of treatment, week, treatment-by-week interaction, and randomization strata and continuous fixed covariates of baseline seated SBP value and baseline seated SBP value by week interaction.

If this primary analysis was statistically significant at the 0.05 level, the statistical test for the second co-primary endpoint (change from baseline in $\mathrm{HbAlc}$ ) was conducted. This analysis also used a similar longitudinal repeated measures analysis except that the continuous fixed covariates were baseline HbA1c and baseline HbAlc by week interaction.

If the comparisons between the dapagliflozin $10 \mathrm{mg}$ treatment group and the placebo group were significant at the 0.05 level for both co-primary endpoints, the statistical tests for the secondary efficacy endpoints were performed. The family-wise type I error rate related to the primary and secondary efficacy endpoints was controlled at the 2 -sided 0.05 level by using a hierarchical closed testing procedure in the following order for change from baseline to Week 12: 
- 24-hr Ambulatory SBP (LOCF)

- $\quad$ Seated DBP (MMRM)

- 24-hr Ambulatory DBP (LOCF)

- Serum uric acid (MMRM)

Data after rescue were excluded from blood pressure (BP) analyses.

\subsubsection{Patient Disposition, Demographic and Baseline Characteristics}

Table 3.2.2.1 shows the disposition of the double-blind treated subjects. All randomized subjects were treated.

Table 3.2.2.1 Subject Disposition for 12-Week Double-blind Treatment Period of Study MB102073 (Source: Clinical Study Report, Table -1)

\begin{tabular}{lccc}
\hline \hline & \multicolumn{3}{c}{ Number (\%) Subjects } \\
\cline { 2 - 4 } & PLA + & DAPA 10 mg + & Total \\
& ACEI/ARB & ACEI/ARB & N = 613 \\
\hline Subjects completing double-blind period, n (\%) & $287(92.3)$ & $281(93.0)$ & $568(92.7)$ \\
Subjects not completing double-blind period, $\mathrm{n}(\%)$ & $24(7.7)$ & $21(7.0)$ & $45(7.3)$ \\
Reason for not completing double-blind period, $\mathrm{n}(\%)$ & & & \\
$\quad$ Adverse event, $\mathrm{n}(\%)$ & $3(1.0)$ & $3(1.0)$ & $6(1.0)$ \\
Subject withdrew consent, $\mathrm{n}(\%)$ & $7(2.3)$ & $9(3.0)$ & $16(2.6)$ \\
Subject no longer met criteria, $\mathrm{n}(\%)$ & $6(1.9)$ & $5(1.7)$ & $11(1.8)$ \\
Lost to follow-up, $\mathrm{n}(\%)$ & $5(1.6)$ & $2(0.7)$ & $7(1.1)$ \\
Lack of efficacy, $\mathrm{n}(\%)$ & 0 & $2(0.7)$ & $2(0.3)$ \\
Other, $\mathrm{n}(\%)$ & $3(1.0)$ & 0 & $3(0.5)$ \\
\hline \hline
\end{tabular}

This table includes all randomized subjects in either treatment group who took at least 1 dose of double-blind study medication. Subjects continuing in the study refers to subjects entering the follow-up period. ACEI $=$ angiotensinconverting enzyme inhibitor; $\mathrm{ARB}=$ angiotensin receptor blocker; DAPA = dapagliflozin; PLA = placebo; $\mathrm{N}=$ number of randomized and treated subjects.

The Applicant reported fairly high completion rates for the 12-week period in the two treatment arms: 93\% (281/302) for DAPA $10 \mathrm{mg}$ and 92\% (287/311) for placebo. The denominators for these results are from the safety analysis set. Considering the small number of dropouts, there was not a substantial imbalance across arms in the reasons for discontinuation.

Table 3.2.2.2 shows selected demographic characteristics for the Randomized Subjects data set. The table was provided by the Applicant. They also reported that $41 \%$ of subjects were in the North America and 21\% in Europe.

Table 3.2.2.2 Demographic Characteristics, Randomized Subjects - MB102073 (Source: Clinical Study Report, Table -2). 


\begin{tabular}{lccc}
\hline & $\begin{array}{c}\text { PLA + } \\
\text { ACEI/ARB } \\
\mathbf{N}=\mathbf{3 1 1}\end{array}$ & $\begin{array}{c}\text { DAPA 10 mg }+ \\
\text { ACEI/ARB } \\
\mathbf{N = 3 0 2}\end{array}$ & $\begin{array}{c}\text { Total } \\
\mathbf{N}=\mathbf{6 1 3}\end{array}$ \\
\hline Mean age, years & 56.2 & 55.6 & 55.9 \\
Age category, $<$ 65 years, $\mathrm{n}(\%)$ & $259(83.3)$ & $260(86.1)$ & $519(84.7)$ \\
Gender, male, $\mathrm{n}(\%)$ & $171(55.0)$ & $179(59.3)$ & $350(57.1)$ \\
Race, $\mathrm{n}(\%)$ & & & \\
$\quad$ White & $241(77.5)$ & $239(79.1)$ & $480(78.3)$ \\
$\quad$ Asian & $54(17.4)$ & $49(16.2)$ & $103(16.8)$ \\
$\quad$ Black-African American & $14(4.5)$ & $12(4.0)$ & $26(4.2)$ \\
$\quad$ Other & $2(0.6)$ & $2(0.7)$ & $4(0.7)$ \\
Mean BMI, kg/m & 30.56 & 31.52 & 31.03 \\
\hline \hline
\end{tabular}

Percentages reported are based on the total number of subjects in each treatment group. ACEI $=$ angiotensinconverting enzyme inhibitor; $\mathrm{ARB}=$ angiotensin receptor blocker; $\mathrm{DAPA}=$ dapagliflozin; $\mathrm{PLA}=$ placebo; $\mathrm{N}=$ number of randomized and treated subjects; $\mathrm{n}=$ number of subjects.

The median baseline duration of T2DM was 7.9 years, and $31.5 \%$ had been diagnosed with T2DM for $>10$ years. The overall mean baseline HbA1c was $8.06 \%$ and the mean baseline fasting plasma glucose (FPG) was $159.33 \mathrm{mg} / \mathrm{dL}$. The median baseline duration of hypertension was 7.0 years, and $24.8 \%$ had been diagnosed with hypertension for $>10$ years. The mean baseline glomerular filtration rate (GFR) was 85.053 and $86.736 \mathrm{~mL} / \mathrm{min} / 1.73 \mathrm{~m}^{2}$ for the dapagliflozin $10 \mathrm{mg}$ and placebo treatment groups, respectively. At baseline, $52.2 \%$ of subjects overall had mild renal impairment (defined as estimated GFR between 60 and $<90$ $\mathrm{mL} / \mathrm{min} / 1.73 \mathrm{~m}^{2}$ ) and $8.6 \%$ of subjects overall had moderate renal impairment (defined as estimated GFR between 30 and $<60 \mathrm{~mL} / \mathrm{min} / 1.73 \mathrm{~m}^{2}$ ).

\subsubsection{Results and Conclusions}

Table 3.2.2.3 shows the Applicant's reported results of the primary and secondary efficacy analysis for Study 2073. The sponsor's primary analysis, which used MMRM imputation, showed DAPA $10 \mathrm{mg}$ to be superior to placebo, which is verified by this reviewer.

Table 3.2.2.3 Adjusted mean change from baseline in Primary and Secondary Efficacy Endpoints at Week 12, Randomized Subjects (MB102073) (Source: Clinical Study Report, Tables 7.2.1-1, 7.2.2-1). 


\begin{tabular}{|c|c|c|c|}
\hline $\begin{array}{l}\text { EFFICACY ENDPOINT } \\
\text { TIMEPOINT }\end{array}$ & & $\begin{array}{c}\mathrm{PIA}+\mathrm{ACEI} / \mathrm{ARB} \\
\mathrm{N}=311\end{array}$ & $\begin{array}{c}\mathrm{DAP} \text { 10MG }+\mathrm{ACEI} / \mathrm{F} \\
\mathrm{N}=302\end{array}$ \\
\hline \multicolumn{4}{|l|}{ PRTMARY EFETCACY EINDPOINTS } \\
\hline \multicolumn{4}{|c|}{$\begin{array}{l}\text { SEATED SYSTOLIC BLOOD PRESSURE (MMHG) } \\
\text { (a) }\end{array}$} \\
\hline WEEK 12 & $\begin{array}{l}\mathrm{n} \\
\text { BASELINE MFAN (SD) } \\
\text { WEFE 12 MEAN (SD) } \\
\text { MFAN CHANGE FROM BASELINE (SD) } \\
\text { ADJ. MFAN CHANGE FROM BASEIINE (SE) } \\
\text { 95\% CONFIDENCE INTERVAL FOR ADJ. MEAN } \\
\text { DIFFERENCE VS. PLACEBO (SE) } \\
\text { 95\% CONFIDENCE INTERVAL FOR DIFFERENCE } \\
\text { P-VALUE VS. PLACEBO }\end{array}$ & $\begin{aligned} 279 & \\
149.53 & (8.047) \\
142.58 & (11.788) \\
-6.94 & (10.697) \\
-7.34 & (0.8812) \\
(-9.07, & -5.61)\end{aligned}$ & $\begin{array}{l}280 \\
149.84(7.527) \\
139.88(12.970) \\
-9.97(11.674) \\
-10.40(0.8822) \\
(-12.13,-8.66) \\
-3.05(0.9251) \\
(-4.87,-1.24) \\
0.0010^{\star}\end{array}$ \\
\hline \multicolumn{4}{|l|}{ HBA1C (\%) (a) } \\
\hline WEEK 12 & 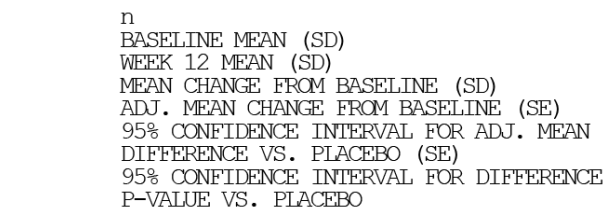 & $\begin{aligned} 279 & \\
8.02 & (0.911) \\
7.97 & (1.105) \\
-0.05 & (0.783) \\
-0.10 & (0.0631) \\
(-0.22, & 0.03)\end{aligned}$ & $\begin{aligned} & 278 \\
& 8.09(1.003) \\
& 7.56(1.005) \\
&-0.52(0.909) \\
&-0.56(0.0633) \\
&(-0.68,-0.43) \\
&-0.46(0.0673) \\
&(-0.59,-0.33) \\
&<0.0001^{\star}\end{aligned}$ \\
\hline \multicolumn{4}{|l|}{ SECONDARY EF'FICACY ENDPOINTS } \\
\hline \multicolumn{4}{|c|}{$\begin{array}{l}\text { 24-HOUR MEAN AMBULATORY SYSTOLIC BLOOD } \\
\begin{array}{llll}\text { PRESSURE (MMHG) } & \text { (LOCF) } & \text { (b) }\end{array}\end{array}$} \\
\hline WEEK 12 & $\begin{array}{l}\text { N\# } \\
\text { BASELINE MEAN (SD) } \\
\text { ADJ. MFAN CHANGE FROM BSL. (SE) } \\
\text { DIFFERENCE VS. PIACEBO (SE) } \\
\text { P-VALUE VS. PLACEBO }\end{array}$ & $\begin{array}{rr}263 & \\
146.64 & (11.732) \\
-6.73 & (1.2427)\end{array}$ & $\begin{array}{rr}267 & \\
145.92 & (11.652) \\
-9.62 & (1.2277) \\
-2.89 & (1.0091) \\
0.0043^{\star}\end{array}$ \\
\hline
\end{tabular}

For the analysis on the secondary efficacy endpoints, the efficacy of DAPA was supported only by the 24-hr Ambulatory SBP according to the sponsor's pre-specified multiplicity adjustment procedure at level of 0.05 . Claims on all other secondary endpoints should not be included in the product label.

The sponsor did subgroup analyses for change in seated SBP and HbA1c at Week 12 were performed for race (Asian, Black or African American, White, Other), age ( $<65$ years, $\geq 65$ years, $\geq 75$ years), gender, ethnicity, geographic region (North America, Latin America, Europe, Asia/Pacific), female age (female $\leq 50$ years, female $>50$ years), and baseline $\mathrm{HbA} 1 \mathrm{c}(<8 \%, \geq$ $8 \%$ to $<9 \%$, and $\geq 9 \%$ ), and baseline seated SBP $(<150 \mathrm{mmHg}, \geq 150 \mathrm{mmHg})$. As shown in Figures 3.2.2.2 and 3.2.2.3, these subgroup analyses showed a consistency in effect across the subgroups, supportive to the efficacy of DAPA across subgroups. No potential treatment interaction ( $p$-value $<0.10$ ) for the co-primary efficacy variable of seated SBP was detected for the subgroups. For the co-primary endpoint $\mathrm{HbA1c}$, the results suggest a potential treatment interaction between subgroup defined by race (interaction test $p$-value $=0.0654$ ). Subjects with a higher baseline HbA1c showed a larger effect of dapagliflozin treatment than subjects with a lower baseline HbA1c. 
Figure 3.2.2.2 Seated Systolic Blood Pressure (mmHg) Adjusted Mean Change for Baseline at Week 12 Evaluation in Subgroups Excluding Data After

$\underset{\text { Subgroup }}{\text { Rescue, Randomized Subjects (MB102073) }}$

Race $=$ Asian

PLA + ACEI/ARB

- DAP 10MG + ACEI/ARB

Race = Black/African American

PLA + ACEI/ARB

DAP $10 M G+A C E I / A R B$

Race $=$ White

PLA + ACEI/ARB

DAP $10 M G+A C E I / A R B$

Race $=$ Othe

PLA + ACEI/ARB

DAP $10 M G+A C E I / A R B$

Gender $=$ Female

PLA + ACEI/ARB

Gender = Male

PLA + ACEI/ARB

DAP $10 M G+A C E I / A R B$

Ethnicity = Hispanic/Latino

DAP $10 M G+A C E I / A R B$

Ethnicity = Non-Hispanic/Latino

$\mathrm{PLA}+\mathrm{ACEI} / \mathrm{ARB}$
$\mathrm{DAP} 10 \mathrm{MG}+\mathrm{ACEI} / \mathrm{ARB}$

Baseline SBP $<150 \mathrm{mmHg}$

Baseline SBP $<150 \mathrm{~mm}$

DAP 10MG + ACEI/ARB

Baseline SBP $>=150 \mathrm{mmHg}$

Baseline SBP > $=150 \mathrm{mmHg}$

DAP 10MG + ACEI/ARB

Age $<65$ yrs

PLA + ACEI/ARB

DAP 10MG + ACEI/ARB

Age $>=65$ yrs

PLA + ACEI/ARB

DAP 10MG + ACEI/ARB

Region $=$ North America

PLA + ACEI/ARB

DAP 10MG + ACEI/ARB

Region $=$ Latin America

PLA + ACEI/ARB

DAP $10 M G+A C E I / A R B$

Region $=$ Europe

PLA + ACEI/ARB

DAP $10 M G+A C E I / A R B$

Region = Asia/Pacific

PAP + ACEI/ARB

Female Age $<=50$ yrs

DAP 10MG + ACEI/ARB

Female Age $>50$ yrs

Female Age $>50$ yrs

DAP 10MG + ACEI/ARB

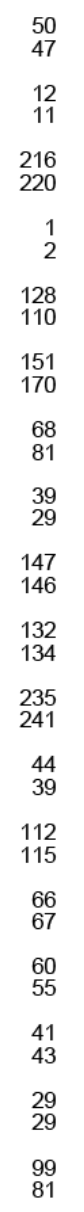

T1
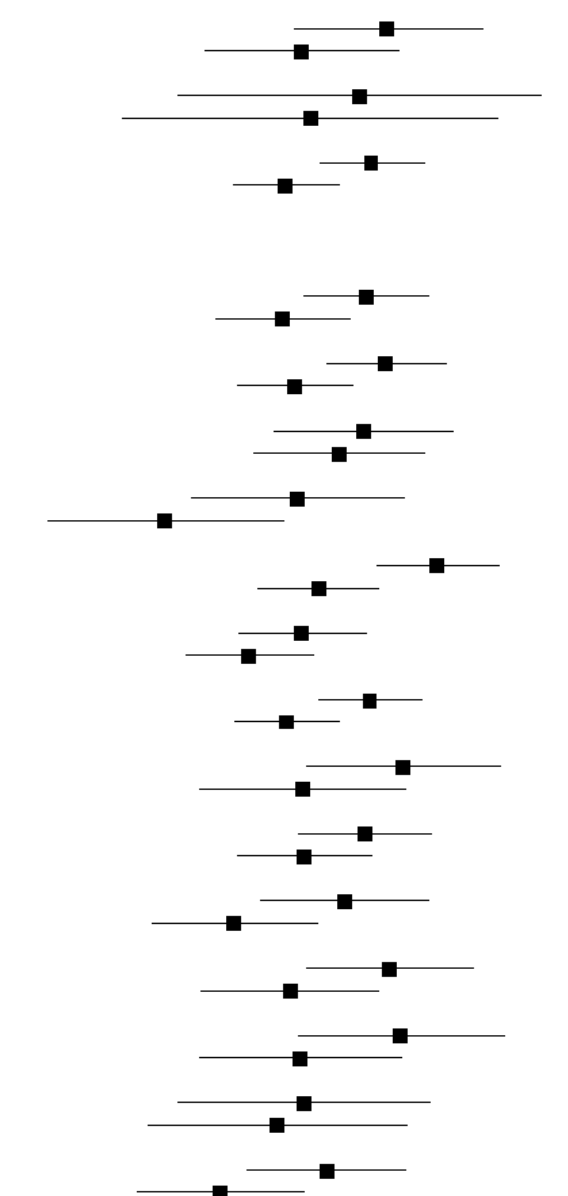

$\begin{array}{llllllllllll}-20 & -18 & -16 & -14 & -12 & -10 & -8 & -6 & -4 & -2 & 0 & 2\end{array}$

Mean Change from Baseline $(\mathrm{Cl})$

$$
\begin{array}{ll}
-6.95 & (-10.27 ;-3.63) \\
-9.99 & (-13.40 ;-6.57) \\
-7.94 & (-14.32 ;-1.56) \\
-9.68 & (-16.28 ;-3.08) \\
-7.52 & (-9.37 ;-5.67) \\
-10.54 & (-12.40 ;-8.68)
\end{array}
$$

$-7.73(-9.94 ;-5.52)$

$-10.65(-13.00 ;-8.30)$

$-7.01(-9.13 ;-4.90)$

$-10.23(-12.26 ;-8.21)$

$-7.81 \quad(-10.97 ;-4.66)$

$-8.68(-11.70 ;-5.66)$

$-10.13(-13.88 ;-6.38)$

$-14.78(-18.91 ;-10.64)$

$-5.19(-7.31 ;-3.06)$

$-9.39 \quad(-11.53 ;-7.26)$

$-9.97(-12.21 ;-7.73)$

$-11.84(-14.07 ;-9.61)$

-7.58
$-10.53(-12.31 ;-5175)$

$-6.41 \quad(-9.83 ;-2.99)$

$-9.95 \quad(-13.58 ;-6.31)$

$\begin{array}{ll}-7.77 & (-10.12 ;-5.43) \\ -9.90 & (-12.23 ;-7.57)\end{array}$

$-8.46(-11.42 ;-5.50)$

$-6.87(-9.81 ;-3.93)$

$-10.39(-13.51 ;-7.28)$

$-6.46 \quad(-10.09 ;-2.83)$

$-10.03(-13.60 ;-6.47)$

$-9.89(-14.33 ;-5.45)$

$-10.84(-15.40 ;-6.28)$

$-9.11(-11.90 ;-6.31)$

Seated Systolic Blood Pressure (mmHg) Adjusted Mean Change from Baseline 


\section{Figure 3.2.2.3 HbA1c (Percent) Adjusted Mean Change from Baseline at Week 12 Evaluation in Subgroups Including Data After Rescue, Randomized Subjects (MB102073)}

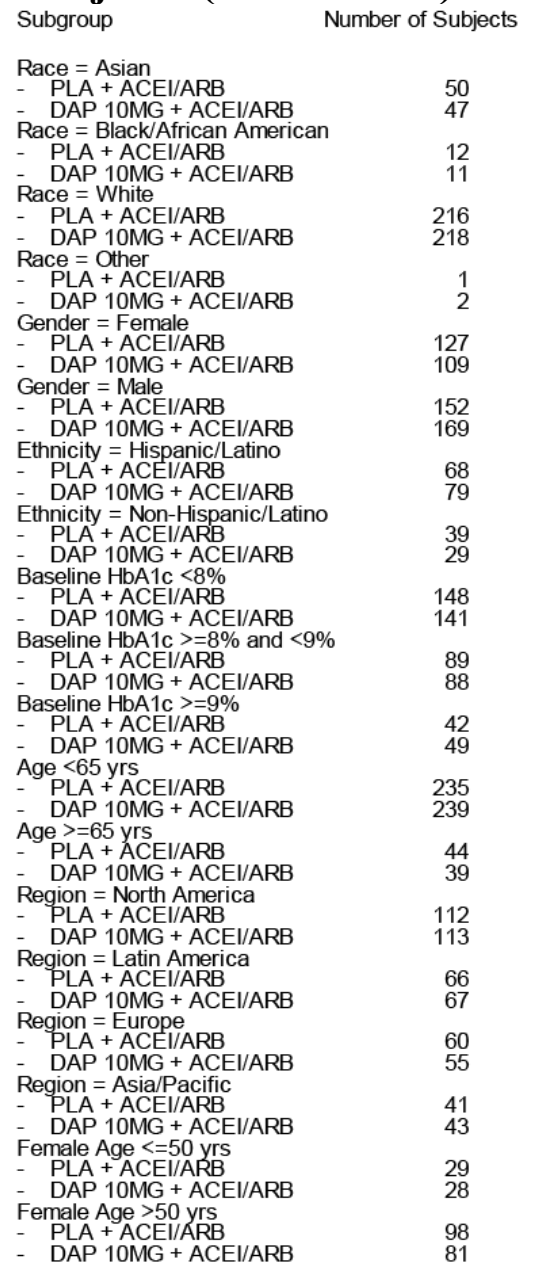

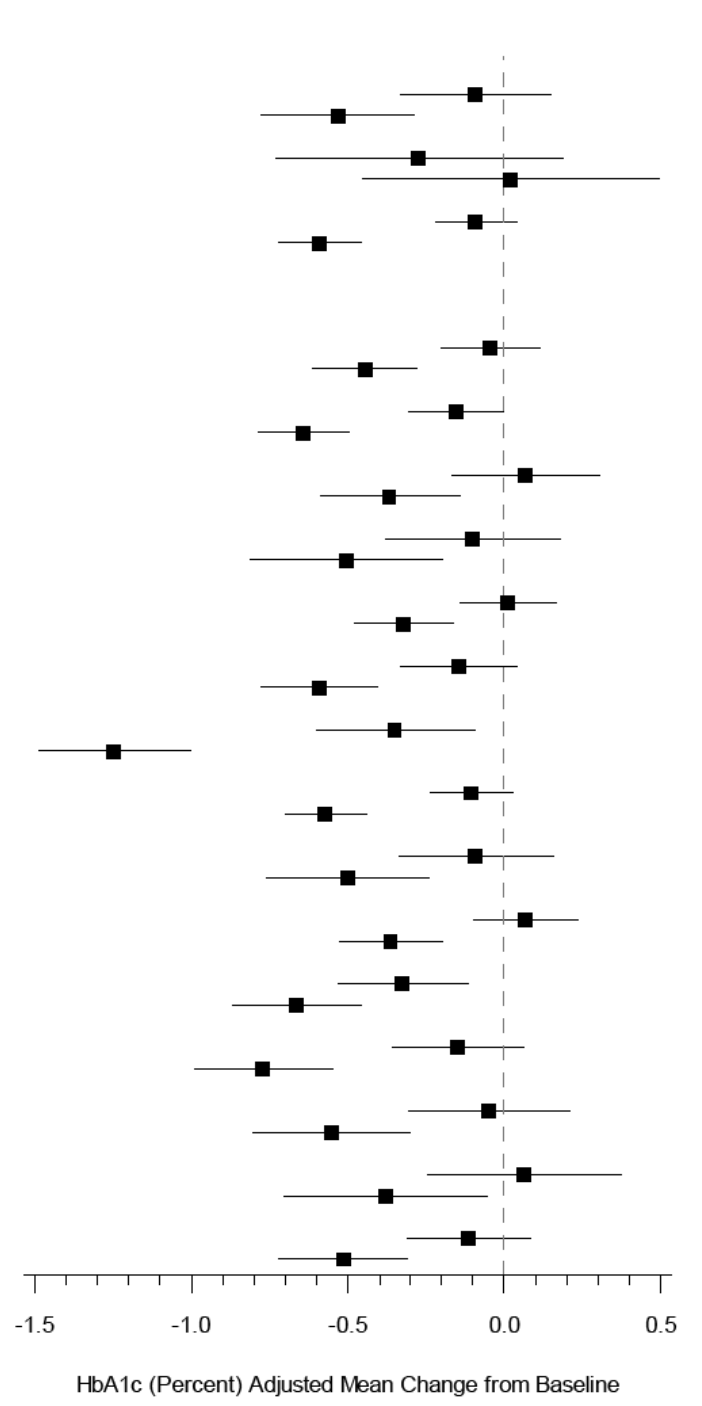

Mean Change from Baseline $(\mathrm{Cl})$

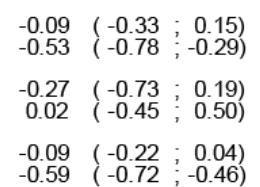

$\begin{array}{lll}-0.04 & (-0.20 ; 0.12) \\ -0.45 & (-0.61 ;-0.28)\end{array}$

$-0.15(-0.30 ; 0.00)$

$-0.64(-0.79 ;-0.50)$

$\begin{array}{rr}0.07 & (-0.17 ; 0.30) \\ -0.36 & (-0.59 ;-0.14)\end{array}$

-0.10
-0.50 $\left(\begin{array}{l}-0.38 ; 0.18) \\ -0.81 ;-0.19)\end{array}\right.$

$0.01 \quad(-0.14 ; 0.17)$

$-0.32(-0.48 ;-0.16)$

$-0.14 \quad(-0.33 ; 0.04)$

$-0.35(-0.60 ;-0.09)$

$1.25(-1.49 ;-1.00)$

$\begin{array}{ll}-0.10 & (-0.23 ; 0.03) \\ -0.57 \quad(-0.70 ;-0.44)\end{array}$

$\begin{array}{ll}-0.09 & (-0.34 ; 0.16) \\ -0.50 & (-0.76 ;-0.24)\end{array}$

0.07
$-0.36 \quad(-0.09 ; 0.24)$
$-0.20)$

$\begin{array}{lll}-0.32 & (-0.53 ;-0.11) \\ -0.66 & (-0.87 ;-0.46)\end{array}$

$\begin{array}{ll}-0.15 & (-0.35 ; 0.06) \\ -0.77 & (-0.99 ;-0.55)\end{array}$

$-0.05(-0.31 ; 0.21)$

$-0.55(-0.80 ;-0.30)$

$\begin{array}{rr}0.07 & (-0.24 ; 0.37) \\ -0.38 & (-0.70 ;-0.05)\end{array}$

$\begin{array}{ll}-0.11 & (-0.31 ; 0.08) \\ -0.51 & (-0.72 ;-0.31)\end{array}$

HbA1c (Percent) Adjusted Mean Change from Baseline

In summary, the data of this study support the efficacy of dapagliflozin $10 \mathrm{mg}$ in reducing HbA1c and systolic blood pressure at Week 12.

\subsubsection{MB102-077 - Patients with Hypertensiuon}

\subsubsection{Study Design and Endpoints}

This was a randomized, double-blind, 2-arm, parallel-group, placebo-controlled Phase 3 study to evaluate the effect of dapagliflozin $10 \mathrm{mg}$, given orally, to subjects with T2DM who have inadequate glycemic control and inadequately-controlled BP, while on a stable dose of an OAD and/or insulin and a stable, effective therapeutic dose of an ACEI or ARB plus an additional antihypertensive medication. 
The primary efficacy objectives were:

- To compare the change from baseline in seated systolic blood pressure (SBP) after 12 weeks of double-blind treatment between the dapagliflozin $10 \mathrm{mg}$ treatment group and the placebo treatment group.

- To compare the change from baseline in glycosolated hemoglobin (HbA1c) after 12 weeks of double-blind treatment between the dapagliflozin $10 \mathrm{mg}$ treatment group and the placebo treatment group.

Figure 3.2.3.1 shows the design for study MB102073.

Figure 3.2.3.1 Overview of the study design, 2077 (Source: Clinical Study Report, Figure 3.1-1)

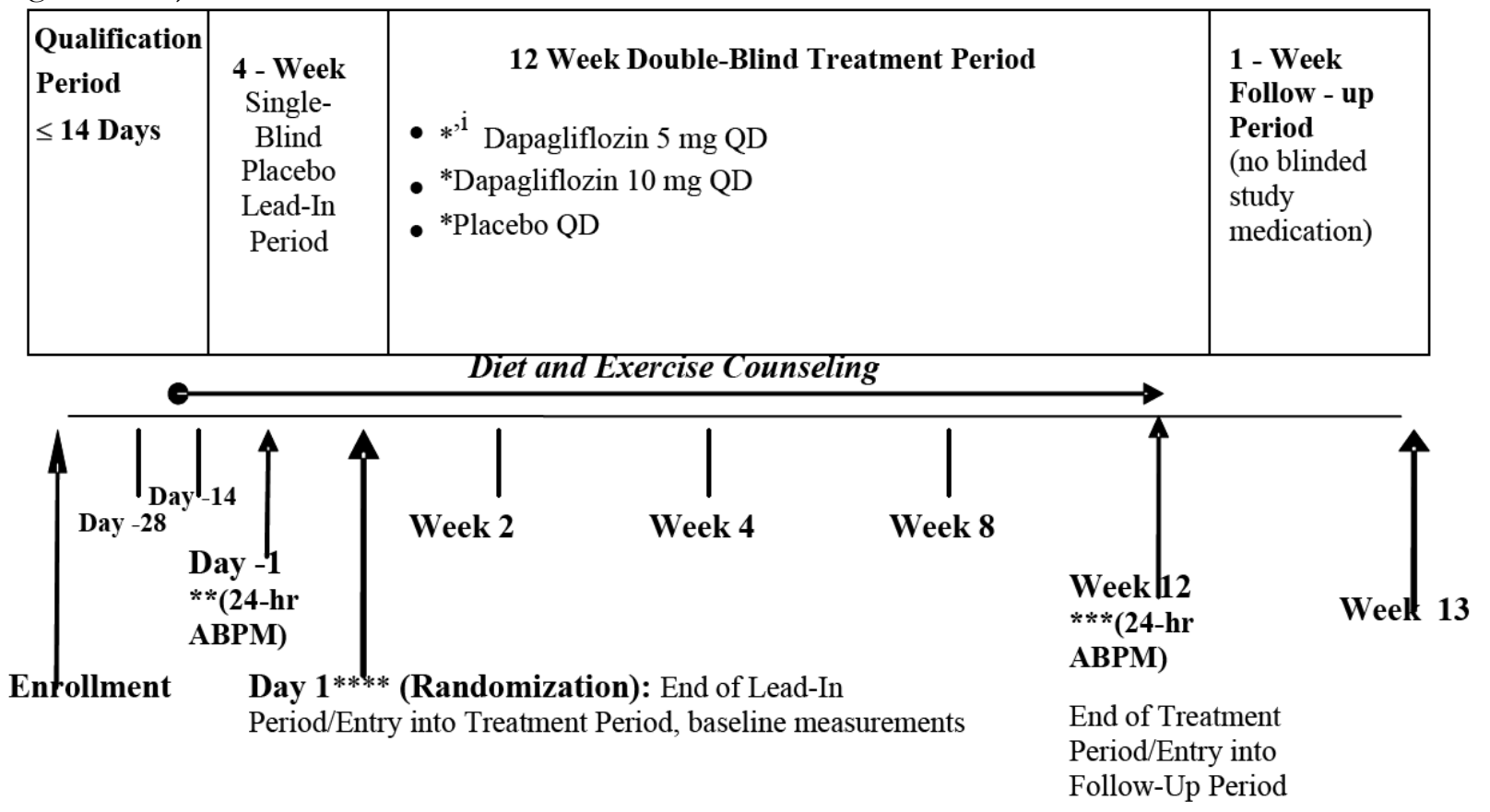

The co-primary endpoints were the changes from baseline at Week 12 in seated SBP and HbA1c. The secondary endpoints were the changes from baseline at Week 12 in 24-hour mean ambulatory SBP, seated DBP, 24-hour mean ambulatory DBP, and serum uric acid.

\subsubsection{Statistical Methodologies}

Randomization was stratified according to the additional antihypertensive medication use (thiazide/thiazide-like diuretic versus calcium channel blocker, beta blocker, alpha adrenergic blocker, or central alpha adrenergic agonist) and according to insulin use at baseline. The Randomized Subjects data set consisted of all randomized subjects who received at least 1 dose of double-blind study medication during the double-blind period. 
The same statistical methods for efficacy analysis and multiplicity procedure for family-wise type 1 error control in 2073 are used in 2077.

\subsubsection{Patient Disposition, Demographic and Baseline Characteristics}

Table 3.2.3.1 shows the disposition of the double-blind treated subjects. All randomized subjects were treated.

Table 3.2.3.1 Subject Disposition for 12-Week Double-blind Treatment Period- MB102077 (Source: Clinical Study Report, Table 1).

\begin{tabular}{lccc}
\hline & \multicolumn{3}{c}{ Number (\%) Subjects } \\
\cline { 2 - 4 } & $\begin{array}{c}\text { PLA + } \\
\text { ACEI/ARB } \\
\text { N = 224 }\end{array}$ & $\begin{array}{c}\text { DAPA 10 mg + } \\
\text { ACEI/ARB }\end{array}$ & $\begin{array}{c}\text { Total } \\
\text { N = 225 }\end{array}$ \\
\hline Subjects completing double-blind period & $202(90.2)$ & $211(93.8)$ & $413(92.0)$ \\
Subjects not completing double-blind period & $22(9.8)$ & $13(5.8)$ & $35(7.8)$ \\
Reason for not completing double-blind period & & & \\
Adverse event & $4(1.8)$ & $1(0.4)$ & $5(1.1)$ \\
Subject withdrew consent & $6(2.7)$ & $4(1.8)$ & $10(2.2)$ \\
Subject no longer met criteria & $1(0.4)$ & $5(2.2)$ & $6(1.3)$ \\
Lost to follow-up & $3(1.3)$ & $2(0.9)$ & $5(1.1)$ \\
Administrative reason by sponsor & $2(0.9)$ & $1(0.4)$ & $3(0.7)$ \\
Lack of efficacy & $2(0.9)$ & 0 & $2(0.4)$ \\
Subject request & $1(0.4)$ & 0 & $1(0.2)$ \\
Other & $3(1.3)$ & 0 & $3(0.7)$ \\
\hline \hline
\end{tabular}

${ }^{a}$ One subject (Subject MB102077-208-70377, received 30 days of double-blind treatment) had missing end-ofstudy disposition information as the site was disbanded before all final queries had been answered. This table includes all randomized subjects in either treatment group who took at least 1 dose of double-blind study medication. Subjects continuing in the study refer to subjects entering the follow-up period. $\mathrm{ACEI}=$ angiotensin-converting enzyme inhibitor; $\mathrm{ARB}=$ angiotensin receptor blocker; DAPA = dapagliflozin; PLA $=$ placebo; $\mathrm{N}=$ number of randomized and treated subjects.

The Applicant reported fairly high completion rates for the 12-week period in the two treatment arms: $94 \%(211 / 225)$ for DAPA $10 \mathrm{mg}$ and $90 \%(202 / 224)$ for placebo. The denominators for these results are from the safety analysis set. Considering the small number of dropouts, there was not a substantial imbalance across arms in the reasons for discontinuation.

Table 3.2.3.2 shows selected demographic characteristics for the Randomized Subjects data set. The table was provided by the Applicant. They also reported that $38 \%$ of subjects were in the North America and 25\% in Europe.

Table 3.2.3.2 Demographic Characteristics, Randomized Subjects - Study MB102077 (Source: Clinical Study Report, Table 2). 


\begin{tabular}{|c|c|c|c|}
\hline & $\begin{array}{c}\text { PLA + } \\
\text { ACEI/ARB } \\
\text { N = 224 }\end{array}$ & $\begin{array}{c}\text { DAPA } 10 \mathrm{mg}+ \\
\text { ACEI/ARB } \\
\text { N }=\mathbf{2 2 5}\end{array}$ & $\begin{array}{c}\text { Total } \\
\mathrm{N}=\mathbf{4 4 9}\end{array}$ \\
\hline Mean age, years & 56.2 & 55.8 & 56.0 \\
\hline Age category, $<65$ years, $\mathrm{n}(\%)$ & $198(88.4)$ & $198(88.0)$ & $396(88.2)$ \\
\hline Gender, male, $\mathrm{n}(\%)$ & $129(57.6)$ & $118(52.4)$ & $247(55.0)$ \\
\hline \multicolumn{4}{|l|}{ Race, $\mathrm{n}(\%)$} \\
\hline White & $157(70.1)$ & $160(71.1)$ & $317(70.6)$ \\
\hline Asian & $38(17.0)$ & $34(15.1)$ & $72(16.0)$ \\
\hline Black/African-American & $17(7.6)$ & $19(8.4)$ & $36(8.0)$ \\
\hline Other & $12(5.4)$ & $12(5.3)$ & $24(5.3)$ \\
\hline Mean BMI, kg/m ${ }^{2}$ & 32.13 & 31.90 & 32.01 \\
\hline
\end{tabular}

The Applicant claimed that the baseline diabetes and hypertension characteristics and baseline renal function were balanced across the dapagliflozin $10 \mathrm{mg}$ and placebo treatment groups. Based on the Applicant, the overall median duration of T2DM was 6.4 years, and $28.1 \%$ had been diagnosed with T2DM for $>10$ years. The overall mean baseline HbA1c was $8.04 \%$ and the mean baseline fasting plasma glucose (FPG) was $160.71 \mathrm{mg} / \mathrm{dL}$. The overall median duration of hypertension was 7.5 years, and $36.7 \%$ had been diagnosed with hypertension for $>10$ years. The mean baseline of estimated glomerular filtration rate (eGFR) was 84.78 and 87.01 $\mathrm{mL} / \mathrm{min} / 1.73 \mathrm{~m}^{2}$ for the dapagliflozin $10 \mathrm{mg}$ and placebo treatment groups, respectively. At baseline, $53.5 \%$ of subjects overall had mild renal impairment (defined as eGFR between 60 and $<90 \mathrm{~mL} / \mathrm{min} / 1.73 \mathrm{~m}^{2}$ ) and $7.8 \%$ of subjects overall had moderate renal impairment (defined as eGFR between 30 and $<60 \mathrm{~mL} / \mathrm{min} / 1.73 \mathrm{~m}^{2}$ ).

Most subjects in the dapagliflozin $10 \mathrm{mg}$ and placebo treatment groups were receiving background metformin therapy $(90.2 \%$ and $92.0 \%$, respectively). Other OAD drugs used in at least $10 \%$ of subjects in the 2 treatment groups were the sulfonylureas, glimepiride $(18.7 \%$ and $20.1 \%$, respectively) and glyburide (14.7\% and $12.9 \%$, respectively). Insulin use was reported for $7.6 \%$ of subjects. Approximately $55 \%$ of subjects in the dapagliflozin $10 \mathrm{mg}$ and placebo groups were receiving a background ACEI (56.0\% and 55.4\%, respectively), with the remaining $45 \%$ receiving a background ARB (44.0\% and $44.2 \%$, respectively). The additional antihypertensive drug consisted of a thiazide or thiazide-like diuretic for 200 subjects (44.5\%) and a calcium channel blocker, beta blocker, central alpha adrenergic agonist or alpha adrenergic blocker for 249 subjects $(55.5 \%)$.

\subsubsection{Results and Conclusions}

Table 3.2.3.3 shows the Applicant's reported results of the primary and secondary efficacy analysis for Study 2077. The primary analysis, which used MMRM imputation, showed DAPA $10 \mathrm{mg}$ to be superior to placebo and is verified by this reviewer. 
Table 3.2.3.3 Seated Systolic Blood Pressure (mmHg) and HbA1c (Percent) Longitudinal Repeated Measures Analysis: 12-Week Double-blind Treatment Period, Excluding Data After Rescue, Randomized Subjects (Study MB102077) (Source: Clinical Study Report, Tables 7.2.1-1, 7.2.2-1).

\begin{tabular}{|c|c|c|c|}
\hline $\begin{array}{l}\text { EFFICACY ENDPOINT } \\
\text { TIMEPOINT }\end{array}$ & & $\begin{array}{c}\mathrm{PIA}+\mathrm{ACEI} / \mathrm{ARB} \\
\mathrm{N}=224\end{array}$ & $\begin{array}{c}\text { DAP 10MG + ACEI/ARB } \\
\mathrm{N}=225\end{array}$ \\
\hline \multicolumn{4}{|c|}{ PRIMARY EFEICACY ENDPOINTS } \\
\hline \multicolumn{4}{|c|}{ SEATED SYSTOLIC BLOOD PRESSURE (a) } \\
\hline WEFK 12 & 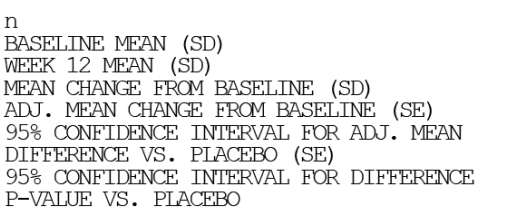 & $\begin{aligned} 199 & \\
151.30 & (6.749) \\
144.08 & (12.833) \\
-7.22 & (11.997) \\
-7.62 & (1.0701) \\
(-9.72, & -5.51)\end{aligned}$ & $\begin{array}{l}205 \\
151.01(7.879) \\
139.69(12.519) \\
-11.33(11.996) \\
-11.90(1.0585) \\
(-13.97,-9.82) \\
-4.28(1.1485) \\
(-6.54,-2.02) \\
0.0002^{\star}\end{array}$ \\
\hline \multicolumn{4}{|l|}{$\mathrm{HBA} 1 \mathrm{C}\left(\frac{\circ}{8}\right)$} \\
\hline WEEK 12 & 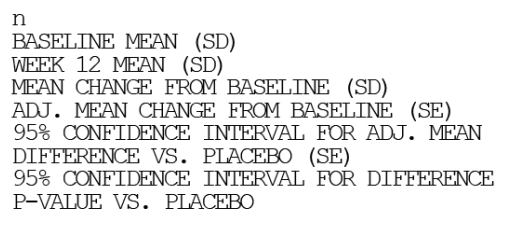 & $\begin{aligned} 197 & \\
8.00 & (0.963) \\
7.98 & (1.175) \\
-0.03 & (0.836) \\
-0.02 & (0.0673) \\
(-0.15, & 0.12)\end{aligned}$ & $\begin{array}{l}204 \\
8.09(0.914) \\
7.43(0.951) \\
-0.66(0.801) \\
-0.63(0.0668) \\
(-0.76,-0.50) \\
-0.61 \quad(0.0773) \\
(-0.76,-0.46) \\
<0.0001^{\star}\end{array}$ \\
\hline \multicolumn{4}{|c|}{ SECONDARY EFFICACY ENDPOINTS } \\
\hline \multicolumn{4}{|c|}{$\begin{array}{l}\text { 24-HOUR MFAN AMBULATORY SYSTOLIC BLOOD } \\
\text { PRESSURE (LOCF) (b) }\end{array}$} \\
\hline WEFK 12 & 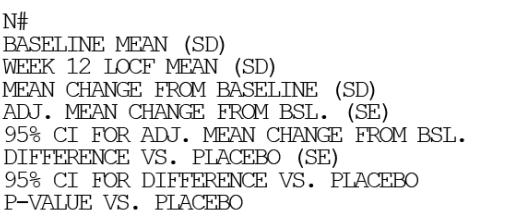 & $\begin{array}{rc}186 & \\
149.20 & (12.675) \\
140.64 & (14.804) \\
-8.55 & (15.379) \\
-6.88 & (1.5793) \\
(-9.98,-3.77)\end{array}$ & $\begin{array}{r}187 \\
146.48(10.436) \\
135.03(13.410) \\
-11.45(13.857) \\
-11.33(1.6031) \\
(-14.48,-8.17) \\
-4.45(1.3680) \\
(-7.14,-1.76) \\
0.0012^{\star}\end{array}$ \\
\hline
\end{tabular}

For the analysis on the secondary efficacy endpoints, the efficacy of DAPA was supported only by the 24-hr Ambulatory SBP according to the sponsor's pre-specified multiplicity adjustment procedure at level of 0.05 .

The sponsor did subgroup analyses for change in seated SBP and HbA1c at Week 12 were performed for race (Asian, Black or African American, White, Other), age ( $<65$ years, $\geq 65$ years, $\geq 75$ years), gender, ethnicity, geographic region (North America, Latin America, Europe, Asia/Pacific), female age (female $\leq 50$ years, female $>50$ years), and baseline HbAlc $(<8 \%, \geq$ $8 \%$ to $<9 \%$, and $\geq 9 \%)$, and baseline seated SBP $(<150 \mathrm{mmHg}, \geq 150 \mathrm{mmHg})$. As shown in Fiures 3.2.3.2 and 3.2.3.3, these subgroup analyses showed a consistency in effect across the subgroups, supportive to the efficacy of DAPA across subgroups. No potential treatment interaction ( $\mathrm{p}$-value $<0.10$ ) for the co-primary efficacy variable of seated SBP was detected for the subgroups. For the co-primary endpoint $\mathrm{HbAlc}$, subjects with a higher baseline $\mathrm{HbAlc}$ showed a larger effect of dapagliflozin treatment than subjects with a lower baseline HbA1c. 
Figure 3.2.3.2 Seated Systolic Blood Pressure (mmHg) Adjusted Mean Change for Baseline at Week 12 Evaluation in Subgroups Excluding Data After

$\underset{\text { Subgroup }}{\text { Rescue, Randomized Subjects (MB102077) }}$

$$
\text { Subgroup }
$$

Race $=$ Asian

PLA + ACEI/ARB

- DAP 10MG + ACEI/ARB

Race $=$ Black $/$ African American

- PLA + ACEI/ARB

- DAP 10MG + ACEI/ARB

Race $=$ White

- PLA + ACEI/ARB

DAP 10MG + ACEI/ARB

Race $=$ Othe

- PLA + ACEI/ARB

DAP 10MG + ACEI/ARB

Gender $=$ Female

PLA + ACEI/ARB

DAP 10MG + ACEI/ARB

Gender $=$ Male

PLA + ACEI/ARB

DAP 1OMG + ACEI/ARB

Ethnicity = Hispanic/Latino

PLA + ACEI/ARB

DAP 10MG + ACEI/ARB

Ethnicity $=$ Non-Hispanic/Latino

PLA + ACEI/ARB

DAP $10 M G+A C E I / A R B$

Baseline SBP $<150 \mathrm{mmHg}$

PLA + ACEI/ARB

DAP $10 M G+A C E I / A R B$

Baseline SBP $>=150 \mathrm{mmHg}$

PLA + ACEI/ARB

DAP 10MG + ACEI/ARB

Age $<65$ yrs

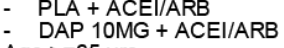

Age $>=65$ yrs

PLA + ACEI/ARB

DAP $10 M G+A C E I / A R B$

Region = North America

- PLA + ACEI/ARB

Region = Latin America

PLA + ACEI/ARB
DAP 10MG + ACEI/ARB

Region = Europe

PLA + ACEI/ARB

Region $=$ Asia $/$ Pacific

PLA + ACEI/ARB

DAP 10MG + ACEI/ARB

Female Age $<=50$ yrs

DAP 10MG + ACEI/ARB

Female Age $>50$ yrs

PLA + ACEI/ARB

34
32
13
16
141
145
11
12
85
98
114
107
38
43
33
34
94
95
105
110
173
182
26
23
75
79
41
50
50
45
33
31
21
28
64
70

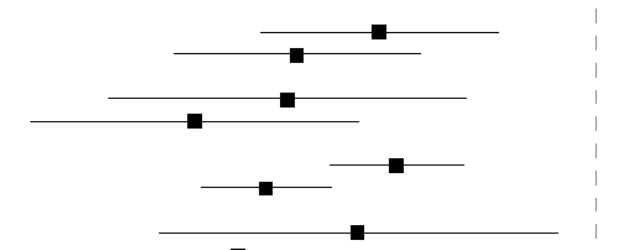

Mean Change from Baseline (CI)

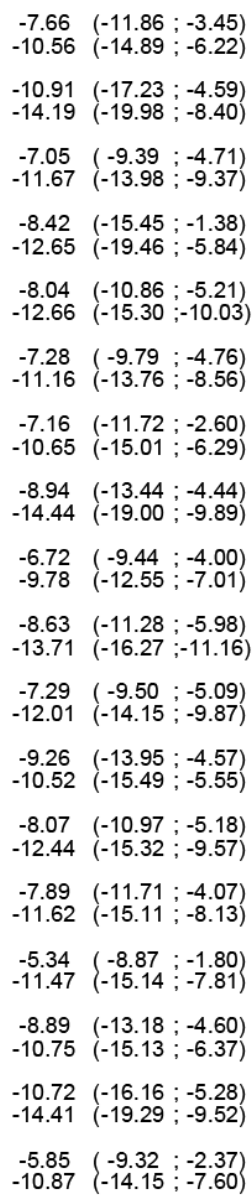

$\begin{array}{lllllllllll}-20 & -18 & -16 & -14 & -12 & -10 & -8 & -6 & -4 & -2 & 0\end{array}$

Seated Systolic Blood Pressure $(\mathrm{mmHg})$ Adjusted Mean Change from Baseline 


\section{Figure 3.2.3.3 HbA1c (Percent) Adjusted Mean Change from Baseline at Week 12 Evaluation in Subgroups, Including Data After Rescue, Randomized}

Subjects (MB102077)

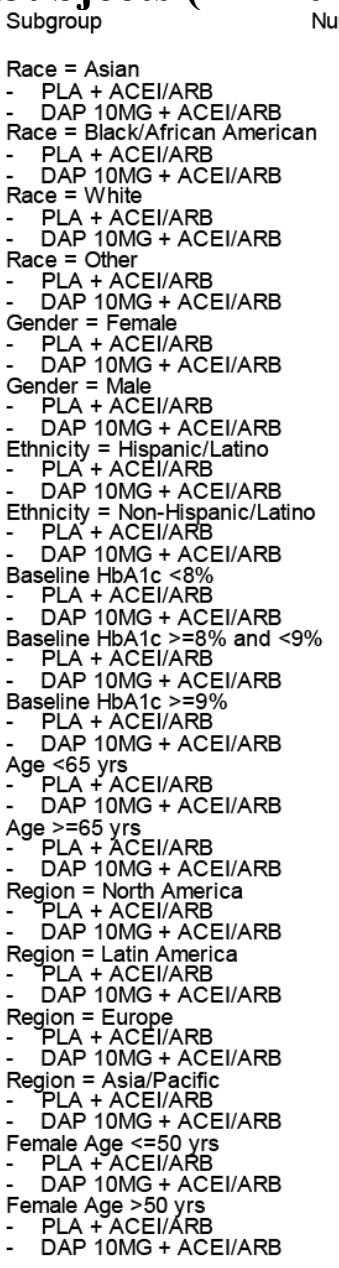

Number of Subjects

34
32
14
16
139
144
10
12
85
97
112
107
38
42
33
34
108
106
56
59
33
39
171
181
26
23
75
78
40
50
49
45
33
31
21
28
64
69

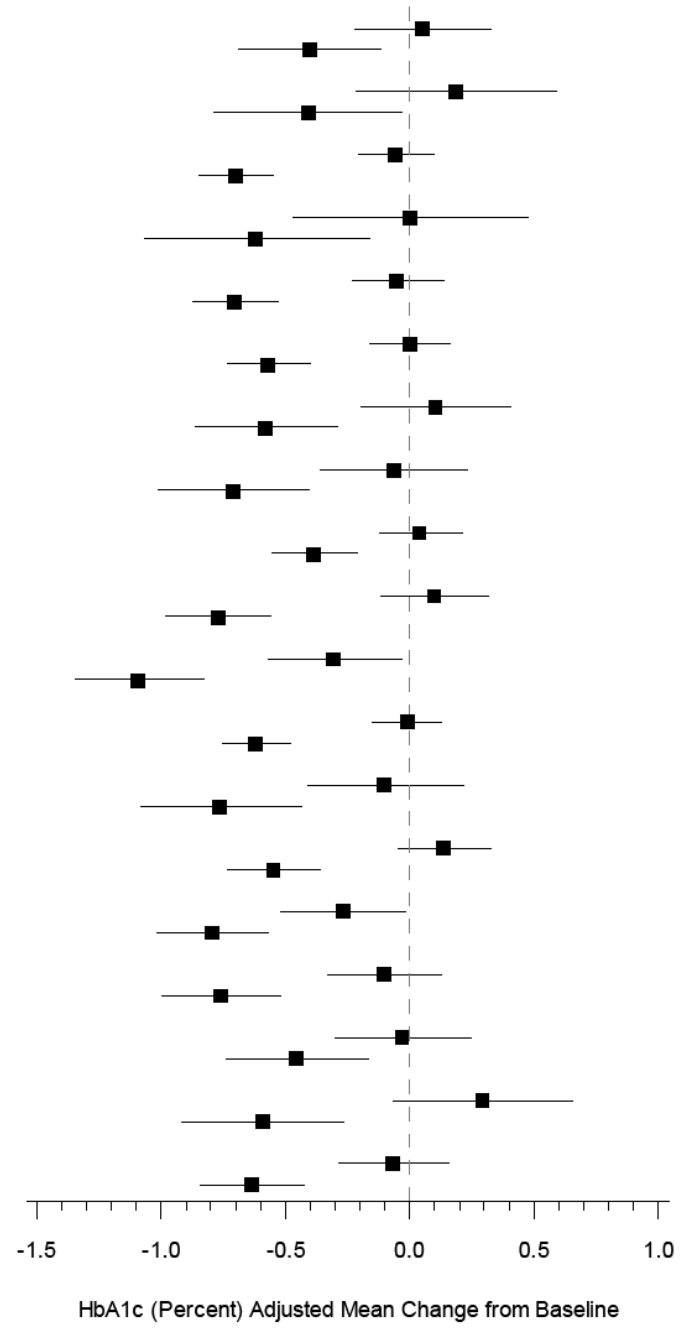

Mean Change from Baseline (CI)

In summary, this study supports the efficacy of DAPA in reducing HbA1c and systolic blood pressure at Week 12.

\subsection{Integrated Analysis of Efficacy in Placebo-Controlled Studies}

In order to provide information on the efficacy of DAPA in a larger group of T2DM subjects, this reviewer performed efficacy analyses based on pooled phase 3 Placebo-controlled studies with endpoints of 24 weeks stratified by study. This assessment of efficacy includes the Phase 3 placebo-controlled studies (except the renal impairment study MB102029, cohort 1 of Study MB102009 and group 2 of Study MB102013, both of which were uncontrolled, and the two 12week studies MB102073 and MB102077 for hypertension patients) plus selected data from the 
initial combination studies (the dapagliflozin plus metformin and placebo plus metformin arms of Studies MB102021 and MB102034).

Two doses, DAPA 5 and $10 \mathrm{mg}$, are selected for the meta-analyses of the pooled 12 phase 3 data. Comparative analyses were stratified by study. The efficacy results of the pooled 12 phase 3 placebo-controlled studies (PC) after 24 weeks of treatment is shown in Table 3.3.1. The mean changes in systolic blood pressure from placebo of the pooled studies after 24 weeks of treatment are similar to that of the two studies MB102073 and MB102077 in hypertension patients after 12 weeks of treatment.

The efficacy of DAPA ( $5 \mathrm{mg}$ and $10 \mathrm{mg}$ ) versus placebo is observed in the integrated analysis for the primary efficacy endpoint HbAlc. Additionally, the Applicant appears to be emphasizing positive effects on blood pressure and body weight as additional potential benefits of the drug. Also, there may be a concern that DAPA increases LDL, which is an undesirable effect.

Integrated analyses were performed to verify the effect/affect of DAPA on these secondary efficacy endpoints (LDL, seated systolic blood pressure, body weight, and FPG). Caution should be taken when interpretating the results, as it is difficult to account for multiplicity. Mainly, these analyses are done for descriptive purposes. As shown in Table 3.3.1, the results support that DAPA is efficacious in reducing FPG, systolic blood pressure, and body weight at Week 24 relative to placebo for both doses of $5 \mathrm{mg}$ and $10 \mathrm{mg}$. The observed mean level of LDL was greater after 24 weeks treatment in patients treated with DAPA ( $5 \mathrm{mg}$ and $10 \mathrm{mg}$ ) compared to baseline while the mean LDL level in placebo patients remained close to the baseline level.

Table 3.3.1 Efficacy Parameters After 24 Weeks of Treatment in Pooled Placebo-Controlled Studies for DAPA $5 \mathrm{mg}$ and $10 \mathrm{mg}$ in Patients with Type 2 Diabetes (FAS/LOCF)

\begin{tabular}{|c|c|c|c|c|c|c|}
\hline \multirow[t]{2}{*}{ Endpoint } & \multicolumn{2}{|c|}{ Placebo } & \multicolumn{2}{|c|}{ DAPA $5 \mathrm{mg}$} & \multicolumn{2}{|c|}{ DAPA $10 \mathrm{mg}$} \\
\hline & $\mathrm{n}$ & & $\mathrm{n}$ & & $\mathrm{n}$ & \\
\hline \multicolumn{7}{|l|}{ HbA1c (\%) } \\
\hline Baseline mean (SD) & 2274 & $8.26(1.03)$ & 996 & $8.44(1.13)$ & 2085 & $8.21(0.97)$ \\
\hline Adj. Mean Change from baseline \pm SE & & $-0.37 \pm 0.02$ & & $-0.88 \pm 0.03$ & & $-0.90 \pm 0.02$ \\
\hline DAPA-P, adjusted LS Mean & & & & -0.51 & & -0.52 \\
\hline$(95 \% \mathrm{CI})$ & & & & $(-0.58,-0.43)$ & & $(-0.58,-0.47)$ \\
\hline$p$-value & & & & $<.0001$ & & $<0.0001$ \\
\hline $\mathrm{HbA1c}<7 \%,(\%)$ & \multicolumn{2}{|c|}{$325(14 \%)$} & \multicolumn{2}{|c|}{$202(20 \%)$} & \multicolumn{2}{|c|}{$418(20 \%)$} \\
\hline \multicolumn{7}{|l|}{ FPG (mg/dL) } \\
\hline Baseline mean (SD) & 2377 & $168.2(47.8)$ & 1009 & $175.2(51.9)$ & 2182 & $165.5 \pm(46.7)$ \\
\hline Adj. Mean Change from baseline \pm SE & & $-4.23 \pm 0.86$ & & $-26.36 \pm 1.32$ & & $-28.75 \pm 0.93$ \\
\hline DAPA-P, adjusted LS Mean & & & & -22.12 & & -24.51 \\
\hline$(95 \% \mathrm{CI})$ & & & & $(-25.27,-18.98)$ & & $(-26.77,-22.25)$ \\
\hline$p$-value & & & & $<.0001$ & & $<0.0001$ \\
\hline \multicolumn{7}{|l|}{ LDL (mg/dL) } \\
\hline Baseline mean (SD) & 1906 & $103.9(38.5)$ & 899 & $113.3(38.3)$ & 1856 & $101.3(38.2)$ \\
\hline Adj. Mean Change from baseline \pm SE & & $-0.11 \pm 0.64$ & & $2.36 \pm 0.93$ & & $3.23 \pm 0.69$ \\
\hline
\end{tabular}




\begin{tabular}{|c|c|c|c|c|c|c|}
\hline $\begin{array}{l}\text { DAPA-P, adjusted LS Mean } \\
(95 \% \mathrm{CI}) \\
\text { p-value }\end{array}$ & & & & $\begin{array}{c}2.47 \\
(0.19,4.74) \\
0.0337\end{array}$ & & $\begin{array}{c}3.34 \\
(1.65,5.03) \\
0.0001\end{array}$ \\
\hline \multicolumn{7}{|l|}{ Body Weight (Kg) } \\
\hline $\begin{array}{l}\text { Baseline mean (SD) } \\
\text { Adj. Mean Change from baseline } \pm \text { SE } \\
\text { DAPA-P, adjusted LS Mean } \\
(95 \% \text { CI) } \\
\text { p-value }\end{array}$ & 2395 & $\begin{array}{c}90.1(19.2) \\
-0.66 \pm 0.07\end{array}$ & 1016 & $\begin{array}{c}86.8(18.9) \\
-2.07 \pm 0.10 \\
-1.40 \\
(-1.64,-1.16) \\
<.0001\end{array}$ & 2203 & $\begin{array}{c}90.9(19.5) \\
-2.48 \pm 0.07 \\
\quad-1.82 \\
(-1.99,-1.65) \\
<.0001\end{array}$ \\
\hline \multicolumn{7}{|l|}{ Systolic Blood Pressure (mmHg) } \\
\hline $\begin{array}{l}\text { Baseline mean (SD) } \\
\text { Adj. Mean Change from baseline } \pm \text { SE } \\
\text { DAPA-P, adjusted LS Mean } \\
(95 \% \mathrm{CI}) \\
\text { p-value }\end{array}$ & 1471 & $\begin{array}{l}129.8(15.1) \\
-0.91 \pm 0.31\end{array}$ & 1015 & $\begin{array}{c}129.3(15.8) \\
-3.19 \pm 0.39 \\
-2.28 \\
(-3.25,-1.32) \\
<.0001\end{array}$ & 1278 & $\begin{array}{c}129.9(16.0) \\
-4.08 \pm 0.35 \\
-3.17 \\
(-4.05,-2.29) \\
<0.0001\end{array}$ \\
\hline
\end{tabular}

Note: SD - standard deviation

Since most of primary analyses used LOCF method for missing observations, this reviewer looked at the missing value percentage in the resubmission of phase 3 studies as shown in Table 3.3.2. The completers are defined as those who completed the efficacy treatment period without rescue medication. The average percent of missing 24 -week data in the placebo-controlled studies is $21 \%$ with a range from $9 \%$ to $47 \%$. 
Table 3.3.2 Information of Data Missing in Resubmitted Phase 3 Trials

\begin{tabular}{|c|c|c|c|c|c|c|c|}
\hline study & week & arm & $\begin{array}{c}\text { Rand. } \\
\text { n }\end{array}$ & $\begin{array}{c}\text { LOCF, } \\
n\end{array}$ & $\begin{array}{c}\text { Completer, } \\
\mathrm{n}\end{array}$ & $\begin{array}{c}\text { Rand.- } \\
\text { Completer, } \mathrm{n}\end{array}$ & $\begin{array}{c}\text { Percent } \\
(\%)\end{array}$ \\
\hline MB102013 & 24 & DAPA $5 \mathrm{mg}$ & 64 & 61 & 49 & 15 & 23 \\
\hline MB102013 & 24 & DAPA $10 \mathrm{mg}$ & 70 & 65 & 55 & 15 & 21 \\
\hline MB102013 & 24 & Placebo & 75 & 72 & 55 & 20 & 27 \\
\hline MB102032 & 24 & DAPA $5 \mathrm{mg}$ & 137 & 133 & 118 & 19 & 14 \\
\hline MB102032 & 24 & DAPA $10 \mathrm{mg}$ & 135 & 132 & 117 & 18 & 13 \\
\hline MB102032 & 24 & Placebo & 137 & 134 & 100 & 37 & 27 \\
\hline MB102014 & 24 & DAPA $5 \mathrm{mg}$ & 137 & 133 & 118 & 19 & 14 \\
\hline MB102014 & 24 & DAPA $10 \mathrm{mg}$ & 135 & 132 & 117 & 18 & 13 \\
\hline MB102014 & 24 & Placebo & 137 & 134 & 100 & 37 & 27 \\
\hline D1690C00005 & 24 & DAPA $5 \mathrm{mg}$ & 145 & 142 & 127 & 18 & 12 \\
\hline D1690C00005 & 24 & DAPA $10 \mathrm{mg}$ & 151 & 150 & 137 & 14 & 9 \\
\hline D1690C00005 & 24 & Placebo & 146 & 143 & 110 & 36 & 25 \\
\hline MB102030 & 24 & DAPA $5 \mathrm{mg}$ & 141 & 140 & 122 & 19 & 13 \\
\hline MB102030 & 24 & DAPA $10 \mathrm{mg}$ & 140 & 140 & 123 & 17 & 12 \\
\hline MB102030 & 24 & Placebo & 139 & 138 & 103 & 36 & 26 \\
\hline D1690C00006 & 24 & DAPA $5 \mathrm{mg}$ & 212 & 210 & 165 & 47 & 22 \\
\hline D1690C00006 & 24 & DAPA $10 \mathrm{mg}$ & 196 & 192 & 158 & 38 & 19 \\
\hline D1690C00006 & 24 & Placebo & 197 & 188 & 121 & 76 & 39 \\
\hline D1690C00004 & 52 & DAPA $2.5-10 \mathrm{mg}$ & 406 & 400 & 321 & 85 & 21 \\
\hline D1690C00004 & 52 & Glipizide & 408 & 401 & 315 & 93 & 23 \\
\hline D1690C00012 & 24 & DAPA $10 \mathrm{mg}$ & 91 & 89 & 82 & 9 & 10 \\
\hline D1690C00012 & 24 & Placebo & 91 & 91 & 82 & 9 & 10 \\
\hline D1690C00010 & 24 & DAPA $10 \mathrm{mg}$ & 225 & 223 & 173 & 52 & 23 \\
\hline D1690C00010 & 24 & Placebo & 226 & 223 & 119 & 107 & 47 \\
\hline MB102021 & 24 & DAPA $5+$ MET & 194 & 185 & 169 & 25 & 13 \\
\hline MB102021 & 24 & DAPA $5 \mathrm{mg}$ & 203 & 196 & 158 & 45 & 22 \\
\hline MB102021 & 24 & MET & 201 & 195 & 148 & 53 & 26 \\
\hline MB102034 & 24 & DAPA $10+$ MET & 211 & 202 & 180 & 31 & 15 \\
\hline MB102034 & 24 & DAPA $10 \mathrm{mg}$ & 219 & 216 & 174 & 45 & 21 \\
\hline MB102034 & 24 & MET & 208 & 203 & 155 & 53 & 25 \\
\hline MB102029 & 24 & DAPA $5 \mathrm{mg}$ & 83 & 83 & 63 & 20 & 24 \\
\hline MB102029 & 24 & DAPA $10 \mathrm{mg}$ & 85 & 82 & 65 & 20 & 24 \\
\hline MB102029 & 24 & Placebo & 84 & 82 & 50 & 34 & 40 \\
\hline MB102073 & 12 & DAPA $10 \mathrm{mg}$ & 302 & 280 & 278 & 24 & 8 \\
\hline MB102073 & 12 & Placebo & 311 & 279 & 278 & 33 & 11 \\
\hline MB102077 & 12 & DAPA $10 \mathrm{mg}$ & 225 & 205 & 204 & 21 & 9 \\
\hline MB102077 & 12 & Placebo & 224 & 199 & 196 & 28 & 13 \\
\hline D1690C00018 & 24 & DAPA $10 \mathrm{mg}$ & 460 & 448 & 359 & 101 & 22 \\
\hline D1690C00018 & 24 & Placebo & 462 & 451 & 293 & 169 & 37 \\
\hline D1690C00019 & 24 & DAPA $10 \mathrm{mg}$ & 482 & 474 & 399 & 83 & 17 \\
\hline D1690C00019 & 24 & Placebo & 482 & 471 & 321 & 161 & 33 \\
\hline
\end{tabular}


Evaluation of Safety

An evaluation of the safety of dapagliflozin presented in this submission is included in the clinical review by Dr. Frank Pucino.

\section{FINDINGS IN SPECIAL/SUBGROUP POPULATIONS}

\subsection{Gender, Race, Age and Geographic Region}

See Section 3 for each individual study.

\subsection{Other Special/Subgroup Populations - Integrated Analysis of HbA1c by Subgroups in Patients Based on Their Baseline eGFR Levels in Placebo- Controlled Studies}

Since dapagliflozin acts via the kidney, the efficacy of the drug may be affected by renal function. Therefore, analyses within different eGFR ranges on HbAlc change from baseline to Week 24 were performed. To evaluate the efficacy of dapagliflozin in a larger group of T2DM subjects based on their renal function, integrated analyses were performed, which included data from study MB102029 of patients with moderate renal impairment and data from the placebocontrolled studies. The integrated analysis of change in HbA1c from baseline to 24 weeks was carried out by the following subgroups:

- baseline eGFR $<30 \mathrm{~mL} / \mathrm{min} / 1.73 \mathrm{~m}^{2}$

- baseline eGFR $<45 \mathrm{~mL} / \mathrm{min} / 1.73 \mathrm{~m}^{2}$

- baseline eGFR 30 to $<45 \mathrm{~mL} / \mathrm{min} / 1.73 \mathrm{~m}^{2}$

- baseline eGFR 45 to $<60 \mathrm{~mL} / \mathrm{min} / 1.73 \mathrm{~m}^{2}$

- baseline eGFR 60 to $<90 \mathrm{~mL} / \mathrm{min} / 1.73 \mathrm{~m}^{2}$

- baseline eGFR $\geq 90 \mathrm{~mL} / \mathrm{min} / 1.73 \mathrm{~m}^{2}$

The integrated analysis of the primary endpoint HbA1c (at Week 24) of the thirteen placebocontrolled studies was performed using ANCOVA analyses (stratified by study) using LOCF method for dealing with missing values. This analysis method is the pre-specified method for the primary endpoint. No statistical adjustments for multiplicity were applied.

This reviewer also computed the percentage of patients who achieved an HbAlc $<7 \%$ at Week 24 for these subgroups.

The subgroup analysis results are summarized in Table 4.1 Across the eGFR categories, the observed treatment effect on HbA1c change from baseline to Week 24 decreased as eGFR decreased for both doses of dapagliflozin. For the eGFR subgroups of 45 to $<60 \mathrm{~mL} / \mathrm{min} / 1.73 \mathrm{~m}^{2}$ or higher, the $95 \%$ confidence intervals for the treatment effect on HbA1c change from baseline to Week 24 ruled out no effect or negative effect on HbA1c. For the eGFR $<45 \mathrm{~mL} / \mathrm{min} / 1.73 \mathrm{~m}^{2}$ subgroup the results are consistent with no or little improvement by dapagliflozin on on HbA1c change from baseline to Week 24. There is no clear dose response of dapagliflozin (DAPA $5 \mathrm{mg}$ and $10 \mathrm{mg}$ ) versus placebo in $\mathrm{HbA} 1 \mathrm{c}$ reduction from baseline to Week 24. HbA1c reduction in 
the eGFR 45 to $<60 \mathrm{~mL} / \mathrm{min} / 1.73 \mathrm{~m}^{2}$ subgroup was smaller than seen overall, with mean difference -0.23 and -0.32 from placebo for the $5 \mathrm{mg}$ and $10 \mathrm{mg}$, respectively, with similar percentage of patients achieving $\mathrm{HbA} 1 \mathrm{c}<7 \%$ at Week 24 in all three arms.

\section{Table 4.1 Subgroup Analaysis in HbA1c for Dapagliflozin (5 mg and $10 \mathrm{mg}$ ) in Patients with Type 2 Diabetes by eGFR Levels (FAS/LOCF)}

\begin{tabular}{|c|c|c|c|c|c|c|}
\hline \multirow{3}{*}{$\begin{array}{l}\text { Endpoint } \\
\text { HbA1c (\%) }\end{array}$} & \multicolumn{2}{|c|}{ Placebo } & \multicolumn{4}{|c|}{ Dapagliflozin } \\
\hline & \multirow[b]{2}{*}{$\mathrm{n}$} & & \multicolumn{2}{|r|}{$5 \mathrm{mg}$} & \multicolumn{2}{|r|}{$10 \mathrm{mg}$} \\
\hline & & & $\mathrm{n}$ & & $\mathrm{n}$ & \\
\hline \multicolumn{7}{|l|}{$\mathrm{eGFR}<30 \mathrm{~mL} / \mathrm{min} / 1.73 \mathrm{~m}^{2}$} \\
\hline $\begin{array}{l}\text { Baseline mean } \pm \text { SE } \\
\text { Adj. Mean Change from baseline } \pm \text { SE } \\
\text { DAPA-P, adjusted LS Mean }(95 \% \mathrm{CI})\end{array}$ & 5 & -- & 4 & -- & 3 & -- \\
\hline \multicolumn{7}{|l|}{ eGFR $<45 \mathrm{~mL} / \mathrm{min} / 1.73 \mathrm{~m}^{2}$} \\
\hline $\begin{array}{l}\text { Baseline mean } \pm \text { SE } \\
\text { Adj. Mean Change from baseline } \pm \text { SE } \\
\text { DAPA-P, adjusted LS Mean }(95 \% \mathrm{CI})\end{array}$ & 60 & $\begin{array}{l}8.24 \pm 0.14 \\
-0.40 \pm 0.19\end{array}$ & 57 & $\begin{array}{l}8.40 \pm 0.16 \\
-0.34 \pm 0.18 \\
0.06(-0.30,0.42)\end{array}$ & 83 & $\begin{array}{l}8.16 \pm 0.10 \\
-0.47 \pm 0.18 \\
-0.07(-0.38,0.24)\end{array}$ \\
\hline HbA1c $<7 \%,(\%)$ & \multicolumn{2}{|c|}{$8(15 \%)$} & \multicolumn{2}{|c|}{$5(9 \%)$} & \multicolumn{2}{|c|}{$5(6 \%)$} \\
\hline \multicolumn{7}{|l|}{ eGFR 30 to $<45 \mathrm{~mL} / \mathrm{min} / 1.73 \mathrm{~m}^{2}$} \\
\hline $\begin{array}{l}\text { Baseline mean } \pm \text { SE } \\
\text { Adj. Mean Change from baseline } \pm \text { SE } \\
\text { DAPA-P, adjusted LS Mean }(95 \% \mathrm{CI})\end{array}$ & 55 & $\begin{array}{l}8.20 \pm 0.015 \\
-0.36 \pm 0.20\end{array}$ & 53 & $\begin{array}{l}8.45 \pm 0.16 \\
-0.38 \pm 0.19 \\
-0.02(-0.39,0.36)\end{array}$ & 80 & $\begin{array}{l}8.16 \pm 0.10 \\
-0.44 \pm 0.18 \\
-0.08(-0.40,0.24)\end{array}$ \\
\hline HbA1c $<7 \%,(\%)$ & \multicolumn{2}{|c|}{$8(15 \%)$} & \multicolumn{2}{|c|}{$5(9 \%)$} & \multicolumn{2}{|c|}{$5(6 \%)$} \\
\hline \multicolumn{7}{|l|}{ eGFR 45 to $<60 \mathrm{~mL} / \mathrm{min} / 1.73 \mathrm{~m}^{2}$} \\
\hline $\begin{array}{l}\text { Baseline mean } \pm \text { SE } \\
\text { Adj. Mean Change from baseline } \pm \text { SE } \\
\text { DAPA-P, adjusted LS Mean }(95 \% \mathrm{CI})\end{array}$ & 278 & $\begin{array}{l}8.16 \pm 0.06 \\
-0.35 \pm 0.07\end{array}$ & 123 & $\begin{array}{l}8.20 \pm 0.09 \\
-0.57 \pm 0.10 \\
-0.23(-0.44,-0.01)\end{array}$ & 234 & $\begin{array}{l}8.20 \pm 0.06 \\
-0.66 \pm 0.08 \\
-0.32(-0.47,-0.16)\end{array}$ \\
\hline HbA1c $<7 \%,(\%)$ & \multicolumn{2}{|c|}{$32(12 \%)$} & \multicolumn{2}{|c|}{$15(12 \%)$} & \multicolumn{2}{|c|}{$33(14 \%)$} \\
\hline \multicolumn{7}{|l|}{ eGFR 60 to $<90 \mathrm{~mL} / \mathrm{min} / 1.73 \mathrm{~m}^{2}$} \\
\hline $\begin{array}{l}\text { Baseline mean } \pm \text { SE } \\
\text { Adj. Mean Change from baseline } \pm \text { SE } \\
\text { DAPA-P, adjusted LS Mean }(95 \% \mathrm{CI})\end{array}$ & 1309 & $\begin{array}{l}8.23 \pm 0.03 \\
-0.34 \pm 0.03\end{array}$ & 543 & $\begin{array}{l}8.39 \pm 0.05 \\
-0.87 \pm 0.05 \\
-0.53(-0.63,-0.43)\end{array}$ & 1183 & $\begin{array}{l}8.17 \pm 0.03 \\
-0.84 \pm 0.04 \\
-0.50(-0.57,-0.43)\end{array}$ \\
\hline HbA1c <7\%, (\%) & \multicolumn{2}{|c|}{$183(14 \%)$} & \multicolumn{2}{|c|}{$111(20 \%)$} & \multicolumn{2}{|c|}{$242(20 \%)$} \\
\hline \multicolumn{7}{|l|}{$\mathrm{eGFR} \geq 90 \mathrm{~mL} / \mathrm{min} / 1.73 \mathrm{~m}^{2}$} \\
\hline $\begin{array}{l}\text { Baseline mean } \pm \text { SE } \\
\text { Adj. Mean Change from baseline } \pm \text { SE } \\
\text { DAPA-P, adjusted LS Mean }(95 \% \mathrm{CI})\end{array}$ & 707 & $\begin{array}{c}78.40 \pm 0.04 \\
-0.36 \pm 0.08\end{array}$ & 356 & $\begin{array}{l}8.58 \pm 0.06 \\
-0.91 \pm 0.09 \\
-0.55(-0.68,-0.42)\end{array}$ & 666 & $\begin{array}{l}8.30 \pm 0.04 \\
-0.99 \pm 0.08 \\
-0.64(-0.74,-0.53)\end{array}$ \\
\hline HbA1c $<7 \%,(\%)$ & \multicolumn{2}{|c|}{$106(15 \%)$} & \multicolumn{2}{|c|}{$76(21 \%)$} & \multicolumn{2}{|c|}{$142(21 \%)$} \\
\hline
\end{tabular}

No other subgroups were analyzed. 


\section{SUMMARY AND CONCLUSIONS}

\subsection{Statistical Issues}

There are issues involving the bias and reliability of an estimate of a treatment difference when carrying forward an intermediate measurement and treating it as an actual measurement at the end point.

The data-missing rates for the three studies are:

$\begin{array}{ll}\text { D1690C00010 (24 weeks) } & \text { DAPA } 10 \mathrm{mg} 23 \% \text {, placebo } 47 \% \\ \text { MB102073 (12 weeks) } & \text { DAPA } 10 \mathrm{mg} 8 \% \text {, placebo } 11 \% \\ \text { MB102077 (12 weeks) } & \text { DAPA } 10 \mathrm{mg} 9 \% \text {, placebo } 13 \%\end{array}$

The average percent of missing 24 -week data in the placebo-controlled studies is $21 \%$ with a range from $9 \%$ to $47 \%$.

The meta-analysis of the integrated placebo-controlled data for the primary efficacy endpoint $\mathrm{HbA1c}$ and interested secondary efficacy endpoints (seated systolic blood pressure, body weight, and FPG) are performed without family-wise type 1 error control.

\subsection{Collective Evidence}

The resubmitted data of phase 3 studies provide convincing evidence that dapagliflozin is efficacious for both of the proposed once daily doses, $5 \mathrm{mg}$ and $10 \mathrm{mg}$. The results from efficacy analyses of the new data from the three studies are consistent with that from other placebocontrolled studies except study MB102029. For majority of the phase 3 studies, the efficacy analyses were based on LOCF as the primary method for accounting for missing data, disregarding observations recorded after any rescue treatment. Results from analyses using MMRM performed by this reviewer to selected studies were consistent with the primary analysis results with LOCF. However, the efficacy of dapagliflozin based on HbA1c reduction is modest.

In all the placebo-controlled studies but study MB102029, both the 5 and $10 \mathrm{mg}$ doses of dapagliflozin were shown to be superior to placebo on the primary endpoint, using the planned primary analysis. The renal impairment study MB102029 failed to show superiority of dapagliflozin, at doses of both the $5 \mathrm{mg}$ and $10 \mathrm{mg}$, to placebo. As seen in Dr. Norton's review dated September 8, 2011, the active-controlled study showed that titrated doses of dapagliflozin and glipizide yielded quite similar results at Week 52. Although dapagliflozin was statistically non-inferior at Week 52, it should be noted that glipizide was clearly superior at some earlier time points.

The efficacy of DAPA ( $5 \mathrm{mg}$ and $10 \mathrm{mg}$ ) versus placebo is observed in the meta-analysis of the integrated placebo-controlled data for the primary efficacy endpoint HbA1c and interested secondary efficacy endpoints (seated systolic blood pressure, body weight, and FPG) without family-wise type 1 error control. However, the results in the LDL change after 24 weeks treatment suggested elevated LDL levels in patients treated with DAPA (5 mg and $10 \mathrm{mg}$ ) while 
the LDL level in placebo arm remained close to the baseline level. This may raise a safety concern.

Subgroups analyses of $\mathrm{HbA} 1 \mathrm{c}$ were conducted based on a pooled patient population from the 13 Phase 3 placebo-controlled studies with the primary endpoint at Week 24. The subgroup analysis suggests that dapagliflozin $(5 \mathrm{mg}$ and $10 \mathrm{mg}$ ) is effective only in subjects with normal renal function or mild impairment (eGFR $\geq 45 \mathrm{~mL} / \mathrm{min} / 1.73 \mathrm{~m}^{2}$ ). The size of the treatment effect should be considered when considering whether treatment with dapagliflozin is appropriate. There is no clear dose response of dapagliflozin (DAPA $5 \mathrm{mg}$ and $10 \mathrm{mg}$ ) versus placebo in $\mathrm{HbA1c}$ reduction from baseline to Week 24.

\subsection{Conclusions and Recommendations}

The resubmitted data of phase 3 studies provide convincing evidence that dapagliflozin is efficacious for both of the proposed once daily doses, $5 \mathrm{mg}$ and $10 \mathrm{mg}$. For majority of the phase 3 studies, the efficacy analyses were based on LOCF as the primary method for accounting for missing data, disregarding observations recorded after any rescue treatment. Results from analyses using MMRM performed by this reviewer to selected studies were consistent with the primary analysis results with LOCF. However, the efficacy of dapagliflozin based on HbA1c reduction is modest.

In all the placebo-controlled studies but study MB102029, both the $5 \mathrm{mg}$ and $10 \mathrm{mg}$ doses of dapagliflozin were shown to be superior to placebo on the primary endpoint, using the planned primary analysis. The renal impairment study MB102029 failed to show superiority of dapagliflozin, at doses of both the $5 \mathrm{mg}$ and $10 \mathrm{mg}$, to placebo. As seen in Dr. Norton's review dated September 8, 2011, the active-controlled study showed that titrated doses of dapagliflozin and glipizide yielded quite similar results at Week 52. Although dapagliflozin was statistically non-inferior at Week 52, it should be noted that glipizide was clearly superior at some earlier time points.

The efficacy of DAPA (5 mg and $10 \mathrm{mg}$ ) versus placebo is observed in the meta-analysis of the integrated placebo-controlled data for the primary efficacy endpoint HbAlc and interested secondary efficacy endpoints (seated systolic blood pressure, body weight, and FPG) without family-wise type 1 error control. However, the results in the LDL change after 24 weeks treatment suggested elevated LDL levels in patients treated with DAPA (5 mg and $10 \mathrm{mg})$ while the LDL level in placebo arm remained close to the baseline level. This may raise a safety concern.

Subgroups analyses of $\mathrm{HbA1c}$ based on a pooled patient population from the 13 Phase 3 placebo-controlled studies with the primary endpoint at Week 24 suggests that dapagliflozin ( 5 $\mathrm{mg}$ and $10 \mathrm{mg}$ ) is effective only in subjects with normal renal function or mild impairment (eGFR $\geq 45 \mathrm{~mL} / \mathrm{min} / 1.73 \mathrm{~m}^{2}$ ). The size of the treatment effect should be considered when considering whether treatment with dapagliflozin is appropriate. There is no clear dose response of dapagliflozin (DAPA $5 \mathrm{mg}$ and $10 \mathrm{mg}$ ) versus placebo in $\mathrm{HbA} 1 \mathrm{c}$ reduction from baseline to Week 24. 


\subsection{Labeling Recommendations}

Labeling Recommendations:

The statistical review addresses statements in the label (section 14) concerning:

1. In Table 4, (b) (4) and should be removed from the table.

2. The last paragraph in 14.1 should be removed because the statement was not evaluated.

3. In Table 9 "In combination with DPP4 Inhibitot (Sitagliptin) with or without Metformin

from the table should be removed

4. In Table 9 "In Combination with Insulin with or without up to 2 Oral Antidiabetic Therapies" the results of removed (b) (4) "FPG" for FORXIGA $5 \mathrm{mg}$ should be 5. The last row in Table 11 appears uncompleted.

6. The text should be removed or modified (b) (4) 
This is a representation of an electronic record that was signed electronically and this page is the manifestation of the electronic signature.

\section{/s/}

WEI LIU

$12 / 13 / 2013$

MARK D ROTHMANN

$12 / 13 / 2013$

I concur

THOMAS J PERMUTT

$12 / 13 / 2013$

I concur. 
U.S. Department of Health and Human Services

Food and Drug Administration

Center for Drug Evaluation and Research

Office of Translational Sciences

Office of Biostatistics

\section{STATISTICAL REVIEW AND EVALUATION \\ Clinical Studies}

NDA/Serial Number:

202-293 / SN 0056

Drug Name:

FORXIGA (dapagliflozin)

Indication(s):

Treatment of type 2 diabetes mellitus (T2DM) in adults as an adjunct to diet and exercise to improve glycemic control

Applicant:

Bristol-Myers Squibb

Date(s):

Submitted November 22, 2011

Original PDUFA Date: October 28, 2011

Extension PDUFA Date: January 28, 2011

Review Priority:

Standard (with 3-month Extension)

Biometrics Division:

Division of Biometrics VII

Statistical Reviewer:

Anita Abraham, Ph.D.

Concurring Reviewers:

Mat Soukup, Ph.D.

Aloka Chakravarty, Ph.D.

Medical Division:

Division of Metabolism and Endocrinology Products (DMEP)

Clinical Team:

Medical Officer: Somya (Verma) Dunn, M.D.

Medical Team Leader: Ilan Irony, M.D.

Project Manager:

Mehreen Hai, M.S. (DMEP)

Keywords: type 2 diabetes mellitus, cardiovascular safety, meta-analysis 


\section{Table of Contents}

1 Executive Summary ..................................................................................................... 5

1.1 Conclusions and Recommendations ............................................................ 5

1.2 Brief Overview of Clinical Studies............................................................ 7

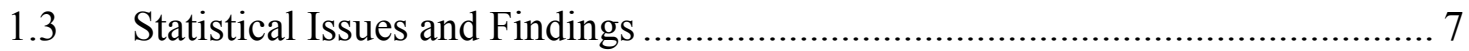

2 Introduction............................................................................................................. 10

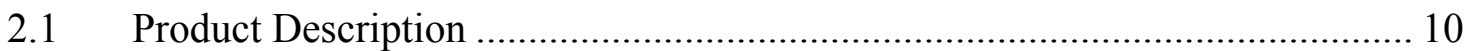

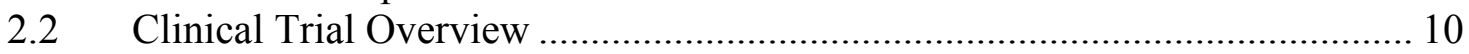

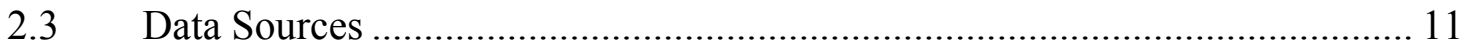

$3 \quad$ Statistical Evaluation ................................................................................................. 14

3.1 Evaluation of Safety......................................................................... 14

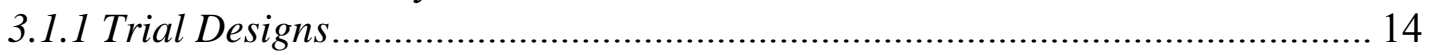

3.1.2 Endpoints and Adjudication Methods ......................................................... 16

3.1.3 Statistical Methodologies............................................................................ 18

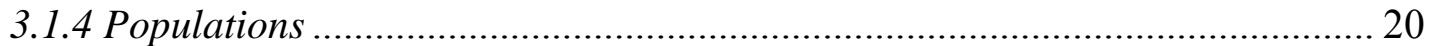

3.1.5 Subject Disposition, Demographics and Baseline Characteristics ................. 21

3.1.6 Results and Conclusions ............................................................................ 23

4 Findings in Special/Subgroup Populations............................................................... 33

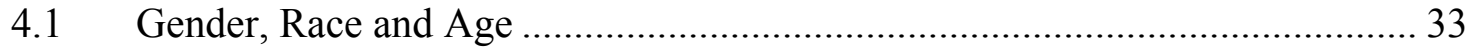

4.2 Other Special/Subgroup Populations ……………………………………....... 35

4.2.1 Baseline BMI, CV Risk Factors, Renal Impairment and Diabetes History ..... 36

4.2.2 Monotherapy and Add-On Trials.................................................................... 37

4.2.3 $10 \mathrm{mg}$ Dosage of Dapagliflozin .............................................................. 38

4.2.4 High-Risk CV Trials: D1690C00018 and D1690C00019 In Relation to All

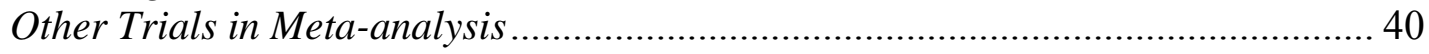

$5 \quad$ Summary and Conclusions............................................................................................. 42

5.1 Statistical Issues and Collective Evidence ................................................... 42

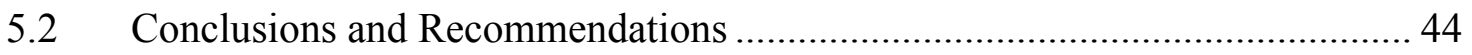

6 References............................................................................................................. 46

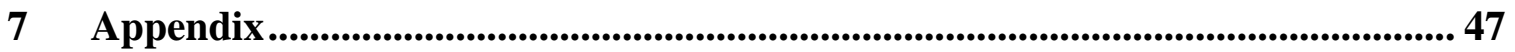

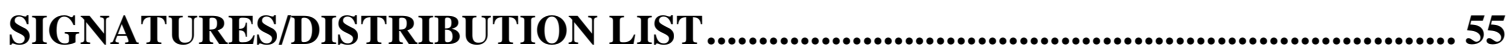




\section{Table of Tables}

Table 1: Meta-Analysis Results for Primary, Secondary, and MACE Composite

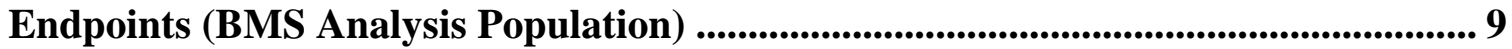

Table 2: List of Trials Included in Original CV Meta-Analysis................................. 12

Table 3: List of Trials Added to Updated Meta-Analysis ......................................... 13

Table 4: Demographics (BMS Analysis Population) ..................................................... 21

Table 5: CV Risk Factors (BMS Analysis Population)................................................ 22

Table 6: Meta-Analysis Results for Primary Composite Endpoint (BMS Analysis

Population)......................................................................................................................................... 24

Table 7: Meta-Analysis Results for Secondary Composite Endpoint (BMS Analysis

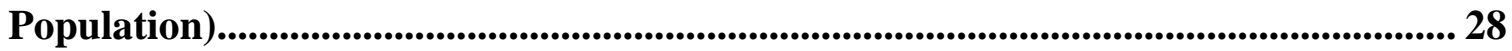

Table 8: Meta-Analysis Results for MACE Composite Endpoint (BMS Analysis

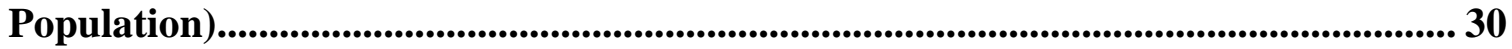

Table 9: Meta-Analysis Risk Difference Results of the Primary Endpoint (BMS Analysis Population) ................................................................................................ 31

Table 10: Comparison of Fixed and Random Effects and Fixed Effects Analysis of Incident Rate Ratio for Primary Composite Endpoint ............................................... 33

Table 11: Meta-Analysis Results of the Primary Composite Endpoint for the Monotherapy Trials and Add-On Therapy Trials (BMS Analysis Population) ....... 37

Table 12: Meta-Analysis Results for 10mg Dose of Dapagliflozin Alone and the Pooled Comparator (BMS Analysis Population excluding MB102021, MB102032 and MB102045)

Table 13: Demographics of All Trials Excluding High-Risk CV Trials and Pooled High -Risk CV Trials (D1690C00018 and D1690C00019)

Table 14: CV Risk Factors of All Trials Excluding High-Risk CV Trials and Pooled High-Risk CV Trials (D1690C00018 and D1690C00019)

Table 15: Meta-Analysis Results for All Trials Excluding High-Risk CV Trials and Pooled High-Risk CV Trials (D1690C00018 and D1690C00019)............................ 42

Table 16: CV Risk Factors by Trial ....................................................................... 50 


\section{Table of Figures}

Figure 1: Percentage of Subjects with Premature Discontinuation of Study Medication by Trial and Treatment Group for Updated and Newly Added Trials (BMS Analysis Population)

Figure 2: Time-to-event Analysis of the Primary Composite Endpoint (BMS Analysis Population) 25

Figure 3: Forest Plot of Hazard Ratios of Individual Trials from Cox Proportional Hazards for Primary CV Composite Endpoint (BMS Analysis Population) 26

Figure 4: Forest Plot of Mantel-Haenszel Incidence Rate Differences of Cardiovascular Events Contributing to Primary Composite Endpoint (BMS Analysis Population)

Figure 5: Forest Plot of Mantel-Haenszel Incidence Rate Differences of Cardiovascular Events Contributing to Secondary Composite Endpoint (BMS Analysis Population)

Figure 6: Forest Plot of Risk Differences by Trial for Primary CV Composite Endpoint (BMS Analysis Population).

Figure 7: Forest Plot of Incidence Rate Difference of Primary Composite Endpoint by Baseline Demographics (BMS Analysis Population). 35

Figure 8: Forest Plot of Incidence Rate Difference of Primary Composite Endpoint by Baseline Risk Factors (BMS Analysis Population). 36

Figure 9: Time-to-event Analysis of the Primary Composite Endpoint for the $10 \mathrm{mg}$ Dose of Dapagliflozin Compared to Pooled Comparator (BMS Analysis Population excluding MB102021, MB102032 and MB102045)

Figure 10: Kaplan Meier Curves of Discontinuation Rates for the 4 Updated Trials (D1690C00004, D1690C00006, D1690C00012, and MB102029).

Figure 11: Assessment of Proportional Hazards Assumption: Log-Log Survival Curve (BMS Analysis Population)

Figure 12: Assessment of Proportional Hazards Assumption: Schoenfeld Residual Plot (BMS Analysis Population). 49 


\section{Executive Summary}

The proposed indication for dapagliflozin is the treatment of type 2 diabetes mellitus (T2DM) in adults as an adjunct to diet and exercise to improve glycemic control. The proposed therapeutic dose of dapagliflozin is $10 \mathrm{mg}$ for most patients and a $5 \mathrm{mg}$ starting dose for patients at risk for volume depletion due to co-existing conditions or concomitant medications, such as loop diuretics. Per the request of the Division of Metabolism and Endocrinology Products this statistical review assesses cardiovascular (CV) related safety endpoints in the randomized phase $2 \mathrm{~b} / 3$ clinical development program of dapagliflozin (Trials D1690C00004, D1690C00005, D1690C00006, D1690C00010, D1690C00012, D1690C00018 and D1690C00019, D1692C00005, MB102008, MB102009, MB102013, MB102014, MB102021, MB102029, MB102030, MB102032, MB102034, MB102035, and MB102045). This review is an update to a previous review, signed September 7, 2011, which was a meta-analysis of 14 of the 19 studies included in this review. This updated review includes additional data from 4 of the previously submitted trials: D1690C00004, D1690C00006, D1690C00012 and MB102029 (contributing an additional 5 events to the primary composite endpoint and an additional 804 person-years since the previous analysis), and data from 5 newly submitted trials: D1690C00010, D1690C00018, D1690C00019, MB102035 and MB102045 (an additional 2455 subjects, contributing 62 events to the primary composite endpoint in 1836 person-years). A separate efficacy evaluation is also being conducted by Dr. Jonathan Norton from DB2. This review focuses solely on the pre-marketing evaluation of cardiovascular safety and supersedes the previous review.

\subsection{Conclusions and Recommendations}

The sponsor conducted a meta-analysis of the cardiovascular events that occurred in 19 Phase $2 b / 3$ trials using dapagliflozin. The FDA Diabetes Guidance $(2008)^{1}$ states that "sponsors should compare the incidence of important cardiovascular events occurring with the investigational agent to [that of] the control group to show that the upper bound of the two-sided 95 percent confidence interval for the estimated risk ratio is less than 1.8." This meta-analysis was the second of a two-part group sequential testing strategy that was pre-specified to control the overall Type I error rate for demonstration of noninferiority with respect to the 1.8 relative risk criterion for incidence of cardiovascular events. As the sponsor had succeeded in the first meta-analysis in demonstrating noninferiority, this meta-analysis used the full one-sided alpha level of 0.025 .

The agreed upon primary endpoint is a composite endpoint, defined as the time to first event of the following adjudicated events: cardiovascular (CV) death, myocardial infarction (MI), stroke, and hospitalization for unstable angina. All CV events were adjudicated by an independent, blinded clinical events committee (CEC) comprised of two cardiologists and two neurologists. The comparator group was comprised of subjects taking either a placebo and/or an active control (metformin, glipizide, insulin, glimepiride, pioglitazone, sitagliptin, hydrochlorthiazide or standard of care). 
Among the 8682 subjects in the Bristol-Myers Squibb (BMS) Analysis population (composed of the safety population of all 19 trials combined), 145 subjects had an event that was counted in the primary composite endpoint (82 of 5498 dapagliflozin subjects $(1.49 \%)$ and 63 of 3184 comparator subjects (1.98\%)). The pre-specified primary analysis of the stratified Cox proportional hazards model yielded a hazard ratio of dapagliflozin versus comparator of 0.82 (95\% CI: $0.59,1.14)$. To incorporate zero event trials in the meta-analysis, the incidence rate difference was considered as a secondary analysis method. Using this method, the incidence rate difference was found to be -0.0036 (95\% CI: -0.0097, 0.0026). Random effects analysis was performed as two of the trials (D1690C00018 and D1690C00019) were from a higher cardiovascular risk patient population than the other trials in the meta-analysis. The results of this analysis provided a slightly lower estimate of the incident rate ratio for the primary composite endpoint with a wider confidence interval: 0.75 (95\% CI: $0.47,1.18)$. With the variability of these trials accounted for in the random effects analysis, the addition of these trials did not change the overall conclusion of the meta-analysis.

A secondary composite endpoint was also evaluated which was comprised of the same $\mathrm{CV}$ endpoints as the primary endpoint and two additional CV endpoints (unplanned coronary revascularization, and hospitalization for heart failure). This endpoint also found no additional risk of $\mathrm{CV}$ events in the dapagliflozin group compared to the comparator group (hazard ratio $(95 \% \mathrm{CI}): 0.73(0.54,0.99)$.

For the updated meta-analysis, the sponsor chose to evaluate a third composite endpoint post-hoc, as an exploratory analysis, which considers only the three endpoints that compose the strict major adverse cardiovascular events (MACE): CV death, myocardial infarction (MI), and stroke. This endpoint also found no additional risk of CV events in the dapagliflozin group compared to the comparator group (hazard ratio ( $95 \% \mathrm{CI}): 0.79$ $(0.54,1.17)$.

Additionally, monotherapy and add-on therapy trials were evaluated separately as a subgroup analysis [hazard ratios $(95 \% \mathrm{CI})$ : monotherapy $0.60(0.25,1.44)$, add-on: 0.86 $(0.58,1.25)]$. To evaluate the proposed therapeutic dose, the $10 \mathrm{mg}$ dose group was separately evaluated and found to have a hazard ratio of 0.85 (95\% CI: $0.59,1.22)$. All subgroup findings were consistent with the primary analysis finding of no additional risk of cardiovascular events in the dapagliflozin group compared to the comparator group.

Based upon the CV risk guidelines set forth in the FDA Diabetes Guidance (2008) ${ }^{1}$, the upper-bound of the $95 \%$ confidence interval of the risk ratio for $\mathrm{CV}$ events in dapagliflozin compared to the pooled comparator, as estimated by the hazard ratio for the primary composite endpoint, meets the stated 1.8 non-inferiority margin. 


\subsection{Brief Overview of Clinical Studies}

In this NDA application, the applicant submitted 19 randomized phase $2 \mathrm{~b}$ and phase 3 trials. Eight trials (D1690C00004, D1690C00005, D1690C00006, D1690C00010, D1690C00012, D1690C00018, D1690C00019, and D1692C00005) were conducted by Astra-Zeneca (AZ) and 11 trials were conducted by BMS. Five trials were newly added to the analysis: trials D1690C00010, D1690C00018, D1690C00019, MB102035 and MB102045. All 19 randomized phase $2 \mathrm{~b} / 3$ trials were conducted in subjects with type 2 diabetes mellitus. Trials D1690C00005, D1690C00006, D1690C00010, D1690C00012, D1690C00018, D1690C00019, MB102009, MB102014 and MB102030 were add-on trials where subjects were on a background therapy and dapagliflozin was added to that therapy. Trial D1690C00004 was a non-inferiority trial where subjects were on metformin and were given titrated doses of dapagliflozin or glipizide. Trials D1692C00005, MB102008, MB102013 and MB102032 were monotherapy trials. Trial MB102029 was a monotherapy trial conducted in subjects with moderate to severe renal impairment. MB102021 and MB102034 were combination therapy trials where subjects were either on dapagliflozin alone, metformin alone or a combination of dapagliflozin and metformin. Trials D1690C00018 and D1690C00019 had different inclusion criteria compared to the other trials, in that they were conducted in subjects with a history of cardiovascular disease. Details of the 19 randomized phase $2 \mathrm{~b} / 3$ trials are provided in Section 3.1.1 and in the previously submitted review.

\subsection{Statistical Issues and Findings}

The primary agreed upon safety endpoint was a composite endpoint consisting of CV death, non-fatal myocardial infarction (MI), non-fatal stroke, and hospitalization due to unstable angina.

In the statistical analysis plan (SAP) agreed upon with the FDA on November 9, 2010, a group sequential testing strategy was pre-specified to control the overall Type I error rate for demonstration of non-inferiority of dapagliflozin to comparator with respect to the incidence of cardiovascular events based on the 1.8 relative risk criterion, as estimated by the hazard ratio. Using a group sequential testing strategy, if the first formal metaanalysis of the primary composite endpoint was found to have an upper-bound less than 1.8 at a one-sided alpha level of 0.01 (corresponding to a $98 \% \mathrm{CI}$ ) then the meta-analysis would meet the FDA CV guidelines ${ }^{1}$, and data from additional studies that have yet to be completed would not need to be included in a second meta-analysis. However, if unsuccessful at this alpha level (0.01), a second meta-analysis would be performed when studies D1690C00018 and D1690C00019 had unblinded and at least 110 patients had adjudicated events for the primary endpoint. The one-sided alpha level for the first metaanalysis was set at 0.01 so that the overall Type I error rate across the 2 analyses was $\leq 0.025$ (one-sided). As the first meta-analysis met the stated guidelines (i.e. the upper bound of the relative risk criterion, estimated by the hazard ratio, for the incidence of $\mathrm{CV}$ events, as measured by the primary composite endpoint, was lower than 1.8), this meta- 
analysis, which includes all data expected, was set at the full one-sided alpha-level of 0.025 .

Phase $2 \mathrm{~b}$ and Phase 3 data from the 19 randomized studies were integrated to evaluate the risk of developing a CV related event as measured by the primary composite endpoint. The data for this second meta-analysis included all the data from the previous metaanalysis, the updated data from 4 studies that were not complete at the time of the previous meta-analysis (contributing an additional 5 events to the primary composite endpoint and an additional 804 person-years since the previous analysis), and 5 additional studies (an additional 2455 subjects, contributing 62 events to the primary composite endpoint in 1836 person-years) that were not included in the previous meta-analysis. Thus, the meta-analysis performed in this review supersedes that of the previous metaanalysis as it includes additional person years from 4 studies and data from 5 additional studies.

The pre-specified primary analysis conducted was a comparison of the time to composite event between the dapagliflozin group and the pooled all comparator group performed using a Cox proportional hazards model with study as stratification factor. The MantelHaenszel $(\mathrm{MH})$ methods for estimating the overall incidence rate ratio via Breslow and Day $(1994)^{2}$, where the estimate was calculated by stratifying for trial, was performed as a pre-specified secondary analysis. The incidence rate difference was also evaluated to include trials with zero events. To further investigate event rates, the risk difference was calculated to determine if a different denominator (number of subjects per group as opposed to number of person-years of exposure) would affect the estimates.

Additionally, random effects methods were employed to determine if the estimate of the incidence rate ratio would be impacted by the heterogeneity of the studies. More details for the statistical methodologies used in this review are provided in Section 3.1.3.

Table 1 presents the results of the meta-analysis for the primary, secondary and post-hoc MACE composite endpoints. The results from the stratified Cox proportional hazards model, the Mantel-Haenszel method incidence rate ratio and incidence rate difference are presented with their associated 95\% confidence intervals. Detailed analysis results of the primary, secondary, and post-hoc MACE composite endpoints are provided in Sections 3.1.6.1, 3.1.6.2 and 3.1.6.3.

Additional subgroup analyses included evaluating the difference between dapagliflozin and comparator event rates based on CV risk factors and demographics. Additionally, monotherapy and add-on therapy trials were evaluated separately as a subgroup analysis [hazard ratios $(95 \% \mathrm{CI})$ : monotherapy $0.60(0.25,1.44)$, add-on: $0.86(0.58,1.25)]$. To evaluate the proposed therapeutic dose, the $10 \mathrm{mg}$ dose group was separately evaluated and found to have a hazard ratio of $0.85(95 \% \mathrm{CI}: 0.59,1.22)$. All results from the subgroup analyses were consistent with the results of the primary analysis, with the exception of trials D1690C00018 and D1690C0019, the high cardiovascular risk trials, where the event rates were nearly equivalent in the dapagliflozin and comparator (standard of care) treatment groups [stratified hazard ratio: 1.07; 95\% CI $(0.64,1.77)$; M$\mathrm{H}$ incident rate difference: $0.0028 ; 95 \% \mathrm{CI}(-0.018,0.024)]$. Detailed results for the sensitivity analyses and subgroup analyses are provided in Section 4. 
Table 1: Meta-Analysis Results for Primary, Secondary, and MACE Composite Endpoints (BMS Analysis Population)

\begin{tabular}{|c|c|c|c|c|c|c|c|c|}
\hline \multicolumn{3}{|c|}{ Primary Composite Endpoint $^{1}$} & \multicolumn{3}{|c|}{$\overline{\text { Secondary Composite Endpoint }{ }^{2}}$} & \multicolumn{3}{|c|}{ MACE Composite Endpoint ${ }^{3}$} \\
\hline & Dapagliflozin & $\begin{array}{l}\text { All } \\
\text { Comparator }\end{array}$ & & Dapagliflozin & $\begin{array}{l}\text { All } \\
\text { Comparator }\end{array}$ & & Dapagliflozin & $\begin{array}{l}\text { All } \\
\text { Comparator }\end{array}$ \\
\hline \multicolumn{9}{|c|}{ Primary Analysis } \\
\hline $\begin{array}{l}\text { Stratified } \\
\text { Hazard Ratio } \\
(\mathbf{9 5 \%} \text { CI) }\end{array}$ & \multicolumn{2}{|c|}{$\begin{array}{c}0.82 \\
(0.58,1.15)\end{array}$} & $\begin{array}{l}\text { Stratified } \\
\text { Hazard Ratio } \\
(95 \% \text { CI) }\end{array}$ & \multicolumn{2}{|c|}{$\begin{array}{c}0.73 \\
(0.54,0.99)\end{array}$} & $\begin{array}{l}\text { Stratified } \\
\text { Hazard Ratio } \\
\text { (95\% CI) }\end{array}$ & \multicolumn{2}{|c|}{$\begin{array}{c}0.79 \\
(0.54,1.17)\end{array}$} \\
\hline \multicolumn{3}{|c|}{ Secondary Analysis } & & \multirow{2}{*}{\multicolumn{2}{|c|}{$\begin{array}{c}0.74 \\
(0.56,0.99)\end{array}$}} & \multirow[b]{2}{*}{$\begin{array}{l}\text { M-H } \\
\text { Incidence } \\
\text { Rate Ratio } \\
\text { (95\% CI) }\end{array}$} & \multirow{2}{*}{\multicolumn{2}{|c|}{$\begin{array}{c}0.79 \\
(0.55,1.15)\end{array}$}} \\
\hline $\begin{array}{l}\text { M-H } \\
\text { Incidence } \\
\text { Rate Ratio } \\
\text { (95\% CI) }\end{array}$ & \multicolumn{2}{|c|}{$\begin{array}{c}0.82 \\
(0.59,1.14)\end{array}$} & $\begin{array}{l}\text { M-H } \\
\text { Incidence } \\
\text { Rate Ratio } \\
\text { (95\% CI) }\end{array}$ & & & & & \\
\hline $\begin{array}{l}\text { Incidence of } \\
\text { Events } \\
\text { events/person- } \\
\text { years } \\
(\%)\end{array}$ & $\begin{array}{l}82 / 5738 \\
(1.42 \%)\end{array}$ & $\begin{array}{l}63 / 3095 \\
(2.03 \%)\end{array}$ & $\begin{array}{l}\text { Incidence of } \\
\text { Events } \\
\text { events/person- } \\
\text { years } \\
(\%)\end{array}$ & $\begin{array}{c}101 / 5728 \\
(1.75 \%)\end{array}$ & $\begin{array}{l}84 / 3085 \\
(2.72 \%)\end{array}$ & $\begin{array}{l}\text { Incidence of } \\
\text { Events } \\
\text { events/person- } \\
\text { years } \\
(\%)\end{array}$ & $\begin{array}{l}62 / 5749 \\
(1.07 \%)\end{array}$ & $\begin{array}{l}49 / 3100 \\
(1.58 \%)\end{array}$ \\
\hline $\begin{array}{l}\text { M-H } \\
\text { Incidence } \\
\text { Rate } \\
\text { Difference } \\
\text { (95\% CI) }\end{array}$ & \multicolumn{2}{|c|}{$\begin{array}{c}-0.0036 \\
(-0.0097,0.0026)\end{array}$} & $\begin{array}{l}\text { M-H } \\
\text { Incidence } \\
\text { Rate } \\
\text { Difference } \\
\text { (95\% CI) }\end{array}$ & \multicolumn{2}{|c|}{$\begin{array}{c}-0.0068 \\
(-0.014,0.00017)\end{array}$} & $\begin{array}{l}\text { M-H } \\
\text { Incidence } \\
\text { Rate } \\
\text { Difference } \\
(95 \% \text { CI })\end{array}$ & \multicolumn{2}{|c|}{$\begin{array}{c}-0.0032 \\
(-0.0086,0.0022)\end{array}$} \\
\hline
\end{tabular}

${ }^{1}$ Primary Composite Endpoint: CV death, myocardial infarction (MI), stroke, and hospitalization for unstable angina.

${ }^{2}$ Secondary Composite Endpoint: CV death, myocardial infarction (MI), stroke, hospitalization for unstable angina, unplanned coronary revascularization, and hospitalization for heart failure.

${ }^{3}$ MACE Composite Endpoint: CV death, myocardial infarction (MI), and stroke

Source: Created by reviewer; reproduction of sponsor analysis in "Supplemental Cardiovascular events meta-analysis report". Dataset: adcv2v2.xpt 


\section{Introduction}

\subsection{Product Description}

Dapagliflozin is a selective, orally administered inhibitor of the human renal sodiumglucose cotransporter, SGLT2. The proposed indication of dapagliflozin is the treatment of type 2 diabetes mellitus (T2DM) in adults as an adjunct to diet and exercise to improve glycemic control. The proposed therapeutic dose of dapagliflozin is $10 \mathrm{mg}$ for most patients and a $5 \mathrm{mg}$ starting dose for patients at risk for volume depletion due to coexisting conditions or concomitant medications, such as loop diuretics.

\subsection{Clinical Trial Overview}

Bristol-Myers Squibb (BMS) conducted a meta-analysis of the cardiovascular events that occurred in all Phase $2 b / 3$ randomized, controlled trials using dapagliflozin (5 Phase $2 b$ trials, 1 Phase $2 b / 3$ trial and 13 Phase 3 trials). The statistical analysis plan used for this updated meta-analysis and the previously submitted meta-analysis was agreed upon by the sponsor and the FDA on November 9, 2010. The summary of design characteristics, the number of subjects per treatment group, and the duration of time included in the CV meta-analysis dataset for these 19 trials are presented in Table 2 and Table 3. Table 2 lists all 14 trials included in the original meta-analysis report with an update as to the trials that are now complete and include updated data (denoted in red). Table 3 lists the information regarding the 5 trials newly added to this updated meta-analysis. Most of the Phase 3 trials consisted of a short-term (ST) double-blind treatment period followed by 1 or 2 site- and subject-blinded or double-blinded long-term (LT) extension periods in which subjects retained the ST assigned treatment.

The original meta-analysis submitted by the sponsor, dated November 17, 2010, included 14 trials, of which 10 trials were complete and had their final database lock (DBL), and four trials which had completed their ST periods and had ongoing LT periods. This update to the meta-analysis was submitted by the sponsor, dated October 18, 2011, and includes 5 additional trials (MB102035, MB102045, D1690C00010, D1690C00018 and D1690C00019), contributing an additional 2455 subjects with 62 events in the primary composite endpoint in 1836 person-years, and additional data from the four trials that were not complete in the previous analysis (MB102029, D1690C00004, D1690C00006, and D1690C00012), contributing an additional 5 events in the primary composite endpoint and an additional 804 person-years since the previous analysis. Of the 19 trials included in this meta-analysis 5 are ongoing (D1690C00004, D1690C00010, D1690C00012, D1690C00018 and D1690C00019) and use a cut-off date of 15-Jul-2011. All events/visits occurring until 15-Jul-2011 were included in the analysis. 
NDA 202-293 (FORXIGA)

\subsection{Data Sources}

The applicant submitted electronic documents and datasets for trials D1690C00004, D1690C00005, D1690C00006, D1690C00012, D1690C00018, D1690C00019, D1692C00005, MB102008, MB102009, MB102013, MB102014, MB102021, MB102029, MB102030, MB102032 and MB102034. In addition, the applicant combined the datasets from these trials into a single cardiovascular meta-analysis dataset (adcv2v2.xpt) which was used for all analyses performed in this review. Clinical study reports (CSRs) of each individual trial were reviewed to evaluate trial protocols.

The following file folders available within the CDER Electronic Document Room (EDR) were utilized in this review:

Location of adcv2v2.xpt file:

- $\quad \mid \backslash C d s e s u b 1 \backslash$ evsprod $\backslash N D A 202293 \backslash 0056 \backslash m 5 \backslash$ datasets $\backslash$ metaanalysis $\backslash$ analysis $\backslash$ datasets

Location of sponsor's updated meta-analysis report:

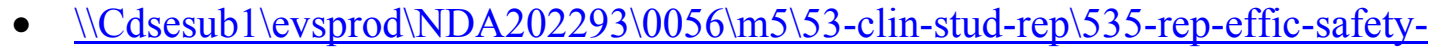
stud \type-ii-diabetes $\backslash 5353$-rep-analys-data-more-one-stud $\backslash$ meta-analysis 
NDA 202-293 (FORXIGA)

Table 2: List of Trials Included in Original CV Meta-Analysis

\begin{tabular}{|c|c|c|c|c|c|c|c|c|c|c|c|c|c|c|c|c|}
\hline \multirow[t]{2}{*}{ Trial ID } & \multirow[t]{2}{*}{$\begin{array}{l}\text { Duration } \\
\text { of Trial }\end{array}$} & \multirow{2}{*}{$\begin{array}{l}\text { Total } \\
\text { Sample } \\
\text { Size** }\end{array}$} & \multicolumn{6}{|c|}{$\begin{array}{l}\text { Dapagliflozin Dosage (mg) } \\
\text { (N per arm) }\end{array}$} & \multicolumn{4}{|c|}{ Co-treatment } & \multicolumn{3}{|c|}{$\begin{array}{l}\text { Control } \\
\text { (N per arm) }\end{array}$} & \multirow{2}{*}{$\begin{array}{l}\text { Data included } \\
\text { in updated } \\
\text { Meta-analysis } \\
\text { dataset }\end{array}$} \\
\hline & & & $1 *$ & 2.5 & 5 & 10 & 20 & 50 & Met & Glim & Ins & Pio & Met & Placebo & Glip & \\
\hline $\begin{array}{l}\text { D1690C00004 } 4^{2 \dagger} \\
\text { (Phase 3) }\end{array}$ & $\begin{array}{l}52+52+ \\
104 \text { weeks }\end{array}$ & 814 & . & . & . & 406 & . & . & 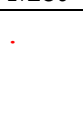 & . & . & . & . & . & 408 & $\begin{array}{l}52+52+\text { at least } \\
52 \text { weeks (up to } \\
65 \text { weeks) }\end{array}$ \\
\hline $\begin{array}{l}\text { D1690C00005 } \\
\text { (Phase 3) }\end{array}$ & $\begin{array}{l}24+24 \\
\text { weeks }\end{array}$ & 596 & . & 154 & 145 & 151 & . & . & . & $\mathrm{X}$ & . & . & . & 146 & . & Complete \\
\hline $\begin{array}{l}\text { D1690C00006† } \\
\text { (Phase 3) }\end{array}$ & $\begin{array}{l}24+24+ \\
56 \text { weeks }\end{array}$ & 807 & . & 202 & 212 & 196 & . & . & . & . & $\mathrm{X}$ & . & . & 197 & . & Complete \\
\hline $\begin{array}{l}\text { D1690C00012† } \\
\text { (Phase 3) }\end{array}$ & $\begin{array}{l}24+78 \\
\text { weeks }\end{array}$ & 182 & . & . & . & 91 & . & . & $X$ & . & . & . & . & 91 & . & $\begin{array}{l}24+\text { at least } 26 \\
\text { weeks (up to } 78 \\
\text { weeks) }\end{array}$ \\
\hline $\begin{array}{l}\text { D1692C00005 } \\
\text { (Phase 2b) }\end{array}$ & 12 weeks & 220 & $59 *$ & 56 & 58 & 52 & . & . & . & . & . & . & . & 54 & . & Complete \\
\hline $\begin{array}{l}\text { MB102008 } \\
\text { (Phase 2b) }\end{array}$ & 12 weeks & 389 & . & 59 & 58 & 47 & 59 & 56 & . & . & . & . & 56 & 54 & . & Complete \\
\hline $\begin{array}{l}\text { MB102009 } \\
\text { (Phase 2b) }\end{array}$ & 12 weeks & 71 & . & . & $\cdot$ & 24 & 24 & . & . & . & $\mathrm{X}$ & . & . & 23 & . & Complete \\
\hline $\begin{array}{l}\text { MB102013 } \\
\text { (Phase 3) }\end{array}$ & $\begin{array}{l}24+78 \\
\text { weeks }\end{array}$ & 485 & . & $\begin{array}{l}65 \\
67 \\
\end{array}$ & $\begin{array}{l}64 \\
68 \\
\end{array}$ & $\begin{array}{l}70 \\
76 \\
\end{array}$ & $\begin{array}{l}\text { AM } \\
\text { PM }\end{array}$ & . & . & . & . & $\cdot$ & . & 75 & . & Complete \\
\hline $\begin{array}{l}\text { MB102014 } \\
\text { (Phase 3) }\end{array}$ & $\begin{array}{l}24+78 \\
\text { weeks }\end{array}$ & 546 & . & 137 & 137 & 135 & . & . & $X$ & . & . & 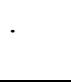 & . & 137 & . & Complete \\
\hline $\begin{array}{l}\text { MB102021 } \\
\text { (Phase 3) }\end{array}$ & 24 weeks & 598 & . & . & $\begin{array}{l}203 \\
194 \\
\end{array}$ & $\begin{array}{l}\text { dapa } \\
\text { dapa }\end{array}$ & ne) & & $\mathrm{X}^{1}$ & . & . & 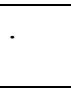 & 201 & . & . & Complete \\
\hline $\begin{array}{l}\text { MB102029† } \\
\text { (Phase 2b/3) }\end{array}$ & $\begin{array}{l}24+28+52 \\
\text { weeks }\end{array}$ & 252 & . & . & 83 & 85 & . & . & . & . & . & ${ }^{\circ}$ & . & 84 & . & Complete \\
\hline $\begin{array}{l}\text { MB102030 } \\
\text { (Phase 3) }\end{array}$ & $\begin{array}{l}24+24 \\
\text { weeks }\end{array}$ & 420 & . & . & 141 & 140 & . & . & . & . & . & $\mathrm{X}$ & . & 139 & . & Complete \\
\hline $\begin{array}{l}\text { MB102032 } \\
\text { (Phase 3) }\end{array}$ & 24 weeks & 210 & $72 *$ & 74 & 68 & - & . & . & . & . & . & . & . & 68 & . & Complete \\
\hline $\begin{array}{l}\text { MB102034 } \\
\text { (Phase 3) }\end{array}$ & 24 weeks & 638 & . & . & . & $\begin{array}{l}219 \\
211\end{array}$ & $\begin{array}{l}\text { apa alc } \\
\text { pa }+n\end{array}$ & & $\mathrm{X}^{1}$ & . & . & . & & 208 & . & Complete \\
\hline
\end{tabular}

* Not included in CV meta-analysis ** Number of subjects included in CV meta-analysis dataset.

${ }^{1}$ Trials MB102021 and MB102034 Have one arm of Dapa +Met, and one arm of Dapa alone

${ }^{2}$ Trial D1690C0004 has titrated doses of Dapa from 2.5 to $10 \mathrm{mg}$ and Glip 5 to 20mg, Dapa dose is calculated as 10mg in analyses

Source: Created by reviewer.

$\dashv$ Trials D1690C00004, D1690C00006, D1690C00012 and MB102029 include updated data since the previous meta-analysis. 
NDA 202-293 (FORXIGA)

Table 3: List of Trials Added to Updated Meta-Analysis

\begin{tabular}{|c|c|c|c|c|c|c|c|c|c|c|}
\hline \multirow[t]{2}{*}{ Trial ID } & \multirow[t]{2}{*}{$\begin{array}{l}\text { Duration } \\
\text { of Trial }\end{array}$} & \multirow[t]{2}{*}{$\begin{array}{l}\text { Total } \\
\text { Sample } \\
\text { Size } \\
\end{array}$} & $\begin{array}{l}\text { Dapagliflozin } \\
\text { Dosage (mg) } \\
\text { (N per arm) }\end{array}$ & \multicolumn{4}{|c|}{ Co-treatment } & \multicolumn{2}{|c|}{$\begin{array}{l}\text { Control } \\
\text { (N per arm) }\end{array}$} & \multirow[t]{2}{*}{$\begin{array}{l}\text { Data included in } \\
\text { Meta-analysis } \\
\text { dataset }\end{array}$} \\
\hline & & & 10 & Met & Sit & $\begin{array}{l}\text { Ins. } \\
\text { Sec. }\end{array}$ & $\begin{array}{l}\text { Standard } \\
\text { of Care }\end{array}$ & HCTZ & Placebo & \\
\hline $\begin{array}{l}\text { MB102035 } \\
\text { (Phase 2b) }\end{array}$ & 12 weeks & 75 & 24 & $\mathrm{X}$ & . & & . & 26 & 25 & Complete \\
\hline $\begin{array}{l}\text { MB102045 } \\
\text { (Phase 2b) }\end{array}$ & 12 weeks & 44 & 23 & $\mathrm{X}$ & . & & & & 21 & Complete \\
\hline $\begin{array}{l}\text { D1690C00010 } \\
\text { (Phase 3) }\end{array}$ & $\begin{array}{l}24+24 \\
\text { weeks }\end{array}$ & 451 & 225 & $\mathrm{X}$ & $\mathrm{X}$ & $+/-$ & & & 226 & $\begin{array}{l}24+\text { at least } 20 \\
\text { weeks (up to } 24 \\
\text { weeks) }\end{array}$ \\
\hline $\begin{array}{l}\text { D1690C00018 } \\
\text { (Phase 3) }\end{array}$ & $\begin{array}{l}24+28 \\
+52 \text { weeks }\end{array}$ & 921 & 459 & . & . & & $\mathrm{X}$ & & 462 & $\begin{array}{l}24+\text { at least } 8 \\
\text { weeks (up to } 52 \\
\text { weeks) }\end{array}$ \\
\hline $\begin{array}{l}\text { D1690C00019 } \\
\text { (Phase 3) }\end{array}$ & $\begin{array}{l}24+28 \\
+52 \text { weeks }\end{array}$ & 963 & 480 & & . & & $\mathrm{X}$ & & 483 & $\begin{array}{l}24+\text { at least } 7 \\
\text { weeks (up to } 52 \\
\text { weeks) }\end{array}$ \\
\hline
\end{tabular}




\section{Statistical Evaluation}

This review is focused on the meta-analysis of cardiovascular (CV) risk. For a complete statistical evaluation of efficacy results, please refer to the review authored by Dr. Jonathan Norton.

\subsection{Evaluation of Safety}

The review of CV safety comprises data from newly-added trials D1690C00010, D1690C00018, D1690C00019, MB102035 and MB102045, in addition to trials D1690C00004, D1690C00005, D1690C00006, D1690C00012, D1692C00005, MB102008, MB102009, MB102013, MB102014, MB102021, MB102029, MB102030, MB102032 and MB102034 which were also included in the original CV meta-analysis review that was submitted on September 7, 2011. New data from trials D1690C00004, D1690C00006, D1690C00012 and MB102029 are also included in this update of the metaanalysis. Based upon interactions with the clinical review team, the following safety review consists of a focused evaluation of $\mathrm{CV}$ endpoints. All comparative analyses are between the randomized treatment groups, dapagliflozin and comparator.

\subsubsection{Trial Designs}

Reviewer's note: This summary of trial designs will only include the five trials that have been added to the meta-analysis since the original submission. Please refer to the original statistical review of the meta-analysis, signed September 7, 2011, for summaries of the other 14 trials.

Trial MB102035 was a Phase 2b, multi-center randomized, double-blind, placebocontrolled 3-arm trial consisting of a12-week treatment duration to evaluate the mode of action of dapagliflozin as an add-on to metformin/SU in adults with T2DM who have inadequate glycemic control on metformin. A total of 75 subjects were randomized in a 1:1:1: fashion to the placebo group, hydrochlorthiazide (HCTZ) $25 \mathrm{mg}$, or $10 \mathrm{mg}$ of dapagliflozin. Study MB102035 was powered to detect a difference in GFR between dapagliflozin dose and placebo or HCTZ. Subjects who discontinue prior to the Week 12 visit were to be followed in the 4-week observational period unless informed consent was withdrawn, subject was detained, the subject was unable to comply with the protocol, etc.

Trial MB102045 was a Phase 2b, multi-center randomized, double-blind, placebocontrolled superiority 2-arm trial consisting of a12-week treatment duration to evaluate the mode of action of dapagliflozin on insulin sensitivity as an add-on therapy to metformin with or without insulin secretagogue in adults with T2DM who are on metformin with or without an insulin secretagogue and have inadequate glycemic control on diet and exercise. A total of 44 subjects were randomized in a 1:1 fashion to the placebo group, or $5 \mathrm{mg}$ dosage of dapagliflozin. Study MB102045 was powered to provide 80\% or greater power to detect a difference in insulin sensitivity between each dapagliflozin and placebo. 
Subjects who discontinue prior to the Week 12 visit were to be followed in the 4-week observational period unless informed consent was withdrawn, subject was detained, the subject was unable to comply with the protocol, etc.

Trial D1690C00010 was a Phase 3, multi-center randomized, double-blind, placebocontrolled superiority trial with a 24 -week short-term treatment period followed by a 24week long-term extension period to evaluate the efficacy and safety of dapagliflozin in combination with sitagliptin (with or without metformin) in adults with T2DM who have inadequate glycemic control on sitagliptin (with or without metformin) therapy alone. A total of 449 subjects were randomized in a 1:1 fashion to the placebo group and $10 \mathrm{mg}$ dosage of dapagliflozin. Trial D1690C00010 was powered to demonstrate the superiority of dapagliflozin compared to placebo based on changes in A1C from baseline to week 24 . The short-term period of this trial was completed and the long-term period was concluding and is included in the meta-analysis up to the cut-off date of July 15, 2011.

Trial D1690C00018 was a Phase 3, multi-center randomized, double-blind, placebocontrolled superiority trial with a 24 -week short-term treatment period followed by a 28 week long-term extension period and a second 52-week long-term extension period (not included in this analysis), to evaluate the efficacy and safety of dapagliflozin $10 \mathrm{mg}$ once daily in adults with T2DM, cardiovascular disease and hypertension, who exhibit inadequate glycemic control on standard of care. Trial D1690C00018 was conducted in 171 centers in Europe, North America, Latin America and Asia, in male subjects $\geq 45$ years of age and female subjects $\geq 50$ years of age diagnosed with T2DM, CVD and hypertension with inadequate glycemic control. A total of 922 subjects were randomized in a 1:1 fashion using 8 strata based on a combination of age, insulin use and time to most recent qualifying $\mathrm{CV}$ event to the placebo group and $10 \mathrm{mg}$ dosages of dapagliflozin. Trial D1690C00018 was powered to provide $90 \%$ or greater power to demonstrate the superiority of dapagliflozin compared to placebo to with a difference of $15 \%$ between dapagliflozin and placebo for changes in clinical benefit using a 3-item endpoint (absolute drop of $0.5 \%$ or more from baseline $\mathrm{HbA} 1 \mathrm{c}$, relative drop of $3 \%$ or more from baseline for total body weight, and an absolute drop of $3 \mathrm{mmHg}$ or more from baseline in seated systolic blood pressure); and to provide $>99 \%$ power to detect a difference of $0.5 \%$ between dapagliflozin and placebo for mean change in HbA1c from baseline to week 24 at a significance level of 0.0125 for both primary objectives. The short-term period of this trial was completed and the long-term period was concluding and is included in the metaanalysis up to the cut-off date of July 15, 2011. Subjects had to be scheduled for a followup visit 3 weeks after discontinuation of investigational product.

Trial D1690C00019 was a Phase 3, multi-center randomized, double-blind, placebocontrolled superiority trial with a 24 -week short-term treatment period followed by a 28 week long-term extension period and a second 52-week long-term extension period (not included in this analysis), to evaluate the efficacy and safety of dapagliflozin $10 \mathrm{mg}$ once daily in adults with T2DM and cardiovascular disease who exhibit inadequate glycemic control on standard of care. Trial D1690C00019 was conducted in 173 centers in Europe, North America, South America and Australia, in male subjects $\geq 45$ years of age and female subjects $\geq 50$ years of age diagnosed with T2DM, and CVD with inadequate 
glycemic control. A total of 922 subjects were randomized in a 1:1 fashion using 8 strata based on a combination of age, insulin use and time to most recent qualifying $\mathrm{CV}$ event to the placebo group and $10 \mathrm{mg}$ dosages of dapagliflozin. Trial D1690C00019 was powered to provide $90 \%$ or greater power to demonstrate the superiority of dapagliflozin compared to placebo to with a difference of $15 \%$ between dapagliflozin and placebo for changes in clinical benefit using a 3 -item endpoint(absolute drop of $0.5 \%$ or more from baseline $\mathrm{HbA1c}$, relative drop of $3 \%$ or more from baseline for total body weight, and an absolute drop of $3 \mathrm{mmHg}$ or more from baseline in seated systolic blood pressure); and provided $>99 \%$ power to detect a difference of $0.5 \%$ between dapagliflozin and placebo for mean change in $\mathrm{HbA1c}$ from baseline to week 24 at a significance level of 0.0125 for both primary objectives. The short-term period of this trial was completed and the long-term period was concluding and is included in the meta-analysis up to the cut-off date of July 15, 2011. Subjects had to be scheduled for a follow-up visit 3 weeks after discontinuation of investigational product.

\subsubsection{Endpoints and Adjudication Methods}

\subsubsection{Primary Composite Endpoint}

The agreed upon primary endpoint is a composite endpoint, defined as the time to first event of the following adjudicated events:

- CV death

- Myocardial infarction (MI)

- Stroke

- Hospitalization for unstable angina

For the primary ST + LT population, time to event was evaluated in days from the date of first randomized dose to the date of the first event. For subjects without events in AZ studies, censoring took place at the earliest of the following: the date of last contact (e.g. date of last study visit) or date of death. For subjects without events in BMS studies, censoring took place at the earliest of the following: end of treatment +30 days, date of death, date of discontinuation due to lost to follow-up. If end of treatment +30 days occurred after 15-Jul-2011, then 15-Jul-2011 was used as censoring date for subjects without events in BMS studies.

\subsubsection{Secondary Composite Endpoint}

The agreed upon secondary endpoint is a composite endpoint, defined as the time to first event of the following adjudicated events:

- CV death

- Myocardial infarction (MI)

- Stroke 
NDA 202-293 (FORXIGA)

- Hospitalization for unstable angina

- Unplanned coronary revascularization

- Hospitalization for heart failure

Time-to-event/censoring and exposure were calculated using similar methods as described for the primary composite endpoint.

\subsubsection{MACE Composite Endpoint}

For the updated meta-analysis, as a post-hoc analysis, the sponsor chose to evaluate an exploratory third composite endpoint which considers only the three endpoints that compose the major adverse cardiovascular events (MACE):

- CV death

- Myocardial infarction (MI)

- Stroke

Time-to-event/censoring and exposure were calculated using similar methods as described for the primary composite endpoint. It should be noted that this endpoint is a subset of the primary composite endpoint.

\subsubsection{Adjudication Methods}

As stated in the previous review, cardiovascular events were adjudicated by an independent Clinical Event Committee (CEC) consisting of two independent cardiologists and two independent neurologists at $\quad$ (b) (4) For 17 of the trials the adjudication was done prospectively (before final trial database locks). Two Phase 2 trials, MB102008 and MB102009, were completed when the adjudication process was initiated, and the adjudication of the events was done retrospectively. The independent adjudication committee was blinded at all times to treatment allocation.

Potential CV events were identified through an in-stream search of all reported adverse events. Standardized MedDRA queries (SMQs) or custom SMQs for adverse events were used to select the events for adjudication. Once a possible CV event was detected, the sponsor contacted the sites and requested the principal investigators to fill in the appropriate case submission report for the respective event which was then sent to the CEC. Additional events could also have been identified by the CEC while reviewing the supporting documentation. 


\subsubsection{Statistical Methodologies}

The following sections, updated from the previously submitted review, detail the statistical methods used in the evaluation of the primary and secondary analyses of the meta-analysis and in the sensitivity analyses included in the review.

\subsubsection{Group Sequential Meta-analysis}

The original meta-analysis conducted by the sponsor was the first part of an agreed upon two-part group sequential testing strategy (Whitehead, 1997) ${ }^{3}$ that was pre-specified to control the Type I error rate for demonstration of non-inferiority with respect to the 1.8 relative risk criterion at an overall one-sided 0.025 level as outlined in the FDA CV guidance.

As agreed upon with the FDA, if the first formal meta-analysis of the primary composite endpoint was found to have an upper-bound less than 1.8 at a one-sided alpha level of 0.01 (corresponding to a $98 \% \mathrm{CI}$ ) then the meta-analysis would meet the guidelines, and data from additional studies that have yet to be completed would not need to be included in a second meta-analysis. However, if unsuccessful at this alpha level (0.01), a second metaanalysis would be performed when studies D1690C00018 and D1690C00019 had unblinded and at least 110 patients had adjudicated events for the primary endpoint. The one-sided alpha level for this final analysis was set at 0.01 so that the overall Type I error rate across the 2 analyses was $\leq 0.025$ (one-sided).

As the first meta-analysis met the stated guidelines, this meta-analysis, which includes all data expected, will use the full one-sided alpha-level of 0.025 .

\subsubsection{Time to Event Analysis}

As in the previous meta-analysis, the agreed upon primary analysis method was to analyze the time to occurrence of CV-related events among all dapagliflozin doses $(\geq 2.5 \mathrm{mg})$ pooled versus all comparator groups pooled using a Cox proportional hazards model stratified by trial and including a dichotomous term for treatment group. To be included in this analysis, a trial had to have at least 1 adjudicated event to contribute to the analysis. The proportional hazards assumption was assessed by plotting the log-log survival curve of the data and the Schoenfeld residuals.

\subsubsection{Analysis of Event Incidence}

As in the previous meta-analysis, agreed upon secondary analyses included the use of Mantel-Haenszel methods estimating the overall incidence rate ratio and incidence rate difference via Breslow and Day (1994) ${ }^{2}$ with asymptotic confidence intervals provided 
using the asymptotic methods described by Robins et al. $(1986)^{4}$. The incidence rate ratio was calculated by stratifying by trial where trials with no events were excluded from the analysis. The incidence rate difference included all trials in the analysis.

\subsubsection{Evaluation of Event Rates}

As in the previous meta-analysis, in addition to the methods used by BMS in their primary and secondary analyses, sensitivity analyses conducted by the FDA included the calculation of the risk difference for the primary and secondary composite endpoints. The calculation of the Mantel-Haenszel method risk difference was included to evaluate the impact of using the denominator of number of subjects per treatment arm (as opposed to number of patient-years of exposure used in the incidence rate difference calculation) on the effect estimates.

\subsubsection{Handling of Trials with Zero Events}

The difference of incidence rates and the difference of events rates were analyzed using Mantel-Haenszel methods to provide analyses that were able to include trials that did not have an adjudicated event. The Cox proportional hazards model and incidence rate ratio exclude trials with zero events.

\subsubsection{Evaluation of Heterogeneity between Trials}

To determine if the event rates in the trials included in the meta-analysis were different from one another, the heterogeneity between the studies was assessed using the Q-statistic to determine in the studies were significantly different from one another and the $\mathrm{I}^{2}$ statistic to determine the amount of heterogeneity present in the studies (Higgins and Thompson, 2002) $)^{5}$.

\subsubsection{Random Effects Analysis}

As two of the trials (D1690C00018 and D1690C00019) were taken from a higher cardiovascular risk patient population than the other trials included in the meta-analysis, random effects analysis was conducted in this review to determine if the estimate for the incidence rate ratio for the primary composite endpoint would change due to this study heterogeneity. Random effects analysis was conducted using the approach of Hardy and Thompson (1996) ${ }^{6}$. 
NDA 202-293 (FORXIGA)

\subsubsection{Populations}

The main analysis population consists of the randomized, controlled, ST + LT periods of all Phase $2 \mathrm{~b}$ and 3 trials of dapagliflozin that have been unblinded by the sponsor at the time of the CV events meta-analysis. The ST + LT data include data following initiation of any rescue therapy. Any dapagliflozin treatment group that does not have a corresponding control group was excluded. All analyses were performed on all subjects who received at least one dose of double-blind study medication during the ST double-blind treatment period (safety population).

The BMS Analysis Population is defined to include all subjects in the 19 trials listed in Table 2 and Table 3. The $1 \mathrm{mg}$ dapagliflozin dosage group was considered sub-therapeutic and was not included in any summary of the pooled dapagliflozin group. The BMS Analysis Population included 8682 subjects: 5498 in the dapagliflozin group and 3184 in the all comparator group (945 standard of care, 739 metformin, 408 glipizide, 335 placebo, 226 sitagliptin, 220 insulin, 146 glimepiride, 139 pioglitazone and 26 hydrochlorothiazide (HCTZ)).

Reviewer's note: In the original meta-analysis review a separate "Sensitivity Analysis Population" excluding short-term 12-week trials and the non-inferiority trial was evaluated. As the previous meta-analysis showed that this "Sensitivity Analysis Population" did not provide results different from the BMS Analysis Population and had less conservative results compared to the BMS Analysis Population, this updated metaanalysis will be based solely on this population.

Reviewer's note: The sponsor performed a sensitivity analysis pooling all short-term (ST) periods of the trials. This analysis was not replicated by the reviewer as it was determined that the long-term risk of cardiovascular events was of primary importance. The long-term (LT) extension periods of these trials maintained the double-blind nature of the trial and continued with the same treatment group assignments and therefore provided extended periods of exposure of the subjects to their assigned treatments. It should be noted that, as in the previous review, the sponsor's results for this analysis were consistent with all other findings of the meta-analysis (i.e. no increased risk of cardiovascular events in dapagliflozin compared to comparator). 


\subsubsection{Subject Disposition, Demographics and Baseline Characteristics}

As shown in Table 4, in the BMS Analysis population, baseline demographics, on average, were similar among the pooled treatment groups. There were more male subjects than female subjects $(55.2 \%$ versus $44.8 \%$ ). Approximately $82.3 \%$ of subjects were White, about $7.9 \%$ were Asian and 3.8\% of subjects were Black or African-American. More than $75 \%$ of subjects in the BMS Analysis population were above 50 years of age, while the mean age was about 58 . More than $60 \%$ of subjects had body mass index (BMI) greater than $30 \mathrm{~kg} / \mathrm{m}^{2}$, while the mean BMI was about $32 \mathrm{~kg} / \mathrm{m}^{2}$.

Reviewer's note: The addition of the two high cardiovascular risk trials, D1690C00018 and D1690C00019, skewed the data from the updated meta-analysis by increasing the percentage of males, the mean age and the mean BMI from what was observed in the previous meta-analysis review.

Table 4: Demographics (BMS Analysis Population)

\begin{tabular}{lll}
\hline & $\begin{array}{l}\text { Dapagliflozin } \\
(N=5498)\end{array}$ & $\begin{array}{l}\text { Combined } \\
\text { Comparator } \\
(N=3184)\end{array}$ \\
\hline Percent Female & $46.02 \%$ & $48.34 \%$ \\
\hline Age+ SD (years) & $57.1 . \pm 10.4$ & $58.4 \pm 10.2$ \\
\hline$\leq 50$ years & $28.1 \%$ & $28.9 \%$ \\
$50-65$ years & $50.6 \%$ & $48.4 \%$ \\
$\geq 65$ years & $20.5 \%$ & $23.5 \%$ \\
\hline BMI+ SD (kg/m $\left.{ }^{2}\right)$ & $31.5 \pm 5.5$ & $31.5 \pm 5.5$ \\
\hline $25-30$ & $30.9 \%$ & $32.4 \%$ \\
$\geq 30$ & $58.7 \%$ & $57.0 \%$ \\
\hline Race and Ethnicity & & \\
\hline White & $81.0 \%$ & $81.2 \%$ \\
Black & $3.4 \%$ & $3.5 \%$ \\
Asian & $9.1 \%$ & $9.7 \%$ \\
\hline Region & & \\
\hline North America & $32.5 \%$ & $29.9 \%$ \\
Latin America & $20.1 \%$ & $19.1 \%$ \\
Europe & $37.2 \%$ & $42.9 \%$ \\
Asia/Pacific & $10.3 \%$ & $8.0 \%$ \\
\hline Source: Created by reviewer. Dataset: adcv2v2.xpt
\end{tabular}

Baseline CV risk factors and complications are presented in Table 5. In the BMS Analysis population, approximately $36.6 \%$ had cardiovascular disease (CVD) at baseline. Furthermore, almost $70 \%$ of subjects were hypertensive at baseline and more than $40 \%$ were current or ex- smoker. Additionally, most subjects had some degree of renal impairment. Table 16 in the Appendix presents the CV risk factors for each trial. In most trials, baseline $\mathrm{CV}$ risk factors were consistent across trials. The major exception to this was in the high cardiovascular risk trials, D1690C00018 and D1690C00019, where all 
subjects in these trials were required to have CV risk factors. Additionally, in trial MB102029, the renal impairment study, a higher percentage (40\%) of subjects, compared to the majority of the trials, had a history of CVD and nearly $90 \%$ of all subjects had a history of hypertension. Also, in trial D1692C00005, the trial in a Japanese population, there was a lower history of CVD (around 3\%) and a much lower rate of renal impairment (nearly $90 \%$ of all subjects had normal renal function). In Table 5, there does appear to be a higher frequency of cardiovascular risk factors in the comparator group compared to dapagliflozin, but this is an artifact of the pooled data and was not observed in the individual trials.

Reviewer's note: The addition of the two high cardiovascular risk trials, D1690C00018 and D1690C00019, skewed the data from the updated meta-analysis by increasing the rate of all CV risk factors from what was observed in the previous meta-analysis review.

Further analysis of the impact of these two trials on the pooled data is provided in Section 4.2.4.

Table 5: CV Risk Factors (BMS Analysis Population)

\section{Dapagliflozin Combined} Comparator

\begin{tabular}{lll} 
& $(N=5498)$ & $(N=3148)$ \\
\hline History of CVD & $33.2 \%$ & $42.3 \%$ \\
\hline $\begin{array}{l}\text { History of } \\
\text { Hypertension }\end{array}$ & $67.6 \%$ & $74.0 \%$ \\
\hline $\begin{array}{l}\text { History of } \\
\text { Dyslipidemia }\end{array}$ & $55.8 \%$ & $60.1 \%$ \\
\hline $\begin{array}{l}\text { History of Congestive } \\
\text { Heart Failure }\end{array}$ & $4.3 \%$ & $5.1 \%$ \\
\hline $\begin{array}{l}\text { Renal Function } \\
\text { Seva }\end{array}$ & &
\end{tabular}

\begin{tabular}{lll}
\hline Renal Function & & \\
Severely Impaired & $0.2 \%$ & $0.2 \%$ \\
Moderately Impaired & $11.9 \%$ & $12.0 \%$ \\
Mildly Impaired & $52.5 \%$ & $53.1 \%$ \\
Normal & $35.4 \%$ & $34.8 \%$ \\
\hline Diabetes Duration & & \\
less than 3 years & $37.9 \%$ & $33.6 \%$ \\
3-10 years & $34.0 \%$ & $35.8 \%$ \\
more than 10 years & $28.2 \%$ & $30.7 \%$ \\
\hline
\end{tabular}

Source: Created by reviewer. Dataset: adcv2v2.xpt

Among the 8,682 subjects in the BMS Analysis Population from the 19 randomized phase $2 \mathrm{~b} / 3$ trials, approximately $25 \%$ (2139/8682) prematurely discontinued study medication. Figure 1 presents the data from the 4 trials that were updated since the previous metaanalysis and the 5 trials that were added since the previous meta-analysis (to see the results from the other trials, please refer to the previous review). The discontinuation rates for the 4 trials that were updated since the previous review are much higher than those found in the 5 newly added trials, however the rate of discontinuation, as evaluated by Kaplan Meier 
curves (included in Figure 10 the Appendix) was consistent with what was seen in other trials. As seen in the previous review, the percentage of subjects with premature discontinuation of study medication tended to be lower in the dapagliflozin treatment groups than in the comparator groups in most of the trials. On average, $23 \%$ of the subjects in the dapagliflozin group discontinued study medication, while $27 \%$ of the subjects in the combined comparator groups prematurely discontinued the study medication.

\section{Figure 1: Percentage of Subjects with Premature Discontinuation of Study Medication by Trial and Treatment Group for Updated and Newly Added Trials (BMS Analysis Population)}

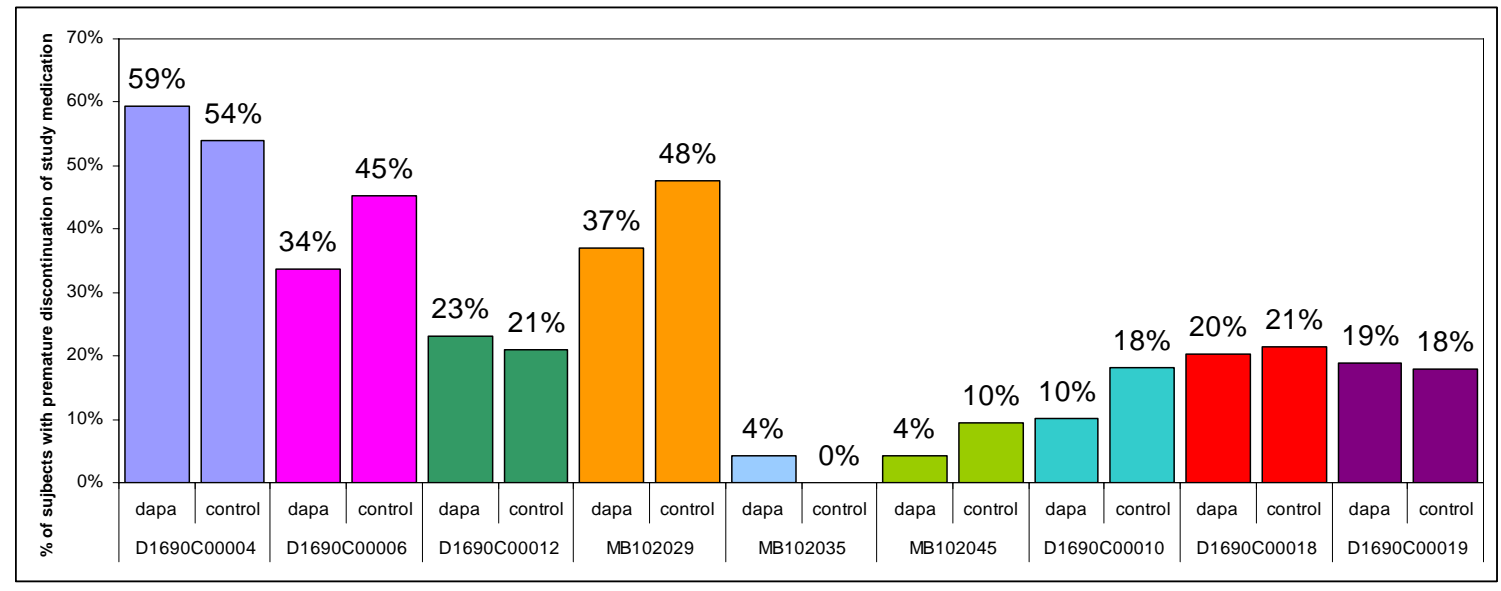

Reviewer's note: As reported in the previous review, trials D1690C00004 and D1690C00006 requested consent from subjects a second time for inclusion in an additional extension period in the trial resulting in inconsistent discontinuation rates. As exclusion of these additional extension periods from the analysis would lead to less conservative estimates of the event rates (due to a higher number of events occurring in the dapagliflozin group vs. the comparator group during this period), the data from these periods remained in the analysis.

\subsubsection{Results and Conclusions}

\subsubsection{Primary Composite Endpoint}

As done in the previous review, to ensure that the Cox proportional hazards method used by BMS was justified, the proportional hazards assumptions of the data were evaluated in the BMS Analysis population by investigating the plots of the log-log survival curve and the Schoenfeld residuals of the data with regards to the primary composite endpoint. Both plots, presented in the Appendix, show that the proportional hazards assumption does hold for this data as the log-log curves for dapagliflozin and comparator are generally parallel to each other and the curve resulting from the plotting of the Schoenfeld residuals does approximately lie on $\mathrm{y}=0$. 
The meta-analysis results for the primary composite endpoint for the BMS Analysis population from this updated analysis and from the previous analysis are presented in Table 6. The results from this analysis yield a higher estimate of the hazard ratio of CV events in the dapagliflozin group compared to the comparator group when compared to the results received in the previous review (stratified hazard ratio of 0.82 compared to 0.67 in previous review), however, there is still a lower overall incidence of $\mathrm{CV}$ events in the dapagliflozin group compared to the comparator group.

Table 6: Meta-Analysis Results for Primary Composite Endpoint (BMS Analysis Population)

\begin{tabular}{|c|c|c|c|c|}
\hline & \multicolumn{2}{|c|}{ Updated Review } & \multicolumn{2}{|c|}{ Previous Review } \\
\hline & Dapagliflozin & $\begin{array}{l}\text { All } \\
\text { Comparator }\end{array}$ & Dapagliflozin & $\begin{array}{l}\text { All } \\
\text { Comparator }\end{array}$ \\
\hline & \multicolumn{4}{|c|}{ Primary Analysis } \\
\hline $\begin{array}{l}\text { Stratified Hazard } \\
\text { Ratio } \\
(95 \% \mathrm{CI}) \\
(98 \% \mathrm{CI})^{*}\end{array}$ & \multicolumn{2}{|c|}{$\begin{array}{c}0.82 \\
(0.58,1.15)\end{array}$} & \multicolumn{2}{|c|}{$\begin{array}{l}0.67 \\
38,1.18)\end{array}$} \\
\hline & \multicolumn{4}{|c|}{ Secondary Analysis } \\
\hline $\begin{array}{l}\text { M-H Incidence Rate } \\
\text { Ratio } \\
(95 \% \text { CI) } \\
(98 \% \text { CI })^{*}\end{array}$ & \multicolumn{2}{|c|}{$\begin{array}{c}0.82 \\
(0.59,1.14)\end{array}$} & \multicolumn{2}{|c|}{$(0.39,1.17)$} \\
\hline $\begin{array}{l}\text { Incidence of Events } \\
\text { events/person-years } \\
(\%)\end{array}$ & $\begin{array}{l}82 / 5738 \\
(1.43 \%)\end{array}$ & $\begin{array}{l}63 / 3095 \\
(2.04 \%)\end{array}$ & $\begin{array}{l}48 / 4344 \\
(1.10 \%)\end{array}$ & $\begin{array}{l}30 / 1849 \\
(1.62 \%)\end{array}$ \\
\hline $\begin{array}{l}\text { M-H Incidence Rate } \\
\text { Difference } \\
(95 \% \mathrm{CI}) \\
(98 \% \mathrm{CI}) *\end{array}$ & \multicolumn{2}{|c|}{$\begin{array}{c}-0.0036 \\
(-0.0097,0.0026)\end{array}$} & \multicolumn{2}{|c|}{-0.0054} \\
\hline
\end{tabular}

Source: Created by reviewer; reproduction of sponsor analysis in "Supplemental Cardiovascular events metaanalysis report". Dataset: adcv2v2.xpt

$* 98 \%$ CIs were calculated for analyses of the primary endpoint in the previous review due to the group sequential meta-analysis. 
Based on the Kaplan-Meier method using the BMS Analysis population, the cumulative probability of developing a CV-related event as measured by the primary composite endpoint is shown in Figure 2. In the combined comparator group, events of the composite $\mathrm{CV}$ safety endpoint occurred earlier than in the dapagliflozin group. Based on the stratified log-rank test stratified by trial, the onset time of event was not statistically significantly different at the one-sided alpha $=0.025$ level between the dapagliflozin group and the comparator group $(p=0.25)$.

Figure 2: Time-to-event Analysis of the Primary Composite Endpoint (BMS Analysis Population)

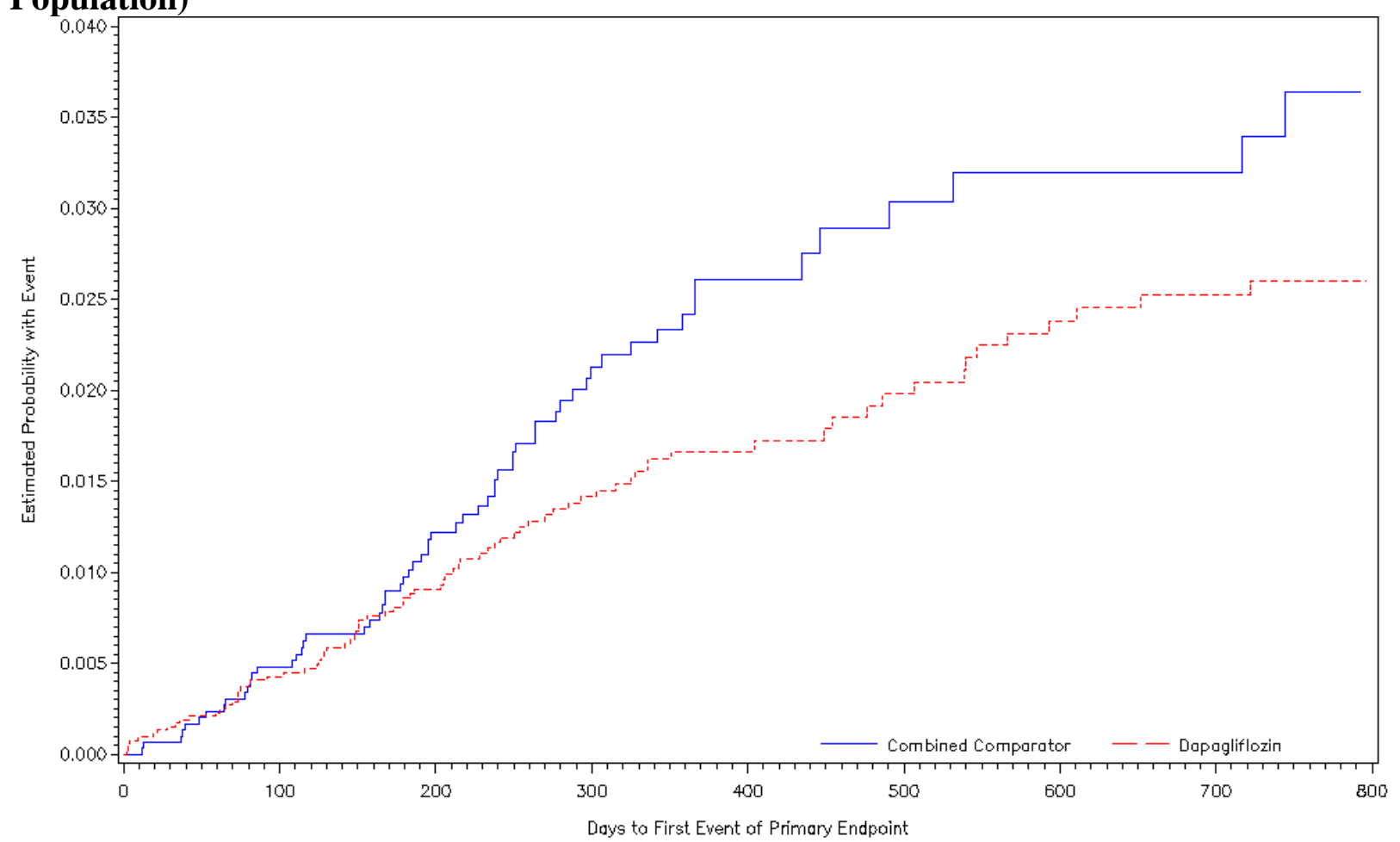

Source: Created by reviewer; reproduction of sponsor analysis in "Supplemental Cardiovascular events metaanalysis report". Dataset: adcv2v2.xpt 
The forest plot of the hazard ratios for all trials included in the BMS Analysis population that had at least one subject who experienced a composite primary endpoint event is presented in Figure 3 (note that no individual trial CI's are presented in trials with zero events in the forest plot). The trials that include updated data since the previous metaanalysis are denoted in red, the newly added trials are denoted in blue and the trials that were complete at the time of the previous meta-analysis are in black. The overall hazard ratio for all trials, stratified by trial, is presented on the bottom of the plot.

Figure 3: Forest Plot of Hazard Ratios of Individual Trials from Cox Proportional Hazards for Primary CV Composite Endpoint (BMS Analysis Population)

$\begin{array}{ll}\text { Trial } & \text { Type of Therapy } \\ \text { D1690C00004 } & \text { Add-on to Metformin (Glipizide) } \\ \text { D1690C00005 } & \text { Add-on to SU } \\ \text { D1690C00006 } & \text { Add-on to Insulin } \\ \text { D1690C00010 } & \text { Add-on to DPP-4 } \\ \text { D1690C00012 } & \text { Add-on to Metformin } \\ \text { D1690C00018 } & \text { Add-on to usual care (CV risk) } \\ \text { D1690C00019 } & \text { Add-on to usual care (CV risk) } \\ \text { D1692C00005* } & \text { Monotherapy } \\ \text { MB102008* } & \text { Monotherapy } \\ \text { MB102013* } & \text { Monotherapy } \\ \text { MB102014 } & \text { Add-on to Metformin } \\ \text { MB102021 } & \text { Combination with Metformin } \\ \text { MB102029 } & \text { Monotherapy (renal impairment) } \\ \text { MB102030* } & \text { Add-on to TZD } \\ \text { MB102034 } & \text { Combination with Metformin } \\ & \\ & \text { Hazard Ratio (Pooled) }\end{array}$

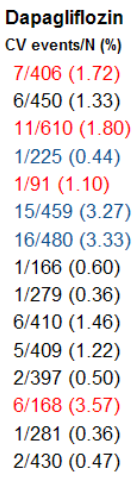

$$
\begin{aligned}
& \text { Comparator } \\
& \text { CV events/N (\%) } \\
& 8 / 408(1.96) \\
& 1 / 146(0.68) \\
& 5 / 197(2.54) \\
& 1 / 225(0.44) \\
& 1 / 91(1.10) \\
& 13 / 462(2.81) \\
& 16 / 483(3.31) \\
& 0 / 54(0.00) \\
& 0 / 110(0.00) \\
& 0 / 75(0.00) \\
& 6 / 137(4.38) \\
& 1 / 201(0.50) \\
& 8 / 84(9.52) \\
& 0 / 139(0.00) \\
& 1 / 208(0.48)
\end{aligned}
$$

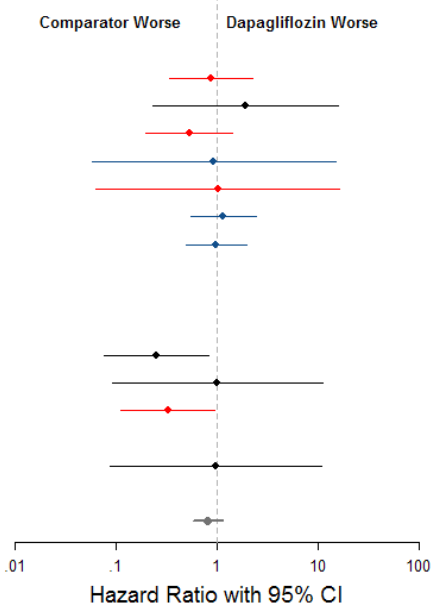

Hazard Ratio estimate $(95 \% \mathrm{Cl})$ $0.874(0.337,2.265)$ $1.929(0.232,16.026)$ $0.537(0.198,1.452)$ $0.941(0.059,15.066)$ $1.026(0.064,16.399)$ $1.165(0.555,2.449)$ $0.989(0.495,1.978)$

$0.252(0.077,0.828)$ $1.011(0.092,11.151)$ $0.328(0.114,0.945)$

$0.979(0.089,10.801)$

$0.67(0.38,1.18)$

* Estimate values are out of range

Legend: red = trials with new data; blue = newly added trials; black = same data as in previous meta-analysis Source: Created by reviewer; reproduction of sponsor analysis in "Supplemental Cardiovascular events metaanalysis report". Dataset: adcv2v2.xpt 
To explore which cardiovascular event components contributed to the composite primary endpoint, the Mantel-Haenszel incidence rate differences for each component of the composite CV safety endpoint were calculated using the pooled BMS Analysis Population. Figure 4 presents a forest plot of these risk differences in reference to the risk difference for the composite primary endpoint. It can be observed in this figure that of the endpoints composing the primary composite endpoint myocardial infarction (MI) has the lowest risk of occurrence in subjects taking dapagliflozin compared to comparator, while the other endpoints are nearly equally likely to occur in either group.

Figure 4: Forest Plot of Mantel-Haenszel Incidence Rate Differences of Cardiovascular Events Contributing to Primary Composite Endpoint (BMS Analysis Population)

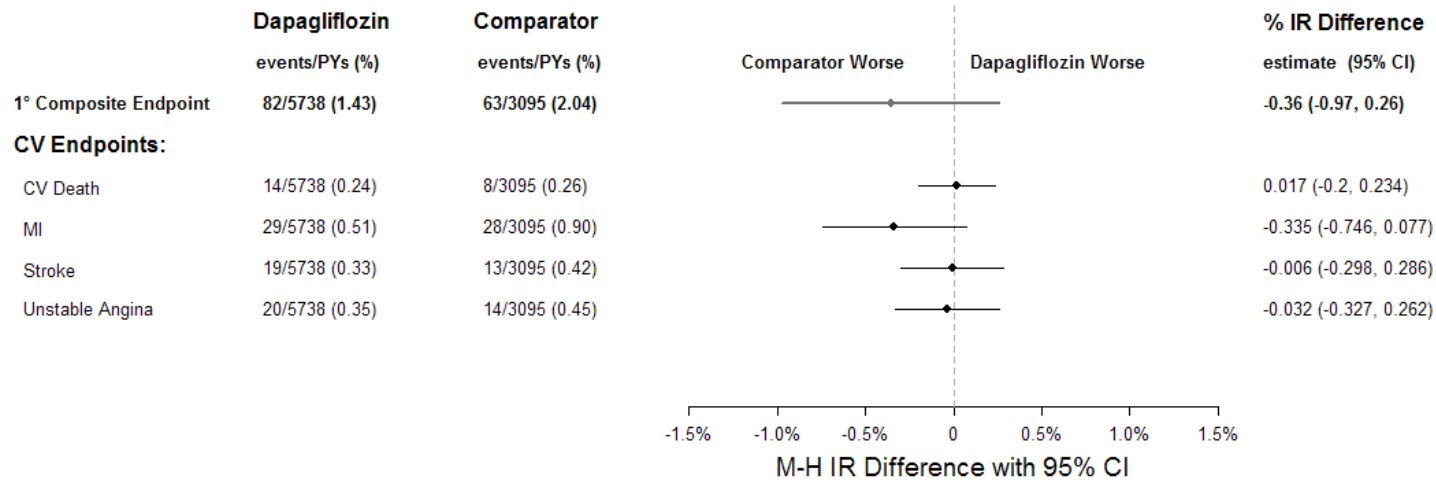

Source: Created by reviewer. Dataset: adcv2v2.xpt 


\subsubsection{Secondary Composite Endpoint}

Recall that the secondary composite endpoint included two additional terms to the composite versus the primary composite endpoint: unplanned coronary revascularization and hospitalization for heart failure. There were 40 additional events in the BMS Analysis Population due to the addition of these terms, 19 in the dapagliflozin group and 21 in the comparator group. The meta-analysis results for the secondary composite endpoint in the BMS Analysis population for this review and for the previous review are presented in Table 7: Meta-Analysis Results for Secondary Composite Endpoint (BMS Analysis Population). Again, while the estimate for the stratified hazard ratio is slightly higher than what was found in the previous review ( 0.73 compared to 0.63$)$, there is a lower risk of the occurrence of an event from the secondary composite endpoint in the dapagliflozin group compared to the comparator group.

Table 7: Meta-Analysis Results for Secondary Composite Endpoint (BMS Analysis Population)

\begin{tabular}{|c|c|c|c|c|}
\hline & \multicolumn{2}{|c|}{ Updated Review } & \multicolumn{2}{|c|}{ Previous Review } \\
\hline & Dapagliflozin & $\begin{array}{l}\text { All } \\
\text { Comparator }\end{array}$ & Dapagliflozin & $\begin{array}{l}\text { All } \\
\text { Comparator }\end{array}$ \\
\hline $\begin{array}{l}\text { Stratified Hazard } \\
\text { Ratio } \\
(95 \% \mathrm{CI}) \\
(98 \% \mathrm{CI}) *\end{array}$ & \multicolumn{2}{|c|}{$\begin{array}{c}0.73 \\
(0.54,0.99)\end{array}$} & \multicolumn{2}{|c|}{$\begin{array}{c}0.63 \\
(0.38,1.03)\end{array}$} \\
\hline $\begin{array}{l}\text { M-H Incidence Rate } \\
\text { Ratio } \\
(95 \% \mathrm{CI}) \\
(98 \% \mathrm{CI}) *\end{array}$ & \multicolumn{2}{|c|}{$\begin{array}{c}0.74 \\
(0.56,0.99)\end{array}$} & \multicolumn{2}{|c|}{$(0.39,1.02)$} \\
\hline $\begin{array}{l}\text { Incidence of Events } \\
\text { events/person-years } \\
(\%)\end{array}$ & $\begin{array}{c}101 / 5728 \\
(1.76 \%)\end{array}$ & $\begin{array}{l}59 / 4339 \\
(1.36 \%)\end{array}$ & $\begin{array}{l}59 / 4339 \\
(1.36 \%)\end{array}$ & $\begin{array}{l}30 / 1849 \\
(1.62 \%)\end{array}$ \\
\hline $\begin{array}{l}\text { M-H Incidence Rate } \\
\text { Difference } \\
(95 \% \mathrm{CI}) \\
(98 \% \mathrm{CI})^{*}\end{array}$ & \multicolumn{2}{|c|}{$\begin{array}{c}-0.0068 \\
(-0.014,0.00017)\end{array}$} & \multicolumn{2}{|c|}{-0.0080} \\
\hline
\end{tabular}

Source: Created by reviewer; reproduction of sponsor analysis in "Supplemental Cardiovascular events metaanalysis report". Dataset: adcv2v2.xpt

$* 98 \%$ CIs were calculated for analyses of the primary endpoint in the previous review due to the group sequential meta-analysis. 
Figure 5 presents a forest plot of these risk differences in reference to the risk difference for the composite secondary endpoint. It can be observed in this figure that of the endpoints composing the secondary composite endpoint, myocardial infarction (MI) and heart failure have a lower risk of occurrence in subjects taking dapagliflozin compared to comparator, while the other endpoints are nearly equally likely to occur in either group.

Figure 5: Forest Plot of Mantel-Haenszel Incidence Rate Differences of Cardiovascular Events Contributing to Secondary Composite Endpoint (BMS Analysis Population)

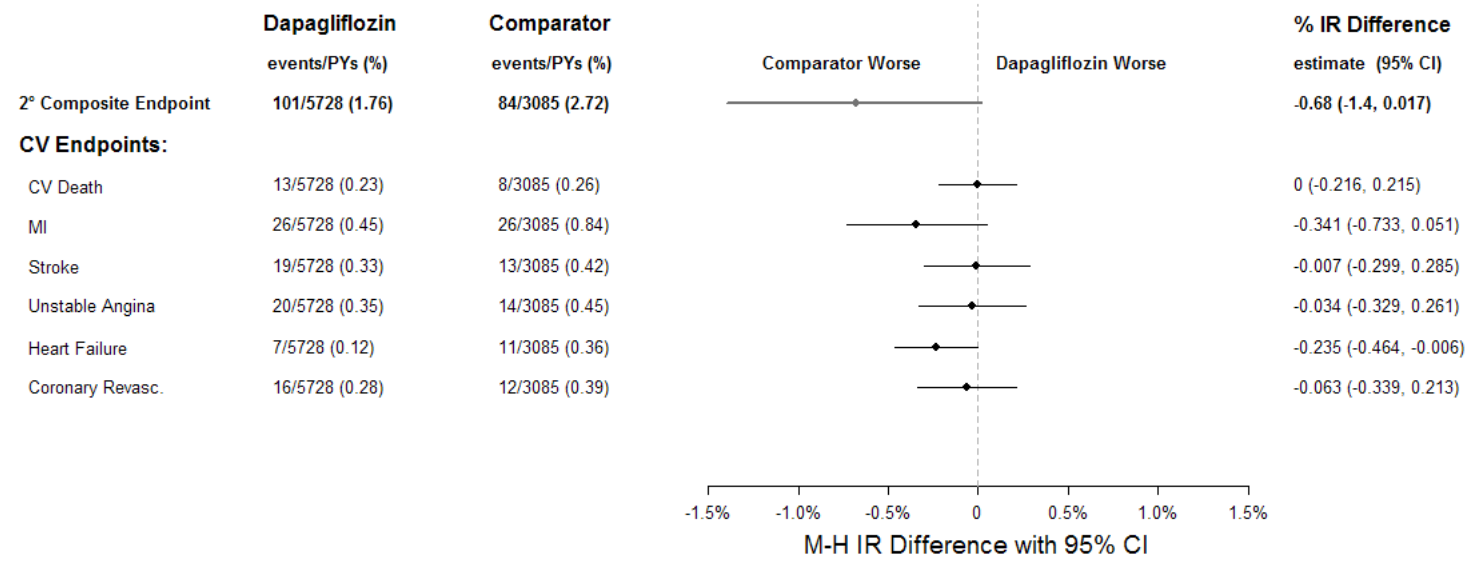

Source: Created by reviewer. Dataset: adcv2v2.xpt 


\subsubsection{MACE Composite Endpoint}

For the updated meta-analysis, the sponsor chose to conduct a post-hoc analysis of a third composite endpoint which considers only the three endpoints that compose the major adverse cardiovascular events (MACE): CV death, myocardial infarction (MI), and stroke, this composite endpoint is a subset of the primary composite endpoint (which includes the additional endpoint of hospitalization due to unstable angina). This analysis is considered to be exploratory in nature as it was proposed after observing results from the primary endpoint analysis. There were 111 events in the BMS Analysis Population due to these 3 components of the primary composite endpoint, 62 in the dapagliflozin group and 49 in the comparator group. The meta-analysis results for the MACE composite endpoint in the BMS Analysis population is presented in Table 8. Again, the estimate for the stratified hazard ratio shows that there is a lower risk of the occurrence of an event from the MACE composite endpoint in the dapagliflozin group compared to the comparator group.

\section{Table 8: Meta-Analysis Results for MACE Composite Endpoint (BMS Analysis} Population)

\begin{tabular}{|c|c|c|}
\hline & Dapagliflozin & $\begin{array}{l}\text { All } \\
\text { Comparator }\end{array}$ \\
\hline $\begin{array}{l}\text { Stratified Hazard } \\
\text { Ratio } \\
(\mathbf{9 8 \%} \text { CI) }\end{array}$ & \multicolumn{2}{|c|}{$\begin{array}{c}0.79 \\
(0.54,1.17)\end{array}$} \\
\hline $\begin{array}{l}\text { M-H Incidence Rate } \\
\text { Ratio } \\
(98 \% \text { CI) }\end{array}$ & \multicolumn{2}{|c|}{$\begin{array}{c}0.79 \\
(0.55,1.15)\end{array}$} \\
\hline $\begin{array}{l}\text { Incidence of Events } \\
\text { events/person-years } \\
(\%)\end{array}$ & $\begin{array}{l}62 / 5749 \\
(1.07 \%)\end{array}$ & $\begin{array}{l}49 / 3100 \\
(1.58 \%)\end{array}$ \\
\hline $\begin{array}{l}\text { M-H Incidence Rate } \\
\text { Difference } \\
\text { (98\% CI) }\end{array}$ & \multicolumn{2}{|c|}{$\begin{array}{c}-0.0032 \\
(-0.0086,0.0022)\end{array}$} \\
\hline
\end{tabular}

Source: Created by reviewer; reproduction of sponsor analysis in "Supplemental Cardiovascular events metaanalysis report". Dataset: adcv2v2.xpt 
NDA 202-293 (FORXIGA)

\subsubsection{Risk Difference}

To determine if using the number of subjects for each treatment as a denominator instead of the number of person-years of exposure, has an impact on the results of the analysis, the risk difference was calculated using fixed effects methods. Table 9 presents the MantelHaenszel risk difference as calculated in the BMS Analysis Population. The results from this analysis are consistent with what was found in calculations of the incidence rate difference.

Table 9: Meta-Analysis Risk Difference Results of the Primary Endpoint (BMS Analysis Population)

\begin{tabular}{|l|c|c|}
\hline & Dapagliflozin & $\begin{array}{l}\text { All } \\
\text { Comparator }\end{array}$ \\
\hline $\begin{array}{l}\text { Event Rates } \\
\text { events/ subjects } \\
\text { (N) }\end{array}$ & $\begin{array}{l}82 / 5498 \\
(1.49 \%)\end{array}$ & $\begin{array}{l}63 / 3184 \\
(1.98 \%)\end{array}$ \\
\hline $\begin{array}{l}\text { M-H Risk } \\
\begin{array}{l}\text { Difference } \\
\text { (95\% CI) }\end{array}\end{array}$ & $(-0.0088,0.0032)$ \\
\hline
\end{tabular}

Source: Created by reviewer. Dataset: adcv2v2.xpt 
Figure 6 presents the forest plot of the risk difference for the primary composite endpoint for all the trials included in the BMS Analysis population. As in the Figure 3, trials that include updated data since the previous meta-analysis are denoted in red, newly added trials are in blue, and trials that were complete at the time of the previous meta-analysis are in black. Confidence intervals are calculated asymptotically. As seen in the previous metaanalysis, the only trial where there is a statistically significant difference (at the unadjusted, nominal $\alpha=0.05$ significance level) between the comparator and the dapagliflozin groups is in trial MB102013 where the dapagliflozin group has a higher risk of a CV event compared to the comparator group. There were no notable differences in this trial population or trial design that could be found to be account for this difference.

\section{Figure 6: Forest Plot of Risk Differences by Trial for Primary CV Composite Endpoint (BMS Analysis Population)}

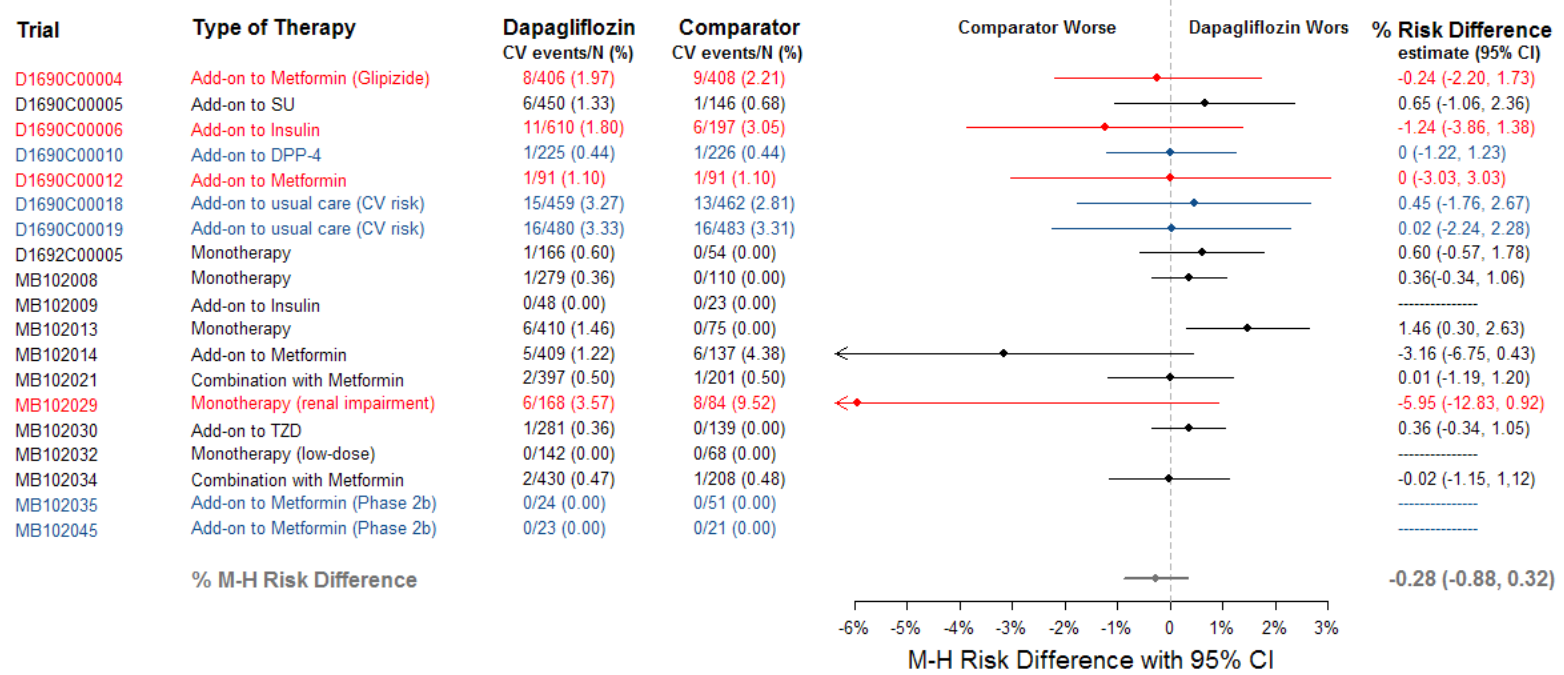

Legend: red = trials with new data; blue = newly added trials; black = same data as in previous meta-analysis Source: Created by reviewer. Dataset: adcv2v2.xpt

\subsubsection{Evaluation of Trial Heterogeneity}

To assess whether heterogeneity was present in the trials included in the evaluation of the primary endpoint, the Q-statistic and $\mathrm{I}^{2}$ were evaluated using the BMS Analysis population. The Q-statistic had a value of 26.45 with an associated p-value of 0.089 , and $\mathrm{I}^{2}=32 \%$. Therefore we could conclude that while there was some heterogeneity between trials with regards to the rate of occurrence of the primary endpoint, the heterogeneity was not significant at an $\alpha$-level of 0.05 , and the fixed effects model used by the sponsor and reported here may be appropriate. 


\subsubsection{Random Effects Analysis}

While the assessment of heterogeneity did not show that there was significant heterogeneity present between the studies included in this meta-analysis, random effects analyses were conducted as the high-risk cardiovascular trials (D1690C00018 and D1690C00019) were from a different patient population and were thought to add increased variability to the analysis. Random effects analysis was conducted using the Hardy and Thompson method. Table 10 presents the results from this analysis and compares it to the results from the fixed effects analysis. As can be observed in the table, the estimate for the incidence risk ratio using the random effects method was found to be somewhat lower compared to that found in the fixed effects analysis; however the confidence interval for this estimate is wider than what was found using fixed effects. Both point estimates are less than 1 and the upper bound of the 95\% confidence intervals are below 1.8 .

Table 10: Comparison of Fixed and Random Effects and Fixed Effects Analysis of Incident Rate Ratio for Primary Composite Endpoint

\begin{tabular}{|l|c|c|}
\hline & \multicolumn{2}{|c|}{ Primary Composite Endpoint } \\
\hline Method & $\begin{array}{c}\text { Mantel - Haenszel } \\
\text { Fixed Effects }\end{array}$ & $\begin{array}{c}\text { Hardy-Thompson } \\
\text { Random Effects }\end{array}$ \\
\hline Incident Rate & 0.82 & 0.75 \\
Ratio & $(0.59,1.14)$ & $(0.47,1.18)$ \\
(95\% CI) & & \\
\hline
\end{tabular}

Source: Created by reviewer. Dataset: adcv2v2.xpt

\section{Findings in Special/Subgroup Populations}

Statistician's note: As all further analyses are exploratory in nature, all estimates will be reported with 95\% confidence intervals (corresponding to an unadjusted, nominal $\alpha=0.05$ significance level). Because of the small number of events which are likely to occur in subgroups, caution is advised when interpreting results of these subgroup analyses. Therefore, these findings should be considered exploratory until confirmation from an independent trial is obtained.

\subsection{Gender, Race and Age}

Evaluations for gender, race and age are presented in the paragraphs that follow. A forest plot combining all results is presented in Figure 7. All incidence rate differences (IRD) were calculated using the Mantel-Haenszel (M-H) method, stratifying by trial. 


\section{Gender}

Among the 8,682 subjects in the BMS Analysis Population, 4,793 (55.2\%) were male subjects and 3,889 (44.8\%) were female subjects. Among the 145 subjects with primary composite endpoint events, 95 subjects were male and 50 were female subjects. The $\mathrm{M}-\mathrm{H}$ incidence rate difference of developing a $\mathrm{CV}$ related event as measured by the primary composite endpoint was lower in the dapagliflozin group for both male and female subjects (IRD $-0.52,95 \% \mathrm{CI}(-1.20,0.17)$ for males and IRD $-0.42,95 \% \mathrm{CI}(-1.26,0.42)$ for females).

\section{Race}

Among the 8,682 subjects in the BMS Analysis Population, 7,146 (82.3\%) were White subjects, 333 (3.8\%) were Black subjects and 689 (7.9\%) were Asian subjects. Among the 145 subjects reported with events of the primary composite endpoint, 126 were White subjects, 5 were Black subjects and 9 were Asian subjects.

For both the White and Black populations, the incidence rate difference of developing a $\mathrm{CV}$-related event as measured by the primary composite endpoint was lower in the dapagliflozin group. Among the White subjects, the IRD was $-0.52,95 \%$ CI $(-1.2,0.17)$. For Black subjects the IRD was $-1.48,95 \% \mathrm{CI}(-4.41,1.45)$. Among Asian subjects there was a statistically significant increased risk of developing a CV event in the dapagliflozin group (IRD: $1.99,95 \% \mathrm{CI}(0.03,3.95))$.

\section{Age}

Among the 8,682 subjects in the BMS Analysis Population, 2,027 (23.3\%) were aged 50 years or younger, 4,376 (50.4\%) were between 51 and 64 years old, 1,967 $(22.7 \%)$ were between age 65 and 74 years old, and $312(3.6 \%)$ were age 75 or older.

In the younger age categories (age $<65$ ), there was a slightly lower risk of experiencing the primary composite endpoint in the dapagliflozin group (age $\leq 50$ IRD: $-0.50,95 \%$ CI ($1.22,0.22)$; age $51-64$ IRD: $-0.45,95 \% \mathrm{CI}(-1.31,0.41)$. In the older age groups the incidence rate difference was higher in the dapagliflozin group, but in both groups this difference was associated with a wide confidence interval (age 65-74 IRD: 0.15, 95\%CI (1.37, 1.66); age $\geq 75$ IRD: 0.24 95\% CI: $(-4.53,5.00))$. 
Figure 7: Forest Plot of Incidence Rate Difference of Primary Composite Endpoint by Baseline Demographics (BMS Analysis Population)

$\begin{array}{lll}\text { Subgroups } & \begin{array}{c}\text { Dapagliflozin } \\ \text { events/PYs } \\ 82 / 5738\end{array} & \begin{array}{c}\text { Comparator } \\ \text { events/PYs } \\ 63 / 3095\end{array} \\ \begin{array}{l}\text { Overall } \\ \text { Male }\end{array} & 55 / 3082 & 40 / 1769 \\ \text { Female } & 27 / 2656 & 23 / 1326 \\ \text { Race } & & \\ \text { White } & 69 / 4919 & 57 / 2642 \\ \text { Black } & 2 / 194 & 3 / 109 \\ \text { Asian } & 8 / 376 & 1 / 206 \\ \text { Age } & 2 / 1323 & \\ <=50 & 40 / 3038 & 4 / 594 \\ 51-64 & 32 / 1193 & 32 / 1608 \\ 65-74 & 8 / 184 & 22 / 789 \\ >=75 & & 5 / 103\end{array}$

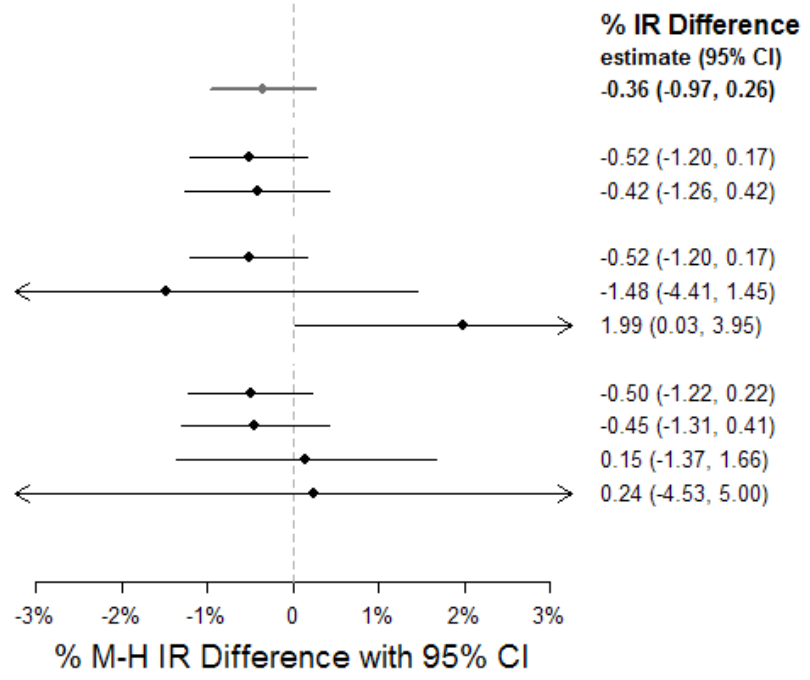

Source: Created by reviewer. Dataset: adcv2v2.xpt

\subsection{Other Special/Subgroup Populations}

In addition to the demographic factors evaluated in the previous section, evaluation of differences in baseline BMI, specific cardiovascular risk factors, baseline renal impairment status and history of diabetes were considered.

To further investigate the sensitivity of the data, two different sets of subgroup analyses, as suggested by the clinical team were conducted. In the first subgroup analysis, results are presented for trials that used dapagliflozin as an add-on therapy and for trials that used dapagliflozin as a monotherapy. In the second subgroup analysis, results are presented for the $10 \mathrm{mg}$ dose alone, the proposed therapeutic dose.

Additionally, as the populations in the high CV risk trials, D1690C00018 and D1690C00019, differed greatly from the other study populations included in the metaanalysis, these two trials were pooled and results were evaluated with regards to the occurrence of the primary composite endpoint. 
NDA 202-293 (FORXIGA)

\subsubsection{Baseline BMI, CV Risk Factors, Renal Impairment and Diabetes History}

To determine if the health history of the subjects had any impact on the relative risk of developing a CV event as determined by the primary composite endpoint, the baseline BMI $(<30, \geq 30)$, history of cardiovascular disease, history of hypertension, history of dyslipidemia, baseline eGFR and duration of diabetes, were evaluated. Based on the M-H incidence rate difference in the BMS Analysis Population of the incidence of the primary composite endpoint, it was found that almost all groups in these analyses had a lower risk of CV events in the dapagliflozin group. The results for these analyses are presented in Figure 8 .

\section{Figure 8: Forest Plot of Incidence Rate Difference of Primary Composite Endpoint by Baseline Risk Factors (BMS Analysis Population)}

$\begin{array}{lcc}\text { Risk Factors } & \begin{array}{c}\text { Dapagliflozin } \\ \text { events/PY } \\ 82 / 5738\end{array} & \begin{array}{c}\text { Comparator } \\ \text { events/PY } \\ 63 / 3095\end{array} \\ \begin{array}{l}\text { Overall } \\ \text { BMI }\end{array} & 38 / 2166 & 20 / 1228 \\ <30 & 44 / 3572 & 43 / 1867 \\ \quad>=30 & 56 / 1822 & 44 / 1171 \\ \text { Hx of CVD } & 26 / 3916 & 19 / 1923 \\ \text { Yes } & & \\ \quad \text { No } & 74 / 4018 & 55 / 2321 \\ \text { Hx of Hypertension } & 8 / 1720 & 8 / 774 \\ \text { Yes } & & \\ \text { No } & 57 / 3375 & 46 / 1898 \\ \text { Hx of Dyslipidemia } & 25 / 2363 & 17 / 1196 \\ \text { Yes } & 24 / 744 & 20 / 363 \\ \text { No } & 37 / 3033 & 32 / 1641 \\ \text { Baseline eGFR } & 21 / 1951 & 11 / 1085 \\ 30-<60 & 16 / 1913 & 8 / 931 \\ 60-<90 & 28 / 2031 & 16 / 1180 \\ >=90 & 38 / 1794 & 39 / 983\end{array}$

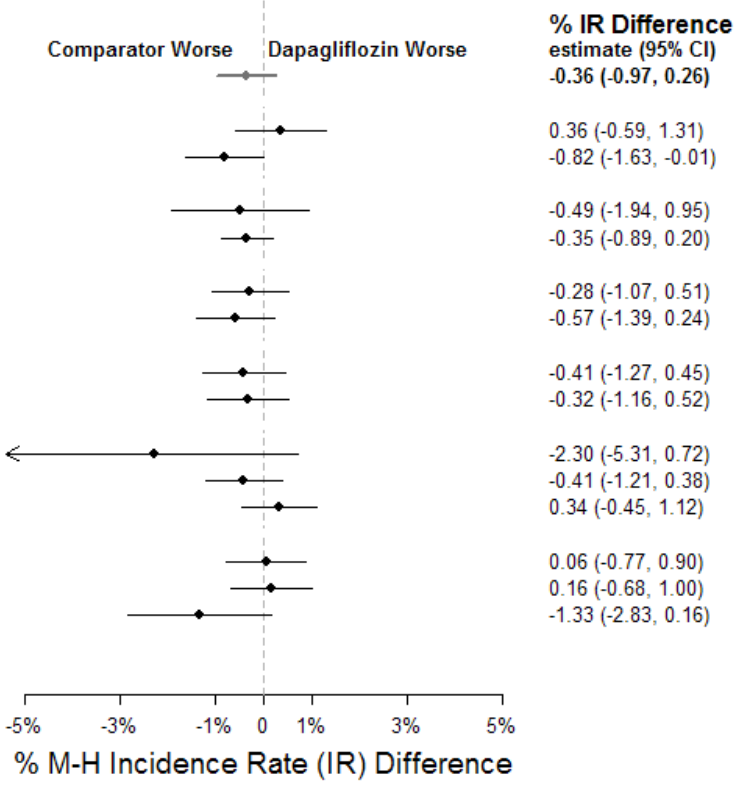

Source: Created by reviewer. Dataset: adcv2v2.xpt 


\subsubsection{Monotherapy and Add-On Trials}

The monotherapy trials from the BMS Analysis Populations (trials D1692C00005, MB102013, MB102029, and MB102032) and the add-on trials from this population (trials D1690C00004, D1690C00005, D1690C00006, D1690C00010, D1690C00012, D1690C00018, D1690C00019, MB102009, MB102014, MB102030, MB102035, and MB102045) were grouped together to observe any difference in the incidence rates of the primary composite endpoint based on trial type. Table 11 presents the results from the meta-analysis of these two subgroups. The hazard rate for the occurrence of the primary endpoint was lower in the dapagliflozin group in both the monotherapy and add-on trials, while the overall lowest incidence of the primary endpoint was in the dapagliflozin group in the monotherapy trials.

Statistician's note: The higher hazard ratio observed in the add-on trials in this subgroup analysis is due to the inclusion of the two high CV-risk trials (D1690C00018 and D1690C00019) in the add-on trials group.

Table 11: Meta-Analysis Results of the Primary Composite Endpoint for the Monotherapy Trials and Add-On Therapy Trials (BMS Analysis Population)

\begin{tabular}{|c|c|c|c|c|}
\hline & \multicolumn{2}{|c|}{ Monotherapy } & \multicolumn{2}{|c|}{ Add-on } \\
\hline & Dapagliflozin & $\begin{array}{l}\text { All } \\
\text { Comparator }\end{array}$ & Dapagliflozin & $\begin{array}{c}\text { All } \\
\text { Comparator }\end{array}$ \\
\hline $\begin{array}{l}\text { Stratified } \\
\text { Hazard Ratio } \\
\text { (95\% CI) }\end{array}$ & \multicolumn{2}{|c|}{$\begin{array}{c}0.60 \\
(0.25,1.44)\end{array}$} & \multicolumn{2}{|c|}{$\begin{array}{c}0.86 \\
(0.58,1.25)\end{array}$} \\
\hline $\begin{array}{l}\text { Event/PY } \\
\text { (\% Incidence) }\end{array}$ & $\begin{array}{l}14 / 1125 \\
(1.24 \%)\end{array}$ & $\begin{array}{c}8 / 311 \\
(2.57 \%)\end{array}$ & $\begin{array}{l}64 / 4200 \\
(1.52 \%)\end{array}$ & $\begin{array}{l}53 / 2579 \\
(2.06 \%)\end{array}$ \\
\hline $\begin{array}{l}\text { M-H Incidence } \\
\text { Rate Difference } \\
\text { (95\% CI) }\end{array}$ & \multicolumn{2}{|c|}{$\begin{array}{c}-0.0099 \\
(-0.027,0.0075)\end{array}$} & \multicolumn{2}{|c|}{$\begin{array}{c}-0.0029 \\
(-0.010,0.0042)\end{array}$} \\
\hline
\end{tabular}

Source: Created by reviewer. Dataset: adcv2v2.xpt 


\subsection{3 $10 \mathrm{mg}$ Dosage of Dapagliflozin}

To investigate the impact of the proposed therapeutic dose on the outcome of cardiovascular events, the subjects taking the $10 \mathrm{mg}$ dosage of dapagliflozin in the BMS Analysis population (excluding trials MB102021, MB102032 and MB102045 which did not include $10 \mathrm{mg}$ dosage groups of dapagliflozin) were isolated in the analysis and compared to the combined comparator group. Table 12 presents the meta-analysis results from the isolation of the $10 \mathrm{mg}$ dosage compared to the pooled comparator groups.

Table 12: Meta-Analysis Results for 10mg Dose of Dapagliflozin Alone and the Pooled Comparator (BMS Analysis Population excluding MB102021, MB102032 and MB102045)

\begin{tabular}{|l|l|l|}
\hline & $\begin{array}{l}\text { Dapagliflozin } \\
\text { 10 mg dose } \\
\text { alone }\end{array}$ & $\begin{array}{l}\text { All } \\
\text { Comparator }\end{array}$ \\
\hline $\begin{array}{l}\text { Hazard Ratio } \\
\text { (95\% CI) }\end{array}$ & \multicolumn{2}{|c|}{0.85} \\
\hline $\begin{array}{l}\text { Event/PY } \\
\text { (\% Incidence) }\end{array}$ & $55 / 3274,1.22)$ \\
\hline $\begin{array}{l}\text { M-H Incidence Rate } \\
\text { Difference } \\
\text { (95\% CI) }\end{array}$ & \multicolumn{2}{|c|}{-0.0032} \\
\hline
\end{tabular}

Source: Created by reviewer. Dataset: adcv2v2.xpt

Based on the Kaplan-Meier method using the BMS Analysis population (excluding MB102021, MB102032 and MB102045), the cumulative probability of developing a CVrelated event as measured by the primary composite endpoint is shown in Figure 9. 
NDA 202-293 (FORXIGA)

Figure 9: Time-to-event Analysis of the Primary Composite Endpoint for the 10 mg Dose of Dapagliflozin Compared to Pooled Comparator (BMS Analysis Population excluding MB102021, MB102032 and MB102045)

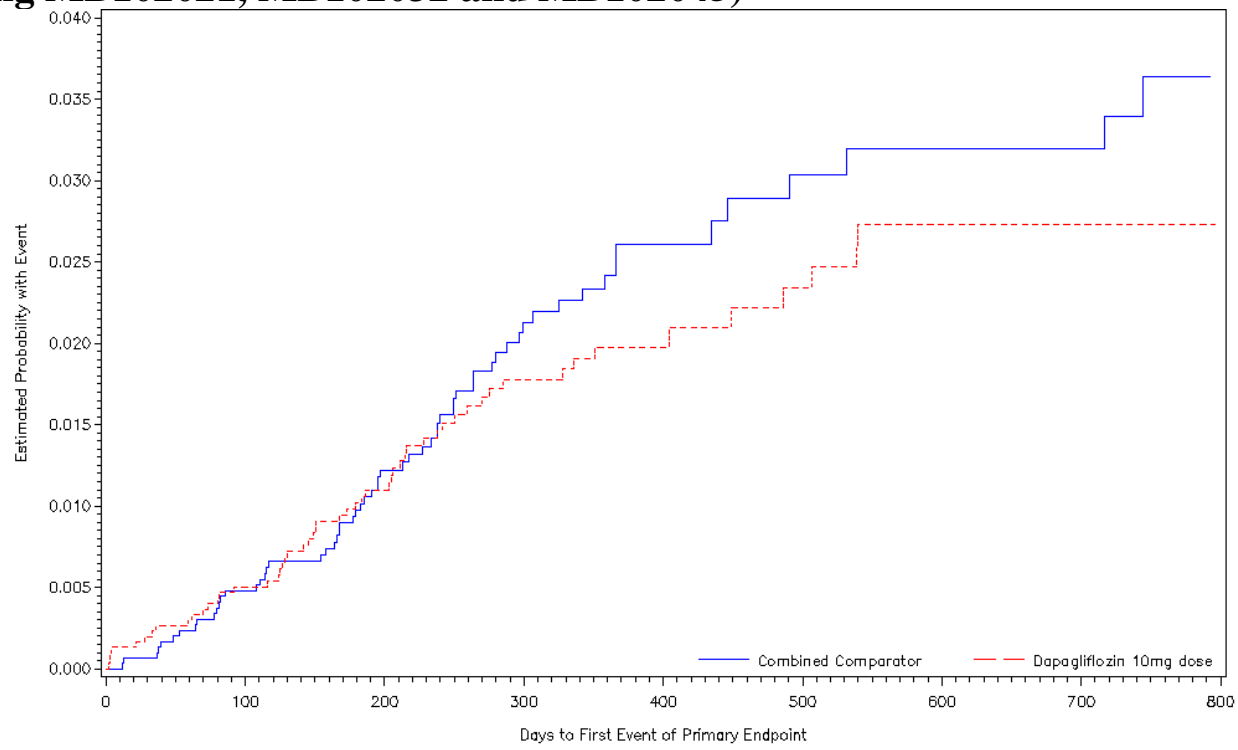

Source: Created by reviewer. Dataset: adcv2v2.xpt 


\subsubsection{High-Risk CV Trials: D1690C00018 and D1690C00019 In Relation to All Other Trials in Meta-analysis}

As trials D1690C00018 and D1690C00019 were conducted in populations that had a history of CV disease, the subjects in these trials tended to differ considerably from those in the rest of the trials included in the meta-analysis. Table 13 presents the demographics for all the trials in the analysis excluding the high CV risk trials alongside the pooled demographics of the two high CV risk trials (D1690C00018 and D1690C00019). The pooled high CV risk trials were composed of 1884 subjects, which had a higher percentage of males, were older, more obese and had a higher percentage of Whites, and a higher percentage of Europeans, compared to all the other trials included in the meta-analysis.

Table 13: Demographics of All Trials Excluding High-Risk CV Trials and Pooled High -Risk CV Trials (D1690C00018 and D1690C00019)

\begin{tabular}{|c|c|c|}
\hline & $\begin{array}{c}\text { All Trials } \\
\text { Excluding High- } \\
\text { Risk CV Trials } \\
\quad(N=6798)\end{array}$ & $\begin{array}{c}\text { High-Risk CV } \\
\text { Trials } \\
\text { (D1690C00018 and } \\
\text { D1690C0001) } \\
\text { combined } \\
(N=1884)\end{array}$ \\
\hline Percent Female & $48.19 \%$ & $32.54 \%$ \\
\hline Age+ SD (years) & $60.0 . \pm 10.5$ & $63.4+7.3$ \\
\hline$\leq 50$ years & $28.9 \%$ & $3.2 \%$ \\
\hline $50-65$ years & $50.0 \%$ & $52.0 \%$ \\
\hline$\geq 65$ years & $21.1 \%$ & $44.8 \%$ \\
\hline BMI+ SD $\left(\mathrm{kg} / \mathrm{m}^{2}\right)$ & $31.6 \pm 5.6$ & $32.8+5.7$ \\
\hline $25-30$ & $31.1 \%$ & $27.2 \%$ \\
\hline$\geq 30$ & $58.4 \%$ & $67.1 \%$ \\
\hline \multicolumn{3}{|l|}{ Race and Ethnicity } \\
\hline White & $80.4 \%$ & $89.1 \%$ \\
\hline Black & $3.8 \%$ & $3.8 \%$ \\
\hline Asian & $8.7 \%$ & $5.3 \%$ \\
\hline \multicolumn{3}{|l|}{ Region } \\
\hline North America & $31.1 \%$ & $33.0 \%$ \\
\hline Latin America & $22.3 \%$ & $10.2 \%$ \\
\hline Europe & $36.6 \%$ & $49.2 \%$ \\
\hline Asia/Pacific & $10.0 \%$ & $7.6 \%$ \\
\hline
\end{tabular}

Source: Created by reviewer. Dataset: adcv2v2.xpt 
Table 14 presents the cardiovascular risk factors for all the trials in the analysis excluding the high CV risk trials alongside those of the two high CV risk trials (D1690C00018 and D1690C00019). As expected, the pooled CV risk factors for D1690C00018 and D1690C00019 are much more common compared to those in all the other trials included in the meta-analysis.

Table 14: CV Risk Factors of All Trials Excluding High-Risk CV Trials and Pooled High-Risk CV Trials (D1690C00018 and D1690C00019)

\begin{tabular}{|c|c|c|}
\hline & $\begin{array}{c}\text { All Trials } \\
\text { Excluding High- } \\
\text { Risk CV Trials } \\
\\
(N=6798)\end{array}$ & $\begin{array}{c}\text { High-Risk CV } \\
\text { Trials } \\
\text { (D1690C00018 and } \\
\text { D1690C0001) } \\
\text { combined } \\
(N=1884)\end{array}$ \\
\hline History of CVD & $19.1 \%$ & $99.5 \%$ \\
\hline $\begin{array}{l}\text { History of } \\
\text { Hypertension }\end{array}$ & $62.7 \%$ & $96.2 \%$ \\
\hline $\begin{array}{l}\text { History of } \\
\text { Dyslipidemia }\end{array}$ & $49.9 \%$ & $84.2 \%$ \\
\hline $\begin{array}{l}\text { History of Congestive } \\
\text { Heart Failure }\end{array}$ & $1.9 \%$ & $14.3 \%$ \\
\hline \multicolumn{3}{|l|}{ Renal Function } \\
\hline Severely Impaired & $0.2 \%$ & $0.1 \%$ \\
\hline Moderately Impaired & $10.5 \%$ & $17.3 \%$ \\
\hline Mildly Impaired & $50.8 \%$ & $59.3 \%$ \\
\hline Normal & $38.5 \%$ & $23.3 \%$ \\
\hline \multicolumn{3}{|l|}{ Smoking History } \\
\hline Never smoked & $59.4 \%$ & $40.3 \%$ \\
\hline Current Smoker & $16.4 \%$ & $14.9 \%$ \\
\hline Former Smoker & $24.2 \%$ & $44.8 \%$ \\
\hline \multicolumn{3}{|l|}{ Diabetes Duration } \\
\hline less than 3 years & $44.0 \%$ & $8.4 \%$ \\
\hline $3-10$ years & $34.6 \%$ & $34.8 \%$ \\
\hline more than 10 years & $21.4 \%$ & $56.8 \%$ \\
\hline
\end{tabular}

Source: Created by reviewer. Dataset: adcv2v2.xpt 
Due to the large number of differences in the population characteristics between these two trials and the other trials in the meta-analysis, a comparison of the results of the metaanalysis of the primary composite endpoint was conducted. The results for this analysis are presented in Table 15. The incidence of CV events in the primary composite endpoint occur at about the same rate in the dapagliflozin group compared to the standard of care group in the high-risk CV trials, however, the incidence of CV events for the primary composite endpoint in the other trials in the meta-analysis occur at a lower rate in the dapagliflozin group compared to the pooled all comparator group.

Table 15: Meta-Analysis Results for All Trials Excluding High-Risk CV Trials and Pooled High-Risk CV Trials (D1690C00018 and D1690C00019)

\begin{tabular}{|c|c|c|c|c|}
\hline & \multicolumn{2}{|c|}{$\begin{array}{l}\text { All Trials Excluding High- } \\
\text { Risk CV Trials }\end{array}$} & \multicolumn{2}{|c|}{$\begin{array}{l}\text { High-Risk CV Trials } \\
\text { (D1690C00018 and } \\
\text { D1690C0001) combined }\end{array}$} \\
\hline & Dapagliflozin & $\begin{array}{l}\text { All } \\
\text { Comparator }\end{array}$ & Dapagliflozin & $\begin{array}{l}\text { Standard of } \\
\text { Care }\end{array}$ \\
\hline $\begin{array}{l}\text { Stratified } \\
\text { Hazard Ratio } \\
(95 \% \text { CI })\end{array}$ & \multicolumn{2}{|c|}{$\begin{array}{c}0.66 \\
(0.42,1.04)\end{array}$} & \multicolumn{2}{|c|}{$\begin{array}{c}1.07 \\
(0.64,1.77)\end{array}$} \\
\hline $\begin{array}{l}\text { Event/PY } \\
\text { (\% Incidence) }\end{array}$ & $\begin{array}{l}51 / 5032 \\
(1.01 \%)\end{array}$ & $\begin{array}{l}34 / 2389 \\
(1.42 \%)\end{array}$ & $\begin{array}{c}31 / 706 \\
(4.39 \%)\end{array}$ & $\begin{array}{c}29 / 706 \\
(4.11 \%)\end{array}$ \\
\hline $\begin{array}{l}\text { M-H } \\
\text { Incidence Rate } \\
\text { Difference } \\
(95 \% \text { CI })\end{array}$ & \multicolumn{2}{|c|}{$\begin{array}{c}-0.0050 \\
(-0.011,0.0007)\end{array}$} & \multicolumn{2}{|c|}{$\begin{array}{c}0.0028 \\
(-0.018,0.024)\end{array}$} \\
\hline
\end{tabular}

Source: Created by reviewer. Dataset: adcv2v2.xpt

\section{Summary and Conclusions}

\subsection{Statistical Issues and Collective Evidence}

The primary agreed upon safety endpoint was a composite endpoint consisting of CV death, non-fatal myocardial infarction (MI), non-fatal stroke, and hospitalization due to unstable angina.

The FDA Diabetes Guidance (2008) ${ }^{1}$ states that "sponsors should compare the incidence of important cardiovascular events occurring with the investigational agent to [that of] the control group to show that the upper bound of the two-sided 95 percent confidence interval for the estimated risk ratio is less than 1.8."

In the statistical analysis plan (SAP) agreed upon with the FDA on November 9, 2010, a group sequential testing strategy was pre-specified to control the overall Type I error rate for demonstration of non-inferiority of dapagliflozin to comparator with respect to the incidence of cardiovascular events based on the 1.8 relative risk criterion, as estimated by 
the hazard ratio. Using a group sequential testing strategy, if the first formal meta-analysis of the primary composite endpoint was found to have an upper-bound less than 1.8 at a one-sided alpha level of 0.01 (corresponding to a $98 \% \mathrm{CI}$ ) then the meta-analysis would meet the FDA CV guidelines ${ }^{1}$, and data from additional studies that have yet to be completed would not need to be included in a second meta-analysis. However, if unsuccessful at this alpha level (0.01), a second meta-analysis would be performed when studies D1690C00018 and D1690C00019 had unblinded and at least 110 patients had adjudicated events for the primary endpoint. The one-sided alpha level for the first metaanalysis was set at 0.01 so that the overall Type I error rate across the 2 analyses was $\leq 0.025$ (one-sided). As the first meta-analysis met the stated guidelines (i.e. the upper bound of the relative risk criterion, estimated by the hazard ratio, for the incidence of $\mathrm{CV}$ events, as measured by the primary composite endpoint, was lower than 1.8), this metaanalysis, which includes all data expected, was set at the full one-sided alpha-level of 0.025 . Additionally, this meta-analysis is meant to supersede the previous review signed on September 7, 2011.

Phase $2 \mathrm{~b}$ and Phase 3 data from the 19 randomized studies were integrated to evaluate the risk of developing a CV related event as measured by the primary composite endpoint. The data for this second meta-analysis included all the data from the previous meta-analysis, the updated data from 4 studies that were not complete at the time of the previous metaanalysis (contributing an additional 5 events to the primary composite endpoint and an additional 804 person-years since the previous analysis), and 5 additional studies (an additional 2455 subjects, contributing 62 events to the primary composite endpoint in 1836 person-years), that were not included in the previous meta-analysis. The pre-specified primary analysis conducted was a comparison of the time to composite event between the dapagliflozin group and the pooled all comparator group performed using a Cox proportional hazards model with study as stratification factor. The Mantel-Haenszel (MH) methods for estimating the overall incidence rate ratio via Breslow and Day $(1994)^{2}$, where the estimate was calculated by stratifying for trial, was performed as a pre-specified secondary analysis. The incidence rate difference was also evaluated to include trials with zero events. To further investigate event rates, the risk difference was calculated to determine if a different denominator (number of subjects per group as opposed to number of person-years of exposure) would affect the estimates. Additionally, random effects methods were employed to determine if the estimate of the incidence rate ratio would be impacted by the heterogeneity of the studies.

Among the 8682 subjects in the BMS Analysis population, 145 subjects had an event that was counted in the primary composite endpoint (82 of 5498 dapagliflozin subjects and 63 of 3184 comparator subjects). The primary analysis of the stratified Cox proportional hazards model found the hazard ratio of dapagliflozin versus comparator to be $0.82(95 \%$ CI: $0.59,1.38)$. To incorporate zero event trials in the meta-analysis, the incidence rate difference was considered as a secondary analysis method. Using this method, the M-H incidence rate difference was found to be -0.0036 (95\% CI: $-0.0097,0.0026)$.

Random effects analysis was performed as two of the trials (D1690C00018 and D1690C00019) were from a higher cardiovascular risk patient population than the other 
trials in the meta-analysis. The results of this analysis provided a slightly lower estimate of the incident rate ratio for the primary composite endpoint with a wider confidence interval: 0.75 ( $95 \%$ CI: $0.47,1.18)$. With the variability of these trials accounted for in the random effects analysis, the addition of these trials did not change the overall conclusion of the meta-analysis.

The secondary composite endpoint was also evaluated which was comprised of the same $\mathrm{CV}$ endpoints as the primary endpoint and two additional $\mathrm{CV}$ endpoints (unplanned coronary revascularization, and hospitalization for heart failure). This endpoint also found no additional risk of $\mathrm{CV}$ events in the dapagliflozin group compared to the comparator group [hazard ratio $(95 \% \mathrm{CI}): 0.73(0.54,0.99)]$.

For the updated meta-analysis, the sponsor chose to evaluate a third composite endpoint which considers only the three endpoints that compose the strict major adverse cardiovascular events (MACE): CV death, myocardial infarction (MI), and stroke. This endpoint also found no additional risk of $\mathrm{CV}$ events in the dapagliflozin group compared to the comparator group [hazard ratio $(95 \% \mathrm{CI}): 0.79(0.54,1.17)]$.

Additional subgroup analyses included evaluating the difference between dapagliflozin and comparator event rates based on CV risk factors, demographics, and monotherapy compared to add-on studies. All results from the subgroup analyses were consistent with the results of the primary analysis.

\subsection{Conclusions and Recommendations}

The sponsor conducted a meta-analysis of the cardiovascular events that occurred in 19 Phase $2 b / 3$ trials using dapagliflozin. This meta-analysis was the second of a two-part group sequential testing strategy that was pre-specified to control the overall Type I error rate for demonstration of non-inferiority with respect to the 1.8 relative risk criterion for incidence of cardiovascular events. As the sponsor had succeeded in the first meta-analysis in demonstrating non-inferiority, this meta-analysis used the full one-sided alpha level of 0.025 .

The primary endpoint is a composite endpoint, defined as the time to first event of the following adjudicated events: CV death, myocardial infarction, stroke, and hospitalization for unstable angina. All CV events were adjudicated by an independent, blinded clinical events committee (CEC) comprised of two cardiologists and two neurologists. The comparator group was comprised of subjects taking either a placebo and/or an active control (metformin, glipizide, insulin, glimepiride, pioglitazone, sitagliptin, hydrochlorthiazide or standard of care).

Among the 8682 subjects in the Bristol-Myers Squibb (BMS) Analysis population (composed of the safety population of all 19 trials combined), 145 subjects had an event that was counted in the primary composite endpoint ( 82 of 5498 dapagliflozin subjects $(1.49 \%)$ and 63 of 3184 comparator subjects $(1.98 \%))$. The pre-specified primary analysis 
of the stratified Cox proportional hazards model yielded a hazard ratio of dapagliflozin versus comparator of 0.82 (95\% CI: $0.59,1.38)$. To incorporate zero event trials in the meta-analysis, the incidence rate difference was considered as a secondary analysis method. Using this method, the incidence rate difference was found to be -0.0036 (95\% CI: -0.0097, 0.0026). Random effects analysis was performed as two of the trials (D1690C00018 and D1690C00019) were from a higher cardiovascular risk patient population than the other trials in the meta-analysis. The results of this analysis provided a slightly lower estimate of the incident rate ratio for the primary composite endpoint with a wider confidence interval: 0.75 (95\% CI: $0.47,1.18)$. With the variability of these trials accounted for in the random effects analysis, the addition of these trials did not change the overall conclusion of the meta-analysis.

A secondary composite endpoint was also evaluated which was comprised of the same CV endpoints as the primary endpoint and two additional CV endpoints (unplanned coronary revascularization, and hospitalization for heart failure). This endpoint also found no additional risk of $\mathrm{CV}$ events in the dapagliflozin group compared to the comparator group (hazard ratio $(95 \% \mathrm{CI}): 0.73(0.54,0.99)$.

For the updated meta-analysis, the sponsor chose to evaluate a third composite endpoint post-hoc, as an exploratory analysis, which considers only the three endpoints that compose the major adverse cardiovascular events (MACE): CV death, myocardial infarction (MI), and stroke. This endpoint also found no additional risk of $\mathrm{CV}$ events in the dapagliflozin group compared to the comparator group (hazard ratio $(95 \% \mathrm{CI}): 0.79(0.54,1.17)$.

Additionally monotherapy and add-on therapy trials were evaluated separately as a subgroup analysis [hazard ratios $(95 \% \mathrm{CI})$ : monotherapy $0.60(0.25,1.44)$, add-on: 0.86 $(0.58,1.25)]$. To evaluate the proposed therapeutic dose, the $10 \mathrm{mg}$ dose group was separately evaluated and found to have a hazard ratio of 0.85 (95\% CI: $0.59,1.22)$. All subgroup findings were consistent with the primary analysis finding of no additional risk of cardiovascular events in the dapagliflozin group compared to the comparator group.

Based upon the CV risk guidelines set forth in the FDA Diabetes Guidance (2008) ${ }^{1}$, the upper-bound of the $95 \%$ confidence interval of the risk ratio for $\mathrm{CV}$ events in dapagliflozin compared to the pooled comparator, as estimated by the hazard ratio for the primary composite endpoint, meets the stated 1.8 non-inferiority margin. 
NDA 202-293 (FORXIGA)

\section{$6 \quad$ References}

1. Food and Drug Administration. Guidance for industry: Diabetes mellitus - evaluating cardiovascular risk in new antidiabetic therapies to treat type 2 diabetes. December 2008.(www.fda.gov/downloads/Drugs/GuidanceComplianceRegulatorylnformation/Guidances/ $\underline{\text { ucm071627.pdf) }}$

2. Breslow NE, Day NE (1994), Statistical Methods in Cancer Research Volume II: The Design and Analysis of Cohort Studies, IARC Scientific Publications, No. 82, New York: Oxford University Press, Inc.

3. Whitehead, Anne. A prospectively planned cumulative meta-analysis applied to a series of concurrent clinical trials. Statistics in Medicine 1997; 16: 2901-2913.

4. Robins J, Breslow N, Greenland S. Estimators of the Mantel-Haenszel variance consistent in both sparse data and large-strata limiting models. Biometrics 1986; 42: 311-23.

5. Higgins, JPT and Thompson SG. Quantifying heterogeneity in a meta-analysis. Statistics in Medicine 2002; 21: 1539-1558.

6. Hardy, RJ and Thompson, SG. A likelihood approach to meta-analysis with random effects. Statistics in Medicine. 1996; 15: 619-629. 
NDA 202-293 (FORXIGA)

\section{$7 \quad$ Appendix}

Figure 10: Kaplan Meier Curves of Discontinuation Rates for the 4 Updated Trials (D1690C00004, D1690C00006, D1690C00012, and MB102029)

Time to Discontinuation for Study D1690C00004

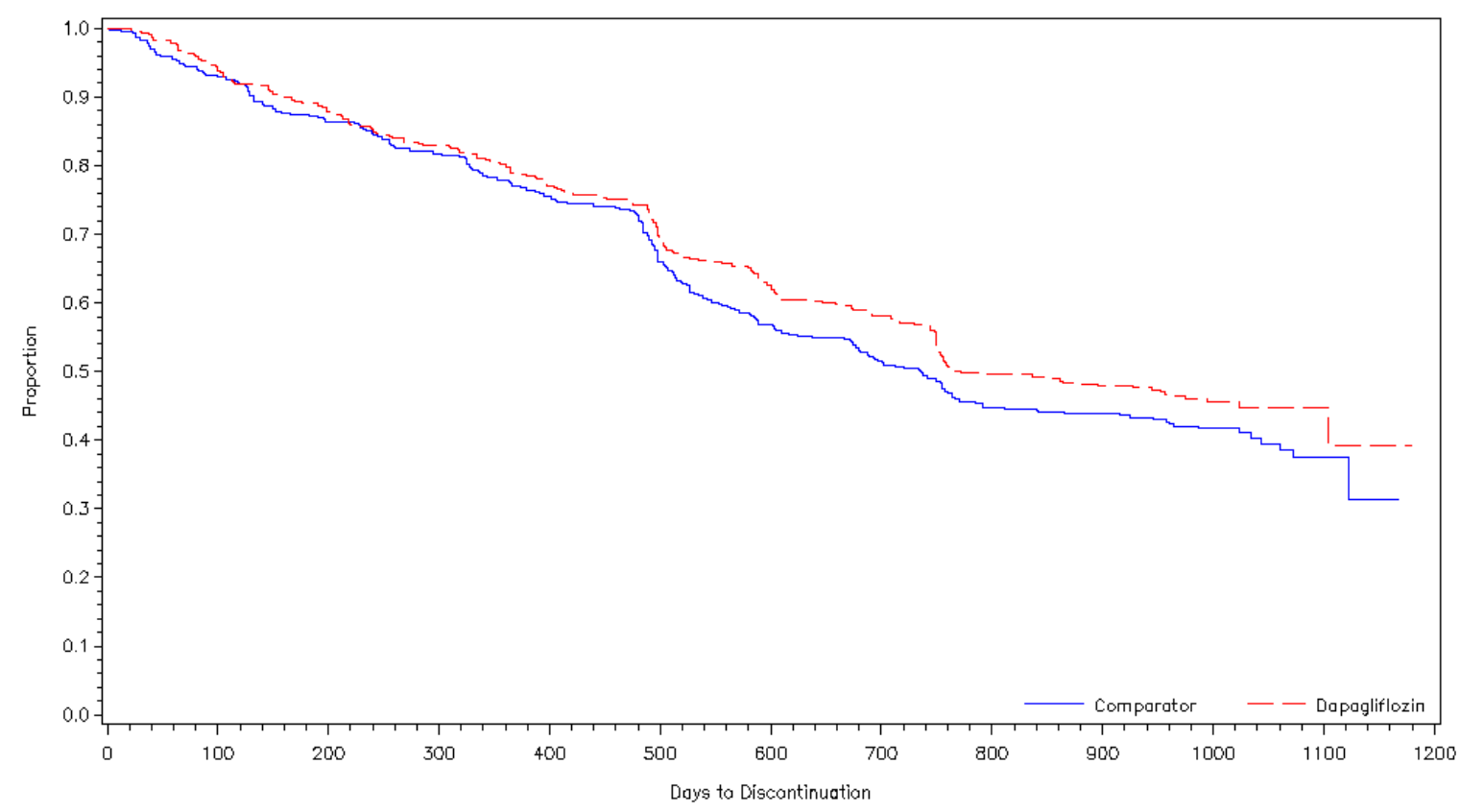

Time to Discontinuation for Study D1690C00006

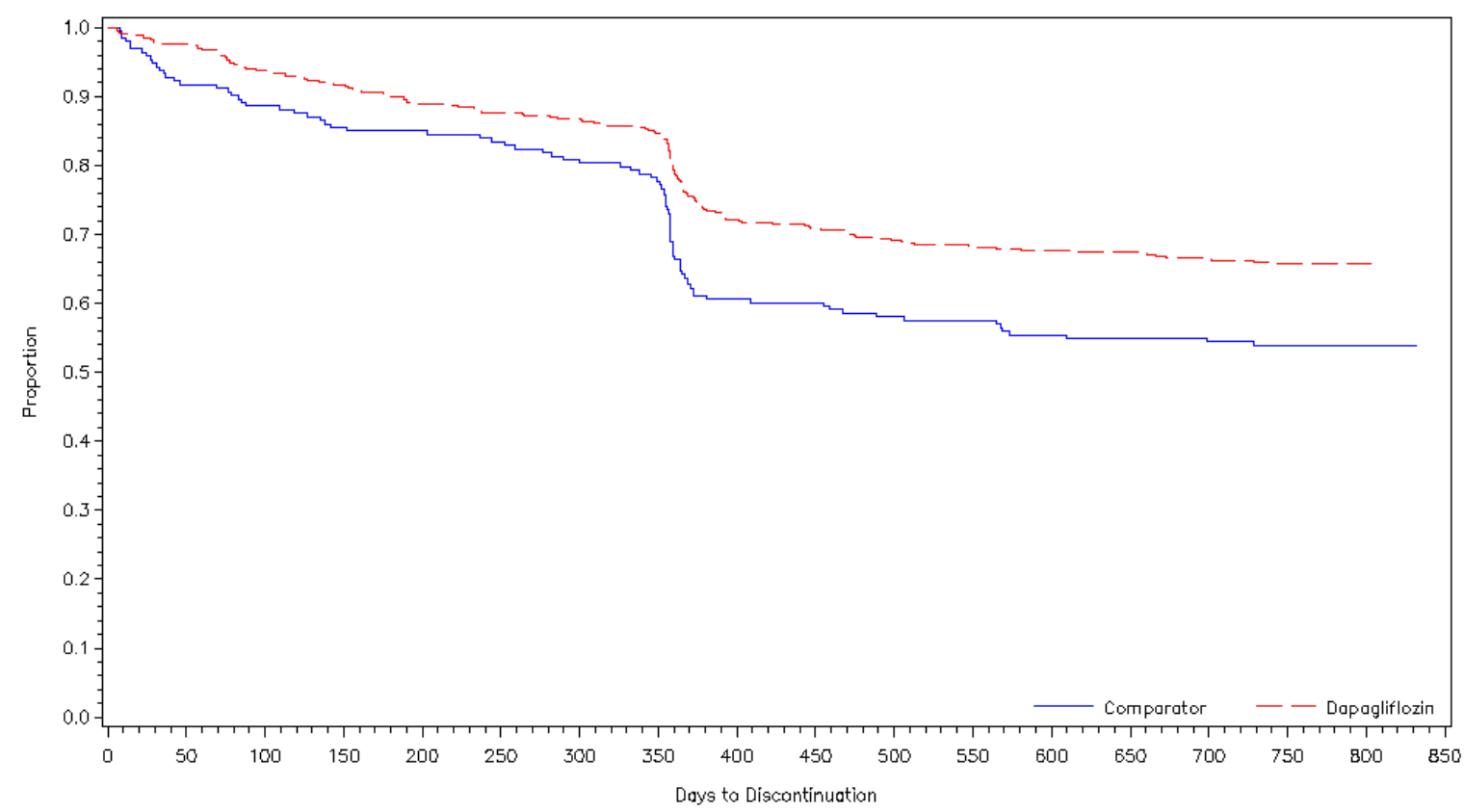


NDA 202-293 (FORXIGA)

Time to Discontinuation for Study D1690C00012

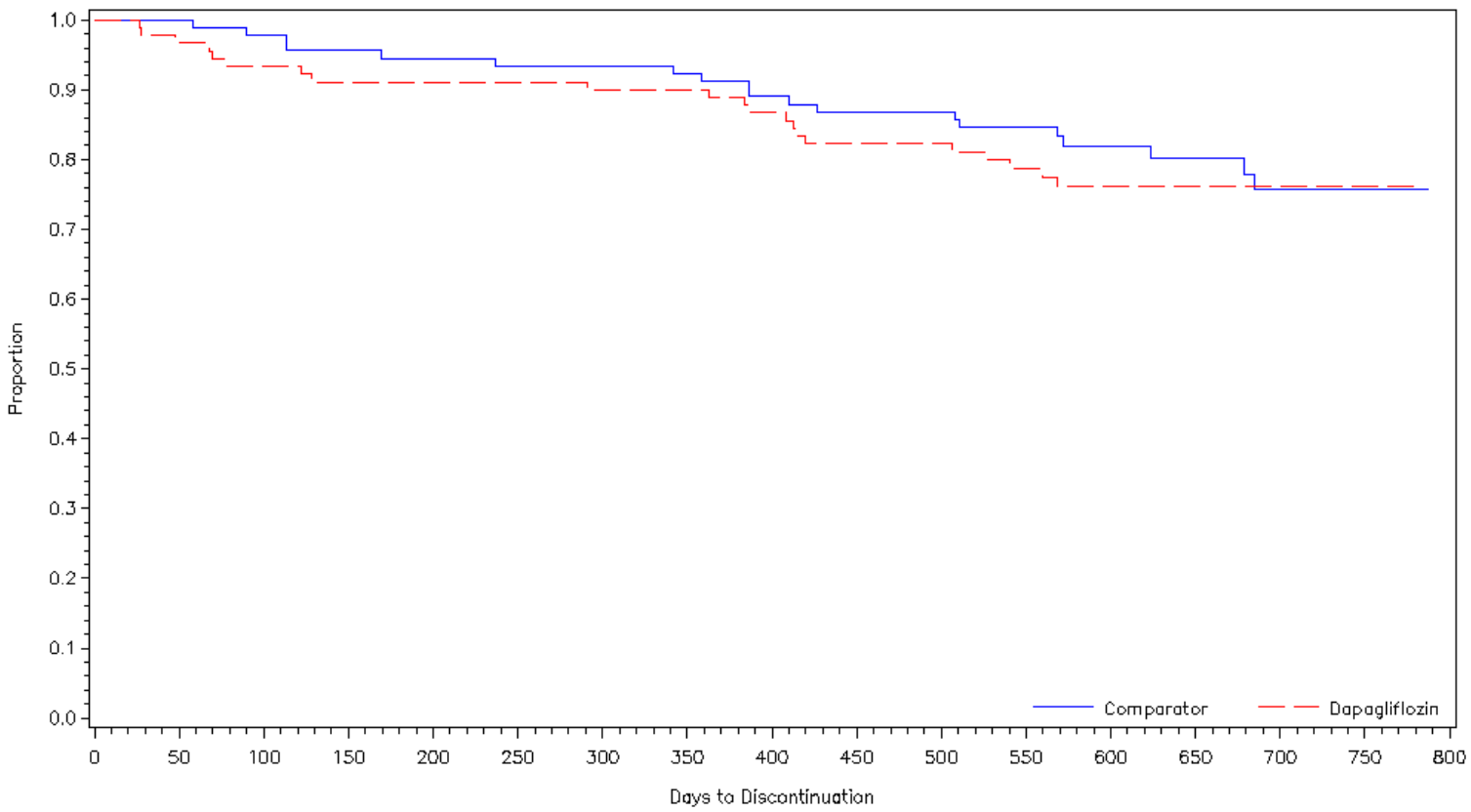

Time to Discontinuation for Study MB102029

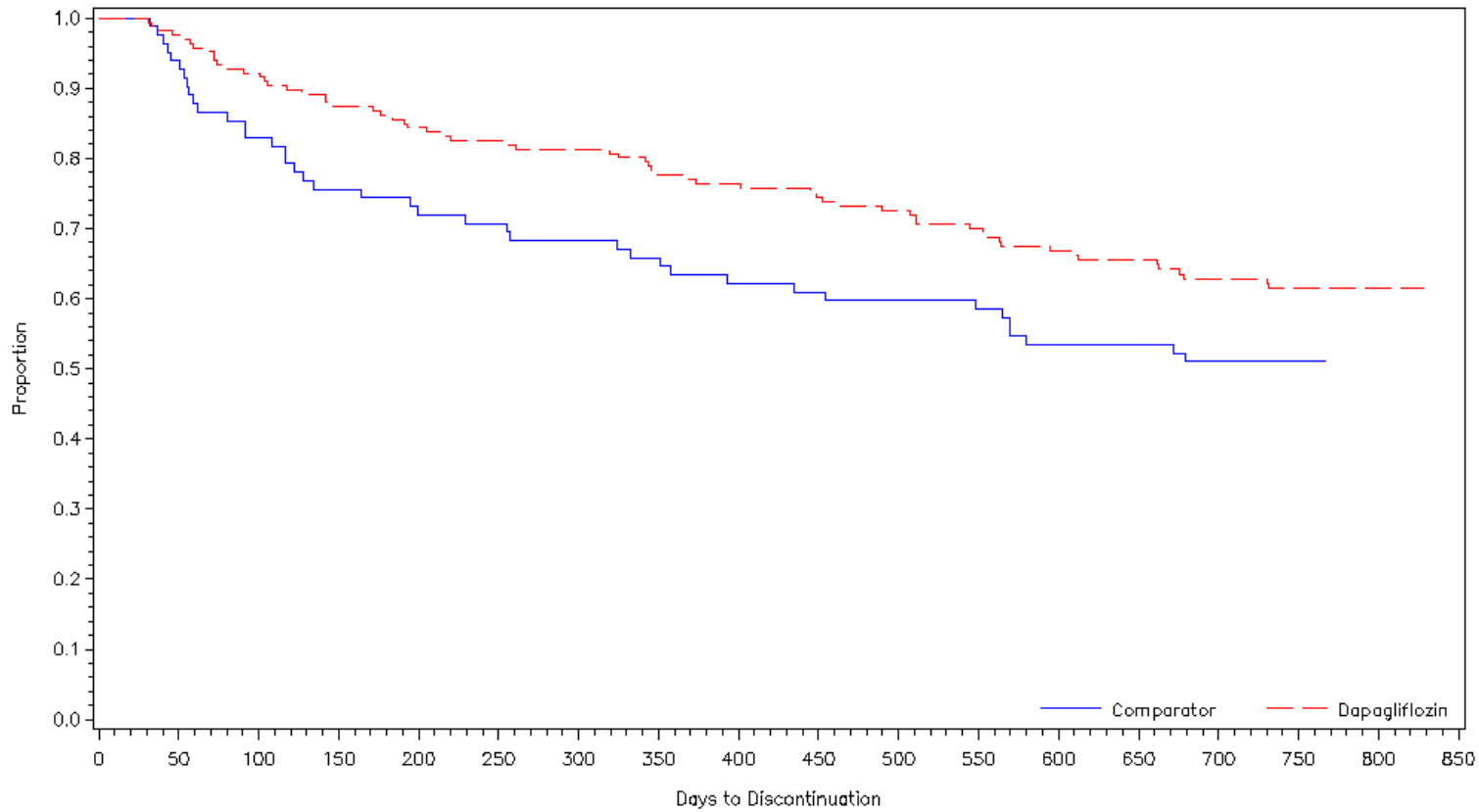


NDA 202-293 (FORXIGA)

Figure 11: Assessment of Proportional Hazards Assumption: Log-Log Survival Curve (BMS Analysis Population)

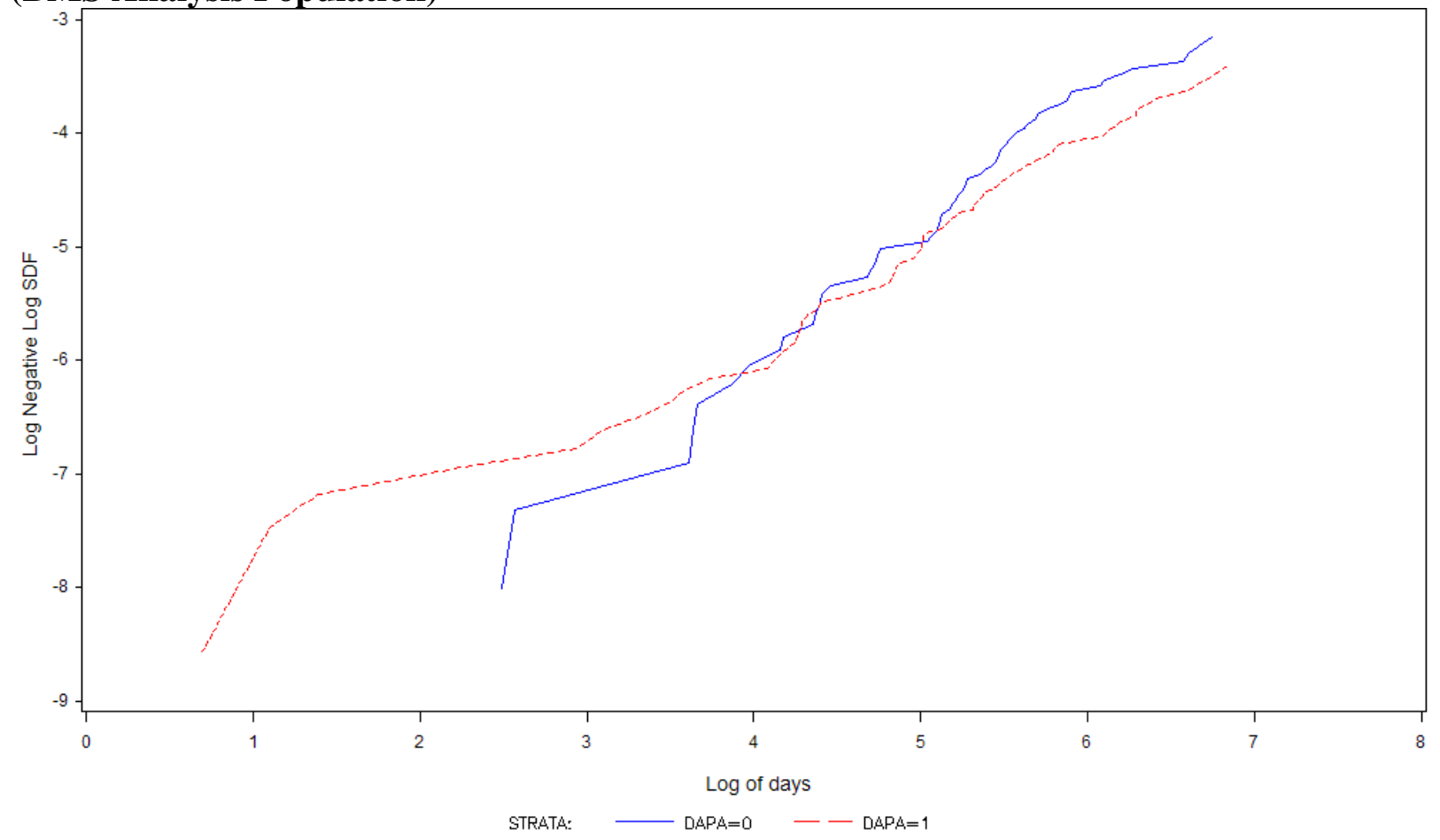

Figure 12: Assessment of Proportional Hazards Assumption: Schoenfeld Residual Plot (BMS Analysis Population)

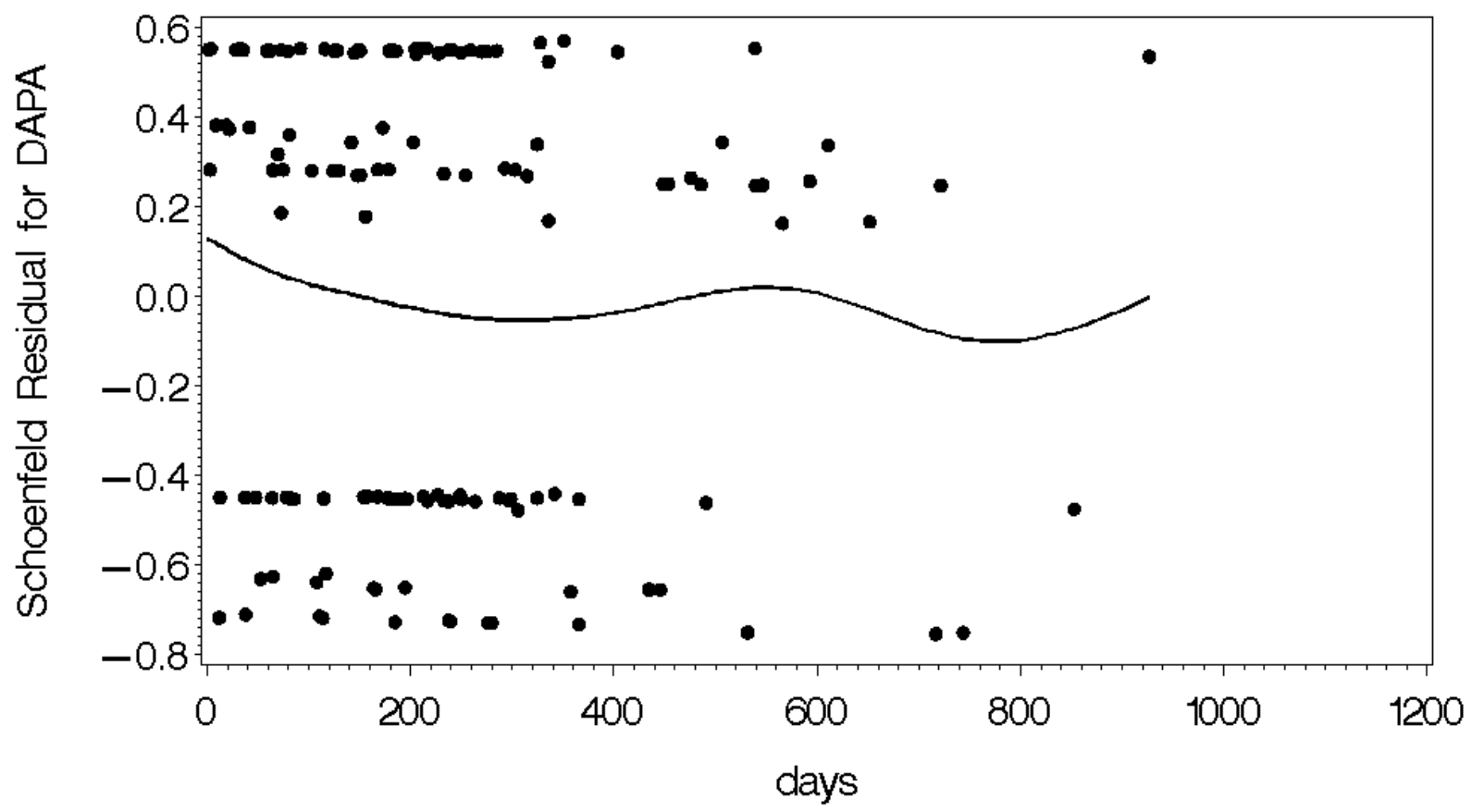


Table 16: CV Risk Factors by Trial

\begin{tabular}{|c|c|c|c|c|c|c|c|c|c|c|}
\hline \multirow[t]{2}{*}{$\%$ of Subjects } & \multicolumn{2}{|c|}{ D1690C00004 } & \multicolumn{2}{|c|}{ D1690C00005 } & \multicolumn{2}{|c|}{ D1690C00006 } & \multicolumn{2}{|c|}{ D1690C00012 } & \multicolumn{2}{|c|}{ D1692C00005 } \\
\hline & Dapa & Comp & Dapa & Comp & Dapa & Comp & Dapa & Comp & Dapa & Comp \\
\hline History of CVD & 18.47 & 19.12 & 35.11 & 37.67 & 32.49 & 38.2 & 24.18 & 28.57 & 3.01 & 3.7 \\
\hline $\begin{array}{l}\text { History of } \\
\text { Hypertension }\end{array}$ & 70.69 & 70.59 & 71.78 & 80.14 & 85.08 & 86.8 & 86.81 & 82.42 & 45.18 & 51.85 \\
\hline $\begin{array}{l}\text { History of } \\
\text { Dyslipidemia }\end{array}$ & 61.33 & 59.31 & 57.11 & 54.79 & 70.16 & 66.5 & 58.24 & 58.24 & 61.45 & 62.96 \\
\hline $\begin{array}{l}\text { History of Congestive } \\
\text { Heart Failure }\end{array}$ & 0.99 & 0.49 & 3.78 & 4.11 & 4.43 & 2.54 & 0 & 0 & 0 & 0 \\
\hline \multicolumn{11}{|l|}{ Renal Function } \\
\hline Severely Impaired & 0.25 & 0.25 & 0 & 0 & 0 & 0 & 0 & 0 & 0 & 0 \\
\hline Moderately Impaired & 4.68 & 5.64 & 8.67 & 16.44 & 16.89 & 13.71 & 1.1 & 5.49 & 0 & 0 \\
\hline Mildly Impaired & 49.26 & 45.1 & 58.67 & 54.79 & 56.72 & 56.35 & 61.54 & 61.54 & 12.05 & 9.26 \\
\hline Normal & 45.81 & 49.02 & 32.67 & 28.77 & 26.39 & 29.95 & 37.36 & 32.97 & 87.95 & 90.74 \\
\hline \multicolumn{11}{|l|}{ Smoking History } \\
\hline Never smoked & 49.01 & 49.02 & 69.2 & 63.01 & 55.25 & 56.35 & 45.05 & 51.65 & 38.55 & 38.89 \\
\hline Current Smoker & 17.98 & 17.89 & 14.51 & 11.64 & 13.28 & 17.77 & 17.58 & 17.58 & 28.92 & 29.63 \\
\hline Former Smoker & 33.00 & 33.09 & 16.29 & 25.34 & 31.31 & 25.89 & 37.36 & 30.77 & 32.53 & 31.48 \\
\hline \multicolumn{11}{|l|}{ Diabetes Duration } \\
\hline less than 3 years & 26.85 & 30.88 & 25.33 & 20.55 & 3.44 & 4.06 & 28.57 & 36.26 & 40.36 & 37.04 \\
\hline $3-10$ years & 55.17 & 48.04 & 48.67 & 55.48 & 30.89 & 27.41 & 50.55 & 51.65 & 47.59 & 51.85 \\
\hline more than 10 years & 17.98 & 21.08 & 26.00 & 23.97 & 65.57 & 68.53 & 20.88 & 12.09 & 12.05 & 11.11 \\
\hline
\end{tabular}




\begin{tabular}{|c|c|c|c|c|c|c|c|c|}
\hline \multirow[t]{2}{*}{ \% of Subjects } & \multicolumn{2}{|c|}{ MB102008 } & \multicolumn{2}{|c|}{ MB102009 } & \multicolumn{2}{|c|}{ MB102013 } & \multicolumn{2}{|c|}{ MB102014 } \\
\hline & Dapa & Comp & Dapa & Comp & Dapa & Comp & Dapa & Comp \\
\hline History of CVD & 8.96 & 4.55 & 18.75 & 34.78 & 11.22 & 9.33 & 13.94 & 7.3 \\
\hline $\begin{array}{l}\text { History of } \\
\text { Hypertension }\end{array}$ & 39.43 & 35.45 & 62.5 & 60.87 & 50.00 & 52.00 & 54.52 & 54.74 \\
\hline $\begin{array}{l}\text { History of } \\
\text { Dyslipidemia }\end{array}$ & 29.75 & 29.09 & 52.08 & 60.87 & 41.71 & 49.33 & 55.01 & 50.36 \\
\hline $\begin{array}{l}\text { History of Congestive } \\
\text { Heart Failure }\end{array}$ & 1.43 & 0.91 & 0 & 0 & 1.46 & 2.67 & 0.24 & 0 \\
\hline \multicolumn{9}{|l|}{ Renal Function } \\
\hline Severely Impaired & 0 & 0 & 0 & 0 & 0 & 0 & 0 & 0 \\
\hline Moderately I & 3.23 & 4.55 & 8.33 & 8.7 & 7.07 & 2.67 & 11.25 & 8.76 \\
\hline Mildly Impaired & 63.80 & 58.18 & 54.17 & 56.52 & 55.85 & 58.67 & 55.26 & 52.55 \\
\hline Normal & 32.97 & 37.27 & 37.50 & 34.78 & 37.07 & 38.67 & 33.50 & 38.69 \\
\hline \multicolumn{9}{|l|}{ Smoking History } \\
\hline $\begin{array}{l}\text { Never/Former } \\
\text { smoked }\end{array}$ & 74.91 & 74.55 & 81.25 & 60.87 & 56.59 & 61.33 & 56.72 & 57.66 \\
\hline Current Smoker & 25.09 & 25.45 & 18.75 & 39.13 & $\begin{array}{l}18.78 \\
24.63\end{array}$ & $\begin{array}{l}14.67 \\
24.00\end{array}$ & $\begin{array}{l}15.16 \\
28.12\end{array}$ & $\begin{array}{l}12.41 \\
29.93\end{array}$ \\
\hline \multicolumn{9}{|l|}{ Diabetes Duration } \\
\hline less than 3 years & 72.04 & 78.18 & 4.17 & 4.35 & 81.95 & 72 & 34.47 & 37.23 \\
\hline $3-10$ years & 25.09 & 17.27 & 35.42 & 39.13 & 15.61 & 25.33 & 46.94 & 46.72 \\
\hline more than 10 years & 2.87 & 4.55 & 60.42 & 56.52 & 2.44 & 2.67 & 18.58 & 16.06 \\
\hline
\end{tabular}




\begin{tabular}{|c|c|c|c|c|c|c|c|c|c|c|}
\hline \multirow[t]{2}{*}{$\%$ of Subjects } & \multicolumn{2}{|c|}{ MB102021 } & \multicolumn{2}{|c|}{ MB102029 } & \multicolumn{2}{|c|}{ MB102030 } & \multicolumn{2}{|c|}{ MB102032 } & \multicolumn{2}{|c|}{ MB102034 } \\
\hline & Dapa & Comp & Dapa & Comp & Dapa & Comp & Dapa & Comp & Dapa & Comp \\
\hline History of CVD & 10.33 & 12.94 & 48.21 & 40.48 & 9.61 & 5.76 & 13.38 & 8.82 & 10 & 11.54 \\
\hline $\begin{array}{l}\text { History of } \\
\text { Hypertension }\end{array}$ & 50.13 & 52.74 & 94.64 & 90.48 & 44.84 & 44.6 & 50.7 & 52.94 & 53.02 & 58.65 \\
\hline $\begin{array}{l}\text { History of } \\
\text { Dyslipidemia }\end{array}$ & 27.2 & 25.37 & 61.9 & 53.57 & 46.26 & 42.45 & 30.28 & 32.35 & 32.09 & 37.5 \\
\hline $\begin{array}{l}\text { History of Congestive } \\
\text { Heart Failure }\end{array}$ & 0.76 & 1 & 10.12 & 11.9 & 0 & 0.72 & 4.23 & 2.94 & 1.86 & 0.96 \\
\hline \multicolumn{11}{|l|}{ Renal Function } \\
\hline Severely Impaired & 0.25 & 0 & 3.57 & 4.76 & 0 & 0 & 0 & 0 & 0 & 0 \\
\hline Moderately Impaired & 6.3 & 3.98 & 92.86 & 89.25 & 6.05 & 5.04 & 4.23 & 4.41 & 5.35 & 6.73 \\
\hline Mildly Impaired & 50.63 & 53.73 & 2.98 & 5.95 & 50.18 & 56.83 & 49.3 & 58.82 & 53.02 & 55.29 \\
\hline Normal & 42.82 & 42.29 & 0.6 & 0 & 43.77 & 38.13 & 46.48 & 36.76 & 41.63 & 37.98 \\
\hline \multicolumn{11}{|l|}{ Smoking History } \\
\hline Never smoked & 69.77 & 67.66 & 50.6 & 54.76 & 65.48 & 64.75 & 63.38 & 66.18 & 65.58 & 72.12 \\
\hline Current Smoker & 13.1 & 11.94 & 10.12 & 22.9 & 12.46 & 17.27 & 18.31 & 8.82 & 17.91 & 14.42 \\
\hline Former Smoker & 17.13 & 20.4 & 39.29 & 33.33 & 22.06 & 17.99 & 18.31 & 25 & 16.51 & 13.46 \\
\hline \multicolumn{11}{|l|}{ Diabetes Duration } \\
\hline less than 3 years & 83.38 & 82.09 & 4.17 & 7.14 & 41.28 & 44.2 & 86.62 & 88.24 & 75.35 & 80.29 \\
\hline $3-10$ years & 14.86 & 15.92 & 20.24 & 22.62 & 43.06 & 38.41 & 11.27 & 10.29 & 20.7 & 16.83 \\
\hline more than 10 years & 1.76 & 1.99 & 75.6 & 70.24 & 15.66 & 17.39 & 2.11 & 1.47 & 3.95 & 2.88 \\
\hline
\end{tabular}

Source: Created by reviewer. Dataset: adcv2v2.xpt 


\begin{tabular}{|c|c|c|c|c|c|c|}
\hline \multirow[t]{2}{*}{$\%$ of Subjects } & \multicolumn{2}{|c|}{ D1690C00010 } & \multicolumn{2}{|c|}{ D1690C00018 } & \multicolumn{2}{|c|}{ D1690C00019 } \\
\hline & Dapa & Comp & Dapa & Comp & Dapa & Comp \\
\hline History of CVD & 21.78 & 19.03 & 98.91 & 99.35 & 100 & 99.79 \\
\hline $\begin{array}{l}\text { History of } \\
\text { Hypertension }\end{array}$ & 62.22 & 64.16 & 99.78 & 99.78 & 92.92 & 92.75 \\
\hline $\begin{array}{l}\text { History of } \\
\text { Dyslipidemia }\end{array}$ & 56.89 & 56.19 & 84.10 & 86.36 & 84.58 & 81.78 \\
\hline $\begin{array}{l}\text { History of Congestive } \\
\text { Heart Failure }\end{array}$ & 0.89 & 0.44 & 12.20 & 13.42 & 17.92 & 13.66 \\
\hline \multicolumn{7}{|l|}{ Renal Function } \\
\hline Severely Impaired & 0 & 0 & 0.22 & 0.22 & 0 & 0 \\
\hline Moderately Impaired & 4.00 & 5.75 & 19.61 & 19.74 & 16.89 & 13.71 \\
\hline Mildly Impaired & 52.44 & 47.35 & 56.43 & 58.13 & 56.72 & 56.35 \\
\hline Normal & 43.56 & 46.90 & 23.75 & 21.91 & 26.39 & 29.95 \\
\hline \multicolumn{7}{|l|}{ Smoking History } \\
\hline Never smoked & 52.00 & 49.02 & 40.74 & 40.91 & 39.17 & 40.37 \\
\hline Current Smoker & 16.89 & 15.93 & 15.25 & 13.42 & 14.58 & 16.15 \\
\hline Former Smoker & 31.11 & 28.76 & 44.01 & 45.45 & 46.25 & 43.27 \\
\hline \multicolumn{7}{|l|}{ Diabetes Duration } \\
\hline less than 3 years & 36.00 & 40.71 & 8.93 & 10.17 & 6.88 & 7.66 \\
\hline $3-10$ years & 50.67 & 44.25 & 35.51 & 33.77 & 31.25 & 38.51 \\
\hline more than 10 years & 13.33 & 15.04 & 55.56 & 56.06 & 61.88 & 53.83 \\
\hline
\end{tabular}




\begin{tabular}{|c|c|c|c|c|}
\hline \multirow[t]{2}{*}{ \% of Subjects } & \multicolumn{2}{|c|}{ MB102035 } & \multicolumn{2}{|c|}{ MB102045 } \\
\hline & Dapa & Comp & Dapa & Comp \\
\hline History of CVD & 4.17 & 19.61 & 4.35 & 4.76 \\
\hline $\begin{array}{l}\text { History of } \\
\text { Hypertension }\end{array}$ & 87.50 & 82.35 & 82.61 & 61.90 \\
\hline $\begin{array}{l}\text { History of } \\
\text { Dyslipidemia }\end{array}$ & 62.50 & 64.71 & 60.87 & 61.90 \\
\hline $\begin{array}{l}\text { History of Congestive } \\
\text { Heart Failure }\end{array}$ & 0 & 0 & 0 & 0 \\
\hline \multicolumn{5}{|l|}{ Renal Function } \\
\hline Severely Impaired & 0 & 0 & 0 & 0 \\
\hline Moderately Impaired & 0 & 1.96 & 13.04 & 4.76 \\
\hline Mildly Impaired & 62.50 & 56.86 & 34.78 & 66.67 \\
\hline Normal & 37.50 & 41.18 & 52.17 & 28.57 \\
\hline \multicolumn{5}{|l|}{ Smoking History } \\
\hline Never smoked & 75.00 & 41.18 & 34.78 & 61.90 \\
\hline Current Smoker & 4.17 & 19.61 & 13.04 & 9.52 \\
\hline Former Smoker & 20.83 & 39.22 & 52.17 & 28.57 \\
\hline \multicolumn{5}{|l|}{ Diabetes Duration } \\
\hline less than 3 years & 20.83 & 31.37 & 4.17 & 7.14 \\
\hline $3-10$ years & 54.17 & 49.02 & 20.24 & 22.62 \\
\hline more than 10 years & 25.00 & 19.61 & 75.6 & 70.24 \\
\hline
\end{tabular}




\section{SIGNATURES/DISTRIBUTION LIST}

Primary Statistical Reviewer: Anita A. Abraham

Date: November 22, 2011

Concurring Reviewers: Mat Soukup, Aloka Chakravarty

Statistical Team Leader: Mat Soukup

Biometrics 7 Division Director: Aloka Chakravarty

cc:

Curtis J. Rosebraugh (Director, Office of Drug Evaluation II)

Mary Parks (Director, Division of Metabolism and Endocrinology)

Ilan Irony (Medical Team Leader)

Somya Dunn (Medical Officer)

Mehreen Hai (Project Manager)

Aloka Chakravarty (Director, Division of Biometrics 7)

Mat Soukup (Division of Biometrics 7)

Anita Abraham (Division of Biometrics 7)

Lillian Patrician (Office of Biostatistics) 
This is a representation of an electronic record that was signed electronically and this page is the manifestation of the electronic signature.

/s/

ANITA A ABRAHAM

$11 / 22 / 2011$

MATTHEW J SOUKUP

$11 / 22 / 2011$

Concur with review

ALOKA G CHAKRAVARTY

$11 / 22 / 2011$ 


\section{STATISTICAL REVIEW AND EVALUATION Clinical Studies}

\author{
NDA/BLA Serial \\ Number: \\ Drug Name: \\ Indication(s): \\ Applicant: \\ Date(s):
}

Review Priority:

Biometrics Division: $\quad$ Division of Biometrics II

Statistical Reviewer: Jonathan Norton, Ph.D.

Concurring Reviewers: Todd Sahlroot, Ph.D.

Medical Division:

Clinical Team:

Project Manager:
202-293/0055,0057,0059

Dapagliflozin propanediol tablets

Improve glycemic control in adults with type 2 diabetes mellitus

Bristol-Myers Squibb

Stamp Date: 12/28/2010

Goal Date: 1/16/2012 (extended)
Division of Metabolism and Endocrinology Products

Somya Dunn, M.D.

Ilan Irony, M.D.

Mehreen Hai

Keywords: Clinical studies, NDA review, add-on design, analysis of covariance, doubleblind, longitudinal data analysis, sensitivity analyses, stratification, missing data 


\section{Table of Contents}

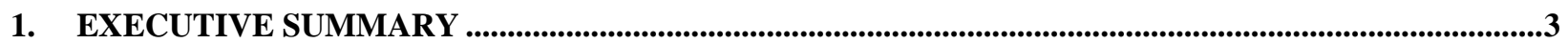

2. INTRODUCTION

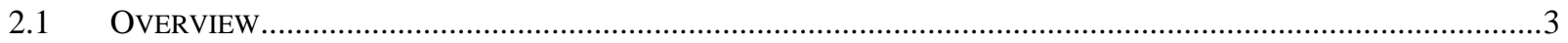

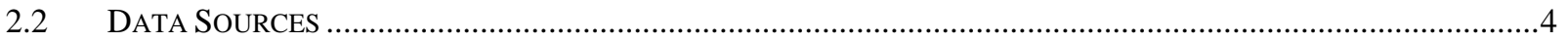

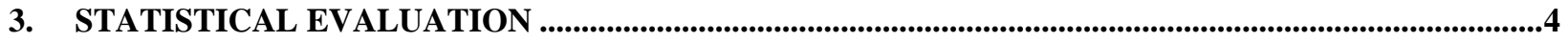

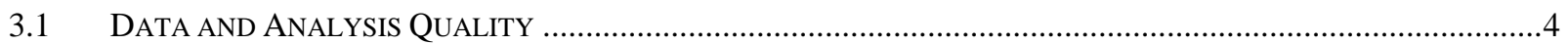

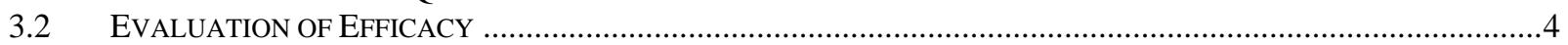

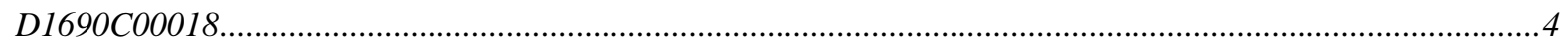

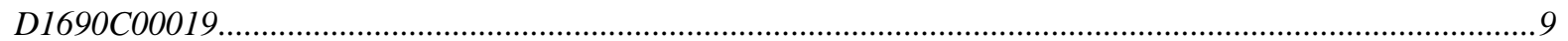

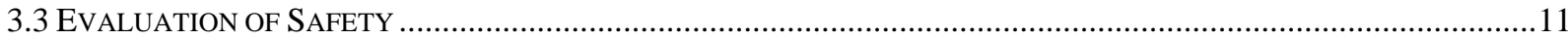

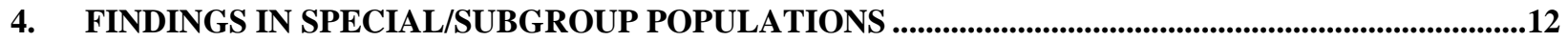

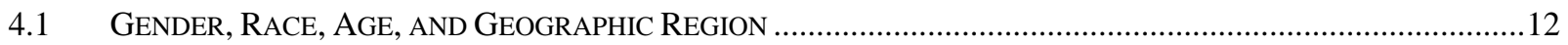

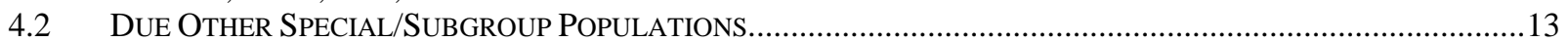

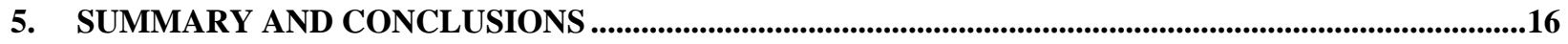

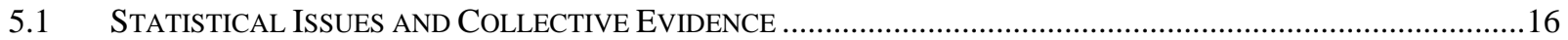

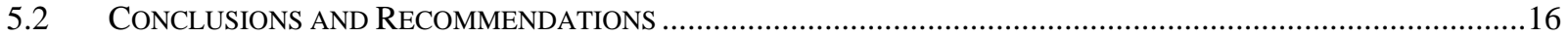




\section{EXECUTIVE SUMMARY}

The Applicant has submitted a new drug application for dapagliflozin propanediol (DAPA) as a treatment for type 2 diabetes mellitus. The original submission included eleven Phase 3 studies. The evidence from these studies was evaluated in my primary statistical review submitted on September 9, 2011, which particularly focused on six of the studies. In an amendment, the Applicant submitted two additional Phase 3 studies in patients with cardiovascular disease.

In my primary review of this NDA, I concluded, "The submitted clinical studies provide strong evidence that dapagliflozin is effective in subjects with normal renal function or mild impairment.” The results from the additional Phase 3 studies provide further support for this conclusion.

\section{INTRODUCTION}

\subsection{Overview}

The Applicant has submitted a new drug application for dapagliflozin propanediol (DAPA) as a treatment for type 2 diabetes mellitus. The original submission included eleven Phase 3 studies. The evidence from these studies was evaluated in my primary statistical review submitted on September 9, 2011, which particularly focused on six of the studies. Since that time the Applicant has submitted two additional Phase 3 studies, shown in Table 1, which are the focus of this amendment.

Table 1: Additional Studies

\begin{tabular}{|l|l|l|l|l|}
\hline $\begin{array}{l}\text { Study ID } \\
\text { (Brief Name) }\end{array}$ & Population & Test Tx & $\begin{array}{l}\text { Background Tx } \\
\text { (Rescue) }\end{array}$ & Comparator(s) \\
\hline $\begin{array}{l}\text { D1690C00018 } \\
\text { (C18) }\end{array}$ & $\begin{array}{l}\text { Patients with } \\
\text { cardiovascular } \\
\text { disease and } \\
\text { hypertension }\end{array}$ & $\begin{array}{l}\text { DAPA 10 } \\
\text { mg }\end{array}$ & $\begin{array}{l}\text { Various OADs } \\
\text { and/or insulin } \\
\text { (Various) }\end{array}$ & Placebo \\
\hline $\begin{array}{l}\text { D1690C00019 } \\
\text { (C19) }\end{array}$ & $\begin{array}{l}\text { Patients with } \\
\text { cardiovascular } \\
\text { disease }\end{array}$ & $\begin{array}{l}\text { DAPA 10 } \\
\text { mg }\end{array}$ & $\begin{array}{l}\text { Various OADs } \\
\text { and/or insulin } \\
\text { (Various) }\end{array}$ & Placebo \\
\hline
\end{tabular}

Notes: Proposed doses are 5, $10 \mathrm{mg}$. DAPA = dapagliflozin. OAD = oral anti-diabetic drug.

The distinct feature of these studies, compared to those previously submitted, is that the patient population had cardiovascular disease. Patients in Study C18 were also required to have hypertension. It is also notable that the subjects were on a variety of background medications, and it was common to be on multiple ones. Furthermore, although there were specific criteria for needing anti-hyperglycemic and/or anti-hypertensive rescue therapy, the rescue therapy itself was subject to investigator discretion. 


\subsection{Data Sources}

The electronic version of this NDA can be found at ॥Cdsesub1levsprod\NDA202293\202293.enx.

\section{STATISTICAL EVALUATION}

\subsection{Data and Analysis Quality}

I did not encounter any major data quality issues in the course of this review. Due to time constraints, however, I did not attempt to reproduce all of the Applicant's key results from the submitted data.

\subsection{Evaluation of Efficacy}

D1690C00018

\section{Study Design and Endpoints}

Figure 1 shows the design for Study C18.

\section{Figure 1: Design for Study C18}

(Source: Figure 1, Clinical Study Report)

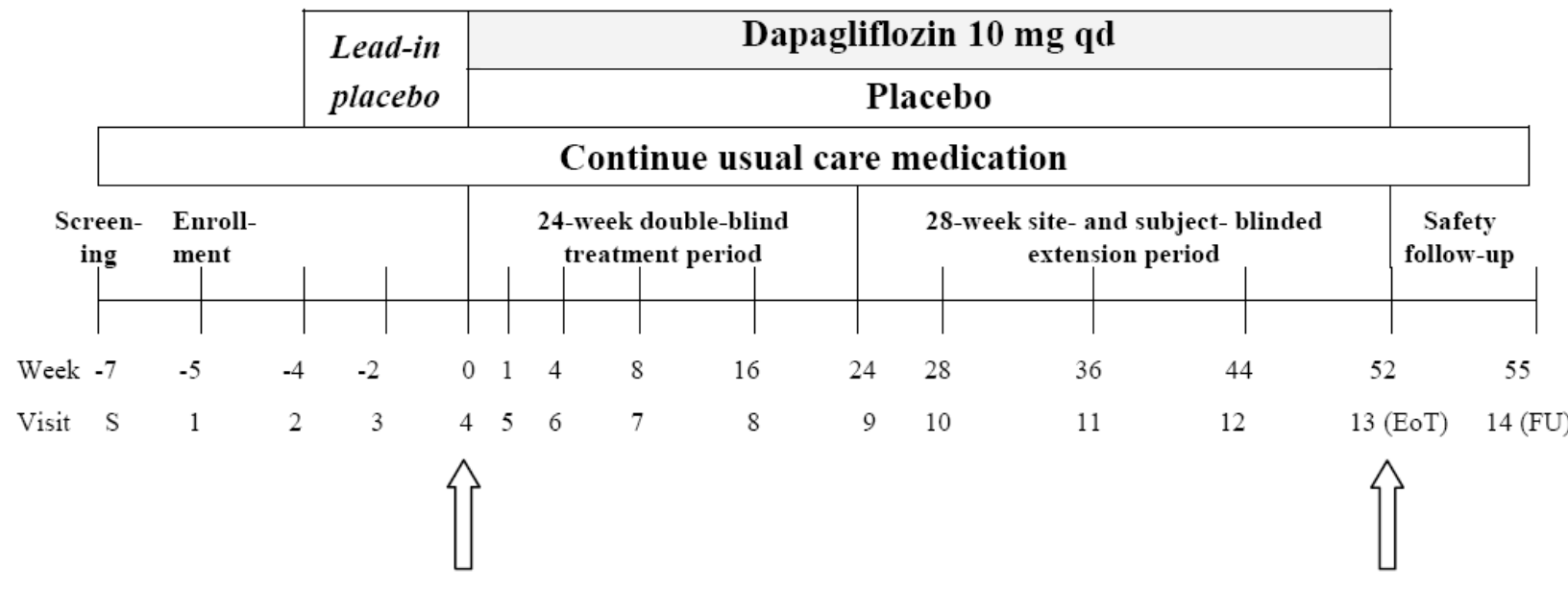

Randomization 1:1

End of randomized treatment

The Applicant prespecified two primary endpoints for this study. The "first" primary endpoint was the change in hemoglobin A1c (HbA1c) at Week 24. The "second" primary endpoint was the proportion of responders who met the following criteria for "clinical benefit" at Week 24:

1) an absolute drop of $0.5 \%$ or more from baseline HbA1c,

2) a relative drop of $3 \%$ or more from baseline in total body weight, and

3) an absolute drop of $3 \mathrm{mmHg}$ or more from baseline in seated systolic blood pressure (SBP). 
The clinical team leader, Ilan Irony, M.D., advised me that this responder definition is not of particular clinical interest. My review will therefore focus on the underlying continuous variables rather than the composite.

\section{Patient Disposition, Demographic and Baseline Characteristics}

The Applicant reported fairly high completion rates for the short-term period in the two treatment arms: 88\% (403/460) for DAPA and 87\% (404/462) for placebo. The denominators for these results are from the safety analysis set. Considering the small number of dropouts, there was not a substantial imbalance across arms in the reasons for discontinuation.

Table 2 summarizes the more common background medications in use by the treated subjects at randomization. Note that many patients used multiple background treatments.

Table 2: Background Medication, Study C18

\begin{tabular}{|l|l|l|}
\hline \multirow{2}{*}{ Metformin } & Placebo (N=462) & DAPA (N=459) \\
\cline { 2 - 3 } & $\mathrm{N}(\%)$ & $\mathrm{N}(\%)$ \\
\hline Sulfonylurea & $344(74 \%)$ & $346(75 \%)$ \\
\hline ACE Inhibitor & $179(39 \%)$ & $179(39 \%)$ \\
\hline Loop Diuretic & $402(87 \%)$ & $402(88 \%)$ \\
\hline Insulin & $88(19 \%)$ & $72(16 \%)$ \\
\hline
\end{tabular}

Table 3 shows selected demographic characteristics for the Full Analysis Set, defined as treated subjects with a baseline value and at least one post-baseline value on an efficacy variable. The table was provided by the Applicant. They also reported that $38 \%$ of subjects were in the United States (29\%) or Canada (9\%). 
Table 3: Demographics, Study C18

(Source: Table 11.1.2.1, Clinical Study Report)

\begin{tabular}{|c|c|c|c|c|c|c|}
\hline \multirow[b]{2}{*}{ AGE } & \multicolumn{2}{|c|}{$\begin{array}{c}\text { PLA } \\
\mathrm{N}=459\end{array}$} & \multicolumn{2}{|c|}{$\begin{array}{c}\mathrm{DAPA} 10 \mathrm{MG} \\
\mathrm{N}=455\end{array}$} & \multicolumn{2}{|c|}{$\begin{array}{c}\text { Total } \\
\mathrm{N}=914\end{array}$} \\
\hline & & & & & & \\
\hline $\mathrm{N}$ & & 459 & & 455 & & 914 \\
\hline MEAN & & 63.0 & & 62.8 & & 62.9 \\
\hline MEDIAN & & 63.0 & & 63.0 & & 63.0 \\
\hline MIN , MAX & 45 & 86 & 46, & 82 & 45 & \\
\hline $\begin{array}{l}Q 1, Q 3 \\
\text { STANDARD DEVIATION }\end{array}$ & 57.0 & $\begin{array}{l}69.0 \\
7.66\end{array}$ & 58.0 & $\begin{array}{l}68.0 \\
6.97\end{array}$ & 58.0 & $\begin{array}{l}68.0 \\
7.32\end{array}$ \\
\hline \multicolumn{7}{|l|}{ AGE CATEGORIZATION (\%) } \\
\hline$<55$ & 74 & $(16.1)$ & 58 & $(12.7)$ & 132 & $(14.4)$ \\
\hline$>=55$ AND $<65$ & 189 & $(41.2)$ & 205 & $(45.1)$ & 394 & $(43.1)$ \\
\hline$>=65$ AND $<75$ & 165 & $(35.9)$ & 164 & $(36.0)$ & 329 & $(36.0)$ \\
\hline$>=75$ & 31 & $(6.8)$ & 28 & $(6.2)$ & 59 & $(6.5)$ \\
\hline NOT REPORTED & 0 & & 0 & & 0 & \\
\hline \multicolumn{7}{|l|}{ GENDER (\%) } \\
\hline MALE & 315 & $(68.6)$ & 309 & $(67.9)$ & 624 & $(68.3)$ \\
\hline FEMALE & 144 & $(31.4)$ & 146 & $(32.1)$ & 290 & $(31.7)$ \\
\hline NOT REPORTED & 0 & & 0 & & 0 & \\
\hline \multicolumn{7}{|l|}{$\operatorname{RACE}(\%)$} \\
\hline WHITE & 391 & $(85.2)$ & 376 & $(82.6)$ & 767 & $(83.9)$ \\
\hline BLACK/AFRICAN AMERICAN & 27 & $(5.9)$ & 26 & $(5.7)$ & 53 & $(5.8)$ \\
\hline ASIAN & 38 & $(8.3)$ & 49 & $(10.8)$ & 87 & $(9.5)$ \\
\hline OTHER & 3 & $(0.7)$ & 4 & $(0.9)$ & 7 & $(0.8)$ \\
\hline NOT REPORTED & 0 & & 0 & & 0 & \\
\hline \multicolumn{7}{|l|}{ ETHNICITY $(\%)$} \\
\hline HISPANIC/LATINO & 97 & $(21.1)$ & 97 & $(21.3)$ & 194 & $(21.2)$ \\
\hline NOT HISPANIC/LATINO & 362 & $(78.9)$ & 358 & $(78.7)$ & 720 & $(78.8)$ \\
\hline NOT REPORTED & 0 & & 0 & & 0 & \\
\hline
\end{tabular}

\section{Statistical Methodologies}

Except where otherwise noted, the statistical methods were similar to those used in the other Phase 3 studies. Randomization was done centrally and was stratified by three binary factors (eight combinations): age ( $<65$ vs. $>=65$ ), use of insulin at randomization, and time since most recent cardiovascular event ( $>1$ year vs. $<=1$ year). The primary analysis set included treated subjects with a baseline and at least one post-baseline value. An analysis of covariance (ANCOVA) model was used for change from baseline variables (including HbA1c) with adjustment for the baseline value and the stratification factors. The age factor was used in a prespecified subgroup analysis. The primary imputation method was last observation carried forward (LOCF), excluding observations after outcome-related rescue (e.g., glycemic rescue for HbA1c). The following sensitivity analyses were also specified for the primary HbA1c endpoint: an analysis of completers, a per-protocol analysis (if more than $10 \%$ of subjects in either arm were excluded from the per-protocol set), a repeated measures analysis that excluded post-rescue observations, and a repeated measures analysis that adjusted for rescue use by including a binary factor. A combination of Bonferroni correction and sequential testing was used to control for multiplicity across primary endpoints, secondary endpoints, and age strata. 


\section{Results and Conclusions}

Figure 2 shows the Applicant's reported results for the primary HbA1c endpoint, change from baseline at Week 24, using LOCF imputation. The placebo-corrected treatment effect was -.46 (standard error $[\mathrm{SE}]=.05$ ), which was significant at $\mathrm{p}<.0001$. For comparison, the metformin add-on trial that I reviewed earlier (MB102014) showed a treatment effect of -.54 (SE = .10) for the $10 \mathrm{mg}$ DAPA dose with LOCF imputation. Note that 75\% of patients in the present study used metformin on background. For further comparison, the insulin add-on study (D1690C00006) showed a treatment effect of -.60 (SE = .07) for the $10 \mathrm{mg}$ dose, again using LOCF. Insulin was used by $52 \%$ of patients in the present study. There is apparently a somewhat attenuated treatment effect in this study compared to these two reference studies, which had younger patients who were not specifically selected for having cardiovascular disease. These differences in treatment effect may be related to the different patient populations, the combined effect of multiple medications, or any number of other causes.

\section{Figure 2: Applicant's Results for Primary HbA1c Endpoint, Study C18}

(Source: Figure 2, Clinical Study Report)

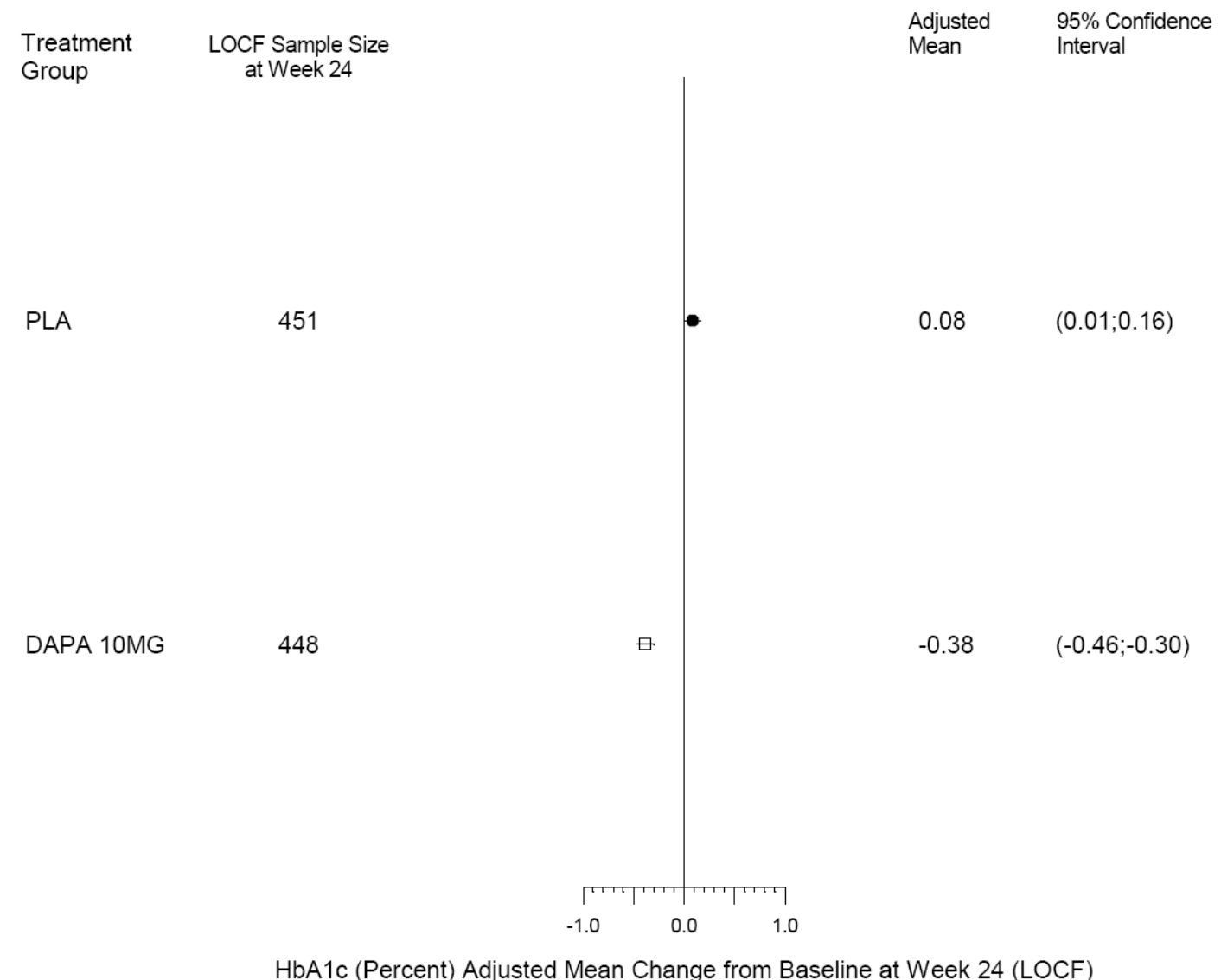

The Applicant's sensitivity analyses for this endpoint yielded similar estimates of the treatment effect to that from the LOCF analysis. They also conducted numerous tests for interaction 
between the treatment effect and various background medications, and did not find any interactions that were even nominally significant (i.e., even before adjustment for multiplicity). My own analyses showed a trend toward lower efficacy for DAPA for patients on ACEinhibitors, but this finding was not replicated in Study C19.

The Applicant reported that a lower proportion of patients on DAPA (8.1\%) received glycemic rescue compared to those on placebo (23.5\%). This statistically-significant difference provides additional evidence that DAPA treatment yielded superior glycemic control.

As stated previously, this review will focus on the components of the composite "clinical benefit" endpoint rather than the endpoint itself. However, it is important for the purpose of multiplicity control that DAPA was reported to be significantly better than placebo overall and in each age stratum.

The Applicant reported results for body weight and seated SBP that support the favorable finding on the primary HbA1c endpoint. Patients on DAPA lost 2.6\% of their body weight at Week 24 compared to $0.3 \%$ for placebo, a statistically-significant difference. In absolute terms, patients on DAPA lost $2.37 \mathrm{~kg}$, compared to a loss of .29 kg for patients on placebo. This magnitude of effect is similar to what was observed in the add-on studies for metformin and insulin. The Applicant also reports that patients on DAPA a showed change in blood pressure of $-2.99 \mathrm{mmHg}$ compared to $-1.03 \mathrm{mmHg}$ on for those on placebo, a statistically-significant difference.

In summary, this study supports the results of the other Phase 3 studies in showing that DAPA was effective in reducing HbA1c and body weight at Week 24. An additional finding of a reduction in blood pressure was also reported. See Section 4 for subgroup analyses of the HbA1c endpoint. 


\section{Study Design and Endpoints}

Figure 3 shows the design for Study C19. The two primary endpoints were the same as in Study C18.

Figure 3: Design for Study C19

(Source: Figure 1, Clinical Study Report)

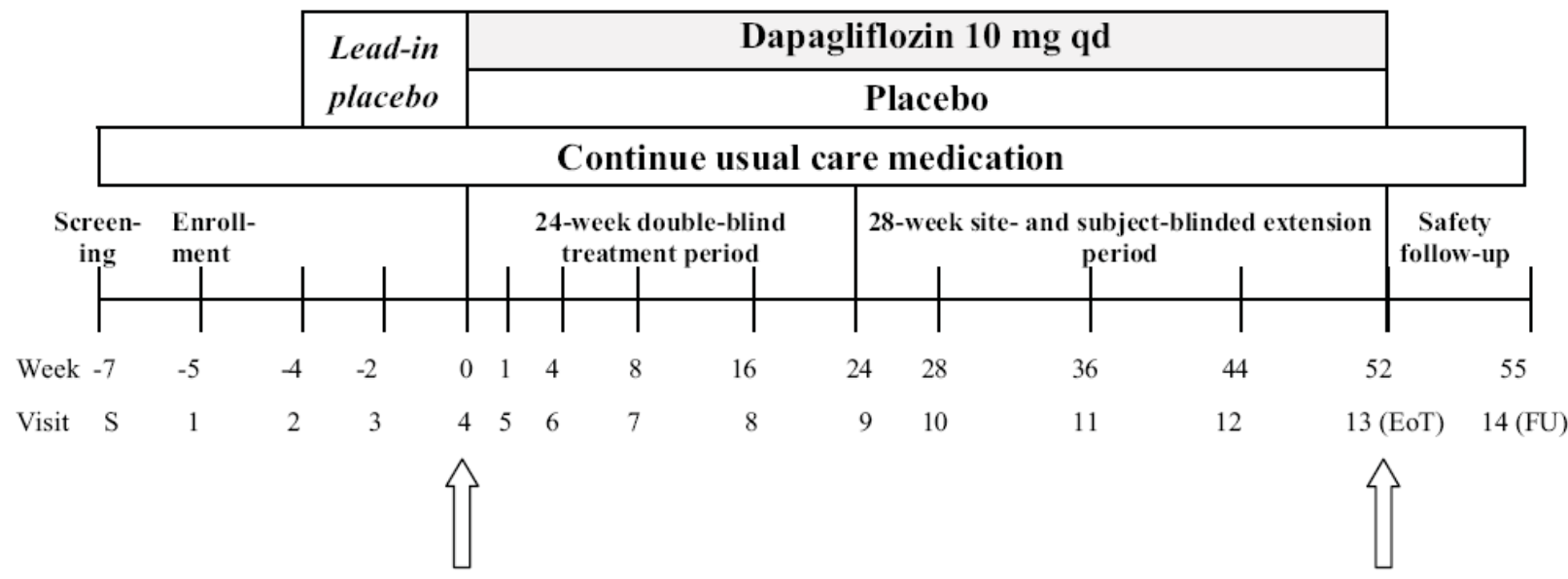

Randomization 1:1

End of randomized treatment

\section{Patient Disposition, Demographic and Baseline Characteristics}

As with Study C18, the reported completion rates were fairly high for the short-term period: $91 \%$ (441/482) for DAPA and 89\% (428/483) for placebo. The denominators are from the safety analysis set. There was not a substantial imbalance in the reasons for discontinuation.

Table 4 summarizes the more common background medications in use by the treated subjects at randomization.

Table 4: Background Medication, Study C19

\begin{tabular}{|l|l|l|}
\hline & Placebo (N=483) & DAPA (N=480) \\
\cline { 2 - 3 } & $\mathrm{N}(\%)$ & $\mathrm{N}(\%)$ \\
\hline Metformin & $355(73 \%)$ & $348(73 \%)$ \\
\hline Sulfonylurea & $159(33 \%)$ & $177(37 \%)$ \\
\hline ACE Inhibitor & $392(81 \%)$ & $391(81 \%)$ \\
\hline Loop Diuretic & $80(17 \%)$ & $101(21 \%)$ \\
\hline Insulin & $290(60 \%)$ & $292(61 \%)$ \\
\hline
\end{tabular}

Table 5, which was provided by the Applicant, shows selected demographic characteristics for the Full Analysis Set. They also reported that $28 \%$ of subjects were in the United States (20\%) or Canada (8\%). 
Table 5: Demographic, Study C19

(Source: Table 11.1.2.1, Clinical Study Report)

\begin{tabular}{|c|c|c|c|c|c|c|}
\hline \multirow[b]{2}{*}{ AGE } & \multicolumn{2}{|c|}{$\begin{array}{c}\text { PLA } \\
\mathrm{N}=482\end{array}$} & \multicolumn{2}{|c|}{$\begin{array}{c}\mathrm{DAPA} 10 \mathrm{MG} \\
\mathrm{N}=480\end{array}$} & \multicolumn{2}{|c|}{$\begin{array}{c}\text { Total } \\
\mathrm{N}=962\end{array}$} \\
\hline & & & & & & \\
\hline $\mathrm{N}$ & & 482 & & 480 & & 962 \\
\hline MEAN & & 63.6 & & 63.9 & & 63.8 \\
\hline MEDIAN & & 64.0 & & 64.0 & & 64.0 \\
\hline MIN , MAX & 47, & 84 & 45, & 84 & 45 & 84 \\
\hline $\begin{array}{l}\text { Q1, Q3 } \\
\text { STANDARD DEVIATION }\end{array}$ & 58.0 & $\begin{array}{l}68.0 \\
7.02\end{array}$ & 58.0, & $\begin{array}{l}69.0 \\
7.60\end{array}$ & 58.0 & $\begin{array}{l}69.0 \\
7.31\end{array}$ \\
\hline \multicolumn{7}{|l|}{ AGE CATEGORIZATION (\%) } \\
\hline$<55$ & 54 & $(11.2)$ & 57 & $(11.9)$ & 111 & $(11.5)$ \\
\hline$>=55$ AND $<65$ & 204 & $(42.3)$ & 196 & $(40.8)$ & 400 & $(41.6)$ \\
\hline$>=65$ AND $<75$ & 196 & $(40.7)$ & 181 & $(37.7)$ & 377 & $(39.2)$ \\
\hline$>=75$ & 28 & $(5.8)$ & 46 & $(9.6)$ & 74 & $(7.7)$ \\
\hline NOT REPORTED & 0 & & 0 & & 0 & \\
\hline \multicolumn{7}{|l|}{ GENDER (\%) } \\
\hline MALE & 323 & $(67.0)$ & 321 & $(66.9)$ & 644 & $(66.9)$ \\
\hline FEMALE & 159 & $(33.0)$ & 159 & $(33.1)$ & 318 & $(33.1)$ \\
\hline NOT REPORTED & 0 & & 0 & & 0 & \\
\hline \multicolumn{7}{|l|}{ RACE (\%) } \\
\hline WHITE & 449 & $(93.2)$ & 454 & $(94.6)$ & 903 & $(93.9)$ \\
\hline BIACK/AFRICAN AMERICAN & 10 & $(2.1)$ & 9 & $(1.9)$ & 19 & $(2.0)$ \\
\hline ASIAN & 7 & $(1.5)$ & 6 & $(1.3)$ & 13 & $(1.4)$ \\
\hline OTHER & 16 & $(3.3)$ & 11 & $(2.3)$ & 27 & $(2.8)$ \\
\hline NOT REPORTED & 0 & & 0 & & 0 & \\
\hline \multicolumn{7}{|l|}{ ETHNICITY (\%) } \\
\hline HISPANIC/LATINO & 38 & $(7.9)$ & 34 & $(7.1)$ & 72 & $(7.5)$ \\
\hline NOT HISPANIC/LATINO & 444 & $(92.1)$ & 446 & $(92.9)$ & 890 & $(92.5)$ \\
\hline NOT REPORTED & 0 & & 0 & & 0 & \\
\hline
\end{tabular}

\section{Statistical Methodologies}

The statistical methods were the same as previously described for Study C18.

\section{Results and Conclusions}

Figure 4 shows the Applicant's reported results for the primary HbA1c endpoint. The placebocorrected treatment effect of $-.40(\mathrm{SE}=.05)$, which was significant at $\mathrm{p}<.0001$, was similar to the effect reported in Study C18. The Applicant's sensitivity analyses yielded similar estimates of the treatment effect to that from the LOCF analysis. They did not find any (even nominally) significant interactions between treatment and background medication. 


\section{Figure 4: Applicant's Results for Primary HbA1c Endpoint, Study C19}

(Source: Figure 2, Clinical Study Report)

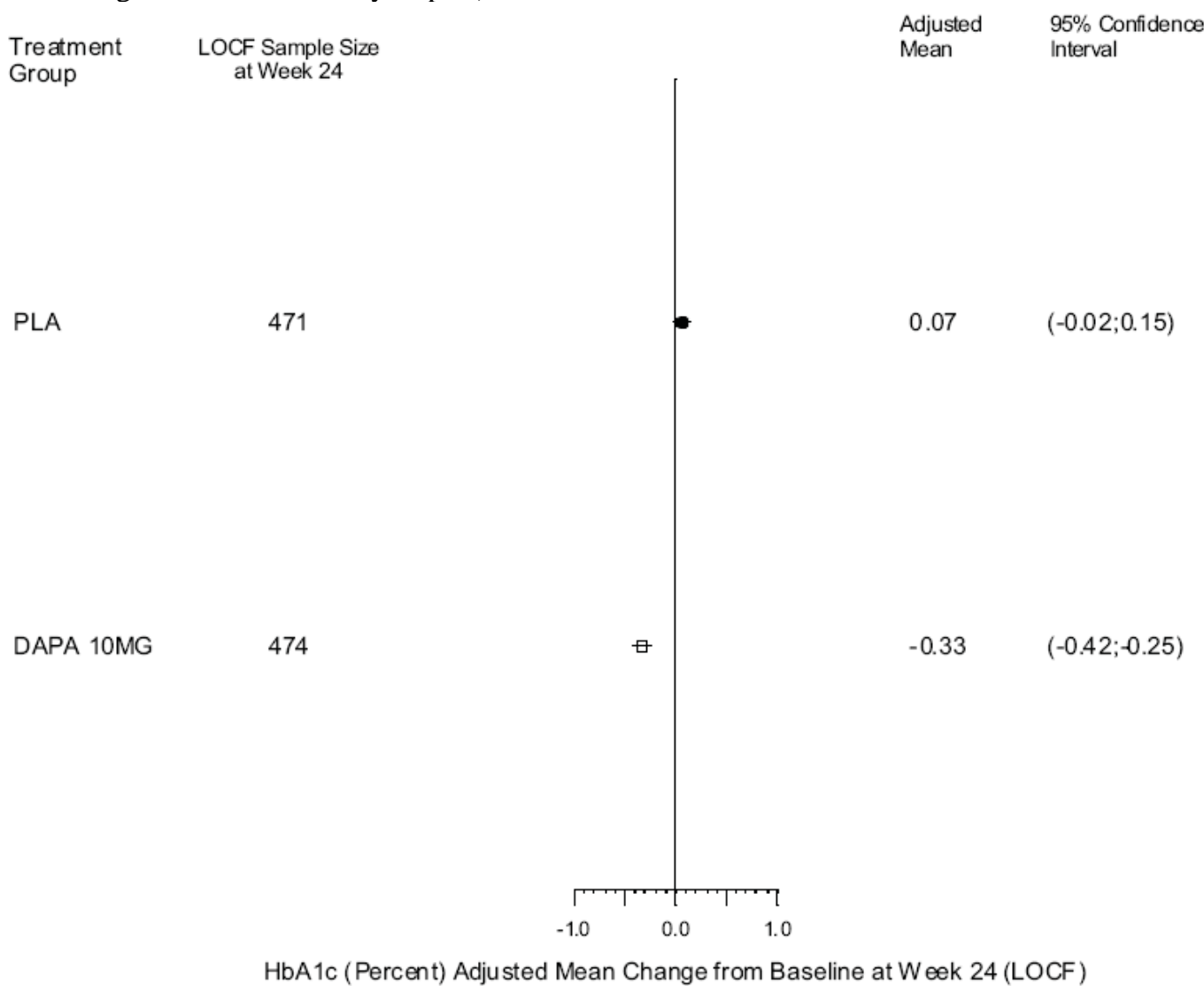

The Applicant also reported that 7.5\% of patients on DAPA used glycemic rescue by Week 23 compared $22.6 \%$ on placebo, a statistically-significant difference. This result is consistent with DAPA providing superior glycemic control.

The Applicant also reported that DAPA was superior to placebo on the composite clinical benefit endpoint, both overall and in each age stratum. In regard to body weight, they report that patients on DAPA lost $2.5 \%$ from baseline at Week 24 , compared to a loss of $0.6 \%$ in the placebo arm (p $<$.0001). In absolute terms, the loss was $2.36 \mathrm{~kg}$ for DAPA and $0.53 \mathrm{~kg}$ for placebo, yielding a treatment effect of $-1.83 \mathrm{~kg}$. This is similar to what was observed in Study C18. The Applicant also reports that subjects on DAPA showed a change in systolic blood pressure of $-2.70 \mathrm{mmHg}$, while those on placebo showed a change of $0.32 \mathrm{mmHg}(\mathrm{p}$-value for difference $=.0002)$.

In summary, this study showed that DAPA was effective in reducing HbA1c, body weight, and blood pressure. See Section 4 for subgroup analyses of the HbA1c endpoint.

\subsection{Evaluation of Safety}

The safety of dapagliflozin was primarily reviewed by Somya Dunn, M.D. Anita Abraham, Ph.D., conducted the statistical review of cardiovascular safety. 


\section{FINDINGS IN SPECIAL/SUBGROUP POPULATIONS}

\subsection{Gender, Race, Age, and Geographic Region}

In regard to gender, for Study C18 the Applicant reported virtually identically treatment effects (DAPA - placebo) for each sex on the primary HbA1c endpoint: -.46 (SE = .06) for men and -.47 (SE = .08) for women. In Study C19 men showed a treatment effect of -.37 (SE = .06), compared to $-.46(\mathrm{SE}=.08)$ for women. The interaction test was not significant in either study.

In both studies, the predominance of white subjects (84\% in C18, 94\% in C19) limits inference about race. The tests for interaction with treatment were not significant. Similarly, the interaction tests for Hispanic ethnicity were also non-significant in these studies.

Age was a factor of particular interest in these studies. Table 6 shows the Applicant's results for HbA1c for Study C18 by age group. The interaction p-value shown (.2065) was non-significant. It is noteworthy that within the DAPA arm the change from baseline is negative (good) and is virtually the same for each age group. In the placebo (i.e., background therapy) arm the apparent trend is for older patients to get worse, but this may be an artifact of the LOCF analysis.

Table 6: Applicant's Results for Primary HbA1c Endpoint by Age, Study C18

(Source: Table 11.2.2.3.1.13, Clinical Study Report)

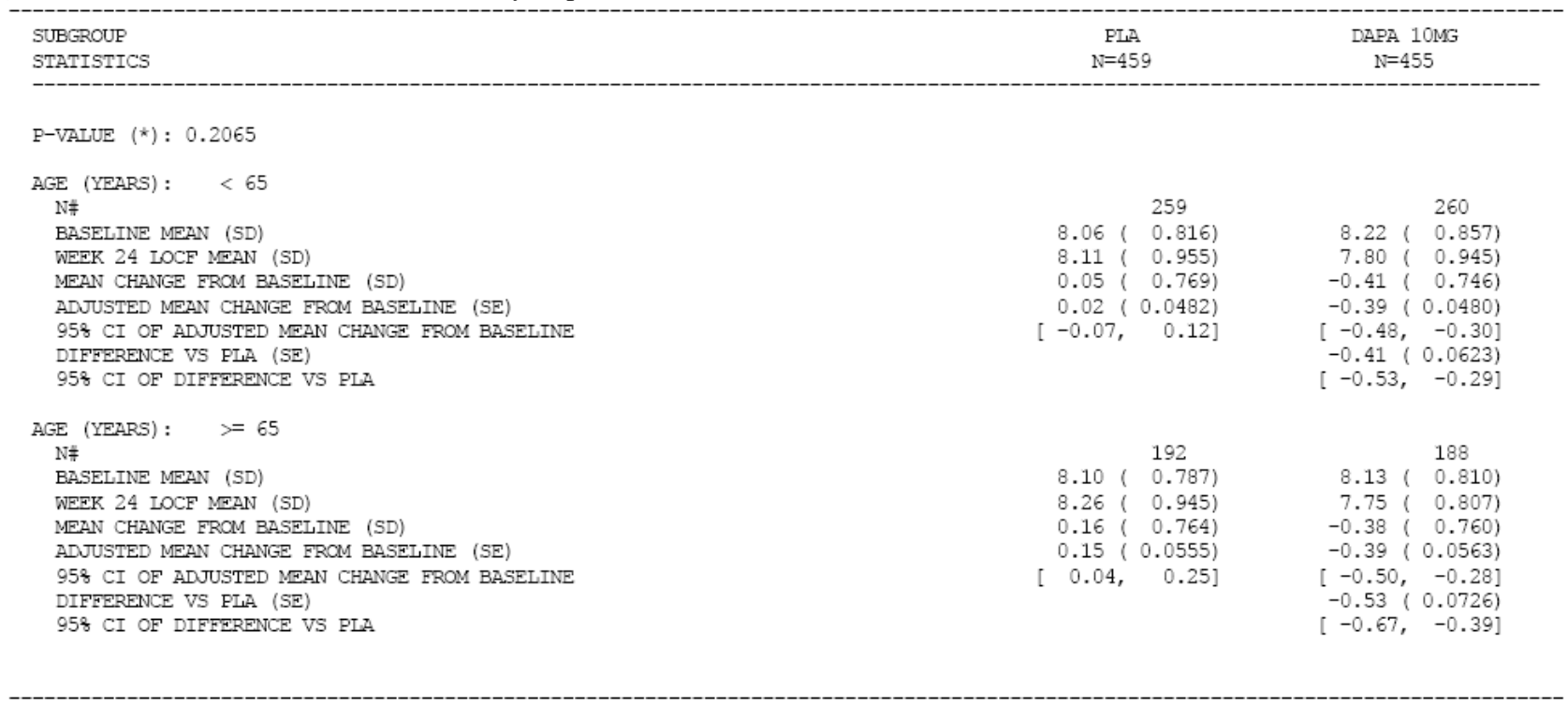


Table 7 shows the corresponding results for Study C19. There appears to be a modest trend for older patients to get lower efficacy from DAPA, but that finding was not replicated in Study C18. The interaction test was again non-significant.

Table 7: Applicant's Results for Primary HbA1c Endpoint by Age, Study C19

(Source: Table 11.2.2.3.1.13, Clinical Study Report)

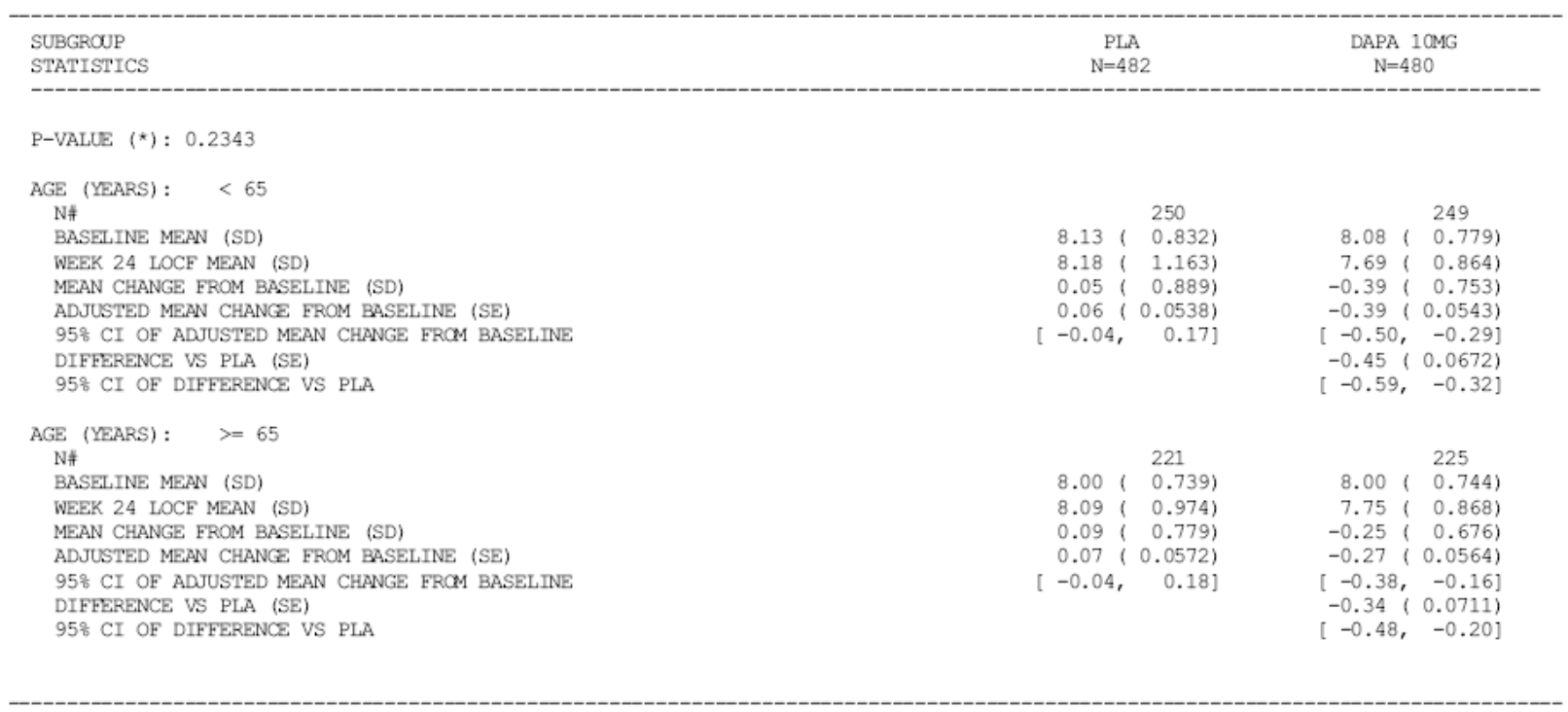

The Applicant defined four geographic regions including "North America”, which was comprised of Canada and the United States. Neither study showed a significant interaction term for region. Focusing on North America, the placebo-corrected treatment effect was -.43 (SE = .08 ) for Study C18 and -.44 (SE = .09) for Study C19. These are similar to the effects observed in the overall study population.

\subsection{Due Other Special/Subgroup Populations}

Since the mechanism of action for DAPA is via the kidneys, there is a particular concern about efficacy in patients who are renally impaired. Table 8, which was provided by the Applicant, shows the results for the primary HbA1c endpoint by severity of renal impairment in Study C18. Renal impairment was measured in this study by the estimated glomerular filtration rate (eGFR). The two patients with an eGFR $<30$ are classified as having severe impairment, while those with an eGFR $>=30$ and $<60$ are classified as moderately impaired. Note that moderately impaired patients had a treatment effect of only -.31 (SE = .11), compared to values of -.50 (SE = .06) and $-.49(\mathrm{SE}=.10)$ in the two categories of patients with better renal function. As a caveat, the Applicant did report a non-significant $p$-value $(\mathrm{p}=.44)$ for a one-degree interaction test between treatment and continuous eGFR, after adjustment for baseline HbA1c and the randomization strata (age, insulin use, time from most recent cardiovascular event). 
Table 8: Primary HbA1c Endpoint by eGFR, Study C18

(Source: Table 11.2.2.3.1.24, Clinical Study Report)

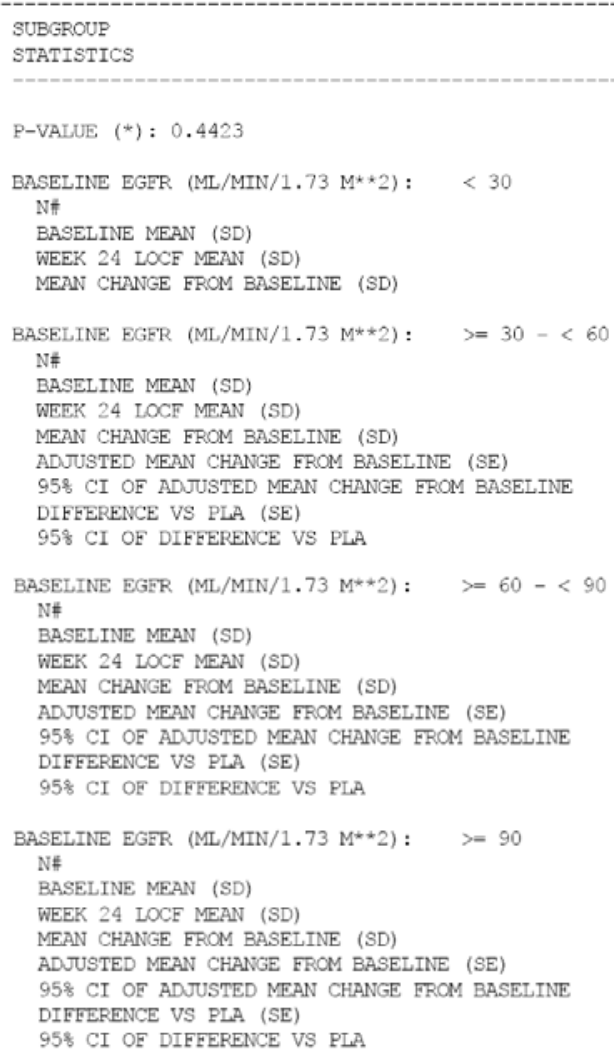


Table 9 shows the corresponding results for Study C19. Patients with moderate renal impairment had a rather modest estimated treatment effect of -.18 $(\mathrm{SE}=.13)$. Again, however, the interaction test was not significant $(\mathrm{p}=.24)$.

Table 9: Primary HbA1c Endpoint by eGFR, Study C19

(Source: Table 11.2.2.3.1.24, Clinical Study Report)

\begin{tabular}{|c|c|c|}
\hline $\begin{array}{l}\text { SUBGROUP } \\
\text { STATISTICS }\end{array}$ & $\begin{array}{c}\text { PIA } \\
\mathrm{N}=482\end{array}$ & $\begin{array}{l}\text { DAPA } 10 \mathrm{MG} \\
\qquad \mathrm{N}=480\end{array}$ \\
\hline P-VALUE $\left(^{*}\right): 0.2393$ & & \\
\hline $\begin{array}{l}\text { BASELINE EGFR (ML/MIN/1.73 M**2): }<30 \\
\text { N\# }\end{array}$ & 0 & 0 \\
\hline $\begin{array}{l}\text { BASELINE EGER (ML/MIN/1.73 M**2): }>=30-<60 \\
\text { N\# } \\
\text { BASELINE MEAN (SD) } \\
\text { WEEK } 24 \text { LOCF MEAN (SD) } \\
\text { MEAN CHANGE FROM BASELINE (SD) } \\
\text { ADJUSTED MEAN CHANGE FROM BASELINE (SE) } \\
\text { 95\% CI OF ADUSTED MEAN CHANGE FROM BASELINE } \\
\text { DIFFERENCE VS PLA (SE) } \\
\text { 95\% CI OF DIFFERENCE VS PLA }\end{array}$ & 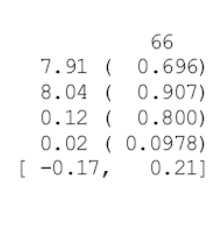 & $\begin{array}{rr}7.97( & 0.672) \\
7.88( & 1.003) \\
-0.09( & 0.728) \\
-0.16( & 0.0906) \\
{[-0.34,} & 0.02] \\
-0.18( & 0.1265) \\
{[-0.43,} & 0.07]\end{array}$ \\
\hline $\begin{array}{l}\text { BASELINE EGER (ML/MIN/1.73 M**2): }>=60-<90 \\
\text { N\# } \\
\text { BASELINE MEAN (SD) } \\
\text { WEEK } 24 \text { LOCF MEAN (SD) } \\
\text { MEAN CHANGE FROM BASELINE (SD) } \\
\text { ADJUSTED MEAN CHANGE FROM BASELINE (SE) } \\
\text { 95\% CI OF ADUSTED MEAN CHANGE FROM BASELINE } \\
\text { DIFFERENCE VS PLA (SE) } \\
\text { 95\% CI OF DIFFERENCE VS PLA }\end{array}$ & 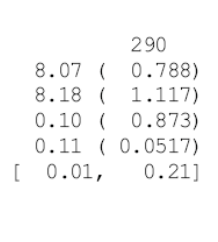 & $\begin{array}{rr} & 292 \\
8.05( & 0.781) \\
7.69( & 0.848) \\
-0.36( & 0.718) \\
-0.37( & 0.0511) \\
{[-0.47,} & -0.27] \\
-0.48( & 0.0623) \\
{[-0.60,} & -0.35]\end{array}$ \\
\hline $\begin{array}{l}\text { BASELINE EGER (ML/MIN/1.73 M**2): >=90 } \\
\text { N\# } \\
\text { BASELINE MEAN (SD) } \\
\text { WEEK } 24 \text { LOCF MEAN (SD) } \\
\text { MEAN CHANGE FROM BASELINE (SD) } \\
\text { ADJUSTED MEAN CHANGE FROM BASELINE (SE) } \\
\text { 95\% CI OF ADJUSTED MEAN CHANGE FROM BASELINE } \\
\text { DIFFERENCE VS PLA (SE) } \\
\text { 95\% CI OF DIFFERENCE VS PLA }\end{array}$ & 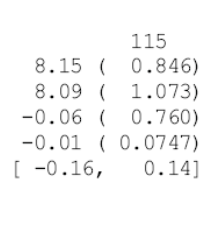 & $\begin{aligned} & 106 \\
8.07( & 0.778) \\
7.69( & 0.801) \\
-0.38( & 0.694) \\
-0.36( & 0.0783) \\
{[-0.51,} & -0.20] \\
-0.35( & 0.1013) \\
{[-0.55,} & -0.15]\end{aligned}$ \\
\hline
\end{tabular}




\section{SUMMARY AND CONCLUSIONS}

\subsection{Statistical Issues and Collective Evidence}

In both of these studies, subjects were on a variety of background medications and were given a variety of rescue therapies. Assuming that the blind was not broken, one can correctly infer that DAPA was superior to placebo in change in HbA1c, as well as other endpoints that were statistically-significant with appropriate multiplicity controls. However, any further interpretation of the trial results should be made with caution. In particular, the observed effect of DAPA treatment on HbA1c is the average over a particular mix of background medications that may or may not reflect clinical practice.

Due to time constraints, this review was somewhat limited relative to what I did for the six key studies in the primary review. My experience with these other studies showed that a positive finding on the primary LOCF analysis was indicative of a real treatment effect for DAPA, perhaps of smaller magnitude then the LOCF estimate might indicate. Based on this experience, I am fairly confident that the statistically-significant differences across treatment arms reported for Studies C18 and C19 are indicative of actual effects, albeit of indeterminate magnitude.

\subsection{Conclusions and Recommendations}

In the primary review of this NDA, I concluded, "The submitted clinical studies provide strong evidence that dapagliflozin is effective in subjects with normal renal function or mild impairment.” Studies C18 and C19 provide additional support for this conclusion. 
This is a representation of an electronic record that was signed electronically and this page is the manifestation of the electronic signature.

/s/

JONATHAN D NORTON

$11 / 21 / 2011$

JON T SAHLROOT

$11 / 21 / 2011$

Concur 


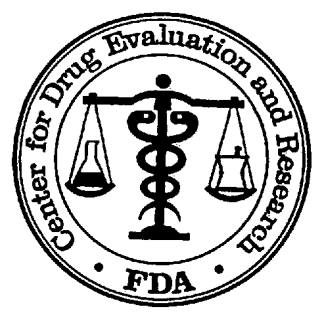

IND/NDA Number:

Drug Name:

Indication(s):

Applicant:

Documents Reviewed:

Review Priority:

Biometrics Division:

Statistical Reviewer:

Concurring Reviewer:

Medical Division:

Reviewing Pharmacologist:

Project Manager:

Keywords:
U.S. Department of Health and Human Services

Food and Drug Administration

Center for Drug Evaluation and Research

Office of Translational Science

Office of Biostatistics
Statistical Review and Evaluation

CARCINOGENICITY STUDIES

NDA 202-293

Dapagliflozin: BMS-512148

104 Week Carcinogenicity in Rats and Mice

Sponsor: BRISTOL MYERS SQUIBB

RESEARCH PKY,WALLINGFORD, CT 06492 USA

Electronic submission, Dated: December 27, 2010

Priority

Division of Biometrics -6

Mohammad Nagem, Ph.D.

Karl Lin, Ph.D.

Division of Metabolism and Endocrinology Products (DMEP)

Mukesh Summan, Ph.D.

Mehreen Hai

Carcinogenicity, Dose response 


\section{Table of Contents}

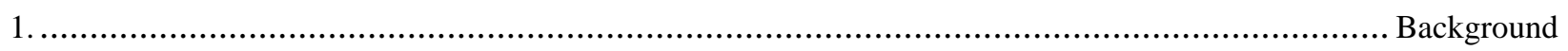

2. Rat Study

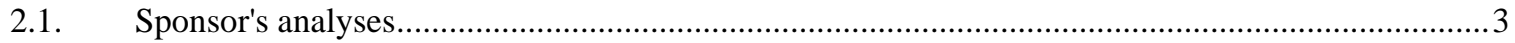

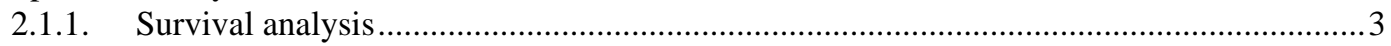

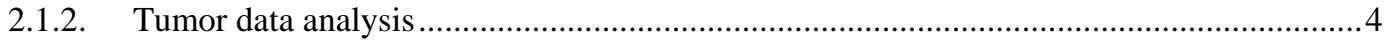

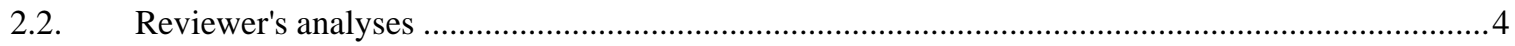

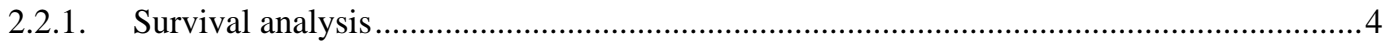

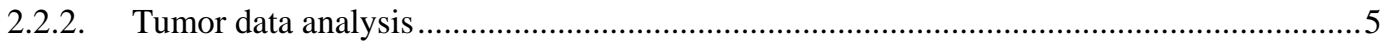

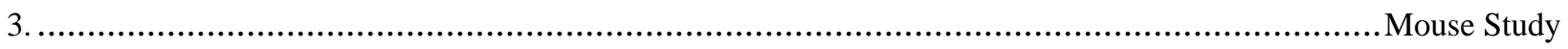

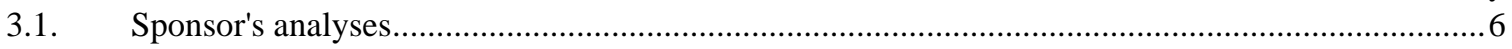

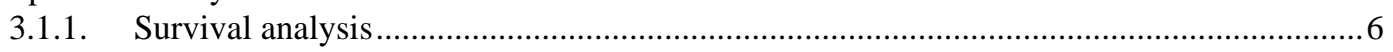

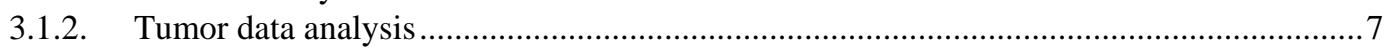

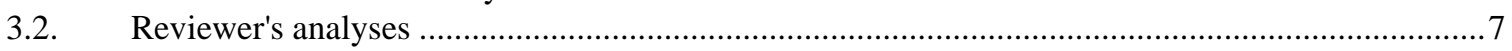

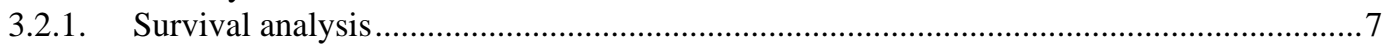

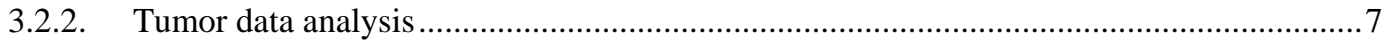

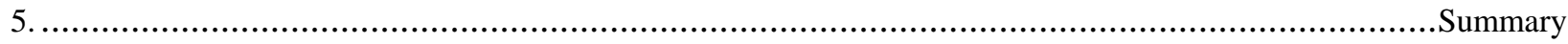

8

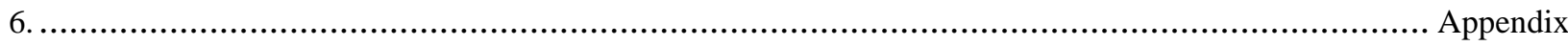




\section{Background}

In this submission the sponsor included reports of two animal carcinogenicity studies, one in rats and one in mice. These studies were intended to assess the carcinogenic potential of BMS-512148, a sodium-dependent glucose transporter 2 (SGLT-2) inhibitor to rats and mice when administered orally at appropriate drug levels for about 104 weeks. Results of this review have been discussed with the reviewing pharmacologist Dr. Summan.

\section{Rat Study}

Two separate experiments were conducted, one in males and one in females. In each of these two experiments there were three treated groups and two control groups. Three hundred and fifty rats were randomly allocated to treated, vehicle and control groups. Each group has 70 rats per sex, the dose levels for treated groups were $0.5,2$ and $10 \mathrm{mg} / \mathrm{kg} /$ day. In this review these dose groups would be referred to as the low, medium and high dose group, respectively. The vehicle control and distilled water control served to generate concurrent control data.

During the administration period all animals were observed for physical and clinical signs three times everyday on normal week days and twice on weekends and holidays. In addition, palpation was performed once a week to detect superficial masses. A complete histopathological examination was performed on all animals from all groups found dead, killed moribund, or sacrificed during or at the end of the experiment.

Terminal sacrifice for the male rats occurred after 89-91 weeks of treatment (days 619-631) and for the female rats occurred after 104 weeks of treatment (days 730-736).

\subsubsection{Survival analysis}

\subsection{Sponsor's analyses}

Survival function of each treatment group was estimated using the Kaplan-Meier product limit method. The dose response relationship ${ }^{1}$ in mortality was tested using similar method as was suggested by Tarone. Pairwise comparisons of control and each treated group were performed using the Log-Rank test. All tests were conducted at one-tailed significance level of 0.05 .

Sponsor's findings: Sponsor's analysis showed survival rates of $29 \%, 31 \%, 48 \%, 43 \%$ and $33 \%$ in Vehicle control, water control, low, medium and high dose groups, respectively in male rats and $40 \%, 49 \%, 44 \%, 36 \%$ and 39\% respectively, in female rats group. Sponsor concluded that there was no statistically significant treatment related effect on the survival in either sex groups, $\mathrm{p}$-value $=0.13$ and $\mathrm{p}$-value $=0.88$ for male and female group respectively.

The survival curves for the male and females rats treated with water control showed consistency with the vehicle control, by comparing their survival rates using a pairwise comparison test. This test was not significant, $\mathrm{p}$-value $=0.6501$ and $\mathrm{p}$-value $=0.4132$ for male and female water control when compared to vehicle control group respectively.

\footnotetext{
${ }^{1}$ In this review, the phrase "dose response relationship" refers to the linear component of the effect of treatment, and not necessarily to a strictly increasing or decreasing mortality or tumor rate as dose increases.
} 


\subsubsection{Tumor data analysis}

The analysis for positive dose response relationship for tumor incidences among controls, low, medium and high dose groups and pairwise comparisons of controls and treated groups were performed using the methods outlined in the paper of Peto et al. (1982). For incidental tumors, the analysis intervals were: weeks 0 - 52, $53-78,79-92$ and the terminal sacrifices for female group, and weeks 0 - 52, $53-78,79-88$ and terminal sacrifice phase for male rats due to high mortality rat in this group. Exact permutation test were used for tumors with less than 10 incidences.

The analysis for dose response relationship was conducted at the significance levels of 0.005 (one tailed-level) for common tumors and 0.025 (one tailed-level) for rare tumors. Pairwise comparisons were conducted at the significance levels of 0.01 (one tailed-level) for common tumors and 0.05 (one tailed-level) for rare tumors.

Common tumors were defined as those with a historical incidence in controls of $1 \%$ or more and rare tumors as less than $1 \%$.

The sponsor used the Vehicle control group as a comparator to the active treatment groups. In addition the sponsor compared both the vehicle control and the water control and concluded that there was no statistically significant differences between these two controls.

Reviewer's comment: The above significance levels for dose response relationship test were suggested by Lin and Rahman (1998) and those for pairwise comparisons were suggested by Haseman (1983) to adjust for multiple testing (to keep the falsepositive rate at the nominal level of approximately $10 \%$ ).

Sponsor's findings: Sponsor's analyses showed no statistically significant positive dose response relationship or pairwise difference between control and any of the treated groups in any of the tested tumor types.

In the entire study, there were only three P-values that fell below 0.05 , and they all involved fibroadenomas for female 'Mammary Protocol' and 'Mammary (Other)' tissues, and the combination. In the assessment of 'Mammary Protocol', the incidence of fibroadenomas was higher in the water control group (10/70) than in the vehicle control group $(2 / 68)(\mathrm{P}=0.0205)$.

\subsection{Reviewer's analyses}

To verify sponsor's analyses and to perform additional analyses suggested by the reviewing pharmacologist, this reviewer independently performed survival and tumor data analyses. Data used in this reviewer's analyses were provided by the sponsor electronically.

\subsubsection{Survival analysis}

The survival distributions of animals in all four treatment groups (Vehicle control and 3 treatment groups) were estimated by the Kaplan-Meier product limit method. The dose response relationship was tested using the likelihood ratio test and homogeneity of survival distributions was tested using the log-rank test. The intercurrent mortality data are given in Tables $1 \mathrm{~A}$ and $1 \mathrm{~B}$ in the appendix for male and female rats, respectively. The KaplanMeier curves for survival rate are given in Figures $1 \mathrm{~A}$ and $1 \mathrm{~B}$ in the appendix for males and females, respectively. Results of the tests for dose response relationship and homogeneity of survivals, are given in Tables $2 \mathrm{~A}$ and $2 \mathrm{~B}$ in 
the appendix for males and females, respectively.

In addition, similar analyses were also conducted using only water control with the 3 active treatment groups. The intercurrent mortality data are given in Tables $1 \mathrm{~A} 0$ and $1 \mathrm{~B} 0$ in the appendix for male and female rats, respectively. Results of the tests for dose response relationship and homogeneity of survivals, are given in Tables $2 \mathrm{~A} 0$ and $2 \mathrm{~B} 0$ in the appendix for males and females, respectively.

Reviewer's findings: The tests showed no statistically significant dose response relationship or differences between the vehicle control or water control and any of the treated groups in survivals across treatment groups in both male and female rats.

\subsubsection{Tumor data analysis}

The tumor data were analyzed for dose response relationship and pairwise comparisons of vehicle control group with each of the treated groups were performed using the Poly-k method described in the paper of Bailer and Portier (1988) and Bieler and Williams (1993). One critical point for Poly-k test is the choice of the appropriate value of $\mathrm{k}$. For long term 104 week standard rat and mouse studies, a value of $\mathrm{k}=3$ is suggested in the literature. Hence, this reviewer used $\mathrm{k}=3$ for the analysis of this data. For the calculation of $\mathrm{p}$-values the exact permutation method was used. The tumor rates and the $\mathrm{p}$-values of the tested tumor types are listed in Tables $3 \mathrm{~A}$ and $3 \mathrm{~B}$ using vehicle control and Tables $3 \mathrm{~A} 0$ and $3 \mathrm{~B} 0$ using water control, in the appendix for males and females, respectively.

Multiple testing adjustment: The adjustment for the multiple dose response relationship testing was done using the criteria developed by Lin and Rahman (1998), which recommend the use of a significance level $\alpha=0.025$ for rare tumors and $\alpha=0.005$ for common tumors for a submission with two species, and the use of a significance level $\alpha=0.05$ for rare tumors and $\alpha=0.01$ for common tumors for a submission with only one species study in order to keep the false-positive rate at the nominal level of approximately $10 \%$. A rare tumor is defined as one in which the published spontaneous tumor rate is less than 1\%. The adjustment for multiple pairwise comparisons was done using the criteria developed by Haseman (1983), which recommend the use of a significance level $\alpha=0.05$ for rare tumors and $\alpha=0.01$ for common tumors, in order to keep the false-positive rate at the nominal level of approximately $10 \%$.

It should be noted that the recommended test levels by Lin and Rahman for the adjustment of multiple testing were originally based on the result of a simulation and an empirical study using the Peto method for dose response relationship analysis. However, some later simulation results by the same authors (Rahman and Lin, 2008) indicated similar usefulness of their recommendation for Poly-3 analysis also.

Reviewer's findings: Following tumor types showed p-values less than or equal to 0.05 either for dose response relationship and/or pairwise comparisons of control and treated groups. 


\section{Tumor Types with P-Values $\leq \mathbf{0 . 0 5}$ for Dose Response Relationship or Pairwise Comparisons Male Rats with Water control}

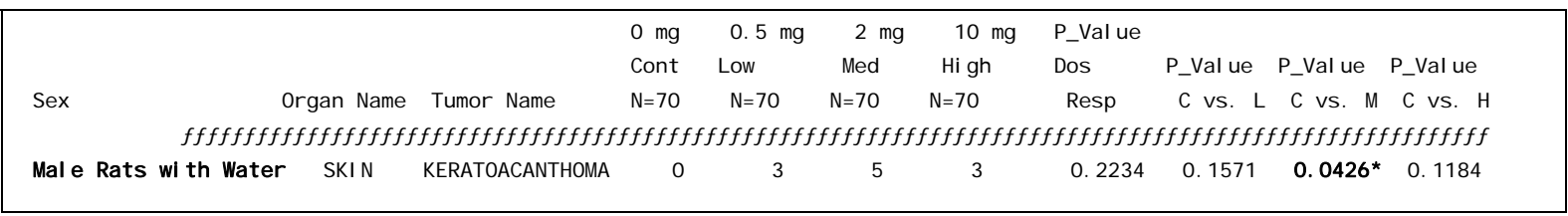

Based on the criteria of adjustment for multiple testing of trends by Lin and Rahman there was no statistically significant dose response relationship. However based on the criteria by Haseman, the pair-wise comparisons of treated groups with the vehicle control and water control, there was statistically significant increased tumor incidence of KERATOACANTHOMA in skin for the medium dose group when compared to the water control in the male rats.

\section{Mouse Study}

Two separate experiments were conducted, one in males and one in females. In each of these two experiments there were three treated groups, a vehicle control and a water control group. Three hundred mice of each sex were randomly allocated to treated and control groups. Each group has 60 mice in each of the sex groups. The vehicle groups consisted of a BMS-512148 at 0 (vehicle control), and a distilled water (water control) group. The treated groups received dose levels of 5, 15 and $40 \mathrm{mg} / \mathrm{kg} /$ day for the males, and 2, 10 and $20 \mathrm{mg} / \mathrm{kg} /$ day for the females. In this review these dose groups would be referred to as the vehicle control, water control, low, medium and high dose group, respectively. Terminal sacrifice for the male and female mice occurred after 104 weeks of treatment (days 729-735).

During the administration period all animals were observed for physical and clinical signs (palpable mass observation, body weight, food consumption and clinical pathology) and post mortem (gross pathology and histology). A complete histopathological examination was performed on all animals from all groups found dead, killed moribund, or sacrificed during or at the end of the experiment.

\subsubsection{Survival analysis}

\subsection{Sponsor's analyses}

Survival data from the mouse study were analyzed using the same statistical methodologies as those that were used to analyze the survival data from the rat study.

Sponsor's findings: The sponsor's analysis showed survival rates of $43 \%, 41 \%, 45 \%, 30 \%$ and $36 \%$, in vehicle control, water control, low, medium, and high dose groups, respectively in males and $47 \%, 35 \%, 33 \%$, $37 \%$ and $42 \%$, respectively in females. Sponsor concluded there was no statistically significant trend toward an increase in mortality in both sexes. No relevant differences between the vehcile controls and water control were found. 


\subsubsection{Tumor data analysis}

Tumor data from the mouse study were also analyzed using the same statistical methodologies as were used to analyze the tumor data from the rat study.

Sponsor's findings: The sponsor's analysis showed no statistically significant positive dose response relationship in both sexes. Pairwise comparisons showed no statistically significant increased incidence of tumor in higher dose groups when compared to the vehicle or water control group, except in two tumor types. One was observed in the assessment of liver hepatocellular adenomas for male mice in the comparison of the vehicle control and water control. Adenomas were found in 4 of the vehicle-control males and 14 of the water-control males ( $p$-value $=0.0087)$. The other was observed in the assessment of lymphatic system lymphomas for female mice in the comparison of the vehicle control and water control $(\mathrm{p}=0.0263)$. Lymphomas were found in 4 of the vehicle-control females and 10 of the water-control females.

\subsection{Reviewer's analyses}

This reviewer independently performed survival and tumor data analyses of the mouse study. For the mouse data analyses this reviewer used similar methodologies as those he used to analyze the data from the rat study. Data used in this reviewer's analyses were provided by the sponsor electronically.

\subsubsection{Survival analysis}

The intercurrent mortality data are given in Tables $4 \mathrm{~A}$ and $4 \mathrm{~B}$ using vehicle control and Tables $4 \mathrm{~A} 0$ and $4 \mathrm{~B} 0$ using water control in the appendix for males and females, respectively. The Kaplan-Meier curves for death rate are given in Figures 2A and 2B in the appendix for males and females, respectively. Results for test of dose response relationship and homogeneity of survivals among treatment groups are given in Tables 5A and 5B in the appendix for males and females, respectively.

Reviewer's findings: The tests showed no statistically significant dose response relationship or differences between the controls and any of the treated groups in survivals across treatment groups in male or female mice.

\subsubsection{Tumor data analysis}

The tumor rates and the p-values of the tumor types tested for dose response relationship and pairwise comparisons of control and treated groups are given in Table $6 \mathrm{~A}$ and $6 \mathrm{~B}$ in the appendix for males and females, respectively.

Reviewer's findings: Following tumor types showed p-values less than or equal to 0.05 either for dose response relationship or pairwise comparisons of control and treated groups. 


\section{Tumor Types with P-Values $\leq \mathbf{0 . 0 5}$ for Dose Response Relationship or Pairwise Comparisons}

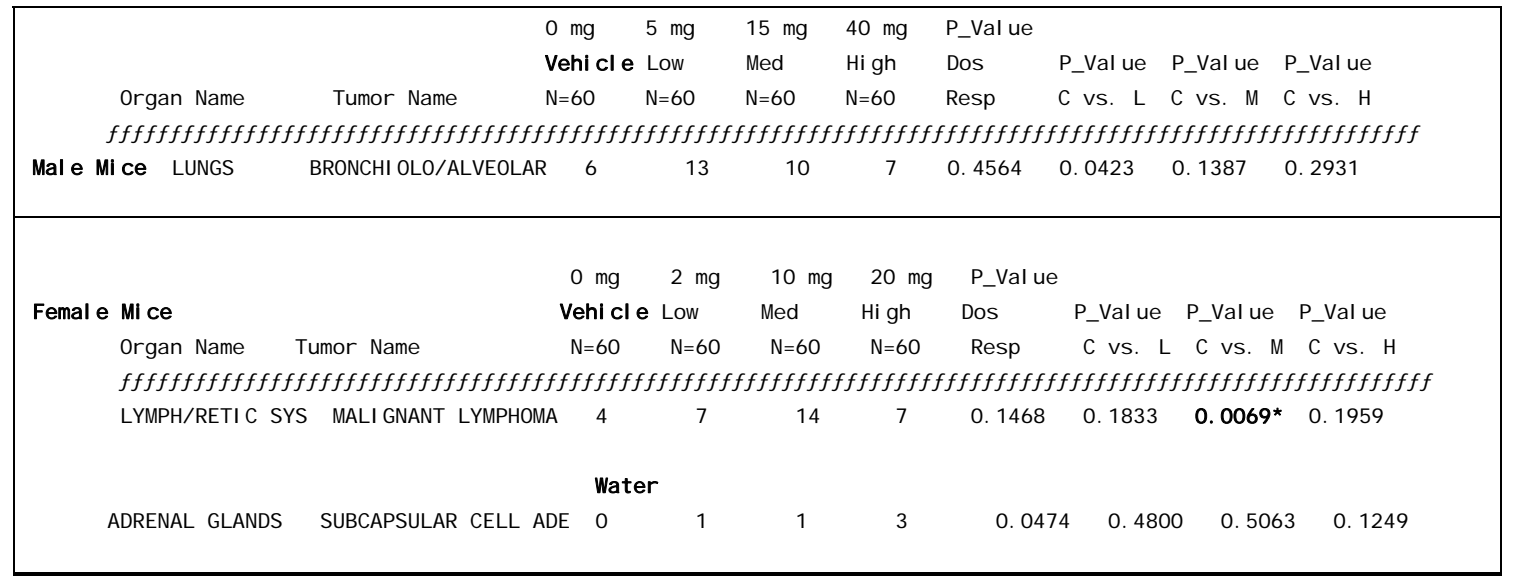

Based on the criteria of Lin and Rahman, the tumor incidences of MALIGNANT LYMPHOMA in LYMPH/RETIC SYS was statistically significant in the medium dose when compared to the vehicle control.

\section{Summary}

In this submission the sponsor included reports of two animal carcinogenicity studies, one in rats and one in mice. These studies were intended to assess the carcinogenic potential of Dapagliflozin (BMS-512148) in rats and mice when administered orally at appropriate drug levels for about 104 weeks.

Rat Study: Two separate experiments were conducted, one in males and one in females. In each of these two experiments there were three treated groups and two control groups. Three hundred and fifty rats each rats of each sex were randomly allocated to treated and control groups. Each group has 70 rats per sex. The dose levels for treated groups were $0.5,2$ and $10 \mathrm{mg} / \mathrm{kg} /$ day. In this review these dose groups would be referred to as the low, medium and high dose group, respectively. The vehicle control and distilled water control served to generate concurrent control data.

The tests showed no statistically significant dose response relationship or differences between the control and any of the treated groups in survivals and in tumor incidences rates across treatment groups in rats in both sex.

Mouse Study: Two separate experiments were conducted, one in males and one in females. In each of these two experiments there were three treated groups and two control groups. Three hundred mice of each sex were randomly allocated to treated and control groups. The control groups with 120 mice received vehicle control and distilled water control. The treated groups received dose levels of 5, 15 and $40 \mathrm{mg} / \mathrm{kg} / \mathrm{day}$ for the male mice, and 2, 10 and $20 \mathrm{mg} / \mathrm{kg} /$ day for the female mice and were in equal size of 60 animals per sex. In this review these dose groups would be referred to as the low, medium and high dose group, respectively.

Based on the criteria of Lin and Rahman, the tumor incidences of the MALIGNANT LYMPHOMA in LYMPH/RETIC SYS was statistically significant in the medium dose when compared to the vehicle control. 
Mohammad Nagem, Ph.D.

Mathematical Statistician

Concur: Karl Lin, Ph.D.

Team Leader, Biometrics-6

cc:

Archival NDA 202-293

Dr. Summan

Dr. Machado

Mehreen Hai

Dr. Lin

Ms. Lillian Patrician 


\section{Appendix}

Table 1A: Intercurrent Mortality Rate Male Rats with Vehicle control

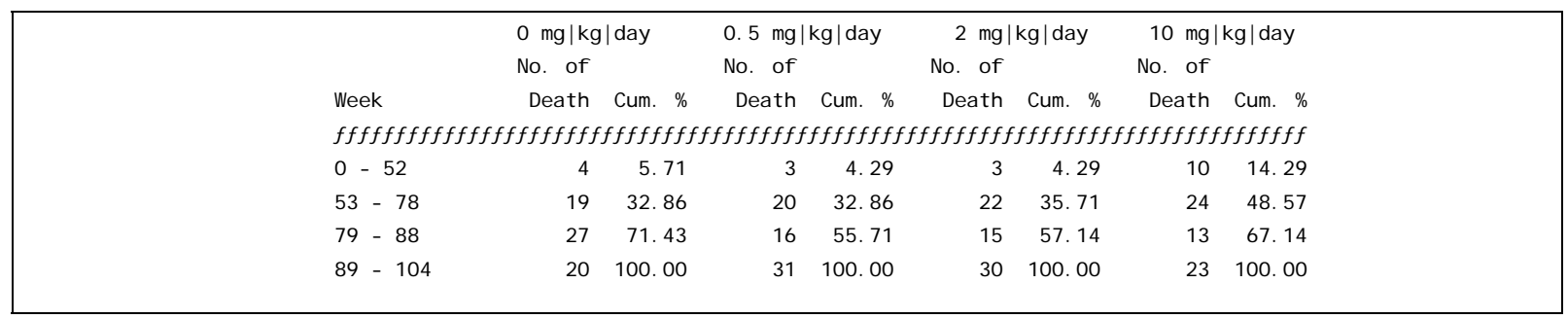

Table 1A0: Intercurrent Mortality Rate Male Rats with Water control

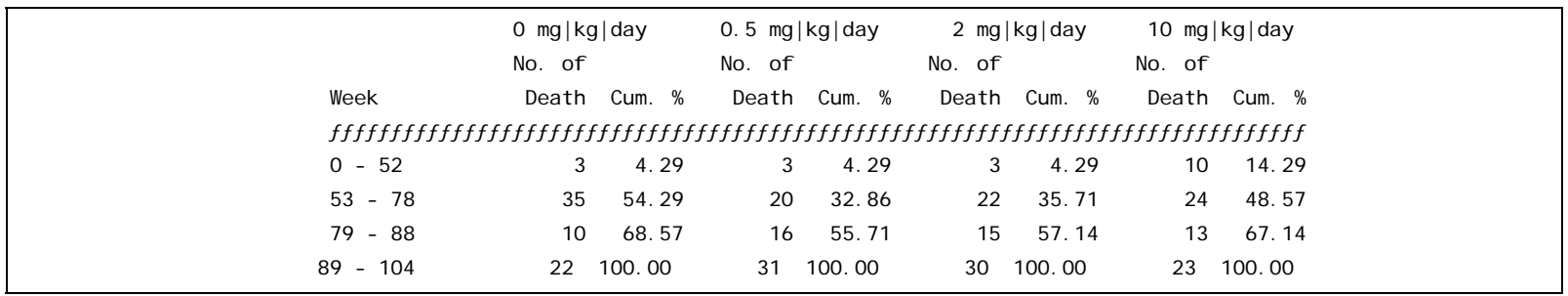

Table 1B: Intercurrent Mortality Rate

Female Rats with Vehicle control

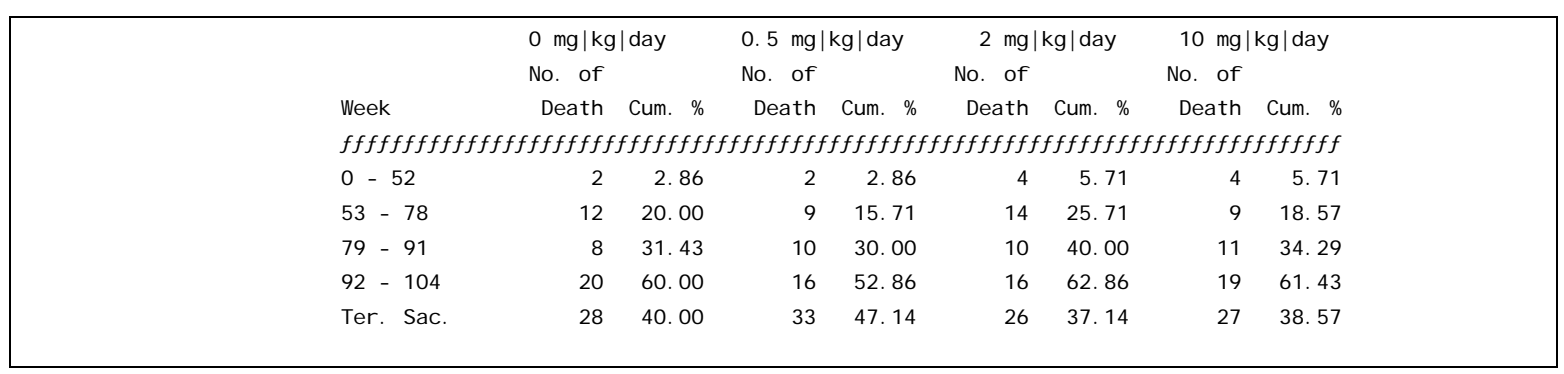

Table 1B0: Intercurrent Mortality Rate

Female Rats with Water control

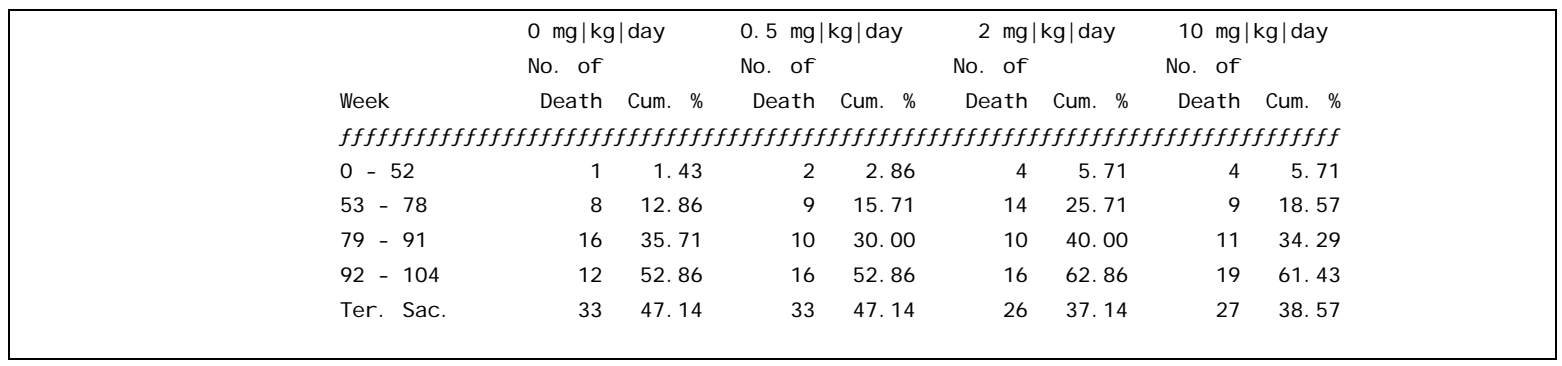


Table 2A: Tests for Dose Response Relationship and Homogeneity of Mortality Male Rats

\begin{tabular}{|lcc|}
\hline \multicolumn{3}{|c|}{ P-value } \\
\hline Test & Using Vehicle & Using Water control \\
\hline Dose Response & 0.1348 & 0.1615 \\
Homogeneity & 0.0702 & 0.0542 \\
\hline
\end{tabular}

Table 2B: Tests for Dose Response Relationship and Homogeneity of Mortality Female Rats

\begin{tabular}{|lcc|}
\hline \multicolumn{3}{|c|}{ P-value } \\
\hline Test & Using Vehicle & Using Water control \\
\hline Dose Response & 0.8162 & 0.5571 \\
Homogeneity & 0.6382 & 0.4461 \\
\hline
\end{tabular}


NDA 202-293 BMS-512148

Page 12 of 26

Table 3A: Tumor Rates and P-Values for Dose Response Relationship and Pairwise Comparisons Male Rats with Vehicle control

\begin{tabular}{|c|c|c|c|c|c|c|c|c|c|}
\hline & & $0 \mathrm{mg}$ & $0.5 \mathrm{mg}$ & $2 \mathrm{~m}$ & 10 & $P_{-}$Val & & & \\
\hline & & Cont & Low & Med & $\mathrm{High}$ & Dos & $P_{-}$Val ue & $P_{-}$Val ue & $P_{\text {_ Val ue }}$ \\
\hline Organ Name & Tumor Name & $N=70$ & $N=70$ & $N=70$ & $N=70$ & Resp & C VS. L & C VS. M & C VS. H \\
\hline$f f f f f f f f f f f f f f f f$ & $f f f f f f f f f f f f f f f f f f f f f f$ & $f f f f f$ & $f f f f f f$ & $f f f f f$ & $f f f f f f$ & $f f f f f f f f$ & $f f f f f f f f$ & $f f f f f f f f$ & $f f f f f f f f$ \\
\hline ADRENAL GLANDS & CORTEX: ADENOMA & 1 & 1 & 1 & 3 & 0.1009 & 0.7764 & 0.7693 & 0.2937 \\
\hline & CORTEX: CARCI NOMA & 0 & 0 & 0 & 1 & 0.2222 & . & . & 0.4746 \\
\hline & MEDULLA BENI GN PHEOC & 12 & 6 & 9 & 9 & 0.4002 & 0.9677 & 0.8645 & 0.7414 \\
\hline & MEDULLA: MALI GNANT $P$ & 0 & 2 & 2 & 0 & 0.7050 & 0.2774 & 0.2619 & . \\
\hline BONE (OTHER) & ODONTOMA: COMPOUND & 0 & 0 & 0 & 1 & 0.2283 & . & . & 0.4833 \\
\hline BRAI N & ASTROCYTOMA & 0 & 1 & 0 & 0 & 0.7540 & 0.5231 & . & . \\
\hline & OLI GODENDROGLI OMA & 0 & 1 & 0 & 0 & 0.7540 & 0.5231 & . & . \\
\hline DUODENUM & ADENOCARCI NOMA & 1 & 1 & 0 & 0 & 0.9410 & 0.7764 & 1.0000 & 1.0000 \\
\hline $\operatorname{EAR}(S)$ & SQUAMOUS CELL CARCIN & 0 & 0 & 0 & 1 & 0.2222 & . & . & 0.4746 \\
\hline EYES & ME L ANOMA & 0 & 0 & 1 & 0 & 0.4841 & . & 0.5156 & . \\
\hline HEART & I NVASI VE/ METASTATIC & 0 & 0 & 1 & 0 & 0.4841 & . & 0.5156 & . \\
\hline & PARAGANGLI OMA & 0 & 0 & 0 & 1 & 0.2222 & . & . & 0.4746 \\
\hline JEJ UNUM & ADENOCARCI NOMA & 0 & 1 & 0 & 1 & 0.2862 & 0.5231 & . & 0.4746 \\
\hline KI DNEYS & HEMANGI OMA & 0 & 0 & 1 & 0 & 0.4841 & . & 0.5156 & . \\
\hline & NEPHROBLASTOMA & 0 & 1 & 0 & 0 & 0.7559 & 0.5303 & . & . \\
\hline & TUBULE ADENOMA & 1 & 0 & 0 & 1 & 0.3964 & 1.0000 & 1.0000 & 0.7282 \\
\hline & TUBULE CARCINOMA & 0 & 0 & 1 & 0 & 0.4841 & . & 0.5156 & . \\
\hline LI VER & HEPATOCELLULAR ADENO & 0 & 2 & 1 & 2 & 0.1600 & 0.2774 & 0.5156 & 0.2209 \\
\hline & HEPATOCELLULAR CARCI & 0 & 0 & 1 & 0 & 0.4841 & . & 0.5156 & . \\
\hline LUNGS & BRONCHI OLO/ ALVEOLAR & 0 & 0 & 1 & 0 & 0.4841 & . & 0.5156 & . \\
\hline LYMPH/RETIC SYS & GRANULOCYTI C LEUKEMI & 0 & 0 & 1 & 0 & 0.4841 & . & 0.5156 & . \\
\hline & HISTIOCYTIC SARCOMA & 1 & 0 & 0 & 0 & 1.0000 & 1.0000 & 1.0000 & 1.0000 \\
\hline & MALI GNANT LYMPHOMA & 0 & 3 & 3 & 0 & 0.8042 & 0.1430 & 0.1430 & . \\
\hline MAMMARY (OTHER) & FI BROADENOMA & 0 & 0 & 1 & 0 & 0.4841 & . & 0.5156 & . \\
\hline MESENTERI C LN & HEMANGI OMA & 1 & 1 & 0 & 1 & 0.4927 & 0.7764 & 1.0000 & 0.7282 \\
\hline MESENTERY / PERI T & SARCOMA (NOS) & 1 & 0 & 0 & 0 & 1.0000 & 1.0000 & 1.0000 & 1.0000 \\
\hline MUSCLE (OTHER) & HEMANGI OSARCOMA & 1 & 0 & 0 & 0 & 1.0000 & 1.0000 & 1.0000 & 1.0000 \\
\hline MUSCLE PROTOCOL & HEMANGI OMA & 0 & 0 & 1 & 0 & 0.4841 & . & 0.5156 & . \\
\hline PANCREAS & ACI NAR CELL ADENOMA & 1 & 2 & 0 & 2 & 0.2732 & 0.5461 & 1.0000 & 0.4612 \\
\hline & ACINAR CELL CARCINOM & 0 & 0 & 1 & 0 & 0.4841 & . & 0.5156 & . \\
\hline & HEMANGI OSARCOMA & 0 & 0 & 1 & 0 & 0.4841 & . & 0.5156 & . \\
\hline & I SLET CELL ADENOMA & 2 & 0 & 1 & 1 & 0.5823 & 1.0000 & 0.8921 & 0.8617 \\
\hline & I SLET CELL CARCI NOMA & 0 & 1 & 0 & 1 & 0.2862 & 0.5231 & . & 0.4746 \\
\hline PARATHYROI D & ADENOMA & 0 & 0 & 1 & 0 & 0.4841 & . & 0.5156 & . \\
\hline PI TUI TARY GLAND & CRANI OPHARYNGI OMA & 0 & 0 & 0 & 1 & 0.2222 & . & . & 0.4746 \\
\hline & PARS DISTALIS-ADENOM & 13 & 8 & 9 & 9 & 0.4850 & 0.9474 & 0.8985 & 0.7865 \\
\hline & PARS I NTERMEDIA: ADE & 0 & 0 & 0 & 1 & 0.2222 & . & . & 0.4746 \\
\hline PREPUT/CLIT GL & CARCI NOMA & 0 & 1 & 0 & 0 & 0.7540 & 0.5231 & . & . \\
\hline SEMI NAL VESI CLE & ADENOMA & 0 & 0 & 1 & 0 & 0.4841 & . & 0.5156 & . \\
\hline SKIN & FI BROMA & 2 & 0 & 1 & 0 & 0.9345 & 1.0000 & 0.8864 & 1.0000 \\
\hline & FI BROSARCOMA & 2 & 0 & 0 & 0 & 1.0000 & 1.0000 & 1.0000 & 1.0000 \\
\hline & HEMANGI OSARCOMA & 1 & 0 & 0 & 0 & 1.0000 & 1.0000 & 1.0000 & 1.0000 \\
\hline & KERATOACANTHOMA & 4 & 3 & 5 & 3 & 0.5468 & 0.8099 & 0.5385 & 0.7301 \\
\hline & SEBACEOUS CELL ADENO & 0 & 1 & 0 & 0 & 0.7540 & 0.5231 & . & . \\
\hline & SQUAMOUS CELL CARCIN & 0 & 0 & 1 & 0 & 0.4841 & . & 0.5156 & . \\
\hline & SQUAMOUS CELL PAPILL & 2 & 0 & 0 & 0 & 1.0000 & 1.0000 & 1.0000 & 1.0000 \\
\hline SPLEEN & HEMANGI OSARCOMA & 0 & 0 & 1 & 0 & 0.4841 & . & 0.5156 & . \\
\hline STOMACH & FORESTOMACH: SQUAMOU & 0 & 1 & 0 & 0 & 0.7540 & 0.5231 & . & . \\
\hline TAI L & KERATOACANTHOMA & 0 & 0 & 2 & 0 & 0.4638 & . & 0.2697 & . \\
\hline TESTES & BENI GN I NTERSTI TI AL & 1 & 0 & 0 & 0 & 1.0000 & 1.0000 & 1.0000 & 1.0000 \\
\hline & MES OT HEL I OMA & 0 & 1 & 0 & 0 & 0.7540 & 0.5231 & . & . \\
\hline THYROI D & C. CELL ADENOMA & 5 & 1 & 3 & 1 & 0.8899 & 0.9889 & 0.8803 & 0.9801 \\
\hline & C. CELL CARCI NOMA & 0 & 1 & 1 & 0 & 0.6060 & 0.5231 & 0.5156 & . \\
\hline & FOLLI CULAR CELL ADEN & 0 & 1 & 1 & 1 & 0.2658 & 0.5231 & 0.5156 & 0.4746 \\
\hline & FOLLI CULAR CELL CARC & 2 & 1 & 1 & 0 & 0.9374 & 0.8916 & 0.8864 & 1.0000 \\
\hline ZYMBAL'S GLAND & CARCI NOMA & 0 & 0 & 1 & 0 & 0.4841 & . & 0.5156 & . \\
\hline
\end{tabular}


Table 3A0: Tumor Rates and P-Values for Dose Response Relationship and Pairwise Comparisons Male Rats with Water control

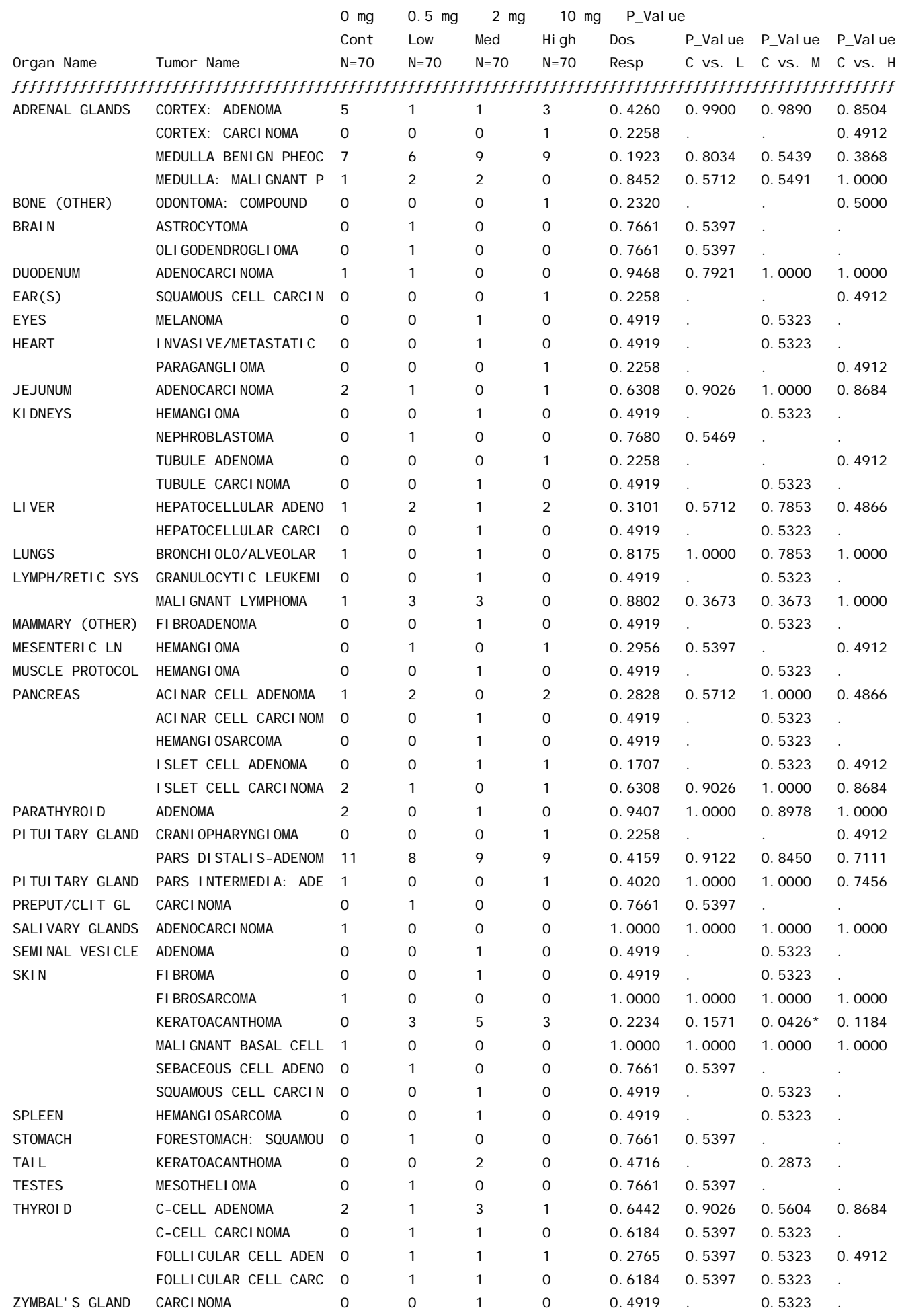


Table 3B: Tumor Rates and P-Values for Dose Response Relationship and Pairwise Comparisons Female Rats with Vehicle control

\begin{tabular}{|c|c|c|c|c|c|c|c|c|c|}
\hline & & $0 \mathrm{mg}$ & $0.5 \mathrm{mg}$ & $2 \mathrm{mg}$ & $10 \mathrm{~m}$ & $P_{-}$Val & & & \\
\hline & & Cont & Low & Med & $\mathrm{High}$ & Dos & $P_{-}$Val u & e $P_{-} V_{a l}$ & ue $P_{-}$Value \\
\hline Organ Name & Tumor Name & $N=70$ & $N=70$ & $N=70$ & $N=70$ & Resp & C vs. & L C VS. & M C V S. \\
\hline$f f f f f f f f f f f f f f f f$ & $f f f f f f f f f f f f f f f f f f f f f f$ & $f f f f f$ & $f f f f$ & $f f f f f$ & 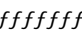 & $f f f f f$ & fffffff & $f f f f f$. & $f f f f f f f$ \\
\hline ADRENAL GLANDS & CORTEX: ADENOMA & 2 & 0 & 0 & 0 & 1.0000 & 1.0000 & 1.0000 & 1.0000 \\
\hline & CORTEX: CARCI NOMA & 0 & 1 & 1 & 1 & 0.2907 & 0.5000 & 0.4757 & 0.4953 \\
\hline & MEDULLA BENIGN PHEOC & 5 & 4 & 5 & 6 & 0.3002 & 0.7557 & 0.5792 & 0.4867 \\
\hline & MEDULLA: MALI GNANT P & 1 & 0 & 1 & 1 & 0.4329 & 1.0000 & 0.7328 & 0.7477 \\
\hline BRAI N & ASTROCYTOMA & 0 & 0 & 1 & 0 & 0.4857 & . & 0.4808 & . \\
\hline DI APHRAGM & HEMANGI OSARCOMA & 0 & 1 & 0 & 0 & 0.7429 & 0.5046 & . & . \\
\hline I LEUM & LEI OMYOSARCOMA & 0 & 0 & 0 & 1 & 0.2488 & . & . & 0.4906 \\
\hline JEJ UNUM & ADENOCARCI NOMA & 0 & 0 & 1 & 0 & 0.4857 & . & 0.4808 & . \\
\hline KI DNEYS & LI POMA & 0 & 0 & 1 & 0 & 0.4833 & . & 0.4757 & . \\
\hline LI VER & HEMANGI OSARCOMA & 0 & 1 & 0 & 1 & 0.3110 & 0.5046 & . & 0.4953 \\
\hline & HEPATOCELLULAR ADENO & 3 & 0 & 2 & 1 & 0.7414 & 1.0000 & 0.7869 & 0.9363 \\
\hline & HEPATOCELLULAR CARCI & 1 & 3 & 0 & 1 & 0.6839 & 0.3089 & 1.0000 & 0.7429 \\
\hline LUNGS & HEMANGI OSARCOMA & 0 & 0 & 0 & 1 & 0.2524 & . & . & 0.4953 \\
\hline LYMPH/RETIC SYS & HISTI OCYTIC SARCOMA & 1 & 0 & 0 & 0 & 1.0000 & 1.0000 & 1.0000 & 1.0000 \\
\hline & LARGE GRANULAR CELL & 0 & 1 & 0 & 1 & 0.3074 & 0.5000 & . & 0.4906 \\
\hline & MALI GNANT LYMPHOMA & 0 & 1 & 1 & 1 & 0.2864 & 0.5000 & 0.4808 & 0.4906 \\
\hline MAMMARY ( OTHER) & ADENOCARCI NOMA & 9 & 9 & 9 & 10 & 0.4235 & 0.5849 & 0.5333 & 0.5170 \\
\hline & ADENOMA & 1 & 0 & 1 & 2 & 0.1881 & 1.0000 & 0.7328 & 0.4929 \\
\hline & FI BROADENOMA & 33 & 36 & 30 & 28 & 0.8269 & 0.3210 & 0.5172 & 0.7656 \\
\hline MAMMARY PROTOCO & ADENOCARCI NOMA & 0 & 3 & 0 & 0 & 0.8337 & 0.1215 & . & . \\
\hline & ADENOMA & 1 & 0 & 0 & 0 & 1.0000 & 1.0000 & 1.0000 & 1.0000 \\
\hline & FI BROADENOMA & 2 & 5 & 2 & 2 & 0.6790 & 0.2342 & 0.6615 & 0.6767 \\
\hline MESENTERY / PERI T & HEMANGI OSARCOMA & 0 & 0 & 1 & 0 & 0.4833 & . & 0.4757 & . \\
\hline MUSCLE (OTHER) & SCHWANNOMA & 1 & 0 & 0 & 0 & 1.0000 & 1.0000 & 1.0000 & 1.0000 \\
\hline OVARIES & BENI GN GRANULOSA CEL & 0 & 0 & 0 & 1 & 0.2488 & . & . & 0.4906 \\
\hline & SERTOLI CELL TUMOR & 1 & 0 & 0 & 0 & 1.0000 & 1.0000 & 1.0000 & 1.0000 \\
\hline & THECOMA & 0 & 1 & 0 & 0 & 0.7416 & 0.5000 & . & . \\
\hline PANCREAS & I SLET CELL ADENOMA & 1 & 2 & 1 & 2 & 0.3404 & 0.5000 & 0.7328 & 0.4857 \\
\hline & I SLET CELL CARCINOMA & 1 & 0 & 0 & 1 & 0.4420 & 1.0000 & 1.0000 & 0.7477 \\
\hline PI TUI TARY GLAND & PARS DISTALIS-ADENOM & 37 & 27 & 29 & 27 & 0.7375 & 0.9371 & 0.7659 & 0.8912 \\
\hline & PARS DISTALIS: CARCI & 7 & 5 & 6 & 5 & 0.6565 & 0.8112 & 0.6571 & 0.8018 \\
\hline & PARS I NTERMEDIA: ADE & 3 & 1 & 0 & 1 & 0.7651 & 0.9410 & 1.0000 & 0.9387 \\
\hline RETROPERI TONEAL & PARAGANGLI OMA & 0 & 1 & 0 & 0 & 0.7416 & 0.5000 & . & . \\
\hline SKIN & FI BROMA & 0 & 1 & 0 & 1 & 0.3116 & 0.5000 & . & 0.4953 \\
\hline & FI BROUS HISTI OCYTOMA & 1 & 1 & 0 & 0 & 0.9342 & 0.7523 & 1.0000 & 1.0000 \\
\hline & MALI GNANT SCHWANNOMA & 0 & 1 & 0 & 0 & 0.7429 & 0.5046 & & . \\
\hline & MYXOSARCOMA & 0 & 0 & 0 & 1 & 0.2524 & . & . & 0.4953 \\
\hline & SEBACEOUS CELL CARCI & 0 & 0 & 0 & 1 & 0.2524 & . & . & 0.4953 \\
\hline & SQUAMOUS CELL CARCIN & 1 & 0 & 0 & 0 & 1.0000 & 1.0000 & 1.0000 & 1.0000 \\
\hline SPLEEN & HEMANGI OSARCOMA & 0 & 0 & 0 & 1 & 0.2524 & . & . & 0.4953 \\
\hline THYROI D & C. CELL ADENOMA & 11 & 13 & 10 & 5 & 0.9778 & 0.4087 & 0.6329 & 0.9697 \\
\hline & C. CELL CARCI NOMA & 2 & 0 & 1 & 0 & 0.9319 & 1.0000 & 0.8597 & 1.0000 \\
\hline & FOLLI CULAR CELL CARC & 0 & 0 & 0 & 1 & 0.2524 & . & . & 0.4953 \\
\hline & GANGL I ONEUROMA & 0 & 0 & 0 & 1 & 0.2524 & . & . & 0.4953 \\
\hline UTERUS W/ CERVI & ENDOMETRIAL ADENOCAR & 0 & 2 & 3 & 2 & 0.2663 & 0.2477 & 0.1076 & 0.2430 \\
\hline & ENDOMETRIAL ADENOMA & 0 & 0 & 1 & 0 & 0.4833 & . & 0.4757 & . \\
\hline & ENDOMETRIAL STROMAL & 10 & 1 & 2 & 3 & 0.8594 & 0.9997 & 0.9967 & 0.9908 \\
\hline & HEMANGI OSARCOMA & 0 & 0 & 0 & 1 & 0.2488 & . & . & 0.4906 \\
\hline & LEI OMYOSARCOMA & 0 & 0 & 1 & 0 & 0.4857 & . & 0.4808 & . \\
\hline & MALI GNANT SCHWANNOMA & 3 & 2 & 1 & 1 & 0.8320 & 0.8183 & 0.9287 & 0.9364 \\
\hline & PAPI LLOMA, SQUAMOUS & 1 & 1 & 1 & 0 & 0.8351 & 0.7523 & 0.7328 & 1.0000 \\
\hline & SQUAMOUS CELL CARCIN & 0 & 0 & 2 & 0 & 0.4906 & . & 0.2287 & . \\
\hline ZYMBAL'S GLAND & CARCI NOMA & 0 & 0 & 0 & 1 & 0.2488 & . & . & 0.4906 \\
\hline
\end{tabular}


Table 3B0: Tumor Rates and P-Values for Dose Response Relationship and Pairwise Comparisons Female Rats with water control

\begin{tabular}{|c|c|c|c|c|c|c|c|c|c|}
\hline & & $0 \mathrm{mg}$ & $0.5 \mathrm{mg}$ & $2 \mathrm{~m}$ & 10 & $P_{\text {_ }}$ Val & & & \\
\hline & & Cont & Low & Med & $\mathrm{High}$ & Dos & $P_{-}$Val ue & $P_{-}$Val ue & $P_{\text {_ Val ue }}$ \\
\hline Organ Name & Tumor Name & $N=70$ & $N=70$ & $N=70$ & $N=70$ & Resp & C VS. L & C Vs. M & C VS. H \\
\hline$f f f f f f f f f f f f f f f f$ & $f f f f f f f f f f f f f f f f f f f f f f$ & $f f f f f f$ & $f f f f f f f$ & $f f f f f f$ & $f f f f f$ & $f f f f f f f$ & $f f f f f f f f$ & $f f f f f f f$ & $f f f f f f f f$ \\
\hline ADRENAL GLANDS & CORTEX: ADENOMA & 5 & 0 & 0 & 0 & 1.0000 & 1.0000 & 1.0000 & 1.0000 \\
\hline & CORTEX: CARCI NOMA & 0 & 1 & 1 & 1 & 0.2907 & 0.5000 & 0.4757 & 0.4953 \\
\hline & MEDULLA BENI GN PHEOC & 6 & 4 & 5 & 6 & 0.3658 & 0.8323 & 0.6790 & 0.5930 \\
\hline & MEDULLA: MALI GNANT P & 0 & 0 & 1 & 1 & 0.1818 & . & 0.4808 & 0.4953 \\
\hline BRAI N & ASTROCYTOMA & 0 & 0 & 1 & 0 & 0.4857 & . & 0.4808 & . \\
\hline DI APHRAGM & HEMANGI OSARCOMA & 0 & 1 & 0 & 0 & 0.7429 & 0.5046 & . & . \\
\hline DUODENUM & ADENOCARCI NOMA & 1 & 0 & 0 & 0 & 1.0000 & 1.0000 & 1.0000 & 1.0000 \\
\hline EYES & IRIS: LEI OMYOMA & 1 & 0 & 0 & 0 & 1.0000 & 1.0000 & 1.0000 & 1.0000 \\
\hline I LEUM & LEI OMYOSARCOMA & 0 & 0 & 0 & 1 & 0.2488 & . & . & 0.4906 \\
\hline JEJ UNUM & ADENOCARCI NOMA & 0 & 0 & 1 & 0 & 0.4857 & . & 0.4808 & . \\
\hline KI DNEYS & LI POMA & 1 & 0 & 1 & 0 & 0.8000 & 1.0000 & 0.7276 & 1.0000 \\
\hline LI VER & HEMANGI OSARCOMA & 0 & 1 & 0 & 1 & 0.3110 & 0.5046 & . & 0.4953 \\
\hline & HEPATOCELLULAR ADENO & 3 & 0 & 2 & 1 & 0.7373 & 1.0000 & 0.7804 & 0.9339 \\
\hline & HEPATOCELLULAR CARCI & 1 & 3 & 0 & 1 & 0.6839 & 0.3089 & 1.0000 & 0.7429 \\
\hline LUNGS & HEMANGI OSARCOMA & 0 & 0 & 0 & 1 & 0.2524 & . & . & 0.4953 \\
\hline LYMPH/RETIC SYS & LARGE GRANULAR CELL & 0 & 1 & 0 & 1 & 0.3074 & 0.5000 & . & 0.4906 \\
\hline & MALI GNANT LYMPHOMA & 1 & 1 & 1 & 1 & 0.5125 & 0.7477 & 0.7280 & 0.7381 \\
\hline MAMMARY (OTHER) & ADENOCARCI NOMA & 10 & 9 & 9 & 10 & 0.4624 & 0.6458 & 0.5947 & 0.5801 \\
\hline & ADENOMA & 1 & 0 & 1 & 2 & 0.1857 & 1.0000 & 0.7280 & 0.4860 \\
\hline & FI BROADENOMA & 43 & 36 & 30 & 28 & 0.9695 & 0.8713 & 0.9453 & 0.9894 \\
\hline MAMMARY PROTOCO & ADENOCARCI NOMA & 0 & 3 & 0 & 0 & 0.8337 & 0.1215 & . & . \\
\hline & FI BROADENOMA & 10 & 5 & 2 & 2 & 0.9789 & 0.9532 & 0.9964 & 0.9970 \\
\hline MESENTERY / PERIT & HEMANGI OSARCOMA & 0 & 0 & 1 & 0 & 0.4833 & . & 0.4757 & . \\
\hline OVARIES & BENI GN GRANULOSA CEL & 0 & 0 & 0 & 1 & 0.2488 & . & . & 0.4906 \\
\hline & THECOMA & 0 & 1 & 0 & 0 & 0.7416 & 0.5000 & . & . \\
\hline PANCREAS & I SLET CELL ADENOMA & 2 & 2 & 1 & 2 & 0.4806 & 0.6911 & 0.8638 & 0.6767 \\
\hline & I SLET CELL CARCI NOMA & 2 & 0 & 0 & 1 & 0.5821 & 1.0000 & 1.0000 & 0.8715 \\
\hline PITUI TARY GLAND & PARS DISTALIS-ADENOM & 40 & 27 & 29 & 27 & 0.8296 & 0.9809 & 0.8962 & 0.9615 \\
\hline & PARS DISTALIS: CARCI & 5 & 5 & 6 & 5 & 0.5109 & 0.6173 & 0.4325 & 0.6052 \\
\hline & PARS I NTERMEDIA: ADE & 0 & 1 & 0 & 1 & 0.3116 & 0.5000 & . & 0.4953 \\
\hline PREPUT/CLIT GL & CARCI NOMA & 1 & 0 & 0 & 0 & 1.0000 & 1.0000 & 1.0000 & 1.0000 \\
\hline RETROPERI TONEAL & PARAGANGLI OMA & 0 & 1 & 0 & 0 & 0.7416 & 0.5000 & . & . \\
\hline SKIN & FI BROMA & 2 & 1 & 0 & 1 & 0.6713 & 0.8750 & 1.0000 & 0.8715 \\
\hline & FIBROUS HISTI OCYTOMA & 0 & 1 & 0 & 0 & 0.7416 & 0.5000 & . & . \\
\hline & MALI GNANT SCHWANNOMA & 0 & 1 & 0 & 0 & 0.7429 & 0.5046 & . & . \\
\hline & MYXOSARCOMA & 0 & 0 & 0 & 1 & 0.2524 & . & . & 0.4953 \\
\hline & SEBACEOUS CELL CARCI & 0 & 0 & 0 & 1 & 0.2524 & . & . & 0.4953 \\
\hline SPLEEN & HEMANGI OSARCOMA & 0 & 0 & 0 & 1 & 0.2524 & . & . & 0.4953 \\
\hline THYROI D & C. CELL ADENOMA & 12 & 13 & 10 & 5 & 0.9804 & 0.4387 & 0.6636 & 0.9759 \\
\hline & C. CELL CARCI NOMA & 3 & 0 & 1 & 0 & 0.9786 & 1.0000 & 0.9285 & 1.0000 \\
\hline & FOLLI CULAR CELL CARC & 0 & 0 & 0 & 1 & 0.2524 & . & . & 0.4953 \\
\hline & GANGLI ONEUROMA & 0 & 0 & 0 & 1 & 0.2524 & . & . & 0.4953 \\
\hline UTERUS W/ CERVI & ENDOMETRIAL ADENOCAR & 1 & 2 & 3 & 2 & 0.3825 & 0.4931 & 0.2737 & 0.4860 \\
\hline & ENDOMETRIAL ADENOMA & 0 & 0 & 1 & 0 & 0.4833 & . & 0.4757 & . \\
\hline & ENDOMETRIAL STROMAL & 3 & 1 & 2 & 3 & 0.3308 & 0.9387 & 0.7804 & 0.6336 \\
\hline & HEMANGI OSARCOMA & 0 & 0 & 0 & 1 & 0.2488 & . & . & 0.4906 \\
\hline & LEI OMYOSARCOMA & 0 & 0 & 1 & 0 & 0.4857 & . & 0.4808 & . \\
\hline & MALI GNANT SCHWANNOMA & 2 & 2 & 1 & 1 & 0.7375 & 0.6841 & 0.8563 & 0.8680 \\
\hline & PAPI LLOMA, SQUAMOUS & 0 & 1 & 1 & 0 & 0.6137 & 0.5000 & 0.4808 & . \\
\hline & SQUAMOUS CELL CARCIN & 1 & 0 & 2 & 0 & 0.7197 & 1.0000 & 0.4640 & 1.0000 \\
\hline ZYMBAL'S GLAND & CARCI NOMA & 0 & 0 & 0 & 1 & 0.2488 & . & . & 0.4906 \\
\hline
\end{tabular}


Table 4A: Intercurrent Mortality Rate in Male Mice with Vehicle control

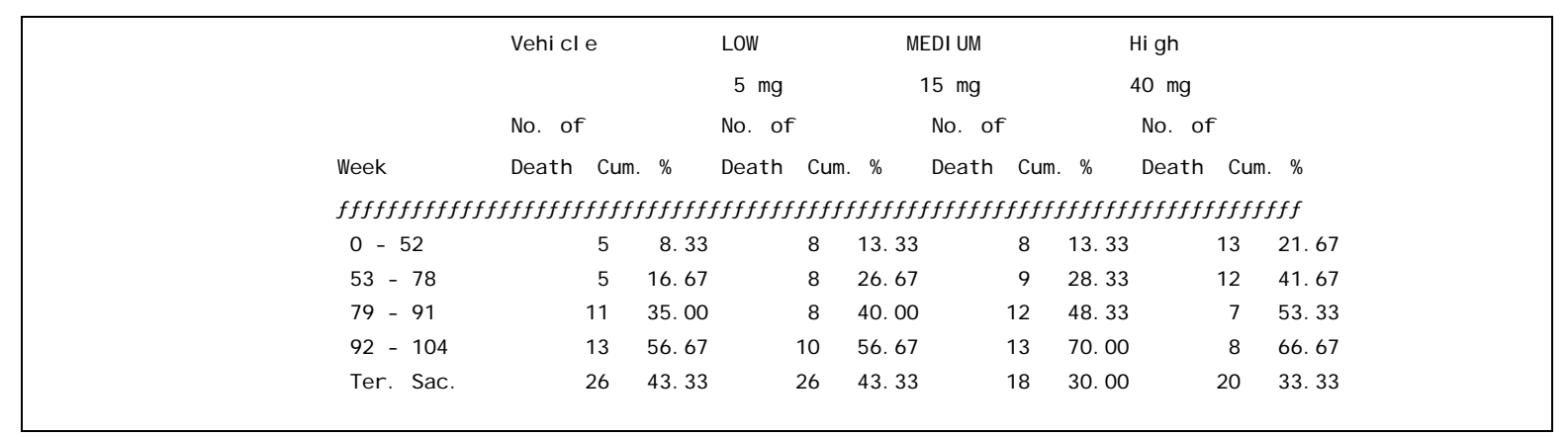

Table 4A0: Intercurrent Mortality Rate in Male Mice with Water control

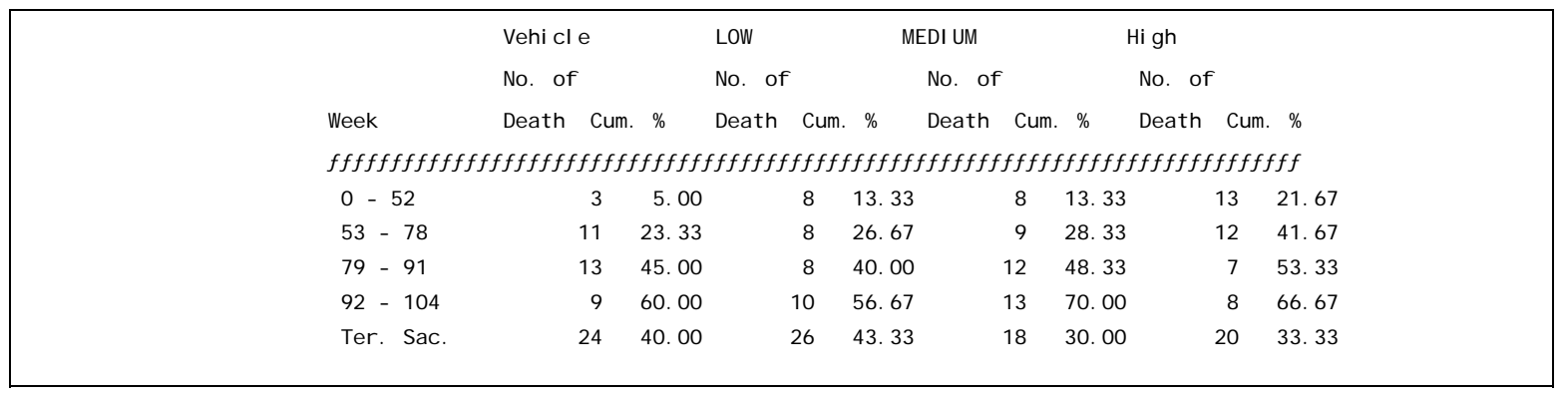

Table 4B: Intercurrent Mortality Rate

Female Mice with Vehicle control

\begin{tabular}{|c|c|c|c|c|c|c|c|c|}
\hline \multirow[b]{2}{*}{ Week } & \multicolumn{2}{|c|}{$\begin{array}{l}0 \mathrm{mg}|\mathrm{kg}| \mathrm{day} \\
\text { No. of }\end{array}$} & \multicolumn{2}{|c|}{$\begin{array}{l}2 \mathrm{mg}|\mathrm{kg}| \mathrm{day} \\
\text { No. of }\end{array}$} & \multicolumn{2}{|c|}{$\begin{array}{l}10 \mathrm{mg} / \mathrm{kg} \mid \mathrm{day} \\
\text { No. of }\end{array}$} & \multicolumn{2}{|c|}{$\begin{array}{l}20 \mathrm{mg}|\mathrm{kg}| \mathrm{day} \\
\text { No. of }\end{array}$} \\
\hline & Death & Cum. $\%$ & Death & Cum. $\%$ & Death & Cum. \% & Deat $h$ & Cum. \% \\
\hline$f f f f f f f f$ & $f f f f f f$ & $f f f f f f f$ & $f f f f f f$ & $f f f f f f$ & $f f f f f f$ & $f f f f f f$ & $f f f f f$ & $f f f f f$ \\
\hline $0 \cdot 52$ & 8 & 13.33 & 12 & 20.00 & 6 & 10.00 & 10 & 16.67 \\
\hline $53 \cdot 78$ & 5 & 21.67 & 6 & 30.00 & 13 & 31.67 & 5 & 25.00 \\
\hline $79 \cdot 91$ & 7 & 33.33 & 11 & 48.33 & 9 & 46.67 & 9 & 40.00 \\
\hline $92 \cdot 104$ & 12 & 53.33 & 12 & 68.33 & 10 & 63.33 & 11 & 58.33 \\
\hline Ter. Sac. & 28 & 46.67 & 19 & 31.67 & 22 & 36.67 & 25 & 41.67 \\
\hline
\end{tabular}


Table 4B0: Intercurrent Mortality Rate Female Mice with Water control

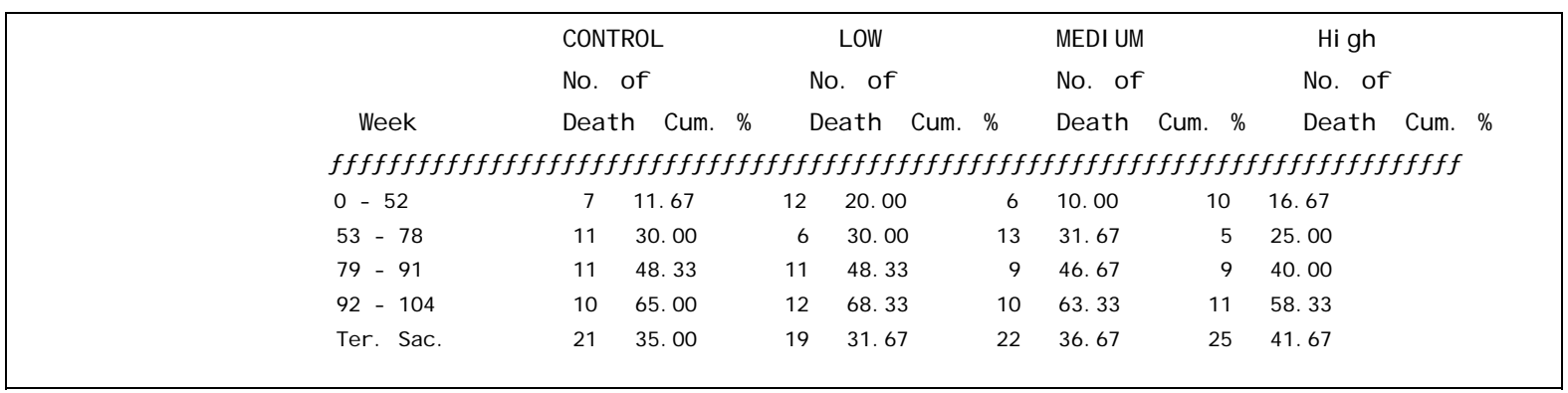

Table 5A: Tests for Dose Response Relationship and Homogeneity of Mortality Male Mice

\begin{tabular}{|lcc|}
\hline \multicolumn{3}{|c|}{ P-value } \\
\hline Test & Using Vehicle & Using Water control \\
\hline Dose Response & 0.0625 & 0.1466 \\
Homogeneity & 0.2005 & 0.3997 \\
\hline
\end{tabular}

Table 5B: Tests for Dose Response Relationship and Homogeneity of Mortality Female Mice

\begin{tabular}{|lcc|}
\hline \multicolumn{3}{|c|}{ P-value } \\
\hline Test & Using Vehicle & Using Water \\
\hline Dose Response & 1.0000 & 0.3513 \\
Homogeneity & 0.3703 & 0.7536 \\
\hline
\end{tabular}


Table 6A: Tumor Rates and P-Values for Dose Response Relationship and Pairwise Comparisons Male Mice with Vehicle control

\begin{tabular}{|c|c|c|c|c|c|c|c|c|c|}
\hline & & $0 \mathrm{mg}$ & $5 \mathrm{mg}$ & $15 \mathrm{mg}$ & $40 \mathrm{mg}$ & P_Val ue & p Value & p Value & P Val ue \\
\hline Organ Name & Tu mor Na me & $N=60$ & $N=60$ & $N=60$ & $N=60$ & Resp & C Vs. L & C Vs. M & C VS. H \\
\hline \multicolumn{10}{|c|}{ } \\
\hline \multirow[t]{2}{*}{ ADRENAL GLANDS } & CORTEX: ADENOMA & 0 & 0 & 0 & 1 & 0.2215 & . & . & 0.4430 \\
\hline & SUBCAPSULAR CELL ADE & 0 & 0 & 1 & 0 & 0.4586 & . & 0.4634 & . \\
\hline BRAI N & OLI GODENDROGL I OMA & 0 & 0 & 1 & 0 & 0.4586 & . & 0.4634 & . \\
\hline \multirow[t]{2}{*}{ EPI DI DYMI DES } & HEMANGI OSARCOMA & 0 & 1 & 0 & 0 & 0.7197 & 0.4824 & . & . \\
\hline & LEYDI G CELL ADENOMA & 1 & 0 & 0 & 0 & 1.0000 & 1.0000 & 1.0000 & 1.0000 \\
\hline GALLBLADDER & ADENOMA & 0 & 0 & 0 & 1 & 0.2166 & . & . & 0.4359 \\
\hline \multirow[t]{2}{*}{ HARDERIAN GL } & ADENOMA & 10 & 5 & 4 & 2 & 0.9740 & 0.9358 & 0.9592 & 0.9918 \\
\hline & CARCI NOMA & 2 & 1 & 1 & 0 & 0.8956 & 0.8613 & 0.8511 & 1.0000 \\
\hline KI DNEYS & TUBULAR ADENOMA & 0 & 0 & 0 & 1 & 0.2166 & . & . & 0.4359 \\
\hline \multirow[t]{4}{*}{ LI VER } & HEMANGI OMA & 2 & 0 & 0 & 0 & 1.0000 & 1.0000 & 1.0000 & 1.0000 \\
\hline & HEMANGI OSARCOMA & 3 & 0 & 4 & 2 & 0.2685 & 1.0000 & 0.4335 & 0.7210 \\
\hline & HEPATOCELLULAR ADENO & 4 & 7 & 7 & 4 & 0.4841 & 0.2204 & 0.2072 & 0.5084 \\
\hline & HEPATOCELLULAR CARCI & 3 & 4 & 2 & 1 & 0.8563 & 0.4609 & 0.7629 & 0.9058 \\
\hline \multirow[t]{2}{*}{ LUNGS } & BRONCHI OLO/ ALVEOLAR & 6 & 13 & 10 & 7 & 0.4564 & 0.0423 & 0.1387 & 0.2931 \\
\hline & & & 2 & 3 & 5 & 0.3252 & 0.9609 & 0.8834 & 0.6150 \\
\hline \multirow[t]{2}{*}{ LYMPH NODE OTHE } & BENI GN MAST CELL NEO & 1 & 0 & 0 & 0 & 1.0000 & 1.0000 & 1.0000 & 1.0000 \\
\hline & HEMANGI OSARCOMA & 0 & 1 & 0 & 0 & 0.7197 & 0.4824 & . & . \\
\hline \multirow[t]{3}{*}{ LYMPH/RETIC SYS } & HISTIOCYTIC SARCOMA & 0 & 1 & 0 & 1 & 0.2651 & 0.4824 & . & 0.4359 \\
\hline & MALI GNANT LYMPHOMA & 3 & 5 & 1 & 3 & 0.6181 & 0.3182 & 0.9189 & 0.5501 \\
\hline & MYELOSARCOMA/ GRANULO & 0 & 1 & 0 & 0 & 0.7197 & 0.4824 & . & . \\
\hline MEDI ASTI NAL TIS & MALI GNANT MESOTHELI O & 0 & 0 & 0 & 1 & 0.2215 & . & . & 0.4430 \\
\hline \multirow[t]{2}{*}{ PANCREAS } & HEMANGI OSARCOMA & 0 & 0 & 0 & 1 & 0.2166 & . & . & 0.4359 \\
\hline & I SLET CELL ADENOMA & 1 & 0 & 1 & 1 & 0.3484 & 1.0000 & 0.7160 & 0.6867 \\
\hline PI TUI TARY GLAND & CRANI OPHARYNGI OMA & 0 & 0 & 0 & 1 & 0.2215 & . & . & 0.4430 \\
\hline PROSTATE & ADENOMA & 1 & 0 & 0 & 0 & 1.0000 & 1.0000 & 1.0000 & 1.0000 \\
\hline \multirow[t]{3}{*}{ SKIN (OTHER) } & FI BROSARCOMA & 0 & 1 & 1 & 0 & 0.5738 & 0.4824 & 0.4699 & . \\
\hline & HEMANGI OSARCOMA & 0 & 1 & 0 & 0 & 0.7197 & 0.4824 & . & . \\
\hline & SQUAMOUS CELL CARCIN & 1 & 0 & 0 & 0 & 1.0000 & 1.0000 & 1.0000 & 1.0000 \\
\hline SKIN PROTOCOL & FI BROSARCOMA & 0 & 1 & 1 & 0 & 0.5738 & 0.4824 & 0.4699 & . \\
\hline SOFT TISSUE & FI BROSARCOMA & 0 & 0 & 0 & 1 & 0.2215 & . & . & 0.4430 \\
\hline SPLEEN & HEMANGI OSARCOMA & 1 & 0 & 1 & 0 & 0.7122 & 1.0000 & 0.7220 & 1.0000 \\
\hline STOMACH & FORESTOMACH: SQUAMOU & 0 & 0 & 1 & 0 & 0.4620 & . & 0.4699 & . \\
\hline STOMACH & GLANDULAR MUCOSA: AD & 1 & 1 & 1 & 0 & 0.7856 & 0.7291 & 0.7160 & 1.0000 \\
\hline \multirow[t]{2}{*}{ TESTES } & BENI GN I NTERSTI TI AL & 0 & 2 & 1 & 2 & 0.1466 & 0.2297 & 0.4699 & 0.1868 \\
\hline & RETE TESTIS: ADENOMA & 1 & 0 & 0 & 0 & 1.0000 & 1.0000 & 1.0000 & 1.0000 \\
\hline THYROI D & FOLLI CULAR CELL ADEN & 0 & 0 & 1 & 1 & 0.1552 & . & 0.4634 & 0.4430 \\
\hline
\end{tabular}


Table 6A0: Tumor Rates and P-Values for Dose Response Relationship and Pairwise Comparisons Male Mice with Water control

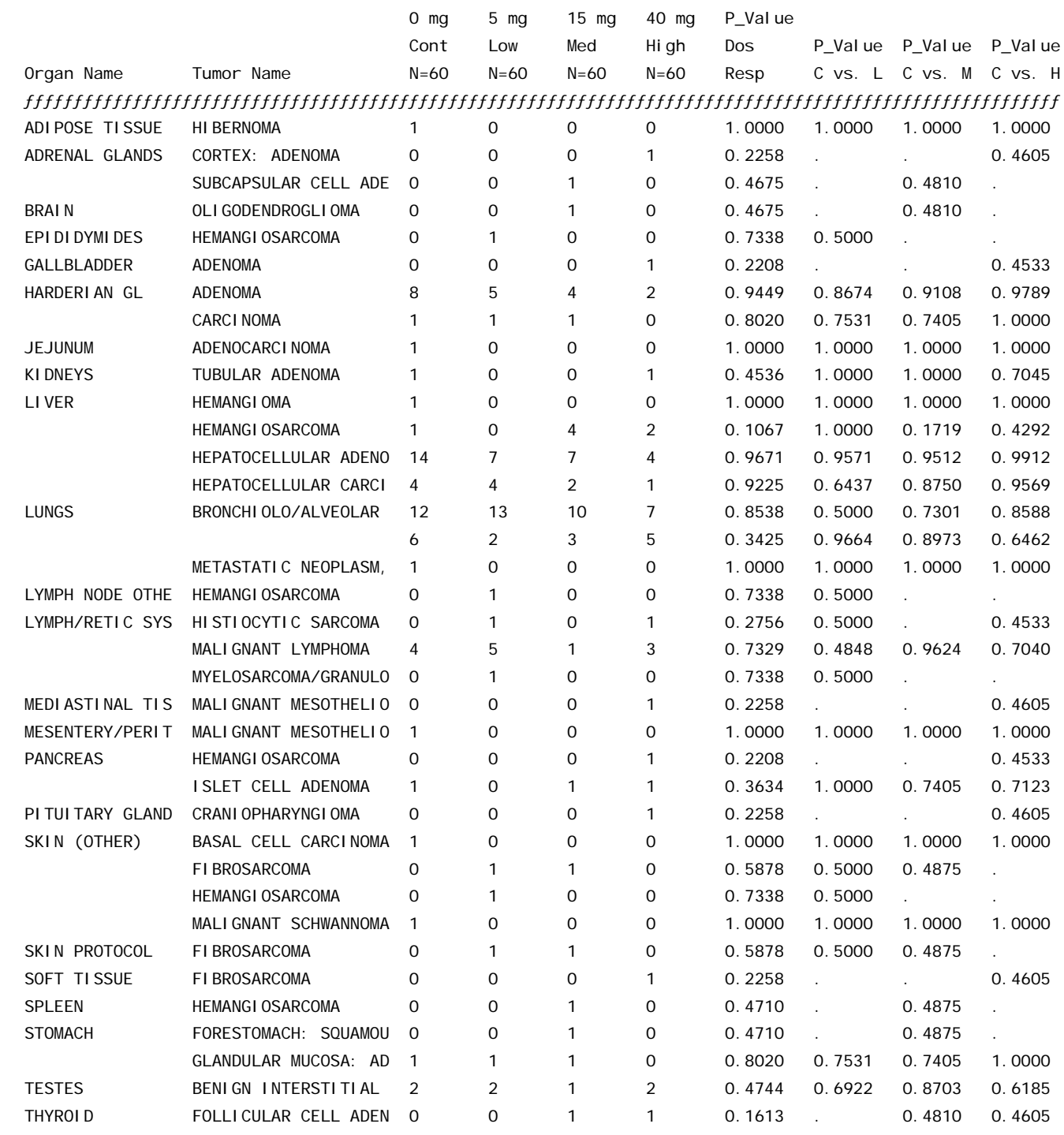


Table 6B: Tumor Rates and P-Values for Dose Response Relationship and Pairwise Comparisons Female Mice with Vehicle control

\begin{tabular}{|c|c|c|c|c|c|c|c|c|c|}
\hline & 0 & $\mathrm{mg}$ & $2 \mathrm{mg}$ & $10 \mathrm{mg}$ & $20 \mathrm{mg}$ & $\begin{array}{c}P_{-} \text {Val ue } \\
\text { Dos }\end{array}$ & p Value & p Value & P_Val ue \\
\hline Organ Name & Tumor Name & $N=60$ & $N=60$ & $N=60$ & $N=60$ & Resp & CVS. L & C Vs. M & C VS. H \\
\hline$f f f f f f f f f f f f f f f$ & $f f f f f f f f f f f f f f f f f f f f$ & $f f f f$ & eft. & 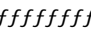 & & $f f$ & $f f f f$ & & \\
\hline ADRENAL GLANDS & MEDULLA: BENI GN NEOP & 0 & 0 & 0 & 2 & 0.0621 & . & . & 0.2292 \\
\hline & SUBCAPSULAR CELL ADE & 1 & 1 & 1 & 3 & 0.1294 & 0.7069 & 0.7346 & 0.2809 \\
\hline & SUBCAPSULAR CELL CAR & 1 & 0 & 0 & 0 & 1.0000 & 1.0000 & 1.0000 & 1.0000 \\
\hline BONE (OTHER) & OSTEOSARCOMA & 1 & 0 & 1 & 1 & 0.4110 & 1.0000 & 0.7346 & 0.7346 \\
\hline DUODENUM & ADENOCARCI NOMA & 0 & 1 & 0 & 0 & 0.7312 & 0.4625 & . & . \\
\hline HARDERIAN GL & ADENOMA & 5 & 5 & 7 & 4 & 0.5944 & 0.5309 & 0.3444 & 0.7209 \\
\hline & CARCI NOMA & 1 & 0 & 1 & 1 & 0.4110 & 1.0000 & 0.7346 & 0.7346 \\
\hline KI DNEYS & MESENCHYMAL TUMOR & 0 & 1 & 0 & 1 & 0.3035 & 0.4625 & . & 0.4819 \\
\hline LI VER & HEMANGI OMA & 1 & 0 & 0 & 0 & 1.0000 & 1.0000 & 1.0000 & 1.0000 \\
\hline & HEMANGI OSARCOMA & 0 & 1 & 0 & 1 & 0.3035 & 0.4625 & . & 0.4819 \\
\hline & HEPATOCELLULAR ADENO & 3 & 1 & 1 & 1 & 0.8269 & 0.9179 & 0.9360 & 0.9328 \\
\hline & HEPATOCELLULAR CARCI & 1 & 0 & 0 & 0 & 1.0000 & 1.0000 & 1.0000 & 1.0000 \\
\hline LUNGS & BRONCHI OLO/ALVEOLAR & 5 & 1 & 3 & 4 & 0.3978 & 0.9782 & 0.8434 & 0.7215 \\
\hline & & 7 & 5 & 1 & 6 & 0.6344 & 0.7145 & 0.9955 & 0.6612 \\
\hline LYMPH/RETIC SYS & HISTIOCYTIC SARCOMA & 5 & 0 & 2 & 2 & 0.6981 & 1.0000 & 0.9294 & 0.9294 \\
\hline & MALI GNANT LYMPHOMA & 4 & 7 & 14 & 7 & 0.1468 & 0.1833 & $0.0069 *$ & 0.1959 \\
\hline MAMMARY (OTHER) & ADENOCARCI NOMA & 0 & 1 & 1 & 2 & 0.1210 & 0.4557 & 0.4881 & 0.2352 \\
\hline & FI BROADENOMA & 0 & 1 & 0 & 0 & 0.7296 & 0.4557 & . & . \\
\hline MAMMARY PROTOCO & ADENOCARCI NOMA & 0 & 1 & 1 & 2 & 0.1210 & 0.4557 & 0.4881 & 0.2352 \\
\hline MESENTERI C LN & HEMANGI OSARCOMA & 0 & 1 & 0 & 0 & 0.7312 & 0.4625 & . & . \\
\hline MUSCLE (OTHER) & HEMANGI OSARCOMA & 0 & 0 & 0 & 1 & 0.2516 & . & . & 0.4819 \\
\hline & RHABDOMY OSARCOMA & 0 & 0 & 1 & 0 & 0.5063 & . & 0.4881 & . \\
\hline OVARIES & CYSTADENOMA & 2 & 2 & 3 & 1 & 0.6905 & 0.6222 & 0.4653 & 0.8657 \\
\hline & HEMANGI OMA & 1 & 0 & 0 & 1 & 0.5094 & 1.0000 & 1.0000 & 0.7410 \\
\hline & HEMANGI OSARCOMA & 1 & 1 & 0 & 0 & 0.9290 & 0.7142 & 1.0000 & 1.0000 \\
\hline & LUTEOMA & 2 & 0 & 0 & 0 & 1.0000 & 1.0000 & 1.0000 & 1.0000 \\
\hline & MALI GNANT GRANULOSA & 0 & 1 & 0 & 0 & 0.7296 & 0.4557 & . & . \\
\hline & SERTOLI CELL TUMOR & 0 & 0 & 0 & 1 & 0.2516 & . & . & 0.4819 \\
\hline & TUBULOSTROMAL ADENOM & 2 & 0 & 0 & 3 & 0.1766 & 1.0000 & 1.0000 & 0.4653 \\
\hline PANCREAS & HEMANGI OMA & 0 & 0 & 1 & 0 & 0.5031 & . & 0.4819 & . \\
\hline PI TUI TARY GLAND & PARS DISTALIS-ADENOM & 0 & 0 & 0 & 1 & 0.2516 & . & . & 0.4819 \\
\hline & PARS DISTALIS: CARCI & 0 & 1 & 0 & 1 & 0.3035 & 0.4625 & . & 0.4819 \\
\hline PREPUT/CLIT GL & HEMANGI OSARCOMA & 1 & 0 & 0 & 0 & 1.0000 & 1.0000 & 1.0000 & 1.0000 \\
\hline SKIN (OTHER) & FI BROSARCOMA & 1 & 0 & 1 & 2 & 0.1852 & 1.0000 & 0.7410 & 0.4726 \\
\hline SKIN (OTHER) & HEMANGI OSARCOMA & 0 & 0 & 1 & 0 & 0.5063 & . & 0.4881 & . \\
\hline & LI POSARCOMA & 0 & 1 & 0 & 0 & 0.7312 & 0.4625 & . & . \\
\hline & MALI GNANT TRI CHOEPIT & 0 & 0 & 0 & 1 & 0.2563 & . & . & 0.4881 \\
\hline SKIN PROTOCOL & FI BROSARCOMA & 1 & 0 & 1 & 2 & 0.1852 & 1.0000 & 0.7410 & 0.4726 \\
\hline SOFT TISSUE & HEMANGI OSARCOMA & 1 & 0 & 0 & 0 & 1.0000 & 1.0000 & 1.0000 & 1.0000 \\
\hline SPLEEN & HEMANGI OSARCOMA & 1 & 0 & 0 & 1 & 0.5031 & 1.0000 & 1.0000 & 0.7346 \\
\hline TAI L & FI BROSARCOMA & 1 & 0 & 1 & 0 & 0.7578 & 1.0000 & 0.7410 & 1.0000 \\
\hline & OSTEOSARCOMA & 1 & 0 & 0 & 0 & 1.0000 & 1.0000 & 1.0000 & 1.0000 \\
\hline THYROI D & C. CELL ADENOMA & 0 & 0 & 1 & 0 & 0.5031 & . & 0.4819 & . \\
\hline & C. CELL CARCI NOMA & 0 & 0 & 1 & 0 & 0.5063 & . & 0.4881 & . \\
\hline & FOLLI CULAR CELL ADEN & 2 & 1 & 0 & 0 & 0.9812 & 0.8439 & 1.0000 & 1.0000 \\
\hline UTERUS W/ CERVI & DECI DUOMA & 1 & 0 & 0 & 0 & 1.0000 & 1.0000 & 1.0000 & 1.0000 \\
\hline & ENDOMETRIAL ADENOMA & 1 & 0 & 0 & 0 & 1.0000 & 1.0000 & 1.0000 & 1.0000 \\
\hline & ENDOMETRIAL CARCI NOM & 2 & 0 & 1 & 1 & 0.6009 & 1.0000 & 0.8657 & 0.8657 \\
\hline & ENDOMETRIAL POLYP & 6 & 1 & 3 & 0 & 0.9819 & 0.9869 & 0.8979 & 1.0000 \\
\hline & HEMANGI OMA & 3 & 2 & 2 & 0 & 0.9544 & 0.7515 & 0.7972 & 1.0000 \\
\hline & HEMANGI OSARCOMA & 3 & 1 & 2 & 0 & 0.9331 & 0.9184 & 0.7972 & 1.0000 \\
\hline & LEI OMYOMA & 1 & 2 & 0 & 1 & 0.6713 & 0.4431 & 1.0000 & 0.7346 \\
\hline & LEI OMYOSARCOMA & 3 & 2 & 1 & 3 & 0.4747 & 0.7515 & 0.9296 & 0.6153 \\
\hline & SQUAMOUS CELL CARCIN & 0 & 0 & 1 & 0 & 0.5031 & . & 0.4819 & . \\
\hline & STROMAL SARCOMA & 3 & 1 & 2 & 0 & 0.9345 & 0.9142 & 0.7972 & 1.0000 \\
\hline
\end{tabular}


Table 6B0: Tumor Rates and P-Values for Dose Response Relationship and Pairwise Comparisons Female Mice with Water control

\begin{tabular}{|c|c|c|c|c|c|c|c|c|c|}
\hline & \multirow{2}{*}{$\begin{array}{l}0 \mathrm{mg} \\
\text { Cont }\end{array}$} & \multirow{2}{*}{$\begin{array}{l}2 \mathrm{mg} \\
\mathrm{Low}\end{array}$} & \multirow{2}{*}{$\begin{array}{l}10 \mathrm{mg} \\
\text { Med }\end{array}$} & $20 \mathrm{mg}$ & \multicolumn{4}{|l|}{$P_{-}$Val ue } \\
\hline & & & & & $\mathrm{High}$ & Dos & $P_{-}$Val ue & $P_{-}$Val ue & $P_{\text {__Val ue }}$ \\
\hline Organ Name & Tumor Name & $N=60$ & $N=60$ & $N=60$ & $N=60$ & Resp & C VS. L & C Vs. M & C VS. H \\
\hline \multicolumn{10}{|c|}{ } \\
\hline \multirow[t]{2}{*}{ ADRENAL GLANDS } & MEDULLA: BENI GN NEOP & 0 & 0 & 0 & 2 & 0.0654 & . & . & 0.2532 \\
\hline & SUBCAPSULAR CELL ADE & 0 & 1 & 1 & 3 & 0.0474 & 0.4800 & 0.5063 & 0.1249 \\
\hline BONE (OTHER) & OSTEOSARCOMA & 0 & 0 & 1 & 1 & 0.1994 & . & 0.5063 & 0.5063 \\
\hline DUODENUM & ADENOCARCI NOMA & 0 & 1 & 0 & 0 & 0.7500 & 0.4868 & . & . \\
\hline \multirow[t]{2}{*}{ HARDERIAN GL } & ADENOMA & 2 & 5 & 7 & 4 & 0.3463 & 0.1939 & 0.0894 & 0.3498 \\
\hline & CARCI NOMA & 1 & 0 & 1 & 1 & 0.4282 & 1.0000 & 0.7595 & 0.7595 \\
\hline KI DNEYS & MESENCHYMAL TUMOR & 0 & 1 & 0 & 1 & 0.3193 & 0.4868 & . & 0.5063 \\
\hline \multirow[t]{3}{*}{ LI VER } & HEMANGI OSARCOMA & 0 & 1 & 0 & 1 & 0.3193 & 0.4868 & . & 0.5063 \\
\hline & HEPATOCELLULAR ADENO & 2 & 1 & 1 & 1 & 0.7312 & 0.8647 & 0.8888 & 0.8844 \\
\hline & HEPATOCELLULAR CARCI & 1 & 0 & 0 & 0 & 1.0000 & 1.0000 & 1.0000 & 1.0000 \\
\hline \multirow[t]{2}{*}{ LUNGS } & BRONCHI OLO/ ALVEOLAR & 3 & 1 & 3 & 4 & 0.2259 & 0.9359 & 0.6861 & 0.5284 \\
\hline & & 8 & 5 & 1 & 6 & 0.7703 & 0.8563 & 0.9988 & 0.8242 \\
\hline \multirow[t]{2}{*}{ LYMPH/RETIC SYS } & HISTI OCYTIC SARCOMA & 3 & 0 & 2 & 2 & 0.4741 & 1.0000 & 0.8204 & 0.8204 \\
\hline & MALI GNANT LYMPHOMA & 10 & 7 & 14 & 7 & 0.5779 & 0.8059 & 0.2571 & 0.8213 \\
\hline \multirow[t]{2}{*}{ MAMMARY ( OTHER) } & ADENOCARCI NOMA & 0 & 1 & 1 & 2 & 0.1322 & 0.4800 & 0.5125 & 0.2595 \\
\hline & FI BROADENOMA & 0 & 1 & 0 & 0 & 0.7484 & 0.4800 & . & . \\
\hline MAMMARY PROTOCO & ADENOCARCI NOMA & 0 & 1 & 1 & 2 & 0.1322 & 0.4800 & 0.5125 & 0.2595 \\
\hline MESENTERI C LN & HEMANGI OSARCOMA & 0 & 1 & 0 & 0 & 0.7500 & 0.4868 & . & . \\
\hline \multirow[t]{2}{*}{ MUSCLE (OTHER) } & HEMANGI OSARCOMA & 0 & 0 & 0 & 1 & 0.2581 & . & . & 0.5063 \\
\hline & RHABDOMY OSARCOMA & 0 & 0 & 1 & 0 & 0.5192 & . & 0.5125 & . \\
\hline \multirow[t]{7}{*}{ OVARIES } & CYSTADENOMA & 1 & 2 & 3 & 1 & 0.5773 & 0.4696 & 0.3173 & 0.7595 \\
\hline & HEMANGI OMA & 0 & 0 & 0 & 1 & 0.2628 & . & . & 0.5125 \\
\hline & HEMANGI OSARCOMA & 0 & 1 & 0 & 0 & 0.7500 & 0.4868 & . & . \\
\hline & LUTEOMA & 1 & 0 & 0 & 0 & 1.0000 & 1.0000 & 1.0000 & 1.0000 \\
\hline & MALI GNANT GRANULOSA & 0 & 1 & 0 & 0 & 0.7484 & 0.4800 & . & . \\
\hline & SERTOLI CELL TUMOR & 0 & 0 & 0 & 1 & 0.2581 & . & . & 0.5063 \\
\hline & TUBULOSTROMAL ADENOM & 2 & 0 & 0 & 3 & 0.1903 & 1.0000 & 1.0000 & 0.5122 \\
\hline \multirow[t]{2}{*}{ PANCREAS } & HEMANGI OMA & 0 & 0 & 1 & 0 & 0.5161 & . & 0.5063 & . \\
\hline & I SLET CELL ADENOMA & 1 & 0 & 0 & 0 & 1.0000 & 1.0000 & 1.0000 & 1.0000 \\
\hline \multirow[t]{2}{*}{ PI TUI TARY GLAND } & PARS DISTALIS-ADENOM & 0 & 0 & 0 & 1 & 0.2581 & . & . & 0.5063 \\
\hline & PARS DISTALIS: CARCI & 0 & 1 & 0 & 1 & 0.3193 & 0.4868 & . & 0.5063 \\
\hline \multirow[t]{4}{*}{ SKIN (OTHER) } & FI BROSARCOMA & 0 & 0 & 1 & 2 & 0.0674 & . & 0.5125 & 0.2532 \\
\hline & HEMANGI OSARCOMA & 0 & 0 & 1 & 0 & 0.5192 & . & 0.5125 & . \\
\hline & LI POSARCOMA & 0 & 1 & 0 & 0 & 0.7500 & 0.4868 & . & . \\
\hline & MALI GNANT TRI CHOEPIT & 0 & 0 & 0 & 1 & 0.2628 & . & . & 0.5125 \\
\hline SKIN PROTOCOL & FI BROSARCOMA & 1 & 0 & 1 & 2 & 0.1981 & 1.0000 & 0.7655 & 0.5096 \\
\hline SPLEEN & HEMANGI OSARCOMA & 0 & 0 & 0 & 1 & 0.2581 & . & . & 0.5063 \\
\hline STOMACH & FORESTOMACH: SQUAMOU & 1 & 0 & 0 & 0 & 1.0000 & 1.0000 & 1.0000 & 1.0000 \\
\hline TAI L & FI BROSARCOMA & 0 & 0 & 1 & 0 & 0.5192 & . & 0.5125 & . \\
\hline THYROI D & C. CELL ADENOMA & 0 & 0 & 1 & 0 & 0.5161 & . & 0.5063 & . \\
\hline & C. CELL CARCINOMA & 0 & 0 & 1 & 0 & 0.5192 & . & 0.5125 & . \\
\hline & FOLLI CULAR CELL ADEN & 0 & 1 & 0 & 0 & 0.7484 & 0.4800 & . & . \\
\hline UTERUS W/ CERVI & ENDOMETRIAL CARCI NOM & 2 & 0 & 1 & 1 & 0.6203 & 1.0000 & 0.8844 & 0.8844 \\
\hline & ENDOMETRIAL POLYP & 2 & 1 & 3 & 0 & 0.8443 & 0.8647 & 0.5240 & 1.0000 \\
\hline & HEMANGI OMA & 3 & 2 & 2 & 0 & 0.9620 & 0.7863 & 0.8281 & 1.0000 \\
\hline & HEMANGI OSARCOMA & 2 & 1 & 2 & 0 & 0.8902 & 0.8700 & 0.7111 & 1.0000 \\
\hline & LEI OMYOMA & 1 & 2 & 0 & 1 & 0.6941 & 0.4800 & 1.0000 & 0.7595 \\
\hline & LEI OMYOSARCOMA & 2 & 2 & 1 & 3 & 0.3661 & 0.6616 & 0.8844 & 0.5122 \\
\hline & SQUAMOUS CELL CARCIN & 0 & 0 & 1 & 0 & 0.5161 & . & 0.5063 & . \\
\hline & STROMAL SARCOMA & 0 & 1 & 2 & 0 & 0.6086 & 0.4800 & 0.2595 & . \\
\hline VAGINA & STROMAL POLYP & 0 & 3 & 0 & 0 & 0.8977 & 0.1153 & . & . \\
\hline
\end{tabular}


Figure 1A: Kaplan-Meier Survival Functions for Male Rats Male Rats

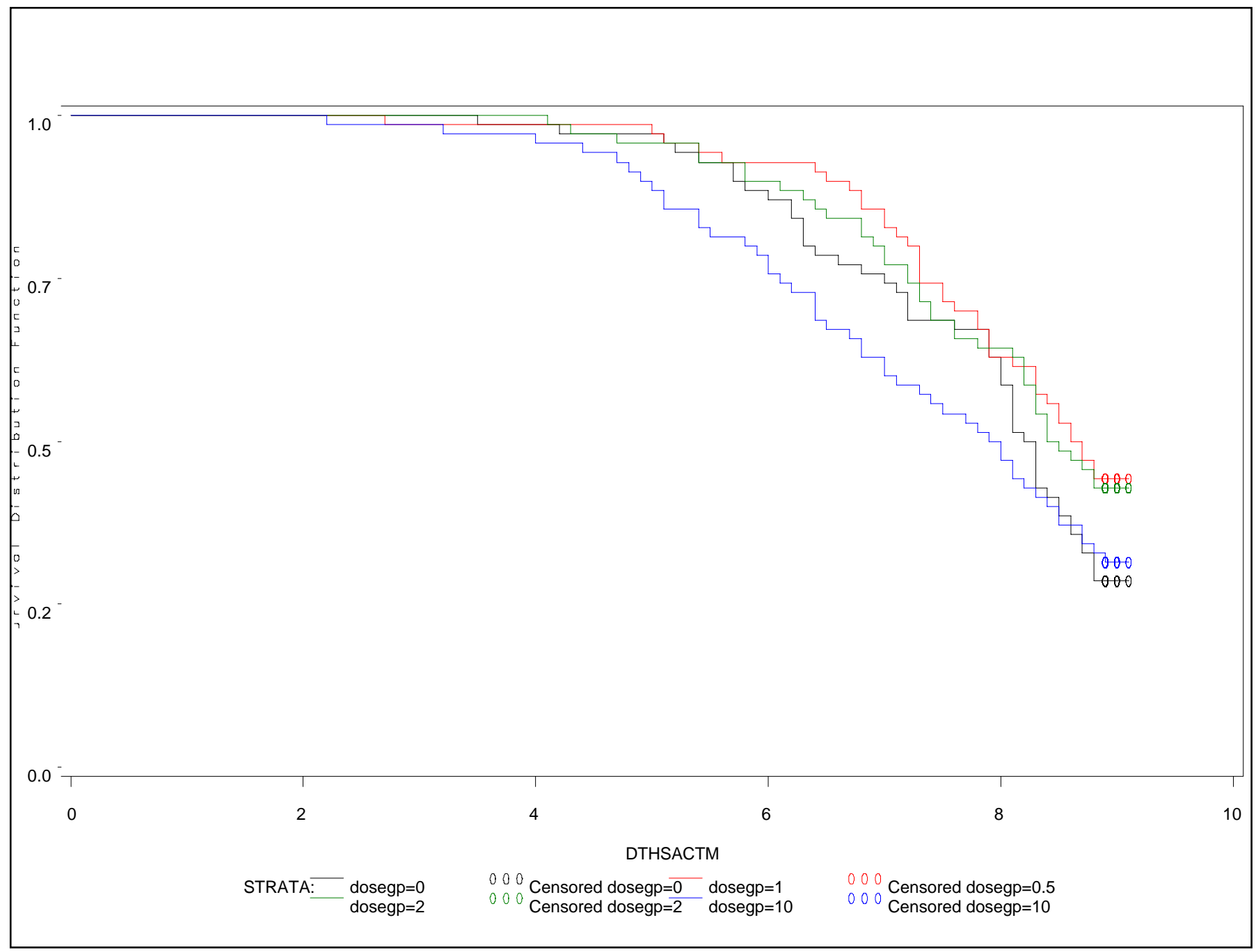

X-Axis: Weeks, Y-Axis: Survival rates 
Figure 1B: Kaplan-Meier Survival Functions for Female Rats Female Rats

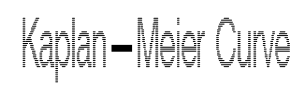

Female Rats With Vehicle-control

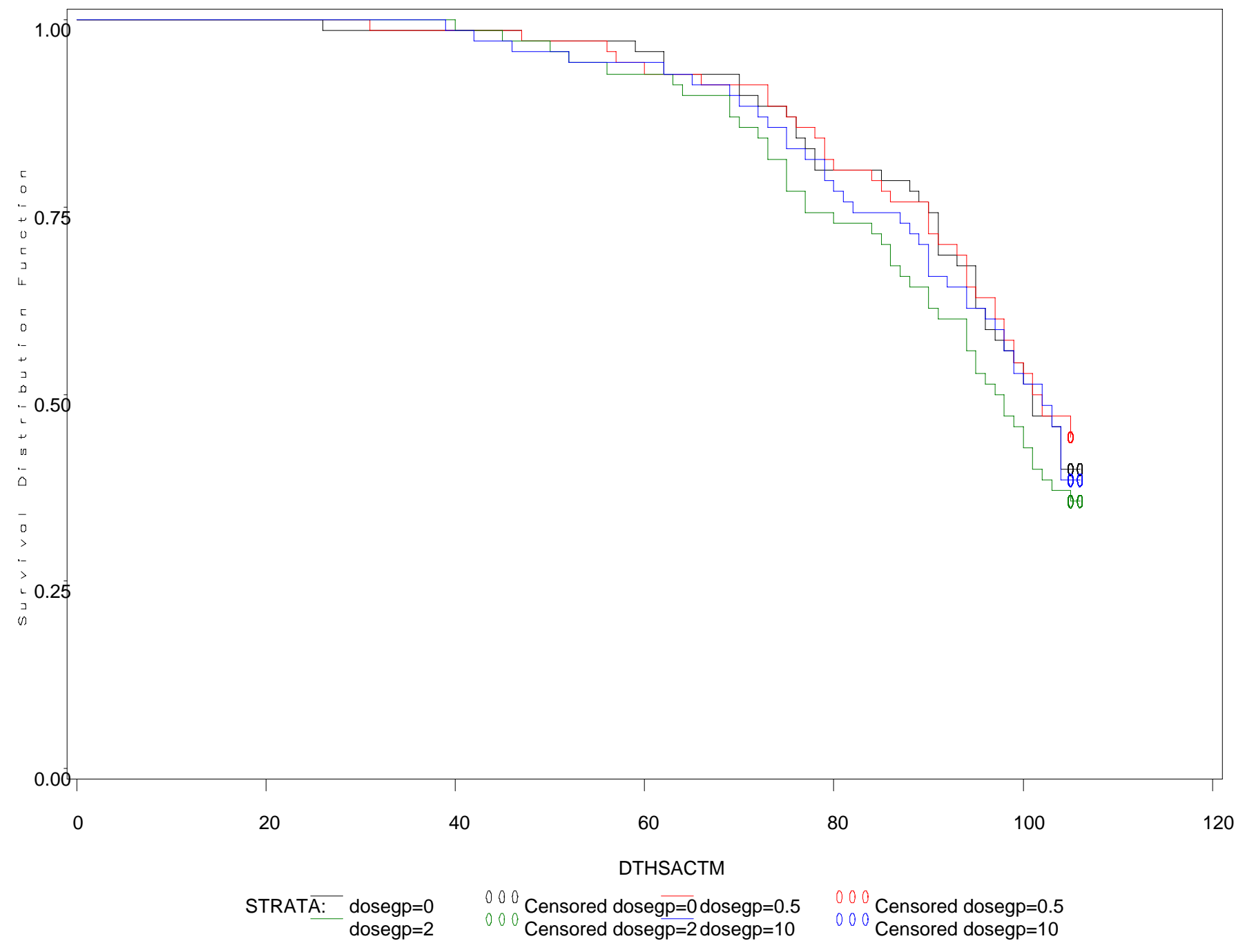

X-Axis: Weeks, Y-Axis: Survival rates 
Figure 2A: Kaplan-Meier Survival Functions for Male Mice Male Mice

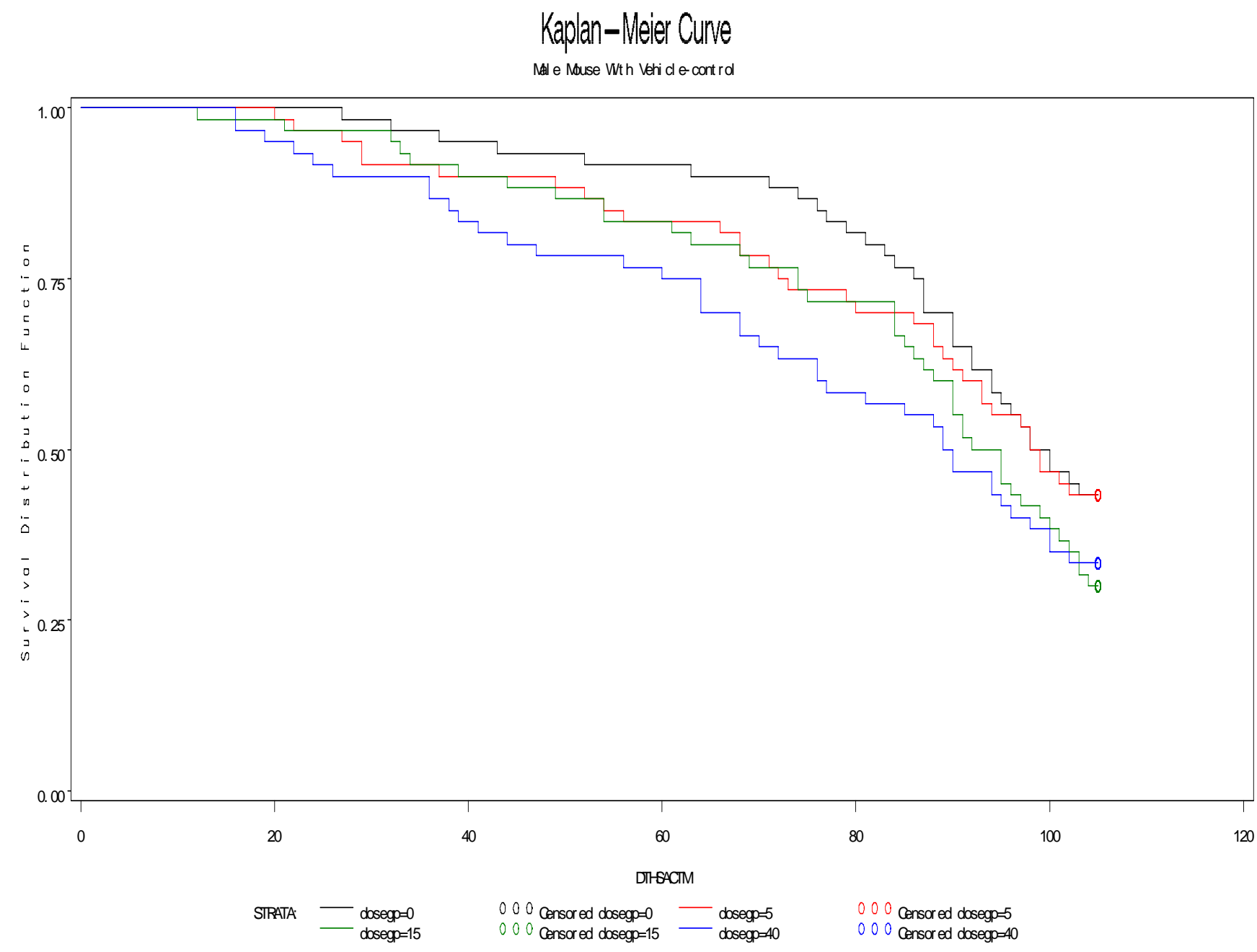

X-Axis: Weeks, Y-Axis: Survival rates 
Figure 2B: Kaplan-Meier Survival Functions for Female Mice Female Mice

\section{Kâplân-Mejề Curive}

Fenal e Nouse W/th Vehi cl e-cont rol

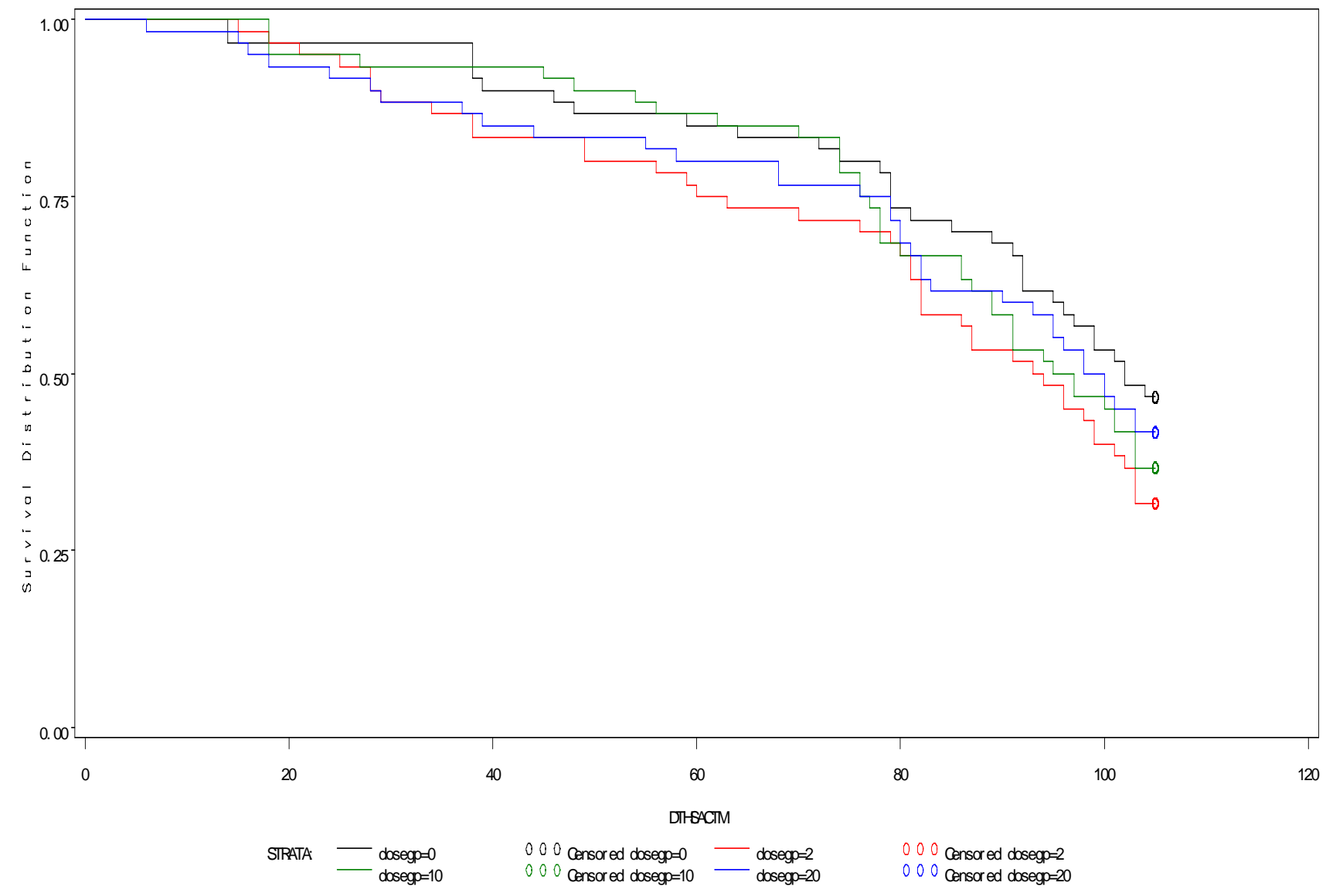

X-Axis: Weeks, Y-Axis: Survival rates 


\section{References:}

1. Peto, R., M.C. Pike, N.E. Day, R.G. Gray, P.N. Lee, S. Parish, J. Peto, Richards, and J.Wahrendorf, “Guidelines for sample sensitive significance test for carcinogenic effects in long-term animal experiments", Long term and short term screening assays for carcinogens: A critical appraisal, International agency for research against cancer monographs, Annex to supplement, World Health Organization, Geneva, 311-426, 1980.

2.. Bailer AJ, Portier CJ (1988). "Effects of treatment-induced mortality and tumor-induced mortality on tests for carcinogenicity in small samples." Biometrics, 44, 417-431.

3.Bieler, G. S. and Williams, R. L. (1993). "Ratio estimates, the delta method, and quantal response tests for increased carcinogenicity". Biometrics $49,793-801$.

4. Cox D. R. "Regression models and life tables", Journal of the Royal Statistical Society, B, 34, 187-220, 1972.

5. Gehan "A generalized Wilcoxon test for comparing arbitrarily singly censored samples", Biometrika, 52, 203$223,1965$.

6. Rahman, M.A. and Lin, K.K., "A Comparison of False Positive Rates of Peto and Poly-3 Methods for Long-Term Carcinogenicity Data Analysis Using Multiple Comparison Adjustment Method Suggested by Lin and Rahman", Journal of Biopharmaceutical Statistics, 18:5, 849-858.

7. Haseman, J, “A re-examination of false-positive rates for carcinogenesis studies", Fundamental and Applied Toxicology, 3: 334-339, 1983.

8. .Lin K.K. and Rahman M.A.," Overall false positive rates in tests for linear trend in tumor incidence in animal carcinogenicity studies of new drugs", Journal of Biopharmaceutical Statistics, 8(1), 1-15, 1998.

9. Tarone RE, "Test for trend in life table analysis”, Biometrika 1975, 62: 679-82 
This is a representation of an electronic record that was signed electronically and this page is the manifestation of the electronic signature.

\section{/s/}

MOHAMED O NAGEM

09/15/2011

KARL K LIN

09/15/2011

Concur with review 


\section{STATISTICAL REVIEW AND EVALUATION Clinical Studies}

\author{
NDA/BLA Serial \\ Number: \\ Drug Name: \\ Indication(s): \\ Applicant: \\ Date(s):
}

Review Priority:

Biometrics Division: Division of Biometrics II

Statistical Reviewer: Jonathan Norton, Ph.D.

Concurring Reviewers: Todd Sahlroot, Ph.D.

Thomas Permutt, Ph.D.

Medical Division:

Clinical Team:

Division of Metabolism and Endocrinology Products

Somya Dunn, M.D.

Ilan Irony, M.D.

Project Manager: $\quad$ Mehreen Hai

Keywords: Active-control/non-inferiority, add-on design, analysis of covariance, clinical studies, double-blind, double-dummy, labeling, longitudinal data analysis, missing data, NDA review, sensitivity analyses, stratification, worst case analysis 


\section{Table of Contents}

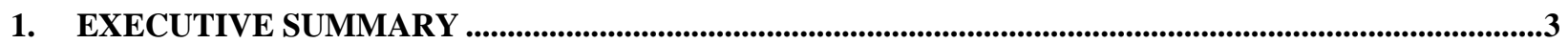

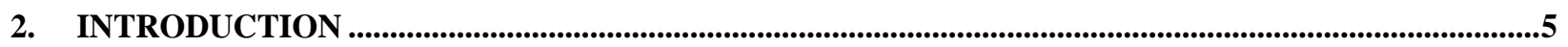

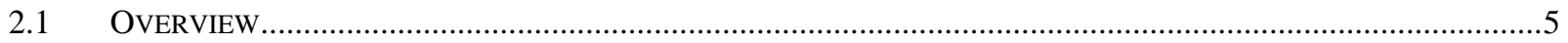

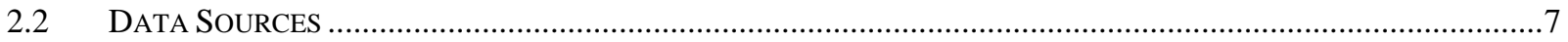

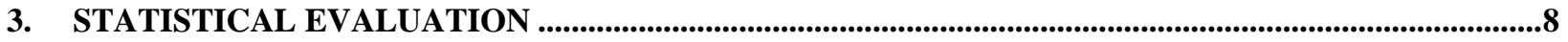

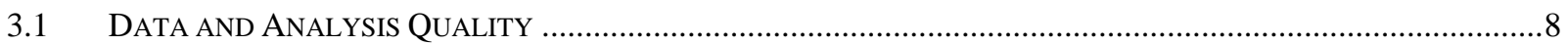

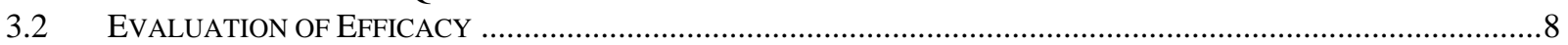

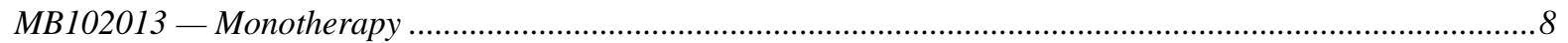

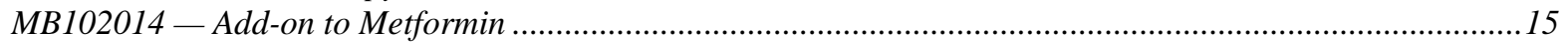

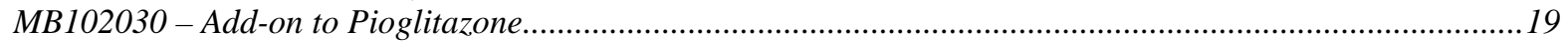

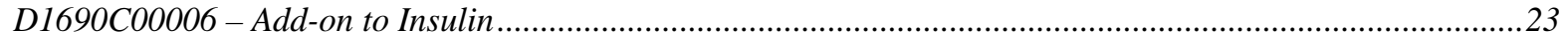

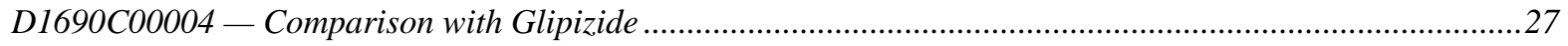

MB102034 - Initial Combination with Metformin ..........................................................................................32

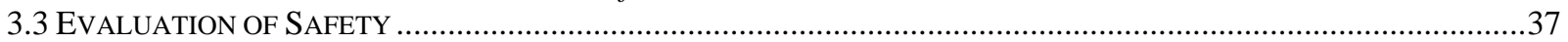

4. FINDINGS IN SPECIAL/SUBGROUP POPULATIONS .............................................................................38

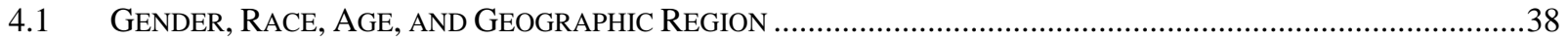

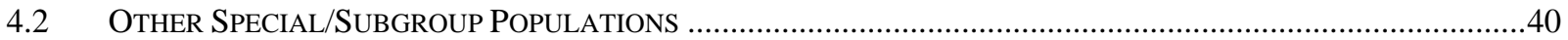

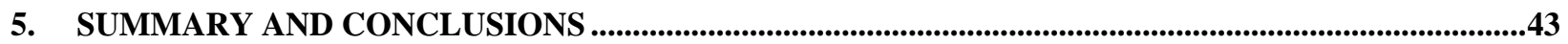

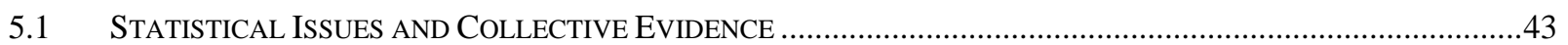

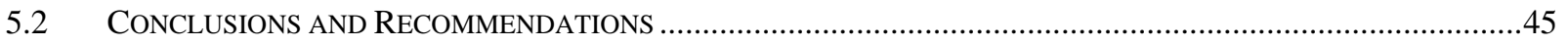

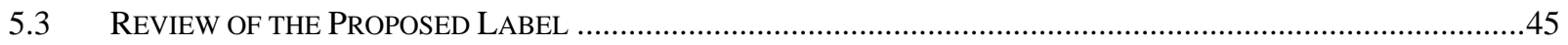

APPENDIX A: REFERENCES..............................................................................................................................48

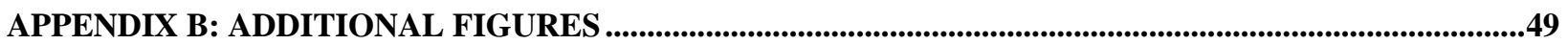




\section{EXECUTIVE SUMMARY}

The Applicant, Bristol-Myers Squibb, has submitted eleven Phase 3 efficacy and safety studies in support of their New Drug Application for dapagliflozin propanediol (DAPA) as a treatment for type 2 diabetes mellitus. The submitted studies provide strong evidence that dapagliflozin is effective in subjects with normal renal function or mild impairment. The dedicated study of subjects with moderate renal impairment did not show statistical evidence of efficacy.

Based on consultation with the medical team leader, I determined that my statistical review would focus primarily on 6 key clinical trials, which are shown in italics in Table 1. Four of these studies were placebo-controlled, and they tested DAPA either as monotherapy (MB102013), or as an add-on therapy to metformin (MB102014), pioglitazone (MB 102030), or insulin (D1690C00006). The fifth study used glipizide as an active control (D1690C00004), with metformin as background therapy. Except for the monotherapy trial, all of the studies randomized patients who had been inadequately controlled by background therapy. Finally, the sixth study tested DAPA as an initial combination with metformin. The primary endpoint in all of these studies was change in HbA1c from baseline. Except for the glipizide-controlled study, which used a 52-week endpoint, the primary endpoint was evaluated at 24 weeks.

I find these studies to provide convincing evidence that DAPA is efficacious for both of the proposed daily doses, $5 \mathrm{mg}$ and $10 \mathrm{mg}$, in patients with at most mild renal impairment. In the four placebo-controlled studies (out of the six that I focused on), both the $5 \mathrm{mg}$ and $10 \mathrm{mg}$ doses of DAPA were shown to be superior to the comparator on the primary endpoint, using the planned primary analysis. The active-controlled study showed that titrated doses of DAPA and glipizide yielded quite similar results at Week 52. Although DAPA was statistically non-inferior at Week 52, it should be noted that glipizide was clearly superior at some earlier time points. (See Figure 13.) Findings for fasting plasma glucose (FPG) also support a finding of efficacy for both doses.

The results in the previous paragraph are based on the planned primary analysis, which used lastobservation-carried-forward (LOCF) imputation, disregarding observations recorded after any rescue treatment. While FDA has implicitly endorsed LOCF imputation for diabetes trials in the past, there is now more awareness in the statistical community of the limitations of this approach. In particular, the argument has been made that LOCF can be anti-conservative (i.e., it sometimes favors the alternative hypothesis more than other approaches) and the findings from the placebo-controlled studies that I reviewed bear this out.

My preferred analysis simply uses the observed values of patients who were rescued. This approach may seem counterintuitive if one believes that rescue treatment makes the subsequent outcomes less relevant to evaluation of the test agent. It has the virtue, however, of respecting the intent-to-treat principle, in the sense that the analysis is based on the randomized treatment rather than the treatment actually received (i.e., planned treatment plus rescue). I conducted this sensitivity analysis for five of the studies that I focused on (excluding C00004, which did not use rescue), and the primary endpoint remained statistically significant for the both the 5 and $10 \mathrm{mg}$ DAPA doses, applicable. The estimated treatment effects were somewhat attenuated, however. 
In regard to the five studies that I did not closely review, the Applicant reported results that broadly support a finding of efficacy for the 5 and $10 \mathrm{mg}$ doses of DAPA. Out of the 11 Phase 3 studies, 10 were successful on the primary endpoint using the pre-specified analysis.

The only failed Phase 3 study was in patients with moderate renal impairment, a result that is consistent with the drug's renal mechanism of action.

As noted earlier, the dedicated renal study in patients with moderate renal impairment (eGFR of 30-59) failed to show statistical significance on the primary endpoint. The Applicant conducted an "ad-hoc" (their term) subgroup analysis of patients with an eGFR of 45-59 that also failed to show statistical significance. They also submitted a pooled analysis of patients from other Phase 3 studies that had moderate renal impairment. I did not closely review the results of the pooled analysis because I considered the dedicated renal study to provide a higher level of evidence. 


\section{INTRODUCTION}

\subsection{Overview}

The Applicant has submitted a new drug application for dapagliflozin (DAPA), for dapagliflozin propanediol as a treatment for type 2 diabetes mellitus. They propose to market the 5 and $10 \mathrm{mg}$ doses, with the $5 \mathrm{mg}$ dose labeled for patients at risk of volume depletion.

The Applicant submitted eleven Phase 3 efficacy and safety studies which are shown in Table 1.

Table 1: Phase 3 Studies

\begin{tabular}{|c|c|c|c|c|}
\hline $\begin{array}{l}\text { Study ID } \\
\text { (Brief Name) }\end{array}$ & Population & Test Tx & $\begin{array}{l}\text { Background Tx } \\
\text { (Rescue) }\end{array}$ & Comparator(s) \\
\hline \multicolumn{5}{|l|}{ Monotherapy } \\
\hline $\begin{array}{l}\text { MB102013 } \\
(2013) \\
\end{array}$ & Drug-naïve & $\begin{array}{l}\text { DAPA 2.5, 5, } \\
10 \mathrm{mg}\end{array}$ & $\begin{array}{l}\text { None } \\
\text { (Metformin) }\end{array}$ & Placebo \\
\hline $\begin{array}{l}\text { MB102032 } \\
(2032)\end{array}$ & Drug-naïve & $\begin{array}{l}\text { DAPA 1, 2.5, } \\
5 \mathrm{mg}\end{array}$ & $\begin{array}{l}\text { None } \\
\text { (Metformin) }\end{array}$ & Placebo \\
\hline \multicolumn{5}{|l|}{ Add-On } \\
\hline $\begin{array}{l}\text { MB102014 } \\
\text { (2014) }\end{array}$ & $\begin{array}{l}\text { Inadequate control on } \\
\text { background }\end{array}$ & $\begin{array}{l}\text { DAPA 2.5, 5, } \\
10 \mathrm{mg}\end{array}$ & $\begin{array}{l}\text { Metformin } \\
\text { (Pioglitazone or } \\
\text { acarbose) }\end{array}$ & Placebo \\
\hline $\begin{array}{l}\text { D1690C00005 } \\
\text { (C00005) }\end{array}$ & $\begin{array}{l}\text { Inadequate control on } \\
\text { background }\end{array}$ & $\begin{array}{l}\text { DAPA 2.5, 5, } \\
10 \mathrm{mg}\end{array}$ & $\begin{array}{l}\text { Glimepiride } \\
\text { (Metformin or } \\
\text { TZD) }\end{array}$ & Placebo \\
\hline $\begin{array}{l}\text { MB102030 } \\
(2030) \\
\end{array}$ & $\begin{array}{l}\text { Inadequate control on } \\
\text { background }\end{array}$ & $\begin{array}{l}\text { DAPA 5, } 10 \\
\mathrm{mg}\end{array}$ & $\begin{array}{l}\text { Pioglitazone } \\
\text { (Metformin or SU) }\end{array}$ & Placebo \\
\hline $\begin{array}{l}\text { D1690C00006 } \\
(C 00006)\end{array}$ & $\begin{array}{l}\text { Inadequate control on } \\
\text { background }\end{array}$ & $\begin{array}{l}\text { DAPA 2.5, 5, } \\
10 \mathrm{mg}\end{array}$ & $\begin{array}{l}\text { Insulin and up to } \\
\text { two oral anti- } \\
\text { diabetics (Insulin } \\
\text { up-titration) }\end{array}$ & Placebo \\
\hline $\begin{array}{l}\text { D1690C00004 } \\
(C 00004)\end{array}$ & $\begin{array}{l}\text { Inadequate control on } \\
\text { background }\end{array}$ & $\begin{array}{l}\text { DAPA 2.5, 5, } \\
10 \mathrm{mg} \\
\text { (titrated) }\end{array}$ & Metformin (None) & Glipizide* \\
\hline $\begin{array}{l}\text { D1690C00012 } \\
(\mathrm{C} 00012)\end{array}$ & $\begin{array}{l}\text { Inadequate control on } \\
\text { background }\end{array}$ & DAPA 10 mg & $\begin{array}{l}\text { Metformin } \\
\text { (Sitagliptin) }\end{array}$ & Placebo** \\
\hline $\begin{array}{l}\text { MB102029 } \\
(2029)\end{array}$ & $\begin{array}{l}\text { Moderate renal } \\
\text { impairment and } \\
\text { inadequate control on } \\
\text { stable regime }\end{array}$ & $\begin{array}{l}\text { DAPA 5, } 10 \\
\text { mg }\end{array}$ & $\begin{array}{l}\text { Any except } \\
\text { metformin (Any } \\
\text { except metformin) }\end{array}$ & Placebo \\
\hline \multicolumn{5}{|c|}{ Initial Combination } \\
\hline $\begin{array}{l}\text { MB102021 } \\
\text { (2021) }\end{array}$ & $\begin{array}{l}\text { Drug-naïve with higher } \\
\text { HbA1c }\end{array}$ & $\begin{array}{l}\text { DAPA } 5 \mathrm{mg}+ \\
\text { metformin }\end{array}$ & $\begin{array}{l}\text { None } \\
\text { (Pioglitazone, } \\
\text { acarbose, or } \\
\text { sitagliptin) } \\
\end{array}$ & $\begin{array}{l}\text { DAPA } 5 \mathrm{mg} \text {, } \\
\text { Metformin }\end{array}$ \\
\hline $\begin{array}{l}\text { MB102034 } \\
(2034)\end{array}$ & $\begin{array}{l}\text { Drug-naïve } \\
\text { with higher HbA1c }\end{array}$ & $\begin{array}{l}\text { DAPA } 10 \text { mg } \\
+ \text { metformin }\end{array}$ & $\begin{array}{l}\text { None } \\
\text { (Pioglitazone, } \\
\text { acarbose, or } \\
\text { sitagliptin) }\end{array}$ & $\begin{array}{l}\text { DAPA } 10 \mathrm{mg} \text {, } \\
\text { Metformin }\end{array}$ \\
\hline
\end{tabular}

Notes: Proposed doses are 5, $10 \mathrm{mg}$. SU = sulfonylurea, TZD = thiazolidinedione. Italics = focus of review.

*Non-inferiority comparison; otherwise superiority.

**Endpoint is change in body weight; otherwise change in HbA1c. 
Based on input from Ilan Irony, M.D., the medical team leader, I determined that my review efforts would be primarily focused on the six studies which are italicized in the table. The studies that start with "MB" were conducted by Bristol-Myers Squib (BMS); those that start with "D" were conducted by AstraZeneca (AZ). DAPA was developed in an alliance between the two firms, but BMS is the Applicant of record.

The Division of Metabolism and Endocrinology Products (DMEP) held an End-of-Phase 2 meeting with representatives of BMS and AZ on September 11, 2007. The Sponsor proposed to bring the $2.5 \mathrm{mg}, 5 \mathrm{mg}$, and $10 \mathrm{mg}$ doses into the Phase 3 program; the Division advised the Sponsor to explore 2 or 3 doses lower than $2.5 \mathrm{mg}$, either in an additional Phase 2 study or in Phase 3. The Division also stated that if 3 doses are carried into Phase 3, then it would be acceptable for some studies to only include 2 of the 3 doses. In regard to patients with renal impairment, the following is noted in the minutes:

The Division requested that the Sponsor perform a dedicated renal safety study in patients with mild, moderate, and severe renal impairment. There should be at least 100 patients per group who complete the study. The duration should be at least one year... Regardless of the magnitude of efficacy in renally impaired patients, the Division stressed the need for a dedicated renal safety study because renal impairment is a common complication of diabetes, and many of these patients will likely receive dapagliflozin, if approved. In addition, the Division stated that this renal safety study could also be designed to capture important efficacy information (e.g., using stratification by background anti-hyperglycemic therapy), and recommended that the Sponsor submit the study protocol for review prior to study initiation.

The Division also advised the Sponsor to conduct "several" six-month extension studies, in addition to the two 18-month studies that had been proposed. In response to a question about whether the proposed clinical program would support inclusion of data on weight loss in the Clinical Studies section of the label, the Division stated that this was possible but that it would be a review issue.

On November 6, 2007, the Division sent an e-mail to clarify the desired composition of the renal study. The e-mail stated that there should be at least 100 DAPA-treated patients with moderate renal impairment and 100 DAPA-treated patients with severe impairment who complete the study. The control arms could have half as many patients in each stratum (2:1 randomization). It would not be necessary for the study to include patients with mild impairment if at least 100 such patients were treated with DAPA in the other studies.

On October 7, 2008, the Division provided comments on protocols for Study 2030 and MB102033, the latter being an insulin add-on study. In regard to Study 2030, the letter stated that the primary efficacy analysis set should be what BMS termed "Randomized Subjects", which includes those randomized subjects with both a baseline and at least post-baseline HbA1c measurement in the short-term treatment period. DMEP also stated that the statistical analysis plan (SAP) should include a sensitivity analysis. Suggested analyses included a mixed model repeated measures (MMRM) model, an analysis of completers who did not take rescue, and one or more methods from White (2001). The rate of rescue was requested as an additional measure of efficacy. In regard to the insulin study, DMEP requested the same analyses, but with an 
insulin up-titration treated as rescue. On October 24, 2008, DMEP sent the same comments for Study 2032 as were given for Study 2030.

In a letter of December 19, 2008, the Division stated that Study C00004 should use randomized subjects as the primary analysis set for the non-inferiority analysis. DMEP also requested justification for the proposed .35\% non-inferiority margin for HbA1c. I was not able to find a record indicating that we agreed to the margin. In addition, the Division suggested that a placebo arm be included in the study; this suggestion was not followed.

A pre-NDA meeting was held on November 9, 2010. In response to a question about the evidence needed to demonstrate durability of glycemic effect beyond the short-term treatment period, DMEP stated, "Conclusions regarding durability of effect may be limited by the voluntary participation in long term extension studies (thus, not preserving the initial randomization) and by large differential proportion of subjects rescued or discontinued, resulting in [sic] substantial amount of data missing.” DMEP also stated that it is not appropriate to include use of rescue as an explanatory variable, and again referred the Sponsor to White (2010).

\subsection{Data Sources}

The electronic version of this NDA can be found at ॥Cdsesub1 levsprod $\backslash$ NDA202293\202293.enx. The Applicant submitted both analysis and tabulation data sets, as well as software code. 


\section{STATISTICAL EVALUATION}

\subsection{Data and Analysis Quality}

I did not encounter any major data quality issues in the course of my review. The Office of Scientific Investigations (OSI) inspected both BMS and AZ, as well as seven clinical investigator sites. Although some violations were found, the OSI reviewer, Susan Leibenhaut, M.D., concluded, "The data are considered reliable in support of the application."

\subsection{Evaluation of Efficacy}

\section{MB102013 - Monotherapy}

\section{Study Design and Endpoints}

Figure 1 illustrates the design for Study 2013, a trial of DAPA as monotherapy. The primary analysis focused on patients in Group 1 who were dosed in the morning (QAM), including patients in the placebo arm.

Figure 1: Design for Study 2013

(Source: Clinical Study Report, Figure 3.1)

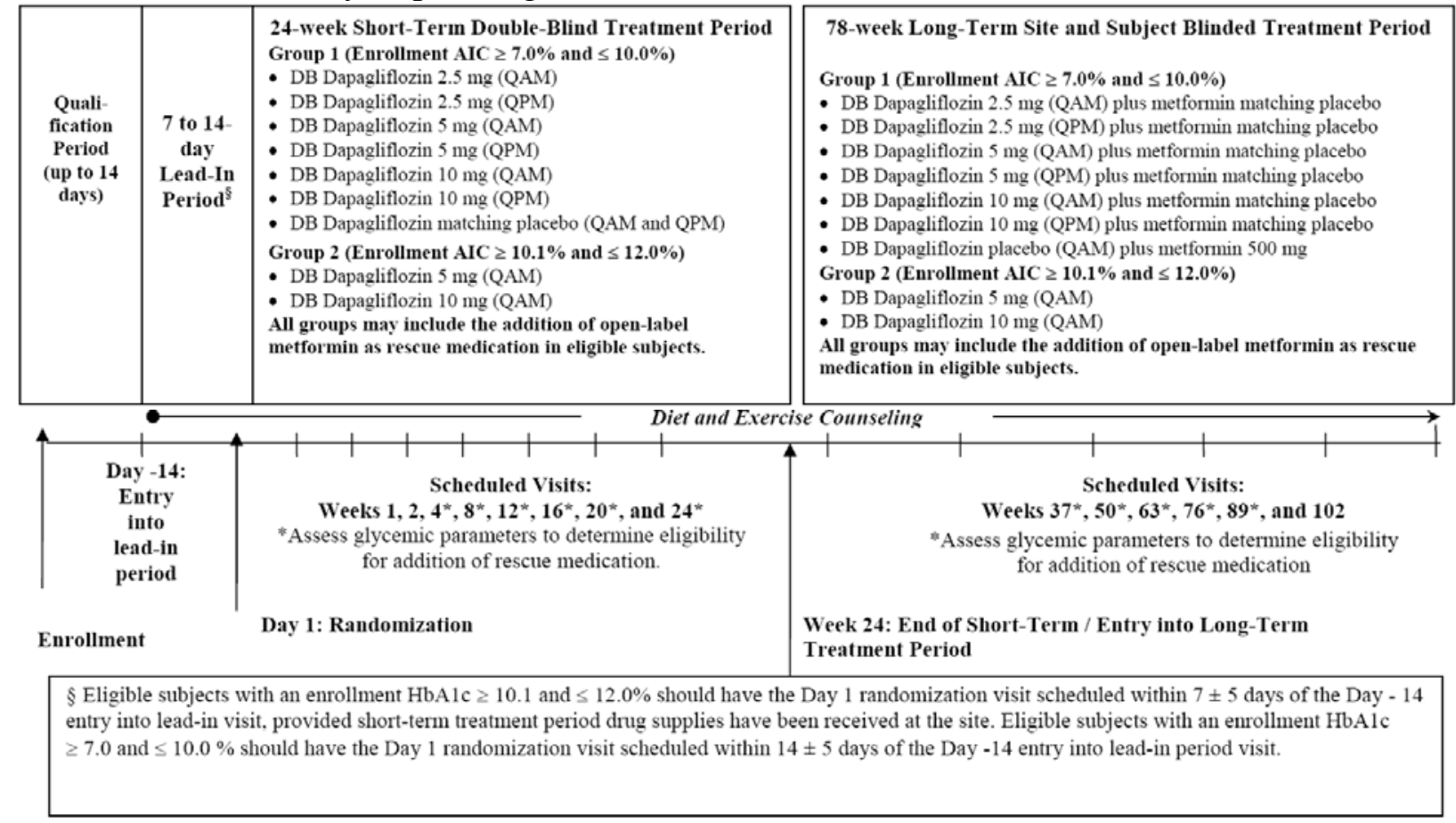

The primary efficacy endpoint was the change in hemoglobin A1c (HbA1c) from baseline to Week 24. Secondary efficacy variables included fasting plasma glucose (FPG), body weight, and therapeutic glycemic response (HbA1c $<7 \%$ ). 


\section{Patient Disposition, Demographic and Baseline Characteristics}

Table 2 shows the disposition of the double-blind treated subjects, in particular whether they completed the short-term period, whether they continued into the long-term extension, and the reasons for discontinuation at each stage. (From this point on, the phrase treated subjects refers to subjects who were randomized and received double-blind treatment.) Although it was produced by the Applicant, I verified the values in the table from the submitted data values. Unless it is otherwise noted, the reader should assume that I reproduced the contents of any tables that I include from a Clinical Study Report.

Table 2: Disposition of Treated Subjects for Study 2013

(Source: Clinical Study Report, Table 5.1B)

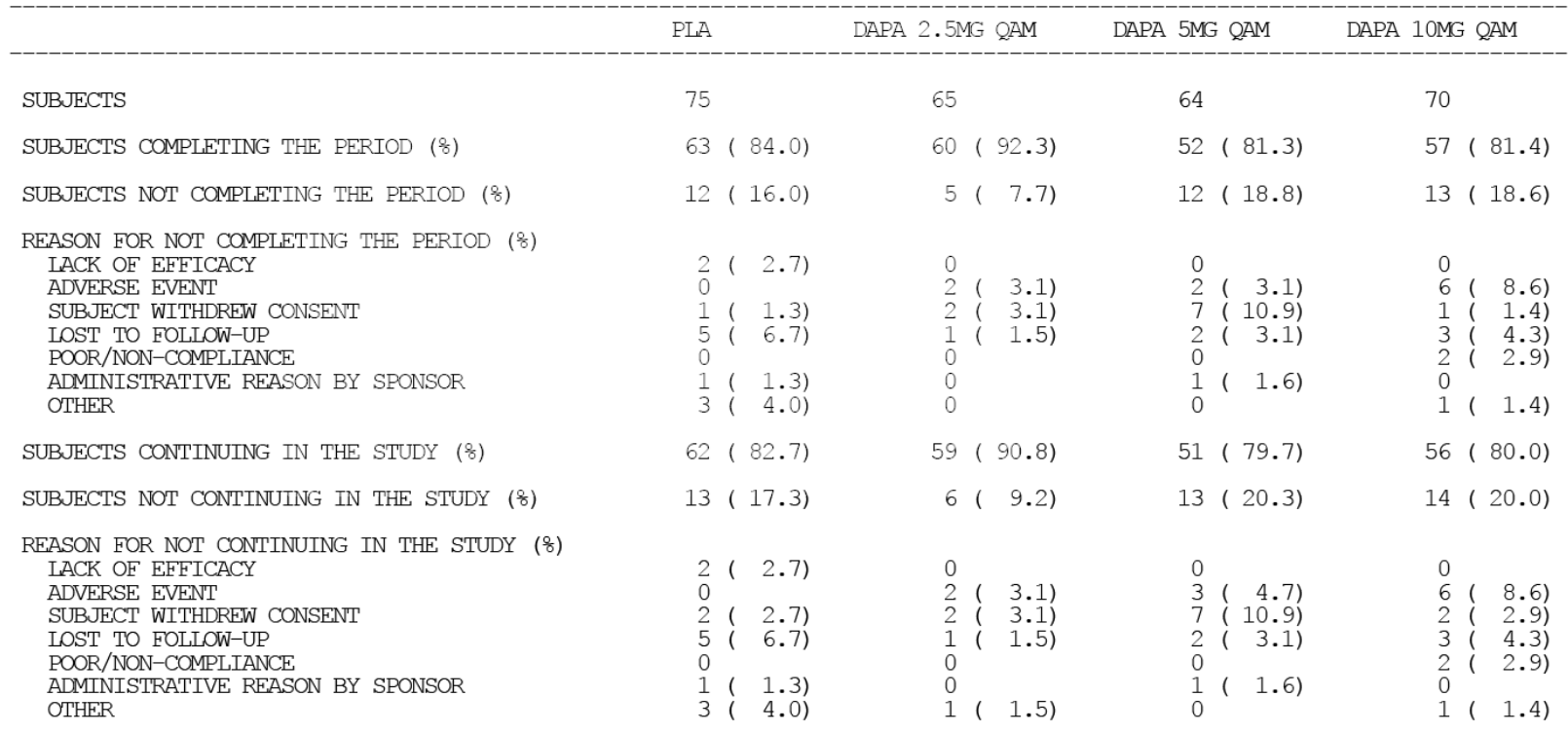

Table 3 shows the demographics of the treated subjects. Note that Table 4 also includes the baseline HbA1c for subjects in the primary analysis set. 
Table 3: Demographics for Treated Subjects, Study 2013

\begin{tabular}{|l|l|l|l|l|}
\hline & $\begin{array}{l}\text { Placebo } \\
\text { (N=75) }\end{array}$ & $\begin{array}{l}\text { DAPA 2.5 mg } \\
\text { (N=65) }\end{array}$ & $\begin{array}{l}\text { DAPA 5 mg } \\
\text { (N=64) }\end{array}$ & $\begin{array}{l}\text { DAPA 10 mg } \\
\text { (N=70) }\end{array}$ \\
\hline Age - Mean (SD) & $53(10)$ & $53(12)$ & $53(11)$ & $51(10)$ \\
\hline $\begin{array}{l}\text { Gender - N (\%) } \\
\text { Male } \\
\text { Female }\end{array}$ & $31(41 \%)$ & $36(55 \%)$ & $31(48 \%)$ & $34(49 \%)$ \\
\hline Race - N (\%) & $44(59 \%)$ & $29(45 \%)$ & $33(52 \%)$ & $36(51 \%)$ \\
$\begin{array}{l}\text { White } \\
\text { Black }\end{array}$ & $71(95 \%)$ & $63(97 \%)$ & $61(95 \%)$ & $63(90 \%)$ \\
Asian & $2(3 \%)$ & $0(0 \%)$ & $1(2 \%)$ & $2(3 \%)$ \\
Other & $2(3 \%)$ & $2(3 \%)$ & $1(2 \%)$ & $3(4 \%)$ \\
\hline $\begin{array}{l}\text { Region - N (\%) } \\
\text { U.S. or Canada } \\
\text { Rest of world }\end{array}$ & $0(0 \%)$ & $0(0 \%)$ & $1(2 \%)$ & $2(3 \%)$ \\
\hline
\end{tabular}

\section{Statistical Methodologies}

The Applicant submitted a Core SAP that includes common analyses for the Phase 3 studies. This SAP specified that, with the exception of Study C00012, the primary endpoint would be the change in HbA1c from baseline to Week $t$, with $t$ being the end of the short-term, double-blind treatment phase. In all but Study C00004, this was Week 24. Last observation carried forward (LOCF) imputation was used, excluding observations taken after rescue medication was given. For the superiority studies (all but C00004), the primary efficacy analysis was an ANCOVA which included treatment arm as a factor and baseline HbA1c as a covariate. The model could include additional terms, in particular factors other than site which were used to stratify the randomization.

The same ANCOVA analysis was used for secondary endpoints which constituted a change from baseline to Week $t$, such as change in FPG. For the proportion of subjects who reached a predefined criterion at Week $t$ (e.g., having been rescued), the planned analysis depended on whether the average number of responders in each treatment group was at least five. When there were sufficient responders, they used the method of Zhang, Tsiatis, and Davidian (2008) and Tsiatis et al. (2007) to adjust for baseline HbA1c. With fewer responders, they used Fisher's exact test and exact confidence intervals.

The Core SAP also specified that multiplicity would be controlled across primary and secondary endpoints using a sequential testing procedure, with the sequence specified for each study. The sequence was to be followed separately for each dose. Considering the large number of studies submitted, I do not consider this type of multiplicity to be a great concern. If a secondary endpoint were found to be significant (without adjustment) in several studies, then this result would be unlikely to be attributable to testing multiple endpoints. 
At first glance, the Core SAP gives the impression that different primary analysis sets (i.e., set of subjects included in the primary efficacy analysis) were used depending on whether the study was conducted by BMS or AZ. After requesting clarification from the Applicant, I determined that the primary analysis set for HbA1c was the same in either case: treated subjects with a baseline value as well as a post-baseline value.

In Study 2013, the randomization was stratified by site, using permuted blocks of size 7 (in Group 1). The LOCF imputation excluded observations after rescue. No additional factors were included in the ANCOVA model. Dunnett's adjustment was used to control for multiplicity across treatment arms.

\section{Results and Conclusions}

\section{Primary Efficacy Endpoint}

Table 4 shows the results of the primary efficacy analysis for Study 2013. The primary analysis, which used LOCF imputation, showed all three DAPA doses to be superior to placebo.

Table 4: Results for Primary Endpoint, Study 2013

(Source: Clinical Study Report, Table 7.2)

\begin{tabular}{|c|c|c|c|c|}
\hline $\begin{array}{l}\text { EFFICACY ENDPOTNT } \\
\text { STATISTICS }\end{array}$ & $\begin{array}{c}\text { PLA } \\
(\mathrm{N}=75)\end{array}$ & $\begin{array}{c}\text { LAPA } \underset{(\mathrm{N}-65)}{2.5 \mathrm{MG}} \text { QAM } \\
\end{array}$ & $\begin{array}{l}\text { DAPA 5MG QAM } \\
(\mathbb{N}=64)\end{array}$ & $\begin{array}{l}\text { DAPA 1OMG QAM } \\
(\mathrm{N}=70)\end{array}$ \\
\hline \multicolumn{5}{|l|}{ PRIMARY EFFICACY ENDPOINT } \\
\hline 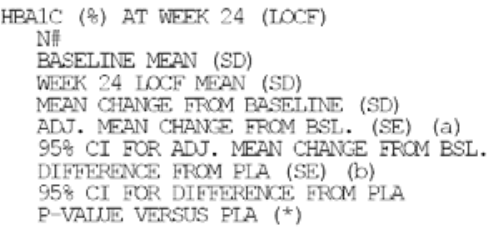 & $\begin{array}{cc}7.79 & (0.831) \\
7.60 & (1.434) \\
-0.19 & (1.154) \\
-0.23(0.1044) \\
(-0.43,-0.02)\end{array}$ & $\begin{array}{cc} & 64 \\
7.91 & (0.892) \\
7.32 & (1.178) \\
-0.59 & (1.090) \\
-0.58(0.1107) \\
(-0.80,-0.36) \\
-0.35(0.1522) \\
(-0.65,-0.05) \\
0.0207\end{array}$ & $\begin{array}{cc} & 61 \\
7.83(0.916) \\
7.09(0.775) \\
-0.74(0.871) \\
-0.77(0.1134) \\
(-0.99,-0.55) \\
-0.54(0.1541) \\
(-0.84,-0.24) \\
0.0005 *\end{array}$ & $\begin{array}{cc}8.01(0.952) \\
7.08(0.751) \\
-0.93(0.857) \\
-0.89(0.1099) \\
(-1.10,-0.67) \\
-0.66(0.1518) \\
(-0.96,-0.36) \\
<.0001\end{array}$ \\
\hline
\end{tabular}

$\mathrm{N}$ is the number of randomized subjects who took at least one dose of double-blind study medication.

N\# is the number of randonized subjects with non-missing baseline and week $t$ (LOCF) valves.

(a) Adjusted mean change from baseline (b) Difference in adjusted mean change from baseline versus PIA

$\left(^{*}\right)$ Significant p-value: Primary endpoint is tested at alpha $=0.019$ applying the Dunnett adjustment.

Analysis of continuous outcomes based on separate ANCovA models with treatment group as an effect and baseline value as a covariate. Both OAM and OPM treatment crouns are included in the same ANCOVA model analvsis.

Note that two sample sizes are listed in the table for each arm: $N$ and $N \# . N$ is the number of treated subjects, as shown, for example, in Table 2 . N\# is the number of subjects included in the primary analysis, which was restricted to those who had both baseline and post-baseline values for HbA1c. FDA agreed to this analysis in advance. As the table shows, a total of 12 treated subjects (out of 274) were excluded from the primary analysis. There was not a notable imbalance between the treatment arms. 
The Applicant reported the results of three sensitivity analyses for the primary endpoint:

1) ANCOVA of observed cases, excluding observations after rescue,

2) Mixed-effects model for repeated measures (MMRM), excluding observations after rescue, and

3) MMRM, including observations after rescue, with a rescue term in the model. I do not believe that sensitivity analysis 3 ) is appropriate. It is not actually possible to adjust for use of rescue medication in the way that the Applicant desires. The problem is that subjects who received rescue differed from the other subjects in at least two ways. First of all, they experienced the effect of rescue treatment, and this is the effect that one would want adjust for if it were possible. Secondly, they also met glycemic criteria in order to be eligible for rescue. This issue is discussed more in Section 5.

As a replacement for \#3, I have included a sensitivity analysis in which the primary HbA1c outcomes are used regardless of rescue treatment, and no statistical adjustment is made for rescue. This approach is also imperfect, but it comes closer to being a true intent-to-treat analysis because it disregards the non-randomized rescue treatment. In this case, my preferred analysis and 3) yield similar estimates of the treatment effect, i.e., the adjustment for rescue is small. The key SAS statement for my analysis is as follows:

Figure 10, which can be found in Appendix B, shows the results for primary LOCF analysis, the Applicant's sensitivity analyses 1) and 2), and my own preferred analysis. For simplicity, only the DAPA $10 \mathrm{mg}$ and placebo arms are shown. Note that the estimates from the dapagliflozin 10 mg arm are fairly consistent, partly due to the fact that no patients were rescued in this arm. Hence the figure can be most easily comprehended by focusing on the placebo arm. The LOCF analysis (green line with squares) shows a reduction in HbA1c of $0.23 \%$ at Week 24 . This analysis assumes that the nine rescued patients (as well as patients who discontinued the study early) would have continued to have the same HbA1c value until the end of the trial. In contrast, the ANCOVA analysis of observed cases (red with circles) shows a reduction of $0.62 \%$. This analysis is particularly favorable to the placebo arm because patients who needed rescue are not included in the week 24 analysis at all. (Each time point is modeled separately). The MMRM analysis which excludes post-rescue observations (black with diamonds) shows a reduction of 0.29\%. Finally, the MMRM analysis which includes post-rescue observations (dark blue with Xs) shows a decrease of $0.45 \%$. This final analysis, which I prefer on theoretical grounds, yields an estimated treatment effect, i.e., difference from placebo, for the $10 \mathrm{mg}$ dose of $0.45 \%$ (95\% confidence interval $=[0.19 \%, 0.72 \%])$. In contrast, the LOCF analysis yields an estimated treatment effect of $0.66 \%$ (95\% confidence interval $=[0.36 \%, 0.96 \%])$ Note that the treatment effect is statistically significant either way. The $5 \mathrm{mg}$ DAPA arm also shows an attenuated, but significant, treatment effect under my sensitivity analysis. See Table 44 in Section 5 for further results from this analysis. 


\section{Secondary Efficacy Endpoints}

Table 5 shows Applicant's results (which I reproduced) for the secondary endpoint of FPG at Week 24. Both the 5 and $10 \mathrm{mg}$ dose of DAPA were significantly different from placebo under the Applicant's sequential testing procedure. Note that all but two treated subjects were included in the analysis set.

Table 5: Results for Fasting Plasma Glucose at Week 24, Study 2013 (Source: Clinical Study Report, Table 7.3.1)

\begin{tabular}{|c|c|c|c|c|}
\hline $\begin{array}{l}\text { EFFICACY ENDPOINT } \\
\text { STATISTICS }\end{array}$ & $\begin{array}{c}\text { PLA } \\
(\mathrm{N}=75)\end{array}$ & DAPA $\underset{(\mathrm{N}=65)}{2.5 \mathrm{MG}} \mathrm{QAM}$ & $\begin{array}{l}\text { DAPA 5MG QAM } \\
(\mathrm{N}=64)\end{array}$ & $\begin{array}{l}\text { DAPA 10MG QAM } \\
\quad(\mathrm{N}=70)\end{array}$ \\
\hline \multicolumn{5}{|l|}{ SECONDARY EFFICACY ENDPOINT } \\
\hline $\begin{array}{l}\text { EPG (MG/DL) AT WEEK } 24 \text { (IOCE) } \\
\text { N\# } \\
\text { BASELINE MEAN (SD) } \\
\text { WEFK } 24 \text { IOCE MEAN (SD) } \\
\text { MEAN CHANGE FROM BASELINE (SD) } \\
\text { ADJ. MEAN CHANGE FROM BSL. (SE) (a) } \\
\text { 95\% CI FOR ADJ. MEAN CHANGE FROM BSL. } \\
\text { DIFHERENCE FROM PLA (SE) (b) } \\
\text { 95\% CI FOR DIFHERENCE FROM PLA } \\
\text { P-VALUE VERSUS PLA (*) }\end{array}$ & \begin{aligned} & \multicolumn{1}{c}{75} \\
& 159.9$(42.08) \\
& 156.7(49.81) \\
&-3.2(33.42) \\
&-4.1(3.906) \\
&(-11.8,3.5)\end{aligned}$ & $\begin{array}{cc} & 65 \\
164.1 \quad(48.01) \\
147.7(64.95) \\
-16.4(51.77) \\
-15.2(4.196) \\
(-23.5,-7.0) \\
-11.1 \quad(5.734) \\
\quad(-22.4,0.2)\end{array}$ & 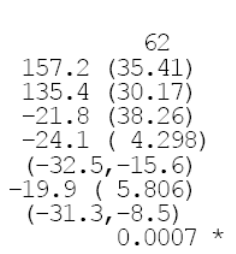 & $\begin{array}{rc} & 70 \\
166.6 & (41.46) \\
135.4 & (25.84) \\
-31.3(35.30) \\
-28.8(4.046) \\
(-36.8,-20.9) \\
-24.7(5.626) \\
(-35.7,-13.6) \\
<.0001\end{array}$ \\
\hline
\end{tabular}

Table 6 shows the results for change in total body weight at Week 24. All arms showed a mean loss of weight from baseline, and none of the DAPA arms were significantly different from placebo. Figure 11 in Appendix B illustrates the change in body weight for this study, as well as the other three placebo-controlled studies that I focused on (2014, 2030, C00006)

\section{Table 6: Results for Total Body Weight at Week 24, Study 2013}

(Source: Clinical Study Report, Table 7.3.2)

\begin{tabular}{|c|c|c|c|c|}
\hline $\begin{array}{l}\text { EFFICACY ENDPOINT } \\
\text { STATISTICS }\end{array}$ & $\underset{(\mathrm{N}=75)}{\mathrm{PLA}}$ & DAPA $\underset{(\mathbb{N}=65)}{2.5 M G}$ QAM & $\underset{(\mathrm{N}=64)}{\operatorname{DAPA} 5 \mathrm{MG} Q \mathrm{QAM}}$ & $\begin{array}{l}\text { DAPA 10MG QAM } \\
(\mathrm{N}=70)\end{array}$ \\
\hline
\end{tabular}

SECONDARY EFFICACY ENDPOINT

TOTAL BODY WEIGHT (KG) AT WEEK 24 (LOCE)

NH

BASELINE MEAN (SD)

WEEK 24 IOCF MEAN (SD)

MEAN CHANGE FROM BASELINE (SD)

ADJ. MEAN CHANGE FROM BSL. (SE) (a)

$95 \%$ CI FOR ADJ. MEAN CHANGE FRCM BSL.

DIFFERENCE FROM PLA (SE) (b)

958 CI FOR DIFFERENCE FFOM PLA

P-VALUE VERSUS PLA (*)
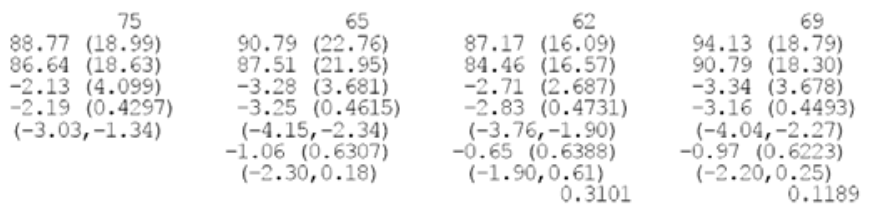

$\mathrm{N}$ is the number of randomized subjects who took at least one dose of double-blind study medication.

$N \#$ is the number of randomized subjects with non-missing baseline and Week $t$ (LOCF) values.

(a) Adjusted mean change from baseline (b) Difference in adjusted mean change from baseline versus PLA

(*) Significant p-value: Secondary endpoints are tested following a secuential testing procedure at alpha=0.05.

Analysis of continuous outcomes based on separate ANCOVA models with treatment group as an effect and baseline value as a covariate.

As I noted earlier, the use of rescue has an impact on the primary analysis that is difficult to account or adjust for. However, it is appropriate to treat rescue as a distinct measure of efficacy. As Table 7 shows, both the 5 and $10 \mathrm{mg}$ doses of DAPA had very low rates of rescue and were significantly better than placebo using an exact test. Note: the pre-specified endpoint was 
“subjects discontinued or rescued for failing to achieve pre-specified glycemic targets”, but in fact all subjects in the numerator were rescued.

Table 7: Subjects Rescued by Week 24, Study 2013

(Source: Clinical Study Report, Table 7.4.3C)

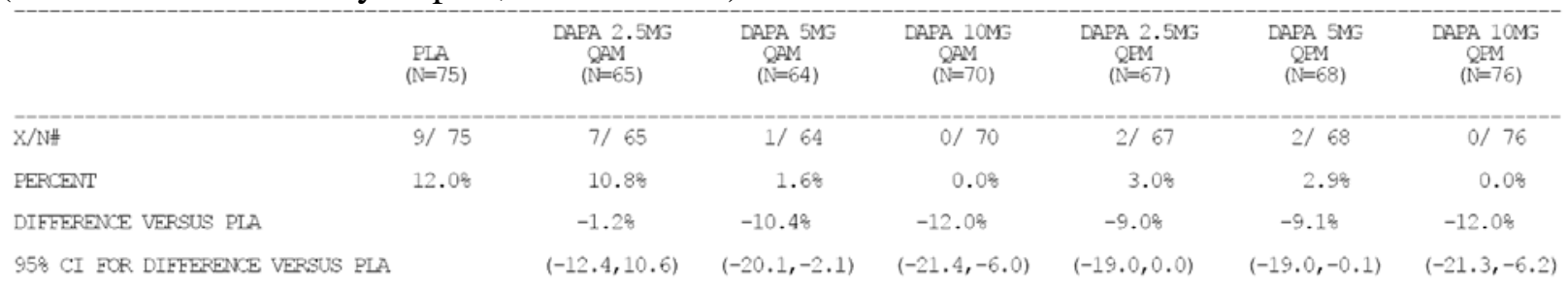

$\mathrm{N}$ is the number of randcmized subjects who took at least one dose of double-blind study medication.

Nif is the number of randimized subjects with non-missing baseline and Week 24 (LOCF) values.

$\mathrm{X}$ is the mumber of responders baseline HoAlc. In case of less than 5 events per treatment group on average, the exact method is used. 


\section{Study Design and Endpoints}

Figure 2 shows the design of Study 2014, which tested DAPA as an add-on to a stable dose of metformin.

\section{Figure 2: Design for Study 2014}

(Source: Clinical Study Report, Figure 3.1)

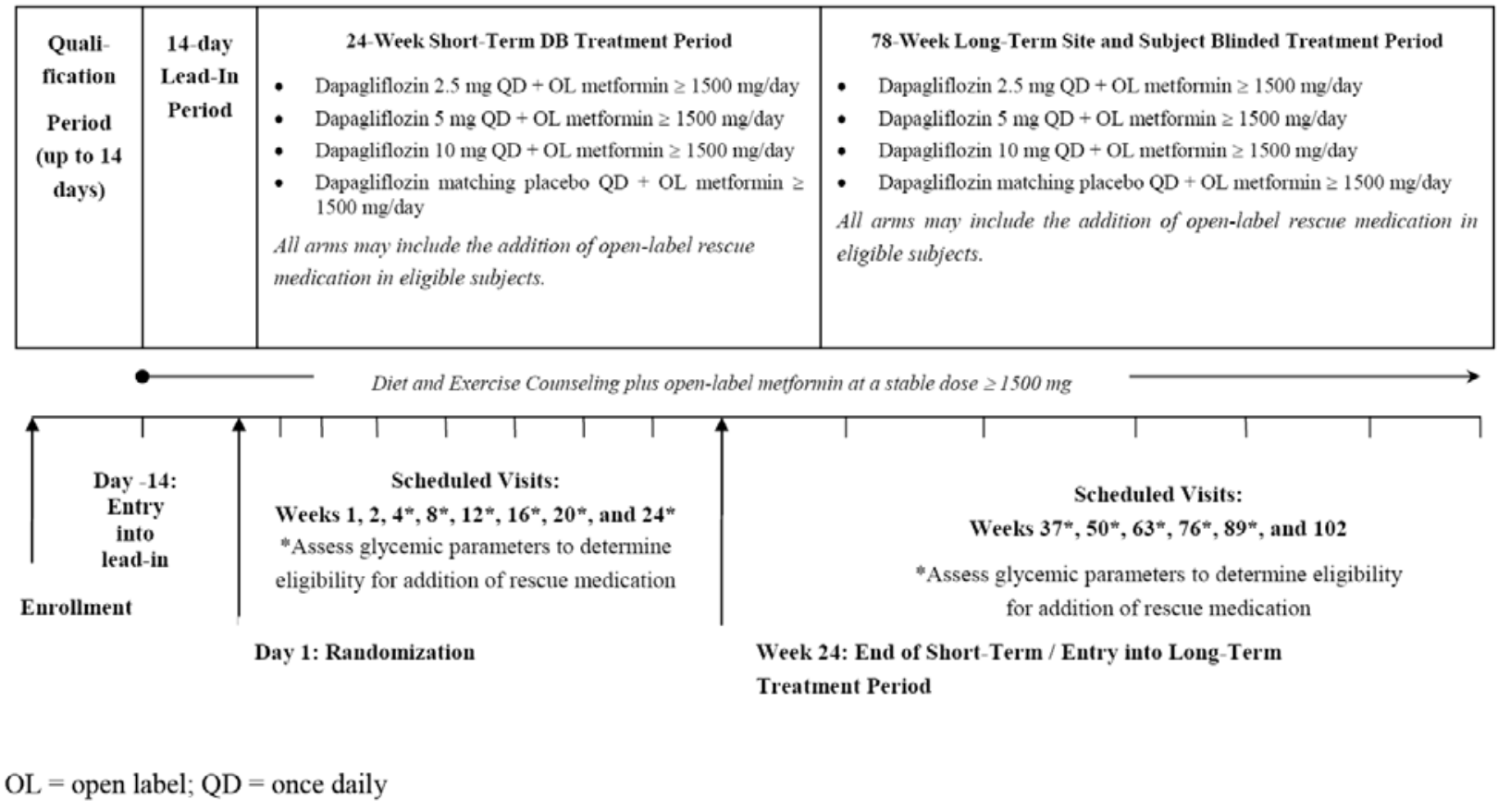

As with Study 2013, the primary efficacy endpoint was change in HbA1c from baseline at Week 24. The same secondary endpoints were used.

\section{Patient Disposition, Demographic and Baseline Characteristics}

Table 8 shows the disposition of treated subjects. 
Table 8: Disposition of Treated Subjects for Study 2014

(Source: Clinical Study Report, Table 5.1B)

\begin{tabular}{|c|c|c|c|c|c|}
\hline & PLA + MET & DAPA 2.5MG + MET & DAPA $5 \mathrm{MG}+\mathrm{MET}$ & DAPA 10MG & $+\mathrm{MET}$ \\
\hline SUBJECTS & 137 & 137 & 137 & 135 & \\
\hline SUBJECTS NOT COMPLETING THE PERIOD (\%) & $18(13.1)$ & $16(11.7)$ & $15(10.9)$ & 14 & $(10.4)$ \\
\hline $\begin{array}{l}\text { REASON FOR NOT COMPLETING THE PERIOD (\%) } \\
\text { LACK OF EFEICACY } \\
\text { ADVERSE EVENT } \\
\text { SUBJECT WITHDREW CONSENT } \\
\text { LOST TO FOLIOW-UP } \\
\text { POOR/NON-COMPLIANCE } \\
\text { SUBJECT NO LONGER MEETS STUDY CRITERIA } \\
\text { ADMINISTRATIVE REASON BY SPONSOR } \\
\text { OTHER }\end{array}$ & $\begin{array}{lll}2 & (1.5) \\
4 & (2.9) \\
6 & (1.4) \\
5 & ( & 3.7) \\
0 & \\
0 & \\
1 & ( & 0.7) \\
0 & \end{array}$ & 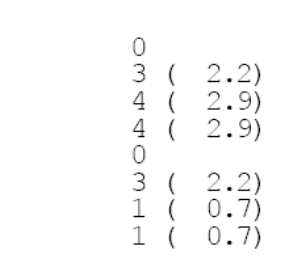 & 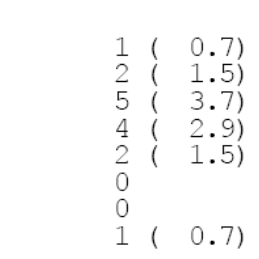 & $\begin{array}{l}0 \\
3 \\
2 \\
5 \\
0 \\
2 \\
1 \\
1\end{array}$ & 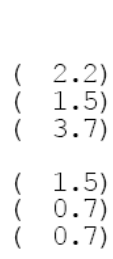 \\
\hline SUBJECTS CONTINUING IN THE STUDY (\%) & $115(83.9)$ & $120(87.6)$ & $122(89.1)$ & 119 & $(88.1)$ \\
\hline
\end{tabular}

Table 9 shows the demographics of the treated subjects.

Table 9: Demographics for Treated Subjects, Study 2014

\begin{tabular}{|l|l|l|l|l|}
\hline & $\begin{array}{l}\text { Placebo } \\
\text { (N=137) }\end{array}$ & $\begin{array}{l}\text { DAPA 2.5 mg } \\
\text { (N=137) }\end{array}$ & $\begin{array}{l}\text { DAPA 5 mg } \\
\text { (N=137) }\end{array}$ & $\begin{array}{l}\text { DAPA 10 mg } \\
\text { (N=135) }\end{array}$ \\
\hline Age - Mean (SD) & $54(10)$ & $55(9)$ & $54(9)$ & $53(10)$ \\
\hline $\begin{array}{l}\text { Gender - N (\%) } \\
\text { Male }\end{array}$ & & & & \\
Female & $76(55 \%)$ & $70(51 \%)$ & $69(50 \%)$ & $77(57 \%)$ \\
\hline Race - N (\%) & $61(45 \%)$ & $67(49 \%)$ & $68(50 \%)$ & $58(43 \%)$ \\
White & & & & \\
Black & $124(91 \%)$ & $117(85 \%)$ & $118(86 \%)$ & $121(90 \%)$ \\
Asian & $2(1 \%)$ & $5(4 \%)$ & $6(4 \%)$ & $4(3 \%)$ \\
Other & $3(2 \%)$ & $3(2 \%)$ & $4(3 \%)$ & $1(1 \%)$ \\
\hline $\begin{array}{l}\text { Region - N (\%) } \\
\text { U.S. or Canada } \\
\text { Rest of world }\end{array}$ & $8(6 \%)$ & $12(9 \%)$ & $9(7 \%)$ & $9(7 \%)$ \\
\hline
\end{tabular}

\section{Statistical Methodologies}

Except where otherwise noted, the statistical methods were the same as for Study 2013. Randomization was by site, using permuted blocks with a size of four. 


\section{Results and Conclusions}

\section{Primary Efficacy Endpoint}

Table 10 shows the results for the primary endpoint for Study 2014. All three DAPA arms were statistically superior to placebo. Twelve treated subjects were excluded from the analysis because they did not have post-baseline HbA1c measurements, but these exclusions were balanced across the treatment arms.

Table 10: Results for Primary Endpoint, Study 2014 (Source: Clinical Study Report, Table 7.2A)

\begin{tabular}{|c|c|c|c|c|}
\hline $\begin{array}{l}\text { EFFICACY ENDPOINI } \\
\text { STATISTICS }\end{array}$ & $\underset{(\mathrm{N}=137)}{\mathrm{PLA}+\mathrm{MET}}$ & $\begin{array}{c}\text { DAPA } 2.5 \mathrm{MG}+\mathrm{MET} \\
(\mathrm{N}=137)\end{array}$ & $\begin{array}{c}\text { DAPA } 5 M G+M E T \\
(N=137)\end{array}$ & DAPA $\underset{(\mathbb{N}=135)}{10 M G+M E T}$ \\
\hline \multicolumn{5}{|l|}{ PRIMARY EFEICACY ENDPOINT } \\
\hline $\begin{array}{l}\text { BAIC (8) AT WEEK } 24 \text { (LOCE) } \\
\text { NH } \\
\text { BASELINE MEAN (SD) } \\
\text { WEEK } 24 \text { IOCF MEAN (SD) } \\
\text { MEAN CHANGE FROM BASELINE (SD) } \\
\text { ADJ. MEAN CHANGE FROM BSL. (SE) (a) } \\
95 \% \text { CI FOR ADU. MEAN CHANGE FROM BSL. } \\
\text { DIFFERENCE FROM PIA + MET (SE) (b) } \\
95 \% \text { CI FOR DIFFERENCE FROM PLA + MET } \\
\left.\text { P-VALUE VS. PLA + MET ( }{ }^{\star}\right)\end{array}$ & $\begin{aligned} 8.11 & (134 \\
7.79 & (1.959) \\
-0.32( & (0.975) \\
-0.30 & (0.0718) \\
(-0.44, & -0.16)\end{aligned}$ & $\begin{array}{cc} & 135 \\
7.99( & 0.897) \\
7.34( & 0.934) \\
-0.65( & 0.882) \\
-0.67(0.0715) \\
(-0.81, & -0.53) \\
-0.38(0.1014) \\
(-0.58, & -0.18) \\
0.0002 \star\end{array}$ & 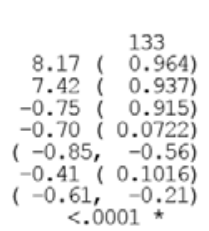 & 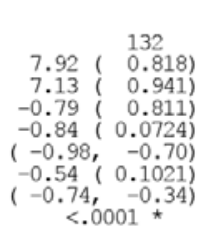 \\
\hline
\end{tabular}

$\mathrm{N}$ is the number of randomized subjects who took at least one dose of double-blind study modication.

N\# is the number of randomized subjects with non-missing baseline and Week $t$ (LOCF) values.

(a) Adjusted mean change from baseline (b) Difference in adjusted mean change from baseline vs. PLA + MET

$(*)$ Significant p-value: Primary endpoint is tested at alpha $=0.019$ applying the Dunnett adjustment,

Figure 12 shows the results of the same sensitivity analyses for this study as were done for Study 2013. Note that unlike that study, Study 2014 had background therapy. Given this difference, it is not surprising that the estimated treatment effect is more consistent across the various analyses. The largest estimated treatment effect, $0.54 \%$, comes from the LOCF analysis; the smallest, $0.38 \%$, comes from the ANCOVA of observed cases. The MMRM analysis which includes post-rescue data yields a treatment effect of $0.44 \%$, with a $95 \%$ confidence interval of $[0.24 \%, 0.63 \%]$. See Table 44 for further results from this analysis.

\section{Secondary Efficacy Endpoints}

Table 11 shows the results for fasting plasma glucose at Week 24. All three DAPA doses showed a significantly larger reduction from baseline than placebo. 
Table 11: Results for Fasting Plasma Glucose at Week 24, Study 2014

(Source: Clinical Study Report, Table 7.3.1)

\begin{tabular}{|c|c|c|c|c|}
\hline $\begin{array}{l}\text { EFFICACY ENDPOINT } \\
\text { STATISTICS }\end{array}$ & $\begin{array}{c}\mathrm{PLA}+\mathrm{MET} \\
(\mathrm{N}=137)\end{array}$ & $\begin{array}{c}\text { DAPA } \\
\text { 2.5MG }+\mathrm{MET} \\
(\mathrm{N}=137)\end{array}$ & $\begin{array}{c}\text { DAPA } 5 \mathrm{MG}+\mathrm{MET} \\
(\mathrm{N}=137)\end{array}$ & $\begin{array}{c}\text { DAPA } \\
(\mathrm{N}=135)\end{array}$ \\
\hline \multicolumn{5}{|l|}{ KEY SECONDARY EFFICACY ENDPOINT } \\
\hline $\begin{array}{l}\text { FPG (MG/DL) AT WEFK } 24 \text { (LOCE) } \\
\text { N\# } \\
\text { BASELINE MFAN (SD) } \\
\text { WEFK } 24 \text { LOCE MEAN (SD) } \\
\text { MEAN CHANGE FROM BASELINE (SD) } \\
\text { ADJ. MEAN CHANGE FROM BSL. (SE) (a) } \\
\text { 95\% CI FOR ADJ. MFAN CHANGE FROM BSL. } \\
\text { DIFHERENCE FROM PLA + MET (SE) (b) } \\
\text { 95\% CI FOR DIFHERENCE FROM PLA + MET } \\
\text { P-VALUE VS. PLA + MET (*) }\end{array}$ & $\begin{array}{rr}165.6( & 46.39) \\
158.3( & 44.61) \\
-7.3( & 41.06) \\
-6.0( & 2.673) \\
(-11.2, & -0.7)\end{array}$ & 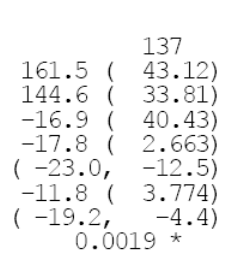 & 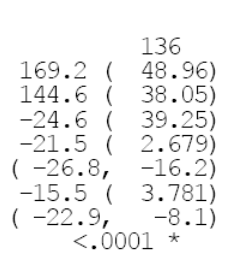 & 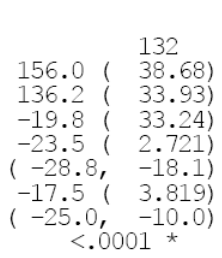 \\
\hline
\end{tabular}

Table 12 shows the results for total body weight at Week 24. All arms showed a reduction in body weight, but the three DAPA arms showed a significantly larger reduction. This is illustrated graphically in Figure 11.

Table 12: Results for Total Body Weight at Week 24, Study 2014

(Source: Clinical Study Report, Table 7.3.2)

\begin{tabular}{|c|c|c|c|c|}
\hline $\begin{array}{l}\text { EFFICACY ENDPOINT } \\
\text { STATISTICS }\end{array}$ & $\begin{array}{c}\mathrm{PLA}+\mathrm{MET} \\
(\mathrm{N}=137)\end{array}$ & 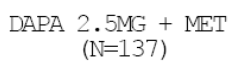 & $\begin{array}{c}\text { DAPA } 5 \mathrm{MG}+\mathrm{MET} \\
(\mathrm{N}=137)\end{array}$ & DAPA $\underset{(N=135)}{10 M G+M E T}$ \\
\hline YY SECONDARY EFFICACY EN & & & & \\
\hline $\begin{array}{l}\text { TOTAL BODY WEIGHT (KG) AT WEEK } 24 \text { (LOCE) } \\
\text { N\# } \\
\text { BASELINE MEAN (SD) } \\
\text { WEEK } 24 \text { LOCE MEAN (SD) } \\
\text { MEAN CHANGE FROM BASELINE (SD) } \\
\text { ADJ. MEAN CHANGE FROM BSL. (SE) (a) } \\
\text { 95\% CI FOR ADJ. MFAN CHANGE FROM BSL. } \\
\text { DIFEERENCE FROM PLA + MET (SE) (b) } \\
\text { 95\% CI FOR DIFFERENCE FROM PLA + MET } \\
\text { P-VALUE VS. PLA + MET (*) }\end{array}$ & $\begin{array}{lc}87.74 & (136 \\
86.81 & (18.242) \\
-0.93 & (1828) \\
-0.89 & (0.2328) \\
(-1.35, & -0.42)\end{array}$ & $\begin{array}{l}84.90(17.773) \\
82.71(17.637) \\
-2.19(2.901) \\
-2.21(0.2357) \\
(-2.67,-1.75) \\
-1.32(0.3344) \\
(-1.98,-0.67) \\
<.0001 *\end{array}$ & $\begin{array}{l}84.73(16.265) \\
81.71(16.143) \\
-3.02(2.651) \\
-3.04(0.2358) \\
(-3.51,-2.58) \\
-2.16(0.3344) \\
(-2.81,-1.50) \\
<.0001 *\end{array}$ & $\begin{array}{l}86.28(17.527) \\
83.42(17.391) \\
-2.86(3.033) \\
-2.86(0.2392) \\
(-3.33,-2.39) \\
-1.97(0.3365) \\
(-2.63,-1.31) \\
<.0001 *\end{array}$ \\
\hline
\end{tabular}

Table 13 shows the proportion of subjects rescued by Week 24. I reproduced the counts but did not attempt to reproduce the Applicant's confidence intervals. Instead I conducted exact tests which showed the three DAPA arms to be superior to placebo ( $\mathrm{p}<.001$ in each case).

Table 13: Subjects Rescued by Week 24, Study 2014

(Source: Clinical Study Report, Table 7.4.2C)

\begin{tabular}{|c|c|c|c|c|}
\hline & $\begin{array}{c}\mathrm{PLA}+\mathrm{MET} \\
(\mathrm{N}=137)\end{array}$ & $\begin{array}{c}\text { DAPA } \underset{(\mathrm{N}=137)}{2.5 \mathrm{MG}}+\mathrm{MET} \\
\end{array}$ & $\begin{array}{c}\text { DAPA } 5 \mathrm{MG}+\mathrm{MET} \\
(\mathrm{N}=137)\end{array}$ & $\begin{array}{c}\text { DAPA } \\
(\mathrm{N}=135)\end{array}$ \\
\hline $\begin{array}{l}\text { X/N\# } \\
\text { PERCENT } \\
\text { PERCENT ADUUSTED FOR BASELINE HBAIC } \\
\text { 95\% CI FOR PERCENT ADJ FOR BSL. HBA1C } \\
\text { DIFEERNCE VS. PLA + MET } \\
\text { 95\% CI FOR DIFEERENCE VS. PLA + MET }\end{array}$ & $\begin{array}{c}22 / 137 \\
16.18 \\
15.0 \% \\
(9.2, \quad 20.7)\end{array}$ & $\begin{array}{c}5 / 137 \\
3.6 \% \\
4.0 \% \\
(\quad 0.9,7.0) \\
-11.08 \\
(-17.6,-4.4)\end{array}$ & $\begin{array}{c}5 / 137 \\
3.6 \% \\
3.1 \% \\
(\quad 0.1,6.1) \\
-11.8 \% \\
(-18.4,-5.3)\end{array}$ & $\begin{array}{c}5 / 135 \\
3.7 \% \\
4.4 \% \\
(1.3,7.6) \\
-10.5 \% \\
(-17.2,-3.9)\end{array}$ \\
\hline
\end{tabular}




\section{Study Design and Endpoints}

Figure 3 illustrates the design of Study 2030, which test DAPA as an add-on to pioglitazone (PIO).

Figure 3: Design for Study 2030 (Source: Clinical Study Report, Figure 3.1)

\begin{tabular}{|c|c|c|c|c|}
\hline $\begin{array}{l}\text { Quali- } \\
\text { fication } \\
\text { Period } \\
\text { (up to } \\
14 \text { days) }\end{array}$ & $\begin{array}{c}\begin{array}{c}\text { Dose } \\
\text { Optimization } \\
\text { Period } \\
\text { (10 weeks) }\end{array} \\
\text { This period is } \\
\frac{\text { only }}{\text { applicable for }} \\
\frac{\text { subjects in }}{\text { Group } 2 .}\end{array}$ & $\begin{array}{l}\text { 14-day } \\
\text { Lead- } \\
\text { In } \\
\text { Period }\end{array}$ & $\begin{array}{l}\text { 24-Wk Short-Term DB Treatment Period } \\
\text { - Dapagliflozin } 5 \mathrm{mg} \mathrm{QD} \mathrm{+} \mathrm{OL} \mathrm{pioglitazone} \\
\geq 30 \mathrm{mg} \mathrm{QD} \\
\text { - Dapagliflozin } 10 \mathrm{mg} \mathrm{QD}+\mathrm{OL} \text { pioglitazone } \\
\geq 30 \mathrm{mg} \mathrm{QD} \\
\text { - Dapagliflozin matching placebo QD + OL } \\
\text { pioglitazone } \geq 30 \mathrm{mg} \mathrm{QD} \\
\text { Dose titration of blinded study medication or open- } \\
\text { label pioglitazone is not permitted at anytime } \\
\text { during the short term period. } \\
\text { All arms may include the addition of open-label } \\
\text { metformin or sulfonylurea rescue medication in } \\
\text { eligible subjects. }\end{array}$ & 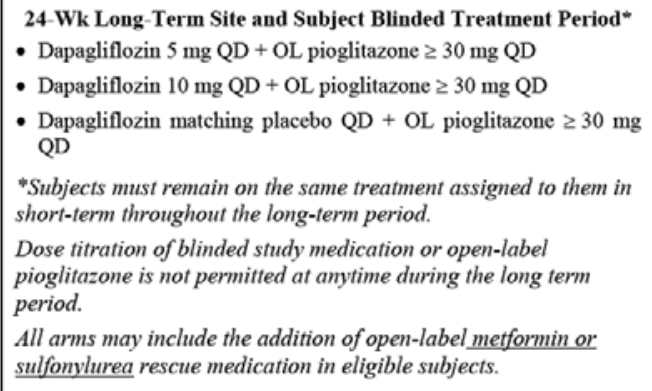 \\
\hline \multicolumn{5}{|c|}{ Diet and Exercise Counseling plus open-label pioglitazone $\geq 30 \mathrm{mg}$ QD } \\
\hline & $T$ & $T$ & 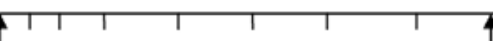 & $T$ \\
\hline & $\begin{array}{l}\text { Entry into dose } \\
\text { ptimization } \\
\text { eriod } \\
\text { This period is } \\
\text { nly applicable } \\
\text { or subjects in } \\
\text { Group 2.) }\end{array}$ & $\begin{array}{l}\text { Day -14: } \\
\text { Entry } \\
\text { into } \\
\text { lead-in } \\
\text { period }\end{array}$ & $\begin{array}{l}\text { Scheduled Visits: } \\
\text { Weeks } 1,2,4^{*}, 8^{*}, 12^{*}, 16^{*} \text {, } \\
20^{*} \text {, and } 24^{*} \\
\text { * Assess glycemic parameters } \\
\text { to determine eligibility for } \\
\text { addition of rescue medication } \\
\text { Dav 1: Randomization }\end{array}$ & $\begin{array}{c}\text { Scheduled Visits: } \\
\text { Weeks } 36^{*} \text { and } 48 \\
\text { *Assess glycemic parameters to } \\
\text { determine eligibility for addition } \\
\text { of rescue medication }\end{array}$ \\
\hline
\end{tabular}

The primary endpoint was the same as in the previous study, change in HbA1c at Week 24. The secondary endpoints were similar to those from the other studies, however 120-minute postchallenge plasma glucose was added.

\section{Patient Disposition, Demographic and Baseline Characteristics}

Table 14 shows the disposition of the treated subjects. 
Table 14: Disposition of Treated Subjects, Study 2030

(Source: Clinical Study Report, Table 5.1B)

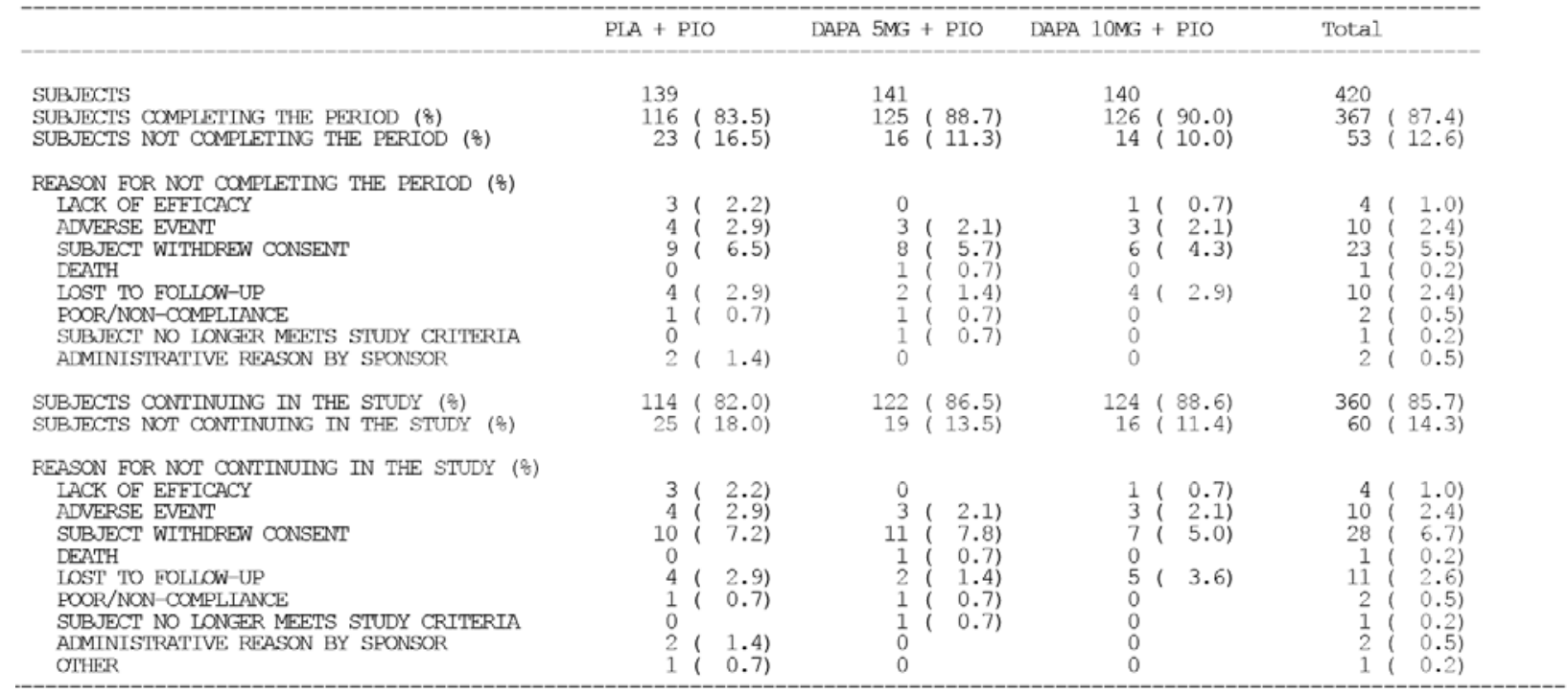

Table 15 shows the demographics of the treated subjects.

Table 15: Demographics for Treated Subjects, Study 2030

\begin{tabular}{|l|l|l|l|}
\hline & $\begin{array}{l}\text { Placebo } \\
\text { (N=139) }\end{array}$ & $\begin{array}{l}\text { DAPA 5 mg } \\
\text { (N=141) }\end{array}$ & $\begin{array}{l}\text { DAPA 10 mg } \\
\text { (N=140) }\end{array}$ \\
\hline Age - Mean (SD) & $53(11)$ & $53(11)$ & $54(10)$ \\
\hline $\begin{array}{l}\text { Gender - N (\%) } \\
\text { Male }\end{array}$ & $71(51 \%)$ & $78(55 \%)$ & $59(42 \%)$ \\
Female & $68(49 \%)$ & $63(45 \%)$ & $81(58 \%)$ \\
\hline Race - N (\%) & & & \\
White & $102(73 \%)$ & $102(72 \%)$ & $101(72 \%)$ \\
Black & $6(4 \%)$ & $9(6 \%)$ & $7(5 \%)$ \\
Asian & $24(17 \%)$ & $26(18 \%)$ & $21(15 \%)$ \\
Other & $7(5 \%)$ & $4(3 \%)$ & $11(8 \%)$ \\
\hline $\begin{array}{l}\text { Region - N (\%) } \\
\text { U.S. or Canada } \\
\text { Rest of world }\end{array}$ & $61(44 \%)$ & $65(46 \%)$ & $62(44 \%)$ \\
\hline
\end{tabular}

\section{Statistical Methodologies}

Except where otherwise noted, the statistical methods were the same as for the previous studies. Central randomization was used in this study. The randomization was not stratified by site, but by pre-enrollment anti-diabetic therapy (two levels). The block size was 3 . 


\section{Results and Conclusions}

\section{Primary Efficacy Endpoint}

Table 16 shows the results for the primary endpoint. Both DAPA doses were statistically superior to placebo.

Table 16: Results for Primary Endpoint, Study 2030

(Source: Clinical Study Report, Table 7.2)

\begin{tabular}{|c|c|c|c|}
\hline $\begin{array}{l}\text { EFFICACY ENDFOINT } \\
\text { STATISTICS }\end{array}$ & $\begin{array}{c}\text { PLA + PIO } \\
(\mathrm{N}=139)\end{array}$ & $\begin{array}{l}\text { DAPA } 5 M G+\text { PIO } \\
(\mathrm{N}=141)\end{array}$ & 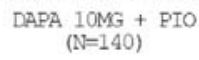 \\
\hline PRIMARY EFFICACY ENDPOINT & & & \\
\hline $\begin{array}{l}\text { HEAIC (\%) AT WEEK } 24 \text { (LOCE) } \\
\text { N\# } \\
\text { BASELINE MEAN (SD) } \\
\text { WEEK } 24 \text { LOCF MEAN (SD) } \\
\text { MEAN CHANGE FRCM BASELINE (SD) } \\
\text { ADUUSTED MEAN CHANGE FROM BASELTNE (SE) } \\
958 \text { CI FOR ADJ. NEAN CHANGE FROM BASELINE } \\
\text { DIFFERENCE FRCM PLA + PIO (SE) } \\
\text { 95\% CI FOR DIFFERENCE FROM PLA + PIO } \\
\text { P-VALUE VS. PLA + PIO (*) }\end{array}$ & $\begin{array}{cc}8.34 & (138) \\
7.93(1.003) \\
-0.42(0.975) \\
-0.42(0.0834) \\
(-0.59,-0.26)\end{array}$ & $\begin{array}{c} \\
8.40(1.00) \\
7.56(1.068) \\
-0.83(0.996) \\
-0.82(0.0828) \\
(-0.99,-0.66) \\
-0.40(0.1175) \\
(-0.63,-0.17) \\
0.0007 \star\end{array}$ & 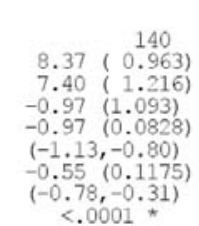 \\
\hline
\end{tabular}

$\mathrm{N}$ is the number of randomized subjects who took at least one dose of double-blind study medication

N\# is the number of randomized subjects with non-missing baseline and Week 24 (LOCE) values.

(*) Significant p-value: Primary endpoint is tested at alpha $=0.027$ applying Dunnett's adjustment,

Based on an ANCOVA models with treatment group as an effect, baseline value and enrollment strata as covariates.

In addition to the planned, primary analysis, I also conducted my preferred sensitivity analysis which included observations taken after rescue. This also showed both DAPA doses to be superior to placebo $(\mathrm{p}<.001)$. The placebo-adjusted treatment effects were somewhat smaller than those found from the primary analysis: -.28 (standard error [SE] = .12) for the $5 \mathrm{mg}$ dose, $.44(\mathrm{SE}=.12)$ for the $10 \mathrm{mg}$ dose. See Table 44 for further results from this analysis.

\section{Secondary Efficacy Endpoints}

Table 17 shows the results for FPG at Week 24. Both DAPA doses showed a greater reduction at Week 24 than placebo.

Table 17: Results for Fasting Plasma Glucose at Week 24, Study 2030 (Source: Clinical Study Report, Table 7.3.3)

\begin{tabular}{|c|c|c|c|}
\hline $\begin{array}{l}\text { EFFICACY ENDPOINT } \\
\text { STATISTICS }\end{array}$ & $\begin{array}{c}\text { PLA + PIO } \\
(\mathrm{N}=139)\end{array}$ & $\begin{array}{c}\text { DAPA 5MG + PIO } \\
(\mathrm{N}=141)\end{array}$ & $\begin{array}{c}\text { DAPA } \\
(\mathrm{N}=140) \\
10 \mathrm{MG}\end{array}$ \\
\hline $\begin{array}{l}\text { EPG (MG/DL) AT WEEK } 24 \text { (LOCE) } \\
\text { N\# } \\
\text { BASELINE MEAN (SD) } \\
\text { WEEK } 24 \text { LOCE MEAN (SD) } \\
\text { MEAN CHANGE FROM BASELINE (SD) } \\
\text { ADUUSTED MEAN CHANGE FROM BASELINE (SE) } \\
\text { 95\% CI FOR ADJ. MEAN CHANGE FROM BASELINE } \\
\text { DIFEERENCE FROM PLA + PIO (SE) } \\
\text { 95\% CI FOR DIFHERENCE FROM PLA + PIO } \\
\text { P-VALUE VS. PLA + PIO (*) }\end{array}$ & $\begin{array}{rc} & 139 \\
160.7 & (46.97) \\
157.4 & (47.70) \\
-3.3 & (40.53) \\
-5.5 & (2.893) \\
(-11.2, & 0.2)\end{array}$ & $\begin{array}{l}168.3(52.17) \\
141.3(33.90) \\
-27.0(45.24) \\
-24.9(2.884) \\
(-30.6,-19.3) \\
-19.5(4.088) \\
(-27.5,-11.4) \\
\quad<.0001 \star\end{array}$ & $\begin{array}{l}164.9(46.34) \\
135.1(37.67) \\
-29.8(44.59) \\
-29.6(2.880) \\
(-35.3,-24.0) \\
-24.1(4.082) \\
(-32.2,-16.1) \\
<.0001 \star\end{array}$ \\
\hline
\end{tabular}

Table 18 shows the Applicant's results for total body weight. In attempting to reproduce these results, I found slightly different values for the adjusted mean change for each treatment arm: 1.65 for placebo, .10 for DAPA $5 \mathrm{mg},-.13$ for DAPA $10 \mathrm{mg}$. However, the estimated treatment 
effects and corresponding confidence intervals were the same. The results are shown graphically in Figure 11.

Table 18: Results for Total Body Weight at Week 24, Study 2030

(Source: Clinical Study Report, Table 7.3.2)

\begin{tabular}{|c|c|c|c|}
\hline $\begin{array}{l}\text { EFFICACY ENDPOINT } \\
\text { STATISTICS }\end{array}$ & $\begin{array}{c}\text { PLA + PIO } \\
(\mathrm{N}=139)\end{array}$ & $\begin{array}{l}\text { DAPA 5MG + PIO } \\
(\mathrm{N}=141)\end{array}$ & $\begin{array}{c}\text { DAPA } \\
(\mathrm{N}=140)\end{array}$ \\
\hline $\begin{array}{l}\text { TOTAL BODY WEIGHT (KG) AT WEEK } 24 \text { (LOCE) } \\
\text { N\# } \\
\text { BASELINE MEAN (SD) } \\
\text { WEEK } 24 \text { LOCE MEAN (SD) } \\
\text { MEAN CHANGE FROM BASELINE (SD) } \\
\text { ADJUSTED MEAN CHANGE FROM BASELINE (SE) } \\
\text { 95\% CI FOR ADJ. MEAN CHANGE FROM BASELINE } \\
\text { DIFHERENCE FROM PLA + PIO (SE) } \\
\text { 95\% CI FOR DIFFERENCE FROM PLA + PIO } \\
\text { P-VALUE VS. PLA + PIO (*) }\end{array}$ & \begin{tabular}{cl} 
& \multicolumn{1}{c}{139} \\
86.40 & $(21.324)$ \\
88.05 & $(21.796)$ \\
1.66 & $(3.009)$ \\
1.64 & $(0.2760)$ \\
$(1.10,2.18)$
\end{tabular} & $\begin{array}{cc} & 140 \\
87.80 & (20.769) \\
87.90 & (21.423) \\
0.11(3.626) \\
0.09(0.2752) \\
(-0.46,0.63) \\
-1.55(0.3896) \\
(-2.32,-0.79) \\
<.0001 \star\end{array}$ & $\begin{array}{cc} & 140 \\
84.82 & (22.177) \\
84.68 & (22.263) \\
-0.13(3.129) \\
-0.14(0.2753) \\
(-0.68,0.40) \\
-1.78(0.3896) \\
(-2.55,-1.02) \\
<.0001 \star\end{array}$ \\
\hline
\end{tabular}

Table 19 shows the Applicant's results for the proportion of subjects rescued in each arm. As with Study 2014, I reproduced the counts but did attempt to reproduce the confidence intervals. As before, I instead conducted exact tests which showed both arms to be statistically superior to placebo $(\mathrm{p}<.05)$.

Table 19: Subjects Rescued by Week 24, Study 2030

(Source: Clinical Study Report, Table 7.4.4B)

\begin{tabular}{|c|c|c|c|}
\hline & $\begin{array}{c}\text { PLA + PIO } \\
(\mathrm{N}=139)\end{array}$ & $\begin{array}{c}\text { DAPA } 5 \mathrm{MG}+\mathrm{PIO} \\
(\mathrm{N}=141)\end{array}$ & $\begin{array}{c}\text { DAPA } \underset{(\mathrm{N}=140)}{10 \mathrm{MG}}+\mathrm{PIO} \\
\end{array}$ \\
\hline $\begin{array}{l}\text { X/N\# } \\
\text { PERCENT } \\
\text { PERCENT ADUUSTED FOR BASELINE HBA1C (SE) } \\
\text { 95\% CI FOR PERCENT ADJ FOR BSL. HBAIC } \\
\text { DIFHERENCE VS. PLA (SE) } \\
\text { 95\% CI FOR DIFEERENCE VS. PLA }\end{array}$ & $\begin{array}{c}16 / 139 \\
11.5 \% \\
11.68(2.595) \\
(6.5,16.7)\end{array}$ & $\begin{array}{c}2 / 141 \\
1.4 \% \\
1.4 \%(0.996) \\
(-0.6,3.3) \\
-10.28(2.779) \\
(-15.6,-4.7)\end{array}$ & $\begin{array}{c}5 / 140 \\
3.6 \% \\
3.7 \%(1.425) \\
(0.9,6.5) \\
-7.9 \%(3.029) \\
(-13.8,-2.0)\end{array}$ \\
\hline
\end{tabular}




\section{Study Design and Endpoints}

Figure 4 illustrates the design of Study C00006, which tested DAPA as on add-on to insulin and up to two oral anti-diabetic drugs (OADs). Insulin up-titrations were allowed in lieu of rescue.

\section{Figure 4: Design for Study C00006}

(Source: Clinical Study Report, Figure 1)

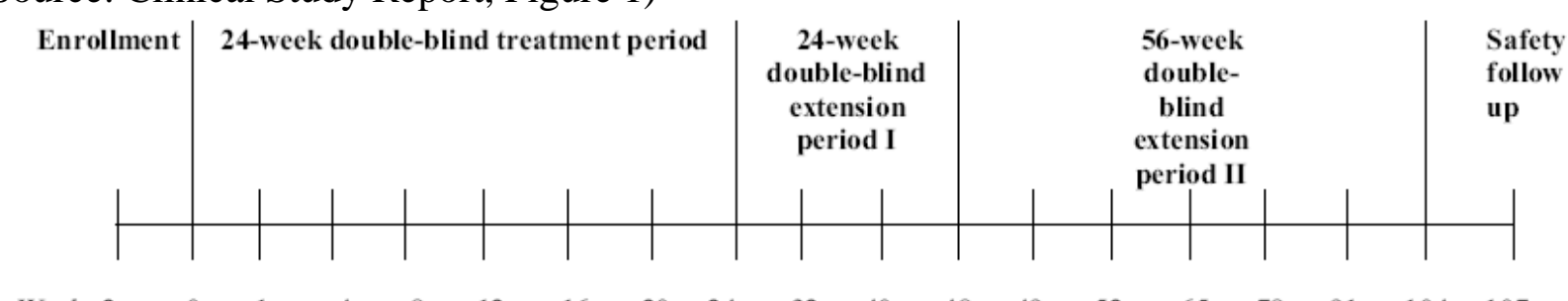

$\begin{array}{lllllllllllllllllll}\text { Week }-2 & 0 & 1 & 4 & 8 & 12 & 16 & 20 & 24 & 32 & 40 & 48 & 49 & 52 & 65 & 78 & 91 & 104 & 107\end{array}$

Visit 1
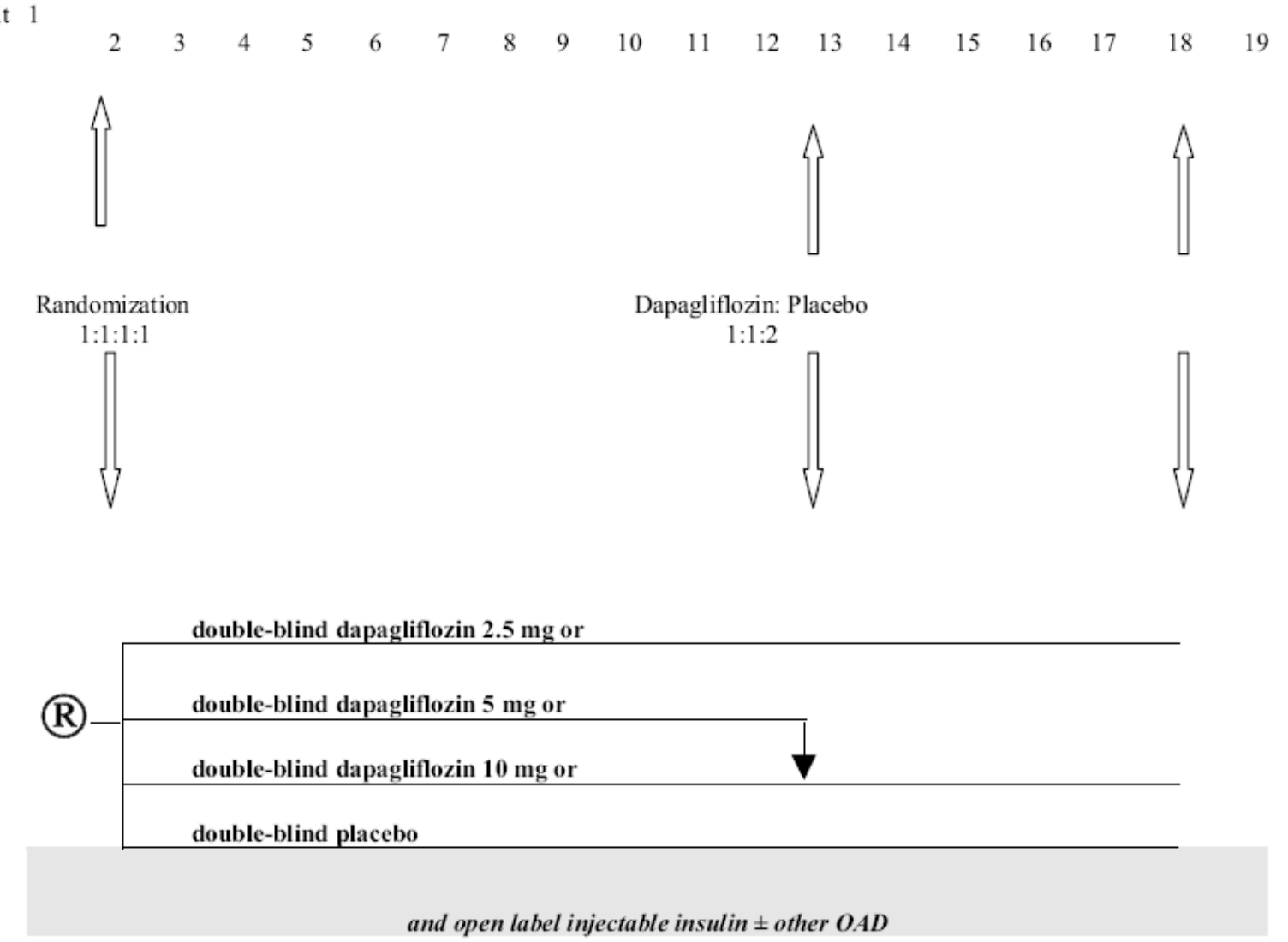

The primary endpoint was change in HbA1c at Week 24. The secondary endpoints were similar to those from the other studies, but also included mean daily insulin use. 


\section{Patient Disposition, Demographic and Baseline Characteristics}

Table 20 shows the disposition of treated subjects. One subject was randomized but not treated.

Table 20: Disposition of Treated Subjects, Study C00006

(Source: Clinical Study Report, Table 9)

\begin{tabular}{|c|c|c|c|c|c|c|c|c|c|c|}
\hline & PLA & + INS & DAPF & $2.5 \mathrm{MG}+\mathrm{INS}$ & DAPA & $5 M G+$ INS & DAPA & $10 \mathrm{MG}+\mathrm{INS}$ & \multicolumn{2}{|c|}{ Total } \\
\hline SUBJECTS & 197 & & 202 & & 212 & & 196 & & 807 & \\
\hline SUBJECTS COMPLETING THE PERIOD (\%) & 168 & $(85.3)$ & 180 & $(89.1)$ & 186 & $(87.7)$ & 178 & $(90.8)$ & 712 & $(88.2)$ \\
\hline SUBJECTS NOT COMPLETING THE PERIOD ( $(8)$ & 29 & $(14.7)$ & 22 & $(10.9)$ & 26 & $(12.3)$ & 18 & $(9.2)$ & 95 & $(11.8)$ \\
\hline \multicolumn{11}{|l|}{ REASON FOR NOT COMPLETING THE PERIOD $\left(\frac{8}{b}\right)$} \\
\hline INCORRECT ENROLNENT & & $(1.0)$ & 1 & $(0.5)$ & 3 & $(1.4)$ & & $(0.5)$ & 7 & $(0.9)$ \\
\hline ADVERSE EVENT & & $(3.0)$ & 5 & $(2.5)$ & 9 & $(4.2)$ & 6 & $(3.1)$ & 26 & $(3.2)$ \\
\hline SUBJECT NO LONGER MEETS STUDY CRITERIA & & $(0.5)$ & 0 & & 0 & & 0 & & 1 & $(0.1)$ \\
\hline SUBJECT WITHDREW CONSENT & 14 & $(7.1)$ & 10 & $(5.0)$ & 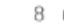 & $(3.8)$ & 6 & $(3.1)$ & 38 & $(4.7)$ \\
\hline LOST TO FOLLOW-UP & & $(0.5)$ & 2 & $(1.0)$ & 1 & $(0.5)$ & 2 & $(1.0)$ & 6 & $(0.7)$ \\
\hline POOR/NON-COMPLIANCE & & $(1.5)$ & 1 & $(0.5)$ & 4 & $(1.9)$ & 3 & $(1.5)$ & 11 & $(1.4)$ \\
\hline SAFETY & 1 & $(0.5)$ & 1 & $(0.5)$ & 0 & & 0 & & 2 & $(0.2)$ \\
\hline DEATH & 0 & & 0 & & 1 & $(0.5)$ & 0 & & 1 & $(0.1)$ \\
\hline OTHER & & $(0.5)$ & 2 & $(1.0)$ & 0 & & 0 & & 3 & $(0.4)$ \\
\hline
\end{tabular}

Table 21 shows the demographics of the treated subjects.

Table 21: Demographics of Treated Subjects, Study C00006

\begin{tabular}{|l|l|l|l|l|}
\hline & $\begin{array}{l}\text { Placebo } \\
\text { (N=197) }\end{array}$ & $\begin{array}{l}\text { DAPA 2.5 mg } \\
\text { (N=202) }\end{array}$ & $\begin{array}{l}\text { DAPA 5 mg } \\
\text { (N=212) }\end{array}$ & $\begin{array}{l}\text { DAPA 10 mg } \\
\text { (N=196) }\end{array}$ \\
\hline Age - Mean (SD) & $59(9)$ & $60(8)$ & $59(8)$ & $59(9)$ \\
\hline $\begin{array}{l}\text { Gender - N (\%) } \\
\text { Male } \\
\text { Female }\end{array}$ & $\begin{array}{l}9(50 \%) \\
99(50 \%)\end{array}$ & $\begin{array}{l}100(50 \%) \\
102(50 \%)\end{array}$ & $100(47 \%)$ & $88(45 \%)$ \\
\hline $\begin{array}{l}\text { Race - N (\%) } \\
\text { White }\end{array}$ & $189(96 \%)$ & $190(94 \%)$ & $201(95 \%)$ & $185(55 \%)$ \\
Black & $7(4 \%)$ & $3(1 \%)$ & $5(2 \%)$ & $5(3 \%)$ \\
$\begin{array}{l}\text { Asian } \\
\text { Other }\end{array}$ & $0(0 \%)$ & $7(3 \%)$ & $3(1 \%)$ & $3(2 \%)$ \\
\hline $\begin{array}{l}\text { Region - N (\%) } \\
\text { U.S. or Canada } \\
\text { Rest of world }\end{array}$ & $1(1 \%)$ & $2(1 \%)$ & $3(1 \%)$ & $3(2 \%)$ \\
\hline
\end{tabular}

\section{Statistical Methodologies}

Except where otherwise noted, the statistical methods were the same as for the previous studies. The randomization was stratified by site and use of OADs $(\mathrm{Y} / \mathrm{N})$. The block size was 4 . The OAD factor was included in the ANCOVA model. The primary analysis excluded data after insulin up-titration. 


\section{Results and Conclusions}

\section{Primary Efficacy Endpoint}

Table 22 shows the results for the primary analysis. All three DAPA doses were shown to be superior to placebo.

Table 22: Results for Primary Endpoint, Study C00006

(Source: Clinical Study Report, Table 20)

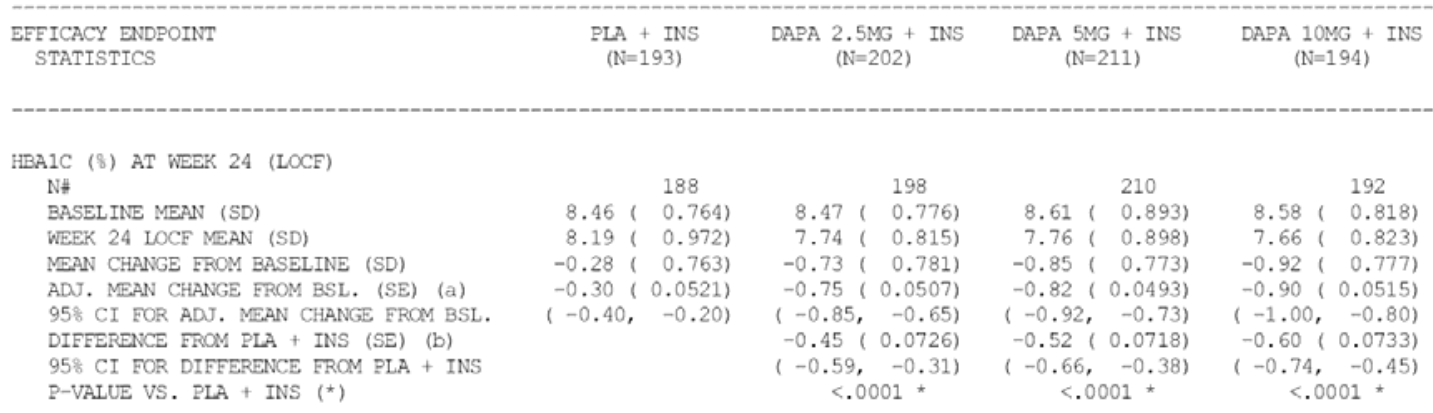

$\mathrm{N}$ is the number of subjects in the full analysis set. $\mathrm{X}$ is the number of responders.

$\mathrm{NF}$ is the number of subjects in the full analysis set with non-missing baseline and Week 24 (IOCF) values.

(a) Adjusted mean change from baseline (b) Difference in adjusted mean change from baseline vs. PLA + INS

(*) Significant p-value: Primary endpoint is tested at alpha=0.019 applying the Dunnett adjustment.

Analysis of continuous outcomes based on separate ANCOVA models with treatment group and stratum as effect and baseline value as a

covariate for each endpoint.

Out of 807 treated subjects, 19 (2\%) were excluded from the primary analysis. Although this is a small percentage, I was surprised to find that 10 of the excluded subjects continued on to the long-term extension. In order to address the impact of the 19 exclusions, I conducted a quasi worst case analysis in which excluded patients in the DAPA arms were given a relatively poor value for the primary endpoint ( $1^{\text {st }}$ quartile) and those in the placebo arm were given a relatively good value $\left(3^{\text {rd }}\right.$ quartile). The three active arms still beat placebo $(\mathrm{p}<.0001)$, with similar estimates for the treatment effects.

I also conducted my preferred sensitivity analysis which included observations taken after uptitration (rescue). This also showed all three DAPA doses to be superior to placebo $(\mathrm{p}<.001)$. The placebo-adjustment treatment effects were slightly smaller than those found from the primary analysis: -.40 for the $2.5 \mathrm{mg}$ dose, -.50 for the $5 \mathrm{mg}$ dose, -.57 for the $10 \mathrm{mg}$ dose (SE = .08 in each case). See Table 44 for more detailed results from this analysis.

\section{Secondary Efficacy Endpoints}

Table 23 shows the results for fasting plasma glucose. Note that 754 subjects (out of 807 treated) were included in the analysis set, which included subjects with both baseline and post-baseline values. 
Table 23: Results for Fasting Plasma Glucose at Week 24, Study C00006 (Source: Clinical Study Report, Table 25)

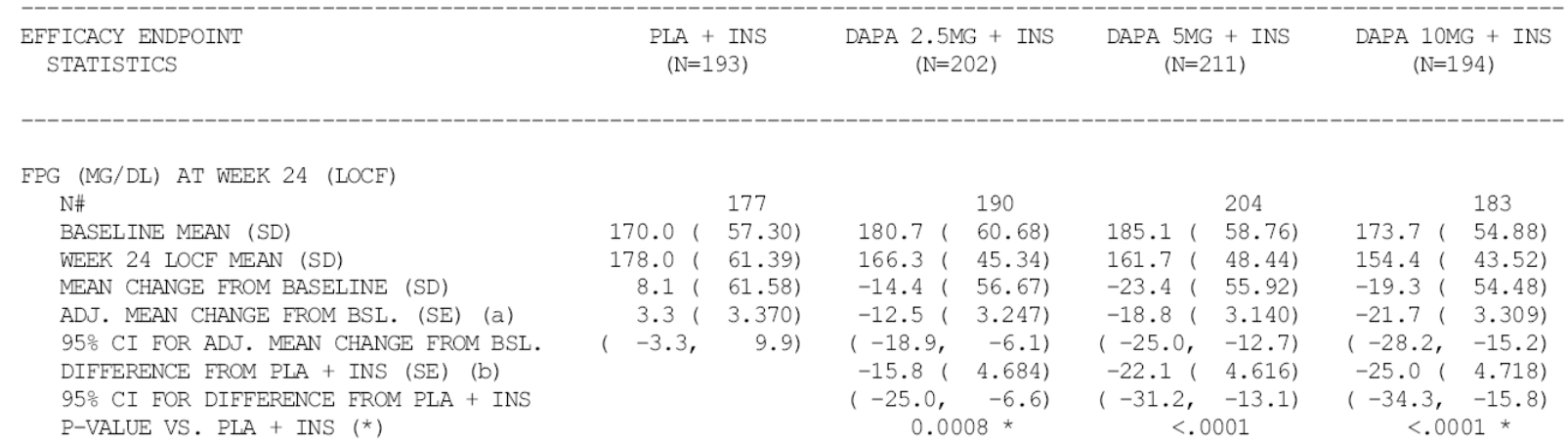

Table 24 shows the results for total body weight at Week 24. All the DAPA arms were superior to placebo. The results are illustrated graphically in Figure 11.

Table 24: Results for Total Body Weight at Week 24, Study C00006 (Source: Clinical Study Report, Table 22)

\begin{tabular}{|c|c|c|c|c|}
\hline $\begin{array}{l}\text { EFFICACY ENDPOINT } \\
\text { STATISTICS }\end{array}$ & $\begin{array}{l}\text { PLA + INS } \\
(\mathrm{N}=193)\end{array}$ & $\begin{array}{c}\text { DAPA } \\
(\mathrm{N}=202)\end{array}$ & $\begin{array}{l}\text { DAPA } 5 \text { MG + INS } \\
(\mathrm{N}=211)\end{array}$ & $\begin{array}{c}\text { DAPA } \\
(\mathrm{N}=194)\end{array}$ \\
\hline \multicolumn{5}{|l|}{ TOTAL BODY WEIGHT (KG) AT WEEK 24 (LOCF) } \\
\hline N\# & 188 & 198 & 210 & 192 \\
\hline BASELINE MEAN (SD) & $94.21(19.491)$ & $93.10(16.754)$ & $93.20(17.377)$ & $94.63(16.829)$ \\
\hline WEEK 24 LOCF MEAN (SD) & $94.22(19.474)$ & $92.13(16.977)$ & $92.22(17.201)$ & $92.96(16.665)$ \\
\hline MEAN CHANGE FROM BASELINE (SD) & $0.01(2.438)$ & $-0.97(2.765)$ & $-0.98(2.507)$ & $-1.68(2.343)$ \\
\hline ADJ. MEAN CHANGE FROM BSL. (SE) (a) & $0.02(0.1833)$ & $-0.98(0.1786)$ & $-0.98(0.1734)$ & $-1.67(0.1814)$ \\
\hline $\begin{array}{l}\text { 95\% CI FOR ADJ. MEAN CHANGE FROM BSL. } \\
\text { DIFFERENCE FROM PLA + INS (SE) (b) }\end{array}$ & $\left(\begin{array}{ll}-0.34, & 0.38\end{array}\right)$ & $\begin{array}{l}(-1.33, \quad-0.63) \\
-1.00(0.2560)\end{array}$ & $\begin{array}{l}(-1.32, \quad-0.64) \\
-1.00(0.2523)\end{array}$ & $\begin{array}{l}(-2.02,-1.31) \\
-1.68(0.2578)\end{array}$ \\
\hline $\begin{array}{l}\text { 95\% CI FOR DIFFERENCE FROM PLA + INS } \\
\text { P-VALUE VS. PLA + INS }\left(^{\star}\right)\end{array}$ & & $\begin{array}{c}(-1.50, \quad-0.49) \\
0.0001 *\end{array}$ & $\begin{array}{c}(-1.50, \quad-0.50) \\
<.0001 \star\end{array}$ & $\begin{array}{c}(-2.19,-1.18) \\
<.0001 *\end{array}$ \\
\hline
\end{tabular}

Table 25 shows the proportion of subjects who either received an up-titration of insulin or discontinued due to lack of glycemic control. As before, I reproduced the Applicant's counts but not the confidence intervals for the proportion. Exact tests showed each DAPA dose to be superior to placebo $(\mathrm{p}<.0001)$. Note: The denominators in this table come from the Full Analysis Set, which included subjects who had a post-baseline value for at least one efficacy variable. For the previous studies, the denominator for rescue was treated subjects. The difference is due to this study being conducted by AZ.

Table 25: Subjects Receiving Insulin Up-Titration or Discontinuing Due to Lack of Glycemic Control, Study C00006

\begin{tabular}{|c|c|c|c|c|}
\hline & $\begin{array}{l}\text { PLA }+ \text { INS } \\
(N=193)\end{array}$ & $\begin{array}{c}\text { DAPA } \\
(\mathrm{N}=202)\end{array}$ & $\begin{array}{l}\text { DAPA } 5 \text { MG + INS } \\
(\mathrm{N}=211)\end{array}$ & $\begin{array}{c}\text { DAPA } \underset{(\mathrm{N}=194)}{10 \mathrm{MG}+\mathrm{INS}} \\
\end{array}$ \\
\hline $\mathrm{X} / \mathrm{N} \#$ & $54 / 193$ & $22 / 202$ & $24 / 211$ & $19 / 194$ \\
\hline PERCENT & $28.0 \%$ & $10.9 \%$ & $11.4 \%$ & $9.8 \%$ \\
\hline PERCENT ADJUSTED & $29.2 \%$ & $11.2 \%$ & $10.6 \%$ & $9.7 \%$ \\
\hline $\begin{array}{l}\text { 95\% CI FOR PERCENT ADUUSTED } \\
\text { DIFFERENCE VS. PLA + INS } \\
\text { 95\% CI FOR DIFFERENCE VS. PLA + INS }\end{array}$ & $(23.0,35.3)$ & 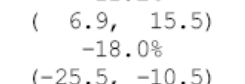 & 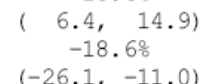 & 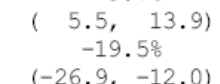 \\
\hline
\end{tabular}




\section{D1690C00004 - Comparison with Glipizide}

\section{Study Design and Endpoints}

Figure 5 shows the design of Study C00004, a comparison of DAPA and glipizide (GLIP) as add-on treatments to metformin. The study did not include rescue medication.

\section{Figure 5: Design of Study C00004}

(Source: Clinical Study Report, Figure 1)

\section{Double-blind treatment period (52 weeks)}

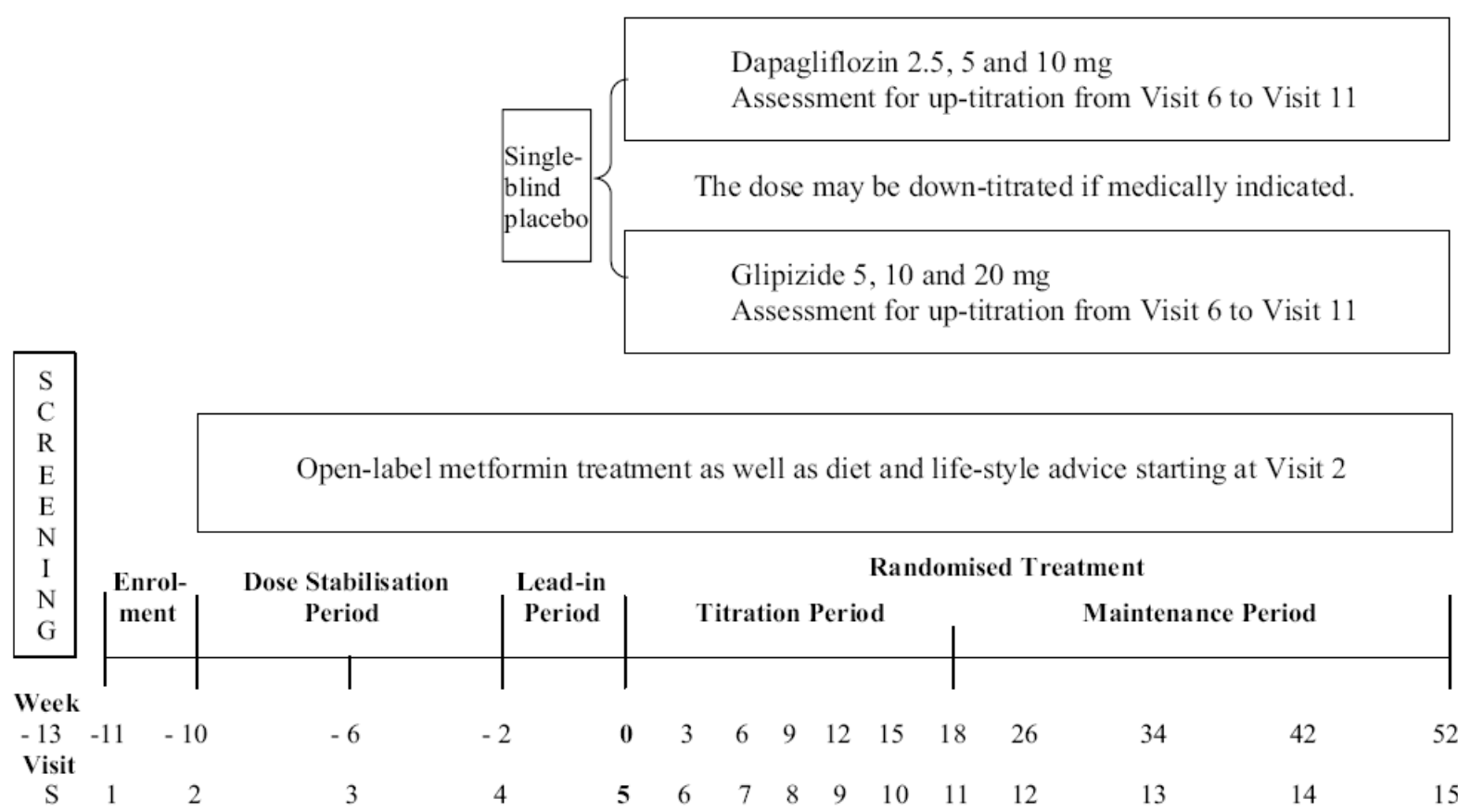

The primary efficacy endpoint was the change in HbA1c at Week 52. Secondary endpoints included change in body weight and the proportion of subjects who had a major hypoglycemic event. FPG was not a key endpoint.

Patient Disposition, Demographic and Baseline Characteristics

Table 26 shows the disposition of the treated subjects. Two subjects were randomized but not treated. 
Table 26: Disposition of Treated Subjects, Study C00004

(Source: Clinical Study Report, Table 8)

\begin{tabular}{|c|c|c|c|c|}
\hline \multicolumn{2}{|c|}{ DAPA + MET } & GLIP + MET & \multicolumn{2}{|c|}{ Total } \\
\hline SUBJECTS & 406 & 408 & 814 & \\
\hline SUBJECTS COMPLETING THE PERIOD (\%) & $322(79.3)$ & $314(77.0)$ & 636 & $(78.1)$ \\
\hline SUBJECTS NOT COMPLETING THE PERIOD (\%) & $84(20.7)$ & $94(23.0)$ & 178 & $(21.9)$ \\
\hline \multicolumn{5}{|l|}{ REASON FOR NOT COMPLETING THE PERIOD (\%) } \\
\hline INCORRECT ENROLMENT & $1(0.2)$ & $1(0.2)$ & 2 & $(0.2)$ \\
\hline ADVERSE EVENT & $33(8.1)$ & $19(4.7)$ & 52 & $(6.4)$ \\
\hline SUBJECT NO LONGER MEETS STUDY CRITERIA & $6(1.5)$ & $27(6.6)$ & 33 & $(4.1)$ \\
\hline SUBJECT WITHDREW CONSENT & $23(5.7)$ & $32(7.8)$ & 55 & $(6.8)$ \\
\hline LOST TO FOLLOW-UP & $3(0.7)$ & $3(0.7)$ & 6 & $(0.7)$ \\
\hline POOR/NON-COMPLIANCE & $5(1.2)$ & $1(0.2)$ & 6 & $(0.7)$ \\
\hline SAFETY & $1(0.2)$ & 0 & 1 & $(0.1)$ \\
\hline DEATH & $1(0.2)$ & $3(0.7)$ & 4 & $(0.5)$ \\
\hline OTHER & $11(2.7)$ & $8(2.0)$ & 19 & $(2.3)$ \\
\hline SUBJECTS CONTINUING IN THE STUDY (\%) & $315(77.6)$ & $309(75.7)$ & 624 & $(76.7)$ \\
\hline SUBJECTS NOT CONTINUING IN THE STUDY ( $\left.\frac{(}{\circ}\right)$ & $91(22.4)$ & $99(24.3)$ & 190 & $(23.3)$ \\
\hline \multicolumn{5}{|l|}{ REASON FOR NOT CONTINUING IN THE STUDY (\%) } \\
\hline INCORRECT ENROLMENT & $1(0.2)$ & $1(0.2)$ & 2 & $(0.2)$ \\
\hline ADVERSE EVENT & $33(8.1)$ & $21(5.1)$ & 54 & $(6.6)$ \\
\hline SUBJECT NO LONGER MEETS STUDY CRITERIA & $6(1.5)$ & $27(6.6)$ & 33 & $(4.1)$ \\
\hline SUBJECT WITHDREW CONSENT & $29(7.1)$ & $34(8.3)$ & 63 & $(7.7)$ \\
\hline LOST TO FOLLOW-UP & $3(0.7)$ & $3(0.7)$ & 6 & $(0.7)$ \\
\hline POOR/NON-COMPLIANCE & $5(1.2)$ & $1(0.2)$ & 6 & $(0.7)$ \\
\hline SAFETY & $1(0.2)$ & 0 & 1 & $(0.1)$ \\
\hline DEATH & $1(0.2)$ & $3(0.7)$ & 4 & $(0.5)$ \\
\hline OTHER & $12(3.0)$ & $9(2.2)$ & 21 & $(2.6)$ \\
\hline
\end{tabular}

Table 27 shows demographics for the treated subjects. Note the absence of domestic or Canadian study sites.

Table 27: Demographics of Treated Subjects, Study C00004

\begin{tabular}{|l|l|l|}
\hline & $\begin{array}{l}\text { DAPA } \\
\text { (N=406) }\end{array}$ & $\begin{array}{l}\text { GLIP } \\
\text { (N=408) }\end{array}$ \\
\hline Age - Mean (SD) & $58(9)$ & $59(10)$ \\
\hline $\begin{array}{l}\text { Gender - N (\%) } \\
\text { Male }\end{array}$ & $226(56 \%)$ & $223(55 \%)$ \\
Female & $180(44 \%)$ & $185(45 \%)$ \\
\hline Race - N (\%) & & \\
White & $331(82 \%)$ & $328(80 \%)$ \\
Black & $27(7 \%)$ & $26(6 \%)$ \\
Asian & $27(7 \%)$ & $34(8 \%)$ \\
Other & $21(5 \%)$ & $20(5 \%)$ \\
\hline $\begin{array}{l}\text { Region - N(\%) } \\
\text { U.S. or Canada } \\
\text { Rest of world }\end{array}$ & $0(0 \%)$ & $0(0 \%)$ \\
& $406(100 \%)$ & $408(100 \%)$ \\
\hline
\end{tabular}




\section{Statistical Methodologies}

The primary analysis was a test of noninferiority (NI), based on an ANCOVA model which included treatment and baseline HbA1c. An NI margin of .35\% was used. I did not find a record indicating that we agreed to this margin, but similar margins have been allowed for diabetes drugs in the past. Moreover, it will be seen that the study would be deemed successful even under a much tighter margin. The primary analysis set was the same as in the other studies. Superiority tests were conducted for the secondary endpoints. The randomization used permuted blocks of size four and was unstratified.

\section{Results and Conclusions}

\section{Primary Efficacy Endpoint}

The results for the primary analysis are shown Table 28. Note that the confidence interval for difference between treatment arms was [-.11, .11]; hence the study showed NI for DAPA even under a quite tight margin.

Table 28: Results for Primary Endpoint, Study C00004

(Source: Clinical Study Report, Table 20)

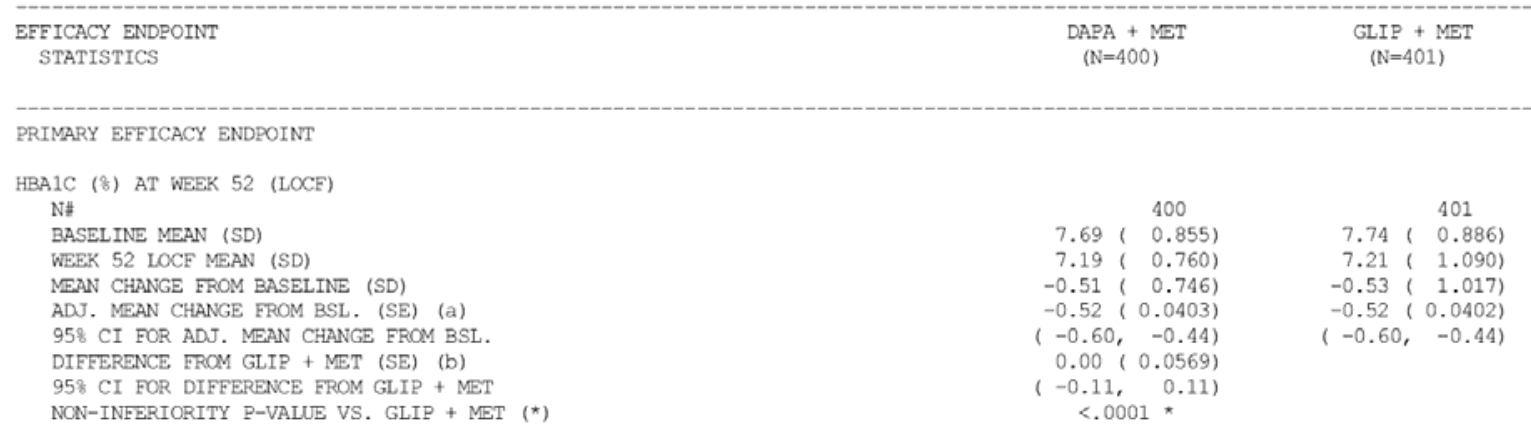

$\mathrm{N}$ is the number of subjects in the full analysis set.

$\mathrm{N} \#$ is the number of subjects in the full analysis set with non-missing baseline and week 52 (LOCF) values.

(a) Adjusted mean change from baseline (b) Difference in adjusted mean change from baseline

$\left.{ }^{*}\right)$ Significant p-value: Primary endpoint is significant (alpha $=0.025$ one-sided) non-inferior if upper limit of $95 \%$ confidence interval is $<0.35 \%$.

Analysis of continuous outcomes based on separate ANCOVA models with treatment group as effect and baseline value as a covariate for each endooint.

The primary analysis excluded 13 treated subjects. In order to investigate the effects of these exclusions, I conducted a quasi - worst case analysis in which excluded patients in the DAPA arms were given a relatively poor value for the primary endpoint (1st quartile) and those in the placebo arm were given a relatively good value (3rd quartile). The results were quite similar to those from the primary analysis. 
The Applicant conducted three sensitivity analyses: a completers-only analysis, a per-protocol analysis, and an MMRM analysis similar to those conducted for other studies. The results were quite similar in all cases. Due to the lack of rescue, the concern I raised in regard to the MMRM analysis of the other studies is moot. Figure 13 shows the results for LOCF imputation and the MMRM analysis. As the figure suggests, GLIP was statistically superior to DAPA at several time points.

\section{Secondary Efficacy Endpoint}

Table 29 shows the results for total body weight at Week 52. Subjects on DAPA lost weight, while those in GLIP gained weight. These results are shown graphically in Figure 6.

Table 29: Results for Total Body Weight at Week 52, Study C00004

(Source: Clinical Study Report, Table 23)

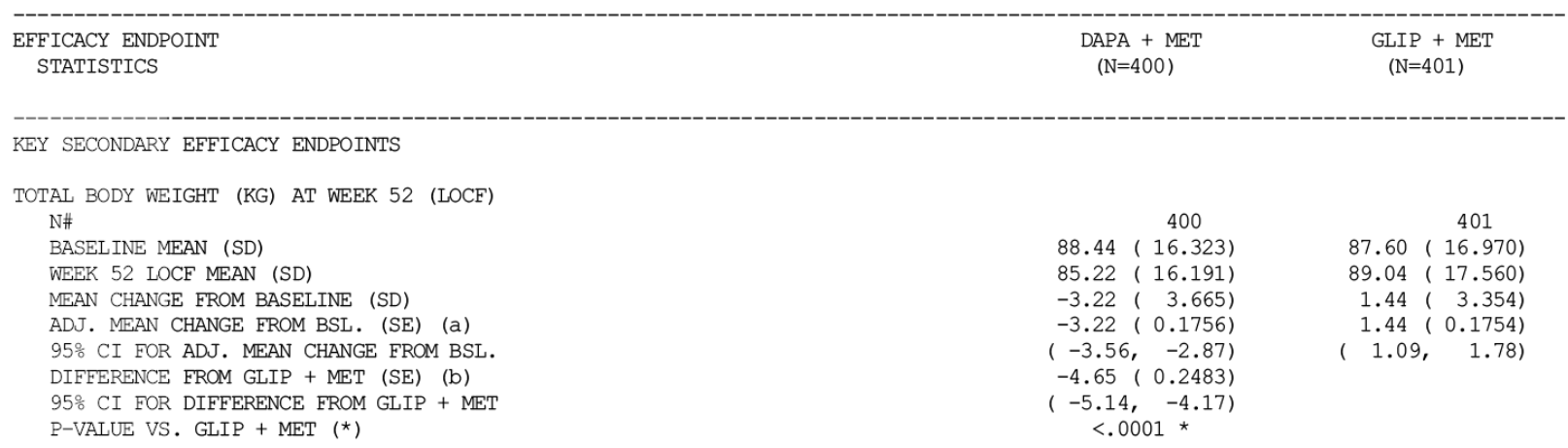


Figure 6: Change in Body Weight at Week 52, Study C00004

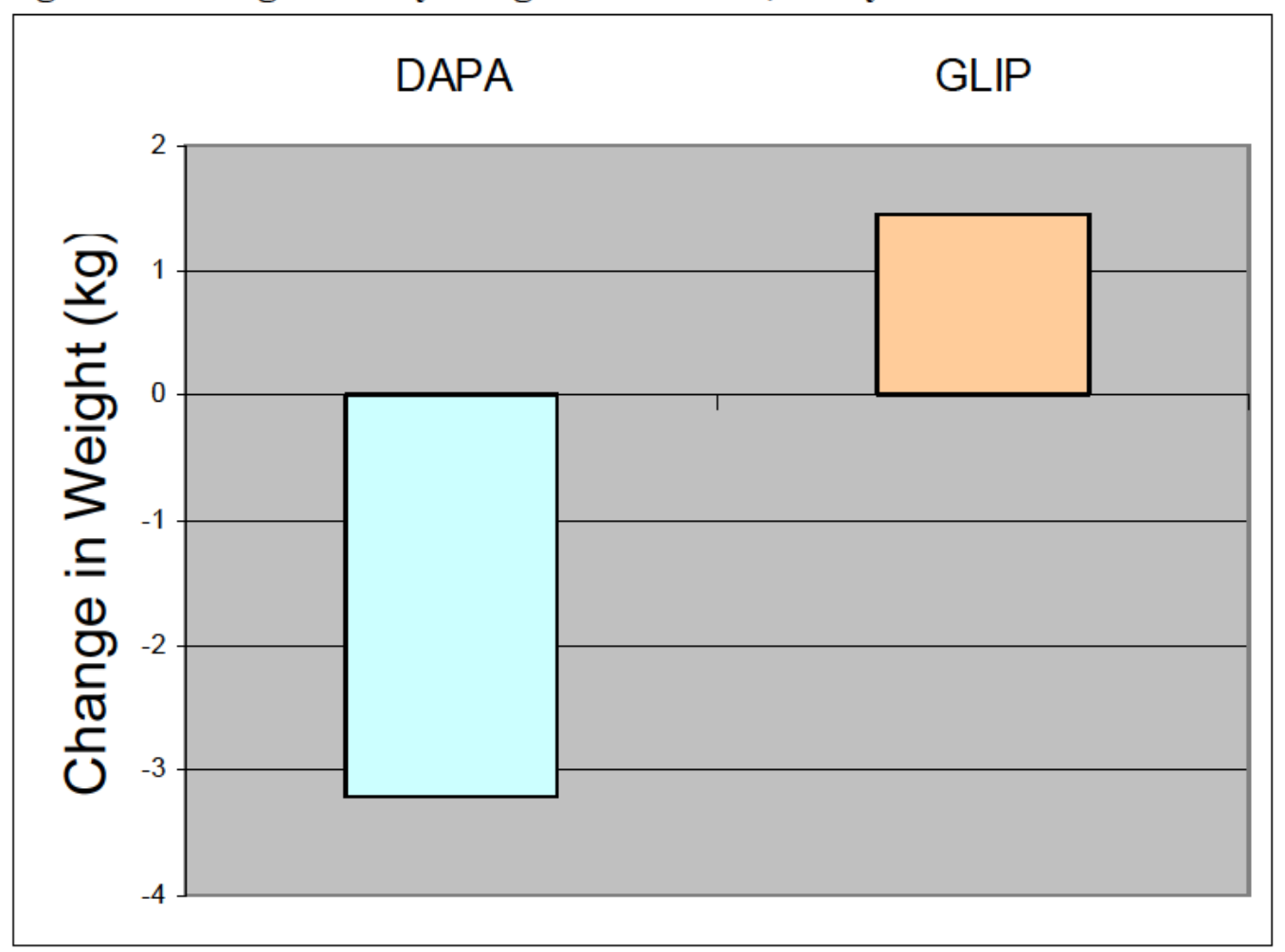




\section{Study Design and Endpoints}

Figure 7 illustrates the design of Study 2034, which tested DAPA in combination with metformin (MET). Patients were excluded if they had received more than 14 days of antihyperglycemic therapy (consecutive or not) in the previous 12 weeks.

Figure 7: Design of Study 2034

(Source: Clinical Study Report, Figure 3.1)

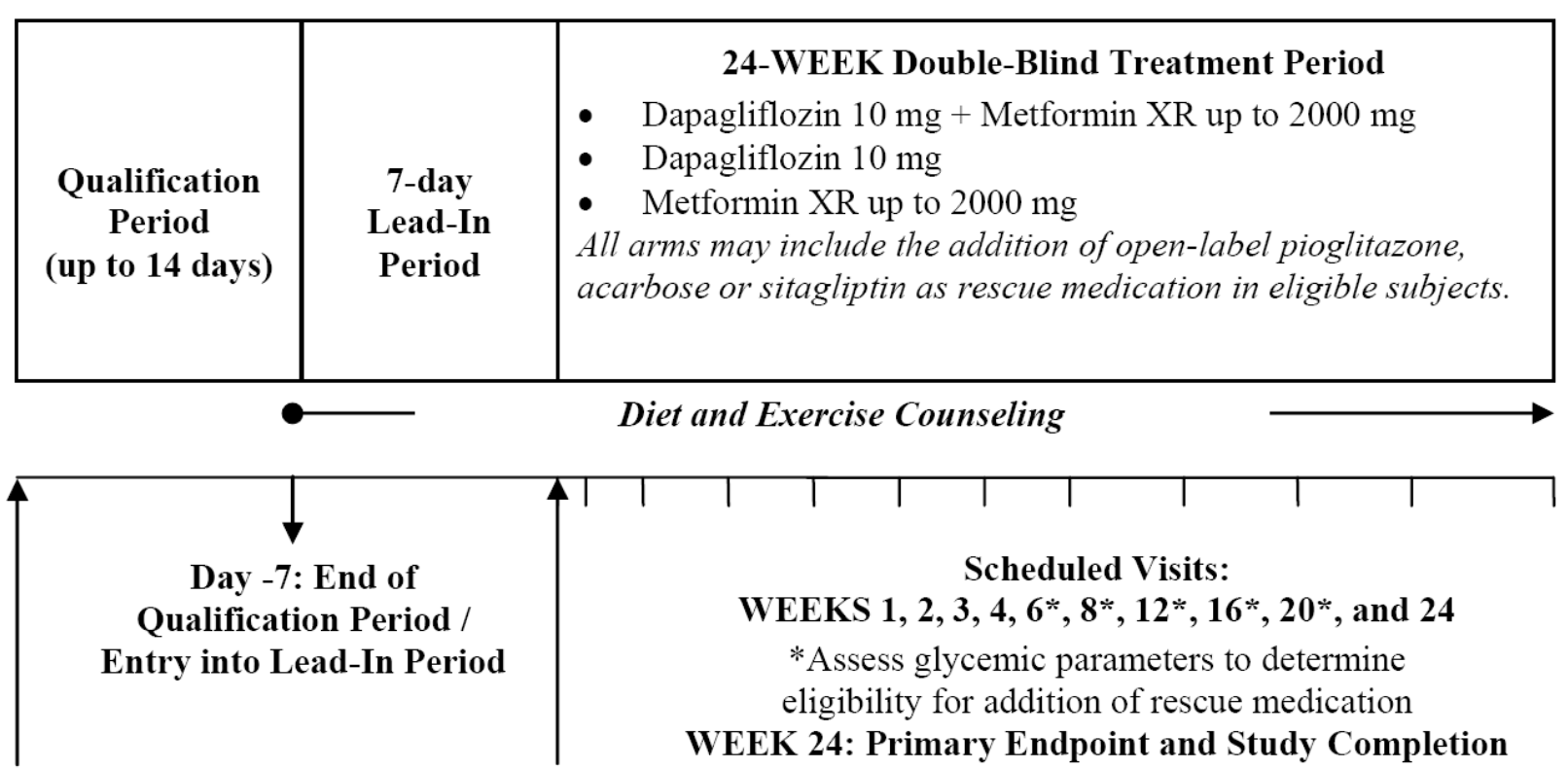

Enrollment

Day 1: Randomization

The primary efficacy endpoint was change in HbA1c at Week 24. In order for study to be deemed positive, the combination of DAPA and MET had to be found statistically superior to each component. The secondary efficacy endpoints were similar to those in the placebocontrolled studies (2013, etc.) 


\section{Patient Disposition, Demographic and Baseline Characteristics}

Three subjects were randomized but not treated.

Table 30 shows the disposition of the treated subjects. Three subjects were randomized but not treated.

Table 30: Disposition of Treated Subjects, Study 2034

(Source: Clinical Study Report, Table 5.1B)

\begin{tabular}{|c|c|c|c|c|c|}
\hline & DAPA $10 \mathrm{MG}+\mathrm{MET}$ & DAPA $10 \mathrm{MG}$ & MET & Total & \\
\hline SUBJECTS & 211 & 219 & 208 & 638 & \\
\hline SUBJECTS COMPLETING THE STUDY ( $\left(\frac{1}{)}\right.$ & $183(86.7)$ & $188(85.8)$ & $181(87.0)$ & 552 & $(86.5)$ \\
\hline SUBJECTS NOT COMPLETING THE STUDY (\%) & $28(13.3)$ & $31(14.2)$ & $27(13.0)$ & 86 & (13.5) \\
\hline $\begin{array}{l}\text { REASON FOR NOT COMPLETING THE STUDY }(\%) \\
\text { LACK OF EFFICACY } \\
\text { ADVERSE EVENT } \\
\text { SUBJECT WITHDREW CONSENT } \\
\text { DEATH } \\
\text { LOST TO FOLLOW-UP } \\
\text { POOR/NON-COMPLIANCE } \\
\text { SUBJECT NO LONGER MEETS STUDY CRITERIA } \\
\text { OTHER }\end{array}$ & 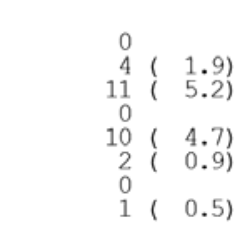 & 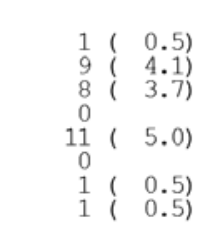 & 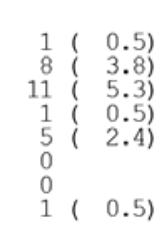 & $\begin{array}{r}2 \\
21 \\
30 \\
1 \\
26 \\
2 \\
1 \\
3\end{array}$ & $\begin{array}{l}0.3) \\
3.31 \\
4.7) \\
0.2) \\
4.1) \\
0.3) \\
0.2) \\
0.5)\end{array}$ \\
\hline
\end{tabular}

Table 31 shows the demographics of the subjects.

Table 31: Demographics of Treated Subjects, Study 2034

\begin{tabular}{|l|l|l|l|}
\hline & $\begin{array}{l}\text { DAPA + MET } \\
\text { (N=211) }\end{array}$ & $\begin{array}{l}\text { DAPA } \\
\text { (N=219) }\end{array}$ & $\begin{array}{l}\text { MET } \\
\text { (N=208) }\end{array}$ \\
\hline Age - Mean (SD) & $51(10)$ & $51(12)$ & $53(10)$ \\
\hline $\begin{array}{l}\text { Gender - N (\%) } \\
\text { Male } \\
\text { Female }\end{array}$ & $106(50 \%)$ & $105(48 \%)$ & $97(47 \%)$ \\
\hline $\begin{array}{l}\text { Race - N (\%) } \\
\text { White }\end{array}$ & $105(50 \%)$ & $114(52 \%)$ & $111(53 \%)$ \\
Black & $174(82 \%)$ & $176(80 \%)$ & $166(80 \%)$ \\
Asian & $11(5 \%)$ & $13(6 \%)$ & $8(4 \%)$ \\
Other & $24(11 \%)$ & $27(12 \%)$ & $31(15 \%)$ \\
\hline $\begin{array}{l}\text { Region - N (\%) } \\
\text { U.S. or Canada } \\
\text { Rest of world }\end{array}$ & $2(1 \%)$ & $3(1 \%)$ & $2(1 \%)$ \\
\hline
\end{tabular}

\section{Statistical Methodologies}

The statistical methods for this study were similar to those for placebo-controlled studies.

Randomization was stratified by site, with a block size of 3. 


\section{Results and Conclusions}

\section{Primary Efficacy Endpoint}

Table 32 shows the results for primary efficacy endpoint. The combination of DAPA and MET was superior to each component. A secondary analysis also showed DAPA to be non-inferior to MET alone.

Table 32: Primary Efficacy Endpoint, Study 2034

(Source: Clinical Study Report, Table 7.2)

\begin{tabular}{|c|c|c|c|}
\hline $\begin{array}{l}\text { EFFICACY ENDPOINT } \\
\text { STATISTICS }\end{array}$ & DAPA $\underset{(\mathrm{N}=211)}{10 \mathrm{MG}+\mathrm{MET}}$ & $\begin{array}{l}\text { DAPA } 10 \mathrm{MG} \\
(\mathrm{N}=219)\end{array}$ & $\underset{(\mathrm{N}=208)}{\mathrm{MET}}$ \\
\hline \multicolumn{4}{|l|}{ PRIMARY EFFICACY ENDPOINT } \\
\hline \multirow{4}{*}{$\begin{array}{l}\text { HBAIC (₹) AT WEEK } 24 \text { (LOCE) } \\
\text { N\# } \\
\text { BASELINE MEAN (SD) } \\
\text { WEEK } 24 \text { LOCE MEAN (SD) } \\
\text { MEAN CHANGE FROM BASELINE (SD) } \\
\text { ADUUSTED MEAN CHANGE FROM BASELINE (SE) } \\
95 \% \text { CI FOR ADJ. MEAN CHANGE FROM BASELTNE } \\
\text { COMBINATION GROUP VS. DAPA 1OMG } \\
\text { DIFFERENCE (SE) (a) } \\
95 \% \text { CI FOR DIFFERENCE } \\
\text { P-VAUE (*) } \\
\text { COMBINATION GROUP VS. MET }\end{array}$} & & & \\
\hline & 202 & & \\
\hline & $\begin{aligned} 9.10 & (1.276) \\
7.10(1.001) & (1.01(1.079) \\
-2.01 & (0.0759) \\
-1.98 & (-2.13,-1.83)\end{aligned}$ & $\begin{array}{r}9.03(1.272) \\
7.59(1.232) \\
-1.44(1.310) \\
-1.45(0.0734) \\
(-1.59,-1.31)\end{array}$ & $\begin{array}{r}9.03(1.295) \\
7.60(1.420) \\
-1.42(1.417) \\
-1.44(0.0757) \\
(-1.59,-1.29)\end{array}$ \\
\hline & $\begin{array}{c}-0.53(0.1056) \\
(-0.74,-0.32) \\
<.0001 *\end{array}$ & & \\
\hline $\begin{array}{l}\text { COMBINATION GROUP VS. MET } \\
\text { DIFFERENCE (SE) (a) } \\
\text { 95\% CI FOR DIFFERENCE } \\
\text { P-VALUE (*) }\end{array}$ & $\begin{array}{l}-0.54(0.1072) \\
(-0.75,-0.33) \\
<.0001 *\end{array}$ & & \\
\hline
\end{tabular}

$\mathrm{N}$ is the number of randomized subjects who took at least one dose of double-blind study medication. $\mathrm{N \#}$ is the number of randomized subjects with non-missing baseline and week $t$ (LOCF) values.

(a) Difference in adjusted mean change from baseline
$(\star)$ Significant p-value: Primary endpoint is tested at alpha $=0.05$ and secondary endipoints are tested following a sequential testing procedure at alpha $=0.05$. Significance is claimed only if DAPA 1OMG + MET is superior to both controls.

Analysis of continuous outcomes based on separate ANCOVA models with treatment group as an effect and baseline value as a covariate.

In addition to the planned, primary analysis, I also conducted my preferred sensitivity analysis which included observations taken after rescue. This also showed the combination of DAPA and MET to be superior to each component $(\mathrm{p}<.001)$. The treatment effects were somewhat smaller than those found in the primary analysis, -.48 (SE = .10) in each case. See Table 45 for further details from this analysis.

\section{Secondary Efficacy Endpoints}

Table 33 shows the results for FPG, which show the combination to be superior to each component. 
Table 33: Results for Fasting Plasma Glucose at Week 24, Study 2034

(Source: Clinical Study Report, Table 7.3.1)

\begin{tabular}{|c|c|c|c|}
\hline $\begin{array}{l}\text { EFFICACY ENDPOINT } \\
\text { STATISTICS }\end{array}$ & DAPA $\underset{(\mathrm{N}=211)}{10 \mathrm{MG}+\mathrm{MET}}$ & $\begin{array}{l}\text { DAPA. 10MG } \\
(\mathrm{N}=219)\end{array}$ & $\begin{array}{c}\text { MET } \\
(\mathrm{N}=208)\end{array}$ \\
\hline $\begin{array}{l}\text { FPG (MG/DL) AT WEEK } 24 \text { (IOCE) } \\
\text { n\# } \\
\text { Nï } \\
\text { BASELINE MEAN (SD) } \\
\text { WEEK } 24 \text { LOCE MEAN (SD) } \\
\text { MEAN CHANGE FROM BASELINE (SD) } \\
\text { ADUUSTED MEAN CHANGE FROM BASELINE (SE) } \\
95 \% \text { CI FOR ADU. MEAN CHANGE FROM BASELINE } \\
\text { COMBINATION GROUP VS. DAPA 1OMG } \\
\text { DIFFERENCE (SE) (a) } \\
\text { 95\% CI FOR DIFFERENE } \\
\text { P-VALE (*) } \\
\text { COMBINATION GROUP VS. MET } \\
\text { DIFFERENC (SE) (a) } \\
\text { 95\% CI FOR DIFERENCE } \\
\text { P-VALUE (*) }\end{array}$ & $\begin{array}{c}\frac{172}{209} \\
189.6(58.21) \\
131.0(34.69) \\
-58.6(52.91) \\
-60.4(2.533) \\
(-65.3,-55.4) \\
-13.9(3.558) \\
(-20.9,-7.0) \\
<.0001 * \\
-25.5(3.590) \\
(-32.6,-18.5) \\
<.0001 *\end{array}$ & $\begin{array}{c}160 \\
216 \\
197.5(62.03) \\
147.8(44.19) \\
-49.6(55.96) \\
-46.4(2.494) \\
(-51.3,-41.5)\end{array}$ & $\begin{array}{c}146 \\
207 \\
189.9(53.72) \\
156.6(47.14) \\
-33.2(46.16) \\
-34.8(2.545) \\
(-39.8,-29.8)\end{array}$ \\
\hline
\end{tabular}

\footnotetext{
$\mathbb{N}$ is the number of randomized subjects who took at least one dose of double-blind study medication. Nil is the number of randiomized subjects with non-missing baseline and Week $t$ (LOCF) values. n\# is the number of subjects with non-missing baseline and week $t$ observed values.

(a) Difference in adjusted mean change from baseline (b) Exploratory encipoint (*) Sionificant p-value: Secondary encipoints are tested following a secouential testing proodure at alpha $=0.05$. Sicmificance is claimed only if DAPA. $10 \mathrm{MG}+\mathrm{MET}$ is superior to both controls based on an ANCOVA model with treatment group as an effect and baseline value as a covariate.
}

Table 34 shows results for body weight. Following the planned analysis, the combination was compared to MET (but not DAPA) and found to be superior. The results are shown graphically in Figure 8.

Table 34: Results for Total Body Weight at Week 24, Study 2034 (Source: Clinical Study Report, Table 7.3.4)

\begin{tabular}{|c|c|c|c|}
\hline $\begin{array}{l}\text { EFFICACY ENDPOINT } \\
\text { STATISTICS }\end{array}$ & $\begin{array}{c}\text { DAPA } \underset{(\mathrm{N}=211)}{10 \mathrm{MG}+\mathrm{MET}} \\
\end{array}$ & $\begin{array}{l}\text { DAPA 10MG } \\
(\mathrm{N}=219)\end{array}$ & $\begin{array}{c}\mathrm{MET} \\
(\mathrm{N}=208)\end{array}$ \\
\hline $\begin{array}{l}\text { TOTAL BODY WEIGHT (KG) AT WEEK } 24 \text { (LOCF) } \\
\text { N\# } \\
\text { BASELINE MEAN (SD) } \\
\text { WEEK } 24 \text { LOCE MEAN (SD) } \\
\text { MEAN CHANGE FROM BASELINE (SD) } \\
\text { ADJUSTED MEAN CHANGE FROM BASELINE (SE) } \\
\text { 95\% CI FOR ADJ. MEAN CHANGE FROM BASELINE } \\
\text { COMBINATION GROUP VS. MET } \\
\text { DIFHERENCE (SE) (a) } \\
\text { 95\% CI FOR DIFFERENCE } \\
\text { P-VALUE (*) }\end{array}$ & $\begin{array}{l}80.56(19.717) \\
85.21(18.863) \\
-3.35(3.788) \\
-3.33(0.2401) \\
(-3.80,-2.86) \\
-1.97(0.3401) \\
(-2.64,-1.30) \\
\quad<.0001 *\end{array}$ & \begin{tabular}{cc} 
& \multicolumn{1}{c}{219} \\
88.53 & $(19.334)$ \\
85.78 & $(18.665)$ \\
-2.75 & $(3.719)$ \\
-2.73 & $(0.2346)$ \\
$(-3.19,-2.27)$
\end{tabular} & $\begin{array}{cl}87.24 & (19.423) \\
85.92 & (19.096) \\
-1.32 & (3.241) \\
-1.36 & (0.2408) \\
(-1.83,-0.89)\end{array}$ \\
\hline
\end{tabular}


Figure 8: Change in Body Weight at Week 24, Study 2034

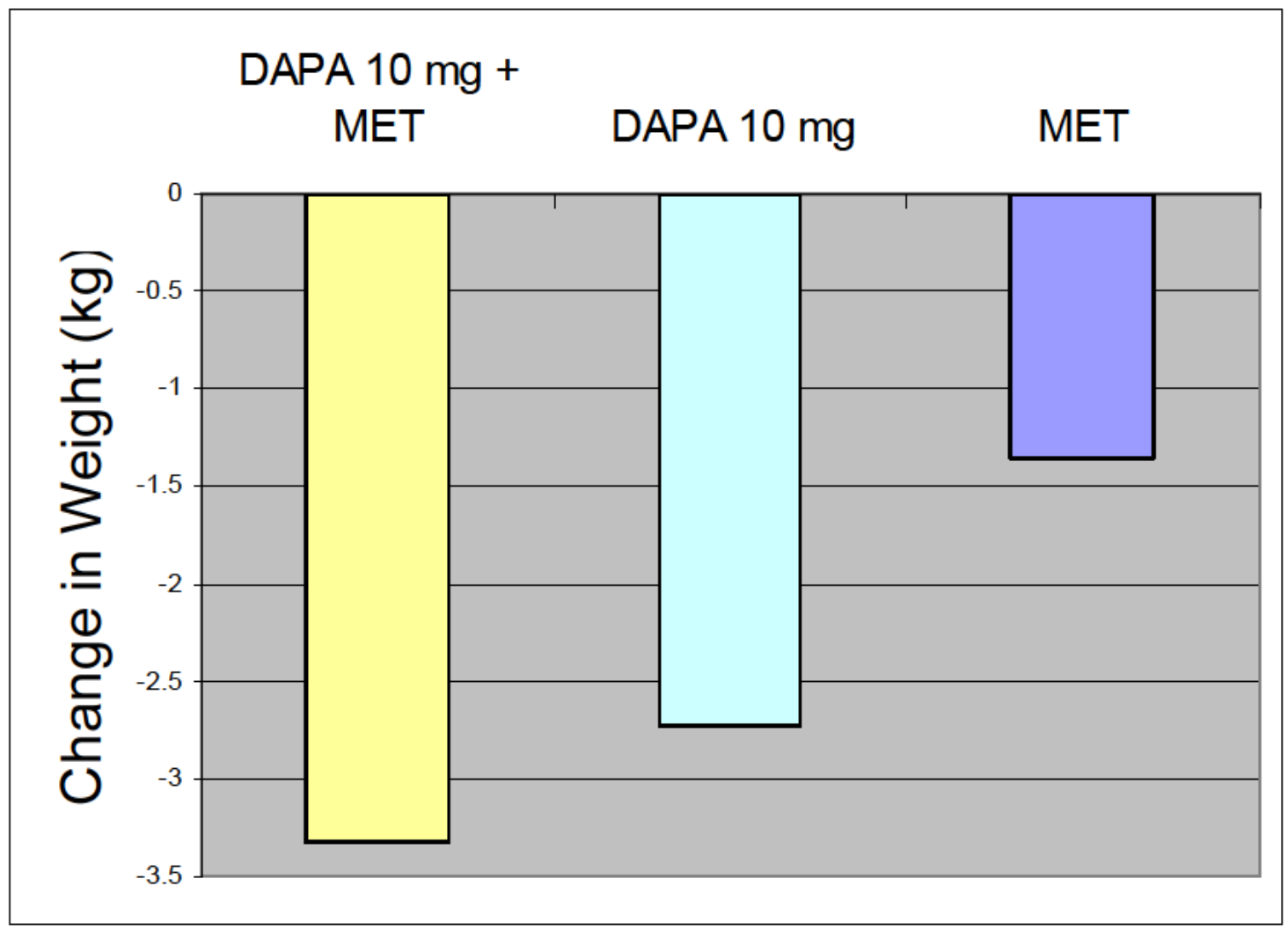

Table 35 shows the proportions of subjects who received rescue or who were discontinued due to lack of glycemic control. As with the other studies which used rescue, I confirmed the counts and conducted exact tests. These tests showed the combination to be superior to each component $(\mathrm{p}<$ .01 in each case).

Table 35: Subjects Rescued or Discontinued Due to Lack of Glycemic Control, Study 2034 (Source: Clinical Study Report, Table 7.4.2C)

\begin{tabular}{|c|c|c|c|}
\hline & $\begin{array}{c}\text { DAPA } 10 \mathrm{MG}+\mathrm{MET} \\
(\mathrm{N}=211)\end{array}$ & $\begin{array}{l}\text { DAPA } 10 \mathrm{MG} \\
(\mathrm{N}=219)\end{array}$ & $\begin{array}{c}\text { MET } \\
(\mathrm{N}=208)\end{array}$ \\
\hline $\mathrm{X} / \mathrm{N} \#$ & $3 / 211$ & $17 / 219$ & $28 / 208$ \\
\hline PERCENT & 1.48 & 7.88 & 13.58 \\
\hline DERCENT ADJUSTED FOR. BASELINE HBA1C (SE) & $1.4 \% \quad(0.815)$ & $7.8 \%(1.770)$ & $13.5 \%(2.345)$ \\
\hline 95\% CI FOR PERCEINT ADUUSTED FOR BASELINE HBA1C & $(-0.2,3.0)$ & $(4.3,11.3)$ & $(8.9,18.1)$ \\
\hline \multicolumn{4}{|l|}{ COMBINATION GROUP VS. DAPA 10MG } \\
\hline DIEFERENCE (SE) & $-6.48(1.951)$ & & \\
\hline 958 CI FOR DIFEERENCE & $(-10.2,-2.6)$ & & \\
\hline \multicolumn{4}{|l|}{ COMBINATION GROUP VS. MET } \\
\hline DIFEERENCE (SE) & $-12.1 \%(2.485)$ & & \\
\hline $95 \%$ CI FOR DIFHERENCE & $(-16.9,-7.2)$ & & \\
\hline
\end{tabular}




\subsection{Evaluation of Safety}

The safety of dapagliflozin was primarily reviewed by Somya Dunn, M.D. Anita Abraham, Ph.D., conducted the statistical review of cardiovascular safety. 


\section{FINDINGS IN SPECIAL/SUBGROUP POPULATIONS}

\subsection{Gender, Race, Age, and Geographic Region}

Due to the large number of studies, I conducted a screening analysis of the primary endpoint in which I tested whether the treatment effect interacted with baseline HbA1c, age (under 65 vs. $65+$ ), gender, race, and region (U.S. and Canada vs. rest of world). In the studies that used fixed doses (all about C00004), I pooled the $5 \mathrm{mg}$ and $10 \mathrm{mg}$ DAPA dose groups and excluded the 2.5 mg dose since it will not be marketed. The race interaction was only tested if there were at least 2 race groups with at least 10 subjects in each (pooled) dose group.

The results are shown in Table 36. In three studies, DAPA a significantly stronger effect (relative to comparator) in patients with higher HbA1c.

Table 36: Screening Subgroup Analysis

\begin{tabular}{|l|l|l|l|l|l|l|}
\hline & $\mathbf{2 0 1 3}$ & $\mathbf{2 0 1 4}$ & $\mathbf{2 0 3 0}$ & $\mathbf{C 0 0 0 0 6}$ & $\mathbf{2 0 3 4}$ & $\mathbf{C 0 0 0 0 4}$ \\
\hline $\begin{array}{l}\text { Baseline } \\
\text { HbA1c }\end{array}$ & $<.0001$ & .12 & $<.0001$ & $\mathbf{. 0 0 8}$ & .71 & .20 \\
\hline Age & .17 &. $\mathbf{0 0 2}$ & .052 & .87 & .68 & .22 \\
\hline Gender & .79 & .63 & .63 & .83 & .90 & .11 \\
\hline Race & N.D. & .91 & .29 & N.D. & .22 & .04 \\
\hline Region & .26 & .50 & .87 & .22 & .13 & N.D. \\
\hline
\end{tabular}

Note: N.D. $=$ not done. Significant p-values are in bold italics.

Table 37 shows the least-squares means for the primary endpoint by race and treatment for Study C00004. There is an apparent trend for black and Asian patients to show higher efficacy on DAPA, adjusted for their response to GLIP. Other studies, however, did not show a pattern of these groups doing better on DAPA. The findings from this study may actually suggest a lack of efficacy for GLIP in these groups.

Table 37: Primary Endpoint by Race, Study C00004

\begin{tabular}{|l|l|l|l|}
\hline \multicolumn{2}{|l}{ Race } & Arm \\
\cline { 3 - 4 } \multicolumn{2}{|l}{ White } & GLIP & DAPA \\
\cline { 2 - 4 } & Adj. Mean (SE) & $-.63(.04)$ & $-.55(.04)$ \\
\hline \multirow{2}{*}{$\begin{array}{l}\text { Black/ African- } \\
\text { American }\end{array}$} & Adj. Mean (SE) & $-.01(.16)$ & $.09(.06)$ \\
\cline { 2 - 4 } Asian & Diff. vs. GLIP & -- & $-.37(.15)$ \\
\hline \multirow{3}{*}{ Other } & Adj. Mean (SE) & $.07(.14)$ & $-.38(.22)$ \\
\cline { 2 - 4 } & Diff. vs. GLIP & -- & $-.25(.15)$ \\
\hline & Adj. Mean (SE) & $-.29(.18)$ & $-.33(.20)$ \\
\cline { 2 - 4 } & Diff. vs. GLIP & -- & $-.55(.18)$ \\
\hline
\end{tabular}

Table 38 shows the primary results for Study 2014 by age group. Notably, this study showed a qualitative interaction. Older patients actually show a trend toward doing worse on DAPA. One reason that older patients may do worse on DAPA is that they tend to have declining renal 
function. However, the interaction remained significant after adjustment for baseline estimated glomerular filtration rate (eGFR). The apparent age-treatment interaction in Study 2014 was not consistently observed in other studies.

Table 38: Primary Endpoint by Age, Study 2014

\begin{tabular}{|l|l|l|l|}
\hline \multicolumn{2}{|l|}{ Race } & Arm \\
\cline { 3 - 4 } & Placebo & DAPA 5/10 mg \\
\hline \multirow{2}{*}{ Age $<65$} & Adj. Mean (SE) & $-.28(.08)$ & $-.85(.05)$ \\
\cline { 2 - 4 } & Diff. vs. Placebo & -- & $-.58(.10)$ \\
\hline \multirow{2}{*}{ Age 65+ } & Adj. Mean (SE) & $-.46(.17)$ & $-.26(.15)$ \\
\cline { 2 - 4 } & Diff. vs. Placebo & -- & $.19(.23)$ \\
\hline
\end{tabular}

Although region did not show up as a significant for any study in the screening analysis, I conducted further analyses due to the regulatory importance of this factor. Having already established a lack of interaction, the interest was in whether the domestic/Canadian studies separately showed efficacy. Table 39 shows the placebo-adjusted means for the placebocontrolled studies by region. Both the 5 and 10 mg DAPA doses consistently showed efficacy in the U.S. and Canada.

Table 39: Placebo-Adj. Mean for Primary Endpoint by Region, Placebo-Controlled Studies

\begin{tabular}{|c|c|c|c|c|}
\hline \multirow{2}{*}{\multicolumn{2}{|c|}{ Study }} & \multicolumn{3}{|c|}{ DAPA Dose } \\
\hline & & $2.5 \mathrm{mg}$ & $5 \mathrm{mg}$ & $10 \mathrm{mg}$ \\
\hline \multirow{2}{*}{$\begin{array}{l}\text { MB102013 } \\
\text { (AM dosing) }\end{array}$} & U.S./Canada & $-.47(.22)^{*}$ & $-.66(.23)^{*}$ & $-.79(.22)^{* *}$ \\
\hline & Rest & $-.19(.21)$ & $-.34(.21)$ & $-.49(.21)^{*}$ \\
\hline \multirow[t]{2}{*}{ MB102014 } & U.S./Canada & $-.39(.18)^{*}$ & $-.53(.17)^{*}$ & $-.59(.17)^{* *}$ \\
\hline & Rest & $-.37(.12)^{*}$ & $-.35(.12)^{*}$ & $-.52(.12) * *$ \\
\hline \multirow[t]{2}{*}{ MB102030 } & U.S./Canada & Not applicable & $-.48(.17)^{*}$ & $-.48(.17)^{*}$ \\
\hline & Rest & Not applicable & $-.37(.16)^{*}$ & $-.61(.16) * *$ \\
\hline \multirow[t]{2}{*}{ D1690C00006 } & U.S./Canada & $-.57(.16)^{* *}$ & $-.60(.15)^{* *}$ & $-.84(.16)^{* *}$ \\
\hline & Rest & $-.41(.08) * *$ & $-.50(.08)^{* *}$ & $-.52(.08) * *$ \\
\hline
\end{tabular}

Note: Standard errors in parentheses. ${ }^{*} \mathrm{p}<.05 * * \mathrm{p}<.001$

Table 40 shows the primary results for Study 2034 by region. The combination of DAPA and MET was superior to each component at both domestic and foreign sites. Study C00004 did not have any domestic or Canadian sites.

Table 40: Primary Endpoint by Region, Study 2034

\begin{tabular}{|l|l|l|l|l|}
\hline \multicolumn{2}{|c|}{} & \multicolumn{3}{c|}{ Arm } \\
\cline { 3 - 5 } \multicolumn{2}{|c|}{} & $\begin{array}{l}\text { DAPA + } \\
\text { MET }\end{array}$ & DAPA & MET \\
\hline \multirow{2}{*}{ U.S. } & Adj. mean (SE) & $-2.28(.12)$ & $-1.51(.11)$ & $-1.62(12)$ \\
\cline { 2 - 5 } & Diff. vs. combin. (SE) & -- & $.77(.17)^{* *}$ & $.66(.18)^{* *}$ \\
\hline \multirow{2}{*}{$\begin{array}{l}\text { Rest of } \\
\text { world }\end{array}$} & Adj. mean (SE) & $-1.74(.09)$ & $-1.40(.09)$ & $-1.28(.09)$ \\
\cline { 2 - 5 } & Diff. vs. combin. (SE) & -- & $.33(.13)^{*}$ & $.45(.13)^{* *}$ \\
\hline
\end{tabular}

$* \mathrm{p}<.05 * * \mathrm{p}<.001$ 


\title{
4.2 Other Special/Subgroup Populations
}

Study 2029 was a failed study in subjects with moderate renal impairment. It was not one of the studies that were originally selected for me to closely review. While preparing for the Advisory Committee meeting, however, I decided that I should reproduce some of the Applicant's results from this study before I presented them. Figure 9 shows the design of the study.

Figure 9: Design of Study 2029

(Source: Clinical Study Report, Figure 3.1)

$\begin{array}{lccc}\text { Lead-in } & \text { Short-Term } & \text { Long-Term } & \text { Site and Subject Blinded } \\ \text { Period } & \text { Double-Blind } & \text { Double-Blind } & \text { Extension Period: } \\ 1 \text { Week } & \text { Period: } 24 \text { Weeks } & \text { Period: 28 Weeks } & 52 \text { Weeks }\end{array}$

\begin{tabular}{|c|c|c|c|}
\hline & Dapagliflozin $10 \mathrm{mg}$ & Dapagliflozin $10 \mathrm{mg}$ & Dapagliflozin $10 \mathrm{mg}$ \\
\hline $\begin{array}{l}\text { T2DM, N }=252 \\
\text { A1C: } 7.0-11.0 \%\end{array}$ & Dapagliflozin $5 \mathrm{mg}$ & Dapagliflozin $5 \mathrm{mg}$ & Dapagliflozin $5 \mathrm{mg}$ \\
\hline $\begin{array}{l}\text { eGFR: } 30 \text { to } \\
59 \mathrm{~mL} / \mathrm{min} / 1.73 \mathrm{~m}^{2}\end{array}$ & Placebo & Placebo & Placebo \\
\hline
\end{tabular}

Week 24

\begin{abstract}
Abbreviations:
$\mathrm{T} 2 \mathrm{DM}=$ type 2 diabetes mellitus

eGFR = estimated glomerular filtration rate

$\mathrm{R}=$ randomized
\end{abstract}

Primary End Point $\triangle \mathrm{A} 1 \mathrm{C}$

Table 41 shows the results for primary endpoint, change in HbA1c at Week 24. Both DAPA doses failed to beat placebo. The observed treatment effects were negligible.

Table 41: Results for Primary Endpoint, Study 2029

(Source: Clinical Study Report, Table 7.1)

\begin{tabular}{|c|c|c|c|}
\hline $\begin{array}{l}\text { EFFICACY ENDPOINT } \\
\text { STATISTICS }\end{array}$ & $\begin{array}{c}\text { Placebo } \\
N=84\end{array}$ & $\begin{array}{l}\text { DAPA 5MG } \\
\mathrm{N}=83\end{array}$ & $\begin{array}{c}\text { DAPA } 10 \mathrm{MG} \\
\mathrm{N}=85\end{array}$ \\
\hline
\end{tabular}

PRIMARY EFFICACY ENDPOINI

HBA1C (8) AT WEEK 24 (IOCF)
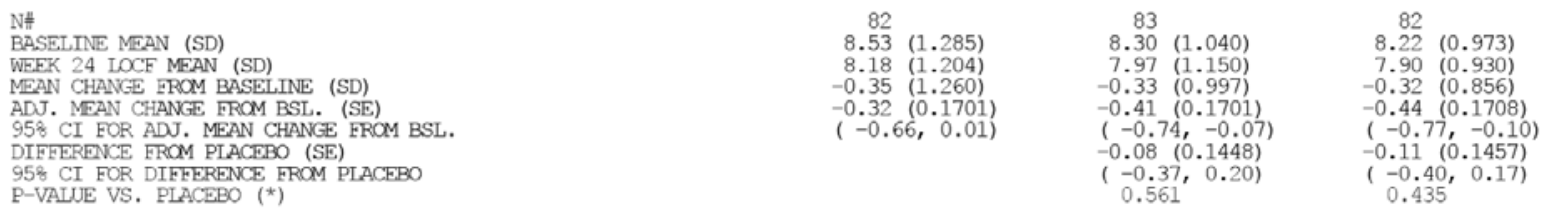

$\mathrm{N}$ is the number of randomized subjects who took at least one dose of double-blind study medication.

$N \#$ is the number of randomized subjects with non-missing baseline and Week $t$ (IDCF) values.

${ }^{*}$ ) Significant p-value: Primary endpoint is tested at alpha=0.027 applying Dunnett's adjustment, and secondary endpoints are tested

Analysis of continuous outcomes based on separate ANCOVA models with treatment group and stratum as effects and baseline values as a covariate. 
The Applicant also conducted an "ad-hoc" (their term) subgroup analysis in patients with stage 3A moderate renal impairment, defined as an eGFR of 45-59. The results of this analysis are shown in Table 42. Although the estimated treatment effects from each DAPA are larger than those seen in the overall study, neither arm was significantly different from placebo. (Note that the confidence intervals include 0.) Even if this "ad-hoc" analysis had succeeded in showing a significant effect, I would have viewed the results skeptically since the study failed on the primary endpoint.

Table 42: Primary Endpoint in Stage 3A Renal Subgroup

(Source: Clinical Study Report, Appendix 38)

\begin{tabular}{|c|c|c|c|}
\hline & $\begin{array}{l}\text { PLACEBO } \\
N=41\end{array}$ & $\begin{array}{l}\text { DAPA 5MG } \\
\quad N=35\end{array}$ & $\begin{array}{l}\text { DAPA } 10 \mathrm{MG} \\
\quad \mathrm{N}=33\end{array}$ \\
\hline \multicolumn{4}{|l|}{ SUMARY STATISTICS } \\
\hline $\begin{array}{l}\text { N\# } \\
\text { BASELINE MEAN (SD) } \\
\text { WEEK } 24 \text { MEAN (SD) } \\
\text { NEAN CHANGE FROM BSL (SD) }\end{array}$ & $\begin{array}{rl}8.78 & 40 \\
8.62(1.318) & (1.201) \\
-0.16(1.374)\end{array}$ & $\begin{array}{r}8.13 \\
7.93(0.928) \\
-0.20(1.086) \\
-0.941)\end{array}$ & $\begin{aligned} & 8.25(32 \\
& 8.03(1.892) \\
&-0.22(1.002) \\
&-0.797)\end{aligned}$ \\
\hline \multicolumn{4}{|l|}{ ADUUSTED CHANGE FRCM BASELINE } \\
\hline $\begin{array}{l}\text { MEAN (SE) } \\
95 \% \text { 2-SIDED CI }\end{array}$ & {$\left[\begin{array}{ll}-0.11, & 0.2339) \\
{[-0.57,} & 0.35]\end{array}\right.$} & {$\left[\begin{array}{cc}-0.47 & (0.2483) \\
{[-0.97,} & 0.02]\end{array}\right.$} & {$\left[\begin{array}{cc}-0.44, & 0.2546) \\
-0.94, & 0.07\end{array}\right]$} \\
\hline \multicolumn{4}{|l|}{ DIFFERENCE IN ADUUSTED CHANGE FROM BASELINE VS PLACEBO } \\
\hline $\begin{array}{l}\text { MEAN (SE) } \\
95 \% \text { 2-SIDED CI }\end{array}$ & & {$\left[\begin{array}{ll}-0.37, & (0.2322) \\
-0.83, & 0.10\end{array}\right]$} & {$\left[\begin{array}{rr}-0.33, & (0.2376) \\
-0.80, & 0.14]\end{array}\right]$} \\
\hline
\end{tabular}

$\mathrm{N}$ is the number of randomized subjects with baseline eGER $>=45$ and $<60 \mathrm{~mL} / \mathrm{min} / 1.73 \mathrm{~m}^{\wedge} 2$

who took at least one dose of double-blind study medication.

$\mathbb{N} \#$ is the number of randomized subjects with baseline eGrR $>45$ and $<60 \mathrm{~mL} / \mathrm{min} / 1.73 \mathrm{~m}^{\wedge}$

and non-missing baseline and week 24 (LOCF) HbAlc values.

Based on an ANCOVA model with treatment groum and stratum as main effects and baseline value as a covariate.

For comparison, Table 43 shows the results for patients with stage 3B impairment, defined as an eGFR of 30 to 44. There were 21 additional patients who did not fall in either subgroup. 
Table 43: Primary Endpoint in Stage 3B Renal Subgroup

\begin{tabular}{|c|c|c|c|}
\hline & $\begin{array}{c}\text { PLACEBO } \\
N=34\end{array}$ & $\begin{array}{c}\text { DAPA } 5 \mathrm{MG} \\
\mathrm{N}=41\end{array}$ & $\begin{array}{l}\text { DAPA } 10 \mathrm{MG} \\
\mathrm{N}=47\end{array}$ \\
\hline \multicolumn{4}{|l|}{ SUMARY STATISTICS } \\
\hline $\begin{array}{l}\text { N\# } \\
\text { BASELINE MEAN (SD) } \\
\text { WEEK } 24 \text { MEAN (SD) } \\
\text { MEAN CHANGE FROM BSL (SD) }\end{array}$ & $\begin{array}{r}8.23 \\
7.79 \\
-0.44\end{array}\left(\begin{array}{l}3.197) \\
-1.034)\end{array}\right.$ & $\begin{aligned} 8.49 & (1.157) \\
7.97 & (1.250) \\
-0.52 & (1.069)\end{aligned}$ & $\begin{aligned} & 45 \\
8.12 & (1.001) \\
7.78 & (0.864) \\
-0.34 & (0.931)\end{aligned}$ \\
\hline \multicolumn{4}{|l|}{ ADUUSTED CHANGE EROM BASELTNE } \\
\hline $\begin{array}{l}\text { MEAN (SE) } \\
95 \% \text { 2-SIDED CI }\end{array}$ & {$\left[\begin{array}{ll}-0.52 & (0.2804) \\
{[-1.08,} & 0.03]\end{array}\right.$} & {$\left[\begin{array}{cc}-0.47( & 0.2712) \\
{[-1.01,} & 0.06]\end{array}\right.$} & {$\left[\begin{array}{cc}-0.45 & (0.2545) \\
{[-0.96,} & 0.05\end{array}\right]$} \\
\hline \multicolumn{4}{|l|}{ DIFFERENCE IN ADUUSTED CHANGE FROM BASELINE VS PLACEBO } \\
\hline $\begin{array}{l}\text { MEAN (SE) } \\
95 \% \text { 2-SIDED CI }\end{array}$ & & {$\left[\begin{array}{ll}0.05 & (0.2107) \\
-0.37, & 0.47]\end{array}\right.$} & {$\left[\begin{array}{cc}0.07 & (0.2074) \\
-0.34, & 0.48\end{array}\right]$} \\
\hline
\end{tabular}

$N$ is the number of randomized subjects with baseline eGRR $\times=30$ and $<45 \mathrm{~mL} / \mathrm{min} / 1.73 \mathrm{~m}^{\wedge} 2$

who took at least one dose of double-blind study medication.

$\mathrm{N} \#$ is the number of randamized subjects with baseline eGrR $\rangle=30$ and $<45 \mathrm{~mL} / \mathrm{min} / 1.73 \mathrm{~m} 2$

and non-missing baseline and week 24 (LOCF) HhAlc values.

Based on an ANCOVA model with treatment group and stratum as main effects and baseline value as a covariate. 


\section{SUMMARY AND CONCLUSIONS}

\subsection{Statistical Issues and Collective Evidence}

The Applicant submitted 11 Phase 3 studies, 10 of which were successful on the primary endpoint using the pre-specified analysis. The only failed Phase 3 study was in patients with moderate renal impairment, a result that is consistent with the drug's renal mechanism of action. I closely reviewed 6 of the 11 studies, and did find any concerns that would lead me to question the positive results. The results of the secondary endpoints were supportive.

Although I do not doubt that there was a non-zero treatment effect for DAPA, the actual magnitude of the treatment effect is uncertain due to missing data and use of rescue medication. For the primary analysis of the HbA1c endpoint the Applicant used LOCF, disregarding observations after rescue.

FDA has accepted LOCF as the primary imputation method for diabetes studies in the past. In February 2008, FDA issued a draft guidance for industry titled Diabetes Mellitus: Developing Drugs and Therapeutic Biologics for Treatment and Prevention. This draft guidance describes LOCF as "easy to apply and transparent" and states that it will "tend to underestimate the true effect of the drug relative to placebo providing a conservative estimate of the drug's effect.” It also states, however, that additional sensitivity analyses should be conducted and, in particular, different ways of handling rescue medication should be examined. More recently, the National Academy of Sciences released a report on missing data which was commissioned by FDA (National Research Council, 2010). The report recommends that, "Single imputation methods like [LOCF] ... should not be used as the primary approach to the treatment of missing data unless the assumptions that underlie them are scientifically justified.” (The report also states that these assumptions will not hold in most circumstances.) In light of this recommendation, it is all the more important to consider alternatives to the prespecified LOCF analysis.

The impact of rescue medication on the analysis merits further discussion. Although rescue therapy is ethically appropriate in some cases, it can complicate statistical inference because it is an additional intervention that occurs after randomization and is not controlled by it. Subjects who received rescue differed from the other subjects in at least two ways. First of all, they experienced the effect of rescue treatment, and this is the effect that one would want adjust for if it were possible. Secondly, they also met glycemic criteria in order to be eligible for rescue. It is not possible to disentangle the effect of rescue from the underlying response to the randomized treatment. If one found, for example, that rescued patients did substantially worse than nonrescued patients, then that could either mean that the rescue treatment was ineffective or that the patients would have had even worse outcomes without it. One way around this problem would be to randomize some rescue-eligible patients to receive sham rescue, but this would undermine the ethical purpose of including rescue treatment in the first place.

As previously noted, for the primary analysis, the Applicant excluded observations after a patient was rescued, carrying forward the pre-rescue values. Since eligibility for rescue indicates a lack of efficacy, it is reasonable from a certain standpoint for the imputed value at the end of study to 
be the poor, pre-rescue value. As the results of these submitted studies have shown, however, this is not a reliably conservative approach.

The Applicant also conducted a sensitivity analysis which incorporated rescue treatment as an independent variable in the linear model. I do not believe that this is appropriate due to the confounding described previously. Instead I have included a sensitivity analysis in which the primary HbA1c outcomes are used regardless of rescue treatment, and no statistical adjustment is made for rescue. This approach is also imperfect, but it comes closer to being a true intent-totreat (ITT) analysis because it disregards the non-randomized rescue treatment. I conducted this sensitivity analysis for five of the studies that I focused on (excluding C00004, which did not use rescue), and the primary endpoint remained statistically significant for the both the 5 and $10 \mathrm{mg}$ DAPA doses, as applicable. The estimated treatment effects were somewhat attenuated, however. Table 44 shows the results of this sensitivity analysis for the four placebo-controlled studies that I focused on.

Table 44: Reviewer's Sensitivity Analysis, Placebo-Controlled Studies

\begin{tabular}{|c|c|c|c|c|c|}
\hline \multicolumn{2}{|l|}{ Study } & \multicolumn{4}{|c|}{ Dapagliflozin Dose } \\
\hline & & Placebo & $2.5 \mathrm{mg}$ & $5 \mathrm{mg}$ & $10 \mathrm{mg}$ \\
\hline \multirow[t]{2}{*}{2013} & $\begin{array}{l}\text { Adj. Mean } \\
\text { (SE) }\end{array}$ &. $.45(.09)$ & $-.72(.10)$ & $-.81(.10)$ & $-.91(.10)$ \\
\hline & \begin{tabular}{|l|} 
Diff. vs. \\
Placebo
\end{tabular} & F- & $-.27(13)^{*}$ & $-.36(.14)^{*}$ & $-.45(.13)^{* *}$ \\
\hline \multirow[t]{2}{*}{2014} & $\begin{array}{l}\text { Adj. Mean } \\
\text { (SE) }\end{array}$ & $-.43(.07)$ & $-.72(.07)$ & $-.70(.07)$ & $-.87(.07)$ \\
\hline & \begin{tabular}{|l|} 
Diff. vs. \\
Placebo
\end{tabular} & F- & $-.29(.10)^{*}$ & $-.27(.10)^{*}$ & $-.44(.10)^{* *}$ \\
\hline \multirow[t]{2}{*}{2030} & $\begin{array}{l}\text { Adj. Mean } \\
\text { (SE) }\end{array}$ & $-.58(.08)$ & N.A. & $-.86(.08)$ & $-1.02(.08)$ \\
\hline & \begin{tabular}{|l} 
Diff. vs. \\
Placebo
\end{tabular} & F- & N.A. & $-.28(.12)^{*}$ & $-.44(.12)^{* *}$ \\
\hline \multirow{2}{*}{ C00006 } & $\begin{array}{l}\text { Adj. Mean } \\
\text { (SE) }\end{array}$ &. $.39(.06)$ & $-.80(.05)$ & $-.89(.05)$ & $-.97(.05)$ \\
\hline & \begin{tabular}{|l|} 
Diff. vs. \\
Placebo
\end{tabular} & - & $-.40(.08)^{* *}$ & $-.50(.08)^{* *}$ & $-.57(.08)^{* *}$ \\
\hline
\end{tabular}

${ }^{*} \mathrm{p}<.05$ vs. placebo. ${ }^{* *} \mathrm{p}<.001$ vs. placebo.

Table 45 shows the results of this sensitivity analysis for Study 2034. The sixth study that I focused on, Study C00004, did not include rescue treatment. 
Table 45: Reviewer's Sensitivity Analysis, Study 2034

\begin{tabular}{|l|l|l|l|}
\hline & \multicolumn{2}{|l|}{ Treatment Arm } & MET \\
\hline & DAPA 10 mg + & DAPA 10 mg & \\
& MET & & $-1.54(.07)$ \\
\hline Adj. Mean (SE) & $-2.02(.07)$ & $-1.54(.07)$ & - \\
\hline Diff. from DAPA & $-.48(.10)^{* *}$ & - & - \\
\hline Diff from. MET & $-.48(.10)^{* *}$ & - & \\
\hline
\end{tabular}

$* * \mathrm{p}<.001$ vs. placebo.

We previously referred the Applicant to White et al. (2001) for advice on handling rescue. This paper actually notes that "Ignoring all data after rescue is likely to be biased because rescued patients are a highly selected group." The only case in which they endorse ignoring such data is when "rescue does not necessarily define a poor outcome". Since rescue was triggered by lack of glycemic control in this case, it would seem to indicate a poor outcome. The authors are also critical of LOCF, and of including rescue as a term in the linear model.

See Section 5.3 for further discussion.

\subsection{Conclusions and Recommendations}

The submitted clinical studies provide strong evidence that dapagliflozin is effective in subjects with normal renal function or mild impairment. The dedicated study of subjects with moderate renal impairment did not show statistical evidence of efficacy.

\subsection{Review of the Proposed Label}

Selections from the Applicant's proposed label language are shown in italics, and my comments are shown in regular type. Note that any references to figures use different numbering than the rest of the report.

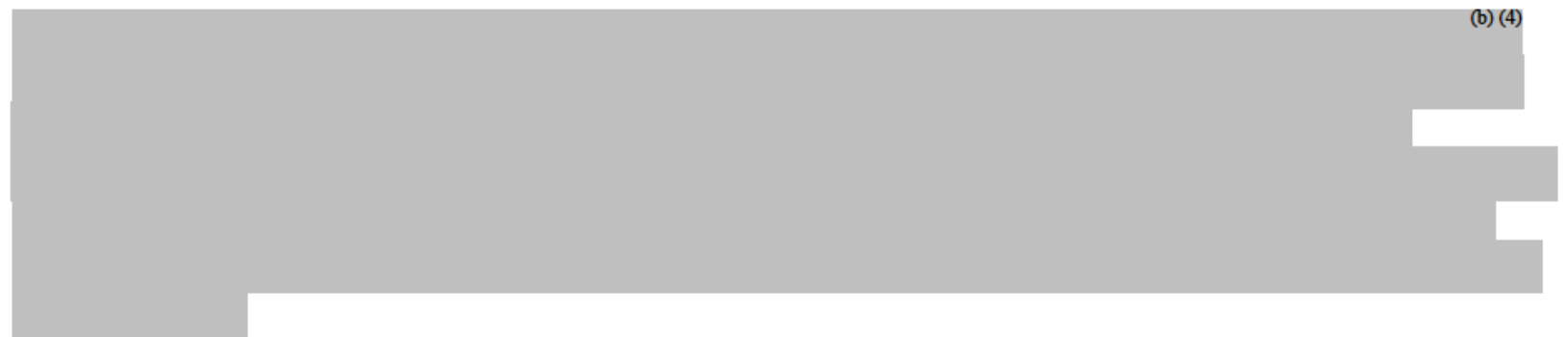

TS) immediately following this page 


\section{APPENDIX A: References}

National Research Council, 2010, The prevention and treatment of missing data in clinical trials. Panel on Handling Missing Data in Clinical Trials, Committee on National Statistics, Division of Behavioral and Social Sciences and Education, Washington, DC: The National Academies Press.

Tsiatis, A., Davidian, M., Zhang, M., Lu, X., 2007, Covariate adjustment for two-sample treatment comparisons in randomized clinical trials: a principled yet flexible approach, Statistics in Medicine, 27:4658-4677.

White, I.R., 2001, Randomized clinical trials with added rescue medication: some approaches to their analysis and interpretation, Statistics in Medicine, 20:2995-3008.

Zhang, M., Tsiatis, A., Davidian, M., 2008, Improving efficiency of inferences in randomized clinical trials using auxiliary covariates, Biometrics, 64:707-715. 


\section{APPENDIX B: Additional Figures}

Note: The following pages will print in landscape mode. 
Figure 10: Sensitivity Analyses for Study 2013 - DAPA 10 mg vs. Placebo

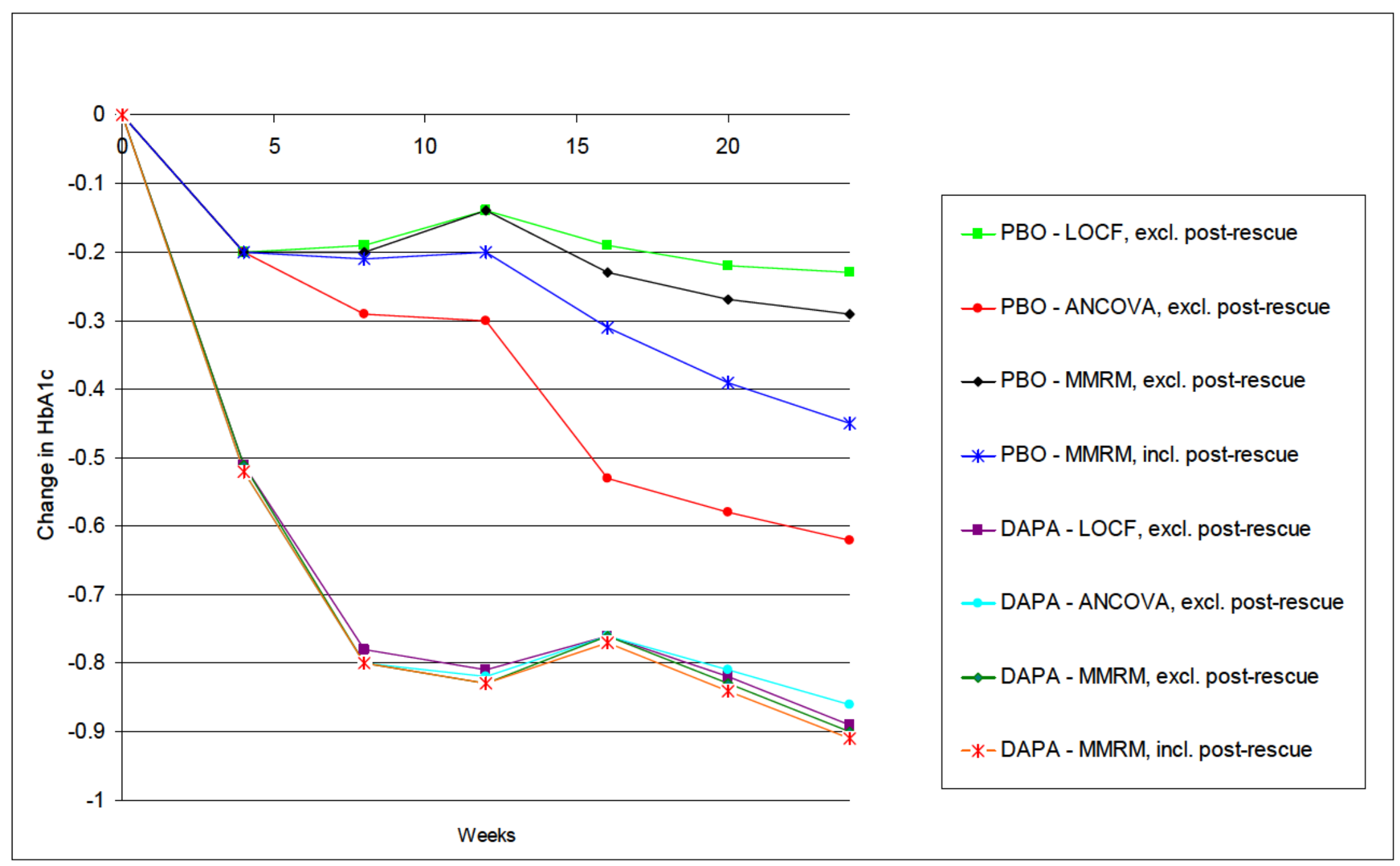


Figure 11: Change in Body Weight at Week 24, Placebo-Controlled Studies

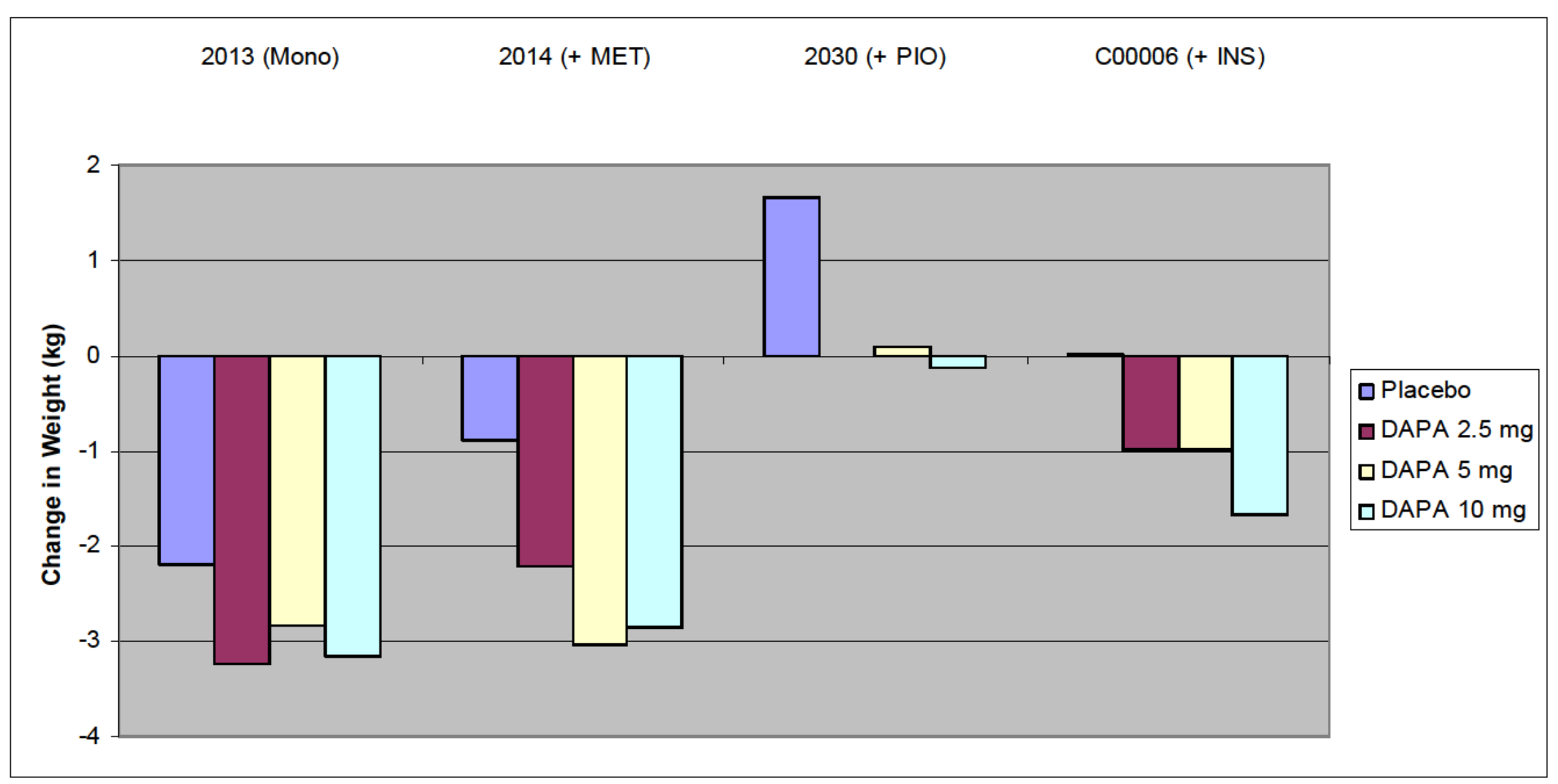


Figure 12: Sensitivity Analysis for Study 2014, DAPA 10 mg vs. Placebo

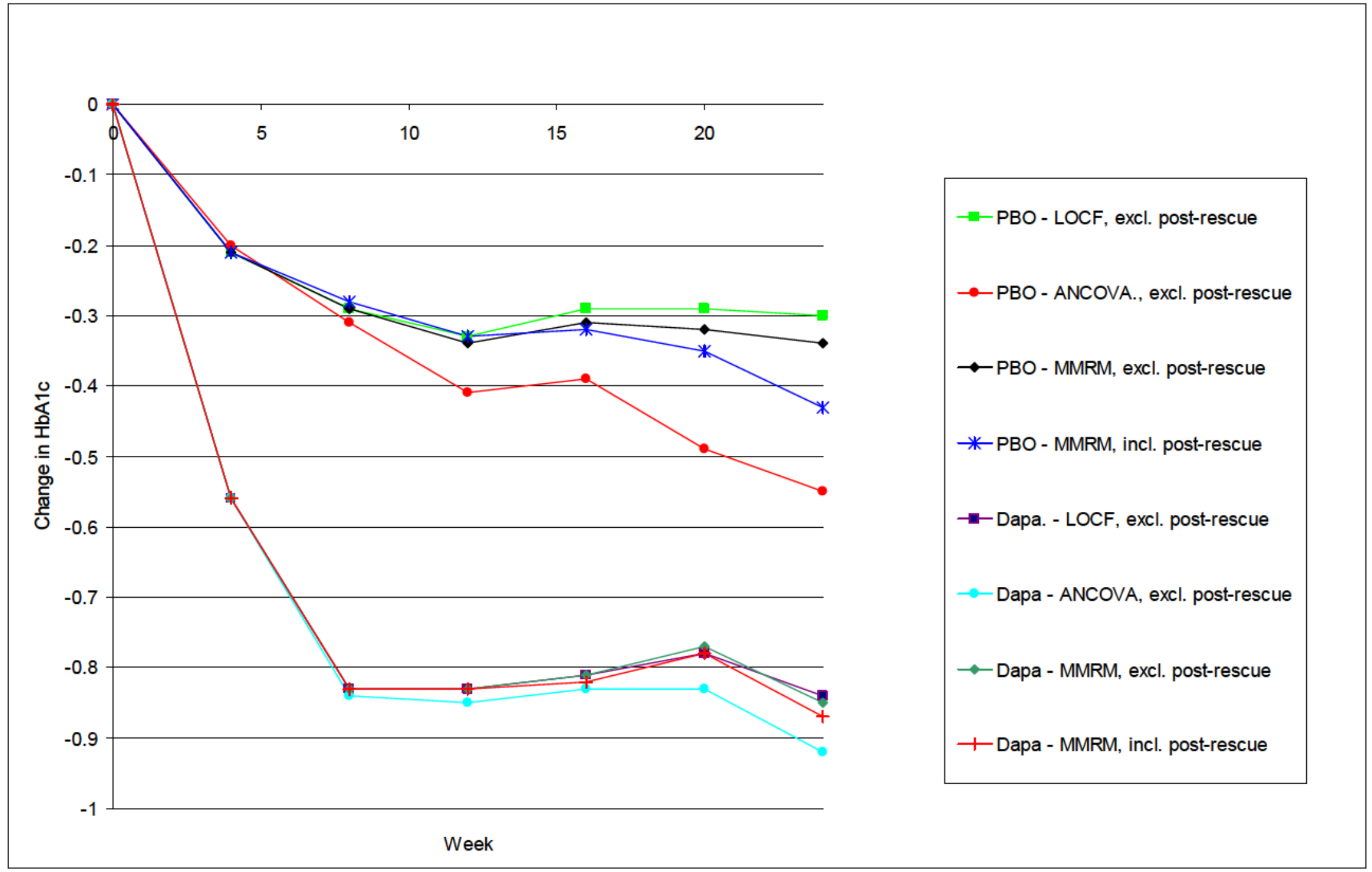


Figure 13: Sensitivity Analysis for Study C00004

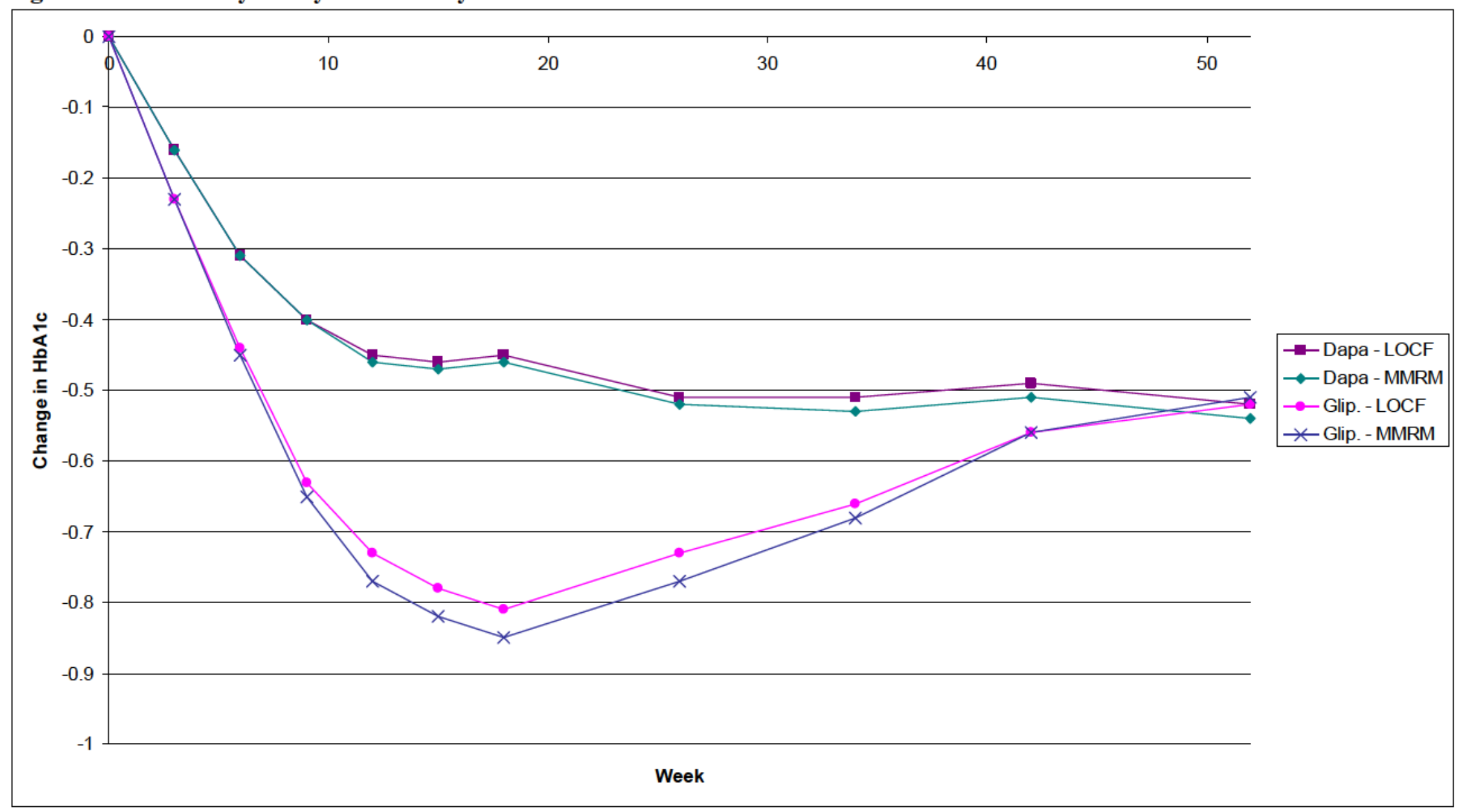


This is a representation of an electronic record that was signed electronically and this page is the manifestation of the electronic signature.

/s/

JONATHAN D NORTON

$09 / 08 / 2011$

JON T SAHLROOT

09/08/2011

concur

THOMAS J PERMUTT

09/09/2011

concur 
U.S. Department of Health and Human Services

Food and Drug Administration

Center for Drug Evaluation and Research

Office of Translational Sciences

Office of Biostatistics

\section{STATISTICAL REVIEW AND EVALUATION \\ Clinical Studies}

NDA/Serial Number:

$202-293$ / SN 000

Drug Name:

(dapagliflozin)

Indication(s):

Treatment of type 2 diabetes mellitus (T2DM) in adults as an adjunct to diet and exercise to improve glycemic control

Applicant:

Date(s):

Review Priority:

Biometrics Division:

Statistical Reviewer:

Concurring Reviewers:

Medical Division:

Clinical Team:

Project Manager:
Bristol-Myers Squibb

Submitted September 7, 2011

Standard

Division of Biometrics VII

Anita Abraham, Ph.D.

Mat Soukup, Ph.D.

Aloka Chakravarty, Ph.D.

Division of Metabolism and Endocrinology Products (DMEP)

Medical Officer: Somya (Verma) Dunn, M.D.

Medical Team Leader: Ilan Irony, M.D.

Mehreen Hai, M.S. (DMEP)

Keywords: type 2 diabetes mellitus, cardiovascular safety, meta-analysis 


\section{Table of Contents}

1 Executive Summary ................................................................................................................ 5

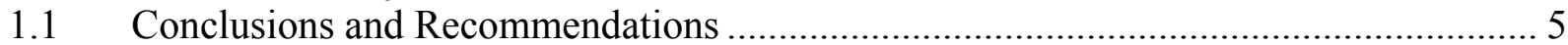

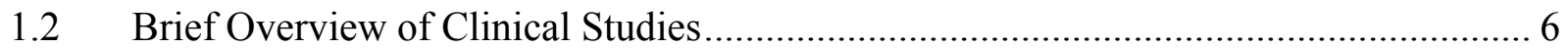

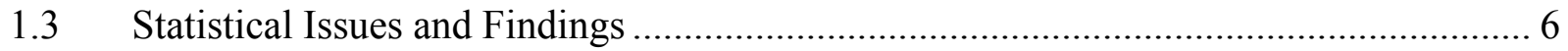

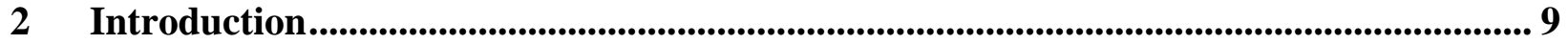

$2.1 \quad$ Product Description ........................................................................................ 9

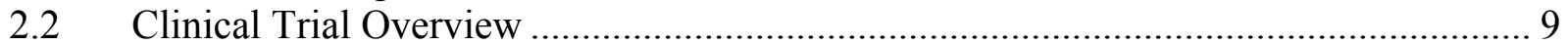

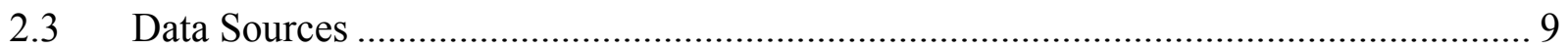

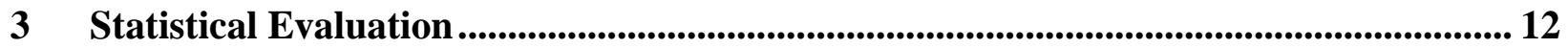

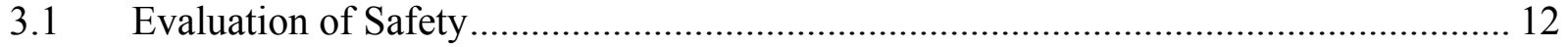

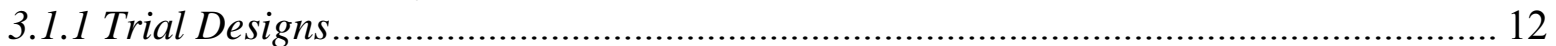

3.1.2 Endpoints and Adjudication Methods ........................................................................ 17

3.1.2.1 Primary Composite Endpoint....................................................................... 17

3.1.2.2 Secondary Composite Endpoint...................................................................... 17

3.1.2.3 Adjudication Methods................................................................................ 17

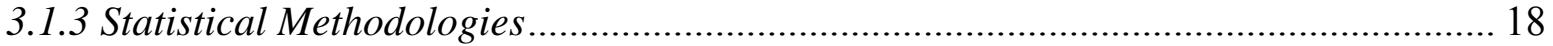

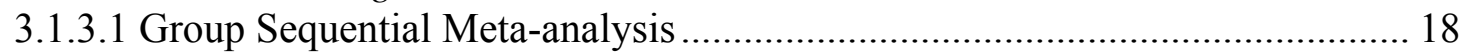

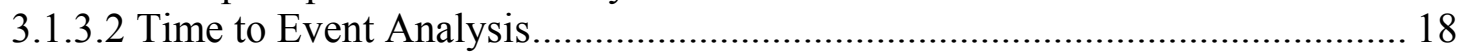

3.1.3.3 Analysis of Event Incidence ...................................................................... 19

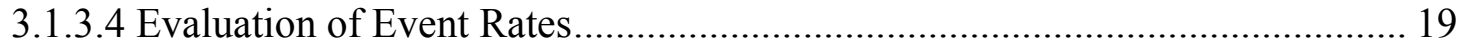

3.1.3.5 Handling of Trials with Zero Events ............................................................. 19

3.1.3.6 Evaluation of Heterogeneity between Trials..................................................... 19

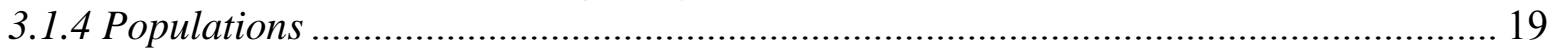

3.1.5 Subject Disposition, Demographics and Baseline Characteristics ............................ 21

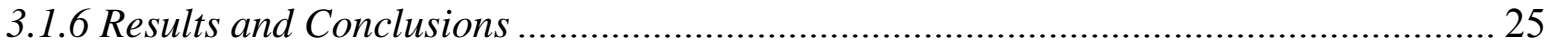

3.1.6.1 Primary Composite Endpoint....................................................................... 25

3.1.6.2 Secondary Composite Endpoint..................................................................... 29

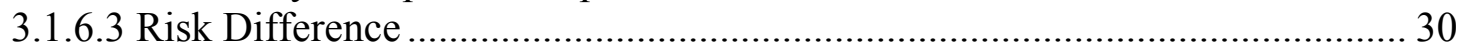

3.1.6.4 Evaluation of Trial Heterogeneity ……………….......................................... 31

$4 \quad$ Findings in Special/Subgroup Populations.......................................................................... 32

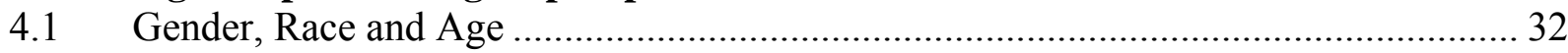

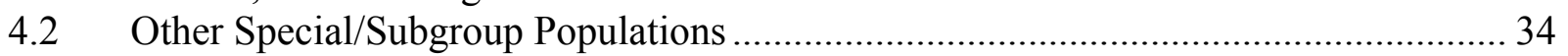

4.2.1 Baseline BMI, CV Risk Factors, Renal Impairment and Diabetes History ................. 35

4.2.2 Monotherapy and Add-On Trials.............................................................................. 36

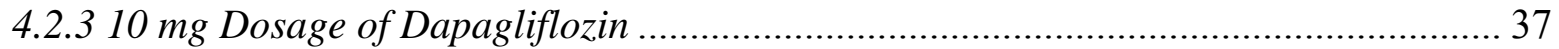

$5 \quad$ Summary and Conclusions................................................................................................... 39

5.1 Statistical Issues and Collective Evidence _.............................................................. 39

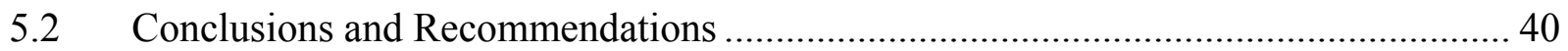

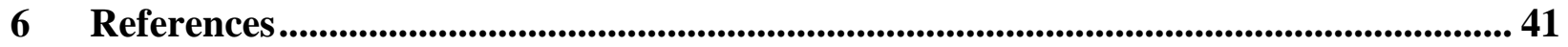

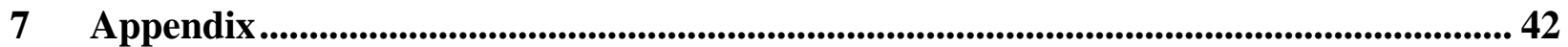

SIGNATURES/DISTRIBUTION LIST _.......................................................................... 53 


\section{Table of Tables}

Table 1: Meta-Analysis Results for Primary and Secondary Composite Endpoints (BMS

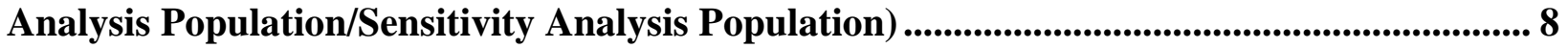

Table 2: List of Trials Included in CV Meta-Analysis .......................................................... 11

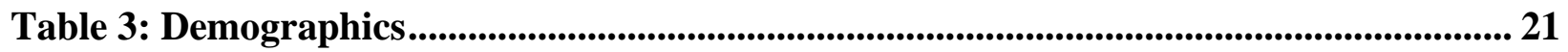

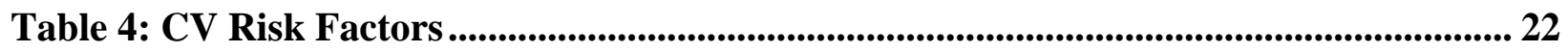

Table 5: Trial Discontinuation Rates by Reason ..................................................................... 24

Table 6: Meta-Analysis Results for Primary Composite Endpoint (BMS Analysis

Population/Sensitvity Analysis Population)........................................................................ 25

Table 7: Meta-Analysis Results for Secondary Composite Endpoint (BMS Analysis Population/Sensitvity Analysis Population).................................................................................. 29

Table 8: Meta-Analysis Risk Difference Results of the Primary Endpoint (BMS Analysis

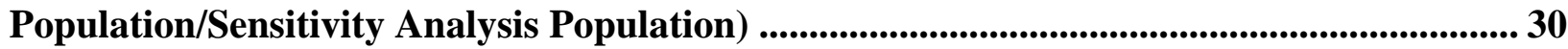

Table 9: Meta-Analysis Results of the Primary Composite Endpoint for the Monotherapy Trials and Add-On Therapy Trials (BMS Analysis Population) .......................................... 36

Table 10: Meta-Analysis Results for 10mg Dose of Dapaglfilozin Alone and the Pooled Comparator (BMS Analysis Population excluding MB102021 and MB102032) .................. 37

Table 11: CV Risk Factors by Trial ................................................................................................ 50 


\section{Table of Figures}

Figure 1: Percentage of Subjects with Premature Discontinuation of Study Medication by Trial and Treatment Group (BMS Analysis Population) ....

Figure 2: Time-to-event Analysis of the Primary Composite Endpoint (BMS Analysis Population)

Figure 3: Forest Plot of Hazard Ratios and 95\% CI of Individual Trials from Cox Proportional Hazards for Primary CV Composite Endpoint and 98\% CI for Overall Stratified Analysis (BMS Analysis Population)

Figure 4: Forest Plot of Mantel-Haenszel Incidence Rate Differences of Cardiovascular Events Contributing to Primary Composite Endpoint (BMS Analysis Population) ............ 28

Figure 5: Forest Plot of Risk Difference and 95\% CI by Trial for Primary CV Composite Endpoint (BMS Meta-analysis Dataset) and 98\% CI for Overall Analysis 31

Figure 6: Forest Plot of Incidence Rate Difference of Primary Composite Endpoint by Baseline Demographics (BMS Analysis Population)

Figure 7: Forest Plot of Incidence Rate Difference of Primary Composite Endpoint by Baseline Risk Factors (BMS Analysis Population) 35

Figure 8: Time-to-event Analysis of the Primary Composite Endpoint for the $10 \mathrm{mg}$ Dose of Dapagliflozin Compared to Pooled Comparator (BMS Analysis Population excluding

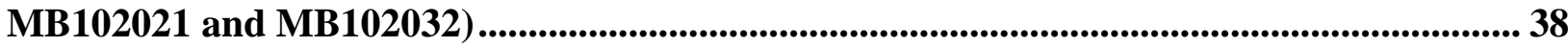

Figure 9: Assessment of Proportional Hazards Assumption: Log-Log Survival Curve (BMS Analysis Population)

Figure 10: Assessment of Proportional Hazards Assumption: Schoenfeld Residual Plot (BMS Analysis Population).

Figure 11: Kaplan Meier Curves of Discontinuation Rates for Each Trial in BMS Analysis

Population. 43 


\section{Executive Summary}

The proposed indication for dapagliflozin is the treatment of type 2 diabetes mellitus (T2DM) in adults as an adjunct to diet and exercise to improve glycemic control. The proposed therapeutic dose of dapagliflozin is $10 \mathrm{mg}$ for most patients and a $5 \mathrm{mg}$ starting dose for patients at risk for volume depletion due to co-existing conditions or concomitant medications, such as loop diuretics. Per the request of the Division of Metabolism and Endocrinology Products this statistical review assesses cardiovascular (CV) related safety endpoints in the randomized phase 2b/3 clinical development program of dapagliflozin (Trials D1690C00004, D1690C00005, D1690C00006, D1690C00012, D1692C00005, MB102008, MB102009, MB102013, MB102014, MB102021, MB102029, MB102030, MB102032 and MB102034). A separate efficacy evaluation is also being conducted by Dr. Jonathan Norton from DB2. This review focuses solely on the pre-marketing evaluation of cardiovascular safety.

\subsection{Conclusions and Recommendations}

The sponsor conducted a meta-analysis of the cardiovascular events that occurred in 14 Phase $2 \mathrm{~b} / 3$ trials using dapagliflozin. This meta-analysis was the first of a two-part group sequential testing strategy that was pre-specified to control the Type I error rate for demonstration of noninferiority with respect to the 1.8 relative risk criterion, as estimated by the hazard ratio, of the investigational agent compared to the control group at a one-sided 0.025 alpha-level. Using this strategy, if the first formal meta-analysis of the primary composite endpoint was found to have an upper-bound less than 1.8 at a one-sided alpha-level of 0.01 (corresponding to a $98 \% \mathrm{CI}$ ) the meta-analysis would meet the CV guidelines outlined in the FDA "Guidance for industry: Diabetes mellitus - evaluating cardiovascular risk in new antidiabetic therapies to treat type 2 diabetes" (December 2008). ${ }^{1}$

The agreed upon primary endpoint is a composite endpoint, defined as the time to first event of the following adjudicated events: CV death, myocardial infarction, stroke, and hospitalization for unstable angina. All CV events were adjudicated by an independent, blinded clinical events committee (CEC) comprised of two cardiologists and two neurologists. The comparator group was comprised of subjects taking either a placebo and/or an active control (metformin, glipizide, insulin, glimepiride, or pioglitazone).

Among the 6228 subjects in the Bristol-Myers Squibb (BMS) Analysis population (composed of the safety population of all 14 trials combined), 78 subjects had an event that was counted in the primary composite endpoint: 48 of 4287 dapagliflozin subjects (1.1\%) and 30 of 1941 comparator subjects $(1.5 \%)$. The pre-specified primary analysis of the stratified Cox proportional hazards model yielded a hazard ratio of dapagliflozin versus comparator to be 0.67 (98\% CI: 0.38, 1.18). The Mantel-Haenszel method was used to calculate the incidence rate ratio and incidence rate difference as pre-specified secondary analyses. The incidence rate ratio was found to be 0.67 (98\% CI: $0.39,1.17)$. The incidence rate difference incorporated zero event trials in the meta-analysis, and was found to be -0.0054 (98\% CI: $-0.013,0.0026)$. These results appear to be consistent across trials and between the subgroups evaluated and are presented in Table 1. 
A secondary composite endpoint was also evaluated which was comprised of the same CV endpoints as the primary endpoint and two additional CV endpoints (unplanned coronary revascularization, and hospitalization for heart failure). This endpoint also found no additional risk of $\mathrm{CV}$ events in the dapagliflozin group compared to the comparator group (hazard ratio (98\% CI): $0.63(0.38,1.03)$.

To further investigate the sensitivity of the analysis, the FDA conducted a sensitivity analysis excluding short-term trials and the non-inferiority trial included in the sponsor's analysis. As displayed in Table 1, this sensitivity analysis found that there was no additional risk of CV events in the dapagliflozin group compared to comparator (hazard ratio: $0.60,98 \% \mathrm{CI}: 0.32$, 1.20). Additionally monotherapy and add-on therapy trials were evaluated separately (hazard ratios $(95 \% \mathrm{CI})$ : monotherapy $0.67(0.27,1.68)$, add-on: $0.64(0.36,1.15))$. To evaluate the proposed therapeutic dose, the $10 \mathrm{mg}$ dose group was separately evaluated and found to have a hazard ratio of $0.69(95 \% \mathrm{CI}: 0.39,1.20)$.

Based upon the 1.8 specific non-inferiority margin set forth in the FDA Diabetes Guidance ${ }^{1}$, the upper-bound of the alpha-adjusted $98 \%$ confidence interval of the hazard ratio meets the 1.8 noninferiority margin.

\subsection{Brief Overview of Clinical Studies}

In this NDA application, the applicant submitted 14 randomized phase $2 \mathrm{~b}$ and phase 3 trials. Four trials (D1690C00004, D1690C00005, D1690C00006, and D1692C00005) were conducted by Astra-Zeneca (AZ) and 10 trials were conducted by BMS. All 14 randomized phase 2b/3 trials were conducted in subjects with type 2 diabetes mellitus. Trials D1690C00005, D1690C00006, D1690C00012, MB102009, MB102014 and MB102030 were add-on trials where subjects were on a background therapy and dapagliflozin was added to that therapy. Trial D1690C00004 was a non-inferiority trial where subjects were on metformin and were given titrated doses of dapagliflozin or glipizide. Trials D1692C00005, MB102008, MB102013 and MB102032 were monotherapy trials. Trial MB102029 was a monotherapy trial conducted in subjects with moderate to severe renal impairment. MB102021 and MB102034 were combination therapy trials where subjects were either on dapagliflozin alone, metformin alone or a combination of dapagliflozin and metformin. Details of the 14 randomized phase $2 b / 3$ trials are provided in Section 3.1.1.

\subsection{Statistical Issues and Findings}

The primary agreed upon safety endpoint was a composite endpoint consisting of CV death, nonfatal myocardial infarction (MI), non-fatal stroke, and hospitalization due to unstable angina.

In the statistical analysis plan (SAP) agreed upon with the FDA on November 9, 2010, a group sequential testing strategy was pre-specified to control the Type I error rate for demonstration of non-inferiority with respect to the 1.8 relative risk criterion, as estimated by the hazard ratio, at an overall one-sided 0.025 level. Using this sequential testing strategy, if the first formal meta- 
analysis of the primary composite endpoint was found to have an upper-bound less than 1.8 at a one-sided alpha level of 0.01 (corresponding to a $98 \% \mathrm{CI}$ ) then the meta-analysis would meet the FDA CV guidelines ${ }^{1}$, and data from additional studies that have yet to be completed would not need to be included in a second meta-analysis. However, if unsuccessful at this alpha level (0.01), a second meta-analysis would be performed when studies D1690C00018 and D1690C00019 are unblinded and at least 110 patients had adjudicated events for the primary endpoint. The one-sided alpha level for this final analysis was set at 0.01 so that the overall Type I error rate across the 2 analyses was $\leq 0.025$ (one-sided).

Phase $2 \mathrm{~b}$ and 3 data from the 14 randomized studies were pooled to evaluate the risk of developing a CV related event as measured by the primary composite endpoint (CV death, nonfatal MI, non-fatal stroke, and hospitalization due to unstable angina). The pre-specified primary analysis conducted was a comparison of incidence between the dapagliflozin group and the pooled all comparator group (including monotherapy, initial combination with metformin, addon to metformin, sulphonylurea (SU), thiazolidinedione (TZD), and insulin studies) was performed using a Cox proportional hazards model with study as stratification factor. The prespecified secondary analyses used the Mantel-Haenszel (MH) method for estimating the overall incidence rate ratio and the incidence rate difference via Breslow and Day $(1994)^{2}$ where the estimate was calculated by stratifying for trial. The incidence rate difference included trials with zero events. To further investigate event rates, the risk difference was calculated to determine if a different denominator (number of subjects per group as opposed to number of person-years of exposure) would affect the estimates. More details for the statistical methodologies used in this review are provided in Section 3.1.2.

Table 1 presents the results of the meta-analysis for the primary and secondary composite endpoints. The results from the stratified Cox proportional hazards model, the Mantel-Haenszel method incidence rate ratio and incidence rate difference are presented with their associated $98 \%$ confidence intervals. Detailed analysis results of the primary and secondary composite endpoints are provided in Sections 3.1.6.1 and 3.1.6.2.

Additional subgroup analyses included evaluating the difference between dapagliflozin and comparator event rates based on $\mathrm{CV}$ risk factors and demographics. Additionally, monotherapy and add-on therapy trials were evaluated separately (hazard ratios $(95 \% \mathrm{CI})$ : monotherapy 0.67 $(0.27,1.68)$, add-on: $0.64(0.36,1.15))$. To evaluate the proposed therapeutic dose, the $10 \mathrm{mg}$ dose group was separately evaluated and found to have a hazard ratio of 0.69 (95\% CI: 0.39 , 1.20). Detailed results for the sensitivity analyses and subgroup analyses are provided in Section 4. 
Table 1: Meta-Analysis Results for Primary and Secondary Composite Endpoints (BMS Analysis Population/Sensitivity Analysis Population)

\begin{tabular}{|c|c|c|c|c|}
\hline & \multicolumn{2}{|c|}{$\begin{array}{c}\text { BMS Analysis } \\
\text { Population } \\
\end{array}$} & \multicolumn{2}{|c|}{$\begin{array}{c}\text { Sensitivity Analysis } \\
\text { Population }\end{array}$} \\
\hline & Dapagliflozin & $\begin{array}{l}\text { All } \\
\text { Comparator }\end{array}$ & Dapagliflozin & $\begin{array}{l}\text { All } \\
\text { Comparator }\end{array}$ \\
\hline & \multicolumn{4}{|c|}{ Primary Composite Endpoint } \\
\hline \multicolumn{5}{|l|}{ Primary Analysis } \\
\hline $\begin{array}{l}\text { Stratified } \\
\text { Hazard Ratio } \\
(98 \% \text { CI) }\end{array}$ & \multicolumn{2}{|c|}{$\begin{array}{c}0.67 \\
(0.38,1.18)\end{array}$} & \multicolumn{2}{|c|}{$\begin{array}{c}0.60 \\
(0.32,1.20)\end{array}$} \\
\hline \multicolumn{5}{|c|}{ Secondary Analysis } \\
\hline $\begin{array}{l}\text { M-H Incidence } \\
\text { Rate Ratio } \\
\text { (98\% CI) }\end{array}$ & \multicolumn{2}{|c|}{$\begin{array}{c}0.67 \\
(0.39,1.17)\end{array}$} & \multicolumn{2}{|c|}{$\begin{array}{c}0.59 \\
(0.31,1.11)\end{array}$} \\
\hline $\begin{array}{l}\text { Incidence of } \\
\text { Events } \\
\text { events/person- } \\
\text { years } \\
(\%)\end{array}$ & $\begin{array}{l}48 / 4344 \\
(1.10 \%)\end{array}$ & $\begin{array}{l}30 / 1849 \\
(1.62 \%)\end{array}$ & $\begin{array}{l}39 / 3616 \\
(1.08 \%)\end{array}$ & $\begin{array}{l}22 / 1225 \\
(1.80 \%)\end{array}$ \\
\hline $\begin{array}{l}\text { M-H Incidence } \\
\text { Rate Difference } \\
\text { (98\% CI) }\end{array}$ & \multicolumn{2}{|c|}{$\begin{array}{c}-0.0054 \\
(-0.013,0.0026)\end{array}$} & \multicolumn{2}{|c|}{$\begin{array}{c}-0.0073 \\
(-0.017,0.0023)\end{array}$} \\
\hline & \multicolumn{4}{|c|}{ Secondary Composite Endpoint } \\
\hline $\begin{array}{l}\text { Stratified } \\
\text { Hazard Ratio } \\
\text { (98\% CI) } \\
\end{array}$ & \multicolumn{2}{|c|}{$\begin{array}{c}0.63 \\
(0.38,1.03)\end{array}$} & \multicolumn{2}{|c|}{$\begin{array}{c}0.55 \\
(0.32,0.96)\end{array}$} \\
\hline $\begin{array}{l}\text { M-H Incidence } \\
\text { Rate Ratio } \\
\text { (98\% CI) }\end{array}$ & \multicolumn{2}{|c|}{$\begin{array}{c}0.63 \\
(0.39,1.02)\end{array}$} & \multicolumn{2}{|c|}{$\begin{array}{c}0.55 \\
(0.32,0.95)\end{array}$} \\
\hline $\begin{array}{l}\text { Incidence of } \\
\text { Events } \\
\text { events/person- } \\
\text { years } \\
(\%)\end{array}$ & $\begin{array}{l}59 / 4339 \\
(1.36 \%)\end{array}$ & $\begin{array}{l}39 / 1843 \\
(2.12 \%)\end{array}$ & $\begin{array}{l}48 / 3610 \\
(1.33 \%)\end{array}$ & $\begin{array}{l}29 / 1221 \\
(2.38 \%)\end{array}$ \\
\hline $\begin{array}{l}\text { M-H Incidence } \\
\text { Rate Difference } \\
\text { (98\% CI) }\end{array}$ & \multicolumn{2}{|c|}{$\begin{array}{c}-0.0080 \\
(-0.017,-0.0011)\end{array}$} & \multicolumn{2}{|c|}{$\begin{array}{c}-0.0109 \\
(-0.022,0.00023)\end{array}$} \\
\hline
\end{tabular}

Source: Created by reviewer; reproduction of sponsor analysis in "Cardiovascular events meta-analysis report" Dataset: adcv.xpt 


\section{Introduction}

\subsection{Product Description}

Dapagliflozin is a selective, orally administered inhibitor of the human renal sodium-glucose cotransporter, SGLT2. The proposed indication of dapagliflozin is the treatment of type 2 diabetes mellitus (T2DM) in adults as an adjunct to diet and exercise to improve glycemic control. The proposed therapeutic dose of dapagliflozin is $10 \mathrm{mg}$ for most patients and a $5 \mathrm{mg}$ starting dose for patients at risk for volume depletion due to co-existing conditions or concomitant medications, such as loop diuretics.

\subsection{Clinical Trial Overview}

Bristol-Myers Squibb (BMS) conducted a meta-analysis of the cardiovascular events that occurred in 14 randomized, controlled trials using dapagliflozin ( 2 Phase $2 \mathrm{~b}$ trials, 1 Phase $2 \mathrm{~b} / 3$ trial and 11 Phase 3 trials). The statistical analysis plan used for this meta-analysis was agreed upon by the sponsor and the FDA on November 9, 2010. The summary of design characteristics, the number of subjects per treatment group, and the duration of time included in the CV metaanalysis dataset for these 14 trials is presented in Table 2. Most of the Phase 3 trials consisted of a short-term (ST) double-blind treatment period followed by 1 or 2 site- and subject-blinded or double-blinded long-term (LT) extension periods in which subjects retained the ST assigned treatment.

Of the 14 trials contributing to the meta-analysis, 10 were completed and had their final database lock (DBL): MB102008, MB102009, D1692C00005, MB102014, MB102021, MB102030, MB102032, MB102034, D1690C00005 and MB102013. Four trials completed their ST periods and were unblinded to the Sponsor (sites, investigators and subjects remained blinded), but their LT periods were ongoing: MB102029 (24 week ST + 28 LT completed), D169000004, D169000006, D169000012. These 4 trials each have a data cutoff date to identify adverse events (AEs) for adjudication and exposure information. The cut-off date for Trial MB102029 corresponds to the 'last subject last visit' for the 1-year data, 25-Jul-2010. The cut-off date for the remaining 3 trials was 30-Jul-2010. All events/visits occurring until 30-Jul-2010 were included in the analysis, and sites were allowed to enter data until 4-Aug-2010. Dates and cutoffs were agreed upon with the FDA.

\subsection{Data Sources}

The applicant submitted electronic documents and datasets for trials D1690C00004, D1690C00005, D1690C00006, D1690C00012, D1692C00005, MB102008, MB102009, MB102013, MB102014, MB102021, MB102029, MB102030, MB102032 and MB102034. In addition, the applicant combined the datasets from these trials into a single cardiovascular meta- 
NDA 202-293

analysis dataset (adcv.xpt) which was used for all analyses performed in this review. Clinical study reports (CSRs) of each individual trial were reviewed to evaluate trial protocols.

The following file folder available within the CDER Electronic Document Room (EDR) was utilized in this review:

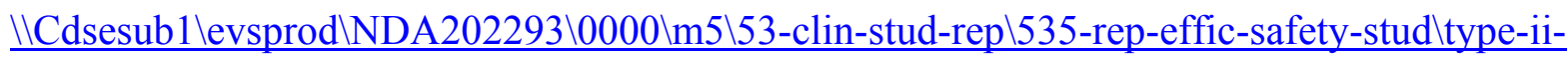
diabetes $\backslash 5353$-rep-analys-data-more-one-stud $\backslash$ meta-analysis 
NDA 202-293

Table 2: List of Trials Included in CV Meta-Analysis

\begin{tabular}{|c|c|c|c|c|c|c|c|c|c|c|c|c|c|c|c|c|}
\hline Trial ID & $\begin{array}{l}\text { Duration } \\
\text { of Trial }\end{array}$ & $\begin{array}{l}\text { Total } \\
\text { Sample }\end{array}$ & $\begin{array}{l}\text { Dap } \\
\text { (N p }\end{array}$ & $\begin{array}{l}\text { gliflo } \\
\text { ar arr }\end{array}$ & in Dc & sage ( & & & Co-tr & atmen & & & $\begin{array}{l}\text { Cont } \\
(\mathbf{N} p\end{array}$ & $\begin{array}{l}\text { ol } \\
\text { arm) }\end{array}$ & & $\begin{array}{l}\text { Data included in } \\
\text { Meta-analysis }\end{array}$ \\
\hline & & & $1 *$ & 2.5 & 5 & 10 & 20 & 50 & Met & Glim & Ins & Pio & Met & Placebo & Glip & \\
\hline $\begin{array}{l}\text { D1690C00004 } \\
\text { (Phase 3) }\end{array}$ & $\begin{array}{l}52+52+ \\
104 \text { weeks }\end{array}$ & 814 & . & . & . & 406 & 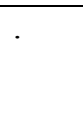 & . & . & . & . & . & . & . & 408 & $\begin{array}{l}52+52+\text { at least } \\
13 \text { weeks (up to } \\
52 \text { weeks) }\end{array}$ \\
\hline $\begin{array}{l}\text { D1690C00005 } \\
\text { (Phase 3) }\end{array}$ & $\begin{array}{l}24+24 \\
\text { weeks }\end{array}$ & 596 & . & 154 & 145 & 151 & . & . & . & $\mathrm{X}$ & . & . & . & 146 & . & Complete \\
\hline $\begin{array}{l}\text { D1690C00006 } \\
\text { (Phase 3) }\end{array}$ & $\begin{array}{l}24+24+ \\
56 \text { weeks }\end{array}$ & 807 & . & 202 & 212 & 196 & . & . & . & & $\mathrm{X}$ & . & . & 197 & . & $\begin{array}{l}24+24+\text { at least } \\
17 \text { weeks (up to } \\
43 \text { weeks) }\end{array}$ \\
\hline $\begin{array}{l}\text { D1690C00012 } \\
\text { (Phase 3) }\end{array}$ & $\begin{array}{l}24+78 \\
\text { weeks }\end{array}$ & 182 & . & . & . & 91 & . & . & $\mathrm{X}$ & . & . & . & . & 91 & . & $\begin{array}{l}24+\text { at least } 13 \\
\text { weeks (up to } 42 \\
\text { weeks) }\end{array}$ \\
\hline $\begin{array}{l}\text { D1692C00005 } \\
\text { (Phase 2b) }\end{array}$ & 12 weeks & 220 & $59 *$ & 56 & 58 & 52 & 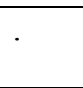 & . & . & . & . & . & . & 54 & . & Complete \\
\hline $\begin{array}{l}\text { MB102008 } \\
\text { (Phase 2b) }\end{array}$ & 12 weeks & 389 & . & 59 & 58 & 47 & 59 & 56 & . & . & . & . & 56 & 54 & . & Complete \\
\hline $\begin{array}{l}\text { MB102009 } \\
\text { (Phase 2b) }\end{array}$ & 12 weeks & 71 & . & . & . & 24 & 24 & . & . & . & $\mathrm{X}$ & . & . & 23 & . & Complete \\
\hline $\begin{array}{l}\text { MB102013 } \\
\text { (Phase 3) }\end{array}$ & $\begin{array}{l}24+78 \\
\text { weeks }\end{array}$ & 485 & . & $\begin{array}{l}65 \\
67 \\
\end{array}$ & $\begin{array}{l}64 \\
68 \\
\end{array}$ & $\begin{array}{l}70 \\
76 \\
\end{array}$ & $\begin{array}{l}\mathrm{AM} \\
\mathrm{PM} \\
\end{array}$ & . & . & . & . & . & . & 75 & . & Complete \\
\hline $\begin{array}{l}\text { MB102014 } \\
\text { (Phase 3) }\end{array}$ & $\begin{array}{l}24+78 \\
\text { weeks }\end{array}$ & 546 & . & 137 & 137 & 135 & . & . & $\mathrm{X}$ & $\cdot$ & . & . & . & 137 & . & Complete \\
\hline $\begin{array}{l}\text { MB102021 } \\
\text { (Phase 3) }\end{array}$ & 24 weeks & 598 & . & & $\begin{array}{l}203 \\
194 \\
\end{array}$ & $\begin{array}{l}\text { dapa } \\
\text { dapa }\end{array}$ & $\begin{array}{l}\text { lone) } \\
\text { met) }\end{array}$ & & $\mathrm{X}^{1}$ & $\cdot$ & . & . & 201 & . & . & Complete \\
\hline $\begin{array}{l}\text { MB102029 } \\
\text { (Phase 2b/3) }\end{array}$ & $\begin{array}{l}24+28+52 \\
\text { weeks }\end{array}$ & 252 & . & . & 83 & 85 & . & . & . & . & . & . & . & 84 & . & $\begin{array}{l}24+28 \text { (complete) } \\
+ \text { up to } 52 \text { weeks }\end{array}$ \\
\hline $\begin{array}{l}\text { MB102030 } \\
\text { (Phase 3) }\end{array}$ & $\begin{array}{l}24+24 \\
\text { weeks }\end{array}$ & 420 & . & . & 141 & 140 & . & . & . & . & . & $\mathrm{X}$ & . & 139 & . & Complete \\
\hline $\begin{array}{l}\text { MB102032 } \\
\text { (Phase 3) }\end{array}$ & 24 weeks & 210 & $72 *$ & 74 & 68 & . & . & . & . & . & . & . & . & 68 & . & Complete \\
\hline $\begin{array}{l}\text { MB102034 } \\
\text { (Phase 3) }\end{array}$ & 24 weeks & 638 & . & & . & $\begin{array}{l}219 \\
211\end{array}$ & $\begin{array}{l}\text { dapa } \\
\text { dapa }+\end{array}$ & $\begin{array}{l}\text { lone) } \\
\text { met) }\end{array}$ & $\mathrm{X}^{1}$ & & . & . & 208 & . & . & Complete \\
\hline
\end{tabular}

* Not included in CV meta-analysis ** Number of subjects included in CV meta-analysis dataset.

${ }^{1}$ Trials MB102021 and MB102034 Have one arm of Dapa +Met, and one arm of Dapa alone

${ }^{2}$ Trial D1690C0004 Has titrated doses of Dapa from 2.5 to $10 \mathrm{mg}$ and Glip 5 to $20 \mathrm{mg}$, Dapa dose is calculated as 10mg in analyses

Source: Created by reviewer. 


\section{Statistical Evaluation}

This review is focused on the meta-analysis of cardiovascular (CV) risk. For a complete statistical evaluation of efficacy results, please refer to the review authored by Dr. Jonathan Norton.

\subsection{Evaluation of Safety}

The review of CV safety comprises data from Trials D1690C00004, D1690C00005, D1690C00006, D1690C00012, D1692C00005, MB102008, MB102009, MB102013, MB102014, MB102021, MB102029, MB102030, MB102032 and MB102034. Based upon interactions with the clinical review team, the following safety review consists of a focused evaluation of $\mathrm{CV}$ endpoints. All comparative analyses are between the randomized treatment groups, dapagliflozin and comparator.

\subsubsection{Trial Designs}

Trial D1690C00004 was a Phase 3, multi-center, randomized, double-blind, active-controlled non-inferiority trial with a 52-week short-term treatment period followed by 2 long-term extension periods, where the sites and subjects remained blinded to treatment, (52 weeks and 104 weeks, respectively) to evaluate the efficacy and safety of dapagliflozin as add-on therapy to metformin compared with glipizide as add-on therapy to metformin in adults with T2DM who have inadequate glycemic control. The 52-week short-term treatment period consisted of a titration period (week 0 to week 18) and a maintenance treatment period (week 19 to week 52). During the titration period, subjects were up-titrated to the optimal effect, or the highest tolerable dose. After completing the 52-week long-term extension period I, subjects still on active study treatment were asked if they would like to continue the study for another 104 weeks. Trial D1690C00004 was conducted at 95 centers in 10 countries, in Europe and South America, in male and female subjects $\geq 18$ years of age. A total of 816 subjects were randomized in a 1:1 fashion to dapagliflozin or glipizide. Trial D1690C00004 was powered to provide $90 \%$ or greater power to demonstrate the non-inferiority of dapagliflozin compared to glipizide with a noninferiority margin of $0.35 \%$ in HbAlc change from baseline to week 52 . Results based on the pre-specified 52 week short-term treatment period up through at least week 65 of the long-term extension period were included in the meta-analysis.

Trial D1690C00005 was a Phase 3, multi-center randomized, double-blind, placebo-controlled superiority trial with a 24 -week short-term treatment period followed by a 24 -week long-term extension period to evaluate the efficacy and safety of dapagliflozin in combination with glimepiride in adults with T2DM who have inadequate glycemic control on glimepiride therapy alone. Trial D1690C00005 was conducted in 100 centers in Europe and Asia, in male and female subjects $\geq 18$ years of age. A total of 597 subjects were randomized in a 1:1:1:1 fashion to the placebo group and $2.5 \mathrm{mg}, 5 \mathrm{mg}$, and $10 \mathrm{mg}$ dosages of dapagliflozin. Trial D1690C00005 was powered to provide $90 \%$ or greater power to demonstrate the superiority of dapagliflozin 
compared to placebo to with a difference of $0.5 \%$ between each dapagliflozin group versus placebo for changes in A1C from baseline to week 24. Both the short-term and long-term periods of this trial were completed and are included in the meta-analysis. Subjects had to be scheduled for a follow-up visit 3 weeks after discontinuation of investigational product.

Trial D1690C00006 was a Phase 3, multi-center randomized, double-blind, placebo-controlled superiority trial with a 24-week short-term treatment period, a 24-week long-term period followed by a second 56-week long term extension period to evaluate the efficacy and safety of dapagliflozin in combination with insulin in adults with T2DM who have inadequate glycemic control on insulin therapy alone. Trial D1690C00006 was conducted in 122 centers in North America and Europe, in male and female subjects $\geq 18$ years of age. A total of 808 subjects were randomized in a 1:1:1:1 fashion to the placebo group and $2.5 \mathrm{mg}, 5 \mathrm{mg}$, and $10 \mathrm{mg}$ dosages of dapagliflozin. Trial D1690C00006 was powered to provide $90 \%$ or greater power to demonstrate the superiority of dapagliflozin compared to placebo with a difference of $0.5 \%$ between each dapagliflozin group versus placebo for changes in A1C from baseline to week 24 . Data from the short-term treatment period and from 42 weeks of the long-term extension period of this study were included in the meta-analysis. Subjects were enrolled to participate in the complete 48-week period (24-week short-term +24 -week long-term extension period 1). After completing the 24-week long-term extension period 1, subjects still on active study treatment (i.e. placebo or dapagliflozin) were asked to continue in the trial for another 56 weeks. When entering long-term extension period 2, subjects on the $5 \mathrm{mg}$ dose of dapagliflozin were switched to the $10 \mathrm{mg}$ dosage. Additionally, the data cut-off for the meta-analysis limited exposure in this study to at least 65 weeks (original 48 week study + at least 17 weeks of LT-extension 2). 70\% of subjects in the safety analysis set continued in the study after completing the long-term extension period 1. Subjects who discontinue have an end-of-treatment visit at latest 7 days after discontinuation and have a follow-up visit 3 weeks after discontinuation of investigational product.

Trial D1690C00012 was a Phase 3, multi-center, randomized, double-blind, placebo-controlled superiority trial with a 24 -week short-term treatment period followed by a 78 -week long term extension period to evaluate the effect of dapagliflozin in combination with metformin on body weight in adults with T2DM who have inadequate glycemic control on metformin alone. Trial D1690C00012 was conducted in 40 centers in Europe, in male and female subjects $\geq 18$ years of age. A total of 182 subjects were randomized in a 1:1 fashion to the placebo group and $10 \mathrm{mg}$ dosage of dapagliflozin. Trial D1690C00006 was powered to provide $90 \%$ or greater power to demonstrate the superiority of dapagliflozin compared to placebo with a mean weight reduction of $2 \mathrm{~kg}$ between dapagliflozin and placebo from baseline to week 24. Data from the short-term period and at least 13 weeks of the on-going LT period were included in the meta-analysis. Subjects who discontinue have an end-of-treatment visit at latest 7 days after discontinuation and have a follow-up visit 3 weeks after discontinuation of investigational product.

Trial D1692C0005 was a Phase 2b, multi-center randomized, double-blind, placebo-controlled superiority trial consisting of a 12 -week treatment duration to evaluate the efficacy and safety of dapagliflozin as monotherapy in Japanese adults with T2DM who have inadequate glycemic control. Trial D1692C00005 was conducted in 26 centers in Japan, in male and female subjects $\geq$ 18 years of age. A total of 279 subjects were randomized in a 1:1:1:1:1 fashion to the placebo 
group, 1, 2.5, 5 or $10 \mathrm{mg}$ dosage of dapagliflozin. Trial D1692C00005 was powered to provide $90 \%$ or greater power to detect a difference in mean change from baseline of $0.54 \%$ for HbA1c between any given dapagliflozin group and placebo. Subjects who discontinue have an end-oftreatment visit at latest 7 days after discontinuation. All randomized subjects entered the 4 -week follow-up period. Subjects in the $1 \mathrm{mg}$ dosage group of dapagliflozin were not included in the meta-analysis as this dosage was considered to be sub-therapeutic.

Trial MB102008 was a Phase 2b, multi-center randomized, double-blind, placebo-controlled superiority 7-arm trial consisting of a12-week treatment duration to evaluate the efficacy and safety of dapagliflozin as monotherapy in adults with T2DM who are treatment naïve and have inadequate glycemic control on diet and exercise. Trial MB102008 was conducted in 133 sites in 4 countries in North America, in male and female subjects $\geq 18$ years of age. A total of 389 subjects were randomized in a 1:1:1:1:1:1:1 fashion to the placebo group, metformin XR $750 / 1500 \mathrm{mg}, 2.5,5,10,20$ or $50 \mathrm{mg}$ dosage of dapagliflozin. Study MB102008 was powered to provide $80 \%$ or greater power to detect a difference in mean difference in A1C of $0.8 \%$ between each dapagliflozin dose and placebo. Subjects who discontinue prior to the Week 12 visit were to be followed in the 4-week observational period unless informed consent was withdrawn, subject was detained, the subject was unable to comply with the protocol, etc.

Trial MB102009 was a Phase 2b trial designed to evaluate the antihyperglycemic effect of two doses of dapagliflozin in subjects with T2DM who were not controlled on combination antihyperglycemic therapy with metformin and/or TZD and SC insulin. The trial consisted of 2 treatment cohorts - only Cohort 2 was included in the meta-analysis. Cohort 2 was comprised of 71 subjects that were randomized 1:1:1 to double-blind treatment with placebo, 10 or $20 \mathrm{mg}$ dosage of dapagliflozin. Study MB102008 was conducted in 26 sites in the United States and Canada, in male and female subjects $\geq 18$ years of age. Trial MB102009 was powered to provide $80 \%$ or greater power to detect a difference in mean difference in $\mathrm{A} 1 \mathrm{C}$ of $0.7 \%$ between each dapagliflozin dose and placebo. Subjects who discontinued study medication prior to the Week 12 visit were to be followed during a 4-week follow-up period.

Trial MB102013 was a Phase 3, multi-center, randomized, double-blind, placebo-controlled superiority trial with a 24 -week short-term treatment period followed by a 78 week long-term extension period to evaluate the effect of dapagliflozin as monotherapy in adults with T2DM who have inadequate glycemic control with diet and exercise. Eligible subjects completing the 24-week short-term double-blind treatment period continued into the 78 week site and subject blinded long-term treatment period. During the LT period, sites and subjects were blinded to treatment assignment, but BMS was not blinded. Trial MB102013 was conducted in 85 sites in North America and Europe in male and female subjects $\geq 18$ years of age. A total of 485 subjects (baseline $\mathrm{HbA} 1 \mathrm{c} \geq 7.0 \%$ and $\leq 10.0 \%$ ) were randomized in a 1:1:1:1:1:1:1 fashion to the placebo group, $2.5 \mathrm{mg}$ QAM, $5 \mathrm{mg}$ QAM, $10 \mathrm{mg}$ QAM, $2.5 \mathrm{mg}$ QPM, $5 \mathrm{mg}$ QPM, or $10 \mathrm{mg}$ QPM dosage of dapagliflozin (QAM= morning dosage, $\mathrm{QPM}=$ evening dosage). Trial MB102013 was powered to provide $90 \%$ or greater power to demonstrate the superiority of dapagliflozin compared to placebo. Data from the short-term treatment period and the long-term period was included in the meta-analysis. Subjects who discontinue have an end-of-treatment visit at latest 7 days after discontinuation and have a follow-up visit 3 weeks after discontinuation of investigational product. 
Trial MB102014 was a Phase 3, multi-center randomized, double-blind, placebo-controlled superiority trial with a 24-week short-term treatment period followed by a 78 week long-term extension period to evaluate the effect of dapagliflozin in combination with metformin in adults with T2DM who have inadequate glycemic control while on metformin alone. Eligible subjects completing the 24-week short-term double-blind treatment period will continue into the 78 week site and subject blinded long-term treatment period. During the LT period, sites and subjects were blinded to treatment assignment, but BMS was not blinded. Trial MB102014 was conducted in 80 sites in North and South America in male and female subjects $\geq 18$ years of age. A total of 546 subjects were randomized in a 1:1:1:1 fashion to the placebo + metformin group, $2.5 \mathrm{mg}, 5 \mathrm{mg}$, or $10 \mathrm{mg}$ dosage of dapagliflozin + metformin group. Study MB102014 was powered to provide $90 \%$ or greater power to demonstrate the superiority of dapagliflozin plus metformin compared to placebo plus metformin. Data from the short-term period and the longterm period was included in the meta-analysis. Subjects who discontinue have an end-oftreatment visit at latest 7 days after discontinuation and have a follow-up visit 3 weeks after discontinuation of investigational product.

Trial MB102021 was a Phase 3, multi-center randomized, double-blind, placebo-controlled superiority study with a 24 -week treatment period to evaluate the effect of dapagliflozin in combination with metformin as initial therapy compared with dapagliflozin monotherapy and metformin monotherapy in adults with T2DM who have inadequate glycemic control. Trial MB102021 was conducted in 105 sites in North America, Asia and Europe in male and female subjects $\geq 18$ years of age. A total of 598 subjects were randomized in a 1:1:1 fashion to the metformin alone, $5 \mathrm{mg}$ dapagliflozin alone, or dapagliflozin $5 \mathrm{mg}+$ metformin group. Trial MB102021 was powered to provide $90 \%$ or greater power to demonstrate the superiority of dapagliflozin plus metformin compared to placebo plus metformin. Subjects who discontinue have an end-of-treatment visit at latest 7 days after discontinuation and have a follow-up visit 3 weeks after discontinuation of investigational product.

Trial MB102029 was a Phase 2/3, multi-center randomized, double-blind, placebo-controlled superiority trial with a 24 -week short-term treatment period followed by a 28 -week long term treatment period and a 52-week long-term extension period (not completed at time of database lock) to evaluate the glycemic efficacy, renal safety, pharmacokinetics, and pharmacodynamics of dapagliflozin in subjects with T2DM and moderate renal impairment who have inadequate glycemic control. Eligible subjects completing the 24-week short-term, double-blind treatment period continued into the site and subject blinded 28 -week long-term treatment period. Eligible subjects completing the 28 -week long-term double-blind treatment period continued into the siteand subject-blinded extension period. Trial MB102029 was conducted in 111 sites in North America, Asia, Australia and Europe in male and female subjects $\geq 18$ years of age. A total of 252 subjects were randomized in a 1:1:1 fashion to the placebo group, $5 \mathrm{mg}$ or $10 \mathrm{mg}$ dapagliflozin dosage group. Trial MB102029 was powered to provide $88 \%$ or greater power to detect a difference of $0.6 \%$ in mean change from baseline in HbA1c between each dapagliflozin treatment group and placebo. All subjects who were discontinued from study medication, continued to complete scheduled visits and selected procedures as follow-up unless they withdrew consent from the trial entirely. During follow-up, subjects continued to complete all trial visits and selected procedures as scheduled through Week 104. 
Trial MB102030 was a Phase 3, multi-center, randomized, double-blind, placebo-controlled superiority study with a 24 -week short-term treatment period followed by a 24 -week long term extension period to evaluate the effect of dapagliflozin in combination with pioglitazone in adults with T2DM who have inadequate glycemic control with pioglitazone alone. Eligible subjects completing the 24-week short-term double-blind treatment period continued into the 24 week site and subject blinded long-term treatment period. During the LT period, sites and subjects were blinded to treatment assignment, but BMS was not blinded. Trial MB102030 was conducted in 105 sites in North and South America and Asia in male and female subjects $\geq 18$ years of age. A total of 420 subjects were randomized in a 1:1:1 fashion to the placebo plus pioglitazone group, $5 \mathrm{mg}$ or $10 \mathrm{mg}$ dosage group of dapagliflozin plus pioglitazone and stratified in 2 groups by their pre-enrollment anti-diabetic therapy. MB102030 was powered to provide $92 \%$ power to detect a difference in means of $0.5 \%$ between each of the dapagliflozin plus pioglitazone treatment groups vs. the placebo plus pioglitazone treatment group. Subjects who discontinue have an end-of-treatment visit at latest 7 days after discontinuation and have a follow-up visit 3 weeks after discontinuation of investigational product.

Trial MB102032 was a Phase 3, multi-center, randomized, double-blind, placebo-controlled superiority trial with a 24-week treatment period to evaluate the effect of dapagliflozin as monotherapy in adults with T2DM who have inadequate glycemic control with diet and exercise. Trial MB102032 was conducted in 68 sites in North America, Asia, Europe and Africa in male and female subjects $\geq 18$ years of age. A total of 280 subjects were randomized in a 1:1:1:1 fashion to the placebo group, $1 \mathrm{mg}, 2.5 \mathrm{mg}$ or $5 \mathrm{mg}$ dosage groups of dapagliflozin. Trial MB102032 was powered to provide $90 \%$ power to detect a difference in means of $0.7 \%$ between each dapagliflozin treatment group (1 mg, $2.5 \mathrm{mg}$ and $5 \mathrm{mg}$ ) and placebo. Subjects who discontinue have an end-of-treatment visit at latest 7 days after discontinuation and have a follow-up visit 4 weeks after discontinuation of investigational product. Subjects in the $1 \mathrm{mg}$ dosage group of dapagliflozin were not included in the meta-analysis as this dosage was considered to be sub-therapeutic.

Trial MB102034 was a Phase 3, multi-center, randomized, double-blind, placebo-controlled superiority trial with a 24-week treatment period to evaluate the effect of dapagliflozin in combination with metformin as initial therapy compared with dapagliflozin monotherapy and metformin monotherapy in adults with T2DM who have inadequate glycemic control. Trial MB102034 was conducted in 131 sites in North America, Asia and Europe in male and female subjects $\geq 18$ years of age. A total of 638 subjects were randomized in a 1:1:1 fashion to the metformin alone, $10 \mathrm{mg}$ dapagliflozin alone, or dapagliflozin $10 \mathrm{mg}+$ metformin group. Trial MB102034 was powered to provide $90 \%$ or greater power to demonstrate the superiority of dapagliflozin plus metformin compared to placebo plus metformin. Subjects who discontinue have an end-of-treatment visit at latest 7 days after discontinuation and have a follow-up visit 3 weeks after discontinuation of investigational product. 


\subsubsection{Endpoints and Adjudication Methods}

\subsubsection{Primary Composite Endpoint}

The primary endpoint is a composite endpoint, defined as the time to first event of the following adjudicated events:

- $\mathrm{CV}$ death

- Myocardial infarction (MI)

- Stroke

- Hospitalization for unstable angina

For the primary ST + LT population, time to event was evaluated in days from the date of first randomized dose to the date of the first event. For subjects without events in AZ studies, censoring took place at the earliest of the following: the date of last contact (e.g. date of last study visit) or date of death. For subjects without events in BMS studies, censoring took place at the earliest of the following: end of treatment +30 days, date of death, date of discontinuation due to lost to follow-up. If end of treatment +30 days occurred after 30-Jul-2010, then 30-Jul2010 was used as censoring date for subjects without events in BMS studies.

\subsubsection{Secondary Composite Endpoint}

The secondary endpoint is a composite endpoint, defined as the time to first event of the following adjudicated events:

- CV death

- Myocardial infarction (MI)

- Stroke

- Hospitalization for unstable angina

- Unplanned coronary revascularization

- Hospitalization for heart failure

Time-to-event/censoring and exposure were calculated using similar methods as described for the primary composite endpoint.

\subsubsection{Adjudication Methods}

Cardiovascular events were adjudicated by an independent Clinical Event Committee (CEC) consisting of two independent cardiologists and two independent neurologists at

For 12 of the trials the adjudication was done prospectively (before final trial database locks). One event in each of the following trials was adjudicated after trial database locks: D1692C00005, MB102034 and MB102021. Two Phase 2 trials, MB102008 and MB102009, were completed when the adjudication process was initiated, and the adjudication of the events 
was done retrospectively. The independent adjudication committee was blinded at all times to treatment allocation.

Potential CV events were identified through an in-stream search of all reported adverse events. Standardized MedDRA queries (SMQs) or custom SMQs for adverse events were used to select the events for adjudication. Once a possible CV event was detected, the sponsor contacted the sites and requested the principal investigators to fill in the appropriate case submission report for the respective event which was then sent to the CEC. Additional events could also have been identified by the CEC while reviewing the supporting documentation.

\subsubsection{Statistical Methodologies}

The following sections detail the statistical methods used in the evaluation of the primary and secondary analyses of the meta-analysis and in the sensitivity analyses included in the review.

\subsubsection{Group Sequential Meta-analysis}

The meta-analysis conducted by the sponsor was the first part of an agreed upon two-part group sequential testing strategy (Whitehead, 1997) ${ }^{3}$ that was pre-specified to control the Type I error rate for demonstration of non-inferiority with respect to the 1.8 relative risk criterion at an overall one-sided 0.025 level as outlined in the FDA CV guidance. ${ }^{1}$

As agreed upon with the FDA, if the first formal meta-analysis of the primary composite endpoint was found to have an upper-bound less than 1.8 at a one-sided alpha level of 0.01 (corresponding to a $98 \% \mathrm{CI}$ ) then the meta-analysis would meet the guidelines, and data from additional studies that have yet to be completed would not need to be included in a second metaanalysis. However, if unsuccessful at this alpha level (0.01), a second meta-analysis would be performed when studies D1690C00018 and D1690C00019 had unblinded and at least 110 patients had adjudicated events for the primary endpoint. The one-sided alpha level for this final analysis was set at 0.01 so that the overall Type I error rate across the 2 analyses was $\leq 0.025$ (one-sided).

Because a one-sided alpha-level of 0.01 is being used to evaluate this meta-analysis, $98 \%$ confidence intervals will be reported for all analyses relating to the primary and secondary objectives of the study. All sensitivity and sub-group analyses that are exploratory in nature will be reported with an associated $95 \% \mathrm{CI}$.

\subsubsection{Time to Event Analysis}

The agreed upon primary analysis method was to analyze the time to occurrence of CV-related events among all dapagliflozin doses $(\geq 2.5 \mathrm{mg})$ pooled versus all comparator groups pooled using a Cox proportional hazards model stratified by trial and including a dichotomous term for treatment group. To be included in this analysis, a trial had to have at least 1 adjudicated event 
to contribute to the analysis. The proportional hazards assumption was assessed by plotting the log-log survival curve of the data and the Schoenfeld residuals.

\subsubsection{Analysis of Event Incidence}

Agreed upon secondary analyses included the use of Mantel-Haenszel methods estimating the overall incidence rate ratio and incidence rate difference via Breslow and Day $(1994)^{2}$ with asymptotic confidence intervals provided using the asymptotic methods described by Robins et al. (1986). ${ }^{4}$ The incidence rate ratio was calculated by stratifying by trial where trials with no events were excluded from the analysis. The incidence rate difference included all trials in the analysis.

\subsubsection{Evaluation of Event Rates}

In addition to the methods used by BMS in their primary and secondary analyses, sensitivity analyses conducted by the FDA included the calculation of the risk difference for the primary and secondary composite endpoints. The calculation of the Mantel-Haenszel method risk difference was included to evaluate the impact of using the denominator of number of subjects per treatment arm (as opposed to number of patient-years of exposure used in the incidence rate difference calculation) on the effect estimates.

\subsubsection{Handling of Trials with Zero Events}

The difference of incidence rates and the difference of events rates were analyzed using MantelHaenszel methods to provide analyses that were able to include trials that did not have an adjudicated event. The Cox proportional hazards model and incidence rate ratio exclude trials with zero events.

\subsubsection{Evaluation of Heterogeneity between Trials}

To determine if the event rates in the trials included in the meta-analysis were different from one another, the heterogeneity between the studies was assessed using the Q-statistic to determine in the studies were significantly different from one another and the $\mathrm{I}^{2}$ statistic to determine the amount of heterogeneity present in the studies (Higgins and Thompson, 2002). ${ }^{5}$

\subsubsection{Populations}

The main analysis population consists of the randomized, controlled, ST + LT periods of all Phase $2 \mathrm{~b}$ and 3 trials of dapagliflozin that have been unblinded by the sponsor at the time of the $\mathrm{CV}$ events meta-analysis. The ST + LT data include data following initiation of any rescue therapy. Any dapagliflozin treatment group that does not have a corresponding control group was 
excluded. All analyses were performed on all subjects who received at least one dose of doubleblind study medication during the ST double-blind treatment period (safety population).

The BMS Analysis Population is defined to include all subjects in the 14 trials listed in Table 2. The $1 \mathrm{mg}$ dapagliflozin dosage group was considered sub-therapeutic and was not included in any summary of the pooled dapagliflozin group. The BMS Analysis Population included 6228 subjects: 4287 in the dapagliflozin group and 1941 in the all comparator group (693 metformin, 408 glipizide, 335 placebo, 220 insulin, 146 glimepiride, and 139 pioglitazone).

To further evaluate the sensitivity of the BMS analysis, the FDA conducted an analysis on a subset of the BMS Analysis Population. The Sensitivity Analysis Population is defined to exclude the short-term, 12-week trials included in the BMS Analysis Population (trials MB102008, MB102009 and D1692C00005) and also excluded the non-inferiority trial, D1690C00004. The Sensitivity Analysis Population had 4734 subjects: 3388 in the dapagliflozin group and 1346 in the all comparator group (637 metformin, 227 placebo, 197 insulin, 146 glimepiride, and 139 pioglitazone). 


\subsubsection{Subject Disposition, Demographics and Baseline Characteristics}

As shown in Table 3, in the BMS Analysis population, baseline demographics were similar among the treatment groups. There were slightly more male subjects than female subjects (51.3\% versus $48.7 \%)$. Approximately $86.4 \%$ of subjects were White, about $9.9 \%$ were Asian and $3.7 \%$ of subjects were Black or African-American. More than $70 \%$ of subjects in the BMS Analysis population were above 50 years of age, while the mean age was about 56 . More than $57 \%$ of subjects had body mass index (BMI) greater than $30 \mathrm{~kg} / \mathrm{m}^{2}$, while the mean BMI was about $31 \mathrm{~kg} / \mathrm{m}^{2}$.

Table 3: Demographics

\begin{tabular}{lll}
\hline & Dapagliflozin & $\begin{array}{l}\text { Combined } \\
\text { Comparator } \\
(N=1941)\end{array}$ \\
\hline Percent Female & $47.50 \%$ & $52.50 \%$ \\
\hline Age+ SD (years) & $55.9 . \pm 10.5$ & $56.5 \pm 10.5$ \\
\hline$\leq 50$ years & $28.1 \%$ & $28.9 \%$ \\
$50-65$ years & $50.6 \%$ & $48.4 \%$ \\
$\geq 65$ years & $20.5 \%$ & $23.5 \%$ \\
\hline BMI+ SD (kg/m $\left.{ }^{2}\right)$ & $31.5 \pm 5.5$ & $31.5 \pm 5.5$ \\
\hline $25-30$ & $30.9 \%$ & $32.4 \%$ \\
$\geq 30$ & $58.7 \%$ & $57.0 \%$ \\
\hline Race and Ethnicity & & \\
\hline White & $81.0 \%$ & $81.2 \%$ \\
Black & $3.4 \%$ & $3.5 \%$ \\
Asian & $9.1 \%$ & $9.7 \%$ \\
\hline Region & & \\
\hline North America & $31.6 \%$ & $27.0 \%$ \\
Latin America & $21.4 \%$ & $21.2 \%$ \\
Europe & $35.7 \%$ & $41.9 \%$ \\
Asia/Pacific & $11.3 \%$ & $9.8 \%$ \\
\hline
\end{tabular}

Source: Created by reviewer. Dataset: adcv.xpt 
Baseline CV risk factors and complications are presented in Table 4. In the BMS Analysis population, approximately $19.4 \%$ had cardiovascular disease (CVD) at baseline. Furthermore, more than $62 \%$ of subjects were hypertensive at baseline and more than $25 \%$ were current or exsmoker. Additionally, most subjects had some degree of renal impairment. Table 11 in the Appendix presents the CV risk factors for each trial. In general, the rate of occurrence of CV risk factors was consistent across trials. However, in trial MB102029, the renal impairment study, a higher percentage of subjects had a history of CVD and nearly $90 \%$ of all subjects had a history of hypertension. Additionally, in trial D1692C00005, the trial in a Japanese population, there was a lower history of CVD (around 3\%) and a much lower rate of renal impairment (nearly $90 \%$ of all subjects had normal renal function).

Table 4: CV Risk Factors

\begin{tabular}{|c|c|c|}
\hline & \multicolumn{2}{|c|}{ BMS Analysis Population } \\
\hline & $\begin{array}{l}\text { Dapagliflozin } \\
(N=4287)\end{array}$ & $\begin{array}{l}\text { Combined } \\
\text { Comparator } \\
(N=1941)\end{array}$ \\
\hline History of CVD & $19.6 \%$ & $18.2 \%$ \\
\hline $\begin{array}{l}\text { History of } \\
\text { Hypertension }\end{array}$ & $61.5 \%$ & $64.3 \%$ \\
\hline $\begin{array}{l}\text { History of } \\
\text { Dyslipidemia }\end{array}$ & $49.4 \%$ & $48.8 \%$ \\
\hline $\begin{array}{l}\text { History of Congestive } \\
\text { Heart Failure }\end{array}$ & $2.2 \%$ & $1.7 \%$ \\
\hline \multicolumn{3}{|l|}{ Renal Function } \\
\hline Severely Impaired & $0.2 \%$ & $0.3 \%$ \\
\hline Moderately Impaired & $11.1 \%$ & $10.7 \%$ \\
\hline Mildly Impaired & $51.1 \%$ & $50.3 \%$ \\
\hline Normal & $37.6 \%$ & $38.8 \%$ \\
\hline \multicolumn{3}{|l|}{ Smoking History } \\
\hline Never smoked & $49.4 \%$ & $53.3 \%$ \\
\hline Current Smoker & $24.1 \%$ & $21.3 \%$ \\
\hline Former Smoker & $3.2 \%$ & $1.4 \%$ \\
\hline \multicolumn{3}{|l|}{ Diabetes Duration } \\
\hline less than 3 years & $44.7 \%$ & $44.7 \%$ \\
\hline $3-10$ years & $33.1 \%$ & $34.2 \%$ \\
\hline more than 10 years & $22.2 \%$ & $21.1 \%$ \\
\hline
\end{tabular}


Among the 6,228 subjects in the BMS Analysis Population from the 14 randomized phase $2 \mathrm{~b} / 3$ trials, approximately $25 \%(1545 / 6228)$ prematurely discontinued study medication. As presented in Figure 1, the percentage of subjects with premature discontinuation of study medication tended to be lower in the dapagliflozin treatment groups than in the comparator groups. On average, $23 \%$ of the subjects in the dapagliflozin group discontinued study medication, while $29 \%$ of the subjects in the combined comparator groups prematurely discontinued the study medication.

Figure 1: Percentage of Subjects with Premature Discontinuation of Study Medication by Trial and Treatment Group (BMS Analysis Population)

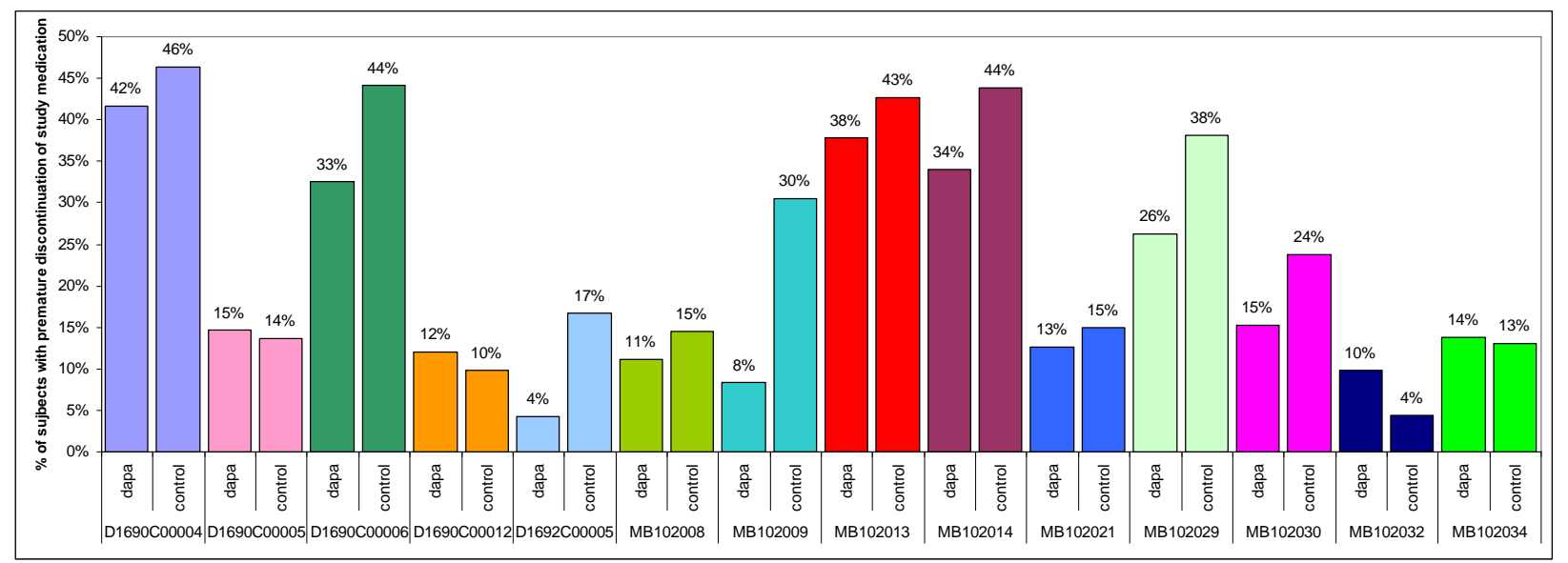

Table 5 presents the discontinuation rates of each trial by reason for discontinuation. In general, it appears that discontinuation rates are fairly consistent between treatment groups for each discontinuation reason in each trial.

Reviewer's note: As many of the trials included in the meta-analysis included both short-term and long-term study periods, the rate of discontinuation during the course of each trial was evaluated using Kaplan-Meier curves (included in the Appendix). While the discontinuation rates appear to be consistent in most of the trials, there were two trials (D1690C00004 and D1690C00006) where consent was requested from subjects a second time for inclusion in an additional extension period in the trial. In these two trials there are increased discontinuation rates at the time of re-consent. To determine if this increased rate of discontinuation had any impact on the evaluation of the primary endpoint, the number of events that occurred after this point in the trials was evaluated: In study D1690C00004, no events occurred in either arm after this point, in study D1690C00006, 5 events occurred in the dapgliflozin arm and 1 event occurred in the comparator. Exclusion of these events from the analysis would result in a less conservative estimate of the event rates, therefore these data remained in the analysis. 
Table 5: Trial Discontinuation Rates by Reason

\begin{tabular}{|c|c|c|c|c|c|c|c|}
\hline Trial & Arms & $\begin{array}{l}\text { Sample } \\
\text { Size }\end{array}$ & $\begin{array}{l}\text { Prematurely } \\
\text { Discontinued } \\
\text { Study } \\
\text { Medicine }\end{array}$ & $\begin{array}{l}\text { Adverse } \\
\text { Event }\end{array}$ & $\begin{array}{l}\text { Subject } \\
\text { Withdrew } \\
\text { Consent }\end{array}$ & $\begin{array}{l}\text { Lack of } \\
\text { Efficacy }\end{array}$ & Other \\
\hline \multirow[t]{2}{*}{ D1690C00004 } & dapagliflozin & 406 & $169(41.6)$ & $39(9.6)$ & $39(9.6)$ & $0(0.0)$ & $91(22.4)$ \\
\hline & glipizide & 408 & $189(46.3)$ & $28(6.9)$ & $42(10.2)$ & $0(0.0)$ & $119(29.2)$ \\
\hline \multirow[t]{2}{*}{ D1690C00005 } & dapagliflozin/glimepiride & 450 & $46(10.2)$ & $12(2.7)$ & $27(6.0)$ & $0(0.0)$ & $7(1.5)$ \\
\hline & placebo/glimepiride & 146 & $20(13.7)$ & $5(3.4)$ & $8(5.5)$ & $0(0.0)$ & $7(4.8)$ \\
\hline \multirow[t]{2}{*}{ D1690C00006 } & dapagliflozin/insulin & 610 & $199(32.6)$ & $37(6.1)$ & $55(9.0)$ & $0(0.0)$ & $107(17.5)$ \\
\hline & placebo/insulin & 197 & $87(44.2)$ & $13(6.6)$ & $23(11.7)$ & $0(0.0)$ & $51(25.9)$ \\
\hline \multirow[t]{2}{*}{ D1690C00012 } & dapagliflozin/metformin & 91 & $11(12.1)$ & $3(3.3)$ & $5(5.5)$ & $0(0.0)$ & $3(3.3)$ \\
\hline & placebo/metformin & 91 & $9(9.9)$ & $0(0.0)$ & $1(1.1)$ & $0(0.0)$ & $8(8.8)$ \\
\hline \multirow[t]{2}{*}{ D1692C00005 } & dapagliflozin & 166 & $7(4.2)$ & $2(1.2)$ & $3(1.8)$ & $0(0.0)$ & $2(1.2)$ \\
\hline & placebo & 54 & $9(16.7)$ & $0(0.0)$ & $1(1.9)$ & $0(0.0)$ & $8(14.8)$ \\
\hline \multirow[t]{3}{*}{ MB102008 } & dapagliflozin & 279 & $31(11.1)$ & $7(2.5)$ & $10(3.6)$ & $0(0.0)$ & $14(5.0)$ \\
\hline & metformin & 56 & $6(10.7)$ & $1(1.8)$ & $1(1.8)$ & $0(0.0)$ & $4(7.1)$ \\
\hline & placebo & 54 & $10(18.5)$ & $1(1.8)$ & $4(7.4)$ & $3(5.6)$ & $2(3.7)$ \\
\hline \multirow[t]{2}{*}{ MB102009 } & dapagliflozin/insulin & 48 & $4(8.3)$ & $2(4.2)$ & $0(0.0)$ & $0(0.0)$ & $2(4.2)$ \\
\hline & placebo/insulin & 23 & $7(30.4)$ & $1(4.3)$ & $2(8.7)$ & $2(8.7)$ & $2(8.7)$ \\
\hline \multirow[t]{2}{*}{ MB102013 } & dapagliflozin & 410 & $155(37.8)$ & $27(6.6)$ & $49(12.0)$ & $30(7.3)$ & $49(12.0)$ \\
\hline & placebo & 75 & $32(42.7)$ & $4(5.3)$ & $7(9.3)$ & $7(9.3)$ & $25(33.3)$ \\
\hline \multirow[t]{2}{*}{ MB102014 } & dapagliflozin/metformin & 409 & $139(34.0)$ & $17(4.2)$ & $29(7.0)$ & $42(10.3)$ & $51(12.5)$ \\
\hline & placebo/metformin & 137 & $60(43.8)$ & $9(6.6)$ & $10(7.3)$ & $29(21.2)$ & $12(8.7)$ \\
\hline \multirow[t]{3}{*}{ MB102021 } & dapagliflozin & 203 & $33(16.2)$ & $6(2.9)$ & $15(7.4)$ & $1(0.03)$ & $12(5.9)$ \\
\hline & dapagliflozin/metformin & 194 & $17(8.7)$ & $2(1.0)$ & $8(4.1)$ & $0(0.0)$ & $7(3.6)$ \\
\hline & placebo/metformin & 201 & $30(14.9)$ & $6(3.0)$ & $9(4.5)$ & $0(0.0)$ & $15(7.4)$ \\
\hline \multirow[t]{2}{*}{ MB102029 } & dapagliflozin & 168 & $44(26.2)$ & $23(13.7)$ & $9(5.4)$ & $1(0.6)$ & $11(6.5)$ \\
\hline & placebo & 84 & $32(38.1)$ & $19(22.6)$ & $5(6.0)$ & $2(2.4)$ & $6(7.1)$ \\
\hline \multirow[t]{2}{*}{ MB102030 } & dapagliflozin/pioglitazone & 281 & $43(15.3)$ & $8(2.8)$ & $19(6.8)$ & $3(1.1)$ & $13(4.6)$ \\
\hline & placebo/pioglitazone & 139 & $33(23.7)$ & $5(3.6)$ & $10(7.2)$ & $5(3.6)$ & $13(9.3)$ \\
\hline \multirow[t]{2}{*}{ MB102032 } & dapagliflozin & 142 & $14(9.9)$ & $1(0.7)$ & $7(4.9)$ & $0(0.0)$ & $6(4.2)$ \\
\hline & placebo & 68 & $3(4.4)$ & $0(0.0)$ & $2(2.9)$ & $1(1.5)$ & $0(0.0)$ \\
\hline \multirow[t]{3}{*}{ MB102034 } & dapagliflozin & 219 & $31(14.0)$ & $9(4.2)$ & $8(3.6)$ & $1(0.2)$ & $13(6.0)$ \\
\hline & dapagliflozin/metformin & 211 & $28(13.3)$ & $4(1.9)$ & $11(5.2)$ & $0(0.0)$ & $13(6.2)$ \\
\hline & placebo/metformin & 208 & $27(13.0)$ & $8(3.8)$ & $11(5.3)$ & $1(0.5)$ & $7(3.4)$ \\
\hline
\end{tabular}




\subsubsection{Results and Conclusions}

\subsubsection{Primary Composite Endpoint}

To ensure that the Cox proportional hazards method used by BMS was justified, the proportional hazards assumptions of the data were evaluated in the BMS Analysis population by investigating the plots of the log-log survival curve and the Schoenfeld residuals of the data with regards to the primary composite endpoint. Both plots, presented in the Appendix, show that the proportional hazards assumption does hold for this data as the log-log curves for dapagliflozin and comparator do not cross and the curve resulting from the plotting of the Schoenfeld residuals does approximately lie on $\mathrm{y}=0$.

The meta-analysis results for the primary composite endpoint in both the BMS Analysis population and the Sensitivity Analysis population are presented in Table 6. The results in both sets of analyses were comparable, with the results in the BMS Analysis population appearing to be slightly more conservative than those in the Sensitivity Analysis population. Both sets of analyses found the incidence of the primary composite endpoint to be lower in the dapagliflozin group compared to the comparator group.

Table 6: Meta-Analysis Results for Primary Composite Endpoint (BMS Analysis Population/Sensitvity Analysis Population)

\begin{tabular}{|c|c|c|c|c|}
\hline & \multicolumn{2}{|c|}{$\begin{array}{l}\text { BMS Analysis } \\
\text { Population }\end{array}$} & \multicolumn{2}{|c|}{$\begin{array}{c}\text { Sensitivity Analysis } \\
\text { Population }\end{array}$} \\
\hline & Dapagliflozin & $\begin{array}{l}\text { All } \\
\text { Comparator }\end{array}$ & Dapagliflozin & $\begin{array}{l}\text { All } \\
\text { Comparator }\end{array}$ \\
\hline \multicolumn{5}{|l|}{ Primary Analysis } \\
\hline $\begin{array}{l}\text { Stratified Hazard } \\
\text { Ratio } \\
\text { (98\% CI) }\end{array}$ & \multicolumn{2}{|c|}{$\begin{array}{c}0.67 \\
(0.38,1.18)\end{array}$} & \multicolumn{2}{|c|}{$\begin{array}{c}0.60 \\
(0.32,1.20)\end{array}$} \\
\hline \multicolumn{5}{|l|}{ Secondary Analysis } \\
\hline $\begin{array}{l}\text { M-H Incidence } \\
\text { Rate Ratio } \\
\text { (98\% CI) }\end{array}$ & \multicolumn{2}{|c|}{$\begin{array}{c}0.67 \\
(0.39,1.17)\end{array}$} & \multicolumn{2}{|c|}{$\begin{array}{c}0.59 \\
(0.31,1.11)\end{array}$} \\
\hline $\begin{array}{l}\text { Incidence of } \\
\text { Events } \\
\text { events/person- } \\
\text { years } \\
\text { (\%) }\end{array}$ & $\begin{array}{l}48 / 4344 \\
(1.10 \%)\end{array}$ & $\begin{array}{l}30 / 1849 \\
(1.62 \%)\end{array}$ & $\begin{array}{l}39 / 3616 \\
(1.08 \%)\end{array}$ & $\begin{array}{l}22 / 1225 \\
(1.80 \%)\end{array}$ \\
\hline $\begin{array}{l}\text { M-H Incidence } \\
\text { Rate Difference } \\
(98 \% \text { CI) }\end{array}$ & \multicolumn{2}{|c|}{$\begin{array}{c}-0.0054 \\
(-0.013,0.0026)\end{array}$} & \multicolumn{2}{|c|}{$\begin{array}{c}-0.0073 \\
(-0.017,0.0023)\end{array}$} \\
\hline
\end{tabular}

Source: Created by reviewer; reproduction of sponsor analysis in "Cardiovascular events meta-analysis report". Dataset: adcv.xpt 
Based on the Kaplan-Meier method using the BMS Analysis population, the cumulative probability of developing a CV-related event as measured by the primary composite endpoint is shown in Figure 2. In the combined comparator group, events of the composite CV safety endpoint occurred earlier than in the dapagliflozin group. Based on the stratified log-rank test stratified by trial, the onset time of event was not statistically significantly different at the onesided alpha $=0.01$ level between the linagliptin group and the comparator group $(p=0.098)$.

\section{Figure 2: Time-to-event Analysis of the Primary Composite Endpoint (BMS Analysis} Population)

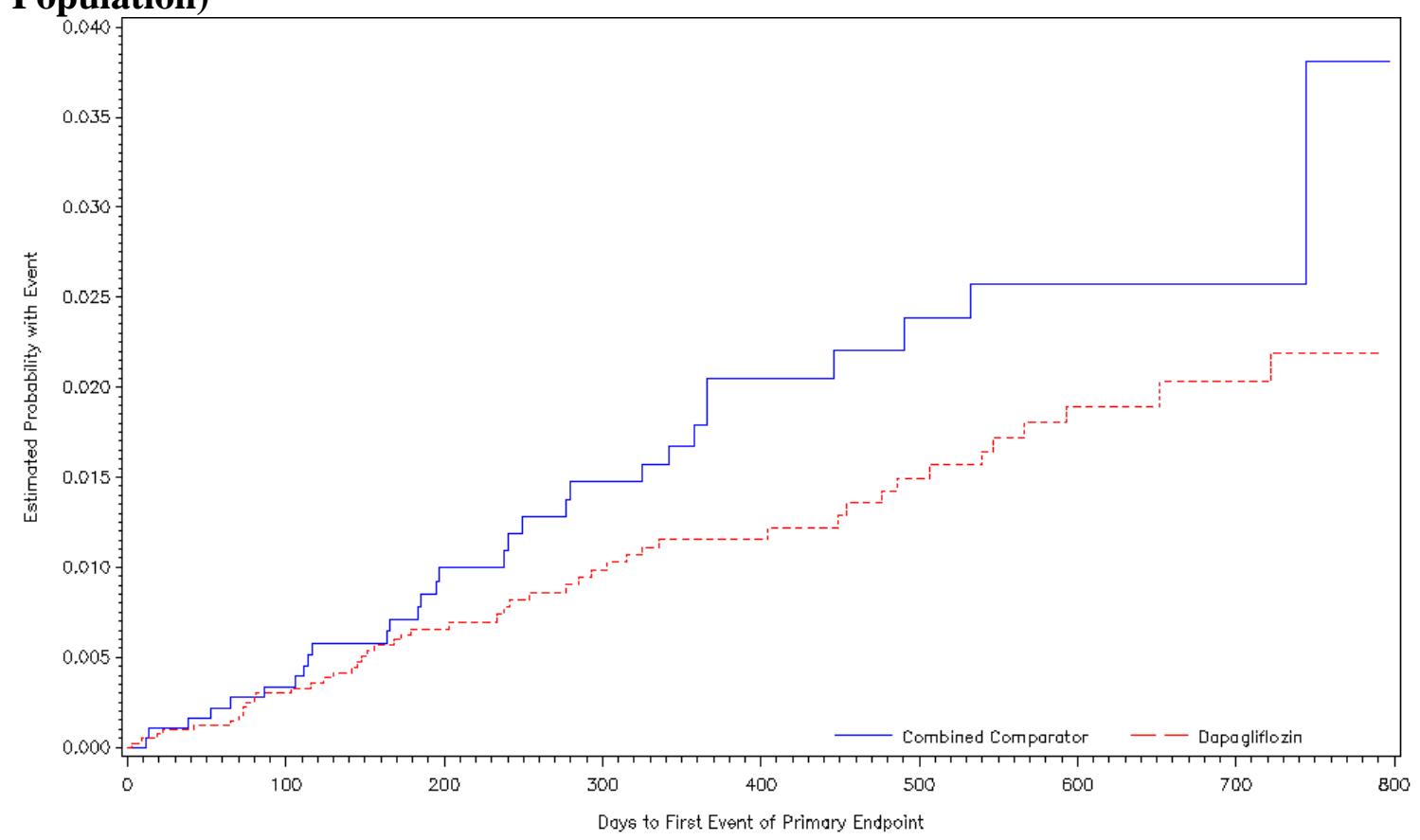

Source: Created by reviewer; reproduction of sponsor analysis in "Cardiovascular events meta-analysis report". Dataset: adcv.xpt 
The forest plot of the hazard ratios for all trials included in the BMS Analysis population that had at least one subject who experienced a composite primary endpoint event is presented in Figure 3 (note that no individual trial CI's are presented in trials with zero events in the forest plot). The trials that used dapagliflozin as an add-on therapy are denoted in red, the combination trials are denoted in blue and the monotherapy trials are in black. The overall hazard ratio for all trials, stratified by trial, is presented on the bottom of the plot.

\section{Figure 3: Forest Plot of Hazard Ratios and 95\% CI of Individual Trials from Cox Proportional Hazards for Primary CV Composite Endpoint and 98\% CI for Overall Stratified Analysis (BMS Analysis Population)}

\begin{tabular}{|c|c|c|c|c|c|c|}
\hline Trial & Type of Therapy & $\begin{array}{l}\text { Dapagliflozin } \\
\text { CV events/N (\%) }\end{array}$ & $\begin{array}{l}\text { Comparator } \\
\text { CV events/N (\%) }\end{array}$ & Comparator Worse & Dapagliflozin Worse & Hazard Ratio (Cl) \\
\hline D1690C00004 & Add-on to Metformin (Glipizide) & $7 / 406(1.72)$ & $8 / 408(1.96)$ & & & $0.868(0.315,2.39)$ \\
\hline D1690C00005 & Add-on to SU & $6 / 450(1.33)$ & $1 / 146(0.68)$ & & 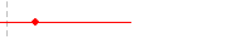 & $1.929(0.232,16.019)$ \\
\hline D1690C00006 & Add-on to Insulin & $11 / 610(1.80)$ & $5 / 197(2.54)$ & & & $0.645(0.224,1.86)$ \\
\hline D1690C00012* & Add-on to Metformin & $0 / 91(0.00)$ & $1 / 91(1.10)$ & & & - \\
\hline D1692C00005* & Monotherapy & $1 / 166(0.60)$ & $0 / 54(0.00)$ & & & - \\
\hline MB102008* & Monotherapy & $1 / 279(0.36)$ & $0 / 110(0.00)$ & & & - \\
\hline MB102013* & Monotherapy & $6 / 410(1.46)$ & $0 / 75(0.00)$ & & & - \\
\hline MB102014 & Add-on to Metformin & $5 / 409(1.22)$ & $6 / 137(4.38)$ & & & $0.253(0.077,0.828)$ \\
\hline MB102021 & Combination with Metformin & $2 / 397(0.50)$ & $1 / 201(0.50)$ & & & $1.011(0.092,11.157)$ \\
\hline MB102029 & Monotherapy (renal impairment) & $6 / 168(3.57)$ & $7 / 84(8.33)$ & & & $0.367(0.123,1.094)$ \\
\hline MB102030* & Add-on to TZD & $1 / 281(0.36)$ & $0 / 139(0.00)$ & & & - \\
\hline \multirow[t]{3}{*}{ MB102034 } & Combination with Metformin & $2 / 430(0.47)$ & $1 / 208(0.48)$ & & & $0.979(0.089,10.807)$ \\
\hline & Hazard Ratio (Pooled) & & & & & $0.67(0.38,1.18)$ \\
\hline & & & & 1 & $\sqrt{100}$ & \\
\hline
\end{tabular}

* estimate values are out of range

Source: Created by reviewer; reproduction of sponsor analysis in "Cardiovascular events meta-analysis report". Dataset: adcv.xpt 
To explore which cardiovascular event components contributed to the composite primary endpoint, the Mantel-Haenszel incidence rate differences for each component of the composite CV safety endpoint were calculated using the pooled BMS Analysis Population. Figure 4 presents a forest plot of these risk differences in reference to the risk difference for the composite primary endpoint.

Figure 4: Forest Plot of Mantel-Haenszel Incidence Rate Differences of Cardiovascular Events Contributing to Primary Composite Endpoint (BMS Analysis Population)

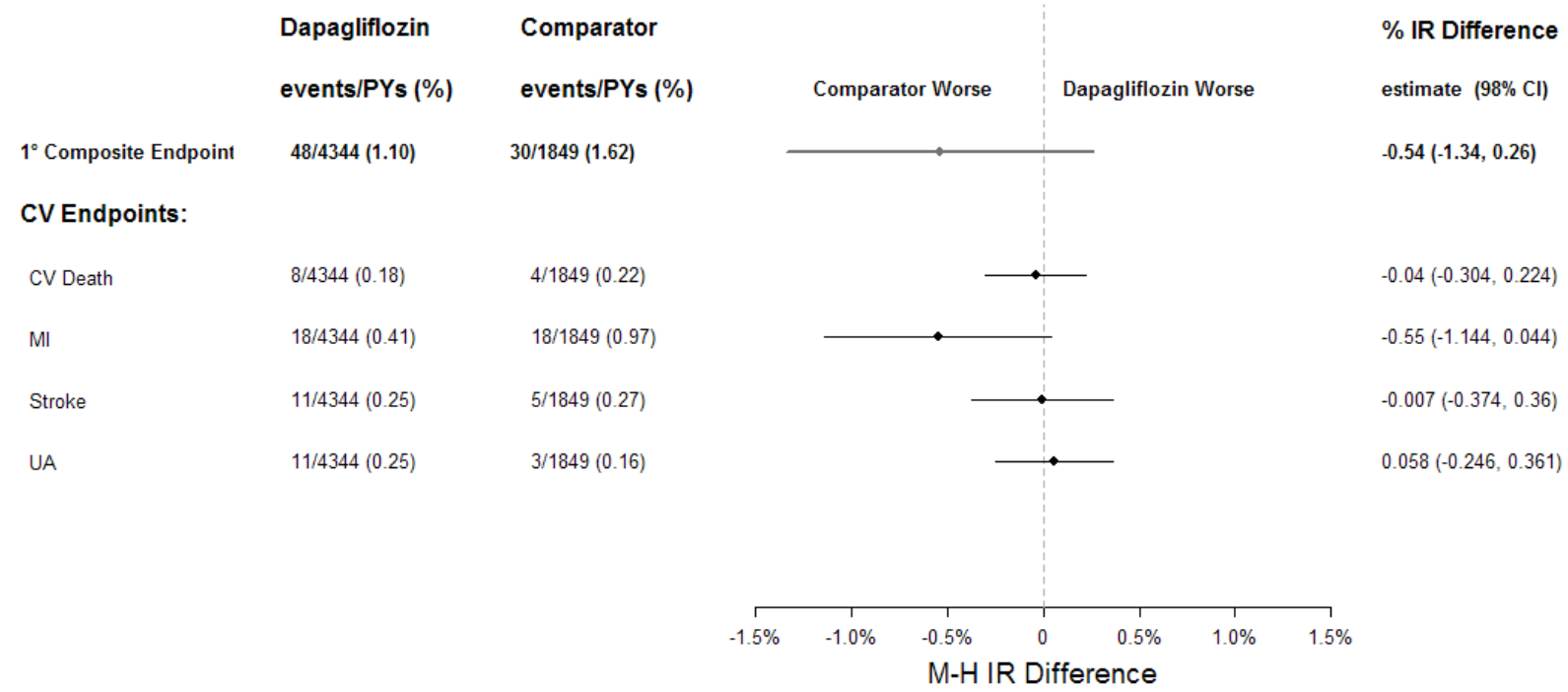

Source: Created by reviewer. Dataset: adcv.xpt 


\subsubsection{Secondary Composite Endpoint}

Recall that the secondary composite endpoint included two additional terms to the composite versus the primary composite endpoint: unplanned coronary revascularization and hospitalization for heart failure. There were 20 additional events in the BMS Analysis Population due to the addition of these terms, 11 in the dapagliflozin group and 9 in the comparator group. The metaanalysis results for the secondary composite endpoint in both the BMS Analysis population and the Sensitivity Analysis population are presented in Table 7. The results in both sets of analyses were again found to be comparable, with the results in the BMS Analysis population appearing to be slightly more conservative than those in the Sensitivity Analysis population.

Table 7: Meta-Analysis Results for Secondary Composite Endpoint (BMS Analysis Population/Sensitvity Analysis Population)

\begin{tabular}{|c|c|c|c|c|}
\hline & \multicolumn{2}{|c|}{$\begin{array}{l}\text { BMS Analysis } \\
\text { Population }\end{array}$} & \multicolumn{2}{|c|}{$\begin{array}{c}\text { Sensitivity Analysis } \\
\text { Population }\end{array}$} \\
\hline & Dapagliflozin & $\begin{array}{l}\text { All } \\
\text { Comparator }\end{array}$ & Dapagliflozin & $\begin{array}{l}\text { All } \\
\text { Comparator }\end{array}$ \\
\hline $\begin{array}{l}\text { Stratified } \\
\text { Hazard Ratio } \\
(\mathbf{9 8 \%} \text { CI) }\end{array}$ & \multicolumn{2}{|c|}{$\begin{array}{c}0.63 \\
(0.38,1.03)\end{array}$} & \multicolumn{2}{|c|}{$\begin{array}{c}0.55 \\
(0.32,0.96)\end{array}$} \\
\hline $\begin{array}{l}\text { M-H Incidence } \\
\text { Rate Ratio } \\
\text { (98\% CI) } \\
\end{array}$ & \multicolumn{2}{|c|}{$\begin{array}{c}0.63 \\
(0.39,1.02)\end{array}$} & \multicolumn{2}{|c|}{$\begin{array}{c}0.55 \\
(0.32,0.95)\end{array}$} \\
\hline $\begin{array}{l}\text { Incidence of } \\
\text { Events } \\
\text { events/person- } \\
\text { years } \\
(\%)\end{array}$ & $\begin{array}{l}59 / 4339 \\
(1.36 \%)\end{array}$ & $\begin{array}{l}39 / 1843 \\
(2.12 \%)\end{array}$ & $\begin{array}{l}48 / 3610 \\
(1.33 \%)\end{array}$ & $\begin{array}{l}29 / 1221 \\
(2.38 \%)\end{array}$ \\
\hline $\begin{array}{l}\text { M-H Incidence } \\
\text { Rate Difference } \\
(98 \% \text { CI) }\end{array}$ & \multicolumn{2}{|c|}{$\begin{array}{c}-0.0080 \\
(-0.017,-0.0011)\end{array}$} & \multicolumn{2}{|c|}{$\begin{array}{c}-0.0109 \\
(-0.022,0.00023)\end{array}$} \\
\hline
\end{tabular}

Source: Created by reviewer; reproduction of sponsor analysis in "Cardiovascular events meta-analysis report".

Dataset: adcv.xpt 


\subsubsection{Risk Difference}

To determine if using the number of subjects for each treatment as a denominator instead of the number of person-years of exposure, has an impact on the results of the analysis, the risk difference was calculated using fixed effects methods. Table 8 presents the Mantel-Haenszel risk difference as calculated in the BMS Analysis Population and in the Sensitivity Analysis Population.

Table 8: Meta-Analysis Risk Difference Results of the Primary Endpoint (BMS Analysis Population/Sensitivity Analysis Population)

\begin{tabular}{|c|c|c|c|c|}
\hline & \multicolumn{2}{|c|}{$\begin{array}{l}\text { BMS Analysis } \\
\text { Population }\end{array}$} & \multicolumn{2}{|c|}{$\begin{array}{c}\text { Sensitivity Analysis } \\
\text { Population }\end{array}$} \\
\hline & Dapagliflozin & $\begin{array}{l}\text { All } \\
\text { Comparator }\end{array}$ & Dapagliflozin & $\begin{array}{l}\text { All } \\
\text { Comparator }\end{array}$ \\
\hline $\begin{array}{l}\text { Event Rates } \\
\text { events/ } \\
\text { subjects (N) }\end{array}$ & $\begin{array}{l}48 / 4287 \\
(1.12 \%)\end{array}$ & $\begin{array}{l}30 / 1941 \\
(1.54 \%)\end{array}$ & $\begin{array}{l}39 / 3388 \\
(1.15 \%)\end{array}$ & $\begin{array}{l}22 / 1346 \\
(1.63 \%)\end{array}$ \\
\hline $\begin{array}{l}\text { M-H Risk } \\
\text { Difference } \\
(98 \% \text { CI) }\end{array}$ & \multicolumn{2}{|c|}{$\begin{array}{c}-0.0043 \\
(-0.012,0.0032)\end{array}$} & \multicolumn{2}{|c|}{$\begin{array}{c}-0.0059 \\
(-0.015,0.0031)\end{array}$} \\
\hline
\end{tabular}

Source: Created by reviewer. Dataset: adcv.xpt

Reviewer's Note: As it was found that the Sensitivity Analysis population resulted in less conservative event rates than the BMS Analysis population for both the primary and secondary endpoint, it was decided that all further evaluations (including subgroup and special population analyses) would be conducted using the BMS Analysis population. 
Figure 5 presents the forest plot of the risk difference for the primary composite endpoint for all the trials included in the BMS Analysis population. Confidence intervals are calculated asymptotically. The only trial where there is a statistically significant difference (at the unadjusted, nominal $\alpha=0.05$ significance level) between the comparator and the dapagliflozin groups is in trial MB102013 where the dapagliflozin group has a higher risk of a CV event compared to the comparator group. There were no notable differences in this trial population or trial design that could be found to be account for this difference.

Figure 5: Forest Plot of Risk Difference and 95\% CI by Trial for Primary CV Composite Endpoint (BMS Meta-analysis Dataset) and 98\% CI for Overall Analysis

\begin{tabular}{|c|c|c|c|c|c|c|}
\hline Trial & Type of Therapy & $\begin{array}{l}\text { Dapagliflozin } \\
\text { CV events/N (\%) }\end{array}$ & $\begin{array}{l}\text { Comparator } \\
\text { CV events/N (\%) }\end{array}$ & Comparator Worse & Dapagliflozin Worse & $\%$ Risk Difference \\
\hline D1690C00004 & Add-on to Metformin (Glipizide) & $7 / 406(1.72)$ & $8 / 408(1.96)$ & - & & $-0.24(-2.08,1.61)$ \\
\hline D1690C00005 & Add-on to SU & $6 / 450(1.33)$ & 1/146 (0.68) & & . & $0.65(-1.06,2.36)$ \\
\hline D1690C00006 & Add-on to Insulin & $11 / 610(1.80)$ & $5 / 197(2.54)$ & & & $-0.73(-3.17,1.70)$ \\
\hline D1690C00012 & Add-on to Metformin & $0 / 91(0.00)$ & $1 / 91(1.10)$ & & & $-1.10(-3.24,1.04)$ \\
\hline D1692C00005 & Monotherapy & $1 / 166(0.60)$ & $0 / 54(0.00)$ & & - & $0.60(-0.57,1.78)$ \\
\hline MB102008 & Monotherapy & $1 / 279(0.36)$ & $0 / 110(0.00)$ & & $\rightarrow$ & $0.36(-0.34,1.06)$ \\
\hline MB102009 & Add-on to Insulin & $0 / 48(0.00)$ & $0 / 23(0.00)$ & & 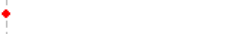 & + \\
\hline MB102013 & Monotherapy & $6 / 410(1.46)$ & $0 / 75(0.00)$ & & - & $1.46(0.30,2.63)$ \\
\hline MB102014 & Add-on to Metformin & $5 / 409(1.22)$ & $6 / 137(4.38)$ & $\leftarrow$ & - & $-3.16(-6.75,0.43)$ \\
\hline MB102021 & Combination with Metformin & $2 / 397(0.50)$ & $1 / 201(0.50)$ & & & $0.01(-1.19,1.20)$ \\
\hline MB102029 & Monotherapy (renal impairment) & $6 / 168(3.57)$ & $7 / 84(8.33)$ & $\leftrightarrow$ & & $-4.76(-11.30,1.78)$ \\
\hline MB102030 & Add-on to TZD & $1 / 281(0.36)$ & $0 / 139(0.00)$ & & $\cdot$ & $0.36(-0.34,1.05)$ \\
\hline MB102032 & Monotherapy (low-dose) & $0 / 142(0.00)$ & $0 / 68(0.00)$ & & $\bullet$ & - \\
\hline \multirow[t]{3}{*}{ MB102034 } & Combination with Metformin & $2 / 430(0.47)$ & 1/208 (0.48) & & - & $-0.02(-1.15,1,12)$ \\
\hline & $\%$ M-H Risk Difference & & & & & $-0.43(-1.18,0.32)$ \\
\hline & & & & $-4 \% \quad-3 \% \quad-2 \% \quad-1 \%$ & $\begin{array}{lllll}0 & 1 \% & 2 \% & 3 \% & 4 \%\end{array}$ & \\
\hline
\end{tabular}

Source: Created by reviewer. Dataset: adcv.xpt

\subsubsection{Evaluation of Trial Heterogeneity}

To assess whether heterogeneity was present in the trials included in the evaluation of the primary endpoint, the Q-statistic and $\mathrm{I}^{2}$ were evaluated using the BMS Analysis population. The $\mathrm{Q}$-statistic had a value of 5.27 with an associated $\mathrm{p}$-value of 0.92 , and $\mathrm{I}^{2}=0 \%$. Therefore we could conclude that there was no heterogeneity between trials with regards to the rate of occurrence of the primary endpoint and the fixed effects model used by the sponsor and reported above is appropriate. 


\section{Findings in Special/Subgroup Populations}

Statistician's note: As all further analyses are exploratory in nature, all estimates will be reported with 95\% confidence intervals (corresponding to an unadjusted, nominal $\alpha=0.05$ significance level). Because of the small number of events which are likely to occur in subgroups, caution is advised when interpreting results of these subgroup analyses. Therefore, these findings should be considered exploratory until confirmation from an independent trial is obtained.

\subsection{Gender, Race and Age}

Evaluations for gender, race and age are presented in the paragraphs that follow. A forest plot combining all results is presented in Figure 6. All incidence rate differences (IRD) were calculated using the Mantel-Haenszel (M-H) method, stratifying by trial.

\section{Gender}

Among the 6,228 subjects in the BMS Analysis Population, 3,173 (50.9\%) were male subjects and 3,055 (49.1\%) were female subjects. Among the 78 subjects with primary composite endpoint events, 43 subjects were male and 35 were female subjects. The $\mathrm{M}-\mathrm{H}$ incidence rate difference of developing a CV related event as measured by the primary composite endpoint was lower in the dapagliflozin group for both male and female subjects (IRD $-0.50,95 \% \mathrm{CI}(-1.55$, $0.56)$ for males and IRD $-0.59,95 \% \mathrm{CI}(-1.39,0.22)$ for females).

\section{Race}

Among the 6,228 subjects in the BMS Analysis Population, 5,048 (81.0\%) were White subjects, $214(3.4 \%)$ were Black subjects and 578 (9.28\%) were Asian subjects. Among the 78 subjects reported with events of the primary composite endpoint, 68 were White subjects, 2 were Black subjects and 5 were Asian subjects.

For both the White and Black populations, the incidence rate difference of developing a CV related event as measured by the primary composite endpoint was lower in the dapagliflozin group. Among the White subjects, this difference was statistically significant (IRD: $-0.77,95 \%$ CI (-1.52, -0.01). Among Asian subjects there was a statistically significant increased risk of developing a CV event in the dapagliflozin group (IRD: $1.69,95 \%$ CI $(0.10,3.28)$ ). For Black subjects the IRD was $-1.50,95 \% \mathrm{CI}(-4.22,1.22)$. 


\section{Age}

Among the 6,228 subjects in the BMS Analysis Population, 1,766 (28.4\%) were aged 50 years or younger, 3,108 (49.9\%) were between 51 and 64 years old, 1,166 (18.7\%) were between age 65 and 74 years old, and $169(2.7 \%)$ were age 75 or older.

In all the age categories, except those ages 75 and older, there was a slightly lower risk of experiencing the primary composite endpoint in the dapagliflozin group (age $\leq 50$ IRD: -0.41 , 95\% CI (-1.09, 0.27); age 51-64 IRD: -0.76, 95\%CI (-1.71, 0.19); age 65-74 IRD: -0.06, 95\%CI $(-1.88,1.76))$. In the oldest age group the incidence rate difference was higher in the dapagliflozin group, but due to the low number of subjects in this group, this difference was associated with a wide confidence interval (IRD: 1.48 95\% CI: $(-6.11,9.07)$ ).

Figure 6: Forest Plot of Incidence Rate Difference of Primary Composite Endpoint by Baseline Demographics (BMS Analysis Population)

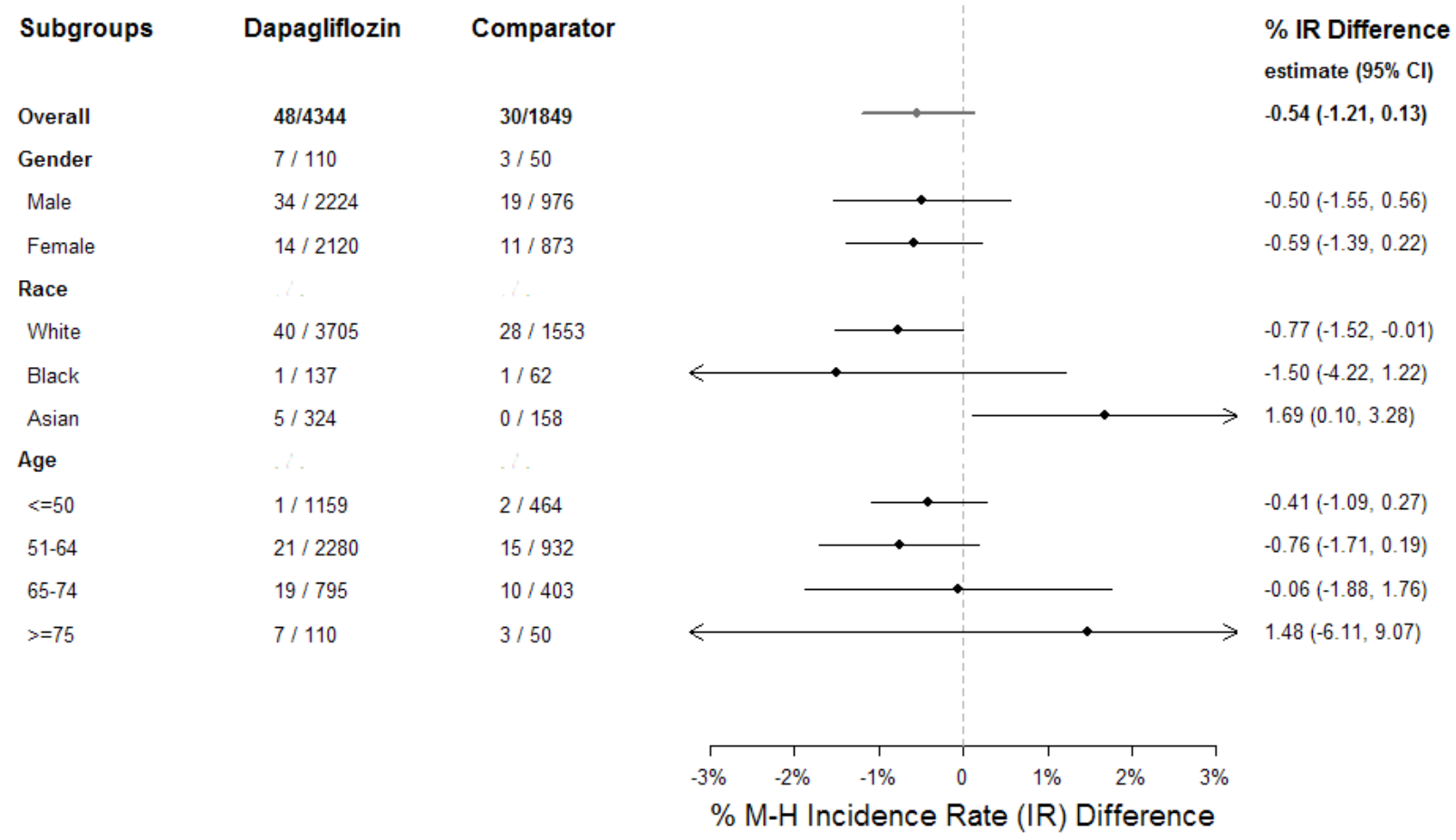




\subsection{Other Special/Subgroup Populations}

In addition to the demographic factors evaluated in the previous section, evaluation of differences in baseline BMI, specific cardiovascular risk factors, baseline renal impairment status and history of diabetes were considered.

To further investigate the sensitivity of the data, two different sets of subgroup analyses were conducted, as suggested by the clinical team. In the first subgroup analysis, results are presented for trials that used dapagliflozin as an add-on therapy and for trials that used dapagliflozin as a monotherapy. In the second subgroup analysis, results are presented for the $10 \mathrm{mg}$ dose alone, the proposed therapeutic dose.

Statistician's note: The sponsor performed a sensitivity analysis pooling all short-term (ST) periods of the trials. This analysis was not replicated by the reviewer as it was determined that the long-term risk of cardiovascular events was of primary importance. The long-term (LT) extension periods of these trials maintained the double-blind nature of the trial and continued with the same treatment group assignments and therefore provided extended periods of exposure of the subjects to their assigned treatments. It should be noted that the sponsor's results for this analysis were consistent with all other findings of the meta-analysis (i.e. no increased risk of cardiovascular events in dapagliflozin compared to comparator). 


\subsubsection{Baseline BMI, CV Risk Factors, Renal Impairment and Diabetes History}

To determine if the health history of the subjects had any impact on the risk of developing a CV event as determined by the primary composite endpoint, the baseline BMI $(<30, \geq 30)$, history of cardiovascular disease, history of hypertension, history of dyslipidemia, baseline eGFR and duration of diabetes, were evaluated. Based on the M-H IRD in the BMS Analysis Population of the incidence of the primary composite endpoint, it was found that all groups in these analyses had a lower risk of CV events in the dapagliflozin group. The results for these analyses are presented in Figure 7.

Figure 7: Forest Plot of Incidence Rate Difference of Primary Composite Endpoint by Baseline Risk Factors (BMS Analysis Population)

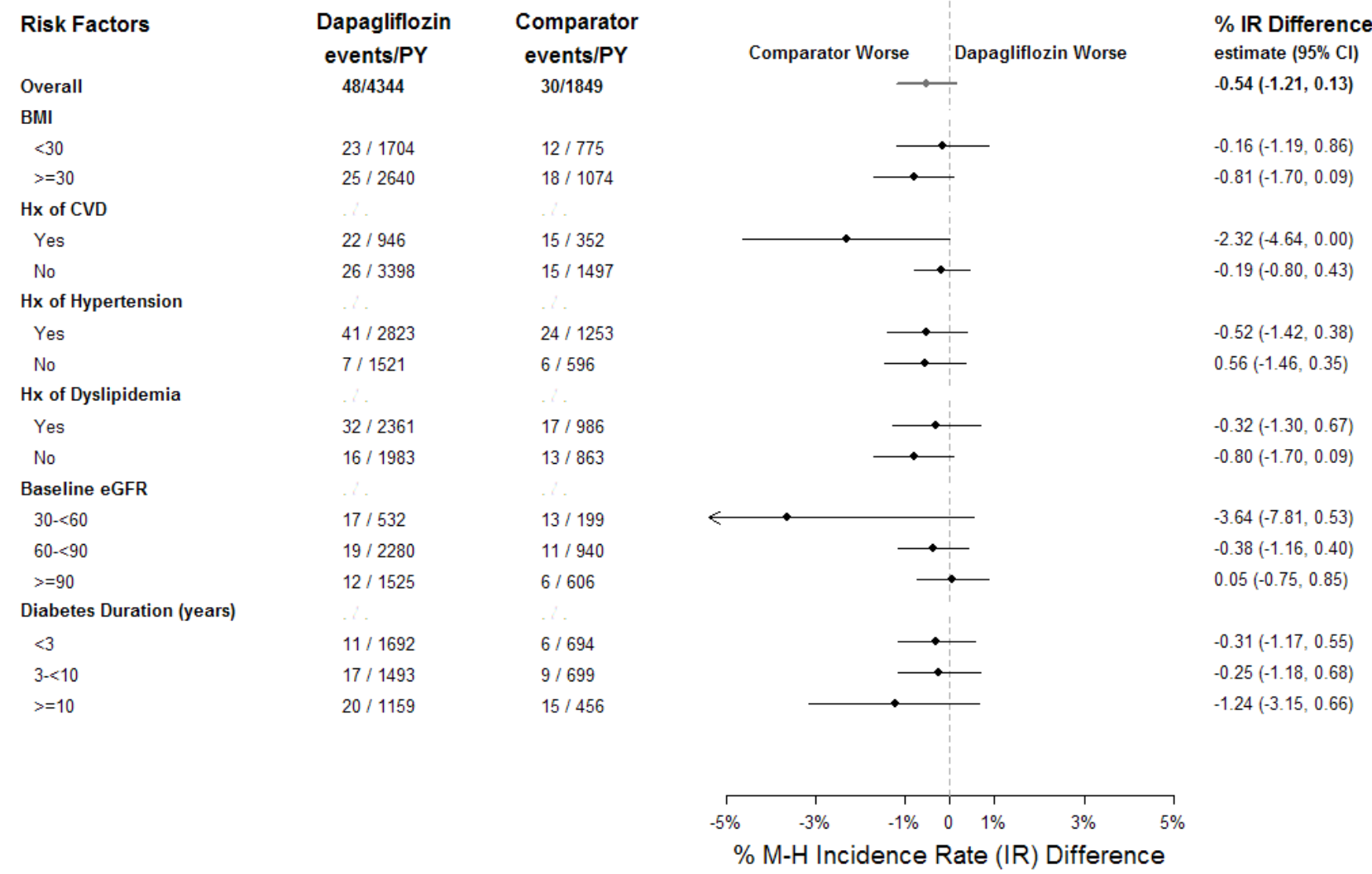




\subsubsection{Monotherapy and Add-On Trials}

The monotherapy trials from the BMS Analysis Populations (trials D1692C00005, MB102013, MB102029 and MB102032) and the add-on trials from this population (trials D1690C00004, D1690C00005, D1690C00006, D1690C00012, MB102009, MB102014 and MB102030) were grouped together to observe any difference in the incidence rates of the primary composite endpoint based on trial type. Table 9 presents the results from the meta-analysis of these two subgroups. The hazard rate for the occurrence of the primary endpoint was comparable in the monotherapy and add-on trials, while the overall lowest incidence of the primary endpoint was in the dapagliflozin group in the add-on trials.

Table 9: Meta-Analysis Results of the Primary Composite Endpoint for the Monotherapy Trials and Add-On Therapy Trials (BMS Analysis Population)

\begin{tabular}{|c|c|c|c|c|}
\hline & \multicolumn{2}{|c|}{ Monotherapy } & \multicolumn{2}{|c|}{ Add-on } \\
\hline & Dapagliflozin & $\begin{array}{l}\text { All } \\
\text { Comparator }\end{array}$ & Dapagliflozin & $\begin{array}{c}\text { All } \\
\text { Comparator }\end{array}$ \\
\hline \begin{tabular}{|l|} 
Stratified \\
Hazard Ratio \\
$(95 \%$ CI)
\end{tabular} & \multicolumn{2}{|c|}{$\begin{array}{c}0.67 \\
(0.27,1.68)\end{array}$} & \multicolumn{2}{|c|}{$\begin{array}{c}0.64 \\
(0.36,1.15)\end{array}$} \\
\hline \begin{tabular}{|l|} 
Event/PY \\
(\% Incidence)
\end{tabular} & $14 / 1060$ & $7 / 282$ & $30 / 2872$ & $21 / 1363$ \\
\hline $\begin{array}{l}\text { M-H Incidence } \\
\text { Rate Difference } \\
\text { (95\% CI) }\end{array}$ & \multicolumn{2}{|c|}{$\begin{array}{c}-0.0079 \\
(-0.026,0.010)\end{array}$} & \multicolumn{2}{|c|}{$\begin{array}{c}-0.0056 \\
(-0.014,0.023)\end{array}$} \\
\hline
\end{tabular}

Source: Created by reviewer. Dataset: adcv.xpt 


\subsubsection{0 mg Dosage of Dapagliflozin}

To investigate the impact of the proposed therapeutic dose on the outcome of cardiovascular events, the subjects taking the $10 \mathrm{mg}$ dosage of dapagliflozin in the BMS Analysis population (excluding trials MB102021 and MB102032 which did not include $10 \mathrm{mg}$ dosage groups of dapagliflozin) were isolated in the analysis and compared to the combined comparator group. Table 10 presents the meta-analysis results from the isolation of the $10 \mathrm{mg}$ dosage compared to the pooled comparator groups.

Table 10: Meta-Analysis Results for 10mg Dose of Dapaglfilozin Alone and the Pooled Comparator (BMS Analysis Population excluding MB102021 and MB102032)

\begin{tabular}{|l|c|c|}
\hline & $\begin{array}{l}\text { Dapagliflozin } \\
\text { 10 mg dose } \\
\text { alone }\end{array}$ & $\begin{array}{l}\text { All } \\
\text { Comparator }\end{array}$ \\
\hline $\begin{array}{l}\text { Hazard Ratio } \\
\text { (95\% CI) }\end{array}$ & \multicolumn{2}{|c|}{0.69} \\
\hline $\begin{array}{l}\text { Event/PY } \\
\text { (\% Incidence) }\end{array}$ & $22 / 2011$ & $1.20)$ \\
\hline $\begin{array}{l}\text { M-H Incidence Rate } \\
\text { Difference }\end{array}$ & \multicolumn{2}{|c|}{$-0.0052 / 1711$} \\
(95\% CI) & $(-0.013,0.0025)$ \\
\hline
\end{tabular}

Source: Created by reviewer. Dataset: adcv.xpt 
Based on the Kaplan-Meier method using the BMS Analysis population (excluding MB102021 and MB102032), the cumulative probability of developing a CV-related event as measured by the primary composite endpoint is shown in Figure 8.

Figure 8: Time-to-event Analysis of the Primary Composite Endpoint for the $10 \mathrm{mg}$ Dose of Dapagliflozin Compared to Pooled Comparator (BMS Analysis Population excluding MB102021 and MB102032)

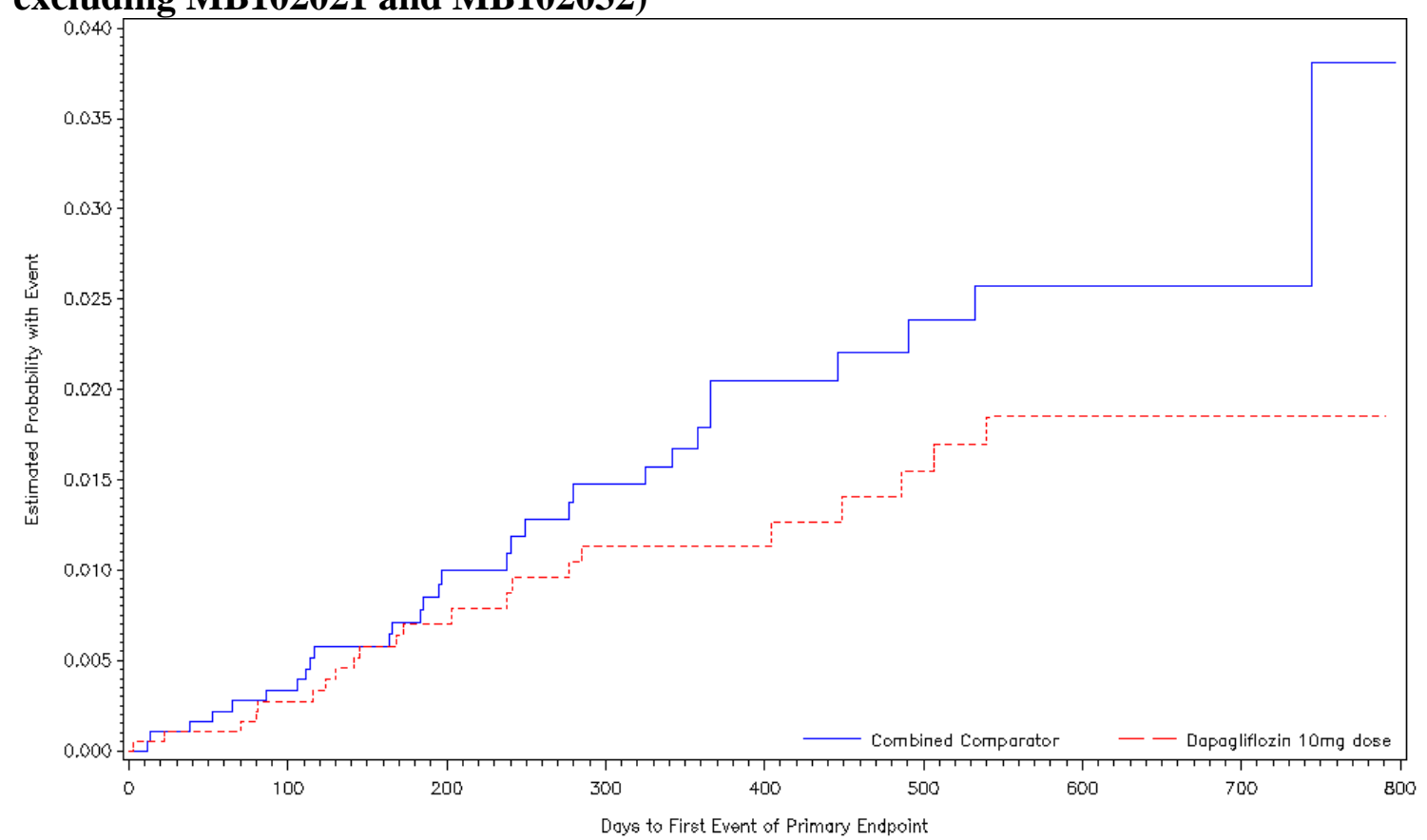

Source: Created by reviewer. Dataset: adcv.xpt 


\section{Summary and Conclusions}

\subsection{Statistical Issues and Collective Evidence}

The primary agreed upon safety endpoint was a composite endpoint consisting of CV death, nonfatal myocardial infarction (MI), non-fatal stroke, and hospitalization due to unstable angina.

In the statistical analysis plan (SAP) agreed upon with the FDA on November 9, 2010, a group sequential testing strategy was pre-specified to control the overall Type I error rate for demonstration of non-inferiority with respect to the 1.8 relative risk criterion, as estimated by the hazard ratio, at a one-sided 0.025 level. Using a group sequential testing strategy, if the first formal meta-analysis of the primary composite endpoint was found to have an upper-bound less than 1.8 at a one-sided alpha level of 0.01 (corresponding to a $98 \% \mathrm{CI}$ ) then the meta-analysis would meet the FDA CV guidelines ${ }^{1}$, and data from additional studies that have yet to be completed would not need to be included in a second meta-analysis. However, if unsuccessful at this alpha level (0.01), a second meta-analysis would be performed when studies D1690C00018 and D1690C00019 had unblinded and at least 110 patients had adjudicated events for the primary endpoint. The one-sided alpha level for this final analysis was set at 0.01 so that the overall Type I error rate across the 2 analyses was $\leq 0.025$ (one-sided).

Phase $2 \mathrm{~b}$ and Phase 3 data from the 14 randomized studies were pooled to evaluate the risk of developing a $\mathrm{CV}$ related event as measured by the primary composite endpoint. The prespecified primary analysis conducted was a comparison of the time to composite event between the dapagliflozin group and the pooled all comparator group (including monotherapy, initial combination with metformin, add-on to metformin, sulphonylurea (SU), thiazolidinedione (TZD), and insulin studies) performed using a Cox proportional hazards model with study as stratification factor. The Mantel-Haenszel (MH) methods for estimating the overall incidence rate ratio via Breslow and Day $(1994)^{2}$ where the estimate was calculated by stratifying for trial was performed as a pre-specified secondary analysis. The incidence rate difference was also evaluated to include trials with zero events. To further investigate event rates, the risk difference was calculated to determine if a different denominator (number of subjects per group as opposed to number of person-years of exposure) would affect the estimates.

Among the 6228 subjects in the BMS Analysis population, 78 subjects had an event that was counted in the primary composite endpoint (48 of 4287 dapagliflozin subjects and 30 of 1941 comparator subjects). The primary analysis of the stratified Cox proportional hazards model found the hazard ratio of dapagliflozin versus comparator to be 0.67 (98\% CI: $0.38,1.18)$. To incorporate zero event trials in the meta-analysis, the incidence rate difference was considered as a secondary analysis method. Using this method, the $\mathrm{M}-\mathrm{H}$ incidence rate difference was found to be -0.0054 (98\% CI: $-0.013,0.0026)$. These results appear to be consistent across trials and between the subgroups evaluated.

The secondary composite endpoint was also evaluated which was comprised of the same CV endpoints as the primary endpoint and two additional CV endpoints (unplanned coronary 
revascularization, and hospitalization for heart failure). This endpoint also found no additional risk of $\mathrm{CV}$ events in the dapagliflozin group compared to the comparator group (hazard ratio (98\% CI): $0.63(0.38,1.03)$.

The sensitivity analysis included the creation of an analysis population (Sensitivity Analysis Population) that excluded the short-term trials and the non-inferiority trial in evaluating the primary and secondary endpoints. Additional subgroup analyses included evaluating the difference between dapagliflozin and comparator event rates based on CV risk factors, demographics, and monotherapy compared to add-on studies. All results from the subgroup analyses were consistent with the results of the primary analysis.

\subsection{Conclusions and Recommendations}

The sponsor conducted a meta-analysis of the cardiovascular events that occurred in 14 Phase $2 \mathrm{~b} / 3$ trials using dapagliflozin. This meta-analysis was the first of a two-part group sequential testing strategy that was pre-specified to control the overall Type I error rate for demonstration of non-inferiority with respect to the 1.8 relative risk criterion at a one-sided 0.025 level. Under this testing approach, a one-sided alpha $=0.01$ level is used for testing primary and secondary endpoints (i.e. $98 \% \mathrm{CI}$ ).

The primary endpoint is a composite endpoint, defined as the time to first event of the following adjudicated events: CV death, myocardial infarction, stroke, and hospitalization for unstable angina. All CV events were adjudicated by an independent, blinded clinical events committee (CEC) comprised of two cardiologists and two neurologists. The comparator group was comprised of subjects taking either a placebo and/or an active control (metformin, glipizide, insulin, glimepiride, or pioglitazone).

Among the 6228 subjects in the Bristol-Myers Squibb (BMS) Analysis population (composed of the safety population of all 14 trials combined), 78 subjects had an event that was counted in the primary composite endpoint: 48 of 4287 dapagliflozin subjects (1.1\%) and 30 of 1941 comparator subjects $(1.5 \%)$. The pre-specified primary analysis of the stratified Cox proportional hazards model yielded a hazard ratio of dapagliflozin versus comparator to be 0.67 (98\% CI: $0.38,1.18)$. To incorporate zero event trials in the meta-analysis, the incidence rate difference was considered as a secondary analysis method. Using this method, the incidence rate difference was found to be -0.0054 (98\% CI: $-0.013,0.0026)$. These results appear to be consistent across trials and between the subgroups evaluated.

A secondary composite endpoint was also evaluated which was comprised of the same CV endpoints as the primary endpoint and two additional CV endpoints (unplanned coronary revascularization, and hospitalization for heart failure). This endpoint also found no additional risk of $\mathrm{CV}$ events in the dapagliflozin group compared to the comparator group (hazard ratio (98\% CI): $0.63(0.38,1.03)$.

To further investigate the sensitivity of the analysis, the FDA conducted a sensitivity analysis excluding short-term trials and the non-inferiority trial included in the sponsor's analysis. This 
sensitivity analysis found that there was no additional risk of $\mathrm{CV}$ events in the dapagliflozin group compared to comparator (hazard ratio: 0.60, 98\% CI: 0.32, 1.20). Additionally monotherapy and add-on therapy trials were evaluated separately as a subgroup analysis (hazard ratios $(95 \% \mathrm{CI})$ : monotherapy $0.67(0.27,1.68)$, add-on: $0.64(0.36,1.15))$. To evaluate the proposed therapeutic dose, the $10 \mathrm{mg}$ dose group was separately evaluated and found to have a hazard ratio of 0.69 (95\% CI: $0.39,1.20)$.

Based upon the 1.8 non-inferiority margin set forth in the FDA Diabetes Guidance $(2008)^{1}$, the upper-bound of the alpha-adjusted $98 \%$ confidence interval of the hazard ratio meets the 1.8 noninferiority margin.

\section{$6 \quad$ References}

1. Food and Drug Administration. Guidance for industry: Diabetes mellitus - evaluating cardiovascular risk in new antidiabetic therapies to treat type 2 diabetes. December 2008. (www.fda.gov/downloads/Drugs/GuidanceComplianceRegulatoryInformation/Guidances/ucm071627. pdf)

2. Breslow NE, Day NE (1994), Statistical Methods in Cancer Research Volume II: The Design and Analysis of Cohort Studies, IARC Scientific Publications, No. 82, New York: Oxford University Press, Inc.

3. Whitehead, Anne. A prospectively planned cumulative meta-analysis applied to a series of concurrent clinical trials. Statistics in Medicine 1997; 16: 2901-2913.

4. Robins J, Breslow N, Greenland S. Estimators of the Mantel-Haenszel variance consistent in both sparse data and large-strata limiting models. Biometrics 1986; 42: 311-23.

5. Higgins, JPT and Thompson SG. Quantifying heterogeneity in a meta-analysis. Statistics in Medicine 2002; 21: 1539-1558.

6. Stinjen T, Hamza TH, Ozdemir P. Random effects meta-analysis of event outcome in the framework of the generalized linear mixed model with applications in sparse data. Statistics in Medicine 2010; 29: 3046-3067. 


\section{$7 \quad$ Appendix}

Figure 9: Assessment of Proportional Hazards Assumption: Log-Log Survival Curve (BMS Analysis Population)

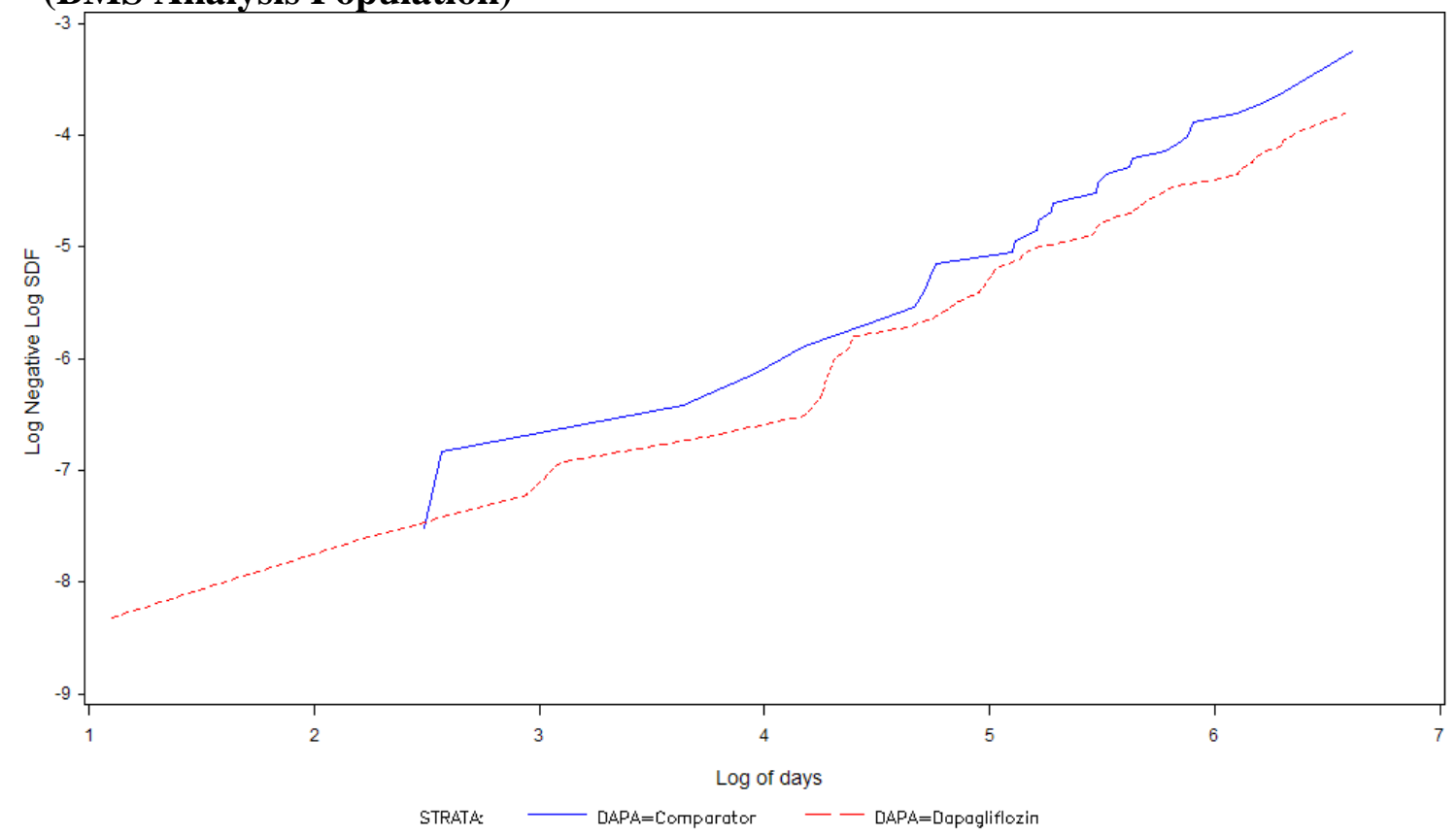

Figure 10: Assessment of Proportional Hazards Assumption: Schoenfeld Residual Plot (BMS Analysis Population)

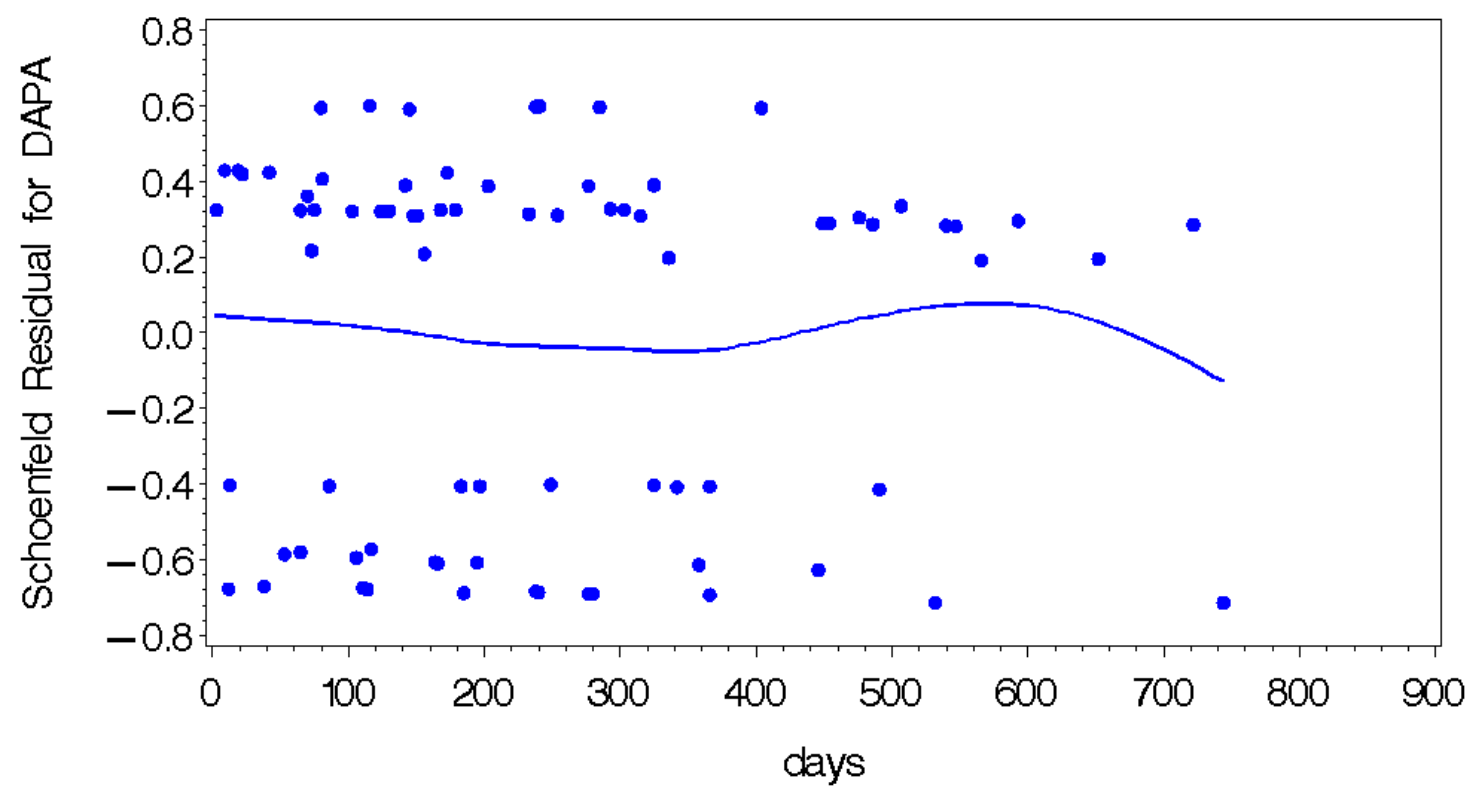


Figure 11: Kaplan Meier Curves of Discontinuation Rates for Each Trial in BMS Analysis Population Time to Discontinuation for Study D1690C00004

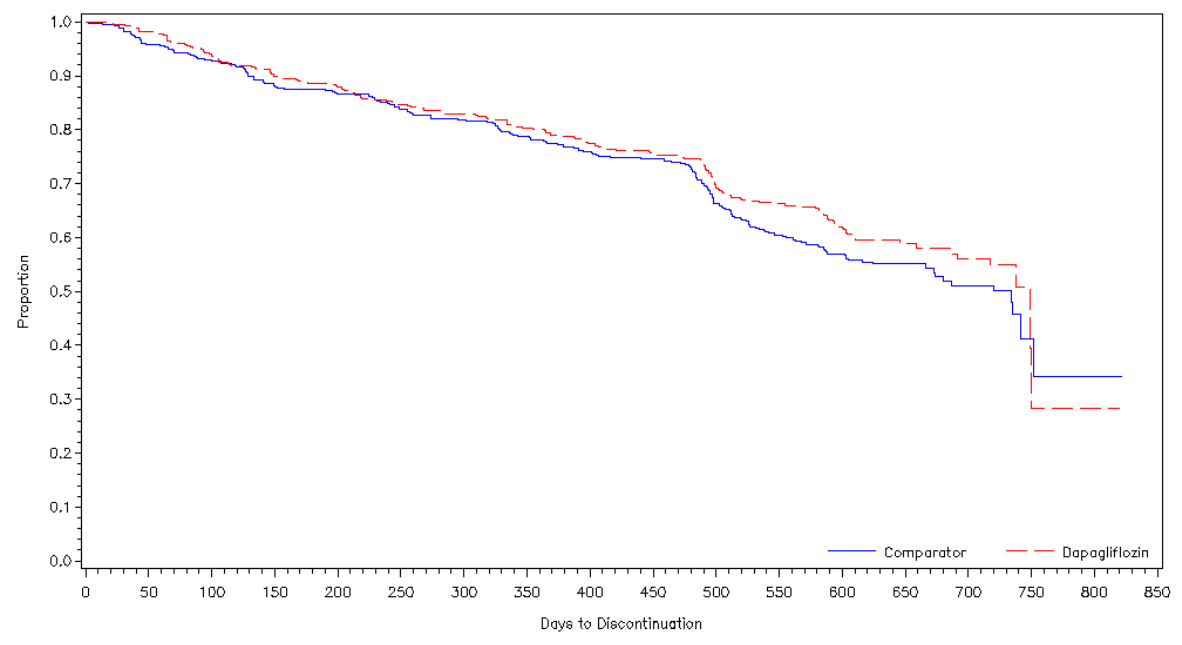

Time to Discontinuation for Study D1690000005

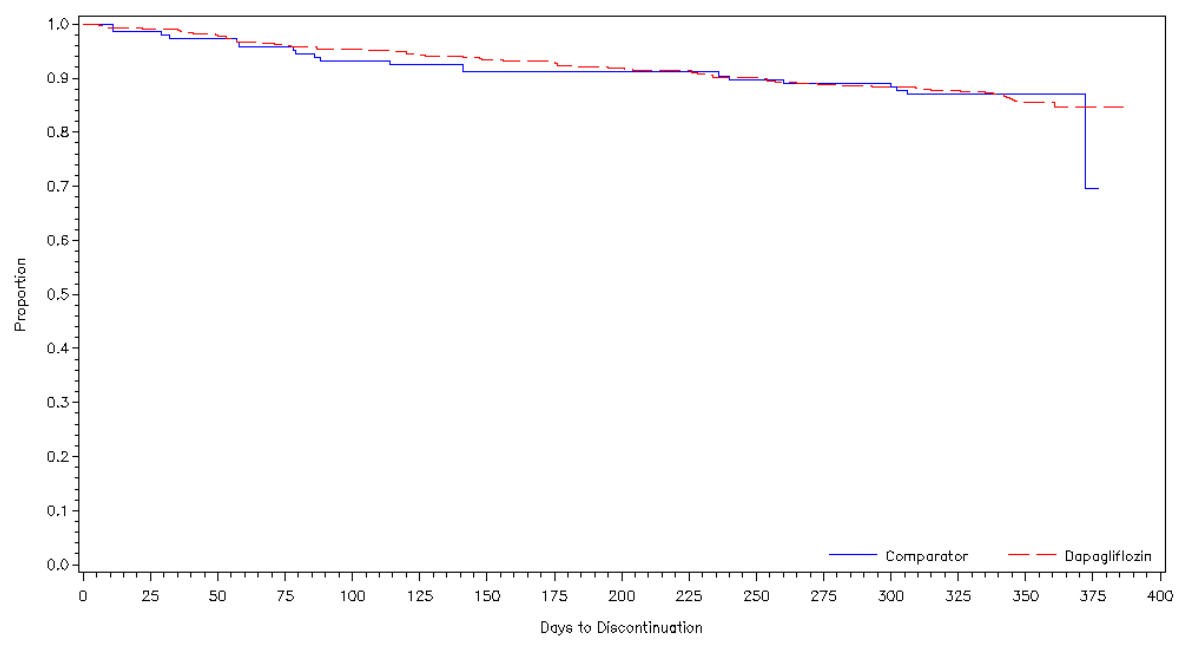


Time to Discontinuation for Study D1690C00006

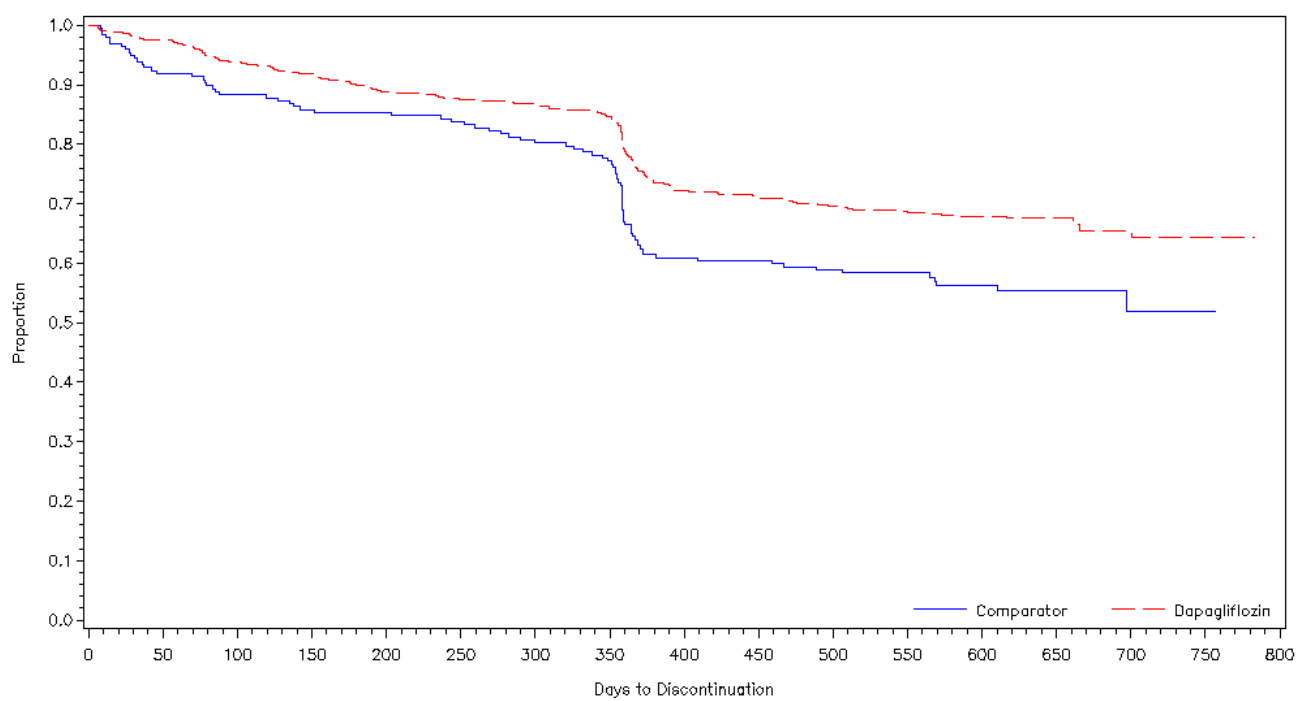

Time to Discontinuation for Study D1690C00012

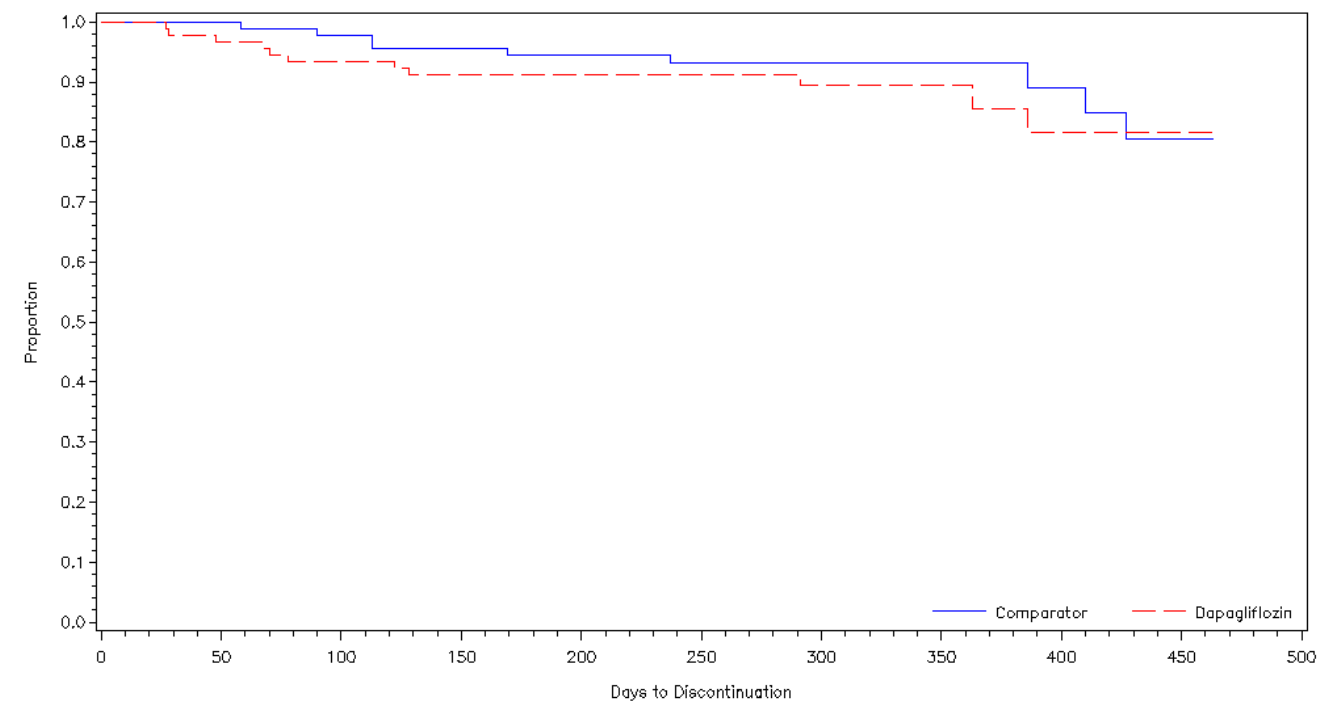


Time to Discontinuation for Study D1692C00005

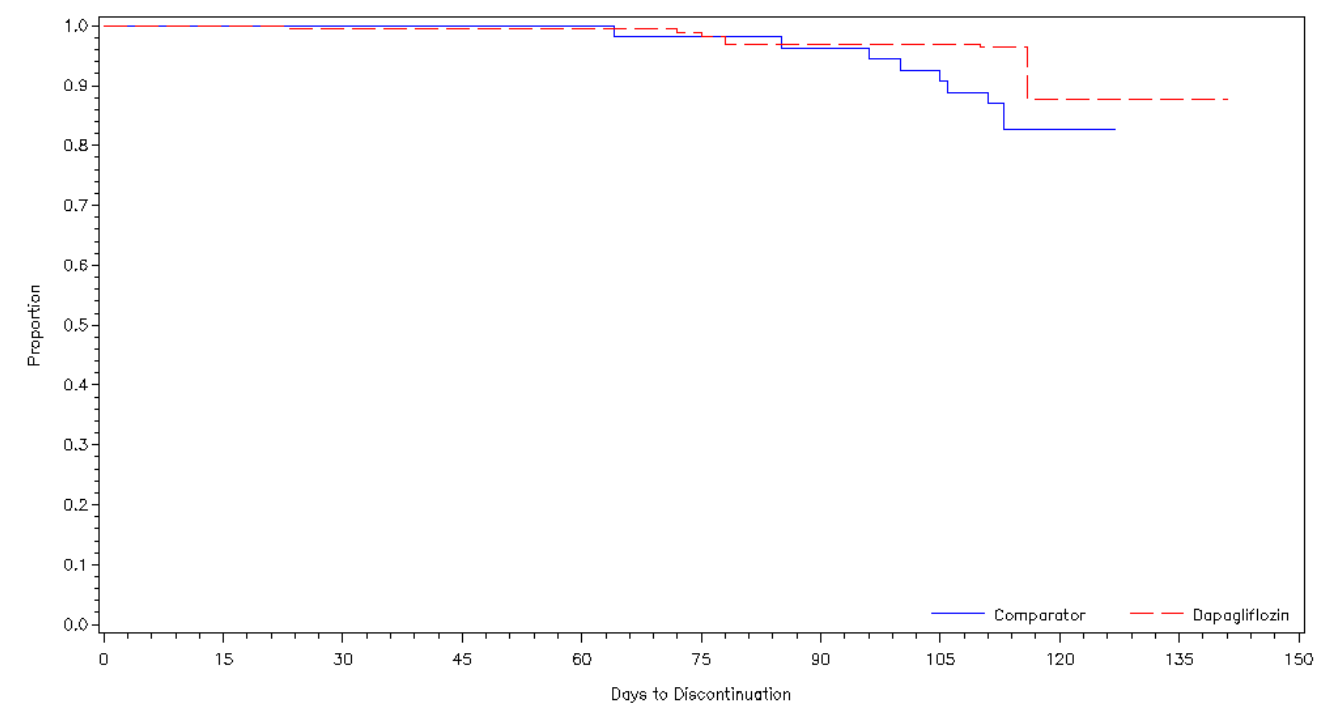

Time to Discontinuation for Study MB102008

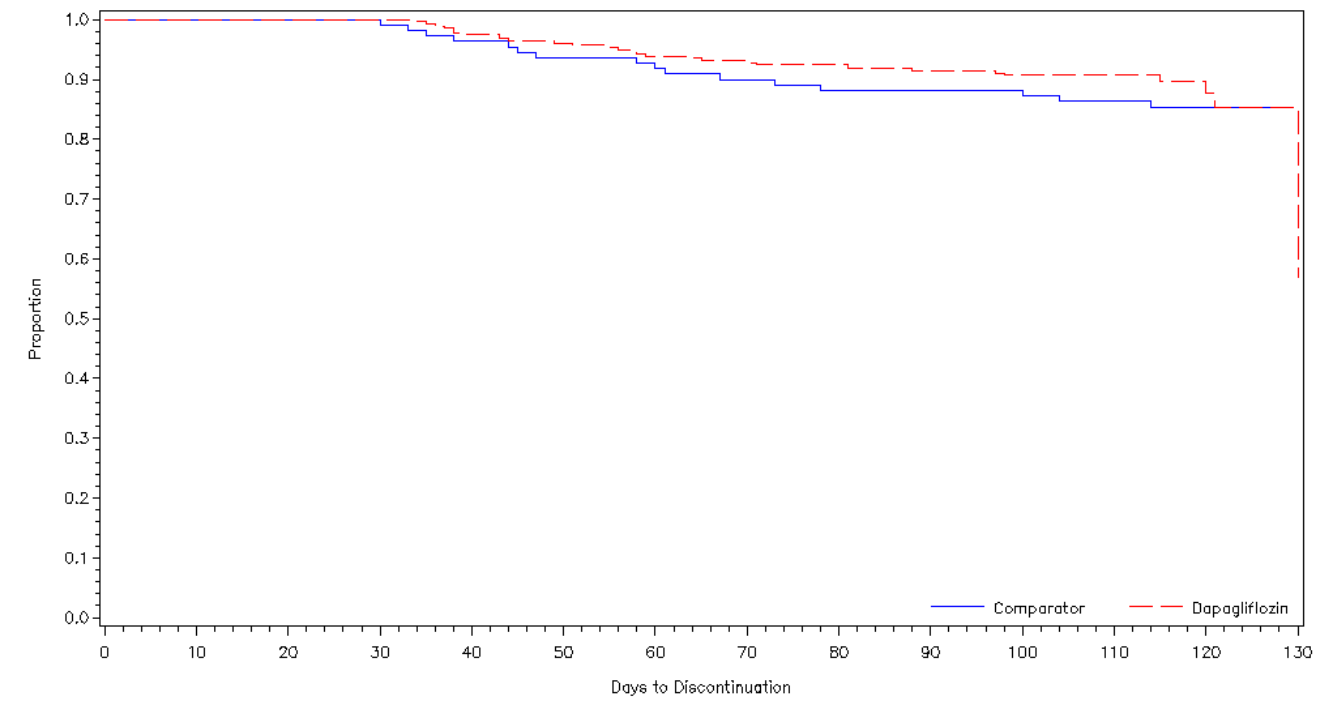


Time to Discontinuation for Study MB102009

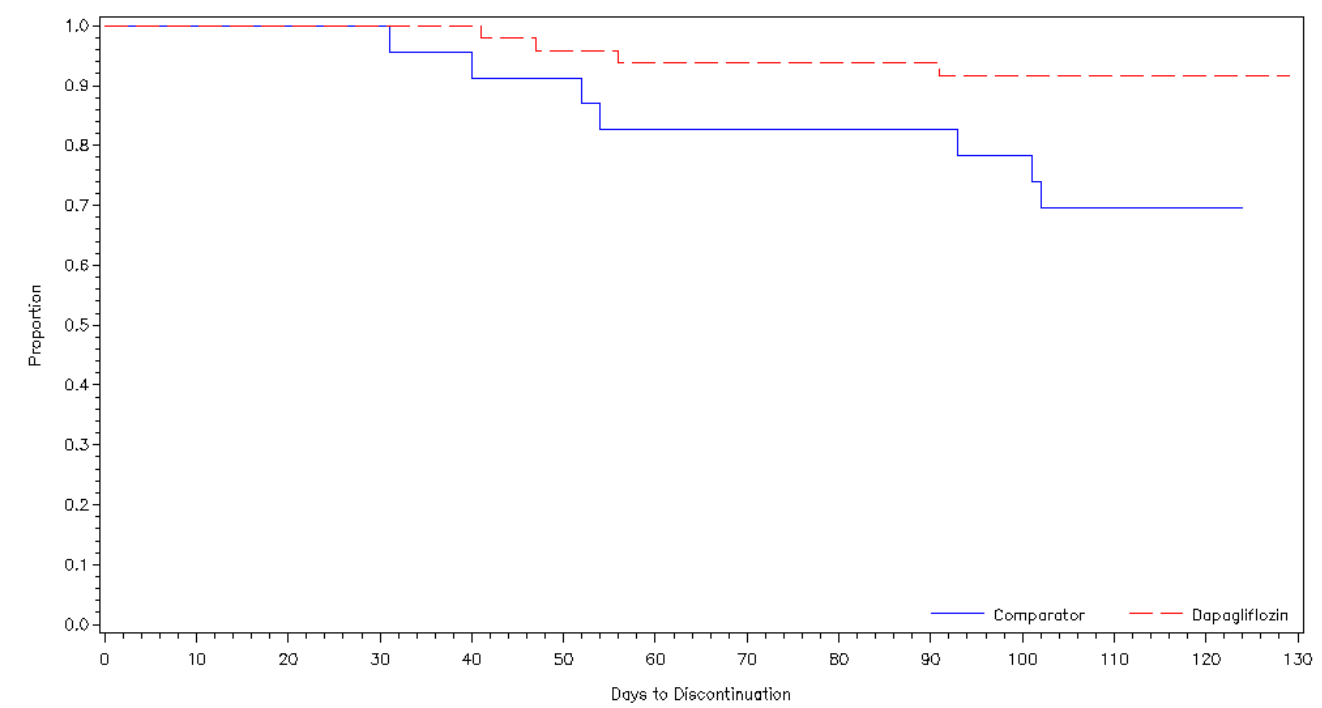

Time to Discontinuation for Study MB102013

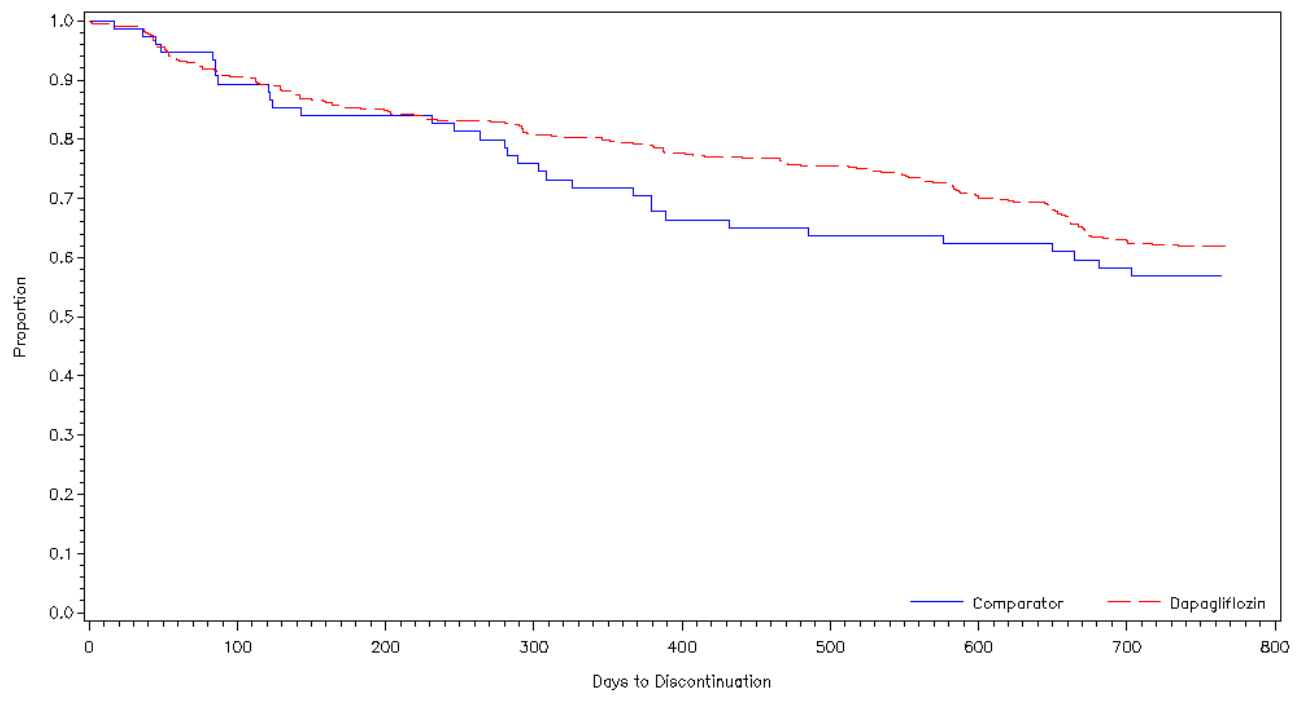


Time to Discontinuation for Study MB102014

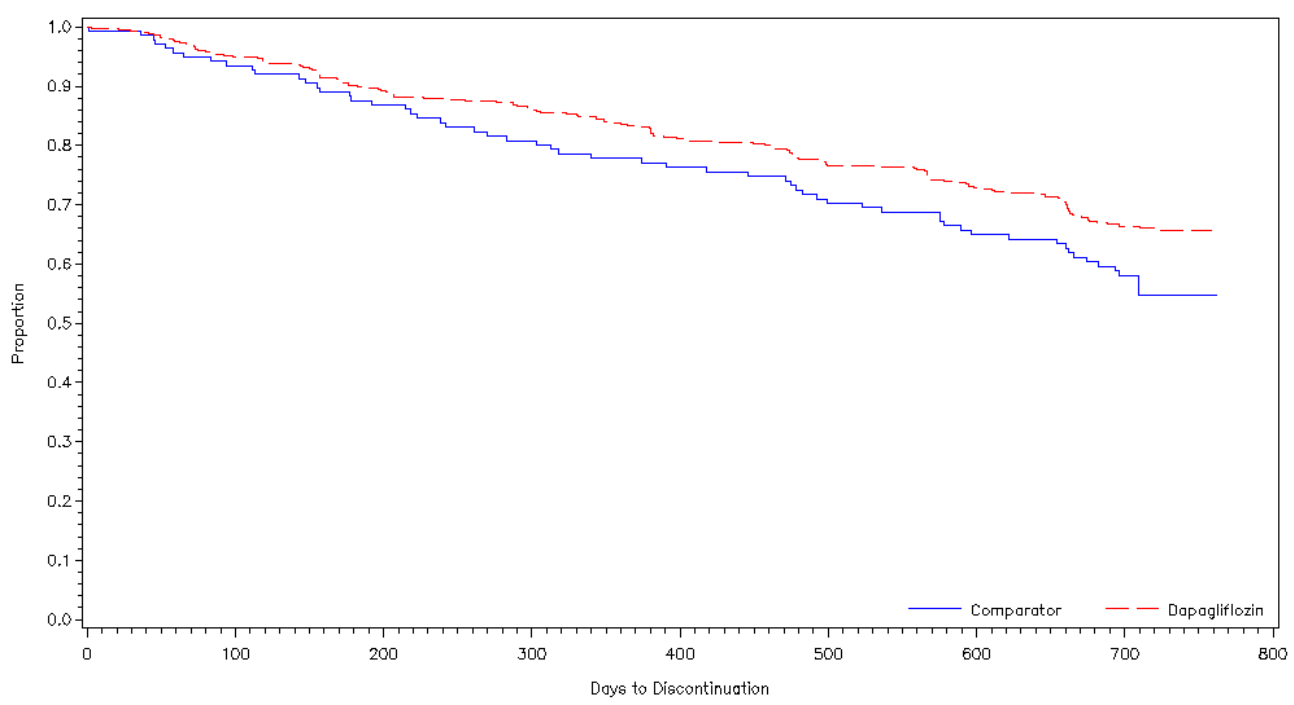

Time to Discontinuation for Study MB102021

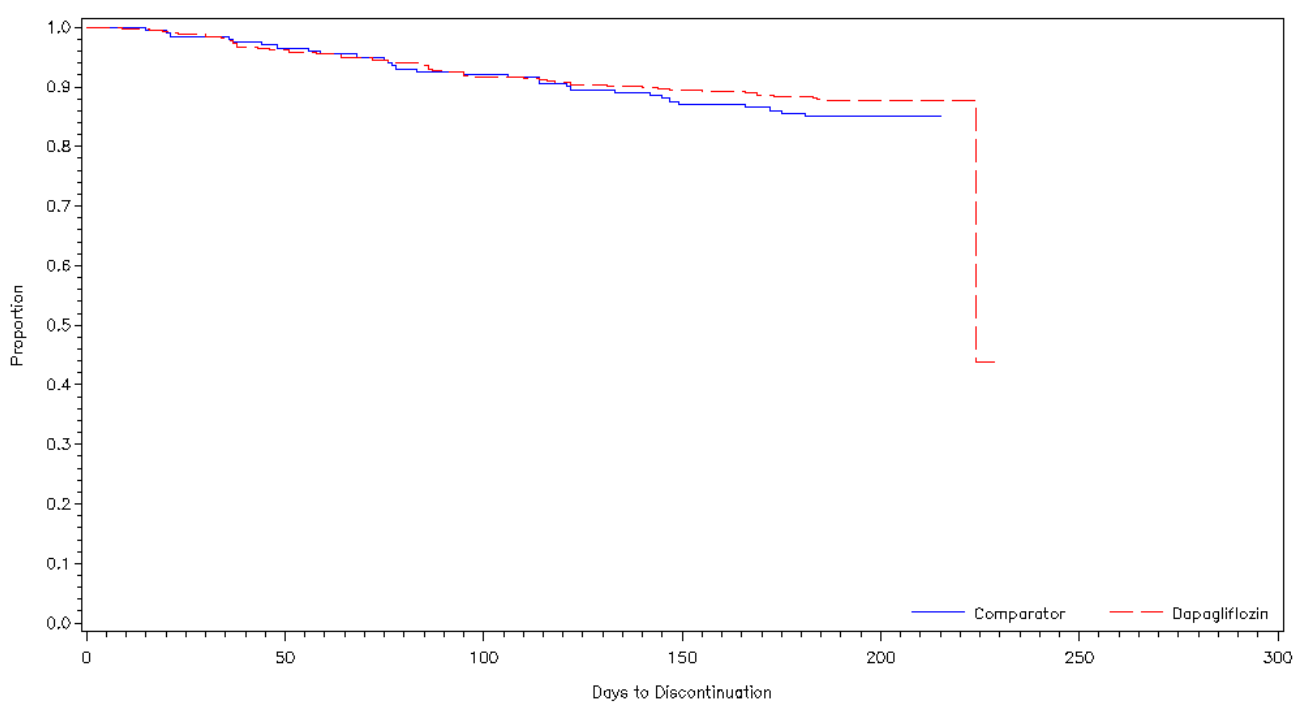


Time to Discontinuation for Study MB102029

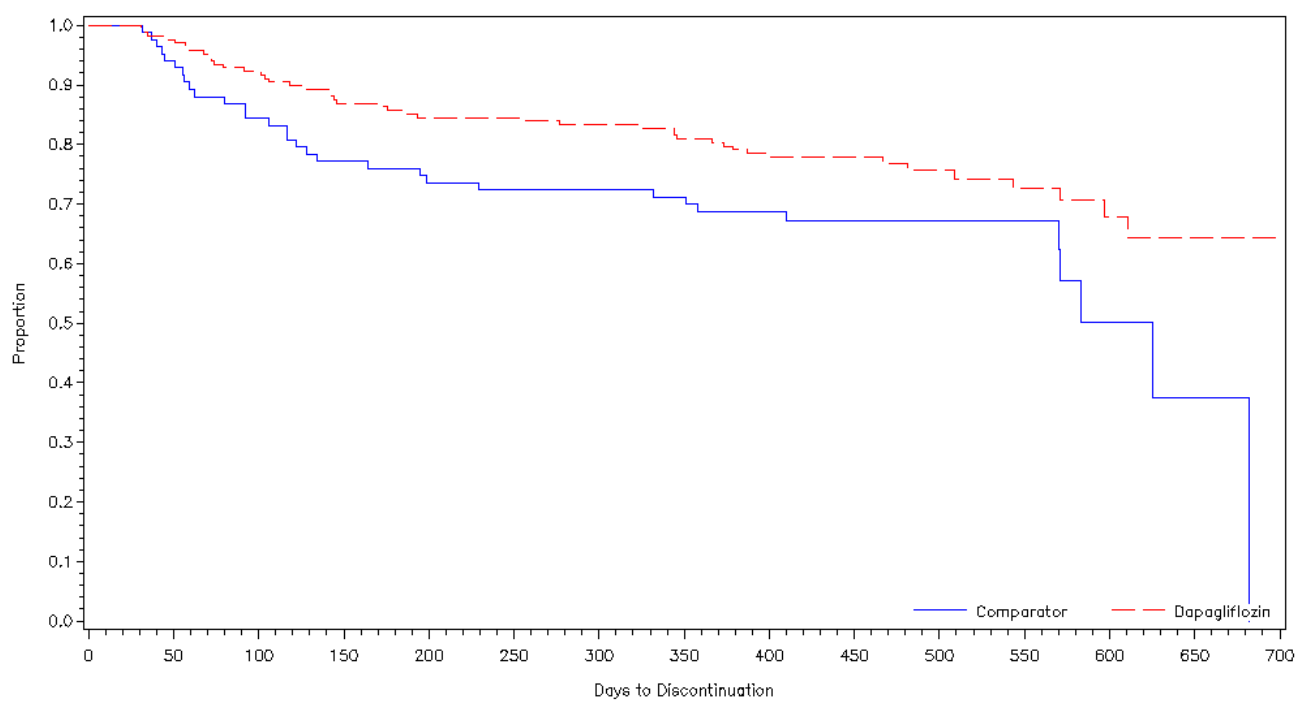

Time to Discontinuation for Study MB102030

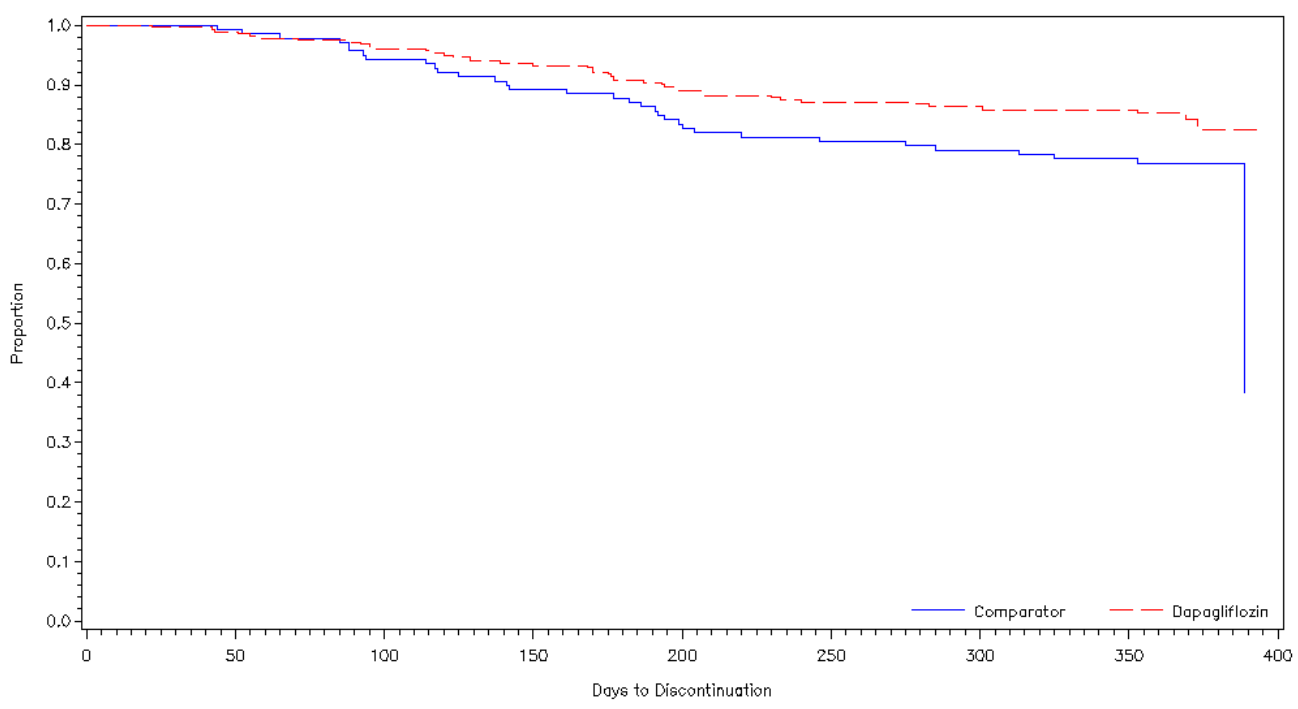


Time to Discontinuation for Study MB102032

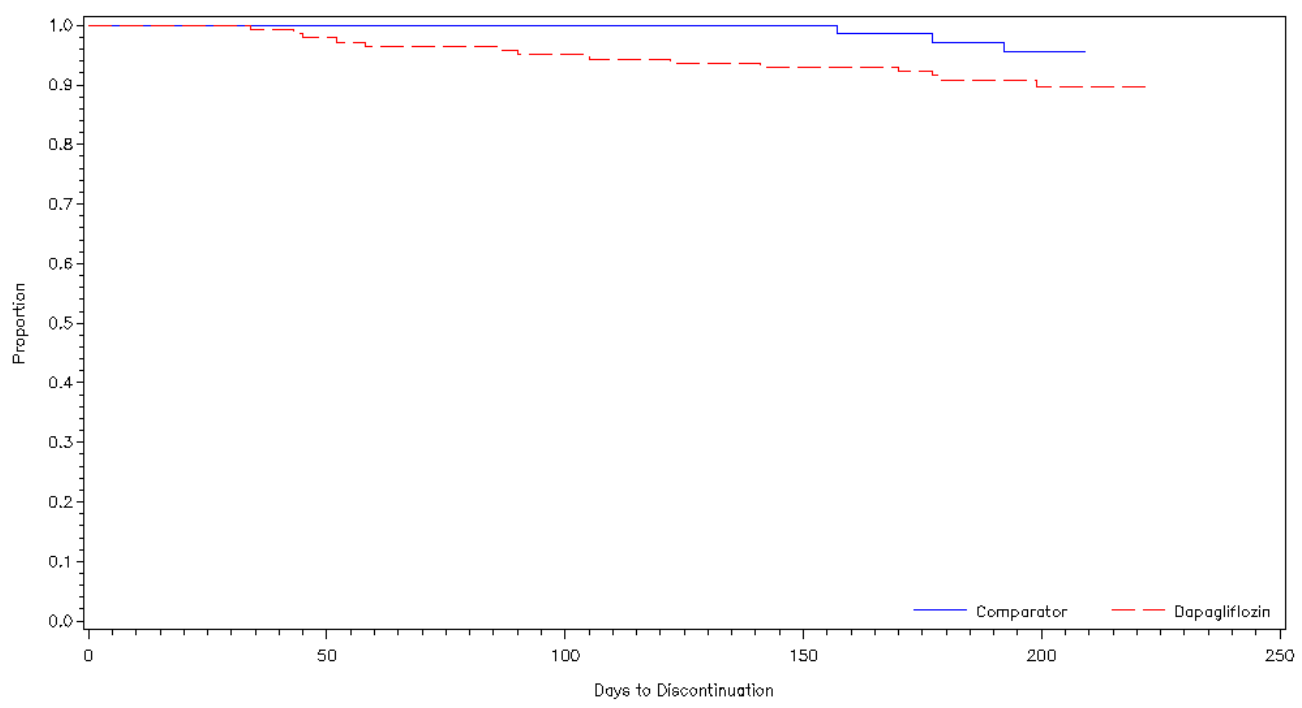

Time to Discontinuation for Study MB102034

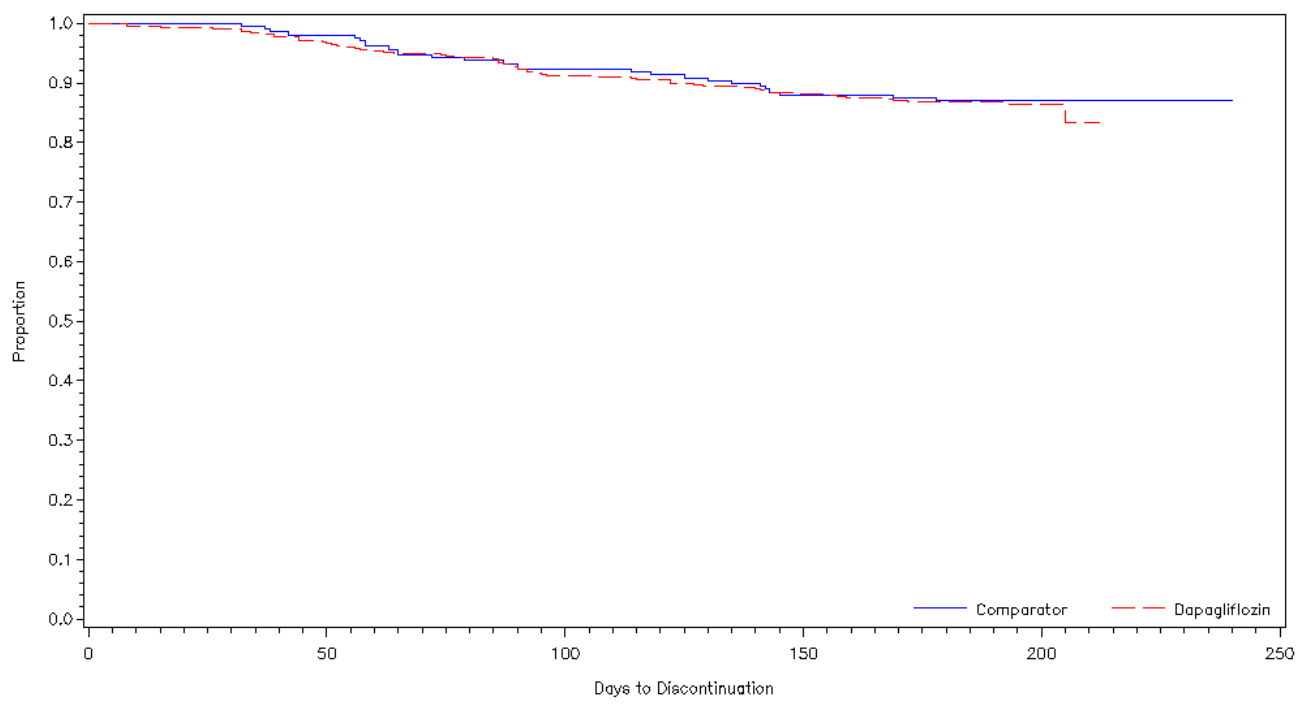


Table 11: CV Risk Factors by Trial

\begin{tabular}{|c|c|c|c|c|c|c|c|c|c|c|}
\hline \multirow[t]{2}{*}{$\%$ of Subjects } & \multicolumn{2}{|c|}{ D1690C00004 } & \multicolumn{2}{|c|}{ D1690C00005 } & \multicolumn{2}{|c|}{ D1690C00006 } & \multicolumn{2}{|c|}{ D1690C00012 } & \multicolumn{2}{|c|}{ D1692C00005 } \\
\hline & Dapa & Comp & Dapa & Comp & Dapa & Comp & Dapa & Comp & Dapa & Comp \\
\hline History of CVD & 18.47 & 19.12 & 35.11 & 37.67 & 32.49 & 38.2 & 24.18 & 28.57 & 3.01 & 3.7 \\
\hline $\begin{array}{l}\text { History of } \\
\text { Hypertension }\end{array}$ & 70.69 & 70.59 & 71.78 & 80.14 & 85.08 & 86.8 & 86.81 & 82.42 & 45.18 & 51.85 \\
\hline $\begin{array}{l}\text { History of } \\
\text { Dyslipidemia }\end{array}$ & 61.33 & 59.31 & 57.11 & 54.79 & 70.16 & 66.5 & 58.24 & 58.24 & 61.45 & 62.96 \\
\hline $\begin{array}{l}\text { History of Congestive } \\
\text { Heart Failure }\end{array}$ & 0.99 & 0.49 & 3.78 & 4.11 & 4.43 & 2.54 & 0 & 0 & 0 & 0 \\
\hline \multicolumn{11}{|l|}{ Renal Function } \\
\hline Severely Impaired & 0.25 & 0.25 & 0 & 0 & 0 & 0 & 0 & 0 & 0 & 0 \\
\hline Moderately Impaired & 4.68 & 5.64 & 8.67 & 16.44 & 16.89 & 13.71 & 1.1 & 5.49 & 0 & 0 \\
\hline Mildly Impaired & 49.26 & 45.1 & 58.67 & 54.79 & 56.72 & 56.35 & 61.54 & 61.54 & 12.05 & 9.26 \\
\hline Normal & 45.81 & 49.02 & 32.67 & 28.77 & 26.39 & 29.95 & 37.36 & 32.97 & 87.95 & 90.74 \\
\hline \multicolumn{11}{|l|}{ Smoking History } \\
\hline Never smoked & 49.01 & 49.02 & 69.2 & 63.01 & 55.25 & 56.35 & 45.05 & 51.65 & 38.55 & 38.89 \\
\hline Current Smoker & 17.98 & 17.89 & 14.51 & 11.64 & 13.28 & 17.77 & 17.58 & 17.58 & 28.92 & 29.63 \\
\hline Former Smoker & 33.00 & 33.09 & 16.29 & 25.34 & 31.31 & 25.89 & 37.36 & 30.77 & 32.53 & 31.48 \\
\hline \multicolumn{11}{|l|}{ Diabetes Duration } \\
\hline less than 3 years & 26.85 & 30.88 & 25.33 & 20.55 & 3.44 & 4.06 & 28.57 & 36.26 & 40.36 & 37.04 \\
\hline $3-10$ years & 55.17 & 48.04 & 48.67 & 55.48 & 30.89 & 27.41 & 50.55 & 51.65 & 47.59 & 51.85 \\
\hline more than 10 years & 17.98 & 21.08 & 26.00 & 23.97 & 65.57 & 68.53 & 20.88 & 12.09 & 12.05 & 11.11 \\
\hline
\end{tabular}




\begin{tabular}{|c|c|c|c|c|c|c|c|c|}
\hline \multirow[t]{2}{*}{ \% of Subjects } & \multicolumn{2}{|c|}{ MB102008 } & \multicolumn{2}{|c|}{ MB102009 } & \multicolumn{2}{|c|}{ MB102013 } & \multicolumn{2}{|c|}{ MB102014 } \\
\hline & Dapa & Comp & Dapa & Comp & Dapa & Comp & Dapa & Comp \\
\hline History of CVD & 8.96 & 4.55 & 18.75 & 34.78 & 11.22 & 9.33 & 13.94 & 7.3 \\
\hline $\begin{array}{l}\text { History of } \\
\text { Hypertension }\end{array}$ & 39.43 & 35.45 & 62.5 & 60.87 & 50.00 & 52.00 & 54.52 & 54.74 \\
\hline $\begin{array}{l}\text { History of } \\
\text { Dyslipidemia }\end{array}$ & 29.75 & 29.09 & 52.08 & 60.87 & 41.71 & 49.33 & 55.01 & 50.36 \\
\hline $\begin{array}{l}\text { History of Congestive } \\
\text { Heart Failure }\end{array}$ & 1.43 & 0.91 & 0 & 0 & 1.46 & 2.67 & 0.24 & 0 \\
\hline \multicolumn{9}{|l|}{ Renal Function } \\
\hline Severely Impaire & 0 & 0 & 0 & 0 & 0 & 0 & 0 & 0 \\
\hline Moderate & 3.23 & 4.55 & 8.33 & 8.7 & 7.07 & 2.67 & 11.25 & 8.76 \\
\hline Mildly Impaired & 63.80 & 58.18 & 54.17 & 56.52 & 55.85 & 58.67 & 55.26 & 52.55 \\
\hline Normal & 32.97 & 37.27 & 37.50 & 34.78 & 37.07 & 38.67 & 33.50 & 38.69 \\
\hline \multicolumn{9}{|l|}{ Smoking History } \\
\hline $\begin{array}{l}\text { Never/Former } \\
\text { smoked }\end{array}$ & 74.91 & 74.55 & 81.25 & 60.87 & 56.59 & 61.33 & 56.72 & 57.66 \\
\hline Current Smoker & 25.09 & 25.45 & 18.75 & 39.13 & $\begin{array}{l}18.78 \\
24.63\end{array}$ & $\begin{array}{l}14.67 \\
24.00\end{array}$ & $\begin{array}{l}15.16 \\
28.12\end{array}$ & $\begin{array}{l}12.41 \\
29.93\end{array}$ \\
\hline \multicolumn{9}{|l|}{ Diabetes Duration } \\
\hline less than 3 years & 72.04 & 78.18 & 4.17 & 4.35 & 81.95 & 72 & 34.47 & 37.23 \\
\hline $3-10$ years & 25.09 & 17.27 & 35.42 & 39.13 & 15.61 & 25.33 & 46.94 & 46.72 \\
\hline more than 10 years & 2.87 & 4.55 & 60.42 & 56.52 & 2.44 & 2.67 & 18.58 & 16.06 \\
\hline
\end{tabular}




\begin{tabular}{|c|c|c|c|c|c|c|c|c|c|c|}
\hline \multirow[t]{2}{*}{ \% of Subjects } & \multicolumn{2}{|c|}{ MB102021 } & \multicolumn{2}{|c|}{ MB102029 } & \multicolumn{2}{|c|}{ MB102030 } & \multicolumn{2}{|c|}{ MB102032 } & \multicolumn{2}{|c|}{ MB102034 } \\
\hline & Dapa & Comp & Dapa & Comp & Dapa & Comp & Dapa & Comp & Dapa & Comp \\
\hline History of CVD & 10.33 & 12.94 & 48.21 & 40.48 & 9.61 & 5.76 & 13.38 & 8.82 & 10 & 11.54 \\
\hline $\begin{array}{l}\text { History of } \\
\text { Hypertension }\end{array}$ & 50.13 & 52.74 & 94.64 & 90.48 & 44.84 & 44.6 & 50.7 & 52.94 & 53.02 & 58.65 \\
\hline $\begin{array}{l}\text { History of } \\
\text { Dyslipidemia }\end{array}$ & 27.2 & 25.37 & 61.9 & 53.57 & 46.26 & 42.45 & 30.28 & 32.35 & 32.09 & 37.5 \\
\hline $\begin{array}{l}\text { History of Congestive } \\
\text { Heart Failure }\end{array}$ & 0.76 & 1 & 10.12 & 11.9 & 0 & 0.72 & 4.23 & 2.94 & 1.86 & 0.96 \\
\hline \multicolumn{11}{|l|}{ Renal Function } \\
\hline Severely Impaired & 0.25 & 0 & 3.57 & 4.76 & 0 & 0 & 0 & 0 & 0 & 0 \\
\hline Moderately Impaired & 6.3 & 3.98 & 92.86 & 89.25 & 6.05 & 5.04 & 4.23 & 4.41 & 5.35 & 6.73 \\
\hline Mildly Impaired & 50.63 & 53.73 & 2.98 & 5.95 & 50.18 & 56.83 & 49.3 & 58.82 & 53.02 & 55.29 \\
\hline Normal & 42.82 & 42.29 & 0.6 & 0 & 43.77 & 38.13 & 46.48 & 36.76 & 41.63 & 37.98 \\
\hline \multicolumn{11}{|l|}{ Smoking History } \\
\hline Never smoked & 69.77 & 67.66 & 50.6 & 54.76 & 65.48 & 64.75 & 63.38 & 66.18 & 65.58 & 72.12 \\
\hline Current Smoker & 13.1 & 11.94 & 10.12 & 22.9 & 12.46 & 17.27 & 18.31 & 8.82 & 17.91 & 14.42 \\
\hline Former Smoker & 17.13 & 20.4 & 39.29 & 33.33 & 22.06 & 17.99 & 18.31 & 25 & 16.51 & 13.46 \\
\hline \multicolumn{11}{|l|}{ Diabetes Duration } \\
\hline less than 3 years & 83.38 & 82.09 & 4.17 & 7.14 & 41.28 & 44.2 & 86.62 & 88.24 & 75.35 & 80.29 \\
\hline $3-10$ years & 14.86 & 15.92 & 20.24 & 22.62 & 43.06 & 38.41 & 11.27 & 10.29 & 20.7 & 16.83 \\
\hline more than 10 years & 1.76 & 1.99 & 75.6 & 70.24 & 15.66 & 17.39 & 2.11 & 1.47 & 3.95 & 2.88 \\
\hline
\end{tabular}

Source: Created by reviewer. Dataset: adcv.xpt 


\section{SIGNATURES/DISTRIBUTION LIST}

Primary Statistical Reviewer: Anita A. Abraham

Date: September 7, 2011

Concurring Reviewers: Mat Soukup, Aloka Chakravarty

Statistical Team Leader: Mat Soukup

Biometrics 7 Division Director: Aloka Chakravarty

cc:

Curtis J. Rosebraugh (Director, Office of Drug Evaluation II)

Mary Parks (Director, Division of Metabolism and Endocrinology)

Ilan Irony (Medical Team Leader)

Somya Dunn (Medical Officer)

Mehreen Hai (Project Manager)

Aloka Chakravarty (Director, Division of Biometrics 7)

Mat Soukup (Division of Biometrics 7)

Anita Abraham (Division of Biometrics 7)

Lillian Patrician (Office of Biostatistics) 
This is a representation of an electronic record that was signed electronically and this page is the manifestation of the electronic signature.

/s/

ANITA A ABRAHAM

09/07/2011

MATTHEW J SOUKUP

09/07/2011

Concur with review

ALOKA G CHAKRAVARTY

09/07/2011 
NDA Number: 202-293

Drug Name: Dapagliflozin
Applicant: Bristol-Myers Squibb Stamp Date: 12/28/2010

NDA/BLA Type: Standard

On initial overview of the NDA/BLA application for RTF:

\begin{tabular}{|c|l|l|l|l|l|}
\hline & \multicolumn{1}{|c|}{ Content Parameter } & Yes & No & NA & Comments \\
\hline 1 & $\begin{array}{l}\text { Index is sufficient to locate necessary reports, tables, data, } \\
\text { etc. }\end{array}$ & $\mathbf{X}$ & & & \\
\hline 2 & $\begin{array}{l}\text { ISS, ISE, and complete study reports are available } \\
\text { (including original protocols, subsequent amendments, etc.) }\end{array}$ & $\mathbf{X}$ & & & \\
\hline 3 & $\begin{array}{l}\text { Safety and efficacy were investigated for gender, racial, } \\
\text { and geriatric subgroups investigated (if applicable). }\end{array}$ & $\mathbf{X}$ & & & \\
\hline 4 & $\begin{array}{l}\text { Data sets in EDR are accessible and do they conform to } \\
\text { applicable guidances (e.g., existence of define.pdf file for } \\
\text { data sets). }\end{array}$ & $\mathbf{X}$ & & \\
\hline
\end{tabular}

\section{IS THE STATISTICAL SECTION OF THE APPLICATION FILEABLE? __ Y}

If the NDA/BLA is not fileable from the statistical perspective, state the reasons and provide comments to be sent to the Applicant.

Please identify and list any potential review issues to be forwarded to the Applicant for the 74day letter. Note: Check boxes pertain to the six studies that I will primarily focus on, which are italicized on next page.

\begin{tabular}{|l|l|l|l|l|}
\hline \hline $\begin{array}{l}\text { Content Parameter (possible review concerns for 74- } \\
\text { day letter) }\end{array}$ & Yes & No & NA & Comment \\
\hline Designs utilized are appropriate for the indications requested. & $\mathbf{X}$ & & & \\
\hline $\begin{array}{l}\text { Endpoints and methods of analysis are specified in the } \\
\text { protocols/statistical analysis plans. }\end{array}$ & $\mathbf{X}$ & & & \\
\hline $\begin{array}{l}\text { Interim analyses (if present) were pre-specified in the protocol } \\
\text { and appropriate adjustments in significance level made. } \\
\text { DSMB meeting minutes and data are available. }\end{array}$ & & $\mathbf{X}$ & \\
\hline $\begin{array}{l}\text { Appropriate references for novel statistical methodology (if } \\
\text { present) are included. }\end{array}$ & $\mathbf{X}$ & & & $\mathbf{X}$ \\
\hline $\begin{array}{l}\text { Safety data organized to permit analyses across clinical trials } \\
\text { in the NDA/BLA. }\end{array}$ & $\begin{array}{l}\text { Not focus of } \\
\text { my review }\end{array}$ \\
\hline $\begin{array}{l}\text { Investigation of effect of dropouts on statistical analyses as } \\
\text { described by applicant appears adequate. }\end{array}$ & $\mathbf{( X )}$ & & $\begin{array}{l}\text { Review issue; } \\
\text { will conduct } \\
\text { own analyses. }\end{array}$ \\
\hline
\end{tabular}


STATISTICS FILING CHECKLIST FOR A NEW NDA/BLA

Phase 3 Studies

\begin{tabular}{|c|c|c|c|c|}
\hline Study ID & Population & Test Tx & $\begin{array}{l}\text { Background } \\
\text { Tx } \\
\text { (Rescue) } \\
\end{array}$ & Comparator(s) \\
\hline \multicolumn{5}{|l|}{ Monotherapy } \\
\hline MB102013 & Drug-naïve & $\begin{array}{l}\text { DAPA } 2.5 \text {, } \\
5,10 \mathrm{mg}\end{array}$ & $\begin{array}{l}\text { None } \\
\text { (Metformin) }\end{array}$ & Placebo \\
\hline MB102032 & Drug-naïve & $\begin{array}{l}\text { DAPA } 1 \text {, } \\
2.5,5 \mathrm{mg}\end{array}$ & $\begin{array}{l}\text { None } \\
\text { (Metformin) }\end{array}$ & Placebo \\
\hline \multicolumn{5}{|l|}{ Add-On } \\
\hline MB102014 & $\begin{array}{l}\text { Inadequate control } \\
\text { on background }\end{array}$ & $\begin{array}{l}\text { DAPA } 2.5, \\
5,10 \mathrm{mg}\end{array}$ & $\begin{array}{l}\text { Metformin } \\
\text { (Pioglitazone or } \\
\text { acarbose) }\end{array}$ & Placebo \\
\hline D1690C00005 & $\begin{array}{l}\text { Inadequate control } \\
\text { on background }\end{array}$ & $\begin{array}{l}\text { DAPA 2.5, } \\
5,10 \mathrm{mg}\end{array}$ & $\begin{array}{l}\text { Glimepiride } \\
\text { (Metformin or } \\
\text { TZD) }\end{array}$ & Placebo \\
\hline MB102030 & $\begin{array}{l}\text { Inadequate control } \\
\text { on background }\end{array}$ & $\begin{array}{l}\text { DAPA 5, } 10 \\
\text { mg }\end{array}$ & $\begin{array}{l}\text { Pioglitazone } \\
\text { (Metformin or } \\
\text { SU) }\end{array}$ & Placebo \\
\hline D1690C00006 & $\begin{array}{l}\text { Inadequate control } \\
\text { on background }\end{array}$ & $\begin{array}{l}\text { DAPA 2.5, } \\
5,10 \mathrm{mg}\end{array}$ & $\begin{array}{l}\text { Insulin and up } \\
\text { to two oral anti- } \\
\text { diabetics } \\
\text { (Insulin up- } \\
\text { titration) }\end{array}$ & Placebo \\
\hline D1690C00004 & $\begin{array}{l}\text { Inadequate control } \\
\text { on background }\end{array}$ & $\begin{array}{l}\text { DAPA 2.5, } \\
5,10 \mathrm{mg} \\
\text { (titrated) }\end{array}$ & $\begin{array}{l}\text { Metformin } \\
\text { (None) }\end{array}$ & Glipizide* \\
\hline D1690C00012 & $\begin{array}{l}\text { Inadequate control } \\
\text { on background }\end{array}$ & $\begin{array}{l}\text { DAPA } 10 \\
\text { mg }\end{array}$ & $\begin{array}{l}\text { Metformin } \\
\text { (Sitagliptin) }\end{array}$ & Placebo** \\
\hline MB102029 & $\begin{array}{l}\text { Moderate renal } \\
\text { impairment and } \\
\text { inadequate control } \\
\text { on stable regime }\end{array}$ & $\begin{array}{l}\text { DAPA 5, } 10 \\
\text { mg }\end{array}$ & $\begin{array}{l}\text { Any except } \\
\text { metformin } \\
\text { (Any except } \\
\text { metformin) }\end{array}$ & Placebo \\
\hline \multicolumn{5}{|c|}{ Initial Combination } \\
\hline MB102021 & $\begin{array}{l}\text { Drug-naïve with } \\
\text { higher HbA1c }\end{array}$ & $\begin{array}{l}\text { DAPA } 5 \mathrm{mg} \\
+ \text { metformin }\end{array}$ & $\begin{array}{l}\text { None } \\
\text { (Pioglitazone, } \\
\text { acarbose, or } \\
\text { sitagliptin) } \\
\end{array}$ & $\begin{array}{l}\text { DAPA } 5 \mathrm{mg} \text {, } \\
\text { metformin }\end{array}$ \\
\hline MB102034 & $\begin{array}{l}\text { Drug-naïve } \\
\text { with higher HbA1c }\end{array}$ & $\begin{array}{l}\text { DAPA } 10 \\
\text { mg }+ \\
\text { metformin }\end{array}$ & $\begin{array}{l}\text { None } \\
\text { (Pioglitazone, } \\
\text { acarbose, or } \\
\text { sitagliptin) }\end{array}$ & $\begin{array}{l}\text { DAPA } 10 \mathrm{mg} \text {, } \\
\text { metformin }\end{array}$ \\
\hline
\end{tabular}

Notes: Proposed doses are 5, $10 \mathrm{mg}$. SU = sulfonylurea, TZD = thiazolidinedione.

*Non-inferiority comparison; otherwise superiority.

*Endpoint is change in body weight; otherwise change in HbA1c. 


\section{STATISTICS FILING CHECKLIST FOR A NEW NDA/BLA}

Additional requests for the 74-day letter (non-filing issues)

These requests apply to all Phase 3 studies:

1. Provide electronic files with the randomization dates and codes (e.g., Appendix 1.9 in study report for MB102013), or describe where these variables can be found.

2. For each study which used block randomization, provide the block size.

3. For each study which used stratified randomization and/or enrollment, describe where the stratification variable can be found in the data files.

4. The protocol for study D1690C00004 refers to a "Study Data Management Plan". Submit this document, and comparable documents for the other Phase 3 studies as applicable. 
This is a representation of an electronic record that was signed electronically and this page is the manifestation of the electronic signature.

/s/

JONATHAN D NORTON

$02 / 17 / 2011$

JON T SAHLROOT

$02 / 17 / 2011$ 
NDA Number: 202293

Drug Name: Dapagliflozin
Applicant: Bristol-Myers Squibb

Stamp Date: $12 / 28 / 2010$

On initial overview of the NDA/BLA application for RTF:

\begin{tabular}{|c|l|c|c|c|l|}
\hline & \multicolumn{1}{|c|}{ Content Parameter } & Yes & No & NA & \multicolumn{1}{|c|}{ Comments } \\
\hline 1 & $\begin{array}{l}\text { Index is sufficient to locate necessary reports, tables, data, } \\
\text { etc. }\end{array}$ & $\mathbf{X}$ & & & eCTD \\
\hline 2 & $\begin{array}{l}\text { ISS, ISE, and complete study reports are available } \\
\text { (including original protocols, subsequent amendments, etc.) }\end{array}$ & $\mathbf{X}$ & $\begin{array}{l}\text { Study report for } \\
\text { meta-analysis } \\
\text { and for studies } \\
\text { included in } \\
\text { meta-analysis } \\
\text { are available }\end{array}$ \\
\hline 3 & $\begin{array}{l}\text { Safety and efficacy were investigated for gender, racial, } \\
\text { and geriatric subgroups investigated. }\end{array}$ & & $\mathbf{X}$ & \\
\hline 4 & $\begin{array}{l}\text { Data sets in EDR are accessible and conform to applicable } \\
\text { guidances (e.g., existence of define.pdf file for data sets). }\end{array}$ & $\mathbf{X}$ & & $\begin{array}{l}\text { Yes in } \\
\text { 5.3.5.3.25.3.1 in } \\
\text { eCTD (adcv.xpt) }\end{array}$ \\
\hline
\end{tabular}

\section{IS THE STATISTICAL SECTION OF THE APPLICATION FILEABLE? YES}

The NDA is filable from a statistics perspective.

Please identify and list any potential review issues to be forwarded to the Applicant for the 74day letter.

No review issues have been noted.

\begin{tabular}{|l|l|l|l|l|}
\hline \hline $\begin{array}{l}\text { Content Parameter (possible review concerns for 74- } \\
\text { day letter) }\end{array}$ & Yes & No & NA & Comment \\
\hline Designs utilized are appropriate for the indications requested. & $\mathbf{X}$ & & & \\
\hline $\begin{array}{l}\text { Endpoints and methods of analysis are specified in the } \\
\text { protocols/statistical analysis plans. }\end{array}$ & $\mathbf{X}$ & $\mathbf{X}$ & & $\begin{array}{l}\text { agreed with } \\
\text { FDA at Type C } \\
\text { meeting on } \\
12 / 4 / 2009\end{array}$ \\
\hline $\begin{array}{l}\text { The primary composite endpoint of adjudicated CV events } \\
\text { including CV death, MI, Stroke and Hospitalization for } \\
\text { unstable angina (i.e. traditional MACE) }\end{array}$ & & $\mathbf{X}$ & $\mathbf{X}$ & \\
\hline $\begin{array}{l}\text { Interim analyses (if present) were pre-specified in the protocol } \\
\text { and appropriate adjustments in significance level made. } \\
\text { DSMB meeting minutes and data are available. }\end{array}$ & & & $\mathbf{X}$ & \\
\hline $\begin{array}{l}\text { Appropriate references for novel statistical methodology (if } \\
\text { present) are included. }\end{array}$ & & & $\mathbf{X}$ & \\
\hline $\begin{array}{l}\text { Safety data organized to permit analyses across clinical trials } \\
\text { in the NDA/BLA. }\end{array}$ & $\mathbf{l}$ & & \\
\hline $\begin{array}{l}\text { Investigation of effect of dropouts on statistical analyses as } \\
\text { described by applicant appears adequate. }\end{array}$ & & & \\
\hline
\end{tabular}




\section{Brief summary of controlled clinical trials}

The following table contains information on the relevant trials contained in the submission.

\begin{tabular}{|c|c|c|c|c|}
\hline $\begin{array}{l}\text { Study } \\
\text { number }\end{array}$ & Design & $\begin{array}{l}\text { Treatment } \\
\text { arms/Sample size }\end{array}$ & Target Population & $\begin{array}{l}\text { Duration of } \\
\text { Short/Long- } \\
\text { Term/Total }\end{array}$ \\
\hline $\begin{array}{l}\text { MB102008 } \\
\text { (Phase 2b) }\end{array}$ & $\begin{array}{l}\text { Randomized, } \\
7 \text { arm study }\end{array}$ & \begin{tabular}{lc} 
Dapa 2.5mg & 59 \\
Dapa 5 mg & 58 \\
Dapa 10 mg & 47 \\
Dapa 20 mg & 59 \\
Dapa 50 mg & 56 \\
Placebo & 54 \\
Metformin & 56 \\
\multicolumn{2}{c}{389 total } \\
\end{tabular} & $\begin{array}{l}\text { Drug naïve subjects } \\
(\mathrm{HbA1c} \geq 7.0 \% \text { and } \leq \\
10.0 \%)\end{array}$ & 12/0/12 weeks \\
\hline $\begin{array}{l}\text { MB102009 } \\
\text { (Phase 2b) }\end{array}$ & $\begin{array}{l}\text { two cohort } \\
\text { study, } 4 \\
\text { groups }\end{array}$ & $\begin{array}{ll}\text { Arm + insulin } & \\
\text { Dapa 10mg } & 24 \\
\text { Dapa 20mg } & 24 \\
\text { Single blind } & \\
\text { Dapa 20mg } & 4 \\
\text { Placebo } & 23 \\
\end{array}$ & $\begin{array}{l}\text { T2DM treated with insulin } \\
\text { and metformin and/or } \\
\text { TZD } \\
\text { (HbA1c } \geq 7.0 \% \text { and } \leq \\
10.0 \%)\end{array}$ & 12/0/12 weeks \\
\hline $\begin{array}{l}\text { MB102013 } \\
\text { (Phase 3) }\end{array}$ & $\begin{array}{l}\text { Randomized } \\
9 \text { arm study } \\
\text { (stratified on } \\
\text { HbA1c } \\
\text { level) }\end{array}$ & $\begin{array}{lr}\text { QAM group } & \\
\text { Dapa 2.5mg } & 65 \\
\text { Dapa 5mg } & 64 \\
\text { Dapa 5mg Grp2 } & 34 \\
\text { Dapa 10mg } & 70 \\
\text { Dapa 10mg Grp2 } 39 \\
\\
\text { QPM group } \\
\text { Dapa 2.5mg } & 67 \\
\text { Dapa 5mg } & 68 \\
\text { Dapa 10mg } & 76 \\
& \\
\text { Placebo } & 75 \\
\end{array}$ & $\begin{array}{l}\text { Drug naïve or minimally } \\
\text { treated subjects } \\
\text { (HbA1c } \geq 7.0 \% \text { and } \leq \\
12.0 \% \text { ) }\end{array}$ & 24/78/102 weeks \\
\hline $\begin{array}{l}\text { MB102014 } \\
\text { (Phase 3) }\end{array}$ & $\begin{array}{l}\text { Randomized } \\
4 \text { arm study }\end{array}$ & $\begin{array}{ll}\text { Arm + metformin } \\
\text { Dapa 2.5mg } & 137 \\
\text { Dapa 5mg } & 137 \\
\text { Dapa 10mg } & 135 \\
\text { Placebo } & 137 \\
\end{array}$ & $\begin{array}{l}\text { Metformin failure } \\
\text { (HbA1c } \geq 7.0 \% \text { and } \leq \\
10.0 \% \text { ) }\end{array}$ & 24/78/102 weeks \\
\hline $\begin{array}{l}\text { MB102021 } \\
\text { (Phase 3) }\end{array}$ & $\begin{array}{l}\text { Randomized } \\
3 \text { arm study }\end{array}$ & $\begin{array}{lc}\text { Dapa 5mg } & 203 \\
\text { Dapa 5mg + } & \\
\text { metformin } & 194 \\
\text { metformin } & 201 \\
\end{array}$ & $\begin{array}{l}\text { Drug naïve or minimally } \\
\text { treated subjects } \\
\text { (HbA1c } \geq 7.5 \% \text { and } \leq \\
12.0 \%)\end{array}$ & 24/0/24 weeks \\
\hline $\begin{array}{l}\text { MB102022 } \\
\text { (D169C00004) } \\
\text { (Phase 3) }\end{array}$ & $\begin{array}{l}\text { Randomized } \\
2 \text { arm study }\end{array}$ & $\begin{array}{l}\text { Dapa } \\
2.5-5-10 \text { mg } 406 \\
\text { Glipizide } \\
\text { 5-10-20 mg } 408 \\
\end{array}$ & $\begin{array}{l}\text { Metformin failure } \\
\text { (HbA1c } \geq 6.5 \% \text { and } \leq \\
10.0 \%)\end{array}$ & $\begin{array}{l}\text { 52/156/208 } \\
\text { weeks }\end{array}$ \\
\hline $\begin{array}{l}\text { MB102028 } \\
\text { (D1690C00005) } \\
\text { (Phase 3) }\end{array}$ & $\begin{array}{l}\text { Randomized } \\
4 \text { arm study }\end{array}$ & $\begin{array}{lc}\text { Arm + Glimepiride } \\
\text { Dapa 2.5mg } & 154 \\
\text { Dapa 5mg } & 145 \\
\text { Dapa 10mg } & 151 \\
\text { Placebo } & 146 \\
\end{array}$ & $\begin{array}{l}\text { Subjects on SU } \\
(\mathrm{HbA1c} \geq 7.0 \% \text { and } \leq \\
10.0 \%)\end{array}$ & 24/24/48 weeks \\
\hline
\end{tabular}


STATISTICS FILING CHECKLIST FOR A NEW NDA/BLA

\begin{tabular}{|c|c|c|c|c|c|}
\hline $\begin{array}{l}\text { MB102029 } \\
\text { (Phase 2b/3) }\end{array}$ & $\begin{array}{l}\text { Randomized } \\
3 \text { arm study }\end{array}$ & $\begin{array}{l}\text { Dapa 5mg } \\
\text { Dapa 10mg } \\
\text { Placebo }\end{array}$ & $\begin{array}{l}83 \\
85 \\
84\end{array}$ & $\begin{array}{l}\text { Moderate renal } \\
\text { impairment, stratified by } \\
\text { type of ongoing diabetes } \\
\text { therapy } \\
\text { (HbA1c } \geq 7.0 \% \text { and } \leq \\
11.0 \% \text { ) }\end{array}$ & 24/28/52weeks \\
\hline $\begin{array}{l}\text { MB102030 } \\
\text { (Phase 3) }\end{array}$ & $\begin{array}{l}\text { Randomized } \\
3 \text { arm study }\end{array}$ & $\begin{array}{l}\text { Dapa }+ \text { pio } \geq 3 \\
\text { Dapa 5mg } \\
\text { Dapa 10mg } \\
\text { Placebo }\end{array}$ & $\begin{array}{c}30 \mathrm{mg} \\
140 \\
141 \\
139\end{array}$ & $\begin{array}{l}\text { TZD failure } \\
(\mathrm{HbA1c} \geq 7.0 \% \text { and } \leq \\
10.5 \%)\end{array}$ & 24/24/48 weeks \\
\hline $\begin{array}{l}\text { MB102032 } \\
\text { (Phase 3) }\end{array}$ & $\begin{array}{l}\text { Randomized } \\
4 \text { arm study }\end{array}$ & $\begin{array}{l}\text { Dapa 1mg } \\
\text { Dapa 2.5mg } \\
\text { Dapa 5mg } \\
\text { Placebo }\end{array}$ & $\begin{array}{l}72 \\
74 \\
68 \\
68\end{array}$ & $\begin{array}{l}\text { Drug naïve or minimally } \\
\text { treated subjects } \\
\text { (HbA1c } \geq 7.0 \% \text { and } \leq \\
10.0 \% \text { ) }\end{array}$ & 24/0/24 weeks \\
\hline $\begin{array}{l}\text { MB102033 } \\
\text { (D1690C00006) } \\
\text { (Phase 3)) }\end{array}$ & $\begin{array}{l}\text { Randomized } \\
4 \text { arm study }\end{array}$ & $\begin{array}{l}\text { Arm + Insulin } \\
\text { IU/day } \\
\text { Dapa } 2.5 \mathrm{mg} \\
\text { Dapa 5mg } \\
\text { Dapa 10mg } \\
\text { Placebo }\end{array}$ & $\begin{array}{l}\geq 30 \\
202 \\
212 \\
196 \\
197\end{array}$ & $\begin{array}{l}\text { Subjects taking injectable } \\
\text { insulin }+/ \text { - other oral anti- } \\
\text { hyperglycemic agent } \\
\text { (HbA1c } \geq 7.5 \% \text { and } \leq \\
10.5 \% \text { ) }\end{array}$ & 24/80/104 weeks \\
\hline $\begin{array}{l}\text { MB102034 } \\
\text { (Phase 3) }\end{array}$ & $\begin{array}{l}\text { Drug naïve } \\
\text { or minimally } \\
\text { treated } \\
\text { subjects }\end{array}$ & $\begin{array}{l}\text { Dapa 10mg } \\
\text { Dapa 10mg + } \\
\text { metformin } \\
\text { Metformin }\end{array}$ & $\begin{array}{l}219 \\
211 \\
208 \\
\end{array}$ & $\begin{array}{l}\text { Drug naïve or minimally } \\
\text { treated subjects } \\
\text { (HbA1c } \geq 7.5 \% \text { and } \leq \\
12.0 \%)\end{array}$ & 24/0/24 weeks \\
\hline $\begin{array}{l}\text { MB102047 } \\
\text { (D1690C00012) } \\
\text { (Phase 3) }\end{array}$ & $\begin{array}{l}\text { Randomized } \\
2 \text { arm study }\end{array}$ & $\begin{array}{l}\text { Dapa } 10 \mathrm{mg}+ \\
\text { metformin }>15 \\
91 \\
\text { Placebo + met } \\
>1500 \mathrm{mg}\end{array}$ & $\begin{array}{l}500 \mathrm{mg} \\
\text { tormin } \\
91\end{array}$ & $\begin{array}{l}\text { Metformin failure } \\
\text { (HbA1c } \geq 6.5 \% \text { and } \leq \\
8.5 \%)\end{array}$ & 24/78/102 weeks \\
\hline $\begin{array}{l}\text { MB102064 } \\
\text { (D1692C00005) } \\
\text { (Phase 2b) }\end{array}$ & $\begin{array}{l}\text { Randomized } \\
5 \text { arm study }\end{array}$ & $\begin{array}{l}\text { Dapa 1mg } \\
\text { Dapa 2.5mg } \\
\text { Dapa 5mg } \\
\text { Dapa 10mg } \\
\text { Placebo } \\
\end{array}$ & $\begin{array}{l}59 \\
56 \\
58 \\
52 \\
54 \\
\end{array}$ & $\begin{array}{l}\text { Japanese subjects with } \\
\text { inadequate control on diet } \\
\text { and exercise } \\
\text { (HbA1c } \geq 7.0 \% \text { and } \leq \\
10.0 \% \text { ) }\end{array}$ & 12/0/12 weeks \\
\hline
\end{tabular}

Anita Abraham, Dr.P.H..

$02 / 14 / 2011$

Reviewing Statistician

Date

Mat Soukup, Ph.D.

$02 / 14 / 2011$

Supervisor/Team Leader

Date 
This is a representation of an electronic record that was signed electronically and this page is the manifestation of the electronic signature.

/s/

ANITA A ABRAHAM

$02 / 16 / 2011$

MATTHEW J SOUKUP

02/16/2011

Concur 